\title{
A Step-Indexed Model of Substructural State
}

\section{Citation}

Ahmed, Amal, Matthew Fluet, and Greg Morrisett. 2005. A Step-Indexed Model of Substructural State. Harvard Computer Science Group Technical Report TR-16-05.

\section{Permanent link}

http://nrs.harvard.edu/urn-3:HUL.InstRepos:25620500

\section{Terms of Use}

This article was downloaded from Harvard University's DASH repository, and is made available under the terms and conditions applicable to Other Posted Material, as set forth at http:// nrs.harvard.edu/urn-3:HUL.InstRepos:dash.current.terms-of-use\#LAA

\section{Share Your Story}

The Harvard community has made this article openly available.

Please share how this access benefits you. Submit a story.

Accessibility 


\title{
A Step-Indexed Model of Substructural State
}

\author{
Amal Ahmed \\ Matthew Fluet
}

Greg Morrisett

TR-16-05

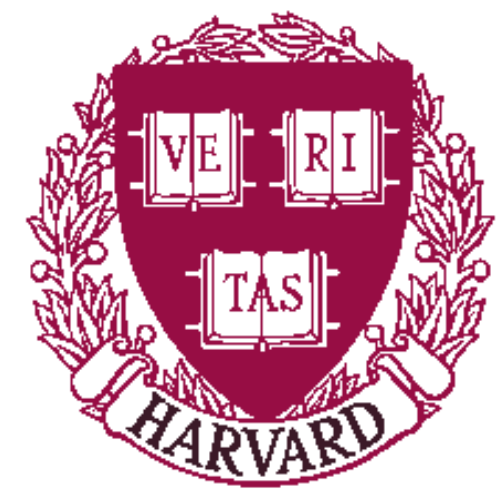

Computer Science Group

Harvard University

Cambridge, Massachusetts 


\title{
A Step-Indexed Model of Substructural State
}

\author{
Amal Ahmed \\ Harvard University \\ amal@eecs.harvard.edu
}

\author{
Matthew Fluet* \\ Cornell University \\ fluet@cs.cornell.edu
}

\author{
Greg Morrisett* \\ Harvard University \\ greg@eecs.harvard.edu
}

\begin{abstract}
The concept of a "unique" object arises in many emerging programming languages such as Clean, CQual, Cyclone, TAL, and Vault. In each of these systems, unique objects make it possible to perform operations that would otherwise be prohibited (e.g., deallocating an object) or to ensure that some obligation will be met (e.g., an opened file will be closed). However, different languages provide different interpretations of "uniqueness" and have different rules regarding how unique objects interact with the rest of the language.

Our goal is to establish a common model that supports each of these languages, by allowing us to encode and study the interactions of the different forms of uniqueness. The model we provide is based on a substructural variant of the polymorphic $\lambda$-calculus, augmented with four kinds of mutable references: unrestricted, relevant, affine, and linear. The language has a natural operational semantics that supports deallocation of references, strong (type-varying) updates, and storage of unique objects in shared references. We establish the strong soundness of the type system by constructing a novel, semantic interpretation of the types.

This technical report is really two documents in one: The first part is a paper appearing in the Tenth ACM SIGPLAN International Conference on Functional Programming (ICFP'05). The second part is a formal development of the language, step-indexed model, and soundness proof referenced in the first part. If you have already read a version of "A Step-Indexed Model of Substructural State", then you should proceed directly to the appendices.
\end{abstract}

\section{Introduction}

Consider the following imperative code fragment, written with SML syntax:

1. fun $f(r 1:$ int ref, $r 2$ : int $r e f):$ int $=$

2. $(r 1:=$ true ;

3. $\quad ! \mathrm{r} 2+42)$

At line 1, we assume ref cells $r 1$ and $r 2$ whose contents are integers. At line 2, we update the first cell with a boolean. Then, at line 3, we read the second cell, using the contents in a context expecting an integer. If the function is called with actual arguments that are different ref cells, then there is nothing in the function that will cause a run-time type error. ${ }^{1}$ Yet, if the same ref cell is passed for each formal argument, then the update on line 2 will change the contents of both $r 1$ and r2, causing a run-time type error to occur at line 3 .

SML (and most imperative languages) reject the above program, because references are unrestricted, that is, they may be freely aliased. In general, reasoning about unrestricted references is hard because we need additional information to understand what other values are affected by an update. In the absence of this information, we must be conservative. For instance, in SML, we must assume that an update to an int ref could affect any other int ref. To ensure type soundness, we must therefore require the type of the ref's contents be preserved by the update. In other words, most type systems can only track invariants on refs, instead of program-point-specific properties. As a result, we are forced to weaken the type of the ref to cover all possible program points. In the example above, we must weaken $r 1$ 's type to "(int + bool) ref" and pay the costs of tagging values, and checking those tags when the pointer is dereferenced.

Unfortunately, in many settings, this weakened invariant is insufficient. Hence, researchers have turned to more powerful systems that do provide a means of ensuring exclusive access to state. In particular, many projects have introduced some form of linearity to "tame" state. Linear logic [15] and other substructural logics give rise to more expressive type systems, because they are designed to precisely account for resources.

\footnotetext{
* This material is based upon work supported by the Air Force Office of Scientific Research under Award No. F49620-03-1-0156 and Award No. F4962001-1-0298 and by the Office of Naval Research under Award No. N00014-01-1-0968. Any opinions, findings, and conclusions or recommendations expressed in this publication are those of the author and do not necessarily reflect the views of these organizations or the U.S. Government.

${ }^{1}$ We assume that values are represented uniformly so that, for instance, unit, booleans, and integers all take up one word of storage.
} 
For instance, the Clean programming language [26] relies upon a form of uniqueness to ensure equational reasoning in the presence of mutable data structures. The Cyclone programming language [17] uses unique pointers to allow finegrained memory management. For example, a unique pointer may be updated from uninitialized to initialized, and its contents may also be deallocated:

1. $\mathrm{x}=\operatorname{malloc}(4) ; / / \mathrm{x}:---*^{\prime} \mathrm{U}$

2. $* \mathrm{x}=3 ; \quad / / \mathrm{x}$ : int $*^{\prime} \mathrm{U}$

3. free $(x) ; \quad / / x$ : undefined

In both of these languages, a unique object may be implicitly discarded, yielding a weak form of uniqueness called affinity.

The Vault programming language [13] uses tracked keys to enforce resource management protocols. For example, the following interface specifies that opening a file returns a new tracked key, which must be present when reading the file, and which is consumed when closing the file:

1. interface IO \{

2. type FILE;

3. tracked $(\$ F)$ FILE open(string) $[+\$ F]$;

4. char read (tracked $(\$ F)$ FILE) [ $\$ F]$;

5. void close (tracked $(\$ F)$ FILE) $[-\$ F] ;\}$

Because tracked keys may be neither duplicated nor discarded, Vault supports a strong form of uniqueness technically termed linearity, which ensures that an opened file must be closed exactly once. Other projects [32, 12] have also incorporated linearity to ensure that memory is reclaimed.

Both forms of uniqueness (linearity and affinity) support strong updates, whereby the type of a stateful object is changed in response to stateful operations. For example, the Cyclone code fragment above demonstrates the type of the unique pointer changing from uninitialized to initialized (with an integer) in response to the assignment. The intuitive understanding is that a unique object cannot be duplicated, and thus there are no aliases to the object; hence, no other portion of the program may observe the change in the object's type, so it is safe to perform a strong update.

Yet, programming in a language with only unique (i.e., linear or affine) objects is much too painful. In such a setting, one can only construct tree-like data structures. Hence, it is not surprising that both Cyclone and Vault allow a programmer to put unique objects in shared objects, with a variety of restrictions to ensure that these mixed objects behave in a safe manner. In fact, understanding the various mechanisms by which unique objects (with strong updates) may safely coexist and mix with shared objects is currently an active area of research [5], though much of it has focused on high-level programming features, often without a complete formal account.

Therefore, it is natural to study a core language with mutable references of all sorts mentioned above: linear, affine, and unrestricted. The study of substructural logics immediately suggests one more sort - relevant, which describes data that may be duplicated but not implicitly discarded. Having made these distinctions, a number of design questions arise: What does it mean to duplicate or to discard a reference? What operations may be safely performed with the different sorts of references? What combinations of sorts for a reference and its contents are safe?

A major contribution of this paper is to answer these questions, giving an integrated design of references for all of these substructural sorts (Section 3). Our design allows unique (linear and affine) values to be stored in shared (unrestricted and relevant) references, while preserving the desirable feature that resources are tracked accurately. Our language extends a core $\lambda$-calculus with a straightforward type system that provides data of each of the substructural sorts mentioned above (Section 2). The key idea, present in other substructural type systems, is to break out the substructural sorts as type "qualifiers." Rather than prove soundness via a syntactic subject-reduction proof, we adopt an approach compatible with that used in Foundational Proof Carrying Code [6, 7]. We construct a step-indexed model (Section 4) where types are interpreted as sets of store description / value pairs, which are further refined using an index representing the number of steps available for future evaluation. We believe this model improves on previous models of mutable state, contributing a compositional notion of aliasing and ownership that directly addresses the subtleties of allowing unique values to be stored in shared references. Furthermore, we achieve a simple model, in comparison to denotational and domain-theoretic approaches, that easily extends to impredicative polymorphism and first-class references. Constructing a (well-founded) set-theoretic model means that our soundness and safety proofs are amenable to formalization in the higher-order logic of Foundational PCC. Hence, our work provides a useful foundation for future extensions of Foundational PCC, which currently only supports unrestricted references, but is an attractive target for source languages wishing to carry high-level security guarantees, enforced by type states and linear resources, through to machine code.

\section{2. $\lambda^{\mathrm{URAL}}$ : A Substructural $\lambda$-Calculus}

Advanced type systems for state rely upon limiting the ordering and number of uses of data and operations to ensure that state is handled in a safe manner. For example, (safely) deallocating a data structure requires that the data structure is never 


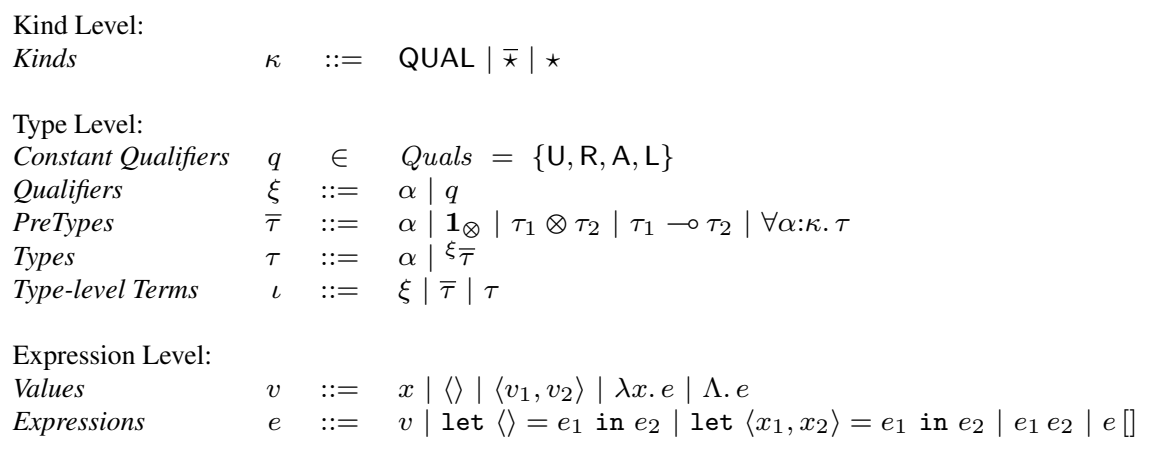

Figure 1. $\lambda^{\text {URAL }}$ Syntax

used in the future. In order to establish this property, a type system may ensure that the data structure is used at most once; after one use, the data structure may be safely deallocated, since there can be no further uses.

A substructural type system provides the core mechanisms necessary to restrict the number and order of uses of data and operations. A conventional type system, such as that employed by the simply-typed $\lambda$-calculus, with a typing judgement like $\Gamma \vdash e: \tau$, satisfies three structural properties:

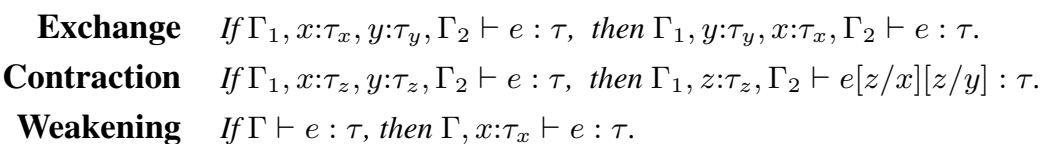

In contrast, a substructural type system is designed so that one or more of these structural properties do not hold in general. Among the most widely studied substructural type systems are the linear type systems [29, 24], derived from Girard's linear logic [15], in which all variables satisfy Exchange, but linearly typed variables satisfy neither Contraction nor Weakening.

In this section, we present a substructural polymorphic $\lambda$-calculus, similar in spirit to Walker's linear lambda calculus [30]. In our calculus, types and variables are qualified as unrestricted $(U)$, relevant $(R)$, affine $(A)$, or linear $(L)$. All variables will satisfy Exchange, while only unrestricted variables will satisfy both Contraction and Weakening, allowing such variables to be used an arbitrary number of times. We will require

- linear variables to satisfy neither Contraction nor Weakening, ensuring that such variables are used exactly once,

- affine variables to satisfy Weakening (but not Contraction), ensuring that such variables are used at most once, and

- relevant variables to satisfy Contraction (but not Weakening), ensuring that such variables are used at least once. ${ }^{2}$

The diagram below demonstrates the relationship between these qualifiers, inducing a lattice ordering $\preceq$.

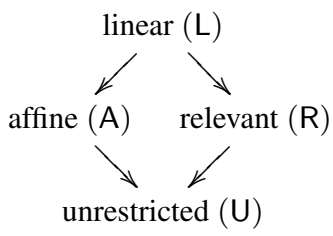

\subsection{Syntax}

Figure 1 presents the syntax for our core calculus, dubbed the $\lambda^{\mathrm{URAL}}$-calculus. Most of the types, expressions, and values are based on a traditional polymorphic $\lambda$-calculus.

Kind and Type Levels We structure our types $\tau$ as a qualifier $\xi$ applied to a pre-type $\bar{\tau}$, yielding the four sorts of types noted above. The qualifier of a type dictates the structural operations that may be applied to values of the type, while the pre-type dictates the introduction and elimination forms. The pre-types $\mathbf{1}_{\otimes}, \tau_{1} \otimes \tau_{2}$, and $\tau_{1} \multimap \tau_{2}$ correspond to the unit, pair, and function types of the polymorphic $\lambda$-calculus.

Polymorphism over qualifiers, pre-types, and types is provided by a single pre-type $\forall \alpha: \kappa$. $\tau$; we introduce a kind level

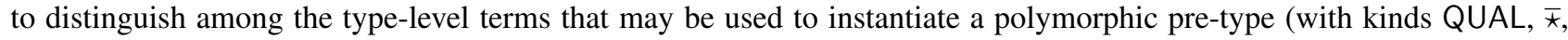
and $\star$ for qualifiers, pre-types, and types, respectively).

\footnotetext{
${ }^{2}$ In the logic community, it is perhaps more accurate to use the qualifier "strict" for such variables. However, "strict" is already an overloaded term in the functional programming community; so, like Walker [30], we use "relevant."
} 
In the appendicies, we also include additive unit $\left(\mathbf{1}_{\circledast}\right)$, additive pair $\left(\tau_{1} \circledast \tau_{2}\right)$, void $(\mathbf{0})$, sum $\left(\tau_{1} \oplus \tau_{2}\right)$, existential $(\exists \alpha: \kappa . \tau)$, and recursive $(\mu \alpha: \bar{x} . \tau)$ pre-types and recursive functions in the calculus, though we elide such constructs in this expository development.

This structuring of types as a qualifier applied to a pre-type follows that of Walker [30], but differs from other presentations of linear lambda calculi that use exactly one modality $(! \tau)$ to distinguish unrestricted from linear types. It seems possible to introduce alternative modalities (e.g, $-\tau$ for affine and $+\tau$ for relevant), but then we would have to consider their interaction (e.g., what does $-!+\tau$ denote?). Also, with four distinct qualifiers, it is natural to introduce qualfier polymorphism, which is best formulated by separating qualifiers from pre-types.

Expression Level Each pre-type has an associated value introduction form. The pattern matching expression forms let \langle\rangle$=e_{1}$ in $e_{2}$ and let $\left\langle x_{1}, x_{2}\right\rangle=e_{1}$ in $e_{2}$ are used to eliminate units $\left(\mathbf{1}_{\otimes}\right)$ and pairs $(\otimes)$, respectively. As usual, a function with pre-type $\tau_{1} \multimap \tau_{2}$ is eliminated via application $e_{1} e_{2}$, while a type-level abstraction $\forall \alpha: \kappa . \tau$ is eliminated via instantiation $e$ [].

Note that expressions are not decorated with type-level terms. This simplifies the semantic model presented in Section 4, where soundness is with respect to typing derivations, and is appropriate for an expressive "internal" language. We leave as an open problem the formulation of appropriate inference and elaboration algorithms yielding derivations in the type system of the next section, which would likely require some type-level annotations on expressions in a "surface" language.

\subsection{Static Semantics}

The goal of the type system for $\lambda^{\text {URAL }}$ is to approximate the requirements of languages like Vault and Cyclone, which ensure that linear values are used exactly once, affine values are used at most once, and relevant values are used at least once. Dually, the type system should ensure that only unrestricted and relevant values are duplicated and only unrestricted and affine values are discarded. To prevent values from being implicitly copied or dropped when their containing value is duplicated or discarded, the type system must also ensure that a (functional) value with a qualifier lower in the lattice may not contain values with qualifiers higher in the lattice. For example, an affine (A) pair may not contain linear (L) components, since we could end up dropping the linear components by dropping the pair, so the type sytem must rule out expressions of type ${ }^{\mathrm{A}}\left({ }^{\mathrm{L}} \bar{\tau}_{1} \otimes{ }^{\mathrm{L}} \bar{\tau}_{2}\right)$.

Despite these requirements, the type system is relatively simple. $\lambda^{\mathrm{URAL}}$ typing judgements have the form $\Delta ; \Gamma \vdash e: \tau$ where the contexts $\Delta$ and $\Gamma$ are defined as follows:

$$
\begin{array}{lll}
\text { Type-level Term Context } & \Delta & ::=\quad \bullet \mid \Delta, \alpha: \kappa \\
\text { Value Context } & \Gamma & ::=\quad|| \Gamma, x: \tau
\end{array}
$$

Thus, $\Delta$ is used to track the set of type-level variables in scope (along with their kinds), whereas $\Gamma$, as usual, is used to track the set of (expression-level) variables in scope (along with their types). There may be at most one occurrence of a type-level variable $\alpha$ in $\Delta$ and, similarly, at most one occurrence of a variable $x$ in $\Gamma$.

Figure 2 presents the $\lambda^{\text {URAL }}$ kinding rules and Figure 3 presents the $\lambda^{\text {URAL }}$ typing rules. In order to ensure the correct relationship between a data structure and its components, we extend the lattice ordering on constant qualifiers to types and contexts (see Figure 4). In the presence of qualifier and type polymorphism, we include the rules $\Delta \vdash \mathrm{U} \preceq \alpha$ and $\Delta \vdash \alpha \preceq \mathrm{L}$, a conservative extension, since $\mathrm{U}$ and $\mathrm{L}$ are the bottom and top of the lattice. A more general approach would incorporate bounded qualifier constraints, which we believe is straightforward, but doing so does not add to the discussion at hand.

As is usual in a substructural setting, our type system relies upon a judgement $\Delta \vdash \Gamma \leadsto \Gamma_{1} \boxplus \Gamma_{2}$ that splits the assumptions in $\Gamma$ between the contexts $\Gamma_{1}$ and $\Gamma_{2}$ (see Figure 5). Splitting the context is necessary to ensure that variables are used appropriately by sub-expressions. Note that $\boxplus$ ensures that an $A$ or $L$ assumption appears in exactly one subcontext. On the other hand, $\mathrm{U}$ and $\mathrm{R}$ assumptions may appear in both sub-contexts, corresponding to implicit duplication of the variables.

The rule (MPair) is representative: the context is split by $\boxplus$ to type each of the pair components, and the types of each component are bounded by the qualifier assigned to the pair. Intuitively, the $L$ and $A$ assumptions in the context are exclusively "owned" by exactly one of the two components. Likewise, in the rule $(\mathrm{Fn})$, the free variables of $\Gamma$, which constitute the closure of the function, must be bounded by the qualifier assigned to the function. Note that the qualifier assigned to a function type is unrelated to the types of the argument and result; rather, it is related to the abstracted components that are used when the function is executed.

The rule (Weak) splits the context into a sub-context used to type the expression $e$ and a discardable sub-context, consisting of $U$ and $A$ variables, that are not required to type the expression. Note that the rule (Weak) acts as a strengthened Weakening property, allowing an arbitrary number of $U$ and $A$ variables to be dropped at once. The corresponding strengthened Contraction property is incorporated into the judgement $\Delta \vdash \Gamma \leadsto \Gamma_{1} \boxplus \Gamma_{2}$, which allows an arbitrary number of $U$ and $R$ variables to be copied at once. 
$\Delta \vdash \iota: \kappa$
$(\operatorname{VarKn}) \frac{\alpha: \kappa \in \Delta}{\Delta \vdash \alpha: \kappa}$
(Qual) $\frac{}{\Delta \vdash q: \text { QUAL }}$
(Type) $\frac{\Delta \vdash \xi: \text { QUAL } \Delta \vdash \bar{\tau}: \bar{x}}{\Delta \vdash \xi_{\bar{\tau}}: \star}$

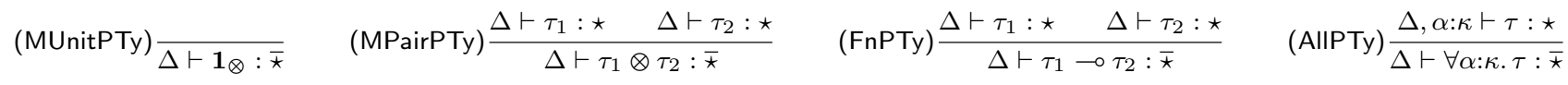

Figure 2. $\lambda^{\text {URAL }}$ Statics (Kinding Rules)

$\Delta ; \Gamma \vdash e: \tau$

$$
\begin{aligned}
& \Delta \vdash \Gamma \leadsto \Gamma_{1} \boxplus \Gamma_{2} \quad \Delta \vdash \xi: \text { QUAL } \\
& (\operatorname{Var}) \frac{\Delta \vdash \tau: \star}{\Delta ; \bullet, x: \tau \vdash x: \tau} \quad \text { (MUnit) } \frac{\Delta \vdash \xi: \text { QUAL }}{\Delta ; \bullet \vdash\langle\rangle:{ }^{\xi} \mathbf{1}_{\otimes}} \quad \text { (MPair) } \frac{\begin{array}{c}
\Delta ; \Gamma_{1} \vdash v_{1}: \tau_{1} \\
\Delta ; \Gamma_{2} \vdash v_{2}: \tau_{2}
\end{array} \quad \Delta \vdash \tau_{1} \preceq \xi}{\Delta ; \Gamma \vdash\left\langle v_{1}, v_{2}\right\rangle:{ }^{\xi}\left(\tau_{1} \otimes \tau_{2}\right)} \\
& (\mathrm{Fn}) \frac{\Delta \vdash \xi: \text { QUAL } \quad \Delta \vdash \Gamma \preceq \xi \quad \Delta ; \Gamma, x: \tau_{1} \vdash e: \tau_{2}}{\Delta ; \Gamma \vdash \lambda x . e:{ }^{\xi}\left(\tau_{1} \multimap \tau_{2}\right)} \quad \text { (All) } \frac{\Delta \vdash \xi: \text { QUAL } \quad \Delta \vdash \Gamma \preceq \xi \quad \Delta, \alpha: \kappa ; \Gamma \vdash e: \tau}{\Delta ; \Gamma \vdash \Lambda . e:{ }^{\xi} \forall \alpha: \kappa . \tau} \\
& \Delta \vdash \Gamma \leadsto \Gamma_{1} \boxplus \Gamma_{2} \\
& \text { (Let-MUnit) } \frac{\Delta ; \Gamma_{1} \vdash e_{1}:{ }^{\xi} \mathbf{1}_{\otimes} \quad \Delta ; \Gamma_{2} \vdash e_{2}: \tau}{\Delta ; \Gamma \vdash \text { let }\langle\rangle=e_{1} \text { in } e_{2}: \tau} \quad \text { (Let-MPair) } \frac{\Delta ; \Gamma_{1} \vdash e_{1}:{ }^{\xi}\left(\tau_{1} \otimes \tau_{2}\right) \quad \Delta ; \Gamma_{2}, x_{1}: \tau_{1}, x_{2}: \tau_{2} \vdash e_{2}: \tau}{\Delta ; \Gamma \vdash \operatorname{let}\left\langle x_{1}, x_{2}\right\rangle=e_{1} \text { in } e_{2}: \tau} \\
& \text { (App) } \frac{\Delta \vdash \Gamma \sim \Gamma_{1} \boxplus \Gamma_{2} \quad \Delta ; \Gamma_{1} \vdash e_{1}:{ }^{\xi}\left(\tau_{1} \multimap \tau_{2}\right) \quad \Delta ; \Gamma_{2} \vdash e_{2}: \tau_{1}}{\Delta ; \Gamma \vdash e_{1} e_{2}: \tau_{2}} \quad \text { (Inst) } \frac{\Delta ; \Gamma \vdash e: \xi_{\forall \alpha: \kappa . \tau} \Delta \vdash \iota: \kappa}{\Delta ; \Gamma \vdash e[]: \tau[\iota / \alpha]} \\
& (\text { Weak }) \frac{\Delta \vdash \Gamma \leadsto \Gamma_{1} \boxplus \Gamma_{2} \quad \Delta ; \Gamma_{1} \vdash e: \tau \quad \Delta \vdash \Gamma_{2} \preceq \mathrm{A}}{\Delta ; \Gamma \vdash e: \tau}
\end{aligned}
$$

Figure 3. $\lambda^{\text {URAL }}$ Static Semantics (Typing Rules)

$$
\begin{aligned}
& \Delta \vdash \xi_{1} \preceq \xi_{2} \\
& \frac{\Delta \vdash \alpha: \text { QUAL }}{\Delta \vdash \mathrm{U} \preceq \alpha} \quad \frac{q_{1} \preceq q_{2}}{\Delta \vdash q_{1} \preceq q_{2}} \quad \frac{\Delta \vdash \alpha: \text { QUAL }}{\Delta \vdash \alpha \preceq \mathrm{L}} \quad \frac{\Delta \vdash \xi: \text { QUAL }}{\Delta \vdash \xi \preceq \xi} \quad \frac{\Delta \vdash \xi_{1} \preceq \xi^{\prime}}{\Delta \vdash \xi_{1} \preceq \xi_{2}} \quad \frac{\Delta \vdash \xi^{\prime} \preceq \xi_{2}}{y^{2}} \\
& \Delta \vdash \tau \preceq \xi \\
& \frac{\Delta \vdash \alpha: \star}{\Delta \vdash \alpha \preceq \mathrm{L}} \quad \frac{\Delta \vdash \bar{\tau}^{\prime}: \star \quad \Delta \vdash \xi^{\prime} \preceq \xi}{\Delta \vdash^{\prime} \bar{\tau}^{\prime} \preceq \xi} \\
& \Delta \vdash \Gamma \preceq \xi \\
& \frac{\Delta \vdash \xi: \text { QUAL }}{\Delta \vdash \bullet \preceq \xi} \quad \frac{\Delta \vdash \Gamma \preceq \xi \quad \Delta \vdash \tau \preceq \xi}{\Delta \vdash \Gamma, x: \tau}
\end{aligned}
$$

Figure 4. $\lambda^{\text {URAL }}$ Statics (Sub-Qual Rules)

$\Delta \vdash \Gamma \leadsto \Gamma_{1} \boxplus \Gamma_{2}$

$\frac{\Delta \vdash \Gamma \leadsto \Gamma_{1} \boxplus \Gamma_{2} \quad \Delta \vdash \tau: \star}{\Delta \vdash \bullet \sim \bullet \boxplus \bullet} \quad \frac{\Delta \vdash \Gamma \sim \Gamma_{1} \boxplus \Gamma_{2} \quad \Delta \vdash \tau: \star}{\Delta \vdash \Gamma, x: \tau \leadsto \Gamma_{1}, x: \tau \boxplus \Gamma_{2}} \quad \frac{\Delta \vdash \Gamma, x: \tau \leadsto \Gamma_{1} \boxplus \Gamma_{2}, x: \tau}{\Delta \vdash \Gamma \sim \Gamma_{1} \boxplus \Gamma_{2} \quad \Delta \vdash \tau \preceq \mathrm{R}} \quad \frac{\Delta \vdash \Gamma, x: \tau \leadsto \Gamma_{1}, x: \tau \boxplus \Gamma_{2}, x: \tau}{\Delta \vdash \Gamma}$

Figure 5. $\lambda^{\text {URAL }}$ Statics (Context Split Rules) 


$\begin{array}{lrll} & \text { Store } s \quad:=\left\{l_{1} \mapsto v_{1}, \ldots, l_{n} \mapsto v_{n}\right\} \\ \text { (let-munit) } & (s, \text { let }\langle\rangle=\langle\rangle \text { in } e) & \longmapsto & (s, e) \\ \text { (let-mpair) } & \left(s, \text { let }\left\langle x_{1}, x_{2}\right\rangle=\left\langle v_{1}, v_{2}\right\rangle \text { in } e\right) & \longmapsto & \left(s, e\left[v_{1} / x_{1}\right]\left[v_{2} / x_{2}\right]\right) \\ (s,(\lambda x . e) v) & \longmapsto & (s, e[v / x]) \\ \text { (app) } & (s,(\Lambda . e)[]) & \longmapsto & (s, e) \\ \text { (inst) } & (s, \text { new } v) & \longmapsto & (s \uplus\{l \mapsto v\}, l) \\ \text { (new) } & (s \uplus\{l \mapsto v\}, \text { free } l) & \longmapsto & (s, v) \\ \text { (free) } & (s \uplus\{l \mapsto v\}, \text { rd } l) & \longmapsto & (s \uplus\{l \mapsto v\},\langle l, v\rangle) \\ \text { (read) } & \left(s \uplus\left\{l \mapsto v_{1}\right\}, \text { wr } l v_{2}\right) & \longmapsto & \left(s \uplus\left\{l \mapsto v_{2}\right\}, l\right) \\ \text { (write) } & \left(s \uplus\left\{l \mapsto v_{1}\right\}, \text { sw } l v_{2}\right) & \longmapsto & \left(s \uplus\left\{l \mapsto v_{2}\right\},\left\langle l, v_{1}\right\rangle\right) \\ \text { (swap) } & (s, e) \longmapsto & \left(s^{\prime}, e^{\prime}\right) \\ \text { (ctxt) } & (s, E[e]) \longmapsto & \left(s^{\prime}, E\left[e^{\prime}\right]\right)\end{array}$

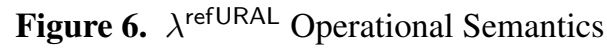

\section{3. $\lambda^{\text {refURAL }}$ : A Substructural $\lambda$-Calculus with References}

Languages like Vault and Cyclone include objects that change state (e.g., file descriptors), so it is natural to include some stateful values. We consider the difficult case of references, which can serve as mutable containers for both functional values and stateful values. Hence, we extend the $\lambda^{\mathrm{URAL}}$-calculus with mutable references, to yield the $\lambda^{\text {refURAL }}$-calculus. The reference pre-type ref $\tau$ may be combined with a qualifier $\xi$ to yield the four sorts ( $U, R, A, L)$ of references discussed earlier. We also introduce operations to allocate $\left(\right.$ new $\left._{q}\right)$ and deallocate (free) references, as well as to read (rd), write (wr), and swap (sw) their contents. Not all of these operations can be safely performed with all sorts of references, as we discuss in Section 3.2. The syntactic extensions to support references are as follows:

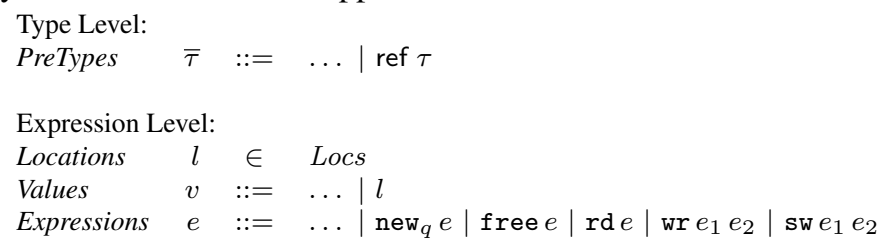

\subsection{Operational Semantics}

Figure 6 gives the small-step operational semantics for $\lambda^{\text {refURAL }}$ as a relation between configurations of the form $(s, e)$, where $s$ is a global store mapping locations to qualifiers and values. ${ }^{3}$ The notation $s_{1} \uplus s_{2}$ denotes the disjoint union of the stores $s_{1}$ and $s_{2}$; the operation is undefined if the domains of $s_{1}$ and $s_{2}$ are not disjoint. We use evaluation contexts $E$ (omitted in this presentation) to lift the primitive rewriting rules to a standard, left-to-right, innermost-to-outermost, call-by-value interpretation of the language.

Most of the rules are standard, so we highlight only those involving references. The expressions new $q$ and free $e$ perform the complementary actions of allocating and deallocating mutable references in the global store. Specifically, the expression new $q$ evaluates $e$ to a value $v$, allocates a fresh (unallocated) location $l$ to store the qualifier $q$ and value $v$, and returns $l$. The expression free $e$ performs the reverse: it evaluates $e$ to a location $l$, deallocates $l$, and returns the value previously stored at $l$.

The expressions for reading and writing a mutable reference implicitly duplicate and discard (respectively) the contents of the reference. The expression $r d e$ evaluates $e$ to a location $l$, duplicates the value $v$ stored at $l$, and returns $\langle l, v\rangle$, leaving the value stored at $l$ unchanged. Meanwhile, wr $e_{1} e_{2}$ evaluates $e_{1}$ to a location $l$ and $e_{2}$ to value $v_{2}$, stores $v_{2}$ at location $l$, discards the value previously stored at $l$, and returns $l$.

In languages with only unrestricted (ML-style) references, it is customary for $r d$ to return only the contents of $l$ and for wr to return \langle\rangle . However, we do not wish to consider reading or writing a linear (resp. affine) reference as the exactly-oneuse (resp. at-least-one-use) of the value. Therefore, the $r d$ and wr (and sw) operations return the location $l$ that was read or written, which remains available for future use. The behavior of ML-style references may be recovered by implicitly discarding the returned location.

\footnotetext{
${ }^{3}$ We write $s^{\text {qual }}(l)$ and $s^{\text {val }}(l)$ for the respective projections of $s(l)$.
} 


\begin{tabular}{|c|c|c|c|c|c|c|}
\hline \multicolumn{2}{|r|}{ Ref } & \multirow[t]{2}{*}{ Ops } & \multicolumn{4}{|c|}{ Contents and Ops } \\
\hline & & & U & $\mathrm{R}$ & A & $\mathrm{L}$ \\
\hline \multirow{2}{*}{ shared \{} & U & $\begin{array}{c}\text { newU } \\
\text { (weak updates) }\end{array}$ & $\begin{array}{l}\text { rd } \\
\text { wr } \\
\text { sw }\end{array}$ & $x$ & $\begin{array}{l}\text { wr } \\
\text { sw }\end{array}$ & $x$ \\
\hline & $\mathrm{R}$ & $\begin{array}{c}\mathrm{new}_{\mathrm{R}} \\
\text { (weak updates) }\end{array}$ & $\begin{array}{l}\text { rd } \\
\text { wr } \\
\text { Sw }\end{array}$ & $\begin{array}{l}\text { rd } \\
\text { sw }\end{array}$ & $\begin{array}{l}\text { wr } \\
\text { sw }\end{array}$ & sw \\
\hline \multirow{2}{*}{ unique \{} & A & $\begin{array}{c}\text { newA } \\
\text { free } \\
\text { (strong updates) }\end{array}$ & $\begin{array}{l}\text { rd } \\
\text { wr } \\
\text { sw }\end{array}$ & $x$ & $\begin{array}{l}\text { wr } \\
\text { sw }\end{array}$ & $x$ \\
\hline & L & $\begin{array}{c}\text { newL } \\
\text { free } \\
\text { (strong updates) }\end{array}$ & $\begin{array}{l}\text { rd } \\
\text { wr } \\
\text { sw }\end{array}$ & $\begin{array}{l}\text { rd } \\
\text { sw }\end{array}$ & $\begin{array}{l}\text { wr } \\
\text { sw }\end{array}$ & sw \\
\hline
\end{tabular}

Figure 7. Operations for Substructural State

The expression sw $e_{1} e_{2}$ combines the operations of dereferencing and updating a mutable reference, but has the attractive property that it neither duplicates nor discards a value. Notice that performing a write or swap operation on a location may change the type of the location's contents. The static semantics will permit weak (type-invariant) updates on all references (with some additional caveats), but will restrict strong (type-varying) updates to unique references.

The reader may well wonder why each reference is "stamped" with a qualifier at its allocation when the remainder of the operational rules are entirely agnostic with respect to a reference's qualifier. Essentially, the qualifier is a form of instrumentation, which, when combined with the semantic model presented in Section 4, allows us to guarantee that linear and relevant references cannot be implicitly discarded. Such a property is difficult to capture exclusively in the operational semantics (i.e., by ensuring that the abstract machine "gets stuck" when a linear or relevant reference is implicitly dropped). On the other hand, the abstract machine does "get stuck" when attempting to access a reference after it has been deallocated.

\subsection{Static Semantics}

As with the type system for $\lambda^{\text {URAL }}$, we would like the type system for $\lambda^{\text {refURAL }}$ to ensure the property that no linear or affine value is implicitly duplicated and no linear or relevant value is implicitly discarded. With that in mind — and noting that only unrestricted and relevant references may be implicitly copied (by the $\Delta \vdash \Gamma \sim \Gamma_{1} \boxplus \Gamma_{2}$ judgement), while only unrestricted and affine references may be implicitly dropped (by the (Weak) rule) - we now answer the questions we laid out in Section 1: What operations may be safely performed with the different sorts of references? What combinations of sorts for a reference and its contents are safe? These answers are summarized in Figure 7.

First, consider what it means to duplicate a reference. Operationally, a reference is a location in the global store. Therefore, duplicating an unrestricted or relevant reference $l$, simply yields two copies of $l$ - while the value stored at $l$ is not duplicated. Since duplicating a shared reference does not alter the uniqueness of its contents, it is not only reasonable but also extremely useful to allow shared references to store unique values. In particular, it permits the sharing of (large) unique data structures without expensive copying.

On the other hand, dropping an unrestricted or affine reference $l$ effectively drops its contents, since this reference may (must, in the case of affine) have been the only copy of $l$. If the contents were a linear or relevant value, then the exactlyone-use and at-least-one-use invariants (respectively) would be violated. Hence, we cannot allow linear and relevant values (which cannot be discarded) to be stored in unrestricted or affine references (which can be discarded).

Considering yet another axis, we note that linear and affine references must be unique. Hence, we can free unique references, and also perform strong updates on them. Shared references, on the other hand, can never be deallocated and can only support weak updates.

As we noted above, the rd operator induces an implicit copy while the wr operator induces an implicit drop. Therefore, whether we can read from or write to a reference depends entirely on the qualifier of its contents: rd is permitted if the contents are unrestricted or relevant (i.e., duplicable), wr is permitted if the contents are unrestricted or affine (i.e., discardable). The operation sw is permitted on any sort of reference, regardless of the qualifier of its contents. As noted above, strong writes and strong swaps, which change the type of the contents of the location, are only permitted on unique references. 


$$
(\operatorname{RefPTy}) \frac{\Delta \vdash \tau: \star}{\Delta \vdash \operatorname{ref} \tau: \star}
$$

$\Delta ; \Gamma \vdash e: \tau$

$$
\begin{aligned}
& (\operatorname{New}(\mathrm{U}, \mathrm{A})) \frac{q \preceq \mathrm{A} \quad \Delta ; \Gamma \vdash e: \tau \quad \Delta \vdash \tau \preceq \mathrm{A}}{\Delta ; \Gamma \vdash \operatorname{new}_{q} e:{ }^{q} \operatorname{ref} \tau} \\
& (\operatorname{New}(\mathrm{R}, \mathrm{L})) \frac{\mathrm{R} \preceq q \quad \Delta ; \Gamma \vdash e: \tau}{\Delta ; \Gamma \vdash \text { new }_{q} e:{ }^{q} \operatorname{ref} \tau} \\
& \text { (Free) } \frac{\Delta ; \Gamma \vdash e:{ }^{\xi} \text { ref } \tau \quad \Delta \vdash \mathrm{A} \preceq \xi}{\Delta ; \Gamma \vdash \text { free } e: \tau} \\
& \text { (Read) } \frac{\Delta ; \Gamma \vdash e:{ }^{\xi} \operatorname{ref} \tau \quad \Delta \vdash \tau \preceq \mathrm{R}}{\Delta ; \Gamma \vdash \operatorname{rd} e:{ }^{\mathrm{L}}\left({ }^{\xi} \text { ref } \tau \otimes \tau\right)} \\
& \begin{array}{c}
\Delta \vdash \Gamma \leadsto \Gamma_{1} \boxplus \Gamma_{2} \\
(\text { Write(Strong) }) \frac{\begin{array}{c}
\Delta ; \Gamma_{1} \vdash e_{1}:{ }^{\xi} \operatorname{ref} \tau_{1} \quad \Delta \vdash \tau_{1} \preceq \mathrm{A} \quad \Delta \vdash \mathrm{A} \preceq \xi \\
\Delta ; \Gamma_{2} \vdash e_{2}: \tau_{2} \quad \Delta \vdash \tau_{2} \preceq \xi
\end{array}}{\Delta ; \Gamma \vdash \operatorname{wr} e_{1} e_{2}:{ }^{\xi} \operatorname{ref} \tau_{2}}
\end{array} \\
& \Delta \vdash \Gamma \sim \Gamma_{1} \boxplus \Gamma_{2} \\
& \Delta ; \Gamma_{1} \vdash e_{1}:{ }^{\xi} \text { ref } \tau_{1} \quad \Delta \vdash \mathrm{A} \preceq \xi \\
& \text { (Swap(Strong)) } \frac{\Delta ; \Gamma_{2} \vdash e_{2}: \tau_{2} \quad \Delta \vdash \tau_{2} \preceq \xi}{\Delta ; \Gamma \vdash \mathrm{sw} e_{1} e_{2}:{ }^{\mathrm{L}}\left({ }^{\xi} \text { ref } \tau_{2} \otimes \tau_{1}\right)} \\
& \begin{array}{c}
\Delta \vdash \Gamma \sim \Gamma_{1} \boxplus \Gamma_{2} \\
\Delta ; \Gamma_{1} \vdash e_{1}: \xi_{\text {ref }} \tau \quad \Delta \vdash \tau \preceq \mathrm{A} \\
\Delta ; \Gamma_{2} \vdash e_{2}: \tau
\end{array} \\
& \Delta \vdash \Gamma \leadsto \Gamma_{1} \boxplus \Gamma_{2} \\
& \Delta ; \Gamma_{1} \vdash e_{1}:{ }^{\xi_{\text {ref }} \tau} \\
& (\operatorname{Swap}(\text { Weak })) \frac{\Delta ; \Gamma_{2} \vdash e_{2}: \tau}{\Delta ; \Gamma \vdash \mathrm{sw} e_{1} e_{2}:{ }^{\mathrm{L}}\left({ }^{\xi} \text { ref } \tau \otimes \tau\right)}
\end{aligned}
$$

Figure 8. $\lambda^{\text {refURAL }}$ Static Semantics (Kinding and Typing Rules)

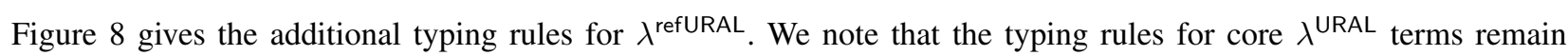
unchanged. There is no rule for locations, as locations are not allowed in the external language. Also note that the (New) and (Free) rules act as the introduction and elimination rules for ${ }^{\xi}$ ref $\tau$ types, while the (Read), (Write), and (Swap) rules maintain an exactly-one-use invariant on references by consuming a value of type ${ }^{\xi}$ ref $\tau_{1}$ and by producing a value of type $\xi_{\text {ref }} \tau_{2}$ (possibly with $\tau_{1}=\tau_{2}$ ).

Finally, we note that wr may be encoded using an explicit sw and an implicit drop: ${ }^{4}$

$$
\begin{aligned}
& \Delta \vdash \Gamma \leadsto \Gamma_{1} \boxplus \Gamma_{2}
\end{aligned}
$$

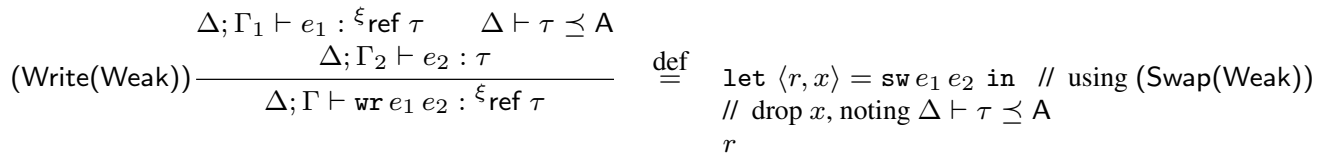

However, rd may not be encoded using an explicit sw and an implicit copy, as a suitable (discardable) dummy value cannot in general be synthesized.

$$
\begin{aligned}
\text { (Read) } \frac{\Delta ; \Gamma \vdash e:{ }^{\xi} \operatorname{ref} \tau \quad \Delta \vdash \tau \preceq \mathrm{R}}{\Delta ; \Gamma \vdash \operatorname{rd} e:{ }^{\mathrm{L}}\left({ }^{\xi} \operatorname{ref} \tau \otimes \tau\right)} \stackrel{\text { def }}{=} & \text { let }\langle r, x\rangle=\operatorname{sw} e ? \text { in } / / \text { where } \Delta ; \Gamma \vdash ?: \tau \\
& / / \operatorname{copy} x, \text { noting } \Delta \vdash \tau \preceq \mathrm{R} \\
& \text { let }\langle r, y\rangle=\operatorname{sw} r x \text { in } / / \text { using (Swap(Weak)) } \\
& / / \text { drop } y, \text { but not necessarily } \Delta \vdash \tau \preceq \mathrm{A} \\
& \langle r, x\rangle
\end{aligned}
$$

\section{A Step-Indexed Model}

We prove the type soundness of $\lambda^{\text {refURAL }}$ in a manner similar to that employed by Appel's Foundational PCC project [6]. The technique uses syntactic logical relations (that is, relations based on the operational semantics) where relations are further refined by an index that, intuitively, records the number of steps available for future evaluation. This stratification is essential for modeling the recursive functions (available via backpatching unrestricted references) and impredicative polymorphism present in the language.

\subsection{Background: A Model of Unrestricted References}

Our model is based on the indexed model of ML-style references by Ahmed, Appel, and Virga [1, 4], henceforth AAV. In their model, the semantic interpretation $\mathcal{T} \llbracket \tau \rrbracket$ of a (closed) type $\tau$ is a set of triples of the form $(k, \Psi, v)$, where, $k$ is a natural number (called the approximation index or step index), $\Psi$ is a (global) store typing that maps locations to (the interpretation of) their designated types, and $v$ is a (closed) value. Intuitively, $(k, \Psi, v) \in \mathcal{T} \llbracket \tau \rrbracket$ says that in any

\footnotetext{
${ }^{4}$ The encoding of a wr typed by the (Write(Strong)) rule makes use of the same term, but an alternate typing derivation.
} 


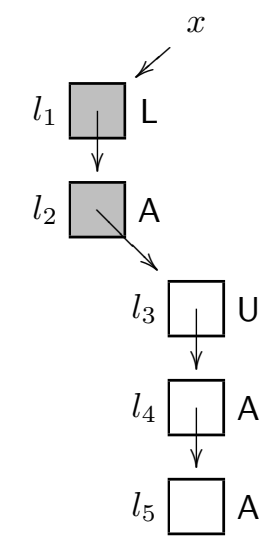

(a) $\left(k, \Psi, \Omega_{1}, x\right) \in \mathcal{T} \llbracket \tau_{1} \rrbracket$

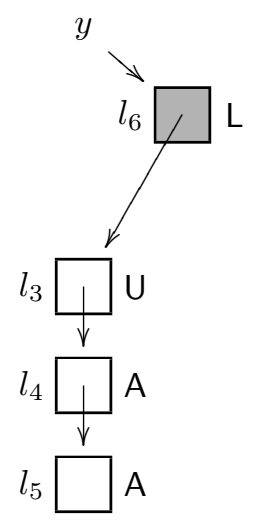

(b) $\left(k, \Psi, \Omega_{2}, y\right) \in \mathcal{T} \llbracket \tau_{2} \rrbracket$

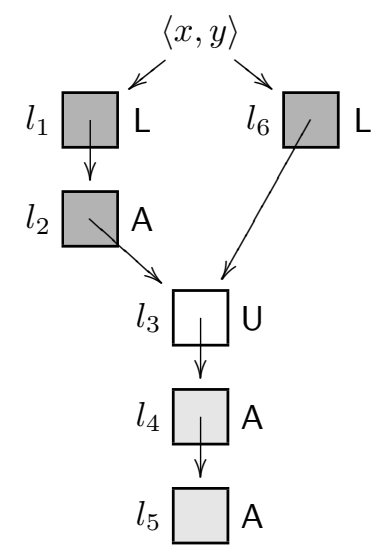

(c) Problem: $\Omega_{1} \uplus \Omega_{2}=$ undefined

Figure 9. Unique References in Shared References: Aliased or Owned?

computation running for no more than $k$ steps, $v$ cannot be distinguished from values of type $\tau$. Furthermore, since dereferencing a location consumes an execution step, in order to determine whether $v$ has type $\tau$ for $k$ steps it suffices to know the type of each store location for $k-1$ steps; hence, $\Psi$ need only specify each location's type to approximation $k-1$. We use a similar indexing approach which is key to ensuring that our model is well-founded (as we shall demonstrate in Section 4.3).

\subsection{Towards a Model of $\lambda^{\text {refURAL }}$}

Aliasing and Ownership Though our model is similar to AAV, the presence of shared and unique references places very different demands on the model, which we illustrate by considering the interpretation of product types in various settings. In a language with only unrestricted references (e.g. AAV), one would say $\left(k, \Psi,\left\langle v_{1}, v_{2}\right\rangle\right) \in \mathcal{T} \llbracket \tau_{1} \otimes \tau_{2} \rrbracket$ if and only if $\left(k, \Psi, v_{1}\right) \in \mathcal{T} \llbracket \tau_{1} \rrbracket$ and $\left(k, \Psi, v_{2}\right) \in \mathcal{T} \llbracket \tau_{2} \rrbracket$, where the store typing $\Psi$ describes every location allocated by the program thus far. In this setting, every location (in $\Psi$ ) may be aliased; hence, the model allows $v_{1}$ and $v_{2}$ to point to data structures that overlap in the heap.

In a language with only linear references [23, 2], however, one must ensure that the set of (linear) locations reachable from $v_{1}$ is disjoint from the set of locations reachable from $v_{2}$. This mirrors the fact that we can only construct tree-like data structures in this setting. Furthermore, it guarantees the safety of strong updates by providing a notion of exclusive ownership. Hence, to model a language with only linear references, it is useful to replace the global store description $\Psi$ with a description of only the accessible (reachable) locations in the store, say $\Omega$. Intuitively, when we write $(k, \Omega, v) \in \mathcal{T} \llbracket \tau \rrbracket$, we intend for $\Omega$ to describe only the subset of store locations that are accessible from, and hence, "owned" by $v$. Thus, one would say $\left(k, \Omega,\left\langle v_{1}, v_{2}\right\rangle\right) \in \mathcal{T} \llbracket \tau_{1} \otimes \tau_{2} \rrbracket$ if and only if $\left(k, \Omega_{1}, v_{1}\right) \in \mathcal{T} \llbracket \tau_{1} \rrbracket$ and $\left(k, \Omega_{2}, v_{2}\right) \in \mathcal{T} \llbracket \tau_{2} \rrbracket$, where the $\Omega$ is the disjoint union of $\Omega_{1}$ and $\Omega_{2}$.

For the $\lambda^{\text {refURAL }}$-calculus, we tried to build a model that supports both aliasing and ownership as follows. We defined the semantic interpretation of a type $\mathcal{T} \llbracket \tau \rrbracket$ as the set of tuples of the form $(k, \Psi, \Omega, v)$ where $\Psi$ describes every $\mathrm{U}$ and $\mathrm{R}$ location allocated by the program and $\Omega$ describes only those $\mathrm{A}$ and $\mathrm{L}$ locations that are reachable from (and owned by) $v$. The interpretation of $\tau_{1} \otimes \tau_{2}$ then naturally yields: $\left(k, \Psi, \Omega,\left\langle v_{1}, v_{2}\right\rangle\right) \in \mathcal{T} \llbracket \tau_{1} \otimes \tau_{2} \rrbracket$ if and only if $\left(k, \Psi, \Omega_{1}, v_{1}\right) \in \mathcal{T} \llbracket \tau_{1} \rrbracket$ and $\left(k, \Psi, \Omega_{2}, v_{2}\right) \in \mathcal{T} \llbracket \tau_{2} \rrbracket$, where the $\Omega$ is the disjoint union of $\Omega_{1}$ and $\Omega_{2}$.

Unfortunately, the above model did not suffice for $\lambda^{\text {refURAL }}$, since it assumes that every unique location reachable from $v$ is exclusively owned by $v$, which is not the case when unique references may be stored in shared references.

Unique References in Shared References: Aliased or Owned? Consider the situation depicted in Figure 9(a) where $x$ maps to $l_{1}$ and locations $l_{1}$ through $l_{5}$ are reachable from $x$. Locations "owned" by $x$ are shaded. Notice that $l_{1}$ and $l_{2}$ are unique locations owned by $x$, while $l_{4}$ and $l_{5}$ are unique locations that $x$ must consider aliased, since they can be reached (from other program subexpressions) via the unrestricted location $l_{3}$. Figure 9(b) depicts such a subexpression, $y$. Note that $y$ maps to $l_{6}$ whose contents alias $l_{3}$, making $l_{4}$ and $l_{5}$ reachable from $y$. 
In $\lambda^{\text {refURAL }}$ we may safely construct the pair $\langle x, y\rangle$ (shown in Figure 9(c)), but the interpretation of $\tau_{1} \otimes \tau_{2}$ that we proposed above prohibits such a pair since locations $l_{4}$ and $l_{5}$ occur in both $\Omega_{1}$ and $\Omega_{2}$, violating the requirement that their domains be disjoint.

To model the $\lambda^{\text {refURAL }}$-calculus, we tried to further refine our model so that the interpretation of a type $\mathcal{T} \llbracket \tau \rrbracket$ is a set of tuples of the form $(k, \Psi, \Omega, \Theta, v)$ where $\Psi$ is as before, but now $\Omega$ describes unique owned locations, (i.e., those reachable from $v$ without indirecting through a shared reference), while $\Theta$ describes unique aliased locations, (i.e., those that cannot be reached without indirecting through a shared cell). The intuition is that the interpretation of $\tau_{1} \otimes \tau_{2}$ splits $\Omega$ into disjoint pieces for each component of the pair, but allows each component to use $\Psi$ and $\Theta$ unchanged.

This proposal, however, is fraught with complications. In particular, whether a unique location belongs in $\Omega$ or $\Theta$ depends on the configuration of the entire program, rather than just the type of the location. This limits the compositionality of the model. For instance, consider $l_{5}$ in Figure 9(c). Clearly $l_{5}$ must appear in $\Theta$ as it is reachable from an unrestricted location. However, if locations $l_{1}, l_{2}, l_{3}$, and $l_{6}$ did not exist, then $l_{5}$ could appear in $\Omega$. In the next section, we propose a far simpler solution that we consider one of the main technical contributions of our work.

\subsection{A Model with Local Store Descriptions}

In our model of the $\lambda^{\text {refURAL }}$-calculus, the semantic interpretation of a type $\mathcal{T} \llbracket \tau \rrbracket$ is a set of tuples of the form $(k, q, \psi, v)$, where the local store description $\psi$ describes only a part of the global store. Intuitively, $\psi$ is the set of "beliefs" about the locations that appear as sub-expressions of the value $v$. Such locations are said to be directly accessible from the value $v$. Conversely, locations that are indirectly accessible from the value $v$ are those locations that are reachable from $v$ only by indirecting through one (or more) references. The local store description $\psi$ says nothing about these indirectly-accessible locations. This enhances the compositionality of our model, making it straightforward to combine local store descriptions with one another.

\subsubsection{Definitions}

We use the meta-variable $\chi$ to denote sets of tuples of the form $(k, q, \psi, v)$ and the meta-variable $\psi$ to denote partial maps from locations $l$ to tuples of the form $(q, \chi) .{ }^{5}$ When $\chi$ corresponds to the semantic interpretation of a type and $(k, q, \psi, v) \in \chi$, we intend that $q$ is the qualifier of $v, \psi$ is the local store description of $v$, and $v$ is a closed value. When $\psi$ corresponds to a local store description and $\psi(l)=(q, \chi)$, we intend that $q$ is the qualifier of the reference and $\chi$ is the semantic interpretation of the type of its contents.

Well-Founded \& Well-Behaved Interpretations If we attempt to naïvely construct a set-theoretic model based on these intentions, we are led to specify:

$$
\begin{aligned}
\text { Type } & =2^{\mathbb{N} \times \text { Quals } \times \text { LocalStoreDesc } \times \text { CValues }} \\
\text { LocalStoreDesc } & =\text { Locs } \rightarrow \text { Quals } \times \text { Type }
\end{aligned}
$$

However, there is a problem with this specification: a simple diagonalization argument will show that the set Type of type interpretations has an inconsistent cardinality (i.e., it's an ill-founded recursive definition).

We can eliminate the inconsistency by stratifying our definitions, making essential use of the approximation index. To simplify the development, we first construct candidate sets, which are well-founded sets of our intended form. Next, we define some useful functions and predicates on these candidate sets. Finally, we construct our semantic interpretations by filtering the candidate sets, making use of the functions and predicates defined in the previous step. Our semantic interpretations impose a number of constraints (e.g., relating the qualifier of a reference to the qualifier of its contents) that are ignored in the construction of the candidate sets.

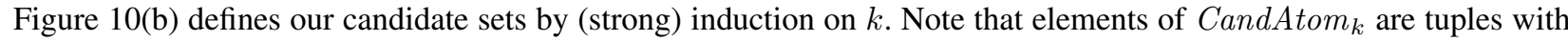
approximation index $j$ strictly less than $k$. Hence, our definitions are well-defined at $k=0$ :

$$
\begin{aligned}
\text { CandAtom }_{0} & =\emptyset \\
\text { CandUberType }_{0} & =\{\emptyset\} \\
\text { CandLocalStoreDesc }_{0} & =\text { Locs } \rightarrow \text { Quals } \times\{\emptyset\}
\end{aligned}
$$

While our candidate sets establish the existence of sets of our intended form, our semantic interpretations will need to be well-behaved in other ways. There are key constraints associated with atoms, pre-types, types, and local store descriptions that will be enforced in our final definitions. Functions and predicates supporting these constraints are given in Figure 10(c).

For any set $\chi$, we define the $k$-approximation of the set (written $\lfloor\chi\rfloor_{k}$ ) as the subset of its elements whose indices are less than $k$; we extend the notion pointwise to local store descriptions $\psi$ (written $\lfloor\psi\rfloor_{k}$ ). Note that $\lfloor\chi\rfloor_{k}$ and $\lfloor\psi\rfloor_{k}$ necessarily yield elements of CandUberType ${ }_{k}$ and CandLocalStoreDesc ${ }_{k}$.

\footnotetext{
${ }^{5}$ We write $\psi^{\text {qual }}(l)$ and $\psi^{\text {type }}(l)$ for the respective projections of $\psi(l)$.
} 
(b)

$$
\begin{aligned}
& \text { CandAtom }_{k} \stackrel{\text { def }}{=}\left\{(j, q, \psi, v) \in \mathbb{N} \times \text { Quals } \times \bigcup_{j<k} \text { CandLocalStoreDesc }_{j} \times \text { CValues } \mid\right. \\
& \left.j<k \wedge \psi \in \text { CandLocalStore Desc }_{j}\right\} \\
& \begin{array}{rll}
\text { CandUberType }_{k} & \stackrel{\text { def }}{=} & 2^{\text {CandAtom }_{k}} \\
\text { CandLocalStoreDesc }_{k} & \stackrel{\text { def }}{=} \text { Locs } \rightarrow \text { Quals } \times \text { CandUberType } \\
k
\end{array} \\
& \text { CandAtom }_{\omega} \stackrel{\text { def }}{=} \bigcup_{k \geq 0} \text { CandAtom }_{k} \\
& \text { CandUberType }_{\omega} \stackrel{\text { def }}{=} 2^{\text {CandAtom }_{\omega}} \supseteq \supseteq \bigcup_{k \geq 0} \text { CandUberType }_{k} \\
& \text { CandLocalStoreDesc } \omega \stackrel{\text { def }}{=} \text { Locs } \rightarrow \text { Quals } \times \text { CandUberType }_{\omega} \supseteq \bigcup_{k \geq 0} \text { CandLocalStoreDesc }_{k}
\end{aligned}
$$

(c)

$$
\begin{aligned}
& \begin{aligned}
\lfloor\chi\rfloor_{k} \stackrel{\text { def }}{=}\{(j, q, \psi, v) \mid j<k \wedge(j, q, \psi, v) \in \chi\} \\
\in \quad \text { CandUberType }_{\omega} \rightarrow \text { CandUberType }_{k}
\end{aligned} \\
& \lfloor\psi\rfloor_{k} \stackrel{\text { def }}{=} \quad\left\{l \mapsto\left(q,\lfloor\chi\rfloor_{k}\right) \mid l \in \operatorname{dom}(\psi) \wedge \psi(l)=(q, \chi)\right\} \\
& \in \quad \text { CandLocalStoreDesc } \omega \rightarrow \text { CandLocalStoreDesc }_{k} \\
& \mathcal{P}(q, \psi) \stackrel{\text { def }}{=} \forall l \in \operatorname{dom}(\psi) \cdot \psi^{\text {qual }}(l) \preceq q \\
& \in \quad \text { Quals } \times \text { CandLocalStoreDesc } \omega \rightarrow \mathbb{P} \\
& \begin{aligned}
\mathcal{R}(\psi) \stackrel{\text { def }}{=} & \forall l \in \operatorname{dom}(\psi) .\left(\psi^{\text {qual }}(l) \preceq \mathrm{A} \Rightarrow \forall\left({ }_{-}, q^{\prime},,_{-},{ }_{-}\right) \in \psi^{\text {type }}(l) . q^{\prime} \preceq \mathrm{A}\right) \\
\in & \text { CandLocalStoreDesc } \omega
\end{aligned}
\end{aligned}
$$

(d)

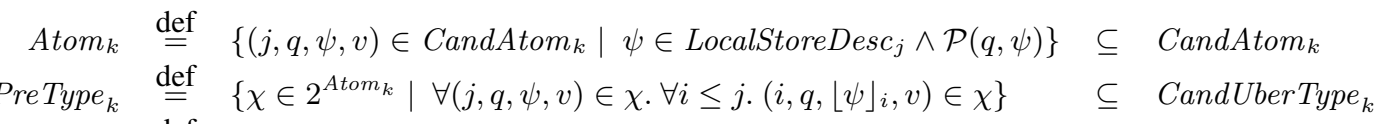

$$
\begin{aligned}
& \text { Type }_{k} \stackrel{\text { def }}{=}\left\{\chi \in \text { PreType }_{k} \mid \exists q^{\prime} \in \text { Quals. } \forall\left({ }_{-}, q,_{-},-\right) \in \chi \cdot q=q^{\prime}\right\} \quad \subseteq \quad \text { CandUberType }_{k} \\
& \text { LocalStoreDesc }_{k} \stackrel{\text { def }}{=}\left\{\psi \in \text { Locs } \rightarrow \text { Quals } \times \text { Type }_{k} \mid \mathcal{R}(\psi)\right\} \\
& \subseteq \quad \text { CandLocalStoreDesc }{ }_{k} \\
& \text { PreType } \stackrel{\text { def }}{=}\left\{\chi \in \text { CandUberType } \omega \mid \forall k \geq 0 .\lfloor\chi\rfloor_{k} \in \text { PreType }_{k}\right\} \supseteq \bigcup_{k \geq 0} \text { PreType }_{k} \\
& \text { Type } \stackrel{\text { def }}{=}\left\{\chi \in \text { CandUberType }_{\omega} \mid \forall k \geq 0 .\lfloor\chi\rfloor_{k} \in \text { Type }_{k}\right\} \quad \supseteq \bigcup_{k \geq 0} \text { Type }_{k}
\end{aligned}
$$

Figure 10. $\lambda^{\text {refURAL }}$ Model (Definitions)

Figure 10(c) defines our semantic interpretations, again by (strong) induction on $k$. Note that our semantic interpretations can be seen as filtering their corresponding candidate sets. Next, we examine each of these filtering constraints.

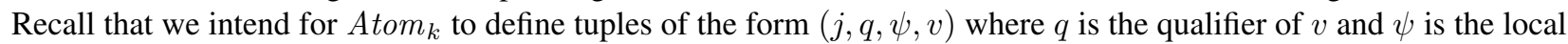
store description of $v$. Filtering CandAtom $_{k}$ by the predicate $\mathcal{P}(q, \psi)$ enforces the requirement that if $v$ is a value with qualifier $q$, then each location directly accessible from $v$ must have a qualifier $q^{\prime}$ such that $q^{\prime} \preceq q$. We further require the local store description $\psi$ to be a member of LocalStoreDesc ${ }_{j}$.

We define Pre Type $_{k}$ as those $\chi \in 2^{\text {Atom }_{k}} \subseteq$ CandUberType $_{k}$ that are closed with respect to a decreasing stepindex. We define Type $_{k}$ by further requiring that all values in $\chi$ share the same qualifier. Looking ahead, we will need to extend our semantic interpretations to a predicate $\operatorname{Comp}(k, \psi, e, \mathcal{T} \llbracket \tau \rrbracket)$, where $e$ is a (closed) expression. Intuitively, an expression $e$ that is indistinguishable from a value of type $\tau$ for $k$ steps must also be indistinguishable for $j<k$ steps. Since we will define the predicate $\operatorname{Comp}(\cdot, \cdot, \cdot, \cdot)$ on elements of Type, we incorporate this closure property into the definition of PreType . $_{\text {. }}$ 


$$
\begin{aligned}
& \mathcal{K} \llbracket \mathrm{QUAL} \rrbracket=\text { Quals } \mathcal{K} \llbracket \bar{\star} \rrbracket=\text { PreType } \mathcal{K} \llbracket \star \rrbracket=\text { Type } \\
& \mathcal{T} \llbracket \Delta \vdash \alpha: \kappa \rrbracket \delta=\delta(\alpha) \\
& \mathcal{T} \llbracket \Delta \vdash q: \mathrm{QUAL} \rrbracket \delta=q \\
& \mathcal{T} \llbracket \Delta \vdash \mathbf{1}_{\otimes}: \nexists \rrbracket \delta=\{(k, q,\{\},\langle\rangle)\} \\
& \mathcal{T} \llbracket \Delta \vdash \tau_{1} \otimes \tau_{2}: \nexists \rrbracket \delta=\left\{\left(k, q, \psi,\left\langle v_{1}, v_{2}\right\rangle\right) \mid \psi=\left(\psi_{1} \odot_{k} \psi_{2}\right) \wedge\right. \\
& \left(k, q_{1}, \psi_{1}, v_{1}\right) \in \mathcal{T} \llbracket \Delta \vdash \tau_{1}: \star \rrbracket \delta \wedge q_{1} \preceq q \wedge \\
& \left.\left(k, q_{2}, \psi_{2}, v_{2}\right) \in \mathcal{T} \llbracket \Delta \vdash \tau_{2}: \star \rrbracket \delta \wedge q_{2} \preceq q\right\} \\
& \mathcal{T} \llbracket \Delta \vdash \tau_{1} \multimap \tau_{2}: \nexists \rrbracket \delta=\left\{\left(k, q_{c}, \psi_{c}, \lambda x . e\right) \mid \psi_{c} \in \text { LocalStoreDesc }_{k} \wedge \mathcal{P}\left(q_{c}, \psi_{c}\right) \wedge\right. \\
& \forall j<k, q_{a}, \psi_{a}, v_{a} \text {. } \\
& \left(j, q_{a}, \psi_{a}, v_{a}\right) \in \mathcal{T} \llbracket \Delta \vdash \tau_{1}: \star \rrbracket \delta \wedge\left(\psi_{c} \odot_{j} \psi_{a}\right) \text { defined } \Rightarrow \\
& \left.\operatorname{Comp}\left(j,\left(\psi_{c} \odot_{j} \psi_{a}\right), e\left[v_{a} / x\right], \mathcal{T} \llbracket \Delta \vdash \tau_{2}: \star \rrbracket \delta\right)\right\} \\
& \mathcal{T} \llbracket \Delta \vdash \forall \alpha: \kappa . \tau: \nexists \rrbracket \delta=\left\{(k, q, \psi, \Lambda . e) \mid \psi \in \text { LocalStoreDesc }_{k} \wedge \mathcal{P}(q, \psi) \wedge\right. \\
& \forall j<k, \mathcal{I} \in \mathcal{K} \llbracket \kappa \rrbracket . \\
& \left.\operatorname{Comp}\left(j,\lfloor\psi\rfloor_{j}, e, \mathcal{T} \llbracket \Delta, \alpha: \kappa \vdash \tau: \star \rrbracket \delta[\alpha \mapsto \mathcal{I}]\right)\right\} \\
& \mathcal{T} \llbracket \Delta \vdash \operatorname{ref} \tau: \nexists \rrbracket \delta=\left\{(k, q,\{l \mapsto(q, \chi)\}, l) \mid \chi=\lfloor\mathcal{T} \llbracket \Delta \vdash \tau: \star \rrbracket \delta\rfloor_{k} \wedge\right. \\
& \left.\left(q \preceq \mathrm{A} \Rightarrow \forall\left({ }_{-}, q^{\prime},{ }_{-},{ }_{-}\right) \in \chi \cdot q^{\prime} \preceq \mathrm{A}\right)\right\} \\
& \mathcal{T} \llbracket \Delta \vdash^{\xi} \bar{\tau}: \star \rrbracket \delta=\{(k, q, \psi, v) \mid q=\mathcal{T} \llbracket \Delta \vdash \xi: \mathrm{QUAL} \rrbracket \delta \wedge \\
& (k, q, \psi, v) \in \mathcal{T} \llbracket \Delta \vdash \bar{\tau}: \nexists \rrbracket \delta\} \\
& \operatorname{Comp}\left(k, \psi_{s}, e_{s}, \chi\right) \stackrel{\text { def }}{=} \forall j<k, s_{s}, \psi_{r}, s_{f}, e_{f} \text {. } \\
& s_{s}:_{k}\left(\psi_{s} \odot_{k} \psi_{r}\right) \wedge\left(s_{s}, e_{s}\right) \longmapsto^{j}\left(s_{f}, e_{f}\right) \wedge \operatorname{irred}\left(s_{f}, e_{f}\right) \Rightarrow \\
& \exists q_{f}, \psi_{f} \text {. } \\
& s_{f}: k-j\left(\psi_{f} \odot_{k-j} \psi_{r}\right) \wedge\left(k-j, q_{f}, \psi_{f}, e_{f}\right) \in \chi
\end{aligned}
$$

Figure 11. $\lambda^{\text {refURAL }}$ Model (Interpretations)

Finally, we define LocalStoreDesc $k$ using the predicate $\mathcal{R}(\psi)$, which requires that every unrestricted or affine location in $\psi$ is mapped to a type with only unrestricted and affine values. The predicate $\mathcal{R}(\psi)$ disallows relevant or linear values as the contents of unrestricted or affine locations (recall Figure 7).

\subsubsection{Semantic Interpretations}

Figure 11 gives our semantic interpretation of kinds $\mathcal{K} \llbracket \kappa \rrbracket$, qualifiers $\mathcal{T} \llbracket q \rrbracket$, pre-types $\mathcal{T} \llbracket \bar{\tau} \rrbracket$, and types $\mathcal{T} \llbracket \tau \rrbracket .^{6}$ The interpretation of the kinds $₹$ and $\star$ are the semantic interpretations Pre Type and Type respectively, while the interpretation of the kind QUAL is the set of (constant) qualifiers Quals.

Units: No Location Beliefs Consider the interpretation of the pre-type $\mathbf{1}_{\otimes}$. Clearly, no locations appear as subexpressions of the value \langle\rangle ; hence, the interpretation of $\mathbf{1}_{\otimes}$ demands an empty local store description \{\} . Furthermore, the value \langle\rangle may be ascribed any qualifier $q$.

References: Single Location Beliefs Next, consider the interpretation of the pre-type ref $\tau$. From the value $l$, the only directly-accessible location is $l$ itself. Hence, the local store description $\psi$ for the location $l$ in the interpretation of ref $\tau$ must take the form $\{l \mapsto(q, \chi)\}$. Furthermore, $\chi$, the semantic interpretation of the type of $l$ 's contents, must match $\mathcal{T} \llbracket \tau \rrbracket$.

Figure 12 graphically depicts the local store description $\psi=\{l \mapsto(q, \mathcal{T} \llbracket \tau \rrbracket)\}$ (slightly abusing notation in the interest of brevity). Our intention is to express the idea that $\psi$ "believes" that $l$ is allocated with qualifier $q$ and contents of type $\tau$, but $\psi$ "believes" nothing about any other location in the store, represented by "?".

\footnotetext{
${ }^{6}$ Since our language supports polymorphic types, we must give the interpretations of type-level terms with free variables. While, technically, we should write $\mathcal{T} \llbracket \Delta \vdash \iota: \kappa \rrbracket \delta$, where the substitution $\delta$ is in the interpretation of the term context $\Delta$ (see $\mathcal{D} \llbracket \Delta \rrbracket$ in Figure 16), we will use the more concise notation $\mathcal{T} \llbracket \iota \rrbracket$ in the text.
} 


$$
(k, q, \psi=\{l \mapsto(q, \mathcal{T} \llbracket \tau \rrbracket)\}, l) \in \mathcal{T} \llbracket \operatorname{ref} \tau \rrbracket
$$

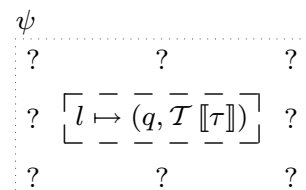

Figure 12. A Local Store Description in $\mathcal{T} \llbracket \operatorname{ref} \tau \rrbracket$

Note that the definition of $\mathcal{T} \llbracket$ ref $\tau \rrbracket$ requires that if $l$ is an unrestricted or affine location, then $\chi$ should never contain local store descriptions that include relevant or linear locations; i.e., the definition of $\mathcal{T} \llbracket$ ref $\tau \rrbracket$ incorporates the predicate $\mathcal{R}(\cdot)$ specialized to $\{l \mapsto(q, \chi)\}$.

Pairs: Compatible Location Beliefs A pair $\left\langle v_{1}, v_{2}\right\rangle$ (such that $\left(k, q_{1}, \psi_{1}, v_{1}\right) \in \mathcal{T} \llbracket \tau_{1} \rrbracket$ and $\left.\left(k, q_{2}, \psi_{2}, v_{2}\right) \in \mathcal{T} \llbracket \tau_{2} \rrbracket\right)$ is in the interpretation of $\tau_{1} \otimes \tau_{2}$ if and only if the pair is ascribed a qualifier greater than that of its components and the two sets of beliefs about the store, $\psi_{1}$ and $\psi_{2}$, can be combined into a single set of beliefs sufficient for safely executing $k$ steps (written $\psi_{1} \odot_{k} \psi_{2}$, see Figure 13). Informally, local store descriptions can be combined only if they are compatible; that is, if the beliefs in one local store description do not contradict the beliefs in the other store description.

$$
\psi_{1} \odot_{k} \psi_{2} \stackrel{\operatorname{def}}{=}\left\{\begin{array}{cl}
\left\{l \mapsto\left\lfloor\psi_{1}\right\rfloor_{k}(l) \mid l \in \operatorname{dom}\left(\psi_{1}\right) \cap \operatorname{dom}\left(\psi_{2}\right)\right\} & \text { if } \forall l \in \operatorname{dom}\left(\psi_{1}\right) \cap \operatorname{dom}\left(\psi_{2}\right) .\left\lfloor\psi_{1}\right\rfloor_{k}(l)=\left\lfloor\psi_{2}\right\rfloor_{k}(l) \\
\uplus\left\{l \mapsto\left\lfloor\psi_{1}\right\rfloor_{k}(l) \mid l \in \operatorname{dom}\left(\psi_{1}\right) \backslash \operatorname{dom}\left(\psi_{2}\right)\right\} & \text { and } \forall l \in \operatorname{dom}\left(\psi_{1}\right) . \mathrm{A} \preceq \psi_{1}^{\text {qual }}(l) \Rightarrow l \notin \operatorname{dom}\left(\psi_{2}\right) \\
\uplus\left\{l \mapsto\left\lfloor\psi_{2}\right\rfloor_{k}(l) \mid l \in \operatorname{dom}\left(\psi_{2}\right) \backslash \operatorname{dom}\left(\psi_{1}\right)\right\} & \text { and } \forall l \in \operatorname{dom}\left(\psi_{2}\right) . \mathrm{A} \preceq \psi_{2}^{\text {qual }}(l) \Rightarrow l \notin \operatorname{dom}\left(\psi_{1}\right) \\
\text { undefined } & \text { otherwise }
\end{array}\right.
$$

Figure 13. $\lambda^{\text {refURAL }}$ Model (Join Partial Function)

Clearly, if $\psi_{1}$ and $\psi_{2}$ have disjoint sets of beliefs about the store, then $\psi_{1} \odot_{k} \psi_{2}$ is defined and equal to the union of their beliefs:

$$
\begin{aligned}
& \left(k, q_{1}, \psi_{1}=\left\{l_{1} \mapsto\left(q_{1}, \mathcal{T} \llbracket \tau_{1} \rrbracket\right)\right\}, l_{1}\right) \in \mathcal{T} \llbracket \llbracket^{q_{1}} \text { ref } \tau_{1} \rrbracket \\
& \left(k, q_{2}, \psi_{2}=\left\{l_{2} \mapsto\left(q_{2}, \mathcal{T} \llbracket \tau_{2} \rrbracket\right)\right\}, l_{2}\right) \in \mathcal{T} \llbracket q^{q_{2}} \text { ref } \tau_{2} \rrbracket
\end{aligned}
$$

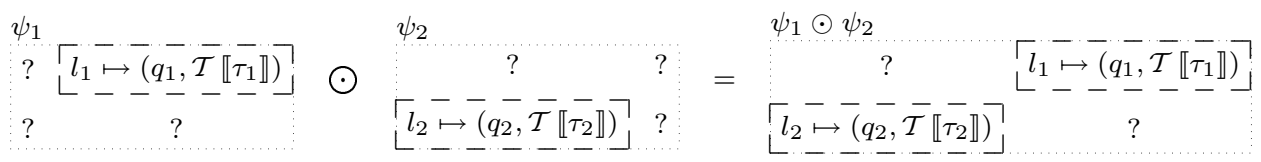

In the more general case, where the same location may be found in the domain of both $\psi_{1}$ and $\psi_{2}$, there are two requirements enforced by the definition of $\psi_{1} \odot_{k} \psi_{2}$.

First, we require that for any location $l$ that is described by both $\psi_{1}$ and $\psi_{2}$, it must be the case that $\psi_{1}$ and $\psi_{2}$ have identical beliefs about $l$ to approximation $k$. Note that $\psi_{1}$ and $\psi_{2}$ must agree on both the qualifier of the location as well as the type of the location's contents:

$$
\begin{aligned}
& \left(k, \mathrm{U}, \psi_{1}=\{l \mapsto(\mathrm{U}, \mathcal{T} \llbracket \tau \rrbracket)\}, l\right) \in \mathcal{T} \llbracket \amalg^{\mathrm{U}} \text { ref } \tau \rrbracket \\
& \left(k, \mathrm{U}, \psi_{2}=\left\{l \mapsto\left(\mathrm{R}, \mathcal{T} \llbracket \tau^{\prime} \rrbracket\right)\right\}, l\right) \in \mathcal{T} \llbracket \mathrm{R}^{\mathrm{ref}} \tau^{\prime} \rrbracket
\end{aligned}
$$

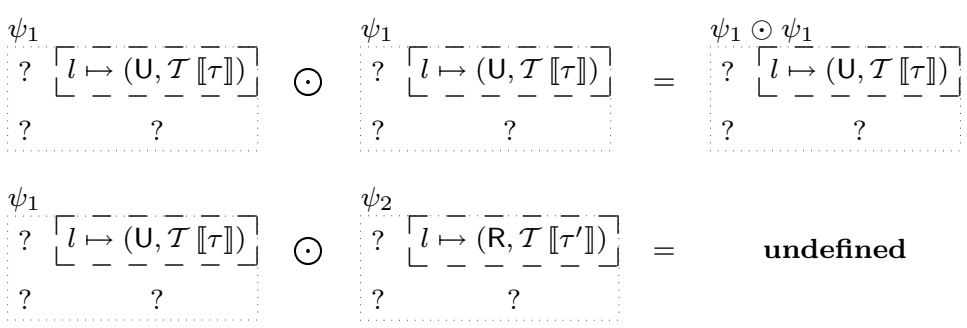

The second requirement is more subtle, having to do with the notion of directly-accessible locations. Suppose that $l_{3}$ is a linear or affine location mapped by $\psi_{b}$. Therefore, a value $v_{b}$ with local store description $\psi_{b}$ must contain $l_{3}$ as a sub-expression. Since $l_{3}$ is linear or affine, this occurrence of $l_{3}$ in the value $v_{b}$ must be the one (and only) occurrence of $l_{3}$ in the entire program state. Now, suppose that $l_{3}$ is also in the domain of a local store description $\psi_{c}$. As before, a value $v_{c}$ with local store description $\psi_{c}$ must contain $l_{3}$ as a sub-expression. If we were to attempt to form the value $\left\langle v_{b}, v_{c}\right\rangle$, then 
we would have a value with two distinct occurrences of $l_{3}$, violating the uniqueness of the location $l_{3}$. Hence, we consider $\psi_{b}$ and $\psi_{c}$ to represent incompatible (contradictory) beliefs about the current store:

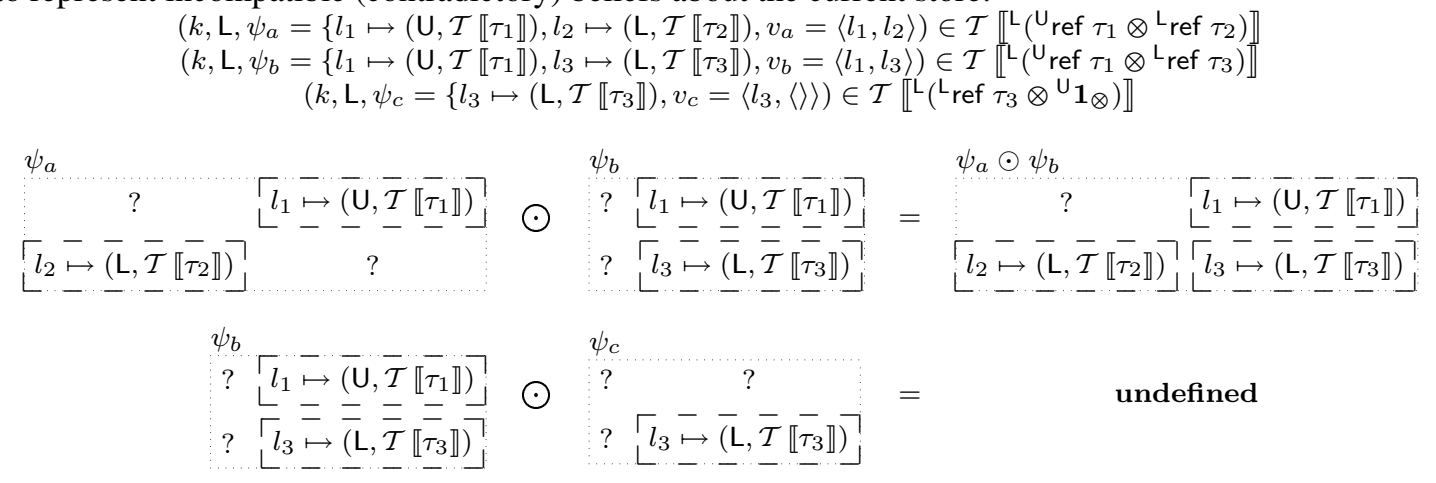

Functions \& Abstractions: Closure Location Beliefs Since functions and abstractions are suspended computations, their interpretations are given in terms of the interpretation of types as computations (see below). A function $\lambda x . e$ with qualifier $q_{c}$ and local store description $\psi_{c}$ (where $\psi_{c}$ describes the locations directly accessible from the function's closure and, hence, must satisfy $\left.\mathcal{P}\left(q_{c}, \psi_{c}\right)\right)$ is in the interpretation of $\tau_{1} \multimap \tau_{2}$ for $k$ steps if, at some point in the future, when there are $j<k$ steps left to execute, and there is an argument $v_{a}$ such that $\left(j,,_{-}, \psi_{a}, v_{a}\right) \in \mathcal{T} \llbracket \tau_{1} \rrbracket$ and the beliefs $\psi_{c}$ and $\psi_{a}$ are compatible, then $e\left[v_{a} / x\right]$ looks like a computation of type $\tau_{2}$ for $j$ steps. The interpretation of $\forall \alpha: \kappa$. $\tau$ is analogous, except that we quantify over (type-level term) interpretations $\mathcal{I} \in \mathcal{K} \llbracket \kappa \rrbracket$.

Store Satisfaction: Tracing Location Beliefs The interpretation of types as computations (Comp) makes use of an auxiliary relation $s::_{k} \quad \psi$ (given in Figure 14), which says that the store $s$ satisfies local store description $\psi$ (to approximation $k$ ). We motivate the definition of $s:_{k} \psi$ by drawing an analogy with the specification of a tracing garbage collector (see Figure 15). As described above, $\psi$ corresponds to (beliefs about) the portion of the store directly accessible from a value (or multiple values, when $\psi$ corresponds to $\odot_{k}$-ed store descriptions). Hence, we can consider $d o m(\psi)$ as a set of root locations. In the definition of $s:_{k} \psi, \mathcal{S}$ corresponds to the set of reachable (root and non-root) locations in the store that would be discovered by the garbage collector. The function $\mathcal{F}_{\psi}$ maps each location in $\mathcal{S}$ to a local store description, while the function $\mathcal{F}_{q}$ maps each location to a qualifier. It is our intention that, for each location $l, \mathcal{F}_{q}(l)$ is an appropriate qualifier and $\mathcal{F}_{\psi}(l)$ is an appropriate local store description for the value $s^{\text {val }}(l)$. Hence, we can consider $\operatorname{dom}\left(\mathcal{F}_{\psi}(l)\right)$ as the set of child locations traced from the contents of $l$.

Having chosen the set $\mathcal{S}$ and the functions $\mathcal{F}_{\psi}$ and $\mathcal{F}_{q}$, we require that they satisfy three criteria. The congruity criteria ensures that our choices are both internally consistent and consistent with the store $s$. The "global" store description $\psi_{*}$ combines the local store descriptions of the roots with the local store descriptions of the contents of every reachable location; the implicit requirement that $\psi_{*}$ is defined ensures that the local beliefs of the roots and individual store contents are all compatible. The clause $\operatorname{dom}\left(\psi_{*}\right)=\mathcal{S}$ requires that $\mathcal{S}$ and $\mathcal{F}_{\psi}$ are chosen such that $\mathcal{S}$ includes all the reachable locations (and not just some of the reachable locations), while the clause $\operatorname{dom}(s) \supseteq \mathcal{S}$ requires that all of the reachable locations are actually in the store. Finally, $\left(j, \mathcal{F}_{q},\left\lfloor\mathcal{F}_{\psi}(l)\right\rfloor_{j}, s^{\text {val }}(l)\right) \in\left\lfloor\psi_{*}^{\text {type }}(l)\right\rfloor_{k}$ ensures that the contents of $l$, with the qualifier assigned by $\mathcal{F}_{q}$ and local store description assigned by $\mathcal{F}_{\psi}$, is in the type assigned by the global store description $\psi_{*}$ (for $j<k$ steps).

The minimality criteria ensures that our choice for the set $\mathcal{S}$ does not contain any locations not reachable from the roots. For example, in Figure 15, including $l_{11}$ in $\mathcal{S}$ would not violate congruity, but would violate minimality. Finally, the reachability criteria ensures that every linear and relevant location is reachable from the roots (and, hence, has not been implicitly discarded).

Computations: Relating Current to Future Beliefs Informally, the interpretation of types as computations $\operatorname{Comp}\left(k, \psi_{s}, e_{s}, \chi\right)$ (see Figure 11) says that if the expression $e_{s}$ (with beliefs $\psi_{s}$, again, corresponding to the locations appearing as sub-expressions of $e_{s}$ ) reaches an irreducible state in less than $k$ steps, then it must have reduced to a value $v_{f}$ (with beliefs $\psi_{f}$ ) that belongs to the type interpretation $\chi$. More precisely, we pick a starting store $s_{s}$ such that $s_{s}:_{k}\left(\psi_{s} \odot_{k} \psi_{r}\right)$, where $\psi_{r}$ is the set of beliefs about the store held by the rest of the computation (alternatively, the set of beliefs held by $e_{s}$ 's continuation). If $\left(s_{s}, e_{s}\right)$ steps to an irreducible configuration $\left(s_{f}, e_{f}\right)$ in $j<k$ steps, then the following conditions hold. First, $e_{f}$ must be a value with a qualifier $q_{f}$ and a set of beliefs $\psi_{f}$ such that $\left(k-j, q_{f}, \psi_{f}, e_{f}\right) \in \chi$. Second, the following two sets of beliefs must be compatible: $\psi_{f}$ (what $e_{f}$ believes) and $\psi_{r}$ (what the rest of the computation believes - note that these beliefs remain unchanged). Third, the final store $s_{f}$ must satisfy the combined set of these beliefs. 


$$
\begin{aligned}
& s:{ }_{k} \psi \stackrel{\text { def }}{=} \exists \mathcal{S}: 2^{\text {Locs }} . \\
& \exists \mathcal{F}_{\psi}: \mathcal{S} \rightarrow \text { LocalStoreDesc. } \\
& \exists \mathcal{F}_{q}: \mathcal{S} \rightarrow \text { Quals. } \\
& \text { let } \psi_{*}=\left(\psi \odot_{k} \bigodot_{k}^{l \in \mathcal{S}} \mathcal{F}_{\psi}(l)\right) \text { in } \\
& \operatorname{dom}\left(\psi_{*}\right)=\mathcal{S} \wedge \operatorname{dom}(s) \supseteq \mathcal{S} \wedge \\
& \forall l \in \mathcal{S} \text {. } \\
& \forall j<k .\left(j, \mathcal{F}_{q}(l),\left\lfloor\mathcal{F}_{\psi}(l)\right\rfloor_{j}, s^{\text {val }}(l)\right) \in\left\lfloor\psi_{*}^{\text {type }}(l)\right\rfloor_{k} \wedge \\
& s^{\text {qual }}(l)=\psi_{*}^{\text {type }}(l) \wedge \\
& \forall \mathcal{S}^{\dagger} \subseteq \mathcal{S} \text {. } \\
& \operatorname{dom}(\psi) \subseteq \mathcal{S}^{\dagger} \wedge\left(\forall l \in \mathcal{S}^{\dagger} . \operatorname{dom}\left(\mathcal{F}_{\psi}(l)\right) \subseteq \mathcal{S}^{\dagger}\right) \Rightarrow \mathcal{S}=\mathcal{S}^{\dagger} \wedge \\
& \forall l \in \operatorname{dom}(s) \text {. } \\
& \mathrm{R} \preceq s^{\text {qual }}(l) \Rightarrow l \in \mathcal{S}
\end{aligned}
$$

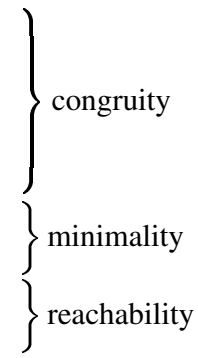

Figure 14. $\lambda^{\text {refURAL }}$ Model (Store Satisfaction)
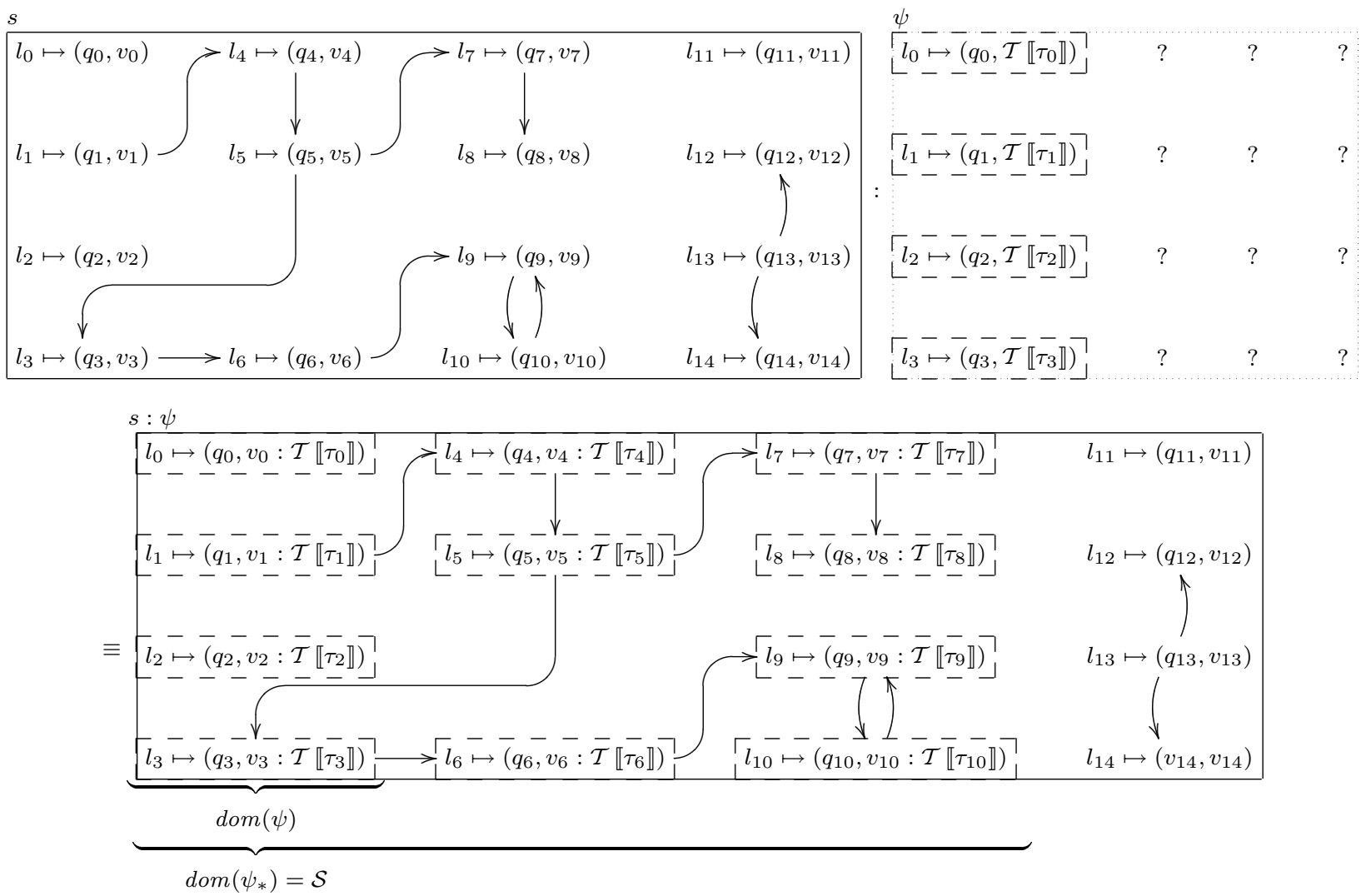

where

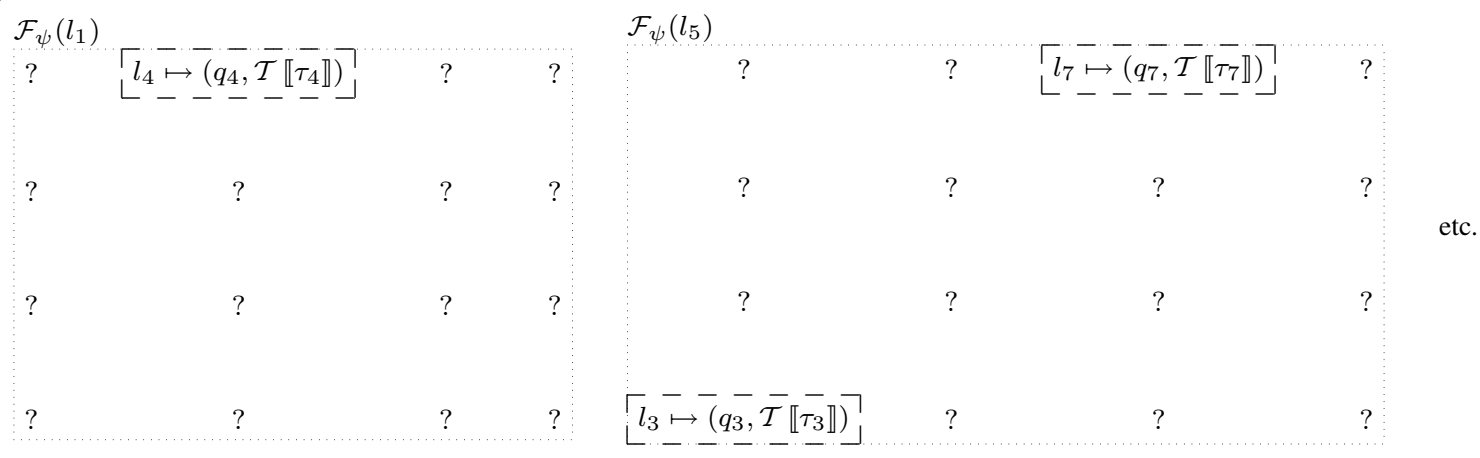

Figure 15. $s: \psi$ Example 


$$
\begin{aligned}
& \mathcal{D} \llbracket \bullet \rrbracket=\{\emptyset\} \\
& \mathcal{D} \llbracket \Delta, \alpha: \kappa \rrbracket=\{\delta[\alpha \mapsto \mathcal{I}] \mid \delta \in \mathcal{D} \llbracket \Delta \rrbracket \wedge \mathcal{I} \in \mathcal{K} \llbracket \kappa \rrbracket\} \\
& \mathcal{G} \llbracket \Delta \vdash \bullet \rrbracket \delta \quad=\quad\{(k, q,\{\}, \emptyset)\} \\
& \mathcal{G} \llbracket \Delta \vdash \Gamma, x: \tau \rrbracket \delta=\left\{(k, q, \psi, \gamma[x \mapsto v]) \mid \psi=\left(\psi_{\Gamma} \odot_{k} \psi_{x}\right) \wedge\right. \\
& \left(k, q_{\Gamma}, \psi_{\Gamma}, \gamma\right) \in \mathcal{G} \llbracket \Delta \vdash \Gamma \rrbracket \delta \wedge q_{\Gamma} \preceq q \wedge \\
& \left.\left(k, q_{x}, \psi_{x}, v\right) \in \mathcal{T} \llbracket \Delta \vdash \tau: \star \rrbracket \delta \wedge q_{x} \preceq q\right\}
\end{aligned}
$$

$$
\begin{aligned}
& \llbracket \Delta ; \Gamma \vdash e: \tau \rrbracket \stackrel{\text { def }}{=} \quad \forall k \geq 0 . \forall \delta, q_{\Gamma}, \psi_{\Gamma}, \gamma \\
& \delta \in \mathcal{D} \llbracket \Delta \rrbracket \wedge\left(k, q_{\Gamma}, \psi_{\Gamma}, \gamma\right) \in \mathcal{G} \llbracket \Delta \vdash \Gamma \rrbracket \delta \Rightarrow \\
& \operatorname{Comp}\left(k, \psi_{\Gamma}, \gamma(e), \mathcal{T} \llbracket \Delta \vdash \tau: \star \rrbracket \delta\right)
\end{aligned}
$$

\section{Figure 16. $\lambda^{\text {refURAL }}$ Model (Additional Interpretations)}

Note that since $\psi_{r}$ is an arbitrary set of beliefs compatible with $\psi_{s}$, one instantiation of $\psi_{r}$ is the local store description that includes all of the shared locations of $\psi_{s}$. By requiring that $\psi_{f}$ and $s_{f}$ are compatible with $\psi_{r}$, we ensure that the types and qualifiers and allocation status of shared locations are preserved.

Judgements: Type Soundness Finally, the semantic interpretation of a typing judgement $\llbracket \Delta ; \Gamma \vdash e: \tau \rrbracket$ (see Figure 16) asserts that for all $k \geq 0$, if $\delta$ is a mapping from type-level variables to an element of the appropriate kind interpretation, and $\gamma$ is a mapping from variables to closed values, and $\psi_{\Gamma}$ is a local store description for the values in the range of $\gamma$, then $\left(k, \psi_{\Gamma}, \gamma(e)\right)$ is in the interpretation of $\tau$ as a computation $\left(\operatorname{Comp}\left(k, \psi_{\Gamma}, \gamma(e), \mathcal{T} \llbracket \tau \rrbracket\right)\right)$.

The appendicies give the proof of the following theorem which shows the soundness of the $\lambda^{\text {refURAL }}$ typing rules with respect to the model.

THEOREM 1. ( $\lambda^{\text {refURAL }}$ Soundness)

$$
\text { If } \Delta ; \Gamma \vdash e: \tau \text {, then } \llbracket \Delta ; \Gamma \vdash e: \tau \rrbracket \text {. }
$$

An immediate corollary is type-safety of $\lambda^{\text {refURAL }}$. Another interesting corollary is that if we evaluate a closed, welltyped term of base type (e.g., $\left.{ }^{q} \mathbf{1}_{\otimes}\right)$ to a value, then the resulting store will have no linear or relevant references.

COROllary 2. ( $\lambda^{\text {refURAL }}$ Safety)

$$
\text { If } \bullet \bullet \vdash e_{1}: \tau \text { and }\left(\{\}, e_{1}\right) \longmapsto *\left(s_{2}, e_{2}\right) \text {, then either } \exists v_{2} . e_{2} \equiv v_{2} \text { or } \exists s_{3}, e_{3} .\left(s_{2}, e_{2}\right) \longmapsto\left(s_{3}, e_{3}\right) \text {. }
$$

\section{Corollary 3. ( $\lambda^{\text {refURAL }}$ Collection)}

$$
\text { If } \bullet ; \bullet e_{1}:{ }^{q} \mathbf{1}_{\otimes} \text { and }\left(\{\}, e_{1}\right) \longmapsto{ }^{*}\left(s_{2}, v_{2}\right) \text {, then } \forall l \in \operatorname{dom}\left(s_{2}\right) . s_{2}^{\text {qual }}(l) \preceq \mathrm{A} .
$$

\section{Proof $\left(\lambda^{\text {refURAL }}\right.$ Safety)}

Suppose $\bullet \bullet \vdash e_{1}: \tau$ and $\left(\{\}, e_{1}\right) \longmapsto^{*}\left(s_{2}, e_{2}\right)$.

If $\neg$ irred $\left(s_{2}, e_{2}\right)$, then $\exists s_{3}, e_{3} .\left(s_{2}, e_{2}\right) \longmapsto\left(s_{3}, e_{3}\right)$.

If $\operatorname{irred}\left(s_{2}, e_{2}\right)$, then $\exists i$. $\left(\{\}, e_{1}\right) \longmapsto^{i}\left(s_{2}, e_{2}\right)$.

Theorem 1 applied to $\bullet ; \bullet \vdash e_{1}: \tau$ yields $\llbracket \bullet ; \bullet \vdash e_{1}: \tau \rrbracket$.

$\llbracket \bullet ; \bullet \vdash e_{1}: \tau \rrbracket$ instantiated with $i+1 \geq 0, \emptyset \in \mathcal{D} \llbracket \bullet \rrbracket$, and $(i+1, \mathrm{U},\{\}, \emptyset) \in \mathcal{G} \llbracket \bullet \rrbracket \emptyset$ yields $\operatorname{Comp}\left(i+1,\{\}, e_{1}, \mathcal{T} \llbracket \bullet \vdash \tau: \star \rrbracket \emptyset\right)$.

$\operatorname{Comp}\left(i+1,\{\}, e_{1}, \mathcal{T} \llbracket \bullet \vdash \tau: \star \rrbracket \emptyset\right)$ instantiated with $i<i+1, s_{1}:_{i+1}\left(\{\} \odot_{i+1}\{\}\right),\left(\{\}, e_{1}\right) \longmapsto^{i}\left(s_{2}, e_{2}\right)$, and $\operatorname{irred}\left(s_{2}, e_{2}\right)$

yields $q_{2}$ and $\psi_{2}$ such that $s_{2}:_{1}\left(\psi_{2} \odot_{1}\{\}\right)$ and $\left(1, q_{2}, \psi_{2}, e_{2}\right) \in \mathcal{T} \llbracket \bullet \vdash \tau: \star \rrbracket \emptyset$.

Recall that $\mathcal{T} \llbracket \bullet \vdash \tau: \star \rrbracket \emptyset \in$ Type and Type $\subseteq$ CandUberType ${ }_{\omega}=2^{\text {CandAtom }}$.

Hence, $\left(1, q_{2}, \psi_{2}, e_{2}\right) \in$ CandAtom $_{\omega}=\bigcup_{k \geq 0}$ CandAtom $_{k}$,

which implies that $e_{2} \in$ CValues and $\exists v_{2} . e_{2} \equiv v_{2}$.

\section{Proof ( $\lambda^{\text {refURAL }}$ Collection)}

Suppose $\bullet ; \vdash e_{1}:{ }^{q} \mathbf{1}_{\otimes}$ and $\left(\{\}, e_{1}\right) \longmapsto{ }^{*}\left(s_{2}, v_{2}\right)$.

By the reasoning above, $\left(1, q_{2}, \psi_{2}, v_{2}\right) \in \mathcal{T} \llbracket \bullet \vdash^{q} \mathbf{1}_{\otimes}: \star \rrbracket \emptyset$, which implies that $q_{2}=q, \psi_{2}=\{\}$, and $v_{2}=\langle\rangle$.

Recall that $s_{2}:_{1}\left(\{\} \odot_{1}\{\}\right) \equiv s_{2}:_{1}\{\} \equiv \exists \mathcal{S}, \mathcal{F}_{\psi}, \mathcal{F}_{q} \ldots$...

The minimality criteria of $s_{2}:_{1}\{\}$ instantiated with $\emptyset \subseteq \mathcal{S}$, $\operatorname{dom}(\{\}) \subseteq \emptyset$, and $\left(\forall l \in \emptyset \cdot \operatorname{dom}\left(\mathcal{F}_{\psi}(l)\right) \subseteq \emptyset\right)$ yields $\mathcal{S}=\emptyset$. 
The reachability criteria of $s_{2}:_{1}\{\}$ yields $\forall l \in \operatorname{dom}\left(s_{2}\right) . \mathrm{R} \preceq s_{2}^{\text {qual }}(l) \Rightarrow l \in \emptyset$, which implies $\forall l \in \operatorname{dom}\left(s_{2}\right) . s_{2}^{\text {qual }}(l) \preceq \mathrm{A}$.

\subsection{Discussion}

A key difference in the model presented here, as compared to previous models of mutable state, is the localization of the store description. Recall that we identify the local store description of a value with those locations that are directly accessible from the value. This is in contrast to the AAV model of unrestricted references [1,4], where the global store description of any value describes every location that has been allocated. It is also in contrast to our previous model of linear references [23, 2], where the store description of a value describes the reachable locations from that value.

The transition from a global store description to a local store description is motivated by the insight that storing a unique object in a shared reference "hides" the unique object in some way. Note that the shared reference must mediate all access to the unique object. The authors have found it hard to construct a model where the store description of a value (in the interpretation of a type) describes the entire store or even the store reachable from the value. When one attempts to describe the entire store, there is a difficulty identifying where the "real" occurrence of a unique location is to be found. When one attempts to describe the reachable store, there is a difficulty defining the $\odot$ relation; it cannot be defined pointwise, and one is required to formally introduce the notions of directly- and indirectly-accessible locations. Furthermore, the reachable store is a property of the actual store, not of the type; hence, it seems better to confine reachability to the store satisfaction relation. We further note that the model of mutable references given in this paper subsumes the models of mutable references cited above. Hence, the technique of localizing the store description subsumes the techniques used by previous approaches.

Although our model of substructural references is different from the previous model of unrestricted references, our model retains the spirit of the step-indexed approach used in Foundational PCC [6, 7] and may be applicable in future extensions of FPCC. This approach, in which the model mixes denotational and operational semantics, offers a number of distinct advantages over a purely syntactic approach to type soundness. One obvious advantage of this approach is that it gives rise to a simpler set of typing rules; note that our typing judgement requires neither a store description component nor a rule for locations. A less obvious advantage of this approach is that it gives rise to stronger meta-

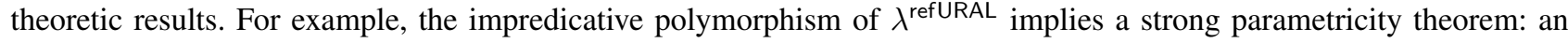
element of $\mathcal{T} \llbracket \forall \alpha: \star . \tau \rrbracket$ behaves uniformly on all elements of Type, which includes elements that do not correspond to the interpretation of any syntactic type. This approach also naturally extends to union and intersection types and to an inclusion interpretation of subtyping. Finally, a (well-founded) set-theoretic model means that soundness and safety proofs are amenable to formalization in the higher-order logic of FPCC.

While we are partial to the step-indexed approach, we acknowledge that there is no fundamental difficulty in adopting a purely syntactic approach to proving the type soundness of substructural state. However, we believe that any proof of type soundness must adopt many of the insights presented here. For example, we conjecture that the typing rule for well-typed configurations would naturally take the form:

$$
\begin{gathered}
\psi_{*}=\psi \odot \bigodot^{l \in \mathcal{S}} \mathcal{F}_{\psi}(l) \\
\begin{array}{c}
\operatorname{dom}\left(\psi_{*}\right)=\mathcal{S} \quad \operatorname{dom}(s) \supseteq \mathcal{S} \\
\forall l \in \mathcal{S} . ; \cdot ; \mathcal{F}_{\psi}(l) \vdash s^{\text {val }}(l): \psi_{*}^{\text {type }}(l) \wedge \\
s^{\text {qual }}(l)=\psi_{*}^{\text {qual }}(l)
\end{array} \\
\qquad s: \psi \\
\vdash(s, e): \tau
\end{gathered}
$$

Note that the judgement $\vdash s: \psi$ mirrors the store satisfaction predicate given in Figure 14. The store typing component complicates the judgement $\Delta ; \Gamma ; \psi \vdash e: \tau$, which must further rely upon an operator $\psi_{1} \odot \psi_{2}=\psi$ to split the locations in $\psi$ between the store typings $\psi_{1}$ and $\psi_{2}$. Splitting the store typing is necessary to ensure that a given unique location is used by at most one sub-expression. The $\odot$ operator in the syntactic approach would need to satisfy many of the same properties as the $\odot_{k}$ operator in the step-indexed approach (e.g., identical beliefs about locations in the common domain and no unique locations in the common domain).

\section{Related Work}

Our $\lambda^{\text {URAL }}$ is most directly influenced by the presentation of substructural type systems by Walker [30], which in turn draws upon the work of Wansbrough and Peyton-Jones [33] and Walker and Watkins [32]. Relative to that work, we have added both relevant and affine qualifiers, which is necessary to account for the varied forms of linearity found in higher-level programming-language proposals. 
A related body of work is that on type systems used to track resource usage $[28,22,33,21,16,19]$. We note that the usage subsumption found in these systems (e.g., a "possibly used many times" variable may be subsumed to appear in a context requiring a "used exactly once" value) is not applicable in our setting (e.g., it is clearly unsound to subsume "ref $\tau$ to ${ }^{\mathrm{L}}$ ref $\tau$ ), due to differences in the interpretation of type qualifiers.

Section 1 noted a number of projects that have introduced some form of linearity to "tame" state. An underlying theme is that linearity and strong updates can be used to provide more effective memory management (c.f. $[10,18,9,8])$.

More recent research has explored other ways in which unique and shared data may be mixed. For example, Cyclone's alias construct [17] takes a unique pointer and returns a shared pointer to the same object, which is available for a limited lexical scope. Vault's focus and CQuals's restrict constructs $[14,5]$ provide the opposite behavior: temporarily giving a linear view of an object of shared type. Both behaviors are of great practical significance.

Our model's semantic interpretations seem strongly related to the logic of Bunched Implications (BI) [20] and Reynolds' separation logic [25]. In particular, our interpretation of $\otimes$ and $\multimap$ resemble the resource semantics for the $*$ and $*$ connectives in BI.

Finally, Boyland and Retert have recently proved the soundness of a variation of Vault by giving an operational semantics of "adoption" [11]. The authors note that adoption may be used to embed a unique pointer within another object; their notion of uniqueness most closely resembles our affine references, as access keys may be dropped.

\section{Conclusion and Future Work}

We have presented the $\lambda^{\text {refURAL }}$-calculus, a substructural polymorphic $\lambda$-calculus with mutable references of unrestricted, relevant, affine, and linear sorts. We motivated the design decisions, gave a type system, and constructed a step-indexed

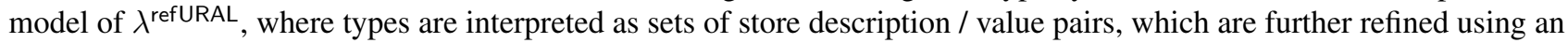
index representing the number of steps available for future evaluation.

In previous work [23, 2], we separated the typing components of a mutable object into two pieces: an unrestricted pointer to the object and a linear capability for accessing the contents of the object. We believe that we can extend the current language and model in the same way. The advantage of this approach is that separating the name of a reference from what it currently holds gives us a model of alias types [27, 31].

As noted in the previous section, allowing a unique pointer to be temporarily treated as shared (and vice versa) can be useful in practice. Understanding how to model these advanced features is a long-term goal of this research. A promising aproach is to model regions as a linear capability to access objects in the region and allow changes in reference qualifiers to be mediated by this capability.

\section{Acknowledgments}

We would like to thank Dan Grossman, Aleks Nanevski, and Dan Wang for their helpful comments.

\section{References}

[1] Amal Ahmed, Andrew W. Appel, and Roberto Virga. An indexed model of impredicative polymorphism and mutable references. Available at http://www.cs.princeton.edu/ appel/papers/impred.pdf, January 2003.

[2] Amal Ahmed, Matthew Fluet, and Greg Morrisett. L $^{3}$ : A linear language with locations. Technical Report TR-24-04, Harvard University, October 2004.

[3] Amal Ahmed, Matthew Fluet, and Greg Morrisett. A step-indexed model of substructural state. Technical Report TR-16-05, Harvard University, July 2005.

[4] Amal Jamil Ahmed. Semantics of Types for Mutable State. PhD thesis, Princeton University, 2004.

[5] Alex Aiken, Jeffrey S. Foster, John Kodumal, and Tachio Terauchi. Checking and inferring local non-aliasing. In Proc. Programming Language Design and Implementation (PLDI), pages 129-140, June 2003.

[6] Andrew W. Appel. Foundational proof-carrying code. In Proc. Logic in Computer Science (LICS), pages 247-258, June 2001.

[7] Andrew W. Appel and David McAllester. An indexed model of recursive types for foundational proof-carrying code. ACM Transactions on Programming Languages and Systems, 23(5):657-683, September 2001.

[8] David Aspinall and Adriana Compagnoni. Heap bounded assembly language. Journal of Automated Reasoning, 31:261-302, 2003.

[9] David Aspinall and Martin Hofmann. Another type system for in-place update. In Proc. European Symposium on Programming (ESOP), pages 36-52, March 2002.

[10] Henry Baker. Lively linear LISP_look ma, no garbage. ACM SIGPLAN Notices, 27(8):89-98, 1992.

[11] John Tang Boyland and William Retert. Connecting effects and uniqueness with adoption. In Proc. Principles of Programming Languages (POPL), pages 283-295, January 2005. 
[12] James Cheney and Greg Morrisett. A linearly typed assembly language. Technical Report 2003-1900, Department of Computer Science, Cornell University, 2003.

[13] Robert DeLine and Manuel Fähndrich. Enforcing high-level protocols in low-level software. In Proc. Programming Language Design and Implementation (PLDI), pages 59-69, June 2001.

[14] Manuel Fähndrich and Robert DeLine. Adoption and focus: Practical linear types for imperative programming. In Proc. Programming Language Design and Implementation (PLDI), pages 13-24, June 2002.

[15] Jean-Yves Girard. Linear logic. Theoretical Computer Science, 50:1-102, 1987.

[16] Jörgen Gustavsson and Josef Svenningsson. A usage analysis with bounded usage polymorphism and subtyping. In Proc. International Workshop on Implementation of Functional Languages (IFL), pages 140-157, September 2001.

[17] Michael Hicks, Greg Morrisett, Dan Grossman, and Trevor Jim. Experience with safe manual memory-management in Cyclone. In Proc. International Symposium on Memory Management (ISMM), pages 73-84, October 2004.

[18] Martin Hofmann. A type system for bounded space and functional in-place update. In Proc. European Symposium on Programming (ESOP), pages 165-179, March 2000.

[19] Atsushi Igarashi and Naoki Kobayashi. Resource usage analysis. In Proc. ACM Principles of Programming Languages (POPL), pages 331-342, January 2002.

[20] Samin Ishtiaq and Peter O'Hearn. BI as an assertion language for mutable data structures. In Proc. Principles of Programming Languages (POPL), pages 14-26, January 2001.

[21] Naoki Kobayashi. Quasi-linear types. In Proc. Principles of Programming Languages (POPL), pages 29-42, January 1999.

[22] Torben Æ. Mogensen. Types for 0, 1 or many uses. In Proc. International Workshop on Implementation of Functional Languages (IFL), pages 112-122, 1998.

[23] Greg Morrisett, Amal Ahmed, and Matthew Fluet. $\mathbf{L}^{3}$ : A linear language with locations. In Proc. International Conference on Typed Lambda Calculi and Applications (TLCA), pages 293-307, April 2005.

[24] Peter W. O'Hearn and John C. Reynolds. From Algol to polymorphic linear lambda-calculus. Journal of the ACM, 47(1):167-223, 2000.

[25] John C. Reynolds. Separation Logic: A Logic for Shared Mutable Data Structures. In Proc. Logic in Computer Science (LICS), pages 55-74, July 2002.

[26] Sjaak Smetsers, Erik Barendsen, Marko C. J. D. van Eekelen, and Rinus J. Plasmeijer. Guaranteeing safe destructive updates through a type system with uniqueness information for graphs. In Dagstuhl Seminar on Graph Transformations in Computer Science, volume 776 of Lecture Notes in Computer Science, pages 358-379. Springer-Verlag, 1994.

[27] Fred Smith, David Walker, and Greg Morrisett. Alias types. In Proc. European Symposium on Programming (ESOP), pages 366-381, March 2000.

[28] David N. Turner, Philip Wadler, and Christian Mossin. Once upon a type. In Proc. Functional Programming Languages and Computer Architecture (FPCA), pages 1-11, June 1995.

[29] Philip Wadler. Linear types can change the world! In Programming Concepts and Methods, April 1990. IFIP TC 2 Working Conference.

[30] David Walker. Substructural type systems. In Benjamin Pierce, editor, Advanced Topics in Types and Programming Languages, chapter 1, pages 3-43. MIT Press, Cambridge, MA, 2005.

[31] David Walker and Greg Morrisett. Alias types for recursive data structures. In Proc. Workshop on Types in Compilation (TIC), pages 177-206, September 2000.

[32] David Walker and Kevin Watkins. On regions and linear types. In Proc. International Conference on Functional Programming (ICFP), pages 181-192, September 2001.

[33] Keith Wansbrough and Simon Peyton-Jones. Once upon a polymorphic type. In Proc. Principles of Programming Languages (POPL), pages 15-28, January 1999. 


\section{Appendix: Formal Development}

The following appendices present a formal development of the language, step-indexed model, and soundness proof described in the main body of this technical report.

Previous research has suggested that linearity and, by extension, the other substructural sorts have been and will continue to be a powerful means of "taming" state and effects in programming languages and type systems. With this in mind, we propose a framework comprised of a core substructural polymorphic $\lambda$-calculus and type system, stepindexed model, and proof of soundness. The entire development of this core language is done with respect to an abstract global stateful world, additional abstract expression forms that interact with the world, and abstract world descriptions, which impart semantic meaning to worlds. Having held these components abstract, the proof establishing the soundness of our step-indexed model is itself parameterized by a collection of requirements that must satisfied by these abstract components. Hence, our methodology is to instantiate the framework by choosing concrete worlds, expression forms, and world descriptions, showing that these concrete components meet the requirements, and discharge any additional proof cases introduced by the new components.

In Appendix A, we use shaded boxes to indicated abstracted components, requirements, and proof cases that depend upon the particular concrete instantiation. In Appendix B, we show a simple instantiation that adds recursive types to the core language. In Appendix $\mathrm{C}$, we show the instantiation for mutable references. 


\section{A Core Language}

\section{A.1 Syntax}

Kind Level:

$\begin{array}{lrll}\text { Kinds } & \kappa & := & \text { QUAL | PRETYPE | TYPE } \mid \kappa_{X} \\ \text { Extended Kinds } & \kappa_{X} & ::= & \ldots\end{array}$

Type Level:

\begin{tabular}{|c|c|c|c|}
\hline Constant Qualifiers & $q$ & $\in$ & Quals $=\{\mathrm{U}, \mathrm{R}, \mathrm{A}, \mathrm{L}\}$ \\
\hline Qualifiers & $\xi$ & $::=$ & $\alpha \mid q$ \\
\hline PreTypes & $\bar{\tau}$ & $::=$ & $\begin{array}{l}\alpha\left|\tau_{1} \multimap \tau_{2}\right| \mathbf{1}_{\otimes}\left|\tau_{1} \otimes \tau_{2}\right| \mathbf{1}_{\circledast}\left|\tau_{1} \circledast \tau_{2}\right| \mathbf{0}\left|\tau_{1} \oplus \tau_{2}\right| \\
\forall \alpha: \kappa . \tau|\exists \alpha: \kappa . \tau| \bar{\tau}_{X}\end{array}$ \\
\hline Extended PreTypes & $\bar{\tau}_{X}$ & $::=$ & .. \\
\hline Types & $\tau$ & $::=$ & $\left.\alpha\right|^{\xi} \bar{\tau}$ \\
\hline Terms & $\iota$ & $::=$ & $\xi|\bar{\tau}| \tau \mid \iota_{X}$ \\
\hline Extended Terms & $\iota_{X}$ & $::=$ & $\ldots$ \\
\hline
\end{tabular}

Expression Level:

\begin{tabular}{|c|c|c|c|}
\hline Values & $v$ & $::=$ & $\begin{array}{l}x|\lambda x . e|\langle\rangle\left|\left\langle v_{1}, v_{2}\right\rangle\right|\langle\langle\rangle\rangle\left|\left\langle\left\langle e_{1}, e_{2}\right\rangle\right\rangle\right| \text { inl } v_{1} \mid \text { inr } v_{2} \mid \\
\Lambda . e|\ulcorner v\urcorner| v_{X}\end{array}$ \\
\hline Extended Values & $v_{X}$ & $::=$ & \\
\hline \multirow{6}{*}{ Expressions } & $e$ & $::=$ & $v \mid$ \\
\hline & & & $e_{1} e_{2}$ \\
\hline & & & $\begin{array}{l}\text { let }\langle\rangle=e_{1} \text { in } e_{2} \mid \text { let }\left\langle x_{1}, x_{2}\right\rangle=e_{1} \text { in } e_{2} \mid \\
\text { fst } e \mid \text { snd } e \mid\end{array}$ \\
\hline & & & abort $e \mid$ case $e$ of inl $x_{1} \Rightarrow e_{1} \|$ inr $x_{2} \Rightarrow e_{2} \mid$ \\
\hline & & & $\begin{array}{l}e[] \mid \text { let }\ulcorner x\urcorner=e_{1} \text { in } e_{2} \mid \\
\text { copy } e \mid \operatorname{drop} e\end{array}$ \\
\hline & & & $e_{X}$ \\
\hline Extended Expressions & $e_{X}$ & $::=$ & $\ldots$ \\
\hline
\end{tabular}

Figure 1: Core Language - Syntax 


\section{A.2 Operational Semantics}

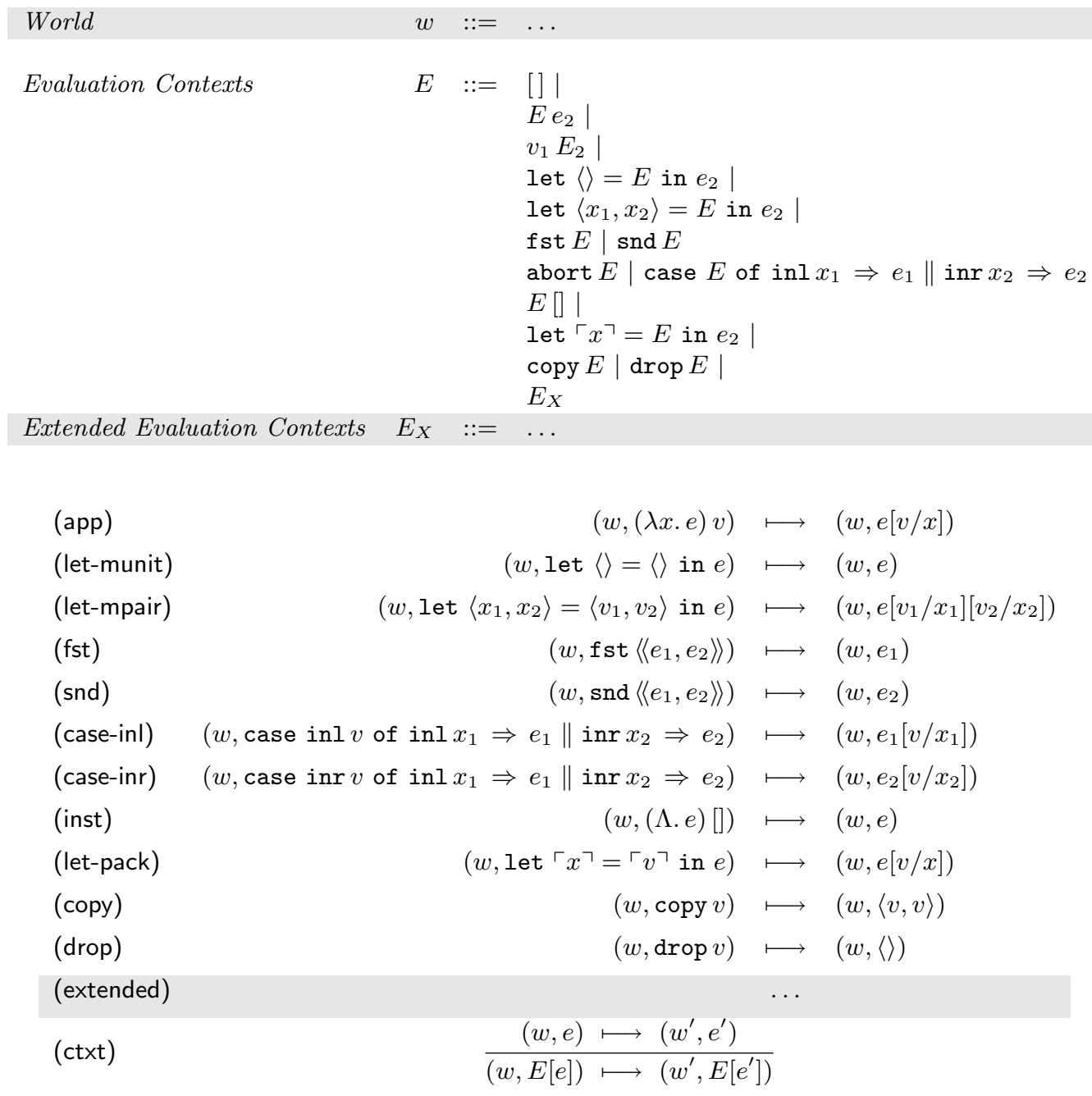

Figure 2: Core Language - Operational Semantics 


\section{A.3 Static Semantics}

Term Context $\Delta \quad:=\bullet \mid \Delta, \alpha: \kappa$

$\Delta \vdash \iota: \kappa$

$$
\begin{gathered}
(\mathrm{VARKN}) \\
\frac{\alpha: \kappa \in \Delta}{\Delta \vdash \alpha: \kappa}
\end{gathered}
$$

(QUAL)

$\overline{\Delta \vdash q: \mathrm{QUAL}}$

(FNPTy)

$\frac{\Delta \vdash \tau_{1}: \text { TYPE } \quad \Delta \vdash \tau_{2}: \text { TYPE }}{\Delta \vdash \tau_{1} \multimap \tau_{2}: \text { PRETYPE }}$

$\frac{(\text { MUnitPTy) }}{\Delta \vdash \mathbf{1}_{\otimes}: \text { PRETYPE }}$

(AUNITPTY)

$\overline{\Delta \vdash \mathbf{1}_{\circledast}: \text { PRETYPE }}$

(VOIDPTy)

$\overline{\Delta \vdash \mathbf{0}: \text { PRETYPE }}$

(AllPTy)

$\Delta, \alpha: \kappa \vdash \tau:$ TYPE

$\overline{\Delta \vdash \forall \alpha: \kappa . \tau: \text { PRETYPE }}$
(MPAIRPTY)
$\frac{\Delta \vdash \tau_{1}: \text { TYPE } \quad \Delta \vdash \tau_{2}: \text { TYPE }}{\Delta \vdash \tau_{1} \otimes \tau_{2}: \text { PRETYPE }}$

(APAIRPTY)

$\frac{\Delta \vdash \tau_{1}: \text { TYPE } \quad \Delta \vdash \tau_{2}: \text { TYPE }}{\Delta \vdash \tau_{1} \circledast \tau_{2}: \text { PRETYPE }}$

(SumPTy)

$\frac{\Delta \vdash \tau_{1}: \text { TYPE } \quad \Delta \vdash \tau_{2}: \text { TYPE }}{\Delta \vdash \tau_{1} \oplus \tau_{2}: \text { PRETYPE }}$

(ExPTy)

$\Delta, \alpha: \kappa \vdash \tau:$ TYPE

$\Delta \vdash \exists \alpha: \kappa . \tau:$ PRETYPE

(UserPTy) ...

(TYPE)

$\frac{\Delta \vdash \xi: \text { QUAL } \quad \Delta \vdash \bar{\tau}: \text { PRETYPE }}{\Delta \vdash^{\xi} \bar{\tau}: \text { TYPE }}$

(UserTerm) ...

Figure 3: Core Language - Static Semantics (I) 


$$
\text { Value Context } \quad \Gamma \quad::=\bullet \mid \Gamma, x: \tau
$$

$\Delta \vdash \Gamma$

$$
\overline{\Delta \vdash \bullet} \quad \frac{\Delta \vdash \Gamma \quad \Delta \vdash \tau: \text { TYPE }}{\Delta \vdash \Gamma, x: \tau}
$$

Figure 4: Core Language - Static Semantics (II)

$\Delta \vdash \xi_{1} \preceq \xi_{2}$

$$
\begin{array}{ccr}
\frac{\Delta \vdash \xi_{2}: \text { QUAL }}{\Delta \vdash \mathrm{U} \preceq \xi_{2}} & \frac{q_{1} \preceq q_{2}}{\Delta \vdash q_{1} \preceq q_{2}} & \frac{\Delta \vdash \xi_{1}: \text { QUAL }}{\Delta \vdash \xi_{1} \preceq \mathrm{L}} \\
\frac{\Delta \vdash \xi: \mathrm{QUAL}}{\Delta \vdash \xi \preceq \xi} & \frac{\Delta \vdash \xi_{1} \preceq \xi^{\prime}}{\Delta \vdash \xi_{1} \preceq \xi_{2}}
\end{array}
$$

$\Delta \vdash \tau \preceq \xi$

$$
\frac{\Delta \vdash \tau: \mathrm{TYPE}}{\Delta \vdash \tau \preceq \mathrm{L}}
$$

$$
\frac{\Delta \vdash \bar{\tau}^{\prime}: \text { PRETYPE } \quad \Delta \vdash \xi^{\prime} \preceq \xi}{\Delta \vdash^{\xi^{\prime}} \bar{\tau}^{\prime} \preceq \xi}
$$

$\Delta \vdash \Gamma \preceq \xi$

$$
\frac{\Delta \vdash \xi: \text { QUAL }}{\Delta \vdash \bullet \preceq \xi} \quad \frac{\Delta \vdash \Gamma \preceq \xi \quad \Delta \vdash \tau \preceq \xi}{\Delta \vdash \Gamma, x: \tau}
$$

Figure 5: Core Language - Static Semantics (III)

$\Delta \vdash \Gamma \leadsto \Gamma_{1} \boxplus \Gamma_{2}$

$$
\begin{gathered}
\frac{\Delta \vdash \Gamma \leadsto \Gamma_{1} \boxplus \Gamma_{2} \quad \Delta \vdash \tau: \text { TYPE }}{\Delta \vdash \bullet \sim \bullet \boxplus \bullet} \quad \frac{\Delta \vdash \Gamma \leadsto \Gamma_{1} \boxplus \Gamma_{2} \quad \Delta \vdash \tau: \text { TYPE }}{\Delta \vdash \Gamma, x: \tau \sim \Gamma_{1} \boxplus \Gamma_{2}, x: \tau} \\
\frac{\Delta \vdash \Gamma \sim \Gamma_{1}, x: \tau \boxplus \Gamma_{2}}{\Delta \vdash \Gamma, x: \tau \leadsto \Gamma_{2} \quad \Delta \vdash \tau \preceq \mathrm{R}} \\
\frac{\Delta \vdash: \tau \boxplus \Gamma_{2}, x: \tau}{\Delta}
\end{gathered}
$$

Figure 6: Core Language - Static Semantics (IV) 


$$
\Delta ; \Gamma \vdash e: \tau
$$

$$
\begin{aligned}
& (\mathrm{VAR}) \\
& \frac{\Delta \vdash \tau: \text { TYPE }}{\Delta ; \bullet, x: \tau \vdash x: \tau}
\end{aligned}
$$

$(\mathrm{FN})$

$\frac{\Delta \vdash \xi: \text { QUAL } \quad \Delta \vdash \Gamma \preceq \xi \quad \Delta ; \Gamma, x: \tau_{1} \vdash e: \tau_{2}}{\Delta ; \Gamma \vdash \lambda x . e:{ }^{\xi}\left(\tau_{1} \multimap \tau_{2}\right)}$

(MUNIT)

$\frac{\Delta \vdash \xi: \text { QUAL }}{\Delta ; \bullet \vdash\langle\rangle::^{\xi} 1 \otimes}$

(MPAIR)

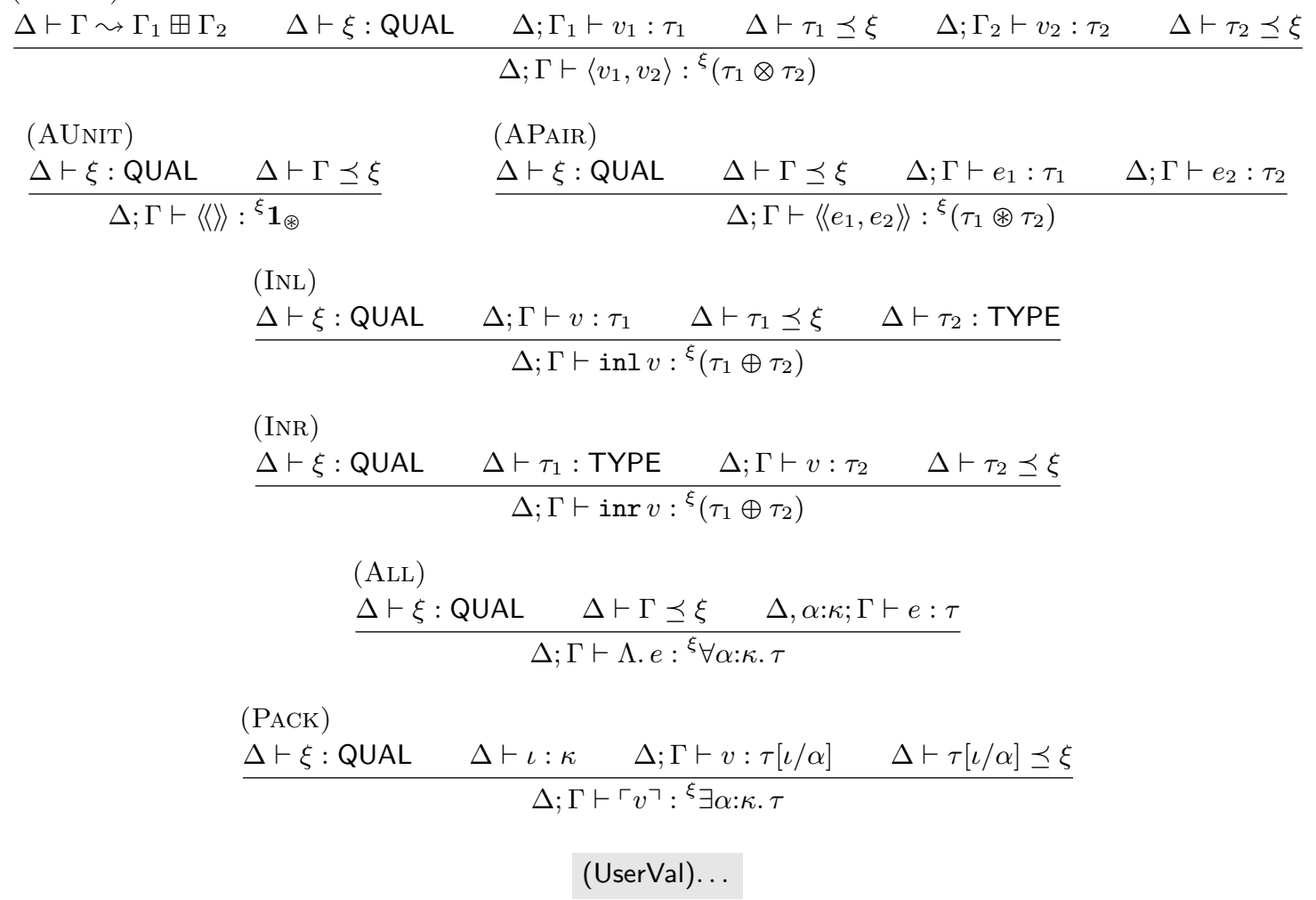

Figure 7: Core Language - Static Semantics (Va) 
$\Delta ; \Gamma \vdash e: \tau$

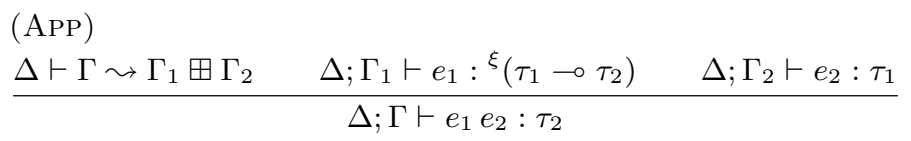

(LET-MUNiT)

$\frac{\Delta \vdash \Gamma \sim \Gamma_{1} \boxplus \Gamma_{2} \quad \Delta ; \Gamma_{1} \vdash e_{1}:{ }^{\xi} \mathbf{1}_{\otimes} \quad \Delta ; \Gamma_{2} \vdash e_{2}: \tau}{\Delta ; \Gamma \vdash \operatorname{let}\langle\rangle=e_{1} \text { in } e_{2}: \tau}$

(LET-MPAIR)

$\frac{\Delta \vdash \Gamma \leadsto \Gamma_{1} \boxplus \Gamma_{2} \quad \Delta ; \Gamma_{1} \vdash e_{1}:{ }^{\xi}\left(\tau_{1} \otimes \tau_{2}\right) \quad \Delta ; \Gamma_{2}, x_{1}: \tau_{1}, x_{2}: \tau_{2} \vdash e_{2}: \tau}{\Delta ; \Gamma \vdash \operatorname{let}\left\langle x_{1}, x_{2}\right\rangle=e_{1} \text { in } e_{2}: \tau}$

$\begin{array}{lll}(\mathrm{FST}) & (\mathrm{SND}) & (\text { ABORT }) \\ \frac{\Delta ; \Gamma \vdash e:{ }^{\xi}\left(\tau_{1} \circledast \tau_{2}\right)}{\Delta ; \Gamma \vdash \mathrm{fst} e: \tau_{1}} & \frac{\Delta ; \Gamma \vdash e:{ }^{\xi}\left(\tau_{1} \circledast \tau_{2}\right)}{\Delta ; \Gamma \vdash \operatorname{snd} e: \tau_{2}} & \frac{\Delta ; \Gamma \vdash e:{ }^{\xi} \quad \Delta \vdash \tau: \text { TYPE }}{\Delta ; \Gamma \vdash \text { abort } e: \tau}\end{array}$

(CASE)

$\frac{\Delta \vdash \Gamma \leadsto \Gamma_{1} \boxplus \Gamma_{2} \quad \Delta ; \Gamma_{1} \vdash e:{ }^{\xi}\left(\tau_{1} \oplus \tau_{2}\right) \quad \Delta ; \Gamma_{2}, x_{1}: \tau_{1}: e_{1}: \tau \quad \Delta ; \Gamma_{2}, x_{2}: \tau_{2}: e_{2}: \tau}{\Delta ; \Gamma \vdash \operatorname{case} e \text { of inl } x_{1} \Rightarrow e_{1} \| \operatorname{inr} x_{2} \Rightarrow e_{2}: \tau}$

(Inst)

$\frac{\Delta ; \Gamma \vdash e:{ }^{\xi} \forall \alpha: \kappa \cdot \tau \quad \Delta \vdash \iota: \kappa}{\Delta ; \Gamma \vdash e[]: \tau[\iota / \alpha]}$

(LET-PACK)

$\frac{\Delta \vdash \Gamma \sim \Gamma_{1} \boxplus \Gamma_{2} \quad \Delta ; \Gamma_{1} \vdash e_{1}:{ }^{\xi} \exists \alpha: \kappa \cdot \tau_{1} \quad \Delta \vdash \Gamma_{2} \quad \Delta \vdash \tau_{2}: \text { TYPE } \quad \Delta, \alpha: \kappa ; \Gamma_{2}, x: \tau_{1} \vdash e_{2}: \tau_{2}}{\Delta ; \Gamma \vdash \text { let }\ulcorner x\urcorner=e_{1} \text { in } e_{2}: \tau_{2}}$

(COPY)

(Drop) (WEAK)

$\frac{\Delta ; \Gamma \vdash e: \tau \quad \Delta \vdash \tau \preceq \mathrm{R}}{\Delta ; \Gamma \vdash \operatorname{copy} e:{ }^{\mathrm{L}}(\tau \otimes \tau)} \quad \frac{\Delta ; \Gamma \vdash e: \tau \quad \Delta \vdash \tau \preceq \mathrm{A}}{\Delta ; \Gamma \vdash \operatorname{drop} e:{ }^{\mathrm{L}} \mathbf{1}_{\otimes}} \quad \frac{\Delta \vdash \Gamma \sim \Gamma_{1} \boxplus \Gamma_{2} \quad \Delta ; \Gamma_{1} \vdash e: \tau \quad \Delta \vdash \Gamma_{2} \preceq \mathrm{A}}{\Delta ; \Gamma \vdash e: \tau}$

(UserExp)...

Figure 8: Core Language - Static Semantics (Vb) 


\section{A.4 Model}

$\begin{array}{lll}\text { PreType/Type Interpretation (Notation) } & \chi & :=\{(k, q, W, v), \ldots\} \\ \text { World Description (Notation) } & W & ::=\ldots\end{array}$

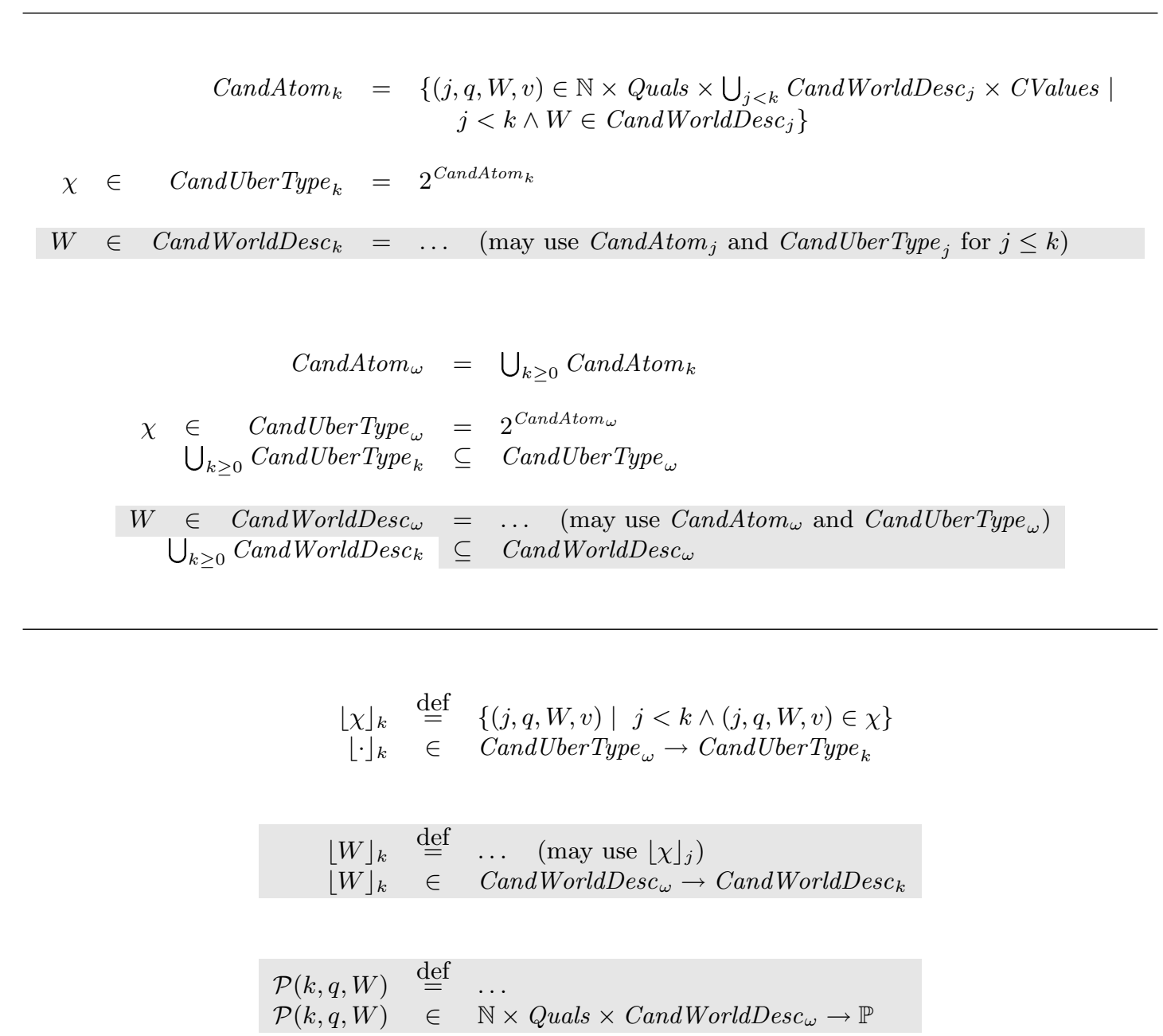

Figure 9: Core Language - Semantic Interpretations (Ia) 


$$
\begin{aligned}
& \text { Atom }_{k}=\left\{(j, q, W, v) \in \text { CandAtom }_{k} \mid W \in \text { WorldDesc }_{j} \wedge \mathcal{P}(k, q, W)\right\} \subseteq \text { CandAtom }_{k} \\
& \chi \in \quad \text { PreType }_{k}=\left\{\chi \in 2^{\text {Atom }_{k}} \mid \forall(j, q, W, v) \in \chi \cdot \forall i \leq j .\left(i, q,\lfloor W\rfloor_{i}, v\right) \in \chi\right\} \quad \subseteq \quad \text { CandUberType }_{k} \\
& \chi \in \quad \text { Type }_{k}=\left\{\chi \in \text { PreType }_{k} \mid \exists q^{\prime} \in \text { Quals. }\left(\left(_{-}, q_{-,-}\right) \in \chi \cdot q=q^{\prime}\right\} \quad \subseteq \quad \text { CandUberType }_{k}\right. \\
& W \in \text { WorldDesc }_{k}=\ldots \quad\left(\text { may use } \text { Atom }_{j} \text { and } \text { PreType }_{j} \text { and } \text { Type }_{j} \text { for } j \leq k\right) \subseteq \text { CandWorldDesc }_{k}
\end{aligned}
$$
$\chi \in \quad$ PreType $=\left\{\chi \in\right.$ CandUberType $_{\omega} \mid \forall k \geq 0 .\lfloor\chi\rfloor_{k} \in$ PreType $\left._{k}\right\}$
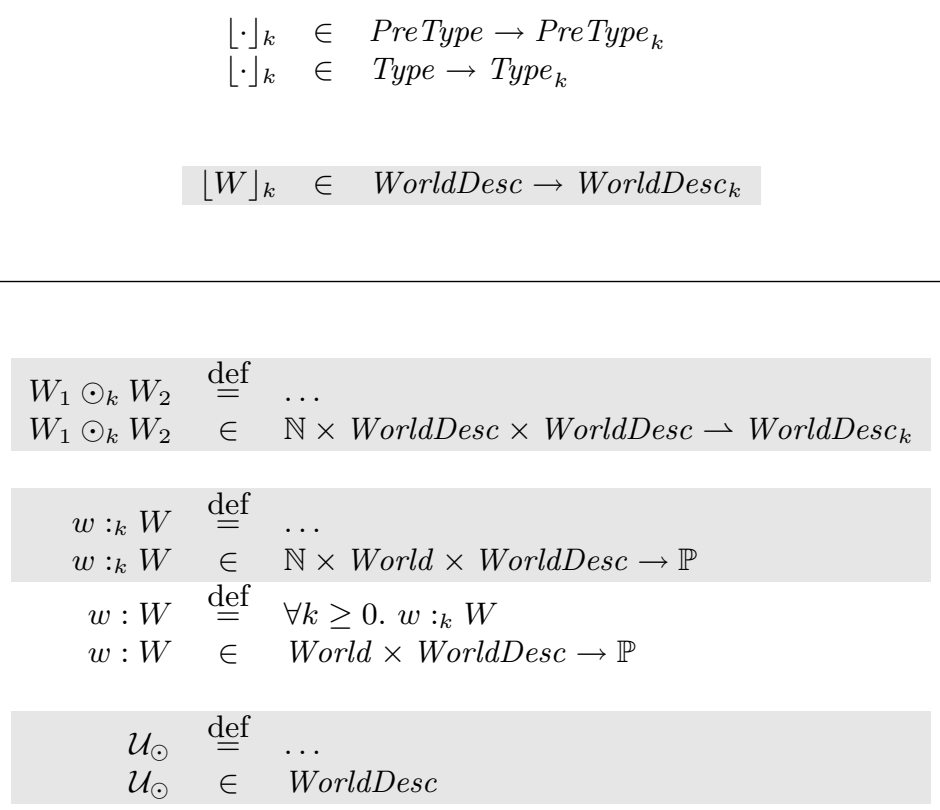

Figure 10: Core Language - Semantic Interpretations (Ib) 


$$
\begin{aligned}
\mathcal{K} \llbracket \mathrm{QUAL} \rrbracket & =\text { Quals } \\
\mathcal{K} \llbracket \mathrm{PRETYPE} \rrbracket & =\text { PreType } \\
\mathcal{K} \llbracket \mathrm{TYPE} \rrbracket & =\text { Type } \\
\mathcal{K} \llbracket \kappa_{X} \rrbracket & =\ldots
\end{aligned}
$$

Figure 11: Core Language - Semantic Interpretations (II) 


$$
\begin{aligned}
& \mathcal{T} \llbracket \frac{\alpha: \kappa \in \Delta}{\Delta \vdash \alpha: \kappa} \rrbracket \delta=\delta(\alpha) \\
& \mathcal{T} \llbracket \frac{}{\Delta \vdash q: \mathrm{QUAL}} \rrbracket \delta=q \\
& \mathcal{T} \llbracket \frac{\Delta \vdash \tau_{1}: \text { TYPE } \quad \Delta \vdash \tau_{2}: \text { TYPE }}{\Delta \vdash \tau_{1} \multimap \tau_{2}: \text { PRETYPE }} \rrbracket \delta=\begin{array}{c}
\left\{\left(k, q_{c}, W_{c}, \lambda x . e\right) \mid W_{c} \in \text { WorldDesc }_{k} \wedge \mathcal{P}\left(k, q_{c}, W_{c}\right) \wedge\right. \\
\forall i<k, q_{a}, W_{a}, v_{a} .
\end{array} \\
& \left(i, q_{a}, W_{a}, v_{a}\right) \in \mathcal{T} \llbracket \Delta \vdash \tau_{1}: \mathrm{TYPE} \rrbracket \delta \wedge \\
& \left(W_{c} \odot_{i} W_{a}\right) \text { defined } \Rightarrow \\
& \left.\operatorname{Comp}\left(i,\left(W_{c} \odot_{i} W_{a}\right), e\left[v_{a} / x\right], \mathcal{T} \llbracket \Delta \vdash \tau_{2}: \text { TYPE } \rrbracket \delta\right)\right\} \\
& \mathcal{T} \llbracket \frac{}{\Delta \vdash \mathbf{1}_{\otimes}: \text { PRETYPE }} \rrbracket \delta=\left\{(k, q, W,\langle\rangle) \mid W \in\left\lfloor\mathcal{U}_{\odot}\right\rfloor_{k}\right\} \\
& \left.\mathcal{T} \llbracket \frac{\Delta \vdash \tau_{1}: \text { TYPE } \quad \Delta \vdash \tau_{2}: \text { TYPE }}{\Delta \vdash \tau_{1} \otimes \tau_{2}: \text { PRETYPE }} \rrbracket \delta=\quad \begin{array}{c}
\left(k, q, W,\left\langle v_{1}, v_{2}\right\rangle\right) \| \\
\left(k, q_{1}, W_{1}, v_{1}\right)
\end{array}\right] \\
& \left(k, q_{1}, W_{1}, v_{1}\right) \in \mathcal{T} \llbracket \Delta \vdash \tau_{1}: \text { TYPE } \rrbracket \delta \wedge \\
& \left(k, q_{2}, W_{2}, v_{2}\right) \in \mathcal{T} \llbracket \Delta \vdash \tau_{2}: \text { TYPE } \rrbracket \wedge \\
& q_{1} \preceq q \wedge q_{2} \preceq q \wedge \\
& \left.\left(W_{1} \odot_{k} W_{2}=W\right)\right\} \\
& \mathcal{T} \llbracket \frac{}{\Delta \vdash \mathbf{1}_{\circledast}: \text { PRETYPE }} \| \delta=\left\{(k, q, W,\langle\langle\rangle\rangle) \mid W \in \operatorname{WorldDesc}_{k} \wedge \mathcal{P}(k, q, W)\right\} \\
& \mathcal{T} \llbracket \frac{\Delta \vdash \tau_{1}: \text { TYPE } \quad \Delta \vdash \tau_{2}: \text { TYPE }}{\Delta \vdash \tau_{1} \circledast \tau_{2}: \text { PRETYPE }} \rrbracket \delta=\begin{array}{c}
\left\{\left(k, q, W,\left\langle\left(e_{1}, e_{2}\right\rangle\right\rangle\right) \mid W \in \text { WorldDesc }_{k} \wedge \mathcal{P}(k, q, W) \wedge\right. \\
\forall i<k .
\end{array} \\
& \operatorname{Comp}\left(i,\lfloor W\rfloor{ }_{i}, e_{1}, \mathcal{T} \llbracket \Delta \vdash \tau_{1}: \text { TYPE } \rrbracket \delta\right) \wedge \\
& \mathcal{T}\left\|\frac{}{\Delta \vdash \mathbf{0}: \text { PRETYPE }}\right\| \delta=\{\} \\
& \left.\operatorname{Comp}\left(i,\lfloor W\rfloor_{i}, e_{2}, \mathcal{T} \llbracket \Delta \vdash \tau_{2}: \operatorname{TYPE} \rrbracket \delta\right)\right\} \\
& \mathcal{T} \llbracket \frac{\Delta \vdash \tau_{1}: \text { TYPE } \quad \Delta \vdash \tau_{2}: \text { TYPE }}{\Delta \vdash \tau_{1} \oplus \tau_{2}: \text { PRETYPE }} \rrbracket \delta=\begin{array}{c}
\left(k, q, W, \text { inl } v_{1}\right) \mid \\
\left(k, q_{1}, W, v_{1}\right) \in
\end{array} \\
& \left.q_{1} \preceq q\right\} \cup \\
& \left\{\left(k, q, W, \text { inr } v_{2}\right) \mid\right. \\
& \left(k, q_{2}, W, v_{2}\right) \in \mathcal{T} \llbracket \Delta \vdash \tau_{2}: \text { TYPE } \rrbracket \delta \wedge \\
& \left.q_{2} \preceq q\right\}
\end{aligned}
$$

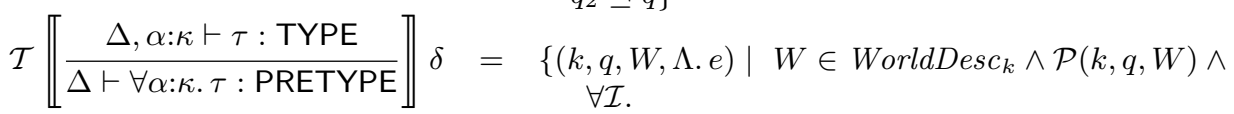

$$
\begin{aligned}
& \mathcal{I} \in \mathcal{K} \llbracket \kappa \rrbracket \Rightarrow \\
& \forall i<k \text {. } \\
& \left.\operatorname{Comp}\left(i,\lfloor W\rfloor_{i}, e, \mathcal{T} \llbracket \Delta, \alpha: \kappa \vdash \tau: \operatorname{TYPE} \rrbracket \delta[\alpha \mapsto \mathcal{I}]\right)\right\}
\end{aligned}
$$

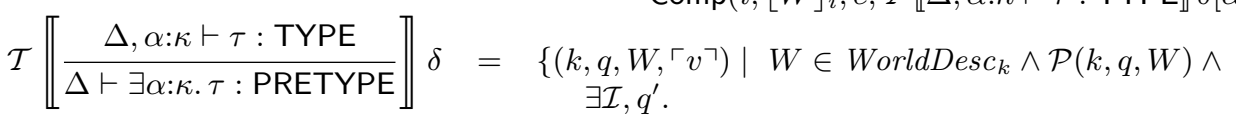

$$
\begin{aligned}
& \mathcal{I} \in \mathcal{K} \llbracket \kappa \rrbracket \wedge \\
& q^{\prime} \preceq q \wedge \\
& \forall i<k \text {. } \\
& \left.\left(i, q^{\prime},\lfloor W\rfloor_{i}, v\right) \in \mathcal{T} \llbracket \Delta, \alpha: \kappa \vdash \tau: \text { TYPE } \rrbracket \delta[\alpha \mapsto \mathcal{I}]\right\}
\end{aligned}
$$

$\mathcal{T} \llbracket($ UserPTy $) \ldots \rrbracket \delta=\ldots$

Figure 12: Core Language - Semantic Interpretations (IIIa) 


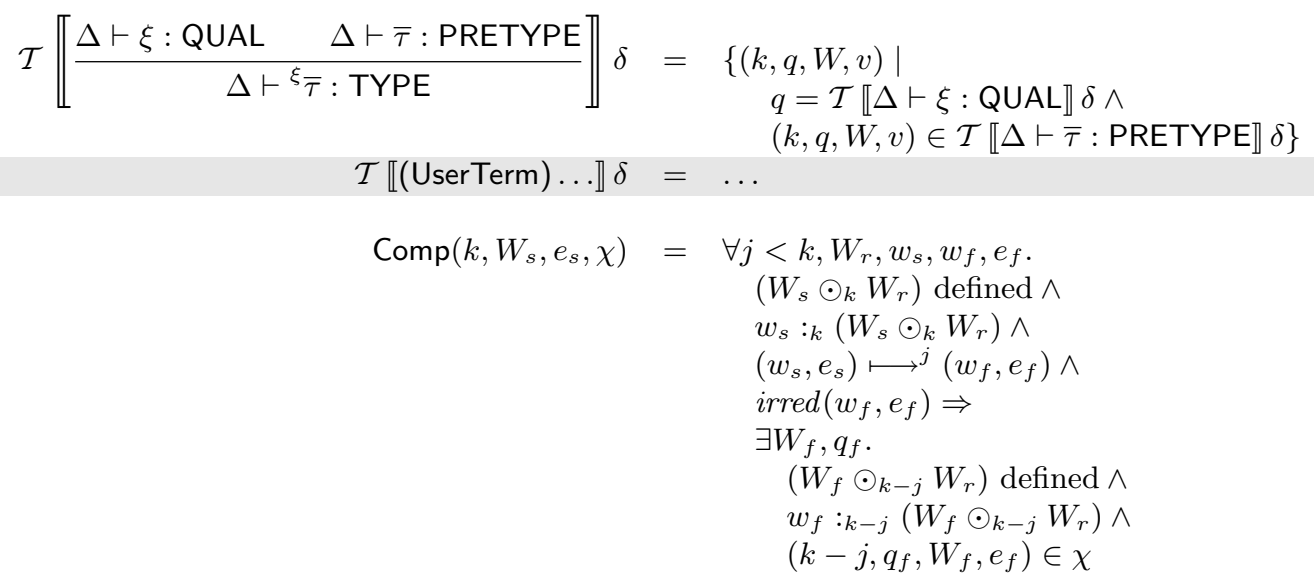

Figure 13: Core Language - Semantic Interpretations (IIIb) 


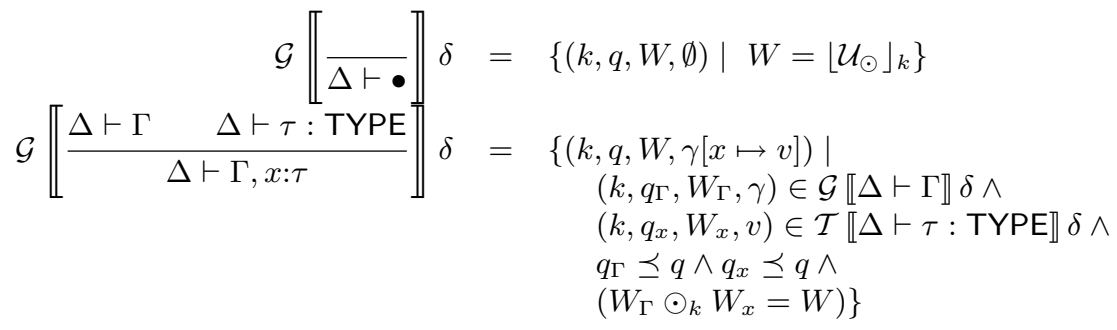

$$
\begin{aligned}
& \llbracket \Delta ; \Gamma \vdash e: \tau \rrbracket=\forall k \geq 0 . \forall \delta, q_{\Gamma}, W_{\Gamma}, \gamma . \\
& \delta \in \mathcal{D} \llbracket \Delta \rrbracket \wedge \\
& \left(k, q_{\Gamma}, W_{\Gamma}, \gamma\right) \in \mathcal{G} \llbracket \Delta \vdash \Gamma \rrbracket \delta \Rightarrow \\
& \operatorname{Comp}\left(k, W_{\Gamma}, \gamma(e), \mathcal{T} \llbracket \Delta \vdash \tau: \text { TYPE } \rrbracket \delta\right)
\end{aligned}
$$

Figure 14: Core Language - Semantic Interpretations (IV) 


\section{A.5 Trivial Facts}

Fact 1

$\forall \chi \in$ CandUberType $_{\omega} . \forall k \geq 0 .\lfloor\chi\rfloor_{k} \subseteq \chi$.

Proof

Immediate from the definition of $\lfloor\cdot\rfloor_{k}$.

Fact 2

$\forall \chi \in$ CandUberType $_{\omega} . \forall k_{1}, k_{2} \geq 0 .\left\lfloor\lfloor\chi\rfloor_{k_{2}}\right\rfloor_{k_{1}}=\lfloor\chi\rfloor_{\min \left(k_{1}, k_{2}\right)}$.

Proof

Immediate from the definition of $\lfloor\cdot\rfloor_{k}$.

Fact 3

Type $\subseteq$ PreType.

Proof

Let $\chi \in$ Type.

Note that $\chi \in$ CandUber Type $_{\omega}$ and $\forall k \geq 0 .\lfloor\chi\rfloor_{k} \in$ Type $_{k}$, which follows from the definition of Type.

Note that $\forall k \geq 0 .\lfloor\chi\rfloor_{k} \in$ PreType $_{k}$, which follows from Type Pre $_{k}$ Type $_{k}$.

Hence, $\chi \in$ CandUberType $_{\omega}$ and $\forall k \geq 0$. $\lfloor\chi\rfloor_{k} \in$ Pre Type $_{k}$.

Thus, $\chi \in$ PreType, which follows from the definition of PreType.

\section{Fact 4}

$$
\begin{aligned}
\text { PreType }_{k}=\left\{\chi \in \text { CandUberType }_{k} \mid\right. \\
\\
\left.\forall(j, q, W, v) \in \chi . W \in \text { WorldDesc }_{j} \wedge \mathcal{P}(j, q, W) \wedge \forall i \leq j .\left(i, q,\lfloor W\rfloor_{i}, v\right) \in \chi\right\} .
\end{aligned}
$$

\section{Proof}

Let

$$
\chi \in \text { PreType }_{k} .
$$

Hence,

$$
\chi \in 2^{\text {Atom }_{k}} \quad \text { and } \quad \forall(j, q, W, v) \in \chi . \forall i \leq j .(i, q,\lfloor W\rfloor i, v) \in \chi,
$$

which follows from the definition of Pre Type ${ }_{k}$.

Note that

$$
\begin{aligned}
& \chi \in 2^{\text {Atom }_{k}} \wedge \forall(j, q, W, v) \in \chi . \forall i \leq j .\left(i, q,\lfloor W\rfloor_{i}, v\right) \in \chi \\
& \Leftrightarrow \forall(j, q, W, v) \in \chi .(j, q, W, v) \in \text { Atom }_{k} \wedge \\
& \forall(j, q, W, v) \in \chi . \forall i \leq j .\left(i, q,\lfloor W\rfloor_{i}, v\right) \in \chi \\
& \text { which follows from the definition of powerset } \\
& \Leftrightarrow \forall(j, q, W, v) \in \chi .(j, q, W, v) \in \text { CandAtom }_{k} \wedge W \in \text { WorldDesc }_{j} \wedge \mathcal{P}(j, q, W) \wedge \\
& \forall(j, q, W, v) \in \chi . \forall i \leq j .\left(i, q,\lfloor W\rfloor_{i}, v\right) \in \chi \\
& \text { which follows from the definition of Atom } k \\
& \Leftrightarrow \forall(j, q, W, v) \in \chi .(j, q, W, v) \in \text { CandAtom }_{k} \wedge \\
& \forall(j, q, W, v) \in \chi . W \in \text { WorldDesc }_{j} \wedge \mathcal{P}(j, q, W) \wedge \\
& \forall(j, q, W, v) \in \chi . \forall i \leq j .\left(i, q,\lfloor W\rfloor_{i}, v\right) \in \chi \\
& \Leftrightarrow \forall(j, q, W, v) \in \chi .(j, q, W, v) \in \text { CandAtom }_{k} \wedge \\
& \forall(j, q, W, v) \in \chi . W \in \text { WorldDesc }_{j} \wedge \mathcal{P}(j, q, W) \wedge \forall i \leq j .\left(i, q,\lfloor W\rfloor_{i}, v\right) \in \chi \\
& \Leftrightarrow \chi \in 2^{\text {CandAtom }_{k}} \wedge \forall(j, q, W, v) \in \chi . W \in \text { WorldDesc }_{j} \wedge \mathcal{P}(j, q, W) \wedge \forall i \leq j .(i, q,\lfloor W\rfloor i, v) \in \chi \\
& \text { which follows from the definition of powerset } \\
& \Leftrightarrow \chi \in \text { CandUberType }_{k} \wedge \forall(j, q, W, v) \in \chi . W \in \text { WorldDesc }_{j} \wedge \mathcal{P}(j, q, W) \wedge \forall i \leq j .(i, q,\lfloor W\rfloor i, v) \in \chi \\
& \text { which follows from the definition of CandUberType }{ }_{k} \text {. }
\end{aligned}
$$


Hence,

$\chi \in$ CandUberType $_{k} \quad$ and $\quad \forall(j, q, W, v) \in \chi . W \in$ WorldDesc $_{j} \wedge \mathcal{P}(j, q, W) \wedge \forall i \leq j .\left(i, q,\lfloor W\rfloor_{i}, v\right) \in \chi$.

Thus

$\chi \in\left\{\chi \in\right.$ CandUberType $_{k} \mid \forall(j, q, W, v) \in \chi . W \in$ WorldDesc $\left._{j} \wedge \mathcal{P}(j, q, W) \wedge \forall i \leq j .\left(i, q,\lfloor W\rfloor_{i}, v\right) \in \chi\right\}$

and

PreType $_{k} \subseteq\left\{\chi \in\right.$ CandUberType $_{k} \mid \forall(j, q, W, v) \in \chi . W \in$ WorldDesc $\left._{j} \wedge \mathcal{P}(j, q, W) \wedge \forall i \leq j .\left(i, q,\lfloor W\rfloor_{i}, v\right) \in \chi\right\}$.

Let

$\chi \in\left\{\chi \in\right.$ CandUberType $_{k} \mid \forall(j, q, W, v) \in \chi . W \in$ WorldDesc $\left._{j} \wedge \mathcal{P}(j, q, W) \wedge \forall i \leq j .\left(i, q,\lfloor W\rfloor_{i}, v\right) \in \chi\right\}$.

Hence,

$\chi \in$ CandUberType $_{k} \quad$ and $\quad \forall(j, q, W, v) \in \chi . W \in$ WorldDesc $_{j} \wedge \mathcal{P}(j, q, W) \wedge \forall i \leq j .\left(i, q,\lfloor W\rfloor_{i}, v\right) \in \chi$.

Note that

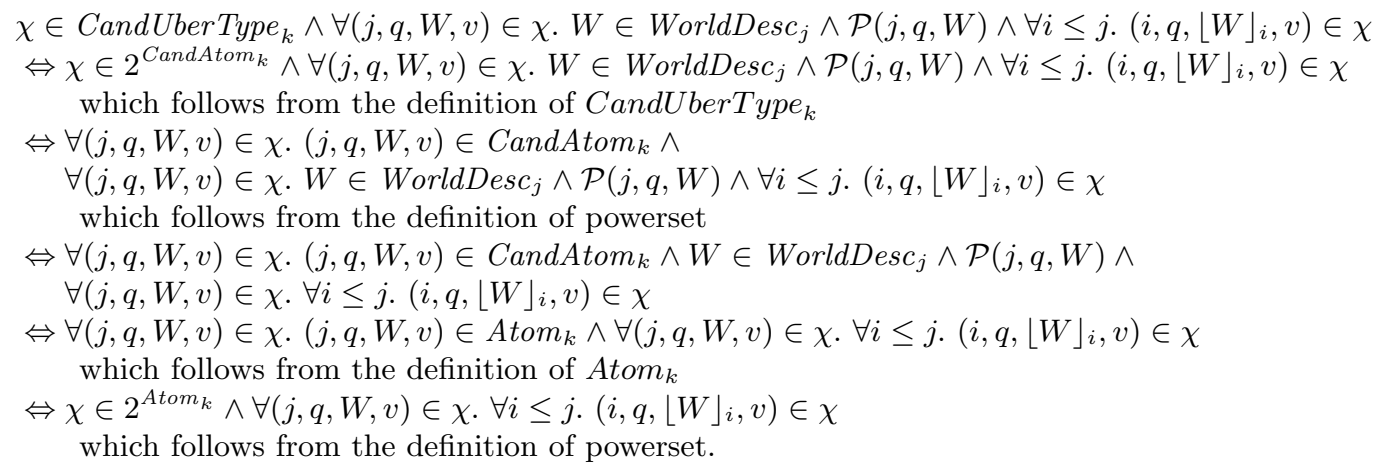

Hence,

$$
\chi \in 2^{\text {Atom }_{k}} \quad \text { and } \quad \forall(j, q, W, v) \in \chi \cdot \forall i \leq j .\left(i, q,\lfloor W\rfloor_{i}, v\right) \in \chi .
$$

Thus

$$
\chi \in \text { PreType }_{k},
$$

which follows from the definition of PreType ${ }_{k}$, and

$\left\{\chi \in\right.$ CandUberType $_{k} \mid \forall(j, q, W, v) \in \chi . W \in$ WorldDesc $\left._{j} \wedge \mathcal{P}(j, q, W) \wedge \forall i \leq j .\left(i, q,\lfloor W\rfloor_{i}, v\right) \in \chi\right\} \subseteq$ PreType $_{k}$.

\section{Fact 5}

If $(j, q, W, v) \in \chi \in$ PreType, then $W \in$ WorldDesc $_{j}$ and $\mathcal{P}(j, q, W)$ and $\forall i \leq j .\left(i, q,\lfloor W\rfloor_{i}, v\right) \in \chi$.

\section{Proof}

Let $(j, q, W, v) \in \chi \in$ PreType.

Note that $\chi \in$ CandUberType $_{\omega}$ and $\forall k \geq 0 .\lfloor\chi\rfloor_{k} \in$ PreType $_{k}$, which follows from the definition of PreType.

Note that $(j, q, W, v) \in\lfloor\chi\rfloor_{j+1}$, which follows from the definition of $\lfloor\cdot\rfloor_{k}$.

Hence, $(j, q, W, v) \in\lfloor\chi\rfloor_{j+1} \in$ Pre Type $_{j+1}$.

By Fact 4, we conclude that $W \in \operatorname{WorldDesc}_{j}$ and $\mathcal{P}(j, q, W)$ and $\forall i \leq j .\left(i, q,\lfloor W\rfloor_{i}, v\right) \in\left\lfloor\lfloor\chi\rfloor_{j+1}\right\rfloor_{i}$.

By Fact 1 , we conclude that $\forall i \leq j .\left(i, q,\lfloor W\rfloor_{i}, v\right) \in \chi$.

\section{Fact 6}


If $(j, q, W, v) \in \chi \in$ Type, then $W \in$ WorldDesc $_{j}$ and $\mathcal{P}(j, q, W)$ and $\forall i \leq j .\left(i, q,\lfloor W\rfloor_{i}, v\right) \in \chi$.

Proof

Immediate from Fact 3 and Fact 5 . 


\section{A.6 Requirements}

1. aprx-idem

$\left\lfloor\lfloor W\rfloor_{k_{2}}\right\rfloor_{k_{1}}=\lfloor W\rfloor_{\min \left(k_{1}, k_{2}\right)}$.

2. models-closed

if $j \leq k$ and $w:_{k} W$, then $w:_{j} W$.

3. models-aprx

$w:_{k} W$ iff $w:_{k}\lfloor W\rfloor_{k}$.

4. join-closed

if $j \leq k$ and $\left(W_{1} \odot_{k} W_{2}=W_{3}\right)$, then $\left(W_{1} \odot_{j} W_{2}=\left\lfloor W_{3}\right\rfloor_{j}\right)$.

5. join-aprx

$\left(W_{1} \odot_{k} W_{2}=W_{3}\right)$ iff $\left(\left\lfloor W_{1}\right\rfloor_{k} \odot_{k} W_{2}=W_{3}\right)$ iff $\left(W_{1} \odot_{k}\left\lfloor W_{2}\right\rfloor_{k}=W_{3}\right)$ iff $\left(\left\lfloor W_{1}\right\rfloor_{k} \odot_{k}\left\lfloor W_{2}\right\rfloor_{k}=W_{3}\right)$.

6. join-commut

if $\left(W_{1} \odot_{k} W_{2}=W_{3}\right)$, then $\left(W_{2} \odot_{k} W_{1}=W_{3}\right)$.

7. join-assocl if $\left(W_{2} \odot_{k} W_{3}=W_{23}\right)$ and $\left(W_{1} \odot_{k} W_{23}=W_{123}\right)$,

then there exists $W_{12}$ such that $\left(W_{1} \odot_{k} W_{2}=W_{12}\right)$ and $\left(W_{12} \odot_{k} W_{3}=W_{123}\right)$.

8. join-assocr

if $\left(W_{1} \odot_{k} W_{1}=W_{12}\right)$ and $\left(W_{12} \odot_{k} W_{3}=W_{123}\right)$,

then there exists $W_{23}$ such that $\left(W_{2} \odot_{k} W_{3}=W_{23}\right)$ and $\left(W_{1} \odot_{k} W_{23}=W_{123}\right)$.

9. join-unit-left

$\left(\mathcal{U}_{\odot} \odot_{k} W=\lfloor W\rfloor k\right)$.

10. qualpred-closed

if $j \leq k$ and $\mathcal{P}(k, q, W)$, then $\mathcal{P}(j, q, W)$.

11. qualpred-aprx

$\mathcal{P}(k, q, W)$ iff $\mathcal{P}\left(k, q,\lfloor W\rfloor_{k}\right)$.

12. qualpred-join

if $\mathcal{P}\left(k, q, W_{1}\right)$ and $\mathcal{P}\left(k, q, W_{2}\right)$ and $\left(W_{1} \odot_{k} W_{2}=W_{3}\right)$, then $\mathcal{P}\left(k, q, W_{3}\right)$.

13. qualpred-qualsub

if $\mathcal{P}(k, q, W)$ and $q \preceq q^{\prime}$, then $\mathcal{P}\left(k, q^{\prime}, W\right)$.

14. qualpred-unr-unit

$\mathcal{P}\left(k, \mathrm{U}, \mathcal{U}_{\odot}\right)$.

15. qualpred-rel-join

if $\mathcal{P}(k, \mathrm{R}, W)$, then $\left.\left(W \odot_{k} W\right)=\lfloor W\rfloor_{k}\right)$.

16. qualpred-aff-models

if $\mathcal{P}\left(k, \mathrm{~A}, W_{1}\right)$ and $\left(W_{1} \odot_{k} W_{2}=W_{3}\right)$ and $w:_{k} W_{3}$, then $w:_{k} W_{2}$.

17. qualpred-lin

$\mathcal{P}(k, \mathrm{~L}, W)$.

Figure 15: Core Language - Requirements 


\section{A.7 Proofs}

\section{A.7.1 Miscellaneous}

\section{Lemma 7 (Core Language: Type-level substitution)}

Let $\Delta, \Delta^{\prime} \vdash \iota_{\alpha}: \kappa_{\alpha}$.

1. If $\Delta, \alpha: \kappa_{\alpha}, \Delta^{\prime} \vdash \iota: \kappa$, then $\Delta, \Delta^{\prime} \vdash \iota\left[\iota_{\alpha} / \alpha\right]: \kappa$.

2. If $\Delta, \alpha: \kappa_{\alpha}, \Delta^{\prime} \vdash \Gamma$, then $\Delta, \Delta^{\prime} \vdash \Gamma\left[\iota_{\alpha} / \alpha\right]$.

3. If $\Delta, \alpha: \kappa_{\alpha}, \Delta^{\prime} \vdash \xi_{1} \preceq \xi_{2}$, then $\Delta, \Delta^{\prime} \vdash \xi_{1}\left[\iota_{\alpha} / \alpha\right] \preceq \xi_{2}\left[\iota_{\alpha} / \alpha\right]$.

4. If $\Delta, \alpha: \kappa_{\alpha}, \Delta^{\prime} \vdash \tau \preceq \xi$, then $\Delta, \Delta^{\prime} \vdash \tau\left[\iota_{\alpha} / \alpha\right] \preceq \xi\left[\iota_{\alpha} / \alpha\right]$.

5. If $\Delta, \alpha: \kappa_{\alpha}, \Delta^{\prime} \vdash \Gamma \preceq \xi$, then $\Delta, \Delta^{\prime} \vdash \Gamma\left[\iota_{\alpha} / \alpha\right] \preceq \xi\left[\iota_{\alpha} / \alpha\right]$.

6. If $\Delta, \alpha: \kappa_{\alpha}, \Delta^{\prime} ; \Gamma \vdash e: \tau$, then $\Delta, \Delta^{\prime} ; \Gamma\left[\iota_{\alpha} / \alpha\right] \vdash e\left[\iota_{\alpha} / \alpha\right]: \tau\left[\iota_{\alpha} / \alpha\right]$.

\section{Proof (Core Language: Type-level substitution)}

Let $\Delta, \Delta^{\prime} \vdash \iota_{\alpha}: \kappa_{\alpha}$.

1. Proceed by induction on the derivation $\Delta, \Delta^{\prime} \vdash \iota: \kappa$.

Case (UserPTy)... :

Case (UserTerm)...:

End Case

2. Proceed by induction on the derivation $\Delta, \Delta^{\prime} \vdash \Gamma$.

3. Proceed by induction on the derivation $\Delta, \Delta^{\prime} \vdash \xi_{1} \preceq \xi_{2}$.

Case $\frac{\Delta, \alpha: \kappa_{\alpha}, \Delta^{\prime} \vdash \xi: \text { QUAL }}{\Delta, \alpha: \kappa_{\alpha}, \Delta^{\prime} \vdash U \preceq \xi}, \frac{\Delta, \alpha: \kappa_{\alpha}, \Delta^{\prime} \vdash \xi: \text { QUAL }}{\Delta, \alpha: \kappa_{\alpha}, \Delta^{\prime} \vdash \xi \preceq \mathrm{L}}, \frac{\Delta, \alpha: \kappa_{\alpha}, \Delta^{\prime} \vdash \xi: \text { QUAL }}{\Delta, \alpha: \kappa_{\alpha}, \Delta^{\prime} \vdash \xi \preceq \xi}:$

Applying Lemma 7.1 to $\Delta, \alpha: \kappa_{\alpha}, \Delta^{\prime} \vdash \xi:$ QUAL, we conclude that $\Delta, \Delta^{\prime} \vdash \xi\left[\iota_{\alpha} / \alpha\right]:$ QUAL. Hence,

$$
\frac{\Delta, \Delta^{\prime} \vdash \xi\left[\iota_{\alpha} / \alpha\right]: \text { QUAL }}{\Delta, \Delta^{\prime} \vdash \mathrm{U} \preceq \xi\left[\iota_{\alpha} / \alpha\right]} \quad \frac{\Delta, \Delta^{\prime} \vdash \xi\left[\iota_{\alpha} / \alpha\right]: \text { QUAL }}{\Delta, \Delta^{\prime} \vdash \xi\left[\iota_{\alpha} / \alpha\right] \preceq \mathrm{L}} \quad \frac{\Delta, \Delta^{\prime} \vdash \xi\left[\iota_{\alpha} / \alpha\right]: \text { QUAL }}{\Delta, \Delta^{\prime} \vdash \xi\left[\iota_{\alpha} / \alpha\right] \preceq \xi\left[\iota_{\alpha} / \alpha\right]}
$$

Case $\frac{q_{1} \preceq q_{2}}{\Delta, \alpha: \kappa_{\alpha}, \Delta^{\prime} \vdash q_{1} \preceq q_{2}}:$ Immediate.

Case $\frac{\Delta, \alpha: \kappa_{\alpha}, \Delta^{\prime} \vdash \xi_{1} \preceq \xi^{\prime} \quad \Delta, \alpha: \kappa_{\alpha}, \Delta^{\prime} \vdash \xi^{\prime} \preceq \xi_{2}}{\Delta, \alpha: \kappa_{\alpha}, \Delta^{\prime} \vdash \xi_{1} \preceq \xi_{2}}$

Applying the induction hypothesis to $\Delta, \alpha: \kappa_{\alpha}, \Delta^{\prime} \vdash \xi_{1} \preceq \xi^{\prime}$, we conclude that $\Delta, \Delta^{\prime} \vdash$ $\xi_{1}\left[\iota_{\alpha} / \alpha\right] \preceq \xi^{\prime}\left[\iota_{\alpha} / \alpha\right]$.

Applying the induction hypothesis to $\Delta, \alpha: \kappa_{\alpha}, \Delta^{\prime} \vdash \xi^{\prime} \preceq \xi_{2}$, we conclude that $\Delta, \Delta^{\prime} \vdash$ $\xi^{\prime}\left[\iota_{\alpha} / \alpha\right] \preceq \xi_{2}\left[\iota_{\alpha} / \alpha\right]$.

Hence

$$
\frac{\Delta, \Delta^{\prime} \vdash \xi_{1}\left[\iota_{\alpha} / \alpha\right] \preceq \xi^{\prime}\left[\iota_{\alpha} / \alpha\right] \quad \Delta, \Delta^{\prime} \vdash \xi^{\prime}\left[\iota_{\alpha} / \alpha\right] \preceq \xi_{2}\left[\iota_{\alpha} / \alpha\right]}{\Delta, \Delta^{\prime} \vdash \xi_{1}\left[\iota_{\alpha} / \alpha\right] \preceq \xi_{2}\left[\iota_{\alpha} / \alpha\right]}
$$

\section{End Case}

4. Proceed by induction on the derivation $\Delta \vdash \tau \preceq \xi$.

Case $\frac{\Delta, \alpha: \kappa_{\alpha}, \Delta^{\prime} \vdash \tau: \text { TYPE }}{\Delta, \alpha: \kappa_{\alpha}, \Delta^{\prime} \vdash \tau \preceq \mathrm{L}}$ :

Applying Lemma 7.1 to $\Delta, \alpha: \kappa_{\alpha}, \Delta^{\prime} \vdash \tau$ : TYPE, we conclude that $\Delta, \Delta^{\prime} \vdash \tau\left[\iota_{\alpha} / \alpha\right]:$ TYPE. Hence,

$$
\frac{\Delta, \Delta^{\prime} \vdash \tau\left[\iota_{\alpha} / \alpha\right]: \text { TYPE }}{\Delta, \Delta^{\prime} \vdash \tau\left[\iota_{\alpha} / \alpha\right] \preceq \mathrm{L}}
$$


Case $\frac{\Delta, \alpha: \kappa_{\alpha}, \Delta^{\prime} \vdash \bar{\tau}^{\prime}: \text { PRETYPE } \quad \Delta, \alpha: \kappa_{\alpha}, \Delta^{\prime} \vdash \xi^{\prime} \preceq \xi}{\Delta, \alpha: \kappa_{\alpha}, \Delta^{\prime} \vdash \xi^{\prime} \bar{\tau}^{\prime} \preceq \xi}:$

Applying Lemma 7.1 to $\Delta, \alpha: \kappa_{\alpha}, \Delta^{\prime} \vdash \bar{\tau}^{\prime}$ : PRETYPE, we conclude that $\Delta, \Delta^{\prime} \vdash \bar{\tau}^{\prime}\left[\iota_{\alpha} / \alpha\right]$ : PRETYPE.

Applying Lemma 7.3 to $\Delta, \alpha: \kappa_{\alpha}, \Delta^{\prime} \vdash \xi^{\prime} \preceq \xi$, we conclude that $\Delta, \Delta^{\prime} \vdash \xi^{\prime}\left[\iota_{\alpha} / \alpha\right] \preceq \xi\left[\iota_{\alpha} / \alpha\right]$. Note that $\xi^{\prime} \bar{\tau}^{\prime}\left[\iota_{\alpha} / \alpha\right] \equiv \xi^{\prime}\left[\iota_{\alpha} / \alpha\right] \bar{\tau}^{\prime}\left[\iota_{\alpha} / \alpha\right]$.

Hence,

$$
\frac{\Delta, \Delta^{\prime} \vdash \bar{\tau}^{\prime}\left[\iota_{\alpha} / \alpha\right]: \text { PRETYPE } \quad \Delta, \Delta^{\prime} \vdash \xi^{\prime}\left[\iota_{\alpha} / \alpha\right] \preceq \xi\left[\iota_{\alpha} / \alpha\right]}{\Delta, \Delta^{\prime} \vdash{ }^{\xi^{\prime}} \bar{\tau}^{\prime}\left[\iota_{\alpha} / \alpha\right] \preceq \xi\left[\iota_{\alpha} / \alpha\right]}
$$

\section{End Case}

5. Proceed by induction on the derivation $\Delta \vdash \Gamma \preceq \xi$.

6. Proceed by induction on the derivation $\Delta ; \Gamma \vdash e: \tau$.

Case (UserVal)... :

Case (UserExp)... :

End Case 


\section{A.7.2 Validity of Kinding Rules}

Lemma 8 (Core Language: $\mathcal{T} \llbracket \Delta \vdash \iota: \kappa \rrbracket \delta \in \mathcal{K} \llbracket \kappa \rrbracket$ )

Let $\delta \in \mathcal{D} \llbracket \Delta \rrbracket$ and $\Delta \vdash \iota: \kappa$.

Then $\mathcal{T} \llbracket \Delta \vdash \iota: \kappa \rrbracket \delta \in \mathcal{K} \llbracket \kappa \rrbracket$.

Proof (Core Language: $\mathcal{T} \llbracket \Delta \vdash \iota: \kappa \rrbracket \delta \in \mathcal{K} \llbracket \kappa \rrbracket$ )

Recall that $\mathcal{K} \llbracket \mathrm{QUAL} \rrbracket=$ Quals $=\{\mathrm{U}, \mathrm{R}, \mathrm{A}, \mathrm{L}\}$; hence, for $\kappa \equiv \mathrm{QUAL}$, it suffices to prove the following: $\mathcal{T} \llbracket \Delta \vdash \xi: \mathrm{QUAL} \rrbracket \delta \in$ Quals.

Recall that $\mathcal{K} \llbracket \mathrm{PRETYPE} \rrbracket=$ PreType; hence, for $\kappa \equiv$ PRETYPE, it suffices to prove the following:

$$
\mathcal{T} \llbracket \Delta \vdash \bar{\tau}: \mathrm{PRETYPE} \rrbracket \delta \in \text { PreType. }
$$

By the definition of PreType, it suffices to prove the following:

$$
\begin{aligned}
& \mathcal{T} \llbracket \Delta \vdash \bar{\tau}: \text { PRETYPE } \rrbracket \delta \in \text { CandUberType }{ }_{\omega} \wedge \\
& \forall k \geq 0 .\lfloor\mathcal{T} \llbracket \Delta \vdash \bar{\tau}: \text { PRETYPE } \rrbracket \delta\rfloor_{k} \in \text { PreType }_{k} .
\end{aligned}
$$

By Fact 4, it suffices to prove the following:

$$
\begin{aligned}
& \mathcal{T} \llbracket \Delta \vdash \bar{\tau}: \mathrm{PRETYPE} \rrbracket \delta \in \text { CandUberType }{ }_{\omega} \wedge \\
& \forall k \geq 0 .\lfloor\mathcal{T} \llbracket \Delta \vdash \bar{\tau}: \mathrm{PRETYPE} \rrbracket \delta\rfloor_{k} \in \text { CandUberType }{ }_{k} \wedge \\
& \forall(j, q, W, v) \in\lfloor\mathcal{T} \llbracket \Delta \vdash \bar{\tau}: \mathrm{PRETYPE} \rrbracket \delta\rfloor_{k} . W \in \text { WorldDesc }_{j} \wedge \mathcal{P}(j, q, W) \wedge \\
& \forall i \leq j .\left(i, q,\lfloor W\rfloor_{i}, v\right) \in\lfloor\mathcal{T} \llbracket \Delta \vdash \bar{\tau}: \mathrm{PRETYPE} \rrbracket \delta\rfloor_{k} .
\end{aligned}
$$

By the fact that $\forall k \geq 0 .\lfloor\cdot\rfloor_{k} \in$ CandUberType $_{\omega} \rightarrow$ CandUberType $_{k}$, it suffices to prove the following:

$$
\begin{aligned}
& \mathcal{T} \llbracket \Delta \vdash \bar{\tau}: \mathrm{PRETYPE} \rrbracket \delta \in \text { CandUberType }_{\omega} \wedge \\
& \forall k \geq 0 . \forall(j, q, W, v) \in\lfloor\mathcal{T} \llbracket \Delta \vdash \bar{\tau}: \mathrm{PRETYPE} \rrbracket \delta\rfloor_{k} . W \in \text { WorldDesc }_{j} \wedge \mathcal{P}(j, q, W) \wedge \\
& \forall i \leq j .\left(i, q,\lfloor W\rfloor_{i}, v\right) \in\lfloor\mathcal{T} \llbracket \Delta \vdash \bar{\tau}: \mathrm{PRETYPE} \rrbracket \delta\rfloor_{k} .
\end{aligned}
$$

By the definition of $\lfloor\cdot\rfloor_{k}$, it suffices to prove the following:

$$
\begin{aligned}
& \mathcal{T} \llbracket \Delta \vdash \bar{\tau}: \text { PRETYPE } \rrbracket \delta \in \text { CandUberType }_{\omega} \wedge \\
& \forall(j, q, W, v) \in \mathcal{T} \llbracket \Delta \vdash \bar{\tau}: \text { PRETYPE } \delta . \quad W \in \text { WorldDesc }_{j} \wedge \mathcal{P}(j, q, W) \wedge \\
& \forall i \leq j .\left(i, q,\lfloor W\rfloor_{i}, v\right) \in \mathcal{T} \llbracket \Delta \vdash \bar{\tau}: \text { PRETYPE } \rrbracket \delta .
\end{aligned}
$$

By the definition of CandUberType ${ }_{\omega}$, it suffices to prove the following:

$$
\begin{aligned}
\mathcal{T} \llbracket \Delta \vdash \bar{\tau}: \mathrm{PRETYPE} \rrbracket \delta \in 2^{\text {CandAtom }_{\omega}} \wedge & \\
\forall(j, q, W, v) \in \mathcal{T} \llbracket \Delta \vdash \bar{\tau}: \mathrm{PRETYPE} \rrbracket \delta . & W \in \text { WorldDesc }_{j} \wedge \mathcal{P}(j, q, W) \wedge \\
& \forall i \leq j .\left(i, q,\lfloor W\rfloor_{i}, v\right) \in \mathcal{T} \llbracket \Delta \vdash \bar{\tau}: \mathrm{PRETYPE} \rrbracket \delta .
\end{aligned}
$$

By the definition of powerset, it suffices to prove the following:

$$
\begin{aligned}
\forall(j, q, W, v) \in \mathcal{T} \llbracket \Delta \vdash \bar{\tau}: \mathrm{PRETYPE} \rrbracket & \delta . \\
\forall(j, q, W, v) \in \mathcal{T} \llbracket \Delta \vdash \bar{\tau}: \mathrm{PRETYPE} \rrbracket & (j, W, v) \in \operatorname{CandAtom}_{\omega} \wedge \\
& \forall i \leq j .\left(i, q,\left\lfloor W \operatorname{World}_{j} \wedge \mathcal{P}(j, q) \in \mathcal{T} \llbracket \Delta \vdash \bar{\tau}: \mathrm{PRETYPE} \rrbracket \delta .\right.\right.
\end{aligned}
$$

By the definition of CandAtom $_{\omega}$, it suffices to prove the following:

$$
\begin{aligned}
\forall(j, q, W, v) \in \mathcal{T} \llbracket \Delta \vdash \bar{\tau}: \mathrm{PRETYPE} \rrbracket & \delta k \geq 0 .(j, q, W, v) \in \text { CandAtom }_{k} \wedge \\
\forall(j, q, W, v) \in \mathcal{T} \llbracket \Delta \vdash \bar{\tau}: \mathrm{PRETYPE} \rrbracket \delta . & W \in \text { WorldDesc }_{j} \wedge \mathcal{P}(j, q, W) \wedge \\
& \forall i \leq j .\left(i, q,\lfloor W\rfloor_{i}, v\right) \in \mathcal{T} \llbracket \Delta \vdash \bar{\tau}: \mathrm{PRETYPE} \rrbracket \delta .
\end{aligned}
$$

By the definition of CandAtom $_{k}$, it suffices to prove the following:

$$
\begin{aligned}
& \forall(j, q, W, v) \in \mathcal{T} \llbracket \Delta \vdash \bar{\tau}: \text { PRETYPE } \rrbracket . \exists k \geq 0 . j \in \mathbb{N} \wedge j<k \wedge q \in \text { Quals } \wedge \\
& W \in \bigcup_{i<k} \text { CandWorldDesc }_{i} \wedge W \in \text { CandWorldDesc }_{j} \wedge \\
& v \in C \text { Values } \wedge \\
& \forall(j, q, W, v) \in \mathcal{T} \llbracket \Delta \vdash \bar{\tau}: \mathrm{PRETYPE} \rrbracket \delta . W \in \text { WorldDesc }_{j} \wedge \mathcal{P}(j, q, W) \wedge \\
& \forall i \leq j .\left(i, q,\lfloor W\rfloor_{i}, v\right) \in \mathcal{T} \llbracket \Delta \vdash \bar{\tau}: \mathrm{PRETYPE} \rrbracket \delta .
\end{aligned}
$$


By logical equivalence, it suffices to prove the following:

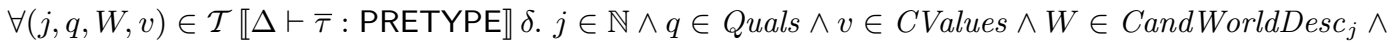

$$
\begin{aligned}
& \exists k \geq 0 . j<k \wedge W \in \bigcup_{i<k} \text { Cand WorldDesc }_{i} \wedge \\
& \forall(j, q, W, v) \in \mathcal{T} \llbracket \Delta \vdash \bar{\tau}: \mathrm{PRETYPE} \rrbracket \delta . W \in \text { WorldDesc }_{j} \wedge \mathcal{P}(j, q, W) \wedge \\
& \forall i \leq j .\left(i, q,\lfloor W\rfloor_{i}, v\right) \in \mathcal{T} \llbracket \Delta \vdash \bar{\tau}: \mathrm{PRETYPE} \rrbracket \delta .
\end{aligned}
$$

Taking $k=j+1$, it suffices to prove the following:

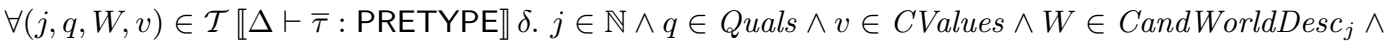

$$
\begin{aligned}
& j<j+1 \wedge W \in \bigcup_{i<j+1} \text { CandWorldDesc }_{i} \wedge \\
& \forall(j, q, W, v) \in \mathcal{T} \llbracket \Delta \vdash \bar{\tau}: \mathrm{PRETYPE} \rrbracket \delta . W \in \text { WorldDesc }_{j} \wedge \mathcal{P}(j, q, W) \wedge \\
& \forall i \leq j .\left(i, q,\lfloor W\rfloor_{i}, v\right) \in \mathcal{T} \llbracket \Delta \vdash \bar{\tau}: \mathrm{PRETYPE} \rrbracket \delta .
\end{aligned}
$$

Since CandWorldDesc $C_{j} \subseteq \bigcup_{i<j+1}$ CandWorldDesc $_{i}$ and $j<j+1$, it suffices to prove the following:

$$
\begin{aligned}
\forall(j, q, W, v) & \in \mathcal{T} \llbracket \Delta \vdash \bar{\tau}: \text { PRETYPE } \llbracket \delta . j \in \mathbb{N} \wedge q \in \text { Quals } \wedge v \in \text { CValues } \wedge W \in \text { CandWorldDesc }_{j} \wedge \\
\forall(j, q, W, v) \in \mathcal{T} \llbracket \Delta \vdash \bar{\tau}: \text { PRETYPE } \delta . & W \in \text { WorldDesc }_{j} \wedge \mathcal{P}(j, q, W) \wedge \\
& \forall i \leq j .\left(i, q,\lfloor W\rfloor_{i}, v\right) \in \mathcal{T} \llbracket \Delta \vdash \bar{\tau}: \text { PRETYPE } \rrbracket .
\end{aligned}
$$

By logical equivalence, it suffices to prove the following:

$$
\begin{aligned}
\forall(j, q, W, v) \in \mathcal{T} \llbracket \Delta \vdash \bar{\tau}: \text { PRETYPE } \rrbracket \delta . & j \in \mathbb{N} \wedge q \in \text { Quals } \wedge v \in \text { CValues } \wedge W \in \text { CandWorldDesc }_{j} \wedge \\
& W \in \text { WorldDesc }_{j} \wedge \mathcal{P}(j, q, W) \wedge \\
& \forall i \leq j .\left(i, q,\lfloor W\rfloor_{i}, v\right) \in \mathcal{T} \llbracket \Delta \vdash \bar{\tau}: \text { PRETYPE } \rrbracket \delta .
\end{aligned}
$$

Since WorldDesc $_{j} \subseteq$ CandWorldDesc $_{j}$, it suffices to prove the following:

$$
\begin{aligned}
\forall(j, q, W, v) \in \mathcal{T} \llbracket \Delta \vdash \bar{\tau}: \text { PRETYPE } \rrbracket . & j \in \mathbb{N} \wedge q \in \text { Quals } \wedge v \in \text { CValues } \wedge \\
& W \in \text { WorldDesc }_{j} \wedge \mathcal{P}(j, q, W) \wedge \\
& \forall i \leq j .\left(i, q,\lfloor W\rfloor_{i}, v\right) \in \mathcal{T} \llbracket \Delta \vdash \bar{\tau}: \text { PRETYPE } \rrbracket \delta .
\end{aligned}
$$

By change of variables, it suffices to prove the following:

$$
\begin{aligned}
\forall(k, q, W, v) \in \mathcal{T} \llbracket \Delta \vdash \bar{\tau}: \text { PRETYPE } \rrbracket & \delta, \quad k \in \mathbb{N} \wedge q \in \text { Quals } \wedge \wedge v \in \text { CValues } \wedge \\
& W \in \text { WorldDesc }_{k} \wedge \mathcal{P}(k, q, W) \wedge \\
& \forall j \leq k .\left(j, q,\lfloor W\rfloor_{j}, v\right) \in \mathcal{T} \llbracket \Delta \vdash \bar{\tau}: \text { PRETYPE } \rrbracket \delta .
\end{aligned}
$$

Since $k \in \mathbb{N}, q \in$ Quals, and $v \in$ CValues is obvious in all cases, it suffices to prove the following:

$$
\begin{aligned}
\forall(k, q, W, v) \in \mathcal{T} \llbracket \Delta \vdash \bar{\tau}: \mathrm{PRETYPE} \rrbracket & \delta . W \in \text { WorldDesc }_{k} \wedge \mathcal{P}(k, q, W) \wedge \\
& \forall j \leq k .\left(j, q,\lfloor W\rfloor_{j}, v\right) \in \mathcal{T} \llbracket \Delta \vdash \bar{\tau}: \mathrm{PRETYPE} \rrbracket \delta .
\end{aligned}
$$

Recall that $\mathcal{K} \llbracket \mathrm{TYPE} \rrbracket=$ Type; hence, for $\kappa \equiv$ TYPE, it suffices to prove the following:

$$
\mathcal{T} \llbracket \Delta \vdash \tau: \text { TYPE } \rrbracket \delta \in \text { Type. }
$$

By the definition of Type, it suffices to prove the following:

$$
\begin{aligned}
& \mathcal{T} \llbracket \Delta \vdash \tau: \text { TYPE } \rrbracket \delta \in \text { CandUberType }_{\omega} \wedge \\
& \forall k \geq 0 .\lfloor\mathcal{T} \llbracket \Delta \vdash \tau: \text { TYPE } \rrbracket \delta\rfloor_{k} \in \text { Type }_{k} .
\end{aligned}
$$

By the definition of Type $_{k}$, it suffices to prove the following:

$$
\begin{aligned}
& \mathcal{T} \llbracket \Delta \vdash \tau: \text { TYPE } \rrbracket \delta \in \text { CandUberType }_{\omega} \wedge \\
& \forall k \geq 0 . \quad\lfloor\mathcal{T} \llbracket \Delta \vdash \tau: \text { TYPE } \rrbracket \delta\rfloor_{k} \in \text { PreType }_{k} \wedge \\
& \quad \exists q^{\prime} \in \text { Quals. } \forall\left(\left(_{-}, q,,_{-},\right) \in\lfloor\mathcal{T} \llbracket \Delta \vdash \tau: \text { TYPE } \rrbracket \delta\rfloor_{k} . q=q^{\prime} .\right.
\end{aligned}
$$

By logical equivalence, it suffices to prove the following:

$$
\begin{aligned}
& \mathcal{T} \llbracket \Delta \vdash \tau: \text { TYPE } \rrbracket \delta \in \text { CandUberType }_{\omega} \wedge \\
& \forall k \geq 0 .\lfloor\mathcal{T} \llbracket \Delta \vdash \tau: \text { TYPE } \rrbracket \delta\rfloor_{k} \in \text { PreType }_{k} \wedge \\
& \forall k \geq 0 . \exists q^{\prime} \in \text { Quals. } \forall\left({ }_{-}, q,,_{-},\right) \in\lfloor\mathcal{T} \llbracket \Delta \vdash \tau: \text { TYPE } \rrbracket \delta\rfloor_{k} . q=q^{\prime} .
\end{aligned}
$$

By the definition of PreType, it suffices to prove the following: 


$$
\begin{aligned}
& \mathcal{T} \llbracket \Delta \vdash \tau: \text { TYPE } \rrbracket \delta \in \text { PreType } \wedge \\
& \forall k \geq 0 . \exists q^{\prime} \in \text { Quals. } \forall\left({ }_{-}, q,_{-},{ }_{-}\right) \in\lfloor\mathcal{T} \llbracket \Delta \vdash \tau: \text { TYPE } \rrbracket \delta\rfloor_{k} . q=q^{\prime} .
\end{aligned}
$$

By the definition of $\lfloor\cdot\rfloor_{k}$, it suffices to prove the following:

$$
\begin{aligned}
& \mathcal{T} \llbracket \Delta \vdash \tau: \text { TYPE } \rrbracket \delta \in \text { PreType } \wedge \\
& \exists q^{\prime} \in \text { Quals. } \forall\left({ }_{-}, q,{ }_{-},-\right) \in \mathcal{T} \llbracket \Delta \vdash \tau: \text { TYPE } \rrbracket \delta . q=q^{\prime} .
\end{aligned}
$$

By logical equivalence, it suffices to prove the following:

$$
\begin{aligned}
\exists q^{\prime} \in \text { Quals. } & \mathcal{T} \llbracket \Delta \vdash \tau: \text { TYPE } \rrbracket \delta \in \text { PreType } \wedge \\
& \forall\left({ }_{-}, q,,_{-},-\right) \in \mathcal{T} \llbracket \Delta \vdash \tau: \text { TYPE } \rrbracket \delta . q=q^{\prime} .
\end{aligned}
$$

By the reasoning above for $\mathcal{T} \llbracket \Delta \vdash \bar{\tau}$ : PRETYPE $\rrbracket \in$ PreType, it suffices to prove the following:

$$
\begin{aligned}
& \exists q^{\prime} \in \text { Quals. } \forall(k, q, W, v) \in \mathcal{T} \llbracket \Delta \vdash \tau: \text { TYPE } \rrbracket \delta . W \in \text { WorldDesc }_{k} \wedge \mathcal{P}(k, q, W) \wedge \\
& \forall j \leq k .\left(j, q,\lfloor W\rfloor_{j}, v\right) \in \mathcal{T} \llbracket \Delta \vdash \bar{\tau}: \text { PRETYPE } \rrbracket \delta \wedge \\
& \forall\left({ }_{-}, q,_{-},{ }_{-}\right) \in \mathcal{T} \llbracket \Delta \vdash \tau: \text { TYPE } \rrbracket \delta . q=q^{\prime} .
\end{aligned}
$$

By logical equivalence, it suffices to prove the following:

$$
\begin{aligned}
\exists q^{\prime} \in \text { Quals. } \forall(k, q, W, v) \in \mathcal{T} \llbracket \Delta \vdash \tau: \text { TYPE } \rrbracket \delta . & W \in W_{\text {orldDesc }} \wedge \mathcal{P}(k, q, W) \wedge \\
& \forall j \leq k .\left(j, q,\lfloor W\rfloor_{j}, v\right) \in \mathcal{T} \llbracket \Delta \vdash \bar{\tau}: \text { PRETYPE } \rrbracket \wedge \\
& q=q^{\prime} .
\end{aligned}
$$

Proceed by induction on the derivation $\Delta \vdash \iota: \kappa$.

$($ VARKN)

Case $\frac{\alpha: \kappa \in \Delta}{\Delta \vdash \alpha: \kappa}:$

Note that $\delta(\alpha) \in \mathcal{K} \llbracket \kappa \rrbracket$, which follows from $\alpha: \kappa \in \Delta$ and $\delta \in \mathcal{D} \llbracket \Delta \rrbracket$.

Hence, $\mathcal{T} \llbracket \Delta \vdash \alpha:$ TYPE $\rrbracket \delta=\delta(\alpha) \in \mathcal{K} \llbracket \kappa \rrbracket$.

Case $\frac{(\mathrm{QUAL})}{\Delta \vdash q: \mathrm{QUAL}}$ :

Hence, $\mathcal{T} \llbracket \Delta \vdash q: \mathrm{QUAL} \rrbracket \delta=q \in$ Quals.

(FNPTY)

Case $\frac{\Delta \vdash \tau_{1}: \text { TYPE } \quad \Delta \vdash \tau_{2}: \text { TYPE }}{\Delta \vdash \tau_{1} \multimap \tau_{2}: \text { PRETYPE }}$

Recall that

$$
\begin{aligned}
& \mathcal{T} \llbracket \frac{\Delta \vdash \tau_{1}: \text { TYPE } \quad \Delta \vdash \tau_{2}: \text { TYPE }}{\Delta \vdash \tau_{1} \multimap \tau_{2}: \text { PRETYPE }} \rrbracket \delta= \\
& \left\{\left(k, q_{c}, W_{c}, \lambda x . e\right) \mid W_{c} \in \text { WorldDesc }_{k} \wedge \mathcal{P}\left(k, q_{c}, W_{c}\right) \wedge\right. \\
& \forall i<k, q_{a}, W_{a}, v_{a} . \\
& \left(i, q_{a}, W_{a}, v_{a}\right) \in \mathcal{T} \llbracket \Delta \vdash \tau_{1}: \text { TYPE } \rrbracket \delta \wedge \\
& \left(W_{c} \odot_{i} W_{a}\right) \text { defined } \Rightarrow \\
& \left.\operatorname{Comp}\left(i,\left(W_{c} \odot_{i} W_{a}\right), e\left[v_{a} / x\right], \mathcal{T} \llbracket \Delta \vdash \tau_{2}: \text { TYPE } \rrbracket \delta\right)\right\}
\end{aligned}
$$

Consider arbitrary $(k, q, W, v) \in \mathcal{T} \llbracket \Delta \vdash \tau_{1} \multimap \tau_{2}:$ PRETYPE $\rrbracket \delta$.

Hence, $v \equiv \lambda x$.e and $W \in$ WorldDesc $_{k}$ and $\mathcal{P}(k, q, W)$.

We are required to show that

- $W \in$ WorldDesc $_{k}$, which follows from above, and

- $\mathcal{P}(k, q, W)$, which follows from above.

Consider arbitrary $j \leq k$.

We are required to show that $\left(j, q,\lfloor W\rfloor_{j}, \lambda x . e\right) \in \mathcal{T} \llbracket \Delta \vdash \tau_{1} \multimap \tau_{2}:$ PRETYPE $\rrbracket \delta$.

Note that $\lfloor W\rfloor_{j} \in$ WorldDesc $_{j}$, which follows from $\lfloor\cdot\rfloor_{j} \in$ WorldDesc $\rightarrow$ WorldDesc $_{j}$.

Note that $\mathcal{P}(j, q,\lfloor W\rfloor j)$, which follows from Req 10 (qualpred-closed) and Req 11 (qualpred-aprx).

Consider arbitrary $i, q_{a}, W_{a}$, and $v_{a}$ such that 
- $i<j$,

- $\left(i, q_{a}, W_{a}, v_{a}\right) \in \mathcal{T} \llbracket \Delta \vdash \tau_{1}: \mathrm{TYPE} \rrbracket \delta$, and,

- $\left(\lfloor W\rfloor_{j} \odot_{i} W_{a}\right)$ defined.

We are required to show that $\operatorname{Comp}\left(i,\left(\lfloor W\rfloor_{j} \odot_{i} W_{a}\right), e\left[v_{a} / x\right], \mathcal{T} \llbracket \Delta \vdash \tau_{2}: \operatorname{TYPE} \rrbracket \delta\right)$.

Instantiate $(k, q, W, \lambda x . e) \in \mathcal{T} \llbracket \Delta \vdash \tau_{1} \multimap \tau_{2}:$ PRETYPE $\rrbracket \delta$ with $i, q_{a}, W_{a}$, and $v_{a}$. Note that

- $i<k$, which follows from $i<j$ and $j \leq k$,

- $\left(i, q_{a}, W_{a}, v_{a}\right) \in \mathcal{T} \llbracket \Delta \vdash \tau_{1}:$ TYPE $\rrbracket \delta$, which follows from above, and

- $\left(W \odot_{i} W_{a}\right)$ defined, which follows from Req 5 (join-aprx) and Req 1 (aprx-idem) and $i<j$ and $\left(\lfloor W\rfloor_{j} \odot_{i} W_{a}\right)$ defined, which in turn follows from above.

Hence, $\operatorname{Comp}\left(i,\left(W \odot_{i} W_{a}\right), e\left[v_{a} / x\right], \mathcal{T} \llbracket \Delta \vdash \tau_{2}: \mathrm{TYPE} \rrbracket \delta\right)$.

Note that $\left(\lfloor W\rfloor_{j} \odot_{i} W_{a}\right) \equiv\left(W \odot_{i} W_{a}\right)$, which follows from Req 5 (join-aprx) and Req 1 (aprx-idem) and $i<j$ and $\left(\lfloor W\rfloor_{j} \odot_{i} W_{a}\right)$ defined, which in turn follows from above.

Hence, $\operatorname{Comp}\left(i,\left(\lfloor W\rfloor_{j} \odot_{i} W_{a}\right), e\left[v_{a} / x\right], \mathcal{T} \llbracket \Delta \vdash \tau_{2}:\right.$ TYPE $\left.\rrbracket \delta\right)$.

Case $\frac{(\text { MUNITPTY })}{\Delta \vdash \mathbf{1}_{\otimes}: \text { PRETYPE }}$ :

Recall that

$$
\mathcal{T} \llbracket \frac{}{\Delta \vdash \mathbf{1}_{\otimes}: \mathrm{PRETYPE}} \rrbracket \delta=\left\{(k, q, W,\langle\rangle) \mid W=\left\lfloor\mathcal{U}_{\odot}\right\rfloor_{k}\right\}
$$

Consider arbitrary $(k, q, W, v) \in \mathcal{T} \llbracket \Delta \vdash \mathbf{1}_{\otimes}:$ PRETYPE $\rrbracket \delta$.

Hence, $v \equiv\langle\rangle$ and $W=\left\lfloor\mathcal{U}_{\odot}\right\rfloor_{k}$.

We are required to show that

- $W \in$ WorldDesc $_{k}$, which follows from $\mathcal{U}_{\odot} \in$ WorldDesc and $\lfloor\cdot\rfloor_{k} \in$ WorldDesc $\rightarrow$ WorldDesc $_{k}$,

- $\mathcal{P}\left(k, q,\left\lfloor\mathcal{U}_{\odot}\right\rfloor_{k}\right)$, which follows from Req 14 (qualpred-unr-unit), Req 13 (qualpred-qualsub) and $\mathrm{U} \preceq q$, and Req 11 (qualpred-aprx).

Consider arbitrary $j \leq k$.

We are required to show that $\left(j, q,\lfloor W\rfloor_{j},\langle\rangle\right) \in \mathcal{T} \llbracket \Delta \vdash \mathbf{1}_{\otimes}:$ PRETYPE $\rrbracket$. Note that

- $\lfloor W\rfloor_{j}=\left\lfloor\mathcal{U}_{\odot}\right\rfloor_{j}$, which follows from Req 1 (aprx-idem) and $j<k$ and $W=\left\lfloor\mathcal{U}_{\odot}\right\rfloor_{k}$, which in turn follows from above.

(MPAIRPTY)

Case $\frac{\Delta \vdash \tau_{1}: \text { TYPE } \quad \Delta \vdash \tau_{2}: \text { TYPE }}{\Delta \vdash \tau_{1} \otimes \tau_{2}: \text { PRETYPE }}$

Recall that

$$
\mathcal{T} \llbracket \frac{\Delta \vdash \tau_{1}: \text { TYPE } \quad \Delta \vdash \tau_{2}: \text { TYPE }}{\Delta \Delta \vdash \tau_{1} \otimes \tau_{2}: \operatorname{PRETYPE}} \rrbracket \delta=\left\{\begin{array}{l}
\left(k, q, W,\left\langle v_{1}, v_{2}\right\rangle\right) \mid \\
\left(k, q_{1}, W_{1}, v_{1}\right) \in \mathcal{T} \llbracket \Delta \vdash \tau_{1}: \text { TYPE } \rrbracket \wedge \wedge \\
\left(k, q_{2}, W_{2}, v_{2}\right) \in \mathcal{T} \llbracket \Delta \vdash \tau_{2}: \text { TYPE } \rrbracket \wedge \\
q_{1} \preceq q \wedge q_{2} \preceq q \wedge \\
\left.\left(W_{1} \odot_{k} W_{2}=W\right)\right\}
\end{array}\right.
$$

Consider arbitrary $(k, q, W, v) \in \mathcal{T} \llbracket \Delta \vdash \tau_{1} \otimes \tau_{2}:$ PRETYPE $\rrbracket \delta$.

Hence, $\quad v \equiv\left\langle v_{1}, v_{2}\right\rangle$ and $\left(k, q_{1}, W_{1}, v_{1}\right) \in \mathcal{T} \llbracket \Delta \vdash \tau_{1}:$ TYPE $\rrbracket \delta$ and $\left(k, q_{2}, W_{2}, v_{2}\right) \quad \in$ $\mathcal{T} \llbracket \Delta \vdash \tau_{2}:$ TYPE $\rrbracket \delta$ and $q_{1} \preceq q$ and $q_{2} \preceq q$ and $\left(W_{1} \odot_{k} W_{2}=W\right)$.

Applying the induction hypothesis to $\Delta \vdash \tau_{1}$ : TYPE, we conclude that $\mathcal{T} \llbracket \Delta \vdash \tau_{1}:$ TYPE $\rrbracket \delta \in$ Type. 
Applying Fact 6 to $\left(k, q_{1}, W_{1}, v_{1}\right) \in \mathcal{T} \llbracket \Delta \vdash \tau_{1}:$ TYPE $\rrbracket \delta \in$ Type, we conclude that $W_{1} \in$ WorldDesc $_{k}$ and $\mathcal{P}\left(k, q_{1}, W_{1}\right)$ and $\forall j \leq k .\left(j, q_{1},\left\lfloor W_{1}\right\rfloor_{j}, v_{1}\right) \in \mathcal{T} \llbracket \Delta \vdash \tau_{1}:$ TYPE $\rrbracket \delta$.

Note that $\mathcal{P}\left(k, q, W_{1}\right)$, which follows from Req 13 (qualpred-qualsub) and $q_{1} \preceq q$.

Applying the induction hypothesis to $\Delta \vdash \tau_{2}$ : TYPE, we conclude that $\mathcal{T} \llbracket \Delta \vdash \tau_{2}:$ TYPE $\rrbracket \delta \in$ Type.

Applying Fact 6 to $\left(k, q_{2}, W_{2}, v_{2}\right) \in \mathcal{T} \llbracket \Delta \vdash \tau_{2}:$ TYPE $\rrbracket \delta \in$ Type, we conclude that $W_{2} \in$ WorldDesc $_{k}$ and $\mathcal{P}\left(k, q_{2}, W_{2}\right)$ and $\forall j \leq k .\left(j, q_{2},\left\lfloor W_{2}\right\rfloor_{j}, v_{2}\right) \in \mathcal{T} \llbracket \Delta \vdash \tau_{2}:$ TYPE $\rrbracket$.

Note that $\mathcal{P}\left(k, q, W_{2}\right)$, which follows from Req 13 (qualpred-qualsub) and $q_{2} \preceq q$.

We are required to show that

- $W \in$ WorldDesc $_{k}$, which follows from $W_{1} \in$ WorldDesc $_{k}$ and $W_{2} \in$ WorldDesc $_{k}$ and $\left(\cdot \odot_{k} \cdot\right) \in$ WorldDesc $\times$ WorldDesc $\rightarrow$ WorldDesc $_{k}$, and

- $\mathcal{P}(k, q, W)$, which follows from Req 12 (qualpred-join).

Consider arbitrary $j \leq k$.

We are required to show that $\left(j, q,\lfloor W\rfloor j,\left\langle v_{1}, v_{2}\right\rangle\right) \in \mathcal{T} \llbracket \Delta \vdash \tau_{1} \otimes \tau_{2}:$ PRETYPE $\rrbracket \delta$. Note that

- $\left(j, q_{1},\left\lfloor W_{1}\right\rfloor_{j}, v_{1}\right) \in \mathcal{T} \llbracket \Delta \vdash \tau_{1}:$ TYPE $\rrbracket \delta$, which follows from above, noting that $j \leq k$,

- $\left(j, q_{2},\left\lfloor W_{2}\right\rfloor_{j}, v_{2}\right) \in \mathcal{T} \llbracket \Delta \vdash \tau_{2}:$ TYPE $\rrbracket \delta$, which follows from above, noting that $j \leq k$,

- $q_{1} \preceq q$,

- $q_{2} \preceq q$, and

- $\left(\left\lfloor W_{1}\right\rfloor_{j} \odot_{j}\left\lfloor W_{2}\right\rfloor_{j}=\lfloor W\rfloor_{j}\right)$, which follows from Req 5 (join-aprx) and $\left(W_{1} \odot_{k} W_{2}=W\right)$, which in turn follows from above.

(AUNITPTy)

Case $\frac{\left(A \cup \mathbf{1}_{\circledast}: \mathrm{PRETYPE}\right.}{\Delta}$ :

Recall that

$$
\mathcal{T} \llbracket \frac{}{\Delta \vdash \mathbf{1}_{\circledast}: \text { PRETYPE }} \rrbracket \delta=\left\{\left(k, q, W,\langle\langle\rangle) \mid W \in \text { WorldDesc }_{k} \wedge \mathcal{P}(k, q, W)\right\}\right.
$$

Consider arbitrary $(k, q, W, v) \in \mathcal{T} \llbracket \Delta \vdash \mathbf{1}_{\circledast}:$ PRETYPE $\rrbracket \delta$.

Hence, $v \equiv\left\langle\langle\rangle\right.$ and $W \in$ WorldDesc $_{k}$ and $\mathcal{P}(k, q, W)$

We are required to show that

- $W \in$ WorldDesc $_{k}$, which follows from above, and

- $\mathcal{P}(k, q, W)$, which follows from above.

Consider arbitrary $j \leq k$.

We are required to show that $\left(j, q,\lfloor W\rfloor_{j},\langle\langle\rangle\rangle\right) \in \mathcal{T} \llbracket \Delta \vdash \mathbf{1}_{\circledast}:$ PRETYPE $\rrbracket \delta$. Note that

- $\lfloor W\rfloor_{j} \in$ WorldDesc $_{j}$, which follows from $\lfloor\cdot\rfloor_{j} \in$ WorldDesc $\rightarrow$ WorldDesc $_{j}$, and

- $\mathcal{P}\left(j, q,\lfloor W\rfloor_{j}\right)$, which follows from Req 10 (qualpred-closed) and Req 11 (qualpred-aprx).

(APAIRPTY)

Case $\frac{\Delta \vdash \tau_{1}: \text { TYPE } \quad \Delta \vdash \tau_{2}: \text { TYPE }}{\Delta \vdash \tau_{1} \circledast \tau_{2}: \text { PRETYPE }}$

Recall that

$$
\mathcal{T} \llbracket \frac{\Delta \vdash \tau_{1}: \text { TYPE } \quad \Delta \vdash \tau_{2}: \text { TYPE }}{\Delta \vdash \tau_{1} \circledast \tau_{2}: \operatorname{PRETYPE}} \rrbracket \delta=\left\{\begin{array}{c}
\left(k, q, W,\left\langle\left\langle e_{1}, e_{2}\right\rangle\right\rangle\right) \mid W \in \operatorname{WorldDesc}_{k} \wedge \mathcal{P}(k, q, W) \wedge \\
\forall i<k . \\
\operatorname{Comp}\left(i,\lfloor W\rfloor_{i}, e_{1}, \mathcal{T} \llbracket \Delta \vdash \tau_{1}: \operatorname{TYPE} \rrbracket \delta\right) \wedge \\
\left.\operatorname{Comp}\left(i,\lfloor W]_{i}, e_{2}, \mathcal{T} \llbracket \Delta \vdash \tau_{2}: \text { TYPE } \rrbracket \delta\right)\right\}
\end{array}\right.
$$


Consider arbitrary $(k, q, W, v) \in \mathcal{T} \llbracket \Delta \vdash \tau_{1} \circledast \tau_{2}:$ PRETYPE $\rrbracket \delta$.

Hence, $v \equiv\left\langle\left\langle e_{1}, e_{2}\right\rangle\right\rangle$ and $W \in$ WorldDesc $_{k}$ and $\mathcal{P}(k, q, W)$.

We are required to show that

- $W \in$ WorldDesc $_{k}$, which follows from above, and

- $\mathcal{P}(k, q, W)$, which follows from above.

Consider arbitrary $j \leq k$.

We are required to show that $\left(j, q,\lfloor W\rfloor_{j},\left\langle\left\langle e_{1}, e_{2}\right\rangle\right\rangle\right) \in \mathcal{T} \llbracket \Delta \vdash \tau_{1} \circledast \tau_{2}:$ PRETYPE $\rrbracket \delta$.

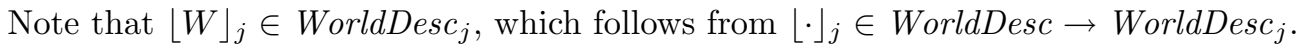

Note that $\mathcal{P}(j, q,\lfloor W\rfloor j)$, which follows from Req 10 (qualpred-closed) and Req 11 (qualpred-aprx).

Consider arbitrary $i$ such that

- $i<j$.

We are required to show that $\operatorname{Comp}\left(i,\left\lfloor\lfloor W\rfloor_{j}\right\rfloor_{i}, e_{1}, \mathcal{T} \llbracket \Delta \vdash \tau_{1}: \operatorname{TYPE} \rrbracket \delta\right)$ and $\operatorname{Comp}\left(i,\left\lfloor\lfloor W\rfloor_{j}\right\rfloor_{i}, e_{2}, \mathcal{T} \llbracket \Delta \vdash \tau_{2}:\right.$ TYPE $\left.\rrbracket \delta\right)$.

Instantiate $\left(k, q, W,\left\langle\left\langle e_{1}, e_{2}\right\rangle\right\rangle\right) \in \mathcal{T} \llbracket \Delta \vdash \tau_{1} \circledast \tau_{2}:$ PRETYPE $\rrbracket \delta$ with $i$.

Note that

- $i<k$, which follows from $i<j$ and $j \leq k$.

Hence, $\operatorname{Comp}\left(i,\lfloor W\rfloor_{i}, e_{1}, \mathcal{T} \llbracket \Delta \vdash \tau_{1}: \operatorname{TYPE} \rrbracket \delta\right)$ and $\operatorname{Comp}\left(i,\lfloor W\rfloor_{i}, e_{2}, \mathcal{T} \llbracket \Delta \vdash \tau_{2}: \operatorname{TYPE} \rrbracket \delta\right)$.

Note that $\left\lfloor\lfloor W\rfloor_{j}\right\rfloor_{i}=\lfloor W\rfloor_{i}$, which follows from Req 1 (aprx-idem) and $i<j$.

Hence, $\operatorname{Comp}\left(i,\left\lfloor\lfloor W\rfloor_{j}\right\rfloor_{i}, e_{1}, \mathcal{T} \llbracket \Delta \vdash \tau_{1}:\right.$ TYPE $\left.\rrbracket \delta\right)$ and $\operatorname{Comp}\left(i,\left\lfloor\lfloor W\rfloor_{j}\right\rfloor_{i}, e_{2}, \mathcal{T} \llbracket \Delta \vdash \tau_{2}:\right.$ TYPE $\left.\rrbracket \delta\right)$.

Case $\frac{(\text { VOIDPTY })}{\Delta \vdash \mathbf{0}: \text { PRETYPE }}$ :

Recall that

$$
\mathcal{T} \llbracket \frac{}{\Delta \vdash \mathbf{0}: \text { PRETYPE }} \rrbracket \delta=\{\}
$$

Vacuous, as there does not exist $(k, q, W, v) \in \mathcal{T} \llbracket \Delta \vdash \mathbf{0}:$ PRETYPE $\rrbracket$.

(SUMPTy)

Case $\frac{\Delta \vdash \tau_{1}: \text { TYPE } \quad \Delta \vdash \tau_{2}: \text { TYPE }}{\Delta \vdash \tau_{1} \oplus \tau_{2}: \text { PRETYPE }}$

Recall that

$$
\mathcal{T} \llbracket \frac{\Delta \vdash \tau_{1}: \text { TYPE } \quad \Delta \vdash \tau_{2}: \text { TYPE }}{\Delta \vdash \tau_{1} \oplus \tau_{2}: \text { PRETYPE }} \rrbracket \delta=\begin{gathered}
\left\{\begin{array}{c}
\left(k, q, W, \text { inl } v_{1}\right) \mid \\
\left.\left(k, q_{1}, W, v_{1}\right) \in \mathcal{T} \llbracket \Delta \vdash \tau_{1}: \text { TYPE } \rrbracket \delta \wedge q_{1} \preceq q\right\} \cup \\
\left\{\left(k, q, W, \text { inr } v_{2}\right) \mid\right. \\
\left.\left(k, q_{2}, W, v_{2}\right) \in \mathcal{T} \llbracket \Delta \vdash \tau_{2}: \text { TYPE } \rrbracket \delta \wedge q_{2} \preceq q\right\}
\end{array}\right.
\end{gathered}
$$

Consider arbitrary $(k, q, W, v) \in \mathcal{T} \llbracket \Delta \vdash \tau_{1} \oplus \tau_{2}:$ PRETYPE $\rrbracket \delta$.

Hence, $v \equiv \operatorname{inl} v_{1}$ or $v \equiv \operatorname{inr} v_{2}$.

Case $v \equiv \operatorname{inl} v_{1}$ :

Hence, $\left(k, q_{1}, W, v_{1}\right) \in \mathcal{T} \llbracket \Delta \vdash \tau_{1}:$ TYPE $\rrbracket \delta$ and $q_{1} \preceq q$.

Applying the induction hypothesis to $\Delta \vdash \tau_{1}:$ TYPE, we conclude that $\mathcal{T} \llbracket \Delta \vdash \tau_{1}:$ TYPE $\rrbracket \in$ Type.

Applying Fact 6 to $\left(k, q_{1}, W, v_{1}\right) \in \mathcal{T} \llbracket \Delta \vdash \tau_{1}:$ TYPE $\rrbracket \delta \in$ Type, we conclude that $W \in$ WorldDesc $_{k}$ and $\mathcal{P}\left(k, q_{1}, W\right)$ and $\forall j \leq k .\left(j, q_{1},\lfloor W\rfloor{ }_{j}, v_{1}\right) \in \mathcal{T} \llbracket \Delta \vdash \tau_{1}:$ TYPE $\rrbracket \delta$.

Note that $\mathcal{P}(k, q, W)$, which follows from Req 13 (qualpred-qualsub) and $q_{1} \preceq q$.

We are required to show that

- $W \in$ WorldDesc $_{k}$, which follows from above, and 
- $\mathcal{P}(k, q, W)$, which follows from above.

Consider arbitrary $j \leq k$.

We are required to show that $\left(j, q,\lfloor W\rfloor_{j}\right.$, inl $\left.v_{1}\right) \in \mathcal{T} \llbracket \Delta \vdash \tau_{1} \oplus \tau_{2}:$ PRETYPE $\rrbracket \delta$. Note that

- $\left(j, q_{1},\lfloor W\rfloor_{j}, v_{1}\right) \in \mathcal{T} \llbracket \Delta \vdash \tau_{1}:$ TYPE $\rrbracket \delta$, which follows from above, noting that $j \leq k$, and

- $q_{1} \preceq q$.

Case $v \equiv \operatorname{inr} v_{2}:$

Symmetric.

\section{End Case}

(AllPTy)

Case $\frac{\Delta, \alpha: \kappa \vdash \tau: \text { TYPE }}{\Delta \vdash \forall \alpha: \kappa \cdot \tau: \text { PRETYPE }}:$

Recall that

$$
\mathcal{T} \llbracket \frac{\Delta, \alpha: \kappa \vdash \tau: \text { TYPE }}{\Delta \vdash \forall \alpha: \kappa \cdot \tau: \text { PRETYPE }} \rrbracket \delta=\left\{\begin{array}{c}
(k, q, W, \Lambda . e) \mid W \in \text { WorldDesc }_{k} \wedge \mathcal{P}(k, q, W) \wedge \\
\forall \mathcal{I} . \\
\mathcal{I} \in \mathcal{K} \llbracket \kappa \rrbracket \Rightarrow \\
\forall i<k . \\
\operatorname{Comp}(i,\lfloor W\rfloor, e, \mathcal{T} \llbracket \Delta, \alpha: \kappa \vdash \tau: \operatorname{TYPE} \rrbracket \delta[\alpha \mapsto \mathcal{I}])\}
\end{array}\right.
$$

Consider arbitrary $(k, q, W, v) \in \mathcal{T} \llbracket \Delta \vdash \forall \alpha: \kappa . \tau:$ PRETYPE $\rrbracket$.

Hence, $v \equiv \Lambda$.e and $W \in$ WorldDesc $_{k}$ and $\mathcal{P}(k, q, W)$.

We are required to show that

- $W \in W_{\text {orldDesc }}$, which follows from above, and

- $\mathcal{P}(k, q, W)$, which follows from above.

Consider arbitrary $j \leq k$.

We are required to show that $\left(j, q,\lfloor W\rfloor_{j}, \Lambda . e\right) \in \mathcal{T} \llbracket \Delta \vdash \forall \alpha: \kappa . \tau: \operatorname{PRETYPE} \rrbracket \delta$.

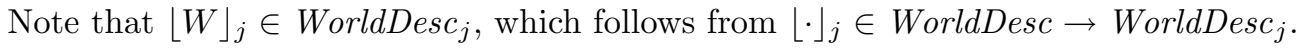

Note that $\mathcal{P}\left(j, q,\lfloor W\rfloor_{j}\right)$, which follows from Req 10 (qualpred-closed) and Req 11 (qualpred-aprx).

Consider arbitrary $\mathcal{I}$ and $i$ such that

- $\mathcal{I} \in \mathcal{K} \llbracket \kappa \rrbracket$, and

- $i<j$.

We are required to show that $\operatorname{Comp}\left(i,\left\lfloor\lfloor W\rfloor_{j}\right\rfloor_{i}, e, \mathcal{T} \llbracket \Delta, \alpha: \kappa \vdash \tau: \operatorname{TYPE} \rrbracket \delta[\alpha \mapsto \mathcal{I}]\right)$.

Instantiate $(k, q, W, \Lambda . e) \in \mathcal{T} \llbracket \Delta \vdash \forall \alpha: \kappa . \tau:$ PRETYPE $\rrbracket \delta$ with $\mathcal{I}$ and $i$. Note that

- $\mathcal{I} \in \mathcal{K} \llbracket \kappa \rrbracket$, which follows from above, and

- $i<k$, which follows from $i<j$ and $j \leq k$.

Hence, $\operatorname{Comp}\left(i,\lfloor W\rfloor_{i}, e, \mathcal{T} \llbracket \Delta, \alpha: \kappa \vdash \tau: \operatorname{TYPE} \rrbracket \delta[\alpha \mapsto \mathcal{I}]\right)$.

Note that $\lfloor W\rfloor_{i} \equiv\left\lfloor\lfloor W\rfloor_{j}\right\rfloor_{i}$, which follows from Req 1 (aprx-idem) and $i<j$.

Hence, $\operatorname{Comp}\left(i,\left\lfloor\lfloor W\rfloor_{j}\right\rfloor_{i}, e, \mathcal{T} \llbracket \Delta, \alpha: \kappa \vdash \tau: \operatorname{TYPE} \rrbracket \delta[\alpha \mapsto \mathcal{I}]\right)$.

(ExPTY)

Case $\frac{\Delta, \alpha: \kappa \vdash \tau: \text { TYPE }}{\Delta \vdash \exists \alpha: \kappa \cdot \tau: \text { PRETYPE }}:$

Recall that

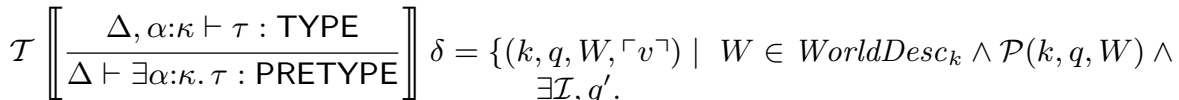

$$
\begin{aligned}
& \mathcal{I} \in \mathcal{K} \llbracket \kappa \rrbracket \wedge \\
& q^{\prime} \preceq q \wedge \\
& \left.\forall i<k .\left(i, q^{\prime},\lfloor W\rfloor_{i}, v\right) \in \mathcal{T} \llbracket \Delta, \alpha: \kappa \vdash \tau: \operatorname{TYPE} \rrbracket \delta[\alpha \mapsto \mathcal{I}]\right\}
\end{aligned}
$$


Consider arbitrary $(k, q, W, v) \in \mathcal{T} \llbracket \Delta \vdash \exists \alpha: \kappa . \tau:$ PRETYPE $\rrbracket \delta$.

Hence, $v \equiv\left\ulcorner v_{\alpha}\right\urcorner$ and $W \in$ WorldDesc $_{k}$ and $\mathcal{P}(k, q, W)$ and there exists $\mathcal{I}_{\alpha}$ and $q_{\alpha}^{\prime}$ such that

- $\mathcal{I}_{\alpha} \in \mathcal{K} \llbracket \kappa \rrbracket$,

- $q_{\alpha}^{\prime} \preceq q$, and

- $\forall i<k .\left(i, q_{\alpha}^{\prime},\lfloor W\rfloor_{i}, v_{\alpha}\right) \in \mathcal{T} \llbracket \Delta, \alpha: \kappa \vdash \tau:$ TYPE $\rrbracket \delta\left[\alpha \mapsto \mathcal{I}_{\alpha}\right]$.

We are required to show that

- $W \in$ WorldDesc $_{k}$, which follows from above, and

- $\mathcal{P}(k, q, W)$, which follows from above.

Consider $j \leq k$.

We are required to show that $\left(j, q,\lfloor W\rfloor_{j},\left\ulcorner v_{\alpha}\right\urcorner\right) \in \mathcal{T} \llbracket \Delta \vdash \exists \alpha: \kappa . \tau:$ PRETYPE $\rrbracket \delta$.

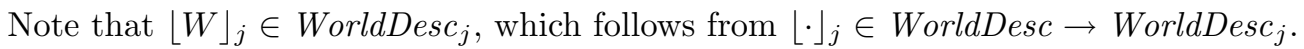

Note that $\mathcal{P}\left(j, q,\lfloor W\rfloor_{j}\right)$, which follows from Req 10 (qualpred-closed) and Req 11 (qualpred-aprx).

Take $\mathcal{I}=\mathcal{I}_{\alpha}$ and $q^{\prime}=q_{\alpha}^{\prime}$. Note that

- $\mathcal{I} \in \mathcal{K} \llbracket \kappa \rrbracket \equiv \mathcal{I}_{\alpha} \in \mathcal{K} \llbracket \kappa \rrbracket$, which follows from above,

- $q^{\prime} \preceq q \equiv q_{\alpha}^{\prime} \preceq q$, which follows from above, and

- $\forall i<j .\left(i, q^{\prime},\left\lfloor\lfloor W\rfloor_{j}\right\rfloor_{i}, v_{\alpha}\right) \in \mathcal{T} \llbracket \Delta, \alpha: \kappa \vdash \tau:$ TYPE $\rrbracket \delta[\alpha \mapsto \mathcal{I}]$ :

Consider arbitrary $i<j$.

We are required to show that $\left(i, q_{\alpha}^{\prime},\left\lfloor\lfloor W\rfloor_{j}\right\rfloor_{i}, v_{\alpha}\right) \in \mathcal{T} \llbracket \Delta, \alpha: \kappa \vdash \tau:$ TYPE $\rrbracket \delta\left[\alpha \mapsto \mathcal{I}_{\alpha}\right]$.

Instantiate $\forall i<k .\left(i, q_{\alpha}^{\prime},\lfloor W\rfloor_{i}, v_{\alpha}\right) \in \mathcal{T} \llbracket \Delta, \alpha: \kappa \vdash \tau:$ TYPE $\rrbracket \delta\left[\alpha \mapsto \mathcal{I}_{\alpha}\right]$ with $i$, noting that $i<k$, which follows from $i<j$ and $j \leq k$.

Hence, $\left(i, q_{\alpha}^{\prime},\lfloor W\rfloor_{i}, v_{\alpha}\right) \in \mathcal{T} \llbracket \Delta, \alpha: \kappa \vdash \tau: \operatorname{TYPE} \rrbracket \delta\left[\alpha \mapsto \mathcal{I}_{\alpha}\right]$.

Note that $\lfloor W\rfloor_{i} \equiv\left\lfloor\lfloor W\rfloor_{j}\right\rfloor_{i}$, which follows from Req 1 (aprx-idem) and $i<j$.

Hence, $\left(i, q_{\alpha}^{\prime},\left\lfloor\lfloor W\rfloor_{j}\right\rfloor_{i}, v_{\alpha}\right) \in \mathcal{T} \llbracket \Delta, \alpha: \kappa \vdash \tau:$ TYPE $\rrbracket \delta\left[\alpha \mapsto \mathcal{I}_{\alpha}\right]$.

\section{Case (UserPTy)...:}

(TYPE)

Case $\frac{\Delta \vdash \xi: \text { QUAL } \quad \Delta \vdash \bar{\tau}: \text { PRETYPE }}{\Delta \vdash \xi_{\bar{\tau}}: \text { TYPE }}$ :

Recall that

$$
\mathcal{T} \llbracket \frac{\Delta \vdash \xi: \mathrm{QUAL} \quad \Delta \vdash \bar{\tau}: \mathrm{PRETYPE}}{\Delta \vdash{ }^{\xi} \bar{\tau}: \mathrm{TYPE}} \rrbracket \delta=\left\{\begin{array}{c}
(k, q, W, v) \mid \\
q=\mathcal{T} \llbracket \Delta \vdash \xi: \mathrm{QUAL} \rrbracket \delta \wedge \\
(k, q, W, v) \in \mathcal{T} \llbracket \Delta \vdash \bar{\tau}: \mathrm{PRETYPE} \rrbracket \delta\}
\end{array}\right.
$$

Applying the induction hypothesis to $\Delta \vdash \xi$ : QUAL, we conclude that $\mathcal{T} \llbracket \Delta \vdash \xi:$ QUAL $\rrbracket \delta \in$ Quals.

Take $q^{\prime}=\mathcal{T} \llbracket \Delta \vdash \xi: \mathrm{QUAL} \rrbracket \delta$.

Consider arbitrary $(k, q, W, v) \in \mathcal{T} \llbracket \Delta \vdash \xi_{\bar{\tau}}:$ TYPE $\rrbracket \delta$.

Hence, $q=\mathcal{T} \llbracket \Delta \vdash \xi: \mathrm{QUAL} \rrbracket \delta$ and $(k, q, W, v) \in \mathcal{T} \llbracket \Delta \vdash \bar{\tau}: \mathrm{PRETYPE} \rrbracket \delta$.

Applying the induction hypothesis to $\Delta \vdash \bar{\tau}:$ PRETYPE, we conclude that $\mathcal{T} \llbracket \Delta \vdash \bar{\tau}:$ PRETYPE $\rrbracket \in$ Pre Type.

Applying Fact 5 to $(k, q, W, v) \in \mathcal{T} \llbracket \Delta \vdash \bar{\tau}:$ PRETYPE $\rrbracket \delta \in$ PreType, we conclude that $W \in$ WorldDesc $_{k}$ and $\mathcal{P}(k, q, W)$ and $\forall \leq k .\left(j, q,\lfloor W\rfloor_{j}, v\right) \in \mathcal{T} \llbracket \Delta \vdash \bar{\tau}:$ PRETYPE $\rrbracket$.

We are required to show that

- $W \in$ WorldDesc $_{k}$, which follows from above,

- $\mathcal{P}(k, q, W)$, which follows from above, 
- $\forall \leq k .\left(j, q,\lfloor W\rfloor_{j}, v\right) \in \mathcal{T} \llbracket \Delta \vdash \bar{\tau}:$ PRETYPE $\rrbracket$, which follows from above, and - $q=\mathcal{T} \llbracket \Delta \vdash \xi: \mathrm{QUAL} \rrbracket \delta$, which follows from above.

Case $($ UserTerm)...:

\section{End Case}




\section{Lemma 9}

Let $\delta \in \mathcal{D} \llbracket \Delta \rrbracket$ and $\Delta \vdash \Gamma$.

Then forall $(k, q, W, \gamma) \in \mathcal{G} \llbracket \Delta \vdash \Gamma \rrbracket \delta$,

$W \in$ WorldDesc $_{k}$ and $\mathcal{P}(k, q, W)$ and if $j \leq k$, then $\left(j, q,\lfloor W\rfloor_{j}, \gamma\right) \in \mathcal{G} \llbracket \Delta \vdash \Gamma \rrbracket \delta$.

\section{Proof}

Proceed by induction on the derivation $\Delta \vdash \Gamma$.

\section{Case $\frac{}{\Delta \vdash \bullet}$ :}

Recall that

$$
\mathcal{G} \llbracket \overline{\Delta \vdash \bullet} \rrbracket \delta=\left\{(k, q, W, \emptyset) \mid W=\left\lfloor\mathcal{U}_{\odot}\right\rfloor_{k}\right\}
$$

Consider arbitrary $(k, q, W, \gamma) \in \mathcal{G} \llbracket \Delta \vdash \bullet \rrbracket \delta$.

Hence, $W \equiv\left\lfloor\mathcal{U}_{\odot}\right\rfloor_{k}$ and $\gamma \equiv \emptyset$.

We are required to show that

- $\left\lfloor\mathcal{U}_{\odot}\right\rfloor_{k} \in$ WorldDesc $_{k}$, which follows from $\mathcal{U}_{\odot} \in$ WorldDesc and $\lfloor\cdot\rfloor_{k} \in$ WorldDesc $\rightarrow$ WorldDesc $_{k}$,

- $\mathcal{P}\left(k, q,\left\lfloor\mathcal{U}_{\odot}\right\rfloor_{k}\right)$, which follows from Req 14 (qualpred-unr-unit), Req 13 (qualpred-qualsub) and $\mathrm{U} \preceq q$, and Req 11 (qualpred-aprx).

Consider arbitrary $j \leq k$.

We are required to show that $\left(j, q,\lfloor W\rfloor_{j}, \emptyset\right) \in \mathcal{G} \llbracket \Delta \vdash \bullet \rrbracket \delta$. Note that

- $\lfloor W\rfloor_{j}=\left\lfloor\mathcal{U}_{\odot}\right\rfloor_{j}$, which follows from Req 1 (aprx-idem) and $j \leq k$ and $W \equiv\left\lfloor\mathcal{U}_{\odot}\right\rfloor_{k}$, which in turn follows from above.

Case $\frac{\Delta \vdash \Gamma \quad \Delta \vdash \tau: \text { TYPE }}{\Delta \vdash \Gamma, x: \tau}$ :

Recall that

$$
\begin{aligned}
& \mathcal{G} \llbracket \frac{\Delta \vdash \Gamma \quad \Delta \vdash \tau: \text { TYPE }}{\Delta \vdash \Gamma, x: \tau} \rrbracket \delta=\left\{\begin{array}{c}
(k, q, W, \gamma[x \mapsto v]) \mid \\
\left(k, q_{\Gamma}, W_{\Gamma}, \gamma\right) \in \mathcal{G} \llbracket \Delta \vdash \Gamma \rrbracket \delta \wedge
\end{array}\right. \\
& \left(k, q_{x}, W_{x}, v\right) \in \mathcal{T} \llbracket \Delta \vdash \tau: \mathrm{TYPE} \rrbracket \delta \wedge \\
& q_{\Gamma} \preceq q \wedge q_{x} \preceq q \wedge \\
& \left.\left(W_{\Gamma} \odot_{k} W_{x}=W\right)\right\}
\end{aligned}
$$

Consider arbitrary $(k, q, W, \gamma) \in \mathcal{G} \llbracket \Delta \vdash \Gamma, x: \tau \rrbracket \delta$.

Hence, $\gamma \equiv \gamma_{1}[x \mapsto v]$ and $\left(k, q_{1}, W_{1}, \gamma_{1}\right) \in \mathcal{G} \llbracket \Delta \vdash \Gamma \rrbracket \delta$ and $\left(k, q_{x}, W_{x}, v\right) \in \mathcal{T} \llbracket \Delta \vdash \tau:$ TYPE $\rrbracket \delta$ and $q_{1} \preceq q$ and $q_{x} \preceq q$ and $\left(W_{1} \odot_{k} W_{x}=W\right)$.

Applying the induction hypothesis to $\Delta \vdash \Gamma$ and $\left(k, q_{1}, W_{1}, \gamma_{1}\right) \in \mathcal{G} \llbracket \Delta \vdash \Gamma \rrbracket \delta$, we conclude that $W_{1} \in$ WorldDesc $_{k}$ and $\mathcal{P}\left(k, q_{1}, W_{1}\right)$ and $\forall j \leq k .\left(j, q_{1},\left\lfloor W_{1}\right\rfloor_{j}, \gamma_{1}\right) \in \mathcal{G} \llbracket \Delta \vdash \Gamma \rrbracket \delta$.

Note that $\mathcal{P}\left(k, q, W_{1}\right)$, which follows from Req 13 (qualpred-qualsub) and $q_{1} \preceq q$.

Applying Lemma 8 to $\Delta \vdash \tau$ : TYPE, we conclude that $\mathcal{T} \llbracket \Delta \vdash \tau:$ TYPE $\rrbracket \in$ Type.

Applying Fact 6 to $\left(k, q_{x}, W_{x}, v\right) \in \mathcal{T} \llbracket \Delta \vdash \tau:$ TYPE $\rrbracket \delta \in$ Type, we conclude that $W_{x} \in$ WorldDesc $_{k}$ and $\mathcal{P}\left(k, q_{x}, W_{x}\right)$ and $\forall j \leq k .\left(j, q_{x},\left\lfloor W_{x}\right\rfloor_{j}, v\right) \in \mathcal{T} \llbracket \Delta \vdash \tau:$ TYPE $\rrbracket \delta$.

Note that $\mathcal{P}\left(k, q_{x}, W_{x}\right)$, which follows from Req 13 (qualpred-qualsub) and $q_{x} \preceq q$.

We are required to show that

- $W \in$ WorldDesc $_{k}$, which follows from $W_{1} \in$ WorldDesc $_{k}$ and $W_{x} \in$ WorldDesc $_{k}$ and $\left(\cdot \odot_{k} \cdot\right) \in$ WorldDesc $\times$ WorldDesc $\rightarrow$ WorldDesc $_{k}$, and

- $\mathcal{P}(k, q, W)$, which follows from Req 12 (qualpred-join). 
Consider arbitrary $j \leq k$.

We are required to show that $\left(j, q,\lfloor W\rfloor_{j}, \gamma_{1}[x \mapsto v]\right) \in \mathcal{G} \llbracket \Delta \vdash \Gamma, x: \tau \rrbracket \delta$. Note that

- $\left(j, q_{1},\left\lfloor W_{1}\right\rfloor_{j}, \gamma_{1}\right) \in \mathcal{G} \llbracket \Delta \vdash \Gamma \rrbracket \delta$, which follows from above, noting that $j \leq k$,

- $\left(j, q_{x},\left\lfloor W_{x}\right\rfloor_{j}, v\right) \in \mathcal{T} \llbracket \Delta \vdash \tau:$ TYPE $\rrbracket \delta$, which follows from above, noting that $j \leq k$,

- $q_{1} \preceq q$,

- $q_{2} \preceq q$, and

- $\left(\left\lfloor W_{1}\right\rfloor_{j} \odot_{j}\left\lfloor W_{x}\right\rfloor_{j}=\lfloor W\rfloor_{j}\right)$, which follows from Req 5 (join-aprx) and $\left(W_{1} \odot_{k} W_{x}=W\right)$, which in turn follows from above.

\section{End Case}


Lemma 10 (Core Language: $\mathcal{T} \llbracket \Delta \vdash \iota: \kappa \rrbracket \delta$ (type-level substitution))

Let $\Delta, \Delta^{\prime} \vdash \iota_{\alpha}: \kappa_{\alpha}$ and $\delta \in \mathcal{D} \llbracket \Delta, \Delta^{\prime} \rrbracket$.

Then $\mathcal{T} \llbracket \Delta, \alpha: \kappa_{\alpha}, \Delta^{\prime} \vdash \iota: \kappa \rrbracket \delta\left[\alpha \mapsto \mathcal{T} \llbracket \Delta, \Delta^{\prime} \vdash \iota_{\alpha}: \kappa_{\alpha} \rrbracket \delta\right]=\mathcal{T} \llbracket \Delta, \Delta^{\prime} \vdash \iota\left[\iota_{\alpha} / \alpha\right]: \kappa \rrbracket \delta$.

Proof (Core Language: $\mathcal{T} \llbracket \Delta \vdash \iota: \kappa \rrbracket \delta$ (type-level substitution))

Let $\Delta, \Delta^{\prime} \vdash \iota_{\alpha}: \kappa_{\alpha}$ and $\delta \in \mathcal{D} \llbracket \Delta, \Delta^{\prime} \rrbracket$.

Proceed by induction on the derivation $\Delta, \alpha: \kappa_{\alpha}, \Delta^{\prime} \vdash \iota: \kappa$.

Case (UserPTy)... :

Case (UserTerm)...:

End Case 


\section{A.7.3 $\preceq$ Properties}

\section{Fact 11}

If $\Delta \vdash \xi_{1} \preceq \xi_{2}$, then $\Delta \vdash \xi_{1}:$ QUAL and $\Delta \vdash \xi_{2}:$ QUAL.

\section{Proof}

Proceed by induction on the derivation $\Delta \vdash \xi_{1} \preceq \xi_{2}$.

\section{Lemma 12 (Core Language: Qual sub-qual)}

Let $\delta \in \mathcal{D} \llbracket \Delta \rrbracket$.

If $\Delta \vdash \xi_{1} \preceq \xi_{2}$ and $q_{1}=\mathcal{T} \llbracket \Delta \vdash \xi_{1}: \mathrm{QUAL} \rrbracket \delta$ and $q_{2}=\mathcal{T} \llbracket \Delta \vdash \xi_{1}: \mathrm{QUAL} \rrbracket \delta$, then $q_{1} \preceq q_{2}$.

\section{Proof}

Proceed by induction on the derivation $\Delta \vdash \xi_{1} \preceq \xi_{2}$.

Case $\frac{\Delta \vdash \xi_{2}: \text { QUAL }}{\Delta \vdash \mathrm{U} \preceq \xi_{2}}$ :

Hence, $q_{1}=\mathcal{T} \llbracket \Delta \vdash \mathrm{U}: \mathrm{QUAL} \rrbracket \delta=\mathrm{U}$.

Applying Lemma 8 to $\Delta \vdash \xi_{2}:$ QUAL, we conclude that $q_{2}=\mathcal{T} \llbracket \Delta \vdash \xi_{2}: \mathrm{QUAL} \rrbracket \delta \in \mathcal{K} \llbracket \mathrm{QUAL} \rrbracket=$ Quals.

Note that $\mathrm{U} \preceq q_{2}$ (for any $q_{2} \in$ Quals).

Case $\frac{q_{1}^{\prime} \preceq q_{2}^{\prime}}{\Delta \vdash q_{1}^{\prime} \preceq q_{1}^{\prime}}$ :

Hence, $q_{1}=\mathcal{T} \llbracket \Delta \vdash q_{1}: \mathrm{QUAL} \rrbracket \delta=q_{1}^{\prime}$ and $q_{2}=\mathcal{T} \llbracket \Delta \vdash q_{2}^{\prime}: \mathrm{QUAL} \rrbracket \delta=q_{2}^{\prime}$.

Note that $q_{1} \preceq q_{2}$, which follows from $q_{1}^{\prime} \preceq q_{2}^{\prime}$ and $q_{1}=q_{1}^{\prime}$ and $q_{2}=q_{2}^{\prime}$.

Case $\frac{\Delta \vdash \xi_{1}: \text { QUAL }}{\Delta \vdash \xi_{1} \preceq \mathrm{L}}$ :

Hence, $q_{2}=\mathcal{T} \llbracket \Delta \vdash \mathrm{L}: \mathrm{QUAL} \rrbracket \delta=\mathrm{L}$.

Applying Lemma 8 to $\Delta \vdash \xi_{1}:$ QUAL, we conclude that $q_{1}=\mathcal{T} \llbracket \Delta \vdash \xi_{1}: \mathrm{QUAL} \rrbracket \delta \in \mathcal{K} \llbracket \mathrm{QUAL} \rrbracket=$ Quals.

Note that $q_{1} \preceq \mathrm{L}$ (for any $q_{1} \in$ Quals).

Case $\frac{\Delta \vdash \xi: \text { QUAL }}{\Delta \vdash \xi \preceq \xi}$ :

Applying Lemma 8 to $\Delta \vdash \xi:$ QUAL, we conclude that $q=q_{1}=q_{2}=\mathcal{T} \llbracket \Delta \vdash \xi: \mathrm{QUAL} \rrbracket \delta \in \mathcal{K} \llbracket \mathrm{QUAL} \rrbracket=$ Quals.

Note that $q \preceq q$ (for any $q \in$ Quals).

Case $\frac{\Delta \vdash \xi_{1} \preceq \xi^{\prime} \quad \Delta \vdash \xi^{\prime} \preceq \xi_{2}}{\Delta \vdash \xi_{1} \preceq \xi_{2}}:$

Let $q^{\prime}=\mathcal{T} \llbracket \Delta \vdash \xi^{\prime}: \mathrm{QUAL} \rrbracket \delta$.

Applying the induction hypothesis to $\Delta \vdash \xi_{1} \preceq \xi^{\prime}$, instantiated with $q_{1}$ and $q^{\prime}$, we conclude that $q_{1} \preceq q^{\prime}$.

Applying the induction hypothesis to $\Delta \vdash \xi^{\prime} \preceq \xi_{2}$, instantiated with $q^{\prime}$ and $q_{2}$, we conclude that $q^{\prime} \preceq q_{2}$.

Hence, $q_{1} \preceq q_{2}$, which follows from $q_{1} \preceq q^{\prime}$ and $q^{\prime} \preceq q_{2}$.

\section{End Case}

\section{Corollary 13 (Core Language: Qual sub-qual)}


Let $\delta \in \mathcal{D} \llbracket \Delta \rrbracket$.

If $\Delta \vdash \xi_{1} \preceq \xi_{2}$ and $q_{1}=\mathcal{T} \llbracket \Delta \vdash \xi_{1}: \mathrm{QUAL} \rrbracket \delta$ and $q_{2}=\mathcal{T} \llbracket \Delta \vdash \xi_{1}: \mathrm{QUAL} \rrbracket \delta$ and $\mathcal{P}\left(k, q_{1}, W\right)$,

then $\mathcal{P}\left(k, q_{2}, W\right)$.

\section{Proof}

Applying Lemma 12 to $\Delta \vdash \xi_{1} \preceq \xi_{2}$ and $q_{1}=\mathcal{T} \llbracket \Delta \vdash \xi_{1}:$ QUAL $\rrbracket \delta$ and $q_{2}=\mathcal{T} \llbracket \Delta \vdash \xi_{1}:$ QUAL $\rrbracket \delta$, we conclude that $q_{1} \preceq q_{2}$.

Hence, $\mathcal{P}\left(k, q_{2}, W\right)$, which follows from Req 13 (qualpred-qualsub) applied to $\mathcal{P}\left(k, q_{1}, W\right)$ and $q_{2} \preceq q_{2}$.

Fact 14

If $\Delta \vdash \tau \preceq \xi^{\prime}$, then $\Delta \vdash \tau:$ TYPE and $\Delta \vdash \xi^{\prime}:$ QUAL.

Proof

Proceed by induction on the derivation $\Delta \vdash \tau \preceq \xi^{\prime}$.

\section{Lemma 15 (Core Language: Type sub-qual)}

Let $\delta \in \mathcal{D} \llbracket \Delta \rrbracket$.

If $\Delta \vdash \tau \preceq \xi^{\prime}$ and $(k, q, W, v) \in \mathcal{T} \llbracket \Delta \vdash \tau: \mathrm{TYPE} \rrbracket \delta$ and $q^{\prime}=\mathcal{T} \llbracket \Delta \vdash \xi^{\prime}: \mathrm{QUAL} \rrbracket \delta$, then $q \preceq q^{\prime}$.

\section{Proof (Core Language: Value context sub-qual)}

Proceed by cases on the derivation $\Delta \vdash \tau \preceq \xi$.

Case $\frac{\Delta \vdash \tau: \mathrm{TYPE}}{\Delta \vdash \tau \preceq \mathrm{L}}$ :

Applying Lemma 8 to $\Delta \vdash \tau:$ TYPE, we conclude that $\mathcal{T} \llbracket \Delta \vdash \tau:$ TYPE $\rrbracket \in$ Type.

Hence, $q \in$ Quals, which follows from $(k, q, W, v) \in \mathcal{T} \llbracket \Delta \vdash \tau:$ TYPE $\rrbracket \in \in$ Type.

Note that $q \preceq \mathrm{L}$ (for any $q \in$ Quals).

Case $\frac{\Delta \vdash \bar{\tau}: \text { PRETYPE } \quad \Delta \vdash \xi \preceq \xi^{\prime}}{\Delta \vdash^{\xi_{\bar{\tau}}} \preceq \xi^{\prime}}$

Note that

$$
\begin{aligned}
(k, q, W, v) \in \mathcal{T} \llbracket \Delta \vdash{ }^{\xi} \bar{\tau}: \mathrm{TYPE} \rrbracket \delta \\
\equiv\{(k, q, W, v) \mid \\
\quad q=\mathcal{T} \llbracket \Delta \vdash \xi: \mathrm{QUAL} \rrbracket \delta \wedge \\
\quad(k, q, W, v) \in \mathcal{T} \llbracket \Delta \vdash \bar{\tau}: \mathrm{PRETYPE} \rrbracket \delta\}
\end{aligned}
$$

Hence $q=\mathcal{T} \llbracket \Delta \vdash \xi:$ QUAL $\rrbracket \delta$.

Applying Lemma 12 to $\Delta \vdash \xi \preceq \xi^{\prime}$, instantiated with $q$ and $q^{\prime}$, we conclude that $q \preceq q^{\prime}$.

\section{End Case}

\section{Corollary 16 (Core Language: Type sub-qual)}

Let $\delta \in \mathcal{D} \llbracket \Delta \rrbracket$.

If $\Delta \vdash \tau \preceq \xi^{\prime}$ and $(k, q, W, v) \in \mathcal{T} \llbracket \Delta \vdash \tau: \mathrm{TYPE} \rrbracket \delta$ and $q^{\prime}=\mathcal{T} \llbracket \Delta \vdash \xi^{\prime}: \mathrm{QUAL} \rrbracket \delta$, then $\mathcal{P}\left(k, q^{\prime}, W\right)$.

\section{Proof}

Applying Fact 14 to $\Delta \vdash \tau \preceq \xi^{\prime}$, we conclude that $\Delta \vdash \tau:$ TYPE.

Applying Lemma 8 to $\Delta \vdash \tau$ : TYPE, we conclude that $\mathcal{T} \llbracket \Delta \vdash \tau:$ TYPE $\rrbracket \delta \in$ Type.

Applying Fact 6 to $(k, q, W, v) \in \mathcal{T} \llbracket \Delta \vdash \tau:$ TYPE $\rrbracket \delta \in$ Type, we conclude that $\mathcal{P}(k, q, W)$.

Applying Lemma 15 to $\Delta \vdash \tau \preceq \xi^{\prime}$ and $(k, q, W, v)$ and $q^{\prime}$, we conclude that $q \preceq q^{\prime}$.

Hence, $\mathcal{P}\left(k, q^{\prime}, W\right)$, which follows from Req 13 (qualpred-qualsub) applied to $\mathcal{P}(k, q, W)$ and $q \preceq q^{\prime}$. 


\section{Fact 17}

If $\Delta \vdash \Gamma \preceq \xi$, then $\Delta \vdash \Gamma$ and $\Delta \vdash \xi^{\prime}:$ QUAL.

\section{Proof}

Proceed by induction on the derivation $\Delta \vdash \Gamma \preceq \xi^{\prime}$.

\section{Lemma 18 (Core Language: Value context sub-qual)}

Let $\delta \in \mathcal{D} \llbracket \Delta \rrbracket$.

If $\Delta \vdash \Gamma \preceq \xi$ and $(k, q, W, \gamma) \in \mathcal{G} \llbracket \Delta \vdash \Gamma \rrbracket \delta$ and $q^{\prime}=\mathcal{T} \llbracket \Delta \vdash \xi: \mathrm{QUAL} \rrbracket \delta$, then $\mathcal{P}\left(k, q^{\prime}, W\right)$.

\section{Proof (Core Language: Value context sub-qual)}

Proceed by induction on the derivation $\Delta \vdash \Gamma \preceq \xi$

Case $\frac{\Delta \vdash \xi^{\prime}: \text { QUAL }}{\Delta \vdash \bullet \preceq \xi^{\prime}}$ :

Note that

$$
\begin{aligned}
(k, q, W, \gamma) & \in \mathcal{G} \llbracket \Delta \vdash \bullet \rrbracket \delta \\
& \equiv\left\{(k, q, W, \emptyset) \mid W=\left\lfloor\mathcal{U}_{\odot}\right\rfloor_{k}\right\} .
\end{aligned}
$$

Hence, $W \equiv\left\lfloor\mathcal{U}_{\odot}\right\rfloor_{k}$ and $\gamma \equiv \emptyset$.

Applying Lemma 8 to $\Delta \vdash \xi^{\prime}:$ QUAL, we conclude that $q^{\prime}=\mathcal{T} \llbracket \Delta \vdash \xi^{\prime}: \mathrm{QUAL} \rrbracket \delta \in \mathcal{K} \llbracket \mathrm{QUAL} \rrbracket=$ Quals.

Note that $\mathcal{P}\left(k, q^{\prime}, W\right)$, which follows from $W \equiv\left\lfloor\mathcal{U}_{\odot}\right\rfloor_{k}$ (which follows from above) and $\mathcal{P}\left(k, q^{\prime},\left\lfloor\mathcal{U}_{\odot}\right\rfloor_{k}\right)$, which follows from Req 13 (qualpred-qualsub) applied to $U \preceq q^{\prime}$ and $\mathcal{P}\left(k, \mathrm{U},\left[\mathcal{U}_{\odot}\right\rfloor_{k}\right)$, which in turn follows from Req 11 (qualpred-aprx) applied to $\mathcal{P}\left(k, \mathrm{U}, \mathcal{U}_{\odot}\right)$, which in turn follows from Req 14 (qualpred-unr-unit).

Case $\frac{\Delta \vdash \Gamma \preceq \xi^{\prime} \quad \Delta \vdash \tau \preceq \xi^{\prime}}{\Delta \vdash \Gamma, x: \tau}:$

Note that

$$
\begin{aligned}
(k, q, W, \gamma) \in \mathcal{G} \llbracket \Delta \vdash \Gamma, x: \tau \rrbracket \delta \\
\equiv\{(k, q, W, \gamma[x \mapsto v]) \mid \\
\left(k, q_{\Gamma}, W_{\Gamma}, \gamma\right) \in \mathcal{G} \llbracket \Delta \vdash \Gamma \rrbracket \delta \wedge \\
\left(k, q_{x}, W_{x}, v\right) \in \mathcal{T} \llbracket \Delta \vdash \tau: \text { TYPE } \rrbracket \delta \wedge \\
q_{\Gamma} \preceq q \wedge q_{x} \preceq q \wedge \\
\left.\left(W_{\Gamma} \odot_{k} W_{x}=W\right)\right\} .
\end{aligned}
$$

Hence, $\gamma \equiv \gamma_{1}[x \mapsto v]$ and $\left(k, q_{1}, W_{1}, \gamma_{1}\right) \in \mathcal{G} \llbracket \Delta \vdash \Gamma \rrbracket \delta$ and $\left(k, q_{x}, W_{x}, v\right) \in \mathcal{T} \llbracket \Delta \vdash \tau:$ TYPE $\rrbracket \delta$ and $q_{1} \preceq q$ and $q_{x} \preceq q$ and $\left(W_{1} \odot_{k} W_{x}=W\right)$.

Applying the induction hypothesis to $\Delta \vdash \Gamma \preceq \xi^{\prime}$, instantiated with $\left(k, q_{1}, W_{1}, \gamma_{1}\right) \in \mathcal{G} \llbracket \Delta \vdash \Gamma \rrbracket \delta$ and $q^{\prime}=\mathcal{T} \llbracket \Delta \vdash \xi^{\prime}: \mathrm{QUAL} \rrbracket \delta$, we conclude that $\mathcal{P}\left(k, q^{\prime}, W_{1}\right)$.

Applying Corollary 16 to $\Delta \vdash \tau \preceq \xi$, instantiated with $\left(k, q_{x}, W_{x}, v\right) \in \mathcal{T} \llbracket \Delta \vdash \tau:$ TYPE $\rrbracket \delta$ and $q^{\prime}=\mathcal{T} \llbracket \Delta \vdash \xi^{\prime}: \mathrm{QUAL} \rrbracket \delta$, we conclude that $\mathcal{P}\left(k, q^{\prime}, W_{x}\right)$.

Note that $\mathcal{P}\left(k, q^{\prime}, W\right)$, which follows from Req 12 (qualpred-join) and $\left(W_{1} \odot_{k} W_{x}=W\right.$ ) (which follows from above) and $\mathcal{P}\left(k, q^{\prime}, W_{1}\right)$ and $\mathcal{P}\left(k, q^{\prime}, W_{x}\right)$, which in turn follows from above.

\section{End Case}




\section{A.7.4 $\boxplus$ Properties}

\section{Fact 19}

If $\Delta \vdash \Gamma \leadsto \Gamma_{1} \boxplus \Gamma_{2}$, then $\Delta \vdash \Gamma_{1}$ and $\Delta \vdash \Gamma_{2}$.

Proof

Proceed by induction on the derivation $\Delta \vdash \Gamma \leadsto \Gamma_{1} \boxplus \Gamma_{2}$.

\section{Lemma 20 (Core Language: $\boxplus$ Properties)}

Let $\delta \in \mathcal{D} \llbracket \Delta \rrbracket$ and $\Delta \vdash \Gamma \leadsto \Gamma_{1} \boxplus \Gamma_{2}$.

If $(k, q, W, \gamma) \in \mathcal{G} \llbracket \Delta \vdash \Gamma \rrbracket \delta$,

then $\Delta \vdash \Gamma_{1}$ and $\Delta \vdash \Gamma_{2}$ and there exists $q_{1}, W_{1}, \gamma_{1}, q_{2}, W_{2}$, and $\gamma_{2}$ such that

- $\left(k, q_{1}, W_{1}, \gamma_{1}\right) \in \mathcal{G} \llbracket \Delta \vdash \Gamma_{1} \rrbracket \delta$,

- $\left(k, q_{2}, W_{1}, \gamma_{2}\right) \in \mathcal{G} \llbracket \Delta \vdash \Gamma_{2} \rrbracket \delta$,

- $\gamma \in \gamma_{1} \boxplus \gamma_{2}$, where

$$
\begin{aligned}
& \gamma_{1} \boxplus \gamma_{2}=\{\gamma \in \operatorname{Vars} \rightarrow \text { CValues } \mid \\
& \operatorname{dom}(\gamma)=\operatorname{dom}\left(\gamma_{1}\right) \cup \operatorname{dom}\left(\gamma_{2}\right) \wedge \\
& \forall z \in \operatorname{dom}\left(\gamma_{1}\right) \cdot \gamma(z)=\gamma_{1}(z) \wedge \\
&\left.\forall z \in \operatorname{dom}\left(\gamma_{2}\right) \cdot \gamma(z)=\gamma_{2}(z)\right\},
\end{aligned}
$$

- $q_{1} \preceq q$,

- $q_{2} \preceq q$, and

- $\left(W_{1} \odot_{k} W_{2}=W\right)$

\section{Proof (Core Language: $\boxplus$ Properties)}

Let $\delta \in \mathcal{D} \llbracket \Delta \rrbracket$ and $\Delta \vdash \Gamma$.

Proceed by induction on the derivation $\Delta \vdash \Gamma \leadsto \Gamma_{1} \boxplus \Gamma_{2}$.

\section{Case $\overline{\Delta \vdash \bullet \sim \bullet \boxplus \bullet}:$}

Note that $\Gamma \equiv \bullet$ and $\Gamma_{1} \equiv \bullet$ and $\Gamma_{2} \equiv \bullet$.

Recall that

$$
\mathcal{G} \llbracket \overline{\Delta \vdash \bullet \bullet} \rrbracket \delta=\left\{(k, q, W, \emptyset) \mid W=\left\lfloor\mathcal{U}_{\odot}\right\rfloor_{k}\right\}
$$

Consider arbitrary $(k, q, W, \gamma) \in \mathcal{G} \llbracket \Delta \vdash \bullet \rrbracket \delta$.

Hence, $W \equiv\left\lfloor\mathcal{U}_{\odot}\right\rfloor_{k}$ and $\gamma \equiv \emptyset$.

Note that

$$
\Delta \vdash \Gamma_{1} \equiv \overline{\Delta \vdash \bullet} \quad \text { and } \quad \Delta \vdash \Gamma_{2} \equiv \overline{\Delta \vdash \bullet} .
$$

Take $q_{1} \preceq q, W_{1}=W, \gamma_{1}=\emptyset, q_{2} \preceq q, W_{2}=W$, and $\gamma_{2}=\emptyset$.

We are required to show that

- $\left(k, q_{1}, W_{1}, \gamma_{1}\right) \in \mathcal{G} \llbracket \Delta \vdash \Gamma_{1} \rrbracket \delta$ $\equiv\left(k, q_{1}, W, \emptyset\right) \in \mathcal{G} \llbracket \Delta \vdash \bullet \rrbracket \delta$, which follows from

- $W=\left\lfloor\mathcal{U}_{\odot}\right\rfloor_{k}$, which follows from above, 
- $\left(k, q_{2}, W_{2}, \gamma_{2}\right) \in \mathcal{G} \llbracket \Delta \vdash \Gamma_{2} \rrbracket \delta$ $\equiv\left(k, q_{2}, W, \emptyset\right) \in \mathcal{G} \llbracket \Delta \vdash \bullet \rrbracket \delta$, which follows from

- $W=\left\lfloor\mathcal{U}_{\odot}\right\rfloor_{k}$, which follows from above,

- $\gamma \in \gamma_{1} \boxplus \gamma_{2}$

$\equiv \emptyset \in \emptyset \boxplus \emptyset$

which follows from

- $\operatorname{dom}(\emptyset)=\operatorname{dom}(\emptyset) \cup \operatorname{dom}(\emptyset)$,

- $\forall z \in \operatorname{dom}(\emptyset) . \emptyset(z)=\emptyset(z)$,

- $\forall z \in \operatorname{dom}(\emptyset) . \emptyset(z)=\emptyset(z)$,

- $q_{1} \preceq q$,

- $q_{2} \preceq q$, and

- $W \equiv\left(W_{1} \odot_{k} W_{2}\right)$

$\equiv W \equiv\left(W \odot_{k} W\right)$

which follows from

$$
\begin{aligned}
& \left(\left\lfloor\mathcal{U}_{\odot}\right\rfloor_{k}=\mathcal{U}_{\odot} \odot_{k} \mathcal{U}_{\odot}\right) \\
& \text { which follows from Req } 9 \text { (join-unit-left) } \\
& \Rightarrow\left(\left\lfloor\mathcal{U}_{\odot}\right\rfloor_{k}=\left\lfloor\mathcal{U}_{\odot}\right\rfloor_{k} \odot_{k}\left\lfloor\mathcal{U}_{\odot}\right\rfloor_{k}\right) \\
& \text { which follows from Req } 5 \text { (join-aprx) } \\
& \equiv\left(W \equiv W \odot_{k} W\right) \\
& \quad \text { which follows from } W=\left\lfloor\mathcal{U}_{\odot}\right\rfloor_{k} .
\end{aligned}
$$

Case $\frac{\Delta \vdash \Gamma^{\prime} \leadsto \Gamma_{1}^{\prime} \boxplus \Gamma_{2} \quad \Delta \vdash \tau: \text { TYPE }}{\Delta \vdash \Gamma^{\prime}, x: \tau \leadsto \Gamma_{1}^{\prime}, x: \tau \boxplus \Gamma_{2}}:$

Note that $\Gamma \equiv \Gamma^{\prime}, x: \tau$ and $\Gamma_{1} \equiv \Gamma_{1}^{\prime}, x: \tau$.

Recall that

$$
\mathcal{G} \llbracket \frac{\Delta \vdash \Gamma^{\prime} \Delta \vdash \tau: \text { TYPE }}{\Delta \vdash \Gamma^{\prime}, x: \tau} \rrbracket \delta=\left\{\begin{array}{l}
(k, q, W, \gamma[x \mapsto v]) \mid \\
\\
\left(k, q_{\Gamma}, W_{\Gamma}, \gamma\right) \in \mathcal{G} \llbracket \Delta \vdash \Gamma \rrbracket \delta \wedge \\
\left(k, q_{x}, W_{x}, v\right) \in \mathcal{T} \llbracket \Delta \vdash \tau: \text { TYPE } \rrbracket \delta \wedge \\
\\
q_{\Gamma} \preceq q \wedge q_{x} \preceq q \wedge \\
\left.\left(W_{\Gamma} \odot_{k} W_{x}=W\right)\right\}
\end{array}\right.
$$

Consider arbitrary $(k, q, W, \gamma) \in \mathcal{G} \llbracket \Delta \vdash \Gamma^{\prime}, x: \tau \rrbracket \delta$.

Hence, $\gamma \equiv \gamma^{\prime}[x \mapsto v]$ and $\left(k, q_{\Gamma^{\prime}}, W_{\Gamma^{\prime}}, \gamma^{\prime}\right) \in \mathcal{G} \llbracket \Delta \vdash \Gamma^{\prime} \rrbracket \delta$ and $\left(k, q_{x}, W_{x}, v\right) \in \mathcal{T} \llbracket \Delta \vdash \tau:$ TYPE $\rrbracket \delta$ and $q_{\Gamma^{\prime}} \preceq q$ and $q_{x} \preceq q$ and $\left(W_{\Gamma^{\prime}} \odot_{k} W_{x}=W\right)$.

Apply the induction hypothesis to $\Delta \vdash \Gamma^{\prime} \leadsto \Gamma_{1}^{\prime} \boxplus \Gamma_{2}$ with $\left(k, q_{\Gamma^{\prime}}, W_{\Gamma^{\prime}}, \gamma^{\prime}\right) \in \mathcal{G} \llbracket \Delta \vdash \Gamma^{\prime} \rrbracket \delta$.

We conclude that $\Delta \vdash \Gamma_{1}^{\prime}$ and $\Delta \vdash \Gamma_{2}$ and there exists $q_{1}^{\prime}, W_{1}^{\prime}, \gamma_{1}^{\prime}, q_{2}, W_{2}$, and $\gamma_{2}$ such that

- $\left(k, q_{1}^{\prime}, W_{1}^{\prime}, \gamma_{1}^{\prime}\right) \in \mathcal{G} \llbracket \Delta \vdash \Gamma_{1}^{\prime} \rrbracket \delta$,

- $\left(k, q_{2}, W_{2}, \gamma_{2}\right) \in \mathcal{G} \llbracket \Delta \vdash \Gamma_{2} \rrbracket \delta$,

- $\gamma^{\prime} \in \gamma_{1}^{\prime} \boxplus \gamma_{2}$,

- $q_{1}^{\prime} \preceq q_{\Gamma^{\prime}}$,

- $q_{2} \preceq q_{\Gamma^{\prime}}$, and

- $\left(W_{1}^{\prime} \odot_{k} W_{2}=W_{\Gamma^{\prime}}\right)$.

Note that

$$
\Delta \vdash \Gamma_{1} \equiv \frac{\Delta \vdash \Gamma_{1}^{\prime} \Delta \vdash \tau: \text { TYPE }}{\Delta \vdash \Gamma_{1}^{\prime}, x: \tau} \quad \text { and } \quad \Delta \vdash \Gamma_{2} .
$$


Note that there exists $W_{1}$ such that $\left(W_{1}^{\prime} \odot_{k} W_{x}=W_{1}\right)$ and $\left(W_{1} \odot_{k} W_{2}=W\right)$, which follows from

$$
\begin{gathered}
\left(W_{1}^{\prime} \odot_{k} W_{2}=W_{\Gamma^{\prime}}\right) \wedge\left(W_{\Gamma^{\prime}} \odot_{k} W_{x}=W\right) \\
\text { which follows from above } \\
\Rightarrow\left(W_{2} \odot_{k} W_{1}^{\prime}=W_{\Gamma^{\prime}}\right) \wedge\left(W_{\Gamma^{\prime}} \odot_{k} W_{x}=W\right) \\
\text { which follows from Req } 6 \text { (join-commut) } \\
\Rightarrow \exists W_{1} .\left(W_{1}^{\prime} \odot_{k} W_{x}=W_{1}\right) \wedge\left(W_{2} \odot_{k} W_{1}=W\right) \\
\text { which follows from Req } 8 \text { (join-assocr) } \\
\Rightarrow \exists W_{1} .\left(W_{1}^{\prime} \odot_{k} W_{x}=W_{1}\right) \wedge\left(W_{1} \odot_{k} W_{2}=W\right) \\
\text { which follows from Req } 6 \text { (join-commut). }
\end{gathered}
$$

Take $q_{1}=\left(q_{1}^{\prime} \sqcup q_{x}\right)$ and $\gamma_{1}=\gamma_{1}^{\prime}[x \mapsto v]$.

We are required to show that

- $\left(k, q_{1}, W_{1}, \gamma_{1}\right) \in \mathcal{G} \llbracket \Delta \vdash \Gamma_{1} \rrbracket \delta$

$\equiv\left(k,\left(q_{1}^{\prime} \sqcup q_{x}\right), W_{1}, \gamma_{1}^{\prime}[x \mapsto v]\right) \in \mathcal{G} \llbracket \Delta \vdash \Gamma_{1}^{\prime}, x: \tau \rrbracket \delta$,

which follows from

- $\left(k, q_{1}^{\prime}, W_{1}^{\prime}, \gamma_{1}^{\prime}\right) \in \mathcal{G} \llbracket \Delta \vdash \Gamma_{1}^{\prime} \rrbracket \delta$, which follows from above,

- $\left(k, q_{x}, W_{x}, v\right) \in \mathcal{T} \llbracket \Delta \vdash \tau:$ TYPE $\delta$, which follows from above,

- $q_{1}^{\prime} \preceq\left(q_{1}^{\prime} \sqcup q_{x}\right)$, which follows from the definition of $\sqcup$,

- $q_{x} \preceq\left(q_{1}^{\prime} \sqcup q_{x}\right)$, which follows from the definition of $\sqcup$,

- $\left(W_{1}^{\prime} \odot_{k} W_{x}=W_{1}\right)$, which follows from above,

- $\left(k, q_{2}, W_{2}, \gamma_{2}\right) \in \mathcal{G} \llbracket \Delta \vdash \Gamma_{2} \rrbracket \delta$, which follows from above,

- $\gamma \in \gamma_{1} \boxplus \gamma_{2}$

$\equiv \gamma^{\prime}[x \mapsto v] \in \gamma_{1}^{\prime}[x \mapsto v] \boxplus \gamma_{2}$,

which follows from

- $\operatorname{dom}\left(\gamma^{\prime}[x \mapsto v]\right)=\operatorname{dom}\left(\gamma_{1}[x \mapsto v]\right) \cup \operatorname{dom}\left(\gamma_{2}\right)$, which follows from

$$
\begin{aligned}
& \operatorname{dom}\left(\gamma^{\prime}[x \mapsto v]\right)=\operatorname{dom}\left(\gamma_{1}^{\prime}[x \mapsto v]\right) \cup \operatorname{dom}\left(\gamma_{2}\right) \\
& \equiv \operatorname{dom}\left(\gamma^{\prime}\right) \cup\{x\}=\operatorname{dom}\left(\gamma_{1}^{\prime}\right) \cup\{x\} \cup \operatorname{dom}\left(\gamma_{2}\right) \\
& \equiv \operatorname{dom}\left(\gamma^{\prime}\right) \cup\{x\}=\operatorname{dom}\left(\gamma^{\prime}\right) \cup\{x\} \\
& \quad \text { which follows from } \gamma^{\prime} \in \gamma_{1}^{\prime} \boxplus \gamma_{2},
\end{aligned}
$$

- $\forall z \in \operatorname{dom}\left(\gamma_{1}^{\prime}[x \mapsto v]\right) . \gamma^{\prime}[x \mapsto v](z)=\gamma_{1}^{\prime}[x \mapsto v](z)$, which follows from $\forall z \in$ $\operatorname{dom}\left(\gamma_{1}^{\prime}\right) \cdot \gamma^{\prime}(z)=\gamma_{1}^{\prime}(z)$, which follows from $\gamma^{\prime} \in \gamma_{1}^{\prime} \boxplus \gamma_{2}$ and $x \notin \operatorname{dom} \Gamma^{\prime}$,

- $\forall z \in \operatorname{dom}\left(\gamma_{2}\right) \cdot \gamma^{\prime}[x \mapsto v](z)=\gamma_{2}(z)$, which follows from $\forall z \in \operatorname{dom}\left(\gamma_{2}\right) \cdot \gamma^{\prime}(z)=\gamma_{2}(z)$, which follows from $\gamma^{\prime} \in \gamma_{1}^{\prime} \boxplus \gamma_{2}$ and $x \notin \operatorname{dom} \Gamma^{\prime}$,

- $q_{1} \preceq q \equiv\left(q_{1}^{\prime} \sqcup q_{x}\right) \preceq q$, which follows from $q_{1}^{\prime} \preceq q_{\Gamma^{\prime}}$ and $q_{\Gamma^{\prime}} \preceq q$ and $q_{x} \preceq q$ and the definition of $\sqcup$,

- $q_{2} \preceq q$, which follows from $q_{2} \preceq q_{\Gamma^{\prime}}$ and $q_{\Gamma^{\prime}} \preceq q$, and

- $\left(W_{1} \odot_{k} W_{2}=W\right)$, which follows from above.

Case $\frac{\Delta \vdash \Gamma^{\prime} \leadsto \Gamma_{1} \boxplus \Gamma_{2}^{\prime} \quad \Delta \vdash \tau: \text { TYPE }}{\Delta \vdash \Gamma^{\prime}, x: \tau \leadsto \Gamma_{1} \boxplus \Gamma_{2}^{\prime}, x: \tau}:$

Symmetric.

Case $\frac{\Delta \vdash \Gamma^{\prime} \leadsto \Gamma_{1}^{\prime} \boxplus \Gamma_{2}^{\prime} \quad \Delta \vdash \tau \preceq \mathrm{R}}{\Delta \vdash \Gamma^{\prime}, x: \tau \leadsto \Gamma_{1}^{\prime}, x: \tau \boxplus \Gamma_{2}^{\prime}, x: \tau}:$

Note that $\Gamma \equiv \Gamma^{\prime}, x: \tau$ and $\Gamma_{1} \equiv \Gamma_{1}^{\prime}, x: \tau$ and $\Gamma_{2}=\Gamma_{2}^{\prime}, x: \tau$.

Recall that 


$$
\mathcal{G} \llbracket \frac{\Delta \vdash \Gamma^{\prime} \quad \Delta \vdash \tau: \text { TYPE }}{\Delta \vdash \Gamma^{\prime}, x: \tau} \rrbracket \delta=\left\{\begin{array}{l}
(k, q, W, \gamma[x \mapsto v]) \mid \\
\\
\left(k, q_{\Gamma}, W_{\Gamma}, \gamma\right) \in \mathcal{G} \llbracket \Delta \vdash \Gamma \rrbracket \delta \wedge \\
\left(k, q_{x}, W_{x}, v\right) \in \mathcal{T} \llbracket \Delta \vdash \tau: \text { TYPE } \rrbracket \delta \wedge \\
q_{\Gamma} \preceq q \wedge q_{x} \preceq q \wedge \\
\left.\left(W_{\Gamma} \odot_{k} W_{x}=W\right)\right\}
\end{array}\right.
$$

Consider arbitrary $(k, q, W, \gamma) \in \mathcal{G} \llbracket \Delta \vdash \Gamma^{\prime}, x: \tau \rrbracket \delta$.

Hence, $\gamma \equiv \gamma^{\prime}[x \mapsto v]$ and $\left(k, q_{\Gamma^{\prime}}, W_{\Gamma^{\prime}}, \gamma^{\prime}\right) \in \mathcal{G} \llbracket \Delta \vdash \Gamma^{\prime} \rrbracket \delta$ and $\left(k, q_{x}, W_{x}, v\right) \in \mathcal{T} \llbracket \Delta \vdash \tau:$ TYPE $\rrbracket \delta$ and $q_{\Gamma^{\prime}} \preceq q$ and $q_{x} \preceq q$ and $\left(W_{\Gamma^{\prime}} \odot_{k} W_{x}=W\right)$.

Note that $\mathcal{P}\left(k, \mathrm{R}, W_{x}\right)$, which follows from Corollary 16 applied to $\Delta \vdash \tau \preceq \mathrm{R}$ and $\left(k, q_{x}, W_{x}, v\right) \in$ $\mathcal{T} \llbracket \Delta \vdash \tau: \mathrm{TYPE} \rrbracket \delta$ and $\mathrm{R}=\mathcal{T} \llbracket \Delta \vdash \mathrm{R}: \mathrm{QUAL} \rrbracket \delta$.

Apply the induction hypothesis to $\Delta \vdash \Gamma^{\prime}$ with $\left(k, q_{\Gamma^{\prime}}, W_{\Gamma^{\prime}}, \gamma^{\prime}\right) \in \mathcal{G} \llbracket \Delta \vdash \Gamma^{\prime} \rrbracket \delta$ and $\Delta \vdash \Gamma^{\prime} \leadsto$ $\Gamma_{1}^{\prime} \boxplus \Gamma_{2}^{\prime}$.

We conclude that $\Delta \vdash \Gamma_{1}^{\prime}$ and $\Delta \vdash \Gamma_{2}^{\prime}$ and there exists $q_{1}^{\prime}, W_{1}^{\prime}, \gamma_{1}^{\prime}, q_{2}^{\prime}, W_{2}^{\prime}$, and $\gamma_{2}^{\prime}$ such that

- $\left(k, q_{1}^{\prime}, W_{1}^{\prime}, \gamma_{1}^{\prime}\right) \in \mathcal{G} \llbracket \Delta \vdash \Gamma_{1}^{\prime} \rrbracket \delta$,

- $\left(k, q_{2}^{\prime}, W_{2}^{\prime}, \gamma_{2}^{\prime}\right) \in \mathcal{G} \llbracket \Delta \vdash \Gamma_{2}^{\prime} \rrbracket \delta$,

- $\gamma^{\prime} \in \gamma_{1}^{\prime} \boxplus \gamma_{2}^{\prime}$,

- $q_{1}^{\prime} \preceq q_{\Gamma^{\prime}}$,

- $q_{2}^{\prime} \preceq q_{\Gamma^{\prime}}$, and

- $\left(W_{1}^{\prime} \odot_{k} W_{2}^{\prime}=W_{\Gamma^{\prime}}\right)$.

Note that

$$
\Delta \vdash \Gamma_{1} \equiv \frac{\Delta \vdash \Gamma_{1}^{\prime} \Delta \vdash \tau: \text { TYPE }}{\Delta \vdash \Gamma_{1}^{\prime}, x: \tau} \quad \text { and } \quad \Delta \vdash \Gamma_{2} \equiv \frac{\Delta \vdash \Gamma_{2}^{\prime} \quad \Delta \vdash \tau: \text { TYPE }}{\Delta \vdash \Gamma_{2}^{\prime}, x: \tau} .
$$

Note that there exists $W_{1}$ and $W_{2}$ such that $\left(W_{1}^{\prime} \odot_{k} W_{x}=W_{1}\right)$ and $\left(W_{2}^{\prime} \odot_{k} W_{x}=W_{2}\right)$ and $\left(W_{1} \odot_{k} W_{2}=W\right)$, which follows from

$$
\begin{aligned}
\left(W_{1}^{\prime} \odot_{k} W_{2}^{\prime}=W_{\Gamma^{\prime}}\right) \wedge\left(W_{\Gamma^{\prime}} \odot_{k} W_{x}=W\right) \wedge \mathcal{P}\left(k, \mathrm{R}, W_{x}\right) & \\
\text { which follows from above } & \left(W_{1}^{\prime} \odot_{k} W_{2}^{\prime}=W_{\Gamma^{\prime}}\right) \wedge\left(W_{\Gamma^{\prime}} \odot_{k} W_{x}=W\right) \wedge\left(W_{x} \odot_{k} W_{x}=\left\lfloor W_{x}\right\rfloor_{k}\right) \\
& \text { which follows from Req } 15 \text { (qualpred-rel-join) } \\
\Rightarrow & \left(W_{1}^{\prime} \odot_{k} W_{2}^{\prime}=W_{\Gamma^{\prime}}\right) \wedge\left(W_{\Gamma^{\prime}} \odot_{k}\left\lfloor W_{x}\right\rfloor_{k}=W\right) \wedge\left(W_{x} \odot_{k} W_{x}=\left\lfloor W_{x}\right\rfloor_{k}\right) \\
& \text { which follows from Req } 5 \text { (join-aprx) } \\
\Rightarrow & \exists W_{z} .\left(W_{2}^{\prime} \odot_{k}\left\lfloor W_{x}\right\rfloor_{k}=W_{z}\right) \wedge\left(W_{1}^{\prime} \odot_{k} W_{z}=W\right) \wedge\left(W_{x} \odot_{k} W_{x}=\left\lfloor W_{x}\right\rfloor_{k}\right) \\
& \text { which follows from Req } 7(\text { join-assocl) } \\
\Rightarrow & \exists W_{2}, W_{z} .\left(W_{2}^{\prime} \odot_{k} W_{x}=W_{2}\right) \wedge\left(W_{x} \odot_{k} W_{2}=W_{z}\right) \wedge\left(W_{1}^{\prime} \odot_{k} W_{z}=W\right) \\
& \text { which follows from Req } 8(\text { join-assocr }) \\
\Rightarrow & \exists W_{1}, W_{2}, W_{z} .\left(W_{2}^{\prime} \odot_{k} W_{x}=W_{2}\right) \wedge\left(W_{1}^{\prime} \odot_{k} W_{x}=W_{1}\right) \wedge\left(W_{1} \odot_{k} W_{2}=W\right) \\
& \text { which follows from Req } 7 \text { (join-assocl) } \\
\equiv & \exists W_{1}, W_{2} .\left(W_{2}^{\prime} \odot_{k} W_{x}=W_{2}\right) \wedge\left(W_{1}^{\prime} \odot_{k} W_{x}=W_{1}\right) \wedge\left(W_{1} \odot_{k} W_{2}=W\right) \\
& \text { which follows from logical equivalence. }
\end{aligned}
$$

Take $q_{1}=\left(q_{1}^{\prime} \sqcup q_{x}\right), \gamma_{1}=\gamma_{1}^{\prime}[x \mapsto v], q_{2}=\left(q_{2}^{\prime} \sqcup q_{x}\right)$, and $\gamma_{2}=\gamma_{2}^{\prime}[x \mapsto v]$.

We are required to show that

- $\left(k, q_{1}, W_{1}, \gamma_{1}\right) \in \mathcal{G} \llbracket \Delta \vdash \Gamma_{1} \rrbracket \delta$

$$
\equiv\left(k,\left(q_{1}^{\prime} \sqcup q_{x}\right),\left(W_{1}^{\prime} \odot_{k} W_{x}\right), \gamma_{1}^{\prime}[x \mapsto v]\right) \in \mathcal{G} \llbracket \Delta \vdash \Gamma_{1}^{\prime}, x: \tau \rrbracket \delta,
$$

which follows from

- $\left(k, q_{1}^{\prime}, W_{1}^{\prime}, \gamma_{1}^{\prime}\right) \in \mathcal{G} \llbracket \Delta \vdash \Gamma_{1}^{\prime} \rrbracket \delta$, which follows from above,

- $\left(k, q_{x}, W_{x}, v\right) \in \mathcal{T} \llbracket \Delta \vdash \tau$ : TYPE $\rrbracket$, which follows from above,

- $q_{1}^{\prime} \preceq\left(q_{1}^{\prime} \sqcup q_{x}\right)$, which follows from the definition of $\sqcup$, 
- $q_{x} \preceq\left(q_{1}^{\prime} \sqcup q_{x}\right)$, which follows from the definition of $\sqcup$,

- $\left(W_{1}^{\prime} \odot_{k} W_{x}=W_{1}\right)$, which follows from above,

- $\left(k, q_{2}, W_{2}, \gamma_{2}\right) \in \mathcal{G} \llbracket \Delta \vdash \Gamma_{2} \rrbracket \delta$

$\equiv\left(k,\left(q_{2}^{\prime} \sqcup q_{x}\right),\left(W_{2}^{\prime} \odot_{k} W_{x}\right), \gamma_{2}^{\prime}[x \mapsto v]\right) \in \mathcal{G} \llbracket \Delta \vdash \Gamma_{2}^{\prime}, x: \tau \rrbracket \delta$, which follows from

- $\left(k, q_{2}^{\prime}, W_{2}^{\prime}, \gamma_{2}^{\prime}\right) \in \mathcal{G} \llbracket \Delta \vdash \Gamma_{2}^{\prime} \rrbracket \delta$, which follows from above,

- $\left(k, q_{x}, W_{x}, v\right) \in \mathcal{T} \llbracket \Delta \vdash \tau:$ TYPE $\rrbracket$, which follows from above,

- $q_{2}^{\prime} \preceq\left(q_{2}^{\prime} \sqcup q_{x}\right)$, which follows from the definition of $\sqcup$,

- $q_{x} \preceq\left(q_{2}^{\prime} \sqcup q_{x}\right)$, which follows from the definition of $\sqcup$,

- $\left(W_{2}^{\prime} \odot_{k} W_{x}=W_{2}\right)$, which follows from above,

- $\gamma \in \gamma_{1} \boxplus \gamma_{2}$

$\equiv \gamma^{\prime}[x \mapsto v] \in \gamma_{1}^{\prime}[x \mapsto v] \boxplus \gamma_{2}^{\prime}[x \mapsto v]$,

which follows from

- $\operatorname{dom}\left(\gamma^{\prime}[x \mapsto v]\right)=\operatorname{dom}\left(\gamma_{1}[x \mapsto v]\right) \cup \operatorname{dom}\left(\gamma_{2}[x \mapsto v]\right)$, which follows from

$\operatorname{dom}\left(\gamma^{\prime}[x \mapsto v]\right)=\operatorname{dom}\left(\gamma_{1}^{\prime}[x \mapsto v]\right) \cup \operatorname{dom}\left(\gamma_{2}^{\prime}[x \mapsto v]\right)$

$\equiv \operatorname{dom}\left(\gamma^{\prime}\right) \cup\{x\}=\operatorname{dom}\left(\gamma_{1}^{\prime}\right) \cup\{x\} \cup \operatorname{dom}\left(\gamma_{2}^{\prime}\right) \cup\{x\}$

$\equiv \operatorname{dom}\left(\gamma^{\prime}\right) \cup\{x\}=\operatorname{dom}\left(\gamma^{\prime}\right) \cup\{x\}$

which follows from $\gamma^{\prime} \in \gamma_{1}^{\prime} \boxplus \gamma_{2}^{\prime}$,

- $\forall z \in \operatorname{dom}\left(\gamma_{1}^{\prime}[x \mapsto v]\right) . \gamma^{\prime}[x \mapsto v](z)=\gamma_{1}^{\prime}[x \mapsto v](z)$, which follows from $\forall z \in$ $\operatorname{dom}\left(\gamma_{1}^{\prime}\right) \cdot \gamma^{\prime}(z)=\gamma_{1}^{\prime}(z)$, which follows from $\gamma^{\prime} \in \gamma_{1}^{\prime} \boxplus \gamma_{2}$ and $x \notin \operatorname{dom} \Gamma^{\prime}$,

- $\forall z \in \operatorname{dom}\left(\gamma_{2}^{\prime}[x \mapsto v]\right) . \gamma^{\prime}[x \mapsto v](z)=\gamma_{2}^{\prime}[x \mapsto v](z)$, which follows from $\forall z \in$ $\operatorname{dom}\left(\gamma_{2}^{\prime}\right) \cdot \gamma^{\prime}(z)=\gamma_{2}^{\prime}(z)$, which follows from $\gamma^{\prime} \in \gamma_{1}^{\prime} \boxplus \gamma_{2}^{\prime}$ and $x \notin \operatorname{dom} \Gamma^{\prime}$,

- $q_{1} \preceq q \equiv\left(q_{1}^{\prime} \sqcup q_{x}\right) \preceq q$, which follows from $q_{1}^{\prime} \preceq q_{\Gamma^{\prime}}$ and $q_{\Gamma^{\prime}} \preceq q$ and $q_{x} \preceq q$ and the definition of $\sqcup$,

- $q_{2} \preceq q \equiv\left(q_{2}^{\prime} \sqcup q_{x}\right) \preceq q$, which follows from $q_{2}^{\prime} \preceq q_{\Gamma^{\prime}}$ and $q_{\Gamma^{\prime}} \preceq q$ and $q_{x} \preceq q$ and the definition of $\sqcup$,

- $\left(W_{1} \odot_{k} W_{2}=W\right)$, which follows from above.

\section{End Case}




\section{A.7.5 Validity of Typing Rules}

\section{Theorem 21 (Core Language Soundness)}

$$
\text { If } \Delta ; \Gamma \vdash e: \tau \text {, then } \Delta ; \Gamma \vdash e: \tau \rrbracket \text {. }
$$

\section{Proof}

By induction on the derivation $\Delta ; \Gamma \vdash e: \tau$.

(VAR)

Case $\frac{\Delta \vdash \tau: \text { TYPE }}{\Delta ; \bullet, x: \tau \vdash x: \tau}$ :

We are required to show $\llbracket \Delta ; \bullet, x: \tau \vdash x: \tau \rrbracket$.

Consider arbitrary $k, \delta, q_{\Gamma}, W_{\Gamma}$, and $\gamma$ such that

- $k \geq 0$,

- $\delta \in \mathcal{D} \llbracket \Delta \rrbracket$, and

- $\left(k, q_{\Gamma}, W_{\Gamma}, \gamma\right) \in \mathcal{G} \llbracket \Delta \vdash \bullet, x: \tau \rrbracket \delta$.

Hence, $\gamma \equiv \gamma_{1}[x \mapsto v]$ and $\left(k, q_{1}, W_{1}, \gamma_{1}\right) \in \mathcal{G} \llbracket \Delta \vdash \bullet \rrbracket \delta$ and $\left(k, q_{x}, W_{x}, v\right) \in \mathcal{T} \llbracket \Delta \vdash \tau:$ TYPE $\rrbracket \delta$ and $q_{1} \preceq q_{\Gamma}$ and $q_{x} \preceq q_{\Gamma}$ and $\left(W_{1} \odot_{k} W_{x}=W_{\Gamma}\right)$.

Hence, $\gamma_{1} \equiv \emptyset$ and $W_{1}=\left\lfloor\mathcal{U}_{\odot}\right\rfloor_{k}$.

Therefore, $\gamma \equiv \emptyset[x \mapsto v]$ and $W_{\Gamma} \equiv\left(\left\lfloor\mathcal{U}_{\odot}\right\rfloor_{k} \odot_{k} W_{x}\right)$.

Let $e_{s}=\gamma(x) \equiv v$ and $W_{s}=W_{\Gamma}$.

We are required to show that $\operatorname{Comp}\left(k, W_{s}, e_{s}, \mathcal{T} \llbracket \Delta \vdash \tau:\right.$ TYPE $\left.\rrbracket \delta\right) \equiv$ $\operatorname{Comp}\left(k, W_{\Gamma}, v, \mathcal{T} \llbracket \Delta \vdash \tau: \mathrm{TYPE} \rrbracket \delta\right)$.

Consider arbitrary $j, W_{r}, w_{s}, w_{f}$, and $e_{f}$ such that

- $j<k$,

- $w_{s}:_{k}\left(W_{s} \odot_{k} W_{r}\right) \equiv w_{s}:_{k}\left(W_{\Gamma} \odot_{k} W_{r}\right)$, noting that

$$
\begin{gathered}
w_{s}: k\left(W_{\Gamma} \odot_{k} W_{r}\right) \\
\equiv w_{s}: k\left(\left(\left\lfloor\mathcal{U}_{\odot}\right\rfloor_{k} \odot_{k} W_{x}\right) \odot_{k} W_{r}\right) \\
\text { which follows from above, }
\end{gathered}
$$

- $\left(w_{s}, e_{s}\right) \equiv\left(w_{s}, v\right) \longmapsto j\left(w_{f}, e_{f}\right)$, and

- $\operatorname{irred}\left(w_{f}, e_{f}\right)$.

Since $v$ is a value, we have $\operatorname{irred}\left(w_{s}, v\right)$.

Hence, $j=0$ and $w_{f} \equiv w_{s}$ and $e_{f} \equiv v$.

Note that $\left(\left(\left\lfloor\mathcal{U}_{\odot}\right\rfloor_{k} \odot_{k} W_{x}\right) \odot_{k} W_{r}\right) \equiv\left(W_{x} \odot_{k} W_{r}\right)$, which follows from

$$
\begin{aligned}
& \left(\left(\left\lfloor\mathcal{U}_{\odot}\right\rfloor_{k} \odot_{k} W_{x}\right) \odot_{k} W_{r}\right) \\
& \equiv\left(\left\lfloor\mathcal{U}_{\odot}\right\rfloor_{k} \odot_{k}\left(W_{x} \odot_{k} W_{r}\right)\right) \\
& \quad \text { which follows from Reqs } 6,7, \text { and } 8 \text { (join-commut, join-assocl, and join-assocr) } \\
& \equiv\left(\mathcal{U}_{\odot} \odot_{k}\left(W_{x} \odot_{k} W_{r}\right)\right) \\
& \text { which follows from Req } 5 \text { (join-aprx) } \\
& \equiv\left\lfloor\left(W_{x} \odot_{k} W_{r}\right)\right\rfloor_{k} \\
& \quad \text { which follows from Req } 9 \text { (join-unit-left) } \\
& \equiv\left(W_{x} \odot_{k} W_{r}\right) \\
& \quad \text { which follows from Req } 4 \text { (join-closed). }
\end{aligned}
$$

Let $W_{f}=W_{x}$ and $q_{f}=q_{x}$.

We are required to show that 
- $w_{f}:_{k-0}\left(W_{f} \odot_{k-0} W_{r}\right)$ $\equiv w_{s}:_{k}\left(W_{x} \odot_{k} W_{r}\right)$, which follows from

$$
\begin{aligned}
& w_{s}:{ }_{k}\left(\left(\left\lfloor\mathcal{U}_{\odot}\right\rfloor_{k} \odot_{k} W_{x}\right) \odot_{k} W_{r}\right) \\
& \text { which follows from above } \\
& \left(\left(\left\lfloor\mathcal{U}_{\odot}\right\rfloor_{k} \odot_{k} W_{x}\right) \odot_{k} W_{r}\right) \equiv\left(W_{x} \odot_{k} W_{r}\right) \\
& \quad \text { which follows from above, }
\end{aligned}
$$

- $\left(k-0, q_{f}, W_{f}, e_{f}\right) \in \mathcal{T} \llbracket \Delta \vdash \tau:$ TYPE $\rrbracket \delta$ $\equiv\left(k, q_{x}, W_{x}, v\right) \in \mathcal{T} \llbracket \Delta \vdash \tau: \mathrm{TYPE} \rrbracket \delta$, which follows from above. 
$(\mathrm{FN})$

Case $\frac{\Delta \vdash \xi: \text { QUAL } \quad \Delta \vdash \Gamma \preceq \xi \quad \Delta ; \Gamma, x: \tau_{1} \vdash e: \tau_{2}}{\Delta ; \Gamma \vdash \lambda x \cdot e:{ }^{\xi} \tau_{1} \multimap \tau_{2}}:$

We are required to show $\llbracket \Delta ; \Gamma \vdash \lambda x . e:{ }^{\xi} \tau_{1} \multimap \tau_{2} \rrbracket$.

Consider arbitrary $k, \delta, q_{\Gamma}, W_{\Gamma}$, and $\gamma$ such that

- $k \geq 0$,

- $\delta \in \mathcal{D} \llbracket \Delta \rrbracket$, and

- $\left(k, q_{\Gamma}, W_{\Gamma}, \gamma\right) \in \mathcal{G} \llbracket \Delta \vdash \Gamma \rrbracket \delta$.

Let $e_{s}=\gamma(\lambda x . e) \equiv \lambda x \cdot \gamma(e)$ and $W_{s}=W_{\Gamma}$.

We are required to show that $\operatorname{Comp}\left(k, W_{s}, e_{s}, \mathcal{T} \llbracket \Delta \vdash \xi_{\tau_{1}} \multimap \tau_{2}:\right.$ TYPE $\left.\rrbracket \delta\right) \equiv$ $\operatorname{Comp}\left(k, W_{\Gamma}, \lambda x \cdot \gamma(e), \mathcal{T} \llbracket \Delta \vdash \xi_{\tau_{1}} \multimap \tau_{2}:\right.$ TYPE $\left.\rrbracket \delta\right)$.

Consider arbitrary $j, W_{r}, w_{s}, w_{f}$, and $e_{f}$ such that

- $j<k$,

- $w_{s}:_{k}\left(W_{s} \odot_{k} W_{r}\right) \equiv w_{s}: k\left(W_{\Gamma} \odot_{k} W_{r}\right)$,

- $\left(w_{s}, e_{s}\right) \equiv\left(w_{s}, \lambda x \cdot \gamma(e)\right) \longmapsto j\left(w_{f}, e_{f}\right)$, and

- $\operatorname{irred}\left(w_{f}, e_{f}\right)$.

Since $\lambda x . \gamma(e)$ is a value, we have $\operatorname{irred}\left(w_{s}, \lambda x . \gamma(e)\right)$.

Hence, $j=0$ and $w_{f} \equiv w_{s}$ and $e_{f} \equiv \lambda x . \gamma(e)$.

Let $W_{f}=W_{\Gamma}$ and $q_{f}=\mathcal{T} \llbracket \Delta \vdash \xi: \mathrm{QUAL} \rrbracket \delta$.

We are required to show that

- $w_{f}: k-0\left(W_{f} \odot_{k-0} W_{r}\right)$

$\equiv w_{s}:_{k}\left(W_{\Gamma} \odot_{k} W_{r}\right)$,

which follows from above,

- $\left(k-0, q_{f}, W_{f}, e_{f}\right) \in \mathcal{T} \llbracket \Delta \vdash \xi_{\tau_{1}} \multimap \tau_{2}:$ TYPE $\rrbracket \delta$

$\equiv\left(k, \mathcal{T} \llbracket \Delta \vdash \xi:\right.$ QUAL $\left.\rrbracket \delta, W_{\Gamma}, \lambda x . \gamma(e)\right) \in \mathcal{T} \llbracket \Delta \vdash \xi_{\tau_{1}} \multimap \tau_{2}:$ TYPE $\rrbracket \delta$

$\equiv\left(k, \mathcal{T} \llbracket \Delta \vdash \xi: \mathrm{QUAL} \rrbracket \delta, W_{\Gamma}, \lambda x \cdot \gamma(e)\right)$

$\in\{(k, q, W, v) \mid$

$q=\mathcal{T} \llbracket \Delta \vdash \xi: \mathrm{QUAL} \rrbracket \delta \wedge$

$\left.(k, q, W, v) \in \mathcal{T} \llbracket \Delta \vdash \tau_{1} \multimap \tau_{2}: \mathrm{PRETYPE} \rrbracket \delta\right\}$

$\equiv\left(k, \mathcal{T} \llbracket \Delta \vdash \xi: \mathrm{QUAL} \rrbracket \delta, W_{\Gamma}, \lambda x \cdot \gamma(e)\right)$

$\in\left\{\left(k, q_{c}, W_{c}, \lambda x . e\right) \mid W_{c} \in\right.$ WorldDesc $_{k} \wedge \mathcal{P}\left(k, q_{c}, W_{c}\right) \wedge$

$q=\mathcal{T} \llbracket \Delta \vdash \xi: \mathrm{QUAL} \rrbracket \delta \wedge$

$\forall i<k, q_{a}, W_{a}, v_{a}$.

$\left(i, q_{a}, W_{a}, v_{a}\right) \in \mathcal{T} \llbracket \Delta \vdash \tau_{1}: \mathrm{TYPE} \rrbracket \delta \wedge$

$\left(W_{c} \odot_{i} W_{a}\right)$ defined $\Rightarrow$

which follows from

$\left.\operatorname{Comp}\left(i,\left(W_{c} \odot_{i} W_{a}\right), e\left[v_{a} / x\right], \mathcal{T} \llbracket \Delta \vdash \tau_{2}: \operatorname{TYPE} \rrbracket \delta\right)\right\}$,

- $\mathcal{T} \llbracket \Delta \vdash \xi: \mathrm{QUAL} \rrbracket \delta=\mathcal{T} \llbracket \Delta \vdash \xi: \mathrm{QUAL} \rrbracket \delta$, which follows trivially,

- $W_{\Gamma} \in$ WorldDesc $_{k}$, which follows from Lemma 9 applied to $\left(k, q_{\Gamma}, W_{\Gamma}, \gamma\right) \in \mathcal{G} \llbracket \Delta \vdash \Gamma \rrbracket \delta$,

- $\mathcal{P}\left(k, \mathcal{T} \llbracket \Delta \vdash \xi: \mathrm{QUAL} \rrbracket \delta, W_{\Gamma}\right)$, which follows from Lemma 18 applied to $\Delta \vdash \Gamma \preceq \xi$ and $\left(k, q_{\Gamma}, W_{\Gamma}, \gamma\right) \in \mathcal{G} \llbracket \Delta \vdash \Gamma \rrbracket \delta$ and $\mathcal{T} \llbracket \Delta \vdash \xi: \mathrm{QUAL} \rrbracket \delta$,

- $\forall i<k, q_{a}, W_{a}, v_{a} . \ldots$

Consider arbitrary $i, q_{a}, W_{a}$, and $v_{a}$ such that

- $i<k$, 
- $\left(i, q_{a}, W_{a}, v_{a}\right) \in \mathcal{T} \llbracket \Delta \vdash \tau_{1}:$ TYPE $\rrbracket \delta$, and

- $\left(W_{\Gamma} \odot_{i} W_{a}\right)$ defined.

We are required to show that $\operatorname{Comp}\left(i,\left(W_{\Gamma} \odot_{i} W_{a}\right), \gamma(e)\left[v_{a} / x\right], \mathcal{T} \llbracket \Delta \vdash \tau_{2}: \operatorname{TYPE} \rrbracket \delta\right)$.

Applying the induction hypothesis to $\Delta ; \Gamma, x: \tau_{1} \vdash e: \tau_{2}$, we conclude that $\llbracket \Delta ; \Gamma, x: \tau_{1} \vdash e: \tau_{2} \rrbracket$.

Instantiate this with $i, \delta,\left(q_{\Gamma} \sqcup q_{a}\right),\left(W_{\Gamma} \odot_{i} W_{a}\right)$, and $\gamma\left[x \mapsto v_{a}\right]$. Note that

- $i \geq 0$,

- $\delta \in \mathcal{D} \llbracket \Delta \rrbracket$, and

- $\left(i,\left(q_{\Gamma} \sqcup q_{a}\right),\left(W_{\Gamma} \odot_{i} W_{a}\right), \gamma\left[x \mapsto v_{a}\right]\right) \in \mathcal{G} \llbracket \Delta \vdash \Gamma, x: \tau_{1} \rrbracket \delta$, which follows from

- $\left(i, q_{\Gamma},\left\lfloor W_{\Gamma}\right\rfloor_{j}, \gamma\right) \in \mathcal{G} \llbracket \Delta \vdash \Gamma \rrbracket \delta$, which follows from Lemma 9 applied to $i<k$ and $\left(k, W_{\Gamma}, \gamma\right) \in \mathcal{G} \llbracket \Delta \vdash \Gamma \rrbracket \delta$,

- $\left(i, q_{a}, W_{a}, v_{a}\right) \in \mathcal{T} \llbracket \Delta \vdash \tau_{1}:$ TYPE $\rrbracket \delta$, which follows from above,

- $q_{\Gamma} \preceq\left(q_{\Gamma} \sqcup q_{a}\right)$, which follows from the definition of $\sqcup$,

- $q_{a} \preceq\left(q_{\Gamma} \sqcup q_{a}\right)$, which follows from the definition of $\sqcup$, and

- $\left(W_{\Gamma} \odot_{i} W_{a}\right)=\left(\left\lfloor W_{\Gamma}\right\rfloor_{i} \odot_{j} W_{a}\right)$, which follows from Req 5 (join-aprx).

Hence, $\operatorname{Comp}\left(i,\left(W_{\Gamma} \odot_{i} W_{a}\right), \gamma\left[x \mapsto v_{a}\right](e), \mathcal{T} \llbracket \Delta \vdash \tau_{2}:\right.$ TYPE $\left.\rrbracket \delta\right)$.

Thus, $\operatorname{Comp}\left(i,\left(W_{\Gamma} \odot_{i} W_{a}\right), \gamma(e)\left[v_{a} / x\right], \mathcal{T} \llbracket \Delta \vdash \tau_{2}:\right.$ TYPE $\left.\rrbracket \delta\right)$. 
(APr)

Case $\frac{\Delta \vdash \Gamma \sim \Gamma_{1} \boxplus \Gamma_{2} \quad \Delta ; \Gamma_{1} \vdash e_{1}: \xi_{\tau_{1}} \multimap \tau_{2} \quad \Delta ; \Gamma_{2} \vdash e_{2}: \tau_{1}}{\Delta ; \Gamma \vdash e_{1} e_{2}: \tau_{2}}:$

We are required to show $\llbracket \Delta ; \Gamma \vdash e_{1} e_{2}: \tau_{2} \rrbracket$.

Consider arbitrary $k, \delta, q_{\Gamma}, W_{\Gamma}$, and $\gamma$ such that

- $k \geq 0$,

- $\delta \in \mathcal{D} \llbracket \Delta \rrbracket$, and

- $\left(k, q_{\Gamma}, W_{\Gamma}, \gamma\right) \in \mathcal{G} \llbracket \Delta \vdash \Gamma \rrbracket \delta$.

Applying Lemma 20 to $\left(k, q_{\Gamma}, W_{\Gamma}, \gamma\right) \in \mathcal{G} \llbracket \Delta \vdash \Gamma \rrbracket \delta$ and $\Delta \vdash \Gamma \leadsto \Gamma_{1} \boxplus \Gamma_{2}$, we conclude that there exist $q_{\Gamma_{1}}, W_{\Gamma_{1}}, \gamma_{1}, q_{\Gamma_{2}}, W_{\Gamma_{2}}$ and $\gamma_{2}$, such that

- $\left(k, q_{\Gamma_{1}}, W_{\Gamma_{1}}, \gamma_{1}\right) \in \mathcal{G} \llbracket \Delta \vdash \Gamma_{1} \rrbracket \delta$,

- $\left(k, q_{\Gamma_{2}}, W_{\Gamma_{2}}, \gamma_{2}\right) \in \mathcal{G} \llbracket \Delta \vdash \Gamma_{2} \rrbracket \delta$,

- $\gamma \in \gamma_{1} \boxplus \gamma_{2}$,

- $q_{\Gamma_{1}} \preceq q_{\Gamma}$,

- $q_{\Gamma_{2}} \preceq q_{\Gamma}$, and

- $\left(W_{\Gamma_{1}} \odot_{k} W_{\Gamma_{2}}=W_{\Gamma}\right)$.

Note that $\gamma\left(e_{1}\right) \equiv \gamma_{1}\left(e_{1}\right)$ and $\gamma\left(e_{2}\right) \equiv \gamma_{2}\left(e_{2}\right)$.

Let $e_{s}=\gamma\left(e_{1} e_{2}\right) \equiv \gamma\left(e_{1}\right) \gamma\left(e_{2}\right) \equiv \gamma_{1}\left(e_{1}\right) \gamma_{2}\left(e_{2}\right)$ and $W_{s}=W_{\Gamma}$.

We are required to show that $\operatorname{Comp}\left(k, W_{s}, e_{s}, \mathcal{T} \llbracket \Delta \vdash \tau_{2}:\right.$ TYPE $\left.\rrbracket \delta\right) \equiv$ $\operatorname{Comp}\left(k, W_{\Gamma}, \gamma_{1}\left(e_{1}\right) \gamma_{2}\left(e_{2}\right), \mathcal{T} \llbracket \Delta \vdash \tau_{2}:\right.$ TYPE $\left.\rrbracket \delta\right)$.

Consider arbitrary $j, W_{r}, w_{s}, w_{f}$, and $e_{f}$ such that

- $j<k$,

- $w_{s}:_{k}\left(W_{s} \odot_{k} W_{r}\right) \equiv w_{s}:_{k}\left(W_{\Gamma} \odot_{k} W_{r}\right)$, noting that

$$
\begin{aligned}
& w_{s}:_{k}\left(W_{\Gamma} \odot_{k} W_{r}\right) \\
& \equiv w_{s}: k\left(\left(W_{\Gamma_{1}} \odot_{k} W_{\Gamma_{2}}\right) \odot_{k} W_{r}\right)
\end{aligned}
$$

which follows from above,

- $\left(w_{s}, e_{s}\right) \equiv\left(w_{s}, \gamma_{1}\left(e_{1}\right) \gamma_{2}\left(e_{2}\right)\right) \longmapsto^{j}\left(w_{f}, e_{f}\right)$, and

- $\operatorname{irred}\left(w_{f}, e_{f}\right)$.

Hence, by inspection of the operational semantics, it follows that there exist $j_{1}, w_{f_{1}}$, and $e_{f_{1}}$ such that

- $\left(w_{s}, \gamma_{1}\left(e_{1}\right)\right) \longmapsto^{j_{1}}\left(w_{f_{1}}, e_{f_{1}}\right)$,

- $\operatorname{irred}\left(w_{f_{1}}, e_{f_{1}}\right)$, and

- $j_{1} \leq j$.

Note that $\left(\left(W_{\Gamma_{1}} \odot_{k} W_{\Gamma_{2}}\right) \odot_{k} W_{r}\right) \equiv\left(W_{\Gamma_{1}} \odot_{k}\left(W_{\Gamma_{2}} \odot_{k} W_{r}\right)\right)$, which follows from $\left(\left(W_{\Gamma_{1}} \odot_{k} W_{\Gamma_{2}}\right) \odot_{k} W_{r}\right)$ $\equiv\left(W_{\Gamma_{1}} \odot_{k}\left(W_{\Gamma_{2}} \odot_{k} W_{r}\right)\right)$

which follows from Reqs 6, 7, and 8 (join-commut, join-assocl, and join-assocr).

Applying the induction hypothesis to $\Delta ; \Gamma_{1} \vdash e_{1}: \xi_{\tau_{1}} \multimap \tau_{2}$, we conclude that $\llbracket \Delta ; \Gamma_{1} \vdash e_{1}:{ }^{\xi} \tau_{1} \multimap \tau_{2} \rrbracket$.

Instantiate this with $k, \delta, q_{\Gamma_{1}}, W_{\Gamma_{1}}$, and $\gamma_{1}$. Note that

- $k \geq 0$, 
- $\delta \in \mathcal{D} \llbracket \Delta \rrbracket$, and

- $\left(k, q_{\Gamma_{1}}, W_{\Gamma_{1}}, \gamma_{1}\right) \in \mathcal{G} \llbracket \Delta \vdash \Gamma_{1} \rrbracket \delta$.

Hence, $\operatorname{Comp}\left(k, W_{\Gamma_{1}}, \gamma_{1}\left(e_{1}\right), \mathcal{T} \llbracket \Delta \vdash{ }^{\xi} \tau_{1} \multimap \tau_{2}:\right.$ TYPE $\left.\rrbracket \delta\right)$.

Instantiate this with $j_{1},\left(W_{\Gamma_{2}} \odot_{k} W_{r}\right), w_{s}, w_{f_{1}}$, and $e_{f_{1}}$. Note that

- $j_{1}<k$, which follows from $j_{1} \leq j$ and $j<k$,

- $w_{s}:_{k}\left(W_{\Gamma_{1}} \odot_{k}\left(W_{\Gamma_{2}} \odot_{k} W_{r}\right)\right)$, which follows from $w_{s}:_{k}\left(\left(W_{\Gamma_{1}} \odot_{k} W_{\Gamma_{2}}\right) \odot_{k} W_{r}\right)$ which follows from above

$\left(\left(W_{\Gamma_{1}} \odot_{k} W_{\Gamma_{2}}\right) \odot_{k} W_{r}\right) \equiv\left(W_{\Gamma_{1}} \odot_{k}\left(W_{\Gamma_{2}} \odot_{k} W_{r}\right)\right)$ which follows from above,

- $\left(w_{s}, \gamma_{1}\left(e_{1}\right)\right) \longmapsto^{j_{1}}\left(w_{f_{1}}, e_{f_{1}}\right)$,

- $\operatorname{irred}\left(w_{f_{1}}, e_{f_{1}}\right)$.

Hence, there exists $W_{f_{1}}$ and $q_{f_{1}}$ such that

- $w_{f_{1}}::_{k-j_{1}}\left(W_{f_{1}} \odot_{k-j_{1}}\left(W_{\Gamma_{2}} \odot_{k} W_{r}\right)\right)$, and

- $\left(k-j_{1}, q_{f_{1}}, W_{f_{1}}, e_{f_{1}}\right)$ $\in \mathcal{T} \llbracket \Delta \vdash \xi_{\tau_{1}} \multimap \tau_{2}:$ TYPE $\rrbracket \delta$

$\equiv\left\{\left(k, q_{c}, W_{c}, \lambda x . e\right) \mid W_{c} \in\right.$ WorldDesc $_{k} \wedge \mathcal{P}\left(k, q_{c}, W_{c}\right) \wedge$ $q=\mathcal{T} \llbracket \Delta \vdash \xi: \mathrm{QUAL} \rrbracket \delta \wedge$

$\forall i<k, q_{a}, W_{a}, v_{a}$.

$\left(i, q_{a}, W_{a}, v_{a}\right) \in \mathcal{T} \llbracket \Delta \vdash \tau_{1}: \mathrm{TYPE} \rrbracket \delta \wedge$

$\left(W_{c} \odot_{i} W_{a}\right)$ defined $\Rightarrow$

$\operatorname{Comp}\left(i,\left(W_{c} \odot_{i} W_{a}\right), e\left[v_{a} / x\right], \mathcal{T} \llbracket \Delta \vdash \tau_{2}:\right.$ TYPE $\left.\left.\rrbracket \delta\right)\right\}$.

Hence, $e_{f_{1}} \equiv \lambda x . e_{f_{11}}$ and $q_{f_{1}}=\mathcal{T} \llbracket \Delta \vdash \xi: \mathrm{QUAL} \rrbracket \delta$.

Note that

$$
\begin{aligned}
\left(w_{s}, e_{s}\right) & \equiv\left(w_{s}, \gamma_{1}\left(e_{1}\right) \gamma_{2}\left(e_{2}\right)\right) \\
& \longmapsto w^{j_{1}}\left(w_{f_{1}}, e_{f_{1}} \gamma_{2}\left(e_{2}\right)\right) \\
& \equiv\left(w_{f_{1}},\left(\lambda x . e_{f_{11}}\right) \gamma_{2}\left(e_{2}\right)\right) \\
& \longmapsto^{j-j_{1}}\left(w_{f}, e_{f}\right)
\end{aligned}
$$

and $\operatorname{irred}\left(w_{f}, e_{f}\right)$.

Hence, by inspection of the operational semantics, it follows that there exist $j_{2}, w_{f_{2}}$, and $e_{f_{2}}$ such that

- $\left(w_{f_{1}}, \gamma_{2}\left(e_{2}\right)\right) \longmapsto{ }^{j_{2}}\left(w_{f_{2}}, e_{f_{2}}\right)$,

- $\operatorname{irred}\left(w_{f_{2}}, e_{f_{2}}\right)$, and

- $j_{2} \leq j-j_{1}$.

Note that $\left(W_{f_{1}} \odot_{k-j_{1}}\left(W_{\Gamma_{2}} \odot_{k} W_{r}\right)\right) \equiv\left(\left\lfloor W_{\Gamma_{2}}\right\rfloor_{k-j_{1}} \odot_{k-j_{1}}\left(W_{f_{1}} \odot_{k-j_{1}} W_{r}\right)\right)$, which follows from $\left(W_{f_{1}} \odot_{k-j_{1}}\left(W_{\Gamma_{2}} \odot_{k} W_{r}\right)\right)$

$\equiv\left(W_{f_{1}} \odot_{k-j_{1}}\left\lfloor\left(W_{\Gamma_{2}} \odot_{k} W_{r}\right)\right\rfloor_{k-j_{1}}\right)$ which follows from Req 5 (join-aprx)

$\equiv\left(W_{f_{1}} \odot_{k-j_{1}}\left(W_{\Gamma_{2}} \odot_{k-j_{1}} W_{r}\right)\right)$ which follows from Req 4 (join-closed)

$\equiv\left(W_{\Gamma_{2}} \odot_{k-j_{1}}\left(W_{f_{1}} \odot_{k-j_{1}} W_{r}\right)\right)$ which follows from Reqs 6,7 , and 8 (join-commut, join-assocl, and join-assocr)

$\equiv\left(\left\lfloor W_{\Gamma_{2}}\right\rfloor_{k-j_{1}} \odot_{k-j_{1}}\left(W_{f_{1}} \odot_{k-j_{1}} W_{r}\right)\right)$ which follows from Req 5 (join-aprx). 
Applying the induction hypothesis to $\Delta ; \Gamma_{2} \vdash e_{2}: \tau_{1}$, we conclude that $\llbracket \Delta ; \Gamma_{2} \vdash e_{2}: \tau_{1} \rrbracket$. Instantiate this with $k-j_{1}, \delta, q_{\Gamma_{2}},\left\lfloor W_{\Gamma_{2}}\right\rfloor_{k-j_{1}}$, and $\gamma_{2}$. Note that

- $k-j_{1} \geq 0$, which follows from $j_{1}<k$,

- $\delta \in \mathcal{D} \llbracket \Delta \rrbracket$, and

- $\left(k-j_{1}, q_{\Gamma_{2}},\left\lfloor W_{\Gamma_{2}}\right\rfloor_{k-j_{1}}, \gamma_{2}\right) \in \mathcal{G} \llbracket \Delta \vdash \Gamma_{2} \rrbracket \delta$, which follows from Lemma 9 applied to $k-j_{1} \leq k$ and $\left(k, q_{\Gamma_{2}}, W_{\Gamma_{2}}, \gamma_{2}\right) \in \mathcal{G} \llbracket \Delta \vdash \Gamma_{2} \rrbracket \delta$.

Hence, $\operatorname{Comp}\left(k-j_{1}, \gamma_{2}\left(e_{2}\right),\left\lfloor W_{\Gamma_{2}}\right\rfloor_{k-j_{1}}, \mathcal{T} \llbracket \Delta \vdash \tau_{1}:\right.$ TYPE $\left.\rrbracket \delta\right)$.

Instantiate this with $j_{2},\left(W_{f_{1}} \odot_{k-j_{1}} W_{r}\right), w_{f_{1}}, w_{f_{2}}$, and $e_{f_{2}}$. Note that

- $j_{2}<k-j_{1}$, which follows from $j_{2} \leq j-j_{1}$ and $j<k$,

- $w_{f_{1}}: k_{-j_{1}}\left(\left\lfloor W_{\Gamma_{2}}\right\rfloor_{k-j_{1}} \odot_{k-j_{1}}\left(W_{f_{1}} \odot_{k-j_{1}} W_{r}\right)\right)$, which follows from

$$
w_{f_{1}}: k-j_{1}\left(W_{f_{1}} \odot_{k-j_{1}}\left(W_{\Gamma_{2}} \odot_{k} W_{r}\right)\right)
$$

which follows from above

$\left(W_{f_{1}} \odot_{k-j_{1}}\left(W_{\Gamma_{2}} \odot_{k} W_{r}\right)\right) \equiv\left(\left\lfloor W_{\Gamma_{2}}\right\rfloor_{k-j_{1}} \odot_{k-j_{1}}\left(W_{f_{1}} \odot_{k-j_{1}} W_{r}\right)\right)$ which follows from above,

- $\left(w_{f_{1}}, \gamma_{2}\left(e_{2}\right)\right) \longmapsto j^{j_{2}}\left(w_{f_{2}}, e_{f_{2}}\right)$, and

- $\operatorname{irred}\left(w_{f_{2}}, e_{f_{2}}\right)$.

Hence, there exists $W_{f_{2}}$ and $q_{f_{2}}$ such that

- $w_{f_{2}}: k-j_{1}-j_{2}\left(W_{f_{2}} \odot_{k-j_{1}-j_{2}}\left(W_{f_{1}} \odot_{k-j_{1}} W_{r}\right)\right)$, and

- $\left(k-j_{1}-j_{2}, q_{f_{2}}, W_{f_{2}}, e_{f_{2}}\right) \in \mathcal{T} \llbracket \Delta \vdash \tau_{1}:$ TYPE $\rrbracket \delta$.

Hence, $e_{f_{2}} \equiv v_{f_{2}}$.

Note that

$$
\begin{aligned}
\left(w_{s}, e_{s}\right) & \equiv\left(w_{s}, \gamma_{1}\left(e_{1}\right) \gamma_{2}\left(e_{2}\right)\right) \\
& \longmapsto^{j_{1}}\left(w_{f_{1}}, e_{f_{1}} \gamma_{2}\left(e_{2}\right)\right) \\
& \equiv\left(w_{f_{1}},\left(\lambda x \cdot e_{f_{11}}\right) \gamma_{2}\left(e_{2}\right)\right) \\
& \longmapsto^{j_{2}}\left(w_{f_{2}},\left(\lambda x \cdot e_{f_{11}}\right) e_{f_{2}}\right) \\
& \equiv\left(w_{f_{2}},\left(\lambda x \cdot e_{f_{11}}\right) v_{f_{2}}\right) \\
& \longmapsto^{1}\left(w_{f_{2}}, e_{f_{11}}\left[v_{f_{2}} / x\right]\right) \\
& \longmapsto^{j 3}\left(w_{f}, e_{f}\right)
\end{aligned}
$$

and $\operatorname{irred}\left(w_{f}, e_{f}\right)$, where $j=j_{1}+j_{2}+1+j_{3}$.

Note that $\left\lfloor\left(\begin{array}{lllll}W_{f_{2}} & \odot_{k-j_{1}-j_{2}} & \left(W_{f_{1}}\right. & \odot_{k-j_{1}} & W_{r}\end{array}\right)\right\rfloor_{k-j_{1}-j_{2}-1} \equiv \quad\left(\begin{array}{lll}W_{f_{1}} & \odot_{k-j_{1}-j_{2}-1}\end{array}\right.$ $\left.\left.\left\lfloor W_{f_{2}}\right\rfloor_{k-j_{1}-j_{2}-1}\right) \odot_{k-j_{1}-j_{2}-1} W_{r}\right)$, which follows from

$$
\begin{aligned}
& \left\lfloor\left(W_{f_{2}} \odot_{k-j_{1}-j_{2}}\left(W_{f_{1}} \odot_{k-j_{1}} W_{r}\right)\right)\right\rfloor_{k-j_{1}-j_{2}-1} \equiv\left(W_{f_{2}} \odot_{k-j_{1}-j_{2}-1}\left(W_{f_{1}} \odot_{k-j_{1}} W_{r}\right)\right) \\
& \quad \text { which follows from Req } 4 \text { (join-closed) } \\
& \equiv\left(W_{f_{2}} \odot_{k-j_{1}-j_{2}-1}\left\lfloor\left(W_{f_{1}} \odot_{k-j_{1}} W_{r}\right)\right\rfloor_{k-j_{1}-j_{2}-1}\right) \\
& \text { which follows from Req } 5 \text { (join-aprx }) \\
& \equiv\left(W_{f_{2}} \odot_{k-j_{1}-j_{2}-1}\left(W_{f_{1}} \odot_{k-j_{1}-j_{2}-1} W_{r}\right)\right) \\
& \quad \text { which follows from Req } 4 \text { (join-closed) } \\
& \equiv\left(\left(W_{f_{1}} \odot_{k-j_{1}-j_{2}-1} W_{f_{2}}\right) \odot_{k-j_{1}-j_{2}-1} W_{r}\right) \\
& \quad \text { which follows from Reqs } 6,7, \text { and } 8(\text { join-commut, join-assocl, and join-assocr }) \\
& \equiv\left(\left(W_{f_{1}} \odot_{k-j_{1}-j_{2}-1}\left\lfloor W_{f_{2}}\right\rfloor_{k-j_{1}-j_{2}-1}\right) \odot_{k-j_{1}-j_{2}-1} W_{r}\right) \\
& \quad \text { which follows from Req } 5 \text { (join-aprx }) .
\end{aligned}
$$

Instantiate $\left(k-j_{1}, q_{f_{1}}, W_{f_{1}}, \lambda x . e_{f_{11}}\right) \in \mathcal{T} \llbracket \Delta \vdash{ }^{\xi} \tau_{1} \multimap \tau_{2}:$ TYPE $\rrbracket \delta$ with $k-j_{1}-j_{2}-1, q_{f_{2}}$, $\left\lfloor W_{f_{2}}\right\rfloor_{k-j_{1}-j_{2}-1}$, and $v_{f_{2}}$. Note that

- $k-j_{1}-j_{2}-1<k-j_{1}$, 
- $\left(k-j_{1}-j_{2}-1, q_{f_{2}},\left\lfloor W_{f_{2}}\right\rfloor_{k-j_{1}-j_{2}-1}, v_{f_{2}}\right) \in \mathcal{T} \llbracket \Delta \vdash \tau_{1}:$ TYPE $\rrbracket \delta$, which follows from Lemma 8 and Fact 6 applied to $k-j_{1}-j_{2}-1 \leq k-j_{1}-j_{2}$ and $\left(k-j_{1}-j_{2}, q_{f_{2}}, W_{f_{2}}, v_{f_{2}}\right) \in$ $\mathcal{T} \llbracket \Delta \vdash \tau_{1}:$ TYPE $\rrbracket \delta$, and

- $\left(W_{f_{1}} \odot_{k-j_{1}-j_{2}-1}\left\lfloor W_{f_{2}}\right\rfloor_{k-j_{1}-j_{2}-1}\right)$ defined, which follows from above.

Hence, $\operatorname{Comp}\left(k-j_{1}-j_{2}-1,\left(W_{f_{1}} \odot_{k-j_{1}-j_{2}-1}\left\lfloor W_{f_{2}}\right\rfloor_{k-j_{1}-j_{2}-1}\right), e_{f_{11}}\left[v_{f_{2}} / x\right], \mathcal{T} \llbracket \Delta \vdash \tau_{2}:\right.$ TYPE $\left.\rrbracket\right)$. Instantiate this with $j_{3}, W_{r}, w_{f_{2}}, w_{f}$, and $e_{f}$. Note that

- $j_{3}<k-j_{1}-j_{2}-1$, which follows from $j_{3}=j-j_{1}-j_{2}-1$ and $j<k$,

- $w_{f_{2}}: k-j_{1}-j_{2}-1\left(\left(W_{f_{1}} \odot_{k-j_{1}-j_{2}-1}\left\lfloor W_{f_{2}}\right\rfloor_{k-j_{1}-j_{2}-1}\right) \odot_{k-j_{1}-j_{2}-1} W_{r}\right)$, which follows from

$w_{f_{2}}: k-j_{1}-j_{2}\left(W_{f_{2}} \odot_{k-j_{1}-j_{2}}\left(W_{f_{1}} \odot_{k-j_{1}} W_{r}\right)\right)$

which follows from above

$\Rightarrow w_{f_{2}}: k-j_{1}-j_{2}-1\left(W_{f_{2}} \odot_{k-j_{1}-j_{2}}\left(W_{f_{1}} \odot_{k-j_{1}} W_{r}\right)\right)$

which follows from Req 2 (models-closed)

$\Leftrightarrow w_{f_{2}}: k-j_{1}-j_{2}-1\left\lfloor\left(W_{f_{2}} \odot_{k-j_{1}-j_{2}}\left(W_{f_{1}} \odot_{k-j_{1}} W_{r}\right)\right)\right\rfloor_{k-j_{1}-j_{2}-1}$

which follows from Req 3 (models-aprx)

$\left\lfloor\left(W_{f_{2}} \odot_{k-j_{1}-j_{2}}\left(W_{f_{1}} \odot_{k-j_{1}} W_{r}\right)\right)\right\rfloor_{k-j_{1}-j_{2}-1}$

$\equiv\left(\left(W_{f_{1}} \odot_{k-j_{1}-j_{2}-1}\left\lfloor W_{f_{2}}\right\rfloor_{k-j_{1}-j_{2}-1}\right) \odot_{k-j_{1}-j_{2}-1} W_{r}\right)$

which follows from above,

- $\left(w_{f_{2}}, e_{f_{11}}\left[v_{f_{2}} / x\right]\right) \longmapsto^{j_{3}}\left(w_{f}, e_{f}\right)$, and

- $\operatorname{irred}\left(w_{f}, e_{f}\right)$.

Hence, there exists $W_{f^{\prime}}$ and $q_{f^{\prime}}$ such that

- $w_{f}:_{k-j_{1}-j_{2}-1-j_{3}}\left(W_{f^{\prime}} \odot_{k-j_{1}-j_{2}-1-j_{3}} W_{r}\right)$, and

- $\left(k-j_{1}-j_{2}-1-j_{3}, q_{f^{\prime}}, W_{f^{\prime}}, e_{f}\right) \in \mathcal{T} \llbracket \Delta \vdash \tau_{2}:$ TYPE $\rrbracket \delta$.

Let $W_{f}=W_{f^{\prime}}$ and $q_{f}=q_{f^{\prime}}$.

We are required to show that

- $w_{f}: k-j\left(W_{f} \odot_{k-j} W_{r}\right)$

$\equiv w_{f}:_{k-j_{1}-j_{2}-1-j_{3}}\left(W_{f^{\prime}} \odot_{k-j_{1}-j_{2}-1-j_{3}} W_{r}\right)$,

which follows from above,

- $\left(k-j, q_{f}, W_{f}, e_{f}\right) \in \mathcal{T} \llbracket \Delta \vdash \tau_{2}:$ TYPE $\rrbracket \delta$

$\equiv\left(k-j_{1}-j_{2}-1-j_{3}, q_{f^{\prime}}, W_{f^{\prime}}, e_{f}\right) \in \mathcal{T} \llbracket \Delta \vdash \tau_{2}:$ TYPE $\rrbracket \delta$,

which follows from above. 
(MUNIT)

Case $\frac{\Delta \vdash \xi: \text { QUAL }}{\Delta ; \bullet \vdash\langle\rangle:{ }^{\xi} \mathbf{1}_{\otimes}}$ :

We are required to show $\llbracket \Delta ; \bullet \vdash\langle\rangle:{ }^{\xi} \mathbf{1}_{\otimes} \rrbracket$.

Consider arbitrary $k, \delta, q_{\Gamma}, W_{\Gamma}$, and $\gamma$ such that

- $k \geq 0$,

- $\delta \in \mathcal{D} \llbracket \Delta \rrbracket$, and

• $\left(k, q_{\Gamma}, W_{\Gamma}, \gamma\right) \in \mathcal{G} \llbracket \Delta \vdash \bullet \rrbracket \delta$.

Hence, $\gamma \equiv \emptyset$ and $W_{\Gamma}=\left\lfloor\mathcal{U}_{\odot}\right\rfloor_{k}$.

Let $e_{s}=\gamma(\langle\rangle) \equiv\langle\rangle$ and $W_{s}=W_{\Gamma}$.

We are required to show that $\operatorname{Comp}\left(k, W_{s}, e_{s}, \mathcal{T} \llbracket \Delta \vdash \xi \mathbf{1}_{\otimes}: \operatorname{TYPE} \rrbracket \delta\right) \quad \equiv$ $\operatorname{Comp}\left(k, W_{\Gamma},\langle\rangle, \mathcal{T} \llbracket \Delta \vdash \xi \mathbf{1}_{\otimes}:\right.$ TYPE $\left.\rrbracket \delta\right)$.

Consider arbitrary $j, W_{r}, w_{s}, w_{f}$, and $e_{f}$ such that

- $j<k$,

- $w_{s}:_{k}\left(W_{s} \odot_{k} W_{r}\right) \equiv w_{s}:_{k}\left(W_{\Gamma} \odot_{k} W_{r}\right)$, noting that

$$
\begin{aligned}
& w_{s}:_{k}\left(W_{\Gamma} \odot_{k} W_{r}\right) \\
& \equiv w_{s}: k\left(\left[\mathcal{U}_{\odot}\right\rfloor_{k} \bigodot_{k} W_{r}\right) \\
& \quad \text { which follows from above, }
\end{aligned}
$$

- $\left(w_{s}, e_{s}\right) \equiv\left(w_{s},\langle\rangle\right) \longmapsto^{j}\left(w_{f}, e_{f}\right)$, and

- $\operatorname{irred}\left(w_{f}, e_{f}\right)$.

Since \langle\rangle is a value, we have $\operatorname{irred}\left(w_{s},\langle\rangle\right)$.

Hence, $j=0$ and $w_{f} \equiv w_{s}$ and $e_{f} \equiv\langle\rangle$.

Let $W_{f}=\left\lfloor\mathcal{U}_{\odot}\right\rfloor_{k}$ and $q_{f}=\mathcal{T} \llbracket \Delta \vdash \xi:$ QUAL $\rrbracket \delta$.

We are required to show that

- $w_{f}:_{k-0}\left(W_{f} \odot_{k-0} W_{r}\right)$ $\equiv w_{s}: k\left(\left\lfloor\mathcal{U}_{\odot}\right\rfloor_{k} \odot_{k} W_{r}\right)$, which follows from above,

- $\left(k-0, q_{f}, W_{f}, e_{f}\right) \in \mathcal{T} \llbracket \Delta \vdash \xi \mathbf{1}_{\otimes}:$ TYPE $\rrbracket \delta$

$$
\begin{gathered}
\equiv\left(k, \mathcal{T} \llbracket \Delta \vdash \xi: \mathrm{QUAL} \rrbracket \delta,\left\lfloor\mathcal{U}_{\odot}\right\rfloor_{k},\langle\rangle\right) \in \mathcal{T} \llbracket \Delta \vdash \mathbf{1}_{\otimes} \rrbracket \delta \\
\equiv\left(k, \mathcal{T} \llbracket \Delta \vdash \xi: \mathrm{QUAL} \rrbracket \delta,\left\lfloor\mathcal{U}_{\odot}\right\rfloor_{k},\langle\rangle\right) \\
\in\{(k, q, W, v) \mid \\
q=\mathcal{T} \llbracket \Delta \vdash \xi: \mathrm{QUAL} \rrbracket \delta \wedge \\
\left.(k, q, W, v) \in \mathcal{T} \llbracket \Delta \vdash \mathbf{1}_{\otimes}: \mathrm{PRETYPE} \rrbracket \delta\right\} \\
\equiv\left(k, \mathcal{T} \llbracket \Delta \vdash \xi: \mathrm{QUAL} \rrbracket \delta,\left\lfloor\mathcal{U}_{\odot}\right\rfloor_{k},\langle\rangle\right) \\
\in\{(k, q, W,\langle\rangle) \mid \\
q=\mathcal{T} \llbracket \Delta \vdash \xi: \mathrm{QUAL} \rrbracket \delta \wedge \\
\left.W=\left\lfloor\mathcal{U}_{\odot}\right\rfloor_{k}\right\},
\end{gathered}
$$

which follows from

- $\mathcal{T} \llbracket \Delta \vdash \xi: \mathrm{QUAL} \rrbracket \delta=\mathcal{T} \llbracket \Delta \vdash \xi: \mathrm{QUAL} \rrbracket \delta$, which follows trivially,

- $\left\lfloor\mathcal{U}_{\odot}\right\rfloor_{k}=\left\lfloor\mathcal{U}_{\odot}\right\rfloor_{k}$, which follows trivially. 
(LET-MUNIT)

Case $\frac{\Delta \vdash \Gamma \leadsto \Gamma_{1} \boxplus \Gamma_{2} \quad \Delta ; \Gamma_{1} \vdash e_{1}:{ }^{\xi} \mathbf{1}_{\otimes} \quad \Delta ; \Gamma_{2} \vdash e_{2}: \tau}{\Delta ; \Gamma \vdash \text { let }\langle\rangle=e_{1} \text { in } e_{2}: \tau}$ :

We are required to show $\llbracket \Delta ; \Gamma \vdash$ let \langle\rangle$=e_{1}$ in $e_{2}: \tau \rrbracket$.

Consider arbitrary $k, \delta, q_{\Gamma}, W_{\Gamma}$, and $\gamma$ such that

- $k \geq 0$,

- $\delta \in \mathcal{D} \llbracket \Delta \rrbracket$, and

- $\left(k, q_{\Gamma}, W_{\Gamma}, \gamma\right) \in \mathcal{G} \llbracket \Delta \vdash \Gamma \rrbracket \delta$.

Applying Lemma 20 to $\left(k, q_{\Gamma}, W_{\Gamma}, \gamma\right) \in \mathcal{G} \llbracket \Delta \vdash \Gamma \rrbracket \delta$ and $\Delta \vdash \Gamma \leadsto \Gamma_{1} \boxplus \Gamma_{2}$, we conclude that there exist $q_{\Gamma_{1}}, W_{\Gamma_{1}}, \gamma_{1}, q_{\Gamma_{2}}, W_{\Gamma_{2}}$, and $\gamma_{2}$, such that

- $\left(k, q_{\Gamma_{1}}, W_{\Gamma_{1}}, \gamma_{1}\right) \in \mathcal{G} \llbracket \Delta \vdash \Gamma_{1} \rrbracket \delta$,

- $\left(k, q_{\Gamma_{2}}, W_{\Gamma_{2}}, \gamma_{2}\right) \in \mathcal{G} \llbracket \Delta \vdash \Gamma_{2} \rrbracket \delta$,

- $\gamma \in \gamma_{1} \boxplus \gamma_{2}$,

- $q_{\Gamma_{1}} \preceq q_{\Gamma}$,

- $q_{\Gamma_{2}} \preceq q_{\Gamma}$, and

- $\left(W_{\Gamma_{1}} \odot_{k} W_{\Gamma_{2}}=W_{\Gamma}\right)$.

Note that $\gamma\left(e_{1}\right) \equiv \gamma_{1}\left(e_{1}\right)$ and $\gamma\left(e_{2}\right) \equiv \gamma_{2}\left(e_{2}\right)$.

Let $e_{s}=\gamma\left(\right.$ let \langle\rangle$=e_{1}$ in $\left.e_{2}\right) \equiv \operatorname{let}\langle\rangle=\gamma\left(e_{1}\right)$ in $\gamma\left(e_{2}\right) \equiv \operatorname{let}\langle\rangle=\gamma_{1}\left(e_{1}\right)$ in $\gamma_{2}\left(e_{2}\right)$ and $W_{s}=W_{\Gamma}$.

We are required to show that $\operatorname{Comp}\left(k, W_{s}, e_{s}, \mathcal{T} \llbracket \Delta \vdash \tau: \mathrm{TYPE} \rrbracket \delta\right) \equiv \operatorname{Comp}\left(k, W_{\Gamma}\right.$, let \langle\rangle$=$ $\gamma_{1}\left(e_{1}\right)$ in $\gamma_{2}\left(e_{2}\right), \mathcal{T} \llbracket \Delta \vdash \tau:$ TYPE $\left.\rrbracket \delta\right)$.

Consider arbitrary $j, W_{r}, w_{s}, w_{f}$, and $e_{f}$ such that

- $j<k$,

- $w_{s}:_{k}\left(W_{s} \odot_{k} W_{r}\right) \equiv w_{s}:_{k}\left(W_{\Gamma} \odot_{k} W_{r}\right)$, noting that

$$
\begin{gathered}
w_{s}:_{k}\left(W_{\Gamma} \odot_{k} W_{r}\right) \\
\equiv w_{s}:_{k}\left(\left(W_{\Gamma_{1}} \odot_{k} W_{\Gamma_{2}}\right) \odot_{k} W_{r}\right) \\
\text { which follows from above, }
\end{gathered}
$$

- $\left(w_{s}, e_{s}\right) \equiv\left(w_{s}\right.$, let \langle\rangle$=\gamma_{1}\left(e_{1}\right)$ in $\left.\gamma_{2}\left(e_{2}\right)\right) \longmapsto j\left(w_{f}, e_{f}\right)$, and

- $\operatorname{irred}\left(w_{f}, e_{f}\right)$.

Hence, by inspection of the operational semantics, it follows that there exist $j_{1}, w_{f_{1}}$, and $e_{f_{1}}$ such that

- $\left(w_{s}, \gamma_{1}\left(e_{1}\right)\right) \longmapsto^{j_{1}}\left(w_{f_{1}}, e_{f_{1}}\right)$,

- $\operatorname{irred}\left(w_{f_{1}}, e_{f_{1}}\right)$, and

- $j_{1} \leq j$.

Note that $\left(\left(W_{\Gamma_{1}} \odot_{k} W_{\Gamma_{2}}\right) \odot_{k} W_{r}\right) \equiv\left(W_{\Gamma_{1}} \odot_{k}\left(W_{\Gamma_{2}} \odot_{k} W_{r}\right)\right)$, which follows from

$$
\begin{aligned}
& \left(\left(W_{\Gamma_{1}} \odot_{k} W_{\Gamma_{2}}\right) \odot_{k} W_{r}\right) \\
& \equiv\left(W_{\Gamma_{1}} \odot_{k}\left(W_{\Gamma_{2}} \odot_{k} W_{r}\right)\right)
\end{aligned}
$$

which follows from Reqs 6, 7, and 8 (join-commut, join-assocl, and join-assocr).

Applying the induction hypothesis to $\Delta ; \Gamma_{1} \vdash e_{1}:{ }^{\xi} \mathbf{1}_{\otimes}$, we conclude that $\llbracket \Delta ; \Gamma_{1} \vdash e_{1}:{ }^{\xi} \mathbf{1}_{\otimes} \rrbracket$. Instantiate this with $k, \delta, q_{\Gamma_{1}}, W_{\Gamma_{1}}$, and $\gamma_{1}$. Note that

- $k \geq 0$, 
- $\delta \in \mathcal{D} \llbracket \Delta \rrbracket$, and

- $\left(k, q_{\Gamma_{1}}, W_{\Gamma_{1}}, \gamma_{1}\right) \in \mathcal{G} \llbracket \Delta \vdash \Gamma_{1} \rrbracket \delta$.

Hence, $\operatorname{Comp}\left(k, W_{\Gamma_{1}}, \gamma_{1}\left(e_{1}\right), \mathcal{T} \llbracket \Delta \vdash^{\xi} \mathbf{1}_{\otimes}: \mathrm{TYPE} \rrbracket \delta\right)$.

Instantiate this with $j_{1},\left(W_{\Gamma_{2}} \odot_{k} W_{r}\right), w_{s}, w_{f_{1}}$, and $e_{f_{1}}$. Note that

- $j_{1}<k$, which follows from $j_{1} \leq j$ and $j<k$,

- $w_{s}:_{k}\left(W_{\Gamma_{1}} \odot_{k}\left(W_{\Gamma_{2}} \odot_{k} W_{r}\right)\right)$, which follows from $w_{s}:_{k}\left(\left(W_{\Gamma_{1}} \odot_{k} W_{\Gamma_{2}}\right) \odot_{k} W_{r}\right)$ which follows from above

$\left(\left(W_{\Gamma_{1}} \odot_{k} W_{\Gamma_{2}}\right) \odot_{k} W_{r}\right) \equiv\left(W_{\Gamma_{1}} \odot_{k}\left(W_{\Gamma_{2}} \odot_{k} W_{r}\right)\right)$ which follows from above,

- $\left(w_{s}, \gamma_{1}\left(e_{1}\right)\right) \longmapsto^{j_{1}}\left(w_{f_{1}}, e_{f_{1}}\right)$, and

- $\operatorname{irred}\left(w_{f_{1}}, e_{f_{1}}\right)$.

Hence, there exists $W_{f_{1}}$ and $q_{f_{1}}$ such that

- $w_{f_{1}}:_{k-j_{1}}\left(W_{f_{1}} \odot_{k-j_{1}}\left(W_{\Gamma_{2}} \odot_{k} W_{r}\right)\right)$, and

- $\left(k-j_{1}, q_{f_{1}}, W_{f_{1}}, e_{f_{1}}\right)$

$\in \mathcal{T} \llbracket \Delta \vdash{ }^{\prime} \mathbf{1}_{\otimes}:$ TYPE $\rrbracket \delta$

$\equiv\{(k, q, W,\langle\rangle) \mid$

$\left.q=\mathcal{T} \llbracket \Delta \vdash \xi: \mathrm{QUAL} \rrbracket \delta \wedge W=\left\lfloor\mathcal{U}_{\odot}\right\rfloor_{k}\right\}$.

Hence, $e_{f_{1}} \equiv\langle\rangle$ and $q_{f_{1}}=\mathcal{T} \llbracket \Delta \vdash \xi: \mathrm{QUAL} \rrbracket \delta$ and $W_{f_{1}} \equiv\left\lfloor\mathcal{U}_{\odot}\right\rfloor_{k-j_{1}}$.

Note that

$$
\begin{aligned}
\left(w_{s}, e_{s}\right) & \equiv\left(w_{s}, \text { let }\langle\rangle=\gamma_{1}\left(e_{1}\right) \text { in } \gamma_{2}\left(e_{2}\right)\right) \\
& \longmapsto{ }^{j_{1}}\left(w_{f_{1}}, \text { let }\langle\rangle=e_{f_{1}} \text { in } \gamma_{2}\left(e_{2}\right)\right) \\
& \equiv\left(w_{f_{1}}, \text { let }\langle\rangle=\langle\rangle \text { in } \gamma_{2}\left(e_{2}\right)\right) \\
& \longmapsto{ }^{1}\left(w_{f_{1}}, \gamma_{2}\left(e_{2}\right)\right) \\
& \longmapsto^{j_{2}}\left(w_{f}, e_{f}\right)
\end{aligned}
$$

and $\operatorname{irred}\left(w_{f}, e_{f}\right)$, where $j=j_{1}+1+j_{2}$.

Note that $\left\lfloor\left(W_{f_{1}} \odot_{k-j_{1}}\left(W_{\Gamma_{2}} \odot_{k} W_{r}\right)\right)\right\rfloor_{k-j_{1}-1} \equiv\left(\left\lfloor W_{\Gamma_{2}}\right\rfloor_{k-j_{1}-1} \odot_{k-j_{1}-1} W_{r}\right)$, which follows from

$$
\begin{gathered}
\left\lfloor\left(W_{f_{1}} \odot_{k-j_{1}}\left(W_{\Gamma_{2}} \odot_{k} W_{r}\right)\right)\right\rfloor_{k-j_{1}-1} \\
\equiv\left\lfloor\left(\left\lfloor\mathcal{U}_{\odot}\right\rfloor_{k-j_{1}} \odot_{k-j_{1}}\left(W_{\Gamma_{2}} \odot_{k} W_{r}\right)\right)\right\rfloor_{k-j_{1}-1} \\
\text { which follows from above } \\
\equiv\left\lfloor\left(\mathcal{U}_{\odot} \odot_{k-j_{1}}\left(W_{\Gamma_{2}} \odot_{k} W_{r}\right)\right)\right\rfloor_{k-j_{1}-1} \\
\text { which follows from Req } 5 \text { (join-aprx) } \\
\equiv\left\lfloor\left\lfloor\left(W_{\Gamma_{2}} \odot_{k} W_{r}\right)\right\rfloor_{k-j_{1}}\right\rfloor_{k-j_{1}-1}{ } \text { (join-unit-left) } \\
\text { which follows from Req } 9 \text { (jom } \\
\equiv\left\lfloor\left(W_{\Gamma_{2}} \odot_{k-j_{1}} W_{r}\right)\right\rfloor_{k-j_{1}-1} \\
\text { which follows from Req } 4 \text { (join-closed) } \\
\equiv\left(W_{\Gamma_{2}} \odot_{k-j_{1}-1} W_{r}\right) \\
\text { which follows from Req } 4 \text { (join-closed) } \\
\equiv\left(\left\lfloor W_{\Gamma_{2}}\right\rfloor_{k-j_{1}-1} \odot_{k-j_{1}-1} W_{r}\right) \\
\text { which follows from Req } 5 \text { (join-aprx). }
\end{gathered}
$$

Applying the induction hypothesis to $\Delta ; \Gamma_{2} \vdash e_{2}: \tau$, we conclude that $\llbracket \Delta ; \Gamma_{2} \vdash e_{2}: \tau \rrbracket$. Instantiate this with $k-j_{1}-1, \delta, q_{\Gamma_{2}},\left\lfloor W_{\Gamma_{2}}\right\rfloor_{k-j_{1}-1}$, and $\gamma_{2}$. Note that

- $k-j_{1}-1 \geq 0$, which follows from $j_{1}+1+j_{2}=j$ and $j<k$,

- $\delta \in \mathcal{D} \llbracket \Delta \rrbracket$, and 
- $\left(k-j_{1}-1, q_{\Gamma_{2}},\left\lfloor W_{\Gamma_{2}}\right\rfloor_{k-j_{1}-1}, \gamma_{2}\right) \in \mathcal{G} \llbracket \Delta \vdash \Gamma_{2} \rrbracket \delta$, which follows from Lemma 9 applied to $k-j_{1}-1 \leq k$ and $\left(k, q_{\Gamma_{2}}, W_{\Gamma_{2}}, \gamma_{2}\right) \in \mathcal{G} \llbracket \Delta \vdash \Gamma_{2} \rrbracket \delta$.

Hence, $\operatorname{Comp}\left(k-j_{1}-1,\left\lfloor W_{\Gamma_{2}}\right\rfloor_{k-j_{1}-1}, \gamma_{2}\left(e_{2}\right), \mathcal{T} \llbracket \Delta \vdash \tau_{1}:\right.$ TYPE $\left.\rrbracket \delta\right)$.

Instantiate this with $j_{2}, W_{r}, w_{f_{1}}, w_{f}$, and $e_{f}$. Note that

- $j_{2}<k-j_{1}-1$, which follows from $j_{2}=j-j_{1}-1$ and $j<k$,

- $w_{f_{1}}:_{k-j_{1}-1}\left(\left\lfloor W_{\Gamma_{2}}\right\rfloor_{k-j_{1}-1} \odot_{k-j_{1}-1} W_{r}\right)$, which follows from

$w_{f_{1}}: k-j_{1}\left(W_{f_{1}} \odot_{k-j_{1}}\left(W_{\Gamma_{2}} \odot_{k} W_{r}\right)\right)$

which follows from above

$\Rightarrow w_{f_{1}}: k-j_{1}-1\left(W_{f_{1}} \odot_{k-j_{1}}\left(W_{\Gamma_{2}} \odot_{k} W_{r}\right)\right)$

which follows from Req 2 (models-closed)

$\Leftrightarrow w_{f_{1}: k-j_{1}-1}\left\lfloor\left(W_{f_{1}} \odot_{k-j_{1}}\left(W_{\Gamma_{2}} \odot_{k} W_{r}\right)\right)\right\rfloor_{k-j_{1}-1}$

which follows from Req 3 (models-aprx)

$\left\lfloor\left(W_{f_{1}} \odot_{k-j_{1}}\left(W_{\Gamma_{2}} \odot_{k} W_{r}\right)\right)\right\rfloor_{k-j_{1}-1} \equiv\left(\left\lfloor W_{\Gamma_{2}}\right\rfloor_{k-j_{1}-1} \odot_{k-j_{1}-1} W_{r}\right)$

which follows from above,

- $\left(w_{f_{1}}, \gamma_{2}\left(e_{2}\right)\right) \longmapsto^{j_{2}}\left(w_{f}, e_{f}\right)$, and

- $\operatorname{irred}\left(w_{f}, e_{f}\right)$.

Hence, there exists $W_{f_{2}}$ and $q_{f_{2}}$ such that

- $w_{f}:_{k-j_{1}-1-j_{2}}\left(W_{f_{2}} \odot_{k-j_{1}-1-j_{2}} W_{r}\right)$, and

- $\left(k-j_{1}-1-j_{2}, q_{f_{2}}, W_{f_{2}}, e_{f}\right) \in \mathcal{T} \llbracket \Delta \vdash \tau:$ TYPE $\rrbracket \delta$.

Let $W_{f}=W_{f_{2}}$ and $q_{f}=q_{f_{2}}$.

We are required to show that

- $w_{f}:_{k-j}\left(W_{f} \odot_{k-j} W_{r}\right)$ $\equiv w_{f}: k-j_{1}-1-j_{2}\left(W_{f_{2}} \odot_{k-j_{1}-1-j_{2}} W_{r}\right)$, which follows from above,

- $\left(k-j, q_{f}, W_{f}, e_{f}\right) \in \mathcal{T} \llbracket \Delta \vdash \tau \rrbracket \delta$ $\equiv\left(k-j_{1}-1-j_{2}, q_{f_{2}}, W_{f_{2}}, e_{f}\right) \in \mathcal{T} \llbracket \Delta \vdash \tau \rrbracket \delta$, which follows from above. 
(MPAIR)

Case $\frac{\Delta \vdash \Gamma \sim \Gamma_{1} \boxplus \Gamma_{2} \quad \Delta \vdash \xi: \text { QUAL } \quad \Delta ; \Gamma_{1} \vdash v_{1}: \tau_{1} \quad \Delta \vdash \tau_{1} \preceq \xi \quad \Delta ; \Gamma_{2} \vdash v_{2}: \tau_{2} \quad \Delta \vdash \tau_{2} \preceq \xi}{\Delta ; \Gamma \vdash\left\langle v_{1}, v_{2}\right\rangle:{ }^{\xi} \tau_{1} \otimes \tau_{2}}:$

We are required to show $\llbracket \Delta ; \Gamma \vdash\left\langle v_{1}, v_{2}\right\rangle:{ }^{\xi} \tau_{1} \otimes \tau_{2} \rrbracket$.

Consider arbitrary $k, \delta, q_{\Gamma}, W_{\Gamma}$, and $\gamma$ such that

- $k \geq 0$,

- $\delta \in \mathcal{D} \llbracket \Delta \rrbracket$, and

- $\left(k, q_{\Gamma}, W_{\Gamma}, \gamma\right) \in \mathcal{G} \llbracket \Delta \vdash \Gamma \rrbracket \delta$.

Applying Lemma 20 to $\left(k, q_{\Gamma}, W_{\Gamma}, \gamma\right) \in \mathcal{G} \llbracket \Delta \vdash \Gamma \rrbracket \delta$ and $\Delta \vdash \Gamma \sim \Gamma_{1} \boxplus \Gamma_{2}$, we conclude that there exist $q_{\Gamma_{1}}, W_{\Gamma_{1}}, \gamma_{1}, q_{\Gamma_{2}}, W_{\Gamma_{2}}$ and $\gamma_{2}$, such that

- $\left(k, q_{\Gamma_{1}}, W_{\Gamma_{1}}, \gamma_{1}\right) \in \mathcal{G} \llbracket \Delta \vdash \Gamma_{1} \rrbracket \delta$,

- $\left(k, q_{\Gamma_{1}}, W_{\Gamma_{2}}, \gamma_{2}\right) \in \mathcal{G} \llbracket \Delta \vdash \Gamma_{2} \rrbracket \delta$,

- $\gamma \in \gamma_{1} \boxplus \gamma_{2}$,

- $q_{\Gamma_{1}} \preceq q_{\Gamma}$,

- $q_{\Gamma_{2}} \preceq q_{\Gamma}$, and

- $\left(W_{\Gamma_{1}} \odot_{k} W_{\Gamma_{2}}=W_{\Gamma}\right)$.

Note that $\gamma\left(v_{1}\right) \equiv \gamma_{1}$ and $\gamma\left(v_{2}\right) \equiv \gamma_{2}\left(v_{2}\right)$.

Let $e_{s}=\gamma\left(\left\langle v_{1}, v_{2}\right\rangle\right) \equiv\left\langle\gamma\left(v_{1}\right), \gamma\left(v_{2}\right)\right\rangle \equiv\left\langle\gamma_{1}\left(v_{1}\right), \gamma_{2}\left(v_{2}\right)\right\rangle$ and $W_{s}=W_{\Gamma}$.

We are required to show that $\operatorname{Comp}\left(k, W_{s}, e_{s}, \mathcal{T} \llbracket \Delta \vdash \xi_{\tau_{1}} \otimes \tau_{2} \rrbracket \delta\right) \quad \equiv$ $\operatorname{Comp}\left(k, W_{\Gamma},\left\langle\gamma_{1}\left(v_{1}\right), \gamma_{2}\left(v_{2}\right)\right\rangle, \mathcal{T} \llbracket \Delta \vdash \xi_{\tau_{1}} \otimes \tau_{2} \rrbracket \delta\right)$.

Consider arbitrary $j, W_{r}, w_{s}, w_{f}, e_{f}$ such that

- $j<k$,

- $w_{s}:_{k}\left(W_{s} \odot_{k} W_{r}\right) \equiv w_{s}:_{k}\left(W_{\Gamma} \odot_{k} W_{r}\right)$, noting that

$$
\begin{gathered}
w_{s}:_{k}\left(W_{\Gamma} \odot_{k} W_{r}\right) \\
\equiv w_{s}: k\left(\left(W_{\Gamma_{1}} \odot_{k} W_{\Gamma_{2}}\right) \odot_{k} W_{r}\right) \\
\text { which follows from above, }
\end{gathered}
$$

- $\left(w_{s}, e_{s}\right) \equiv\left(w_{s},\left\langle\gamma_{1}\left(v_{1}\right), \gamma_{2}\left(v_{2}\right)\right\rangle\right) \longmapsto^{j}\left(w_{f}, e_{f}\right)$, and

- $\operatorname{irred}\left(w_{f}, e_{f}\right)$.

Since $\left\langle\gamma_{1}\left(v_{1}\right), \gamma_{2}\left(v_{2}\right)\right\rangle$ is a value, we have $\operatorname{irred}\left(w_{s},\left\langle\gamma_{1}\left(v_{1}\right), \gamma_{2}\left(v_{2}\right)\right\rangle\right)$.

Hence, $j=0$ and $w_{f} \equiv w_{s}$ and $e_{f} \equiv\left\langle\gamma_{1}\left(v_{1}\right), \gamma_{2}\left(v_{2}\right)\right\rangle$.

Note that $\left(\left(W_{\Gamma_{1}} \odot_{k} W_{\Gamma_{2}}\right) \odot_{k} W_{r}\right) \equiv\left(W_{\Gamma_{1}} \odot_{k}\left(W_{\Gamma_{2}} \odot_{k} W_{r}\right)\right)$, which follows from

$$
\begin{aligned}
& \left(\left(W_{\Gamma_{1}} \odot_{k} W_{\Gamma_{2}}\right) \odot_{k} W_{r}\right) \\
& \equiv\left(W_{\Gamma_{1}} \odot_{k}\left(W_{\Gamma_{2}} \odot_{k} W_{r}\right)\right)
\end{aligned}
$$

which follows from Reqs 6, 7, and 8 (join-commut, join-assocl, and join-assocr).

Applying the induction hypothesis to $\Delta ; \Gamma_{1} \vdash v_{1}: \tau_{1}$, we conclude that $\llbracket \Delta ; \Gamma_{1} \vdash v_{1}: \tau_{1} \rrbracket$. Instantiate this with $k, \delta, q_{\Gamma_{1}}, W_{\Gamma_{1}}$, and $\gamma_{1}$. Note that

- $k \geq 0$,

- $\delta \in \mathcal{D} \llbracket \Delta \rrbracket$, and

- $\left(k, q_{\Gamma_{1}}, W_{\Gamma_{1}}, \gamma_{1}\right) \in \mathcal{G} \llbracket \Delta \vdash \Gamma_{1} \rrbracket \delta$.

Hence, $\operatorname{Comp}\left(k, W_{\Gamma_{1}}, \gamma_{1}\left(v_{1}\right), \mathcal{T} \llbracket \Delta \vdash \tau_{1}:\right.$ TYPE $\left.\rrbracket \delta\right)$.

Instantiate this with $0,\left(W_{\Gamma_{2}} \odot_{k} W_{r}\right), w_{s}, w_{s}$, and $\gamma_{1}\left(v_{1}\right)$. Note that 
- $0<k$, which follows from $j=0$ and $j<k$,

- $w_{s}:_{k}\left(W_{\Gamma_{1}} \odot_{k}\left(W_{\Gamma_{2}} \odot_{k} W_{r}\right)\right)$, which follows from

$$
w_{s}: k\left(\left(W_{\Gamma_{1}} \odot_{k} W_{\Gamma_{2}}\right) \odot_{k} W_{r}\right)
$$

which follows from above

$\left(\left(W_{\Gamma_{1}} \odot_{k} W_{\Gamma_{2}}\right) \odot_{k} W_{r}\right) \equiv\left(W_{\Gamma_{1}} \odot_{k}\left(W_{\Gamma_{2}} \odot_{k} W_{r}\right)\right)$

which follows from above,

- $\left(w_{s}, \gamma_{1}\left(v_{1}\right)\right) \longmapsto^{0}\left(w_{s}, \gamma_{1}\left(v_{1}\right)\right)$, and

- $\operatorname{irred}\left(w_{s}, \gamma_{1}\left(v_{1}\right)\right)$, which follows from the fact that $\gamma_{1}\left(v_{1}\right)$ is a value.

Hence, there exists $W_{f_{1}}$ and $q_{f_{1}}$ such that

- $w_{s}:_{k-0}\left(W_{f_{1}} \odot_{k-0}\left(W_{\Gamma_{2}} \odot_{k} W_{r}\right)\right)$, and

- $\left(k-0, q_{f_{1}}, W_{f_{1}}, \gamma_{1}\left(v_{1}\right)\right) \in \mathcal{T} \llbracket \Delta \vdash \tau_{1}:$ TYPE $\rrbracket \delta$.

Note that $\left(W_{f_{1}} \odot_{k}\left(W_{\Gamma_{2}} \odot_{k} W_{r}\right)\right) \equiv\left(W_{\Gamma_{2}} \odot_{k}\left(W_{f_{1}} \odot_{k} W_{r}\right)\right)$, which follows from

$\left(W_{f_{1}} \odot_{k}\left(W_{\Gamma_{2}} \odot_{k} W_{r}\right)\right)$

$\equiv\left(W_{\Gamma_{2}} \odot_{k}\left(W_{f_{1}} \odot_{k} W_{r}\right)\right)$

which follows from Reqs 6, 7, and 8 (join-commut, join-assocl, and join-assocr).

Applying the induction hypothesis to $\Delta ; \Gamma_{2} \vdash v_{2}: \tau_{2}$, we conclude that $\llbracket \Delta ; \Gamma_{2} \vdash v_{2}: \tau_{2} \rrbracket$. Instantiate this with $k, \delta, q_{\Gamma_{2}}, W_{\Gamma_{2}}$, and $\gamma_{2}$. Note that

- $k \geq 0$,

- $\delta \in \mathcal{D} \llbracket \Delta \rrbracket$, and

- $\left(k, q_{\Gamma_{2}}, W_{\Gamma_{2}}, \gamma_{2}\right) \in \mathcal{G} \llbracket \Delta \vdash \Gamma_{2} \rrbracket \delta$.

Hence, $\operatorname{Comp}\left(k, W_{\Gamma_{2}}, \gamma_{2}\left(v_{2}\right), \mathcal{T} \llbracket \Delta \vdash \tau_{2}:\right.$ TYPE $\left.\rrbracket \delta\right)$.

Instantiate this with $0,\left(W_{f_{1}} \odot_{k} W_{r}\right), w_{s}, w_{s}$, and $\gamma_{2}\left(v_{2}\right)$. Note that

- $0<k$, which follows from $j=0$ and $j<k$,

- $w_{s}:_{k}\left(W_{\Gamma_{2}} \odot_{k}\left(W_{f_{1}} \odot_{k} W_{r}\right)\right)$, which follows from

$$
w_{s}:_{k}\left(W_{f_{1}} \odot_{k}\left(W_{\Gamma_{2}} \odot_{k} W_{r}\right)\right)
$$

which follows from above

$\left(W_{f_{1}} \odot_{k}\left(W_{\Gamma_{2}} \odot_{k} W_{r}\right)\right) \equiv\left(W_{\Gamma_{2}} \odot_{k}\left(W_{f_{1}} \odot_{k} W_{r}\right)\right)$

which follows from above,

- $\left(w_{s}, \gamma_{2}\left(v_{2}\right)\right) \longmapsto{ }^{0}\left(w_{s}, \gamma_{2}\left(v_{2}\right)\right)$, and

- $\operatorname{irred}\left(w_{s}, \gamma_{2}\left(v_{2}\right)\right)$, which follows from the fact that $\gamma_{2}\left(v_{2}\right)$ is a value.

Hence, there exists $W_{f_{2}}$ and $q_{f_{2}}$ such that

- $w_{s}: k-0\left(W_{f_{2}} \odot_{k-0}\left(W_{f_{1}} \odot_{k} W_{r}\right)\right)$, and

- $\left(k-0, q_{f_{2}}, W_{f_{2}}, \gamma_{2}\left(v_{2}\right)\right) \in \mathcal{T} \llbracket \Delta \vdash \tau_{2}:$ TYPE $\rrbracket \delta$.

Note that $\left(W_{f_{2}} \odot_{k}\left(W_{f_{1}} \odot_{k} W_{r}\right)\right) \equiv\left(\left(W_{f_{1}} \odot_{k} W_{f_{2}}\right) \odot_{k} W_{r}\right)$, which follows from

$$
\begin{aligned}
& \left(W_{f_{2}} \odot_{k}\left(W_{f_{1}} \odot_{k} W_{r}\right)\right) \\
& \equiv\left(\left(W_{f_{1}} \odot_{k} W_{f_{2}}\right) \odot_{k} W_{r}\right)
\end{aligned}
$$

which follows from Reqs 6, 7, and 8 (join-commut, join-assocl, and join-assocr).

Let $W_{f}=\left(W_{f_{1}} \odot_{k} W_{f_{2}}\right)$ and $q_{f}=\mathcal{T} \llbracket \Delta \vdash \xi: \mathrm{QUAL} \rrbracket \delta$.

We are required to show that 
- $w_{f}: k_{-0}\left(W_{f} \odot_{k-0} W_{r}\right)$ $\equiv w_{s}: k\left(\left(W_{f_{1}} \odot_{k} W_{f_{2}}\right) \odot_{k} W_{r}\right)$,

which follows from

$$
\begin{aligned}
& w_{s}: k\left(W_{f_{2}} \odot_{k}\left(W_{f_{1}} \odot_{k} W_{r}\right)\right) \\
& \text { which follows from above } \\
& \left(W_{f_{2}} \odot_{k}\left(W_{f_{1}} \odot_{k} W_{r}\right)\right) \equiv\left(\left(W_{f_{1}} \odot_{k} W_{f_{2}}\right) \odot_{k} W_{r}\right) \\
& \quad \text { which follows from above, }
\end{aligned}
$$

- $\left(k-0, q_{f}, W_{f}, e_{f}\right) \in \mathcal{T} \llbracket \Delta \vdash \xi_{\tau_{1}} \otimes \tau_{2}:$ TYPE $\rrbracket \delta$

$\equiv\left(k, \mathcal{T} \llbracket \Delta \vdash \xi: \mathrm{QUAL} \rrbracket \delta,\left(W_{f_{1}} \odot_{k} W_{f_{2}}\right),\left\langle\gamma_{1}\left(v_{1}\right), \gamma_{2}\left(v_{2}\right)\right\rangle\right)$ $\in\{(k, q, W, v) \mid$

$q=\mathcal{T} \llbracket \Delta \vdash \xi: \mathrm{QUAL} \rrbracket \delta \wedge$

$(k, q, W, v) \in \mathcal{T} \llbracket \Delta \vdash \tau_{1}\langle\tau,\rangle \rrbracket \rrbracket \delta$

$\equiv\left(k, \mathcal{T} \llbracket \Delta \vdash \xi: \mathrm{QUAL} \rrbracket \delta,\left(W_{f_{1}} \odot_{k} W_{f_{2}}\right),\left\langle\gamma_{1}\left(v_{1}\right), \gamma_{2}\left(v_{2}\right)\right\rangle\right)$

$\in\left\{\left(k, q, W,\left\langle v_{1}, v_{2}\right\rangle\right) \mid\right.$

$q=\mathcal{T} \llbracket \Delta \vdash \xi: \mathrm{QUAL} \rrbracket \delta \wedge$

$\left(k, q_{1}, W_{1}, v_{1}\right) \in \mathcal{T} \llbracket \Delta \vdash \tau_{1}: \mathrm{TYPE} \rrbracket \delta \wedge$

$\left(k, q_{2}, W_{2}, v_{2}\right) \in \mathcal{T} \llbracket \Delta \vdash \tau_{2}: \mathrm{TYPE} \rrbracket \delta \wedge$

$q_{1} \preceq q \wedge q_{2} \preceq q \wedge$

$\left.\left(W_{1} \odot_{k} W_{2}=W\right)\right\}$,

which follows from

- $\mathcal{T} \llbracket \Delta \vdash \xi: \mathrm{QUAL} \rrbracket \delta=\mathcal{T} \llbracket \Delta \vdash \xi: \mathrm{QUAL} \rrbracket \delta$, which follows trivially,

- $\left(k, q_{f_{1}}, W_{f_{1}}, \gamma\left(v_{1}\right)\right) \in \mathcal{T} \llbracket \Delta \vdash \tau_{1}:$ TYPE $\rrbracket \delta$, which follows from above,

- $\left(k, q_{f_{2}}, W_{f_{2}}, \gamma\left(v_{2}\right)\right) \in \mathcal{T} \llbracket \Delta \vdash \tau_{2}:$ TYPE $\rrbracket \delta$, which follows from above,

- $q_{f_{1}} \preceq \mathcal{T} \llbracket \Delta \vdash \xi: \mathrm{QUAL} \rrbracket \delta$, which follows from Lemma 15 applied to $\Delta \vdash \tau \preceq \xi$ and $\left(k, q_{f_{1}}, W_{f_{1}}, \gamma\left(v_{1}\right)\right) \in \mathcal{T} \llbracket \Delta \vdash \tau_{1}:$ TYPE $\rrbracket \delta$ and $\mathcal{T} \llbracket \Delta \vdash \xi: \mathrm{QUAL} \rrbracket$,

- $q_{f_{2}} \preceq \mathcal{T} \llbracket \Delta \vdash \xi:$ QUAL $\delta$, which follows from Lemma 15 applied to $\Delta \vdash \tau \preceq \xi$ and $\left(k, q_{f_{2}}, W_{f_{2}}, \gamma\left(v_{2}\right)\right) \in \mathcal{T} \llbracket \Delta \vdash \tau_{2}:$ TYPE $\rrbracket \delta$ and $\mathcal{T} \llbracket \Delta \vdash \xi: \mathrm{QUAL} \rrbracket$, and

- $\left(W_{f_{1}} \odot_{k} W_{f_{2}}\right)=\left(W_{f_{1}} \odot_{k} W_{f_{2}}\right)$, which follows trivially. 
(LET-MPAIR)

Case $\frac{\Delta \vdash \Gamma \sim \Gamma_{1} \boxplus \Gamma_{2} \quad \Delta ; \Gamma_{1} \vdash e_{1}:{ }^{\xi} \tau_{1} \otimes \tau_{2} \quad \Delta ; \Gamma_{2}, x_{1}: \tau_{1}, x_{2}: \tau_{2} \vdash e_{2}: \tau}{\Delta ; \Gamma \vdash \operatorname{let}\left\langle x_{1}, x_{2}\right\rangle=e_{1} \text { in } e_{2}: \tau}:$

We are required to show $\llbracket \Delta ; \Gamma \vdash$ let $\left\langle x_{1}, x_{2}\right\rangle=e_{1}$ in $e_{2}: \tau \rrbracket$.

Consider arbitrary $k, \delta, q_{\Gamma}, W_{\Gamma}$, and $\gamma$ such that

- $k \geq 0$,

- $\delta \in \mathcal{D} \llbracket \Delta \rrbracket$, and

- $\left(k, q_{\Gamma}, W_{\Gamma}, \gamma\right) \in \mathcal{G} \llbracket \Delta \vdash \Gamma \rrbracket \delta$.

Applying Lemma 20 to $\left(k, q_{\Gamma}, W_{\Gamma}, \gamma\right) \in \mathcal{G} \llbracket \Delta \vdash \Gamma \rrbracket \delta$ and $\Delta \vdash \Gamma \leadsto \Gamma_{1} \boxplus \Gamma_{2}$, we conclude that there exist $q_{\Gamma_{1}}, W_{\Gamma_{1}}, \gamma_{1}, q_{\Gamma_{2}}, W_{\Gamma_{2}}$, and $\gamma_{2}$, such that

- $\left(k, q_{\Gamma_{1}}, W_{\Gamma_{1}}, \gamma_{1}\right) \in \mathcal{G} \llbracket \Delta \vdash \Gamma_{1} \rrbracket \delta$,

- $\left(k, q_{\Gamma_{2}}, W_{\Gamma_{2}}, \gamma_{2}\right) \in \mathcal{G} \llbracket \Delta \vdash \Gamma_{2} \rrbracket \delta$,

- $\gamma \in \gamma_{1} \boxplus \gamma_{2}$,

- $q_{\Gamma_{1}} \preceq q_{\Gamma}$,

- $q_{\Gamma_{2}} \preceq q_{\Gamma}$, and

- $\left(W_{\Gamma_{1}} \odot_{k} W_{\Gamma_{2}}=W_{\Gamma}\right)$.

Note that $\gamma\left(e_{1}\right) \equiv \gamma_{1}\left(e_{1}\right)$ and $\gamma\left(e_{2}\right) \equiv \gamma_{2}\left(e_{2}\right)$.

Let $e_{s}=\gamma\left(\operatorname{let}\left\langle x_{1}, x_{2}\right\rangle=e_{1}\right.$ in $\left.e_{2}\right) \equiv \operatorname{let}\left\langle x_{1}, x_{2}\right\rangle=\gamma\left(e_{1}\right)$ in $\gamma\left(e_{2}\right) \equiv \operatorname{let}\left\langle x_{1}, x_{2}\right\rangle=$ $\gamma_{1}\left(e_{1}\right)$ in $\gamma_{2}\left(e_{2}\right)$ and $W_{s}=W_{\Gamma}$.

We are required to show that $\operatorname{Comp}\left(k, W_{s}, e_{s}, \mathcal{T} \llbracket \Delta \vdash \tau: \operatorname{TYPE} \rrbracket \delta\right) \equiv \operatorname{Comp}\left(k, W_{\Gamma}\right.$, let $\left\langle x_{1}, x_{2}\right\rangle=$ $\gamma_{1}\left(e_{1}\right)$ in $\gamma_{2}\left(e_{2}\right), \mathcal{T} \llbracket \Delta \vdash \tau:$ TYPE $\left.\rrbracket \delta\right)$.

Consider arbitrary $j, W_{r}, w_{s}, w_{f}$, and $e_{f}$ such that

- $j<k$,

- $w_{s}:_{k}\left(W_{s} \odot_{k} W_{r}\right) \equiv w_{s}:_{k}\left(W_{\Gamma} \odot_{k} W_{r}\right)$, noting that

$$
\begin{gathered}
w_{s}:_{k}\left(W_{\Gamma} \odot_{k} W_{r}\right) \\
\equiv w_{s}:_{k}\left(\left(W_{\Gamma_{1}} \odot_{k} W_{\Gamma_{2}}\right) \odot_{k} W_{r}\right) \\
\text { which follows from above, }
\end{gathered}
$$

- $\left(w_{s}, e_{s}\right) \equiv\left(w_{s}\right.$, let $\left\langle x_{1}, x_{2}\right\rangle=\gamma_{1}\left(e_{1}\right)$ in $\left.\gamma_{2}\left(e_{2}\right)\right) \longmapsto^{j}\left(w_{f}, e_{f}\right)$, and

- $\operatorname{irred}\left(w_{f}, e_{f}\right)$.

Hence, by inspection of the operational semantics, it follows that there exist $j_{1}, w_{f_{1}}$, and $e_{f_{1}}$ such that

- $\left(w_{s}, \gamma_{1}\left(e_{1}\right)\right) \longmapsto{ }^{j_{1}}\left(w_{f_{1}}, e_{f_{1}}\right)$,

- $\operatorname{irred}\left(w_{f_{1}}, e_{f_{1}}\right)$, and

- $j_{1} \leq j$.

Note that $\left(\left(W_{\Gamma_{1}} \odot_{k} W_{\Gamma_{2}}\right) \odot_{k} W_{r}\right) \equiv\left(W_{\Gamma_{1}} \odot_{k}\left(W_{\Gamma_{2}} \odot_{k} W_{r}\right)\right)$, which follows from

$$
\begin{aligned}
& \left(\left(W_{\Gamma_{1}} \odot_{k} W_{\Gamma_{2}}\right) \odot_{k} W_{r}\right) \\
& \equiv\left(W_{\Gamma_{1}} \odot_{k}\left(W_{\Gamma_{2}} \odot_{k} W_{r}\right)\right)
\end{aligned}
$$

which follows from Reqs 6, 7, and 8 (join-commut, join-assocl, and join-assocr).

Applying the induction hypothesis to $\Delta ; \Gamma_{1} \vdash e_{1}: \xi_{\tau_{1}} \otimes \tau_{2}$, we conclude that $\llbracket \Delta ; \Gamma_{1} \vdash e_{1}:{ }^{\xi} \tau_{1} \otimes \tau_{2} \rrbracket$.

Instantiate this with $k, \delta, q_{\Gamma_{1}}, W_{\Gamma_{1}}$, and $\gamma_{1}$. Note that 
- $k \geq 0$,

- $\delta \in \mathcal{D} \llbracket \Delta \rrbracket$, and

- $\left(k, q_{\Gamma_{1}}, W_{\Gamma_{1}}, \gamma_{1}\right) \in \mathcal{G} \llbracket \Delta \vdash \Gamma_{1} \rrbracket \delta$.

Hence, $\operatorname{Comp}\left(k, W_{\Gamma_{1}}, \gamma_{1}\left(e_{1}\right), \mathcal{T} \llbracket \Delta \vdash \xi_{\tau_{1}} \otimes \tau_{2}:\right.$ TYPE $\left.\rrbracket \delta\right)$.

Instantiate this with $j_{1},\left(W_{\Gamma_{2}} \odot_{k} W_{r}\right), w_{s}, w_{f_{1}}$, and $e_{f_{1}}$. Note that

- $j_{1}<k$, which follows from $j_{1} \leq j$ and $j<k$,

- $w_{s}:_{k}\left(W_{\Gamma_{1}} \odot_{k}\left(W_{\Gamma_{2}} \odot_{k} W_{r}\right)\right)$, which follows from

$$
\begin{array}{r}
w_{s}:_{k}\left(\left(W_{\Gamma_{1}} \odot_{k} W_{\Gamma_{2}}\right) \odot_{k} W_{r}\right) \\
\text { which follows from above }
\end{array}
$$

$\left(\left(W_{\Gamma_{1}} \odot_{k} W_{\Gamma_{2}}\right) \odot_{k} W_{r}\right) \equiv\left(W_{\Gamma_{1}} \odot_{k}\left(W_{\Gamma_{2}} \odot_{k} W_{r}\right)\right)$

which follows from above,

- $\left(w_{s}, \gamma_{1}\left(e_{1}\right)\right) \longmapsto^{j_{1}}\left(w_{f_{1}}, e_{f_{1}}\right)$, and

- $\operatorname{irred}\left(w_{f_{1}}, e_{f_{1}}\right)$.

Hence, there exists $W_{f_{1}}$ and $q_{f_{1}}$ such that

- $w_{f_{1}}:_{k-j_{1}}\left(W_{f_{1}} \odot_{k-j_{1}}\left(W_{\Gamma_{2}} \odot_{k} W_{r}\right)\right)$, and

- $\left(k-j_{1}, q_{f_{1}}, W_{f_{1}}, e_{f_{1}}\right)$

$\in \mathcal{T} \llbracket \Delta \vdash \xi_{\tau_{1}} \otimes \tau_{2}:$ TYPE $\rrbracket \delta$

$\equiv\left\{\left(k, q, W,\left\langle v_{1}, v_{2}\right\rangle\right) \mid\right.$

$q=\mathcal{T} \llbracket \Delta \vdash \xi: \mathrm{QUAL} \rrbracket \delta \wedge$

$\left(k, q_{1}, W_{1}, v_{1}\right) \in \mathcal{T} \llbracket \Delta \vdash \tau_{1}:$ TYPE $\| \wedge \wedge$

$\left(k, q_{2}, W_{2}, v_{2}\right) \in \mathcal{T} \llbracket \Delta \vdash \tau_{2}: \mathrm{TYPE} \rrbracket \delta \wedge$

$q_{1} \preceq q \wedge q_{2} \preceq q \wedge$

$\left.\left(W_{1} \odot_{k} W_{2}=W\right)\right\}$.

Hence, $e_{f_{1}} \equiv\left\langle v_{f_{11}}, v_{f_{12}}\right\rangle$ and $q_{f_{1}}=\mathcal{T} \llbracket \Delta \vdash \xi: \mathrm{QUAL} \rrbracket \delta$, and $\left(k-j_{1}, q_{f_{11}}, W_{f_{11}}, v_{f_{11}}\right) \in$ $\mathcal{T} \llbracket \Delta \vdash \tau_{1}:$ TYPE $\rrbracket \delta$ and $\left(k-j_{1}, q_{f_{12}}, W_{f_{12}}, v_{f_{12}}\right) \in \mathcal{T} \llbracket \Delta \vdash \tau_{2}:$ TYPE $\rrbracket \delta$ and $q_{f_{11}} \preceq q_{f_{1}}$ and $q_{f_{12}} \preceq q_{f_{1}}$ and $\left(W_{f_{11}} \odot_{k-j_{1}} W_{f_{12}}=W_{f_{1}}\right)$.

Note that

$$
\begin{aligned}
\left(w_{s}, e_{s}\right) & \equiv\left(w_{s}, \text { let }\left\langle x_{1}, x_{2}\right\rangle=\gamma_{1}\left(e_{1}\right) \text { in } \gamma_{2}\left(e_{2}\right)\right) \\
& \longmapsto^{j}\left(w_{f_{1}}, \text { let }\left\langle x_{1}, x_{2}\right\rangle=e_{f_{1}} \text { in } \gamma_{2}\left(e_{2}\right)\right) \\
& \equiv\left(w_{f_{1}}, \text { let }\left\langle x_{1}, x_{2}\right\rangle=\left\langle v_{f_{11}}, v_{f_{12}}\right\rangle \text { in } \gamma_{2}\left(e_{2}\right)\right) \\
& \longmapsto^{1}\left(w_{f_{1}}, \gamma_{2}\left(e_{2}\right)\left[v_{f_{11}} / x_{1}\right]\left[v_{f_{12}} / x_{2}\right]\right) \\
& \longmapsto^{j_{2}}\left(w_{f}, e_{f}\right)
\end{aligned}
$$

and $\operatorname{irred}\left(w_{f}, e_{f}\right)$, where $j=j_{1}+1+j_{2}$.

Note that $\left\lfloor\left(W_{f_{1}} \odot_{k-j_{1}}\left(W_{\Gamma_{2}} \odot_{k} W_{r}\right)\right)\right\rfloor_{k-j_{1}-1} \equiv\left(\left(\left(W_{\Gamma_{2}} \odot_{k-j_{1}-1} W_{f_{11}}\right) \odot_{k-j_{1}-1} W_{f_{12}}\right) \odot_{k-j_{1}-1} W_{r}\right)$, which follows from

$$
\begin{aligned}
& \left\lfloor\left(W_{f_{1}} \odot_{k-j_{1}}\left(W_{\Gamma_{2}} \odot_{k} W_{r}\right)\right)\right\rfloor_{k-j_{1}-1} \\
& \equiv\left\lfloor\left(\left(W_{f_{11}} \odot_{k-j_{1}} W_{f_{12}}\right) \odot_{k-j_{1}}\left(W_{\Gamma_{2}} \odot_{k} W_{r}\right)\right)\right\rfloor_{k-j_{1}-1} \\
& \quad \text { which follows from } W_{f_{1}}=\left(W_{f_{11}} \odot_{k} W_{f_{12}}\right) \\
& \equiv\left(\left(W_{f_{11}} \odot_{k-j_{1}} W_{f_{12}}\right) \odot_{k-j_{1}-1}\left(W_{\Gamma_{2}} \odot_{k} W_{r}\right)\right) \\
& \quad \text { which follows from Req } 4 \text { (join-closed }) \\
& \equiv\left(\left(\left(W_{\Gamma_{2}} \odot_{k-j_{1}-1} W_{f_{11}}\right) \odot_{k-j_{1}-1} W_{f_{12}}\right) \odot_{k-j_{1}-1} W_{r}\right)
\end{aligned}
$$

which follows from Reqs 4, 5, 6, 7, and 8 (join-closed, join-aprx, join-commut, join-assocl, and join-assocr).

Applying the induction hypothesis to $\Delta ; \Gamma_{2}, x_{1}: \tau_{1}, x_{2}: \tau_{2} \vdash e_{2}: \tau$, we conclude that $\llbracket \Delta ; \Gamma_{2}, x: \tau_{1}, x: \tau_{2} \vdash e_{2}: \tau \rrbracket$.

Instantiate this with $k-j_{1}-1, \delta,\left(\left(q_{\Gamma_{2}} \sqcap q_{f_{11}}\right) \sqcap q_{f_{12}}\right),\left(\left(W_{\Gamma_{2}} \odot_{k-j_{1}-1} W_{f_{11}}\right) \odot_{k-j_{1}-1} W_{f_{12}}\right)$, and $\gamma_{2}\left[x_{1} \mapsto v_{f_{11}}\right]\left[x_{2} \mapsto v_{f_{12}}\right]$. Note that 
- $k-j_{1}-1 \geq 0$, which follows from $j_{1}+1+j_{2}=j$ and $j<k$,

- $\delta \in \mathcal{D} \llbracket \Delta \rrbracket$, which follows from above,

- $\left(k-j_{1}-1,\left(\left(q_{\Gamma_{2}} \sqcap q_{f_{11}}\right) \sqcap q_{f_{12}}\right),\left(\left(W_{\Gamma_{2}} \odot_{k-j_{1}-1} W_{f_{11}}\right) \odot_{k-j_{1}-1} W_{f_{12}}\right), \gamma_{2}\left[x_{1} \mapsto v_{f_{11}}\right]\left[x_{2} \mapsto\right.\right.$ $\left.\left.v_{f_{12}}\right]\right) \in \mathcal{G} \llbracket \Delta \vdash \Gamma_{2}, x_{1}: \tau_{1}, x_{2}: \tau_{2} \rrbracket \delta$, which follows from

- $\left(k-j_{1}-1,\left(q_{\Gamma_{2}} \sqcap q_{f_{11}}\right),\left(W_{\Gamma_{2}} \odot_{k-j_{1}-1} W_{f_{11}}\right), \gamma_{2}\left[x_{1} \mapsto v_{f_{11}}\right]\right) \in \mathcal{G} \llbracket \Delta \vdash \Gamma_{2}, x_{1}: \tau_{1} \rrbracket \delta$, which follows from

- $\left(k-j_{1}-1, q_{\Gamma_{2}},\left\lfloor W_{\Gamma_{2}}\right\rfloor_{k-j_{1}-1}, \gamma_{2}\right) \in \mathcal{G} \llbracket \Delta \vdash \Gamma_{2} \rrbracket \delta$, which in turn follows from Lemma 9 applied to $k-j_{1}-1 \leq k$ and $\left(k, q_{\Gamma_{2}}, W_{\Gamma_{2}}, \gamma_{2}\right) \in \mathcal{G} \llbracket \Delta \vdash \Gamma_{2} \rrbracket \delta$,

- $\left(k-j_{1}-1, q_{f_{11}},\left\lfloor W_{f_{11}}\right\rfloor_{k-j_{1}-1}, v_{f_{11}}\right) \in \mathcal{T} \llbracket \Delta \vdash \tau_{1}:$ TYPE $\rrbracket \delta$, which follows from Fact 6 applied to $\left(k-j_{1}, q_{f_{11}}, W_{f_{11}}, v_{f_{11}}\right) \in \mathcal{T} \llbracket \Delta \vdash \tau_{1}:$ TYPE $\rrbracket \in$ Type (which follows from Lemma 8) instantiated with $k-j_{1}-1$, noting that $k-j_{1}-1 \leq k-j_{1}$,

- $q_{\Gamma_{2}} \preceq\left(q_{\Gamma_{2}} \sqcap q_{f_{11}}\right)$, which follows from the definition of $\sqcap$,

- $q_{f_{11}} \preceq\left(q_{\Gamma_{2}} \sqcap q_{f_{11}}\right)$, which follows from the definition of $\sqcap$, and

- $\left(W_{\Gamma_{2}} \odot_{k-j_{1}-1} W_{f_{11}}=\left(\left\lfloor W_{\Gamma_{2}}\right\rfloor_{k-j_{1}-1} \odot_{k-j_{1}-1}\left\lfloor W_{f_{11}}\right\rfloor_{k-j_{1}-1}\right)\right)$, which follows from $\left(W_{\Gamma_{2}} \odot_{k-j_{1}-1} W_{f_{11}}\right)$ $\equiv\left(\left\lfloor W_{\Gamma_{2}}\right\rfloor_{k-j_{1}-1} \odot_{k-j_{1}-1}\left\lfloor W_{f_{11}}\right\rfloor_{k-j_{1}-1}\right)$ which follows from Req 5 (join-aprx), and

- $\left(k-j_{1}-1, q_{f_{12}},\left\lfloor W_{f_{12}}\right\rfloor_{k-j_{1}-1}, v_{f_{12}}\right) \in \mathcal{T} \llbracket \Delta \vdash \tau_{2}:$ TYPE $\rrbracket \delta$, which follows from Fact 6 applied to $\left(k-j_{1}, q_{f_{12}}, W_{f_{12}}, v_{f_{12}}\right) \in \mathcal{T} \llbracket \Delta \vdash \tau_{2}:$ TYPE $\rrbracket \in$ Type (which follows from Lemma 8) instantiated with $k-j_{2}-1$, noting that $k-j_{2}-1 \leq k-j_{2}$,

- $\left(q_{\Gamma_{2}} \sqcap q_{f_{11}}\right) \preceq\left(\left(q_{\Gamma_{2}} \sqcap q_{f_{11}}\right) \sqcap q_{f_{12}}\right)$, which follows from the definition of $\sqcap$,

- $q_{f_{12}} \preceq\left(\left(q_{\Gamma_{2}} \sqcap q_{f_{11}}\right) \sqcap q_{f_{12}}\right)$, which follows from the definition of $\sqcap$, and

- $\left(\left(W_{\Gamma_{2}} \odot_{k-j_{1}-1} W_{f_{11}}\right) \odot_{k-j_{1}-1} W_{f_{12}}=\left(\left\lfloor\left(\begin{array}{lll}W_{\Gamma_{2}} & \odot_{k-j_{1}-1} & W_{f_{11}}\end{array}\right\rfloor_{k-j_{1}-1} \odot_{k-j_{1}-1}\right.\right.\right.$ $\left.\left.\left\lfloor W_{f_{12}}\right\rfloor_{k-j_{1}-1}\right)\right)$, which follows from

$$
\begin{aligned}
& \left(\left(W_{\Gamma_{2}} \odot_{k-j_{1}-1} W_{f_{11}}\right) \odot_{k-j_{1}-1} W_{f_{12}}\right) \\
& \equiv\left(\left\lfloor\left(W_{\Gamma_{2}} \odot_{k-j_{1}-1} W_{f_{11}}\right)\right\rfloor_{k-j_{1}-1} \bigodot_{k-j_{1}-1}\left\lfloor W_{f_{12}}\right\rfloor_{k-j_{1}-1}\right) \\
& \quad \text { which follows from Req } 5 \text { (join-aprx). }
\end{aligned}
$$

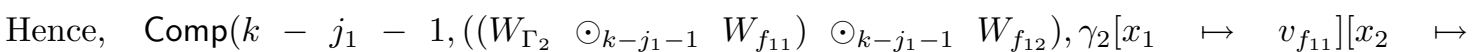
$\left.v_{f_{12}}\right], \mathcal{T} \llbracket \Delta \vdash \tau:$ TYPE $\left.\rrbracket \delta\right)$

Instantiate this with $j_{2}, W_{r}, w_{f_{1}}, w_{f}$, and $e_{f}$. Note that

- $j_{2}<k-j_{1}-1$, which follows from $j_{2}=j-j_{1}-1$ and $j<k$,

- $w_{f_{1}}:_{k-j_{1}-1}\left(\left(\left(W_{\Gamma_{2}} \odot_{k-j_{1}-1} W_{f_{11}}\right) \odot_{k-j_{1}-1} W_{f_{12}}\right) \odot_{k-j_{1}-1} W_{r}\right)$, which follows from

$w_{f_{1}}: k-j_{1}\left(W_{f_{1}} \odot_{k-j_{1}}\left(W_{\Gamma_{2}} \odot_{k} W_{r}\right)\right)$

which follows from above

$\Rightarrow w_{f_{1}}: k_{k-j_{1}-1}\left(W_{f_{1}} \odot_{k-j_{1}}\left(W_{\Gamma_{2}} \odot_{k} W_{r}\right)\right)$

which follows from Req 2 (models-closed)

$\Leftrightarrow w_{f_{1}}:_{k-j_{1}-1}\left\lfloor\left(W_{f_{1}} \odot_{k-j_{1}}\left(W_{\Gamma_{2}} \odot_{k} W_{r}\right)\right)\right\rfloor_{k-j_{1}-1}$

which follows from Req 3 (models-aprx)

$\left\lfloor\left(W_{f_{1}} \odot_{k-j_{1}}\left(W_{\Gamma_{2}} \odot_{k} W_{r}\right)\right)\right\rfloor_{k-j_{1}-1} \equiv\left(\left(\left(W_{\Gamma_{2}} \odot_{k-j_{1}-1} W_{f_{11}}\right) \odot_{k-j_{1}-1} W_{f_{12}}\right) \odot_{k-j_{1}-1} W_{r}\right)$ which follows from above,

- $\left(w_{f_{1}}, \gamma_{2}\left[x_{1} \mapsto v_{f_{11}}\right]\left[x_{1} \mapsto v_{f_{12}}\right]\left(e_{2}\right)\right) \equiv\left(w_{f_{1}}, \gamma_{2}\left(e_{2}\right)\left[v_{f_{11}} / x_{1}\right]\left[v_{f_{12}} / x_{2}\right]\right) \longmapsto{ }^{j_{2}}\left(w_{f}, e_{f}\right)$,

- $\operatorname{irred}\left(w_{f}, e_{f}\right)$.

Hence, there exists $W_{f_{2}}$ and $q_{f_{2}}$ such that

- $w_{f}:_{k-j_{1}-1-j_{2}}\left(W_{f_{2}} \odot_{k-j_{1}-1-j_{2}} W_{r}\right)$, and 
- $\left(k-j_{1}-1-j_{2}, q_{f_{2}}, W_{f_{2}}, e_{f}\right) \in \mathcal{T} \llbracket \Delta, \vdash \tau: \mathrm{TYPE} \rrbracket \delta$.

Let $W_{f}=W_{f_{2}}$ and $q_{f}=q_{f_{2}}$.

We are required to show that

- $w_{f}: k-j\left(W_{f} \odot_{k-j} W_{r}\right)$ $\equiv w_{f}:_{k-j_{1}-1-j_{2}}\left(W_{f_{2}} \odot_{k-j_{1}-1-j_{2}} W_{r}\right)$, which follows from above,

- $\left(k-j, q_{f}, W_{f}, e_{f}\right) \in \mathcal{T} \llbracket \Delta \vdash \tau \rrbracket \delta$ $\equiv\left(k-j_{1}-1-j_{2}, q_{f_{2}}, W_{f_{2}}, e_{f}\right) \in \mathcal{T} \llbracket \Delta \vdash \tau \rrbracket \delta$, which follows from above. 
(AUNIT)

Case $\frac{\Delta \vdash \xi: \text { QUAL } \quad \Delta \vdash \Gamma \preceq \xi}{\Delta ; \Gamma \vdash\left\langle\langle\rangle:{ }^{\xi} \mathbf{1}_{\circledast}\right.}:$

We are required to show $\llbracket \Delta ; \Gamma \vdash\langle\langle\rangle\rangle:{ }^{\xi} \mathbf{1}_{\circledast} \rrbracket$.

Consider arbitrary $k, \delta, q_{\Gamma}, W_{\Gamma}$, and $\gamma$ such that

- $k \geq 0$,

- $\delta \in \mathcal{D} \llbracket \Delta \rrbracket$, and

- $\left(k, q_{\Gamma}, W_{\Gamma}, \gamma\right) \in \mathcal{G} \llbracket \Delta \vdash \Gamma \rrbracket \delta$.

Let $e_{s}=\gamma\left(\langle\langle\rangle) \equiv\langle\langle\rangle\rangle\right.$ and $W_{s}=W_{\Gamma}$.

We are required to show that $\operatorname{Comp}\left(k, W_{s}, e_{s}, \mathcal{T} \llbracket \Delta \vdash{ }^{\xi} \mathbf{1}_{\circledast}: \operatorname{TYPE} \rrbracket \delta\right) \equiv$ $\operatorname{Comp}\left(k, W_{\Gamma},\langle\rangle, \mathcal{T} \llbracket \Delta \vdash{ }^{\xi} \mathbf{1}_{\circledast}:\right.$ TYPE $\left.\rrbracket \delta\right)$.

Consider arbitrary $j, W_{r}, w_{s}, w_{f}$, and $e_{f}$ such that

- $j<k$,

- $w_{s}:_{k}\left(W_{s} \odot_{k} W_{r}\right) \equiv w_{s}:_{k}\left(W_{\Gamma} \odot_{k} W_{r}\right)$,

- $\left(w_{s}, e_{s}\right) \equiv\left(w_{s},\langle\rangle\right) \longmapsto j\left(w_{f}, e_{f}\right)$, and

- $\operatorname{irred}\left(w_{f}, e_{f}\right)$.

Since $\langle\langle\rangle\rangle$ is a value, we have $\operatorname{irred}\left(w_{s},\langle\langle\rangle\rangle\right)$.

Hence, $j=0$ and $w_{f} \equiv w_{s}$ and $e_{f} \equiv\langle\langle\rangle\rangle$.

Let $W_{f}=W_{\Gamma}$ and $q_{f}=\mathcal{T} \llbracket \Delta \vdash \xi: \mathrm{QUAL} \rrbracket \delta$.

We are required to show that

- $w_{f}: k-0\left(W_{f} \odot_{k-0} W_{r}\right)$ $\equiv w_{s}:_{k}\left(W_{\Gamma} \odot_{k} W_{r}\right)$, which follows from above,

- $\left(k-0, q_{f}, W_{f}, e_{f}\right) \in \mathcal{T} \llbracket \Delta \vdash^{\xi} \mathbf{1}_{\circledast}:$ TYPE $\rrbracket \delta$

$\equiv\left(k, \mathcal{T} \llbracket \Delta \vdash \xi:\right.$ QUAL $\left.\rrbracket \delta, W_{\Gamma},\langle\langle\rangle\rangle\right) \in \mathcal{T} \llbracket \Delta \vdash^{\xi} \mathbf{1}_{\circledast}:$ TYPE $\rrbracket \delta$

$\equiv\left(k, \mathcal{T} \llbracket \Delta \vdash \xi: \mathrm{QUAL} \rrbracket \delta, W_{\Gamma},\langle\langle\rangle)\right.$

$\in\{(k, q, W, v) \mid$

$q=\mathcal{T} \llbracket \Delta \vdash \xi: \mathrm{QUAL} \rrbracket \delta \wedge$

$\left.(k, q, W, v) \in \mathcal{T} \llbracket \Delta \vdash \mathbf{1}_{\circledast}: \mathrm{PRETYPE} \rrbracket \delta\right\}$

$\equiv\left(k, \mathcal{T} \llbracket \Delta \vdash \xi: \mathrm{QUAL} \rrbracket \delta, W_{\Gamma},\langle\langle\rangle\rangle\right)$

$\in\left\{(k, q, W,\langle\langle\rangle\rangle) \mid W \in\right.$ WorldDesc $_{k} \wedge \mathcal{P}(k, q, W) \wedge$

$q=\mathcal{T} \llbracket \Delta \vdash \xi: \mathrm{QUAL} \rrbracket \delta\}$,

which follows from

- $\mathcal{T} \llbracket \Delta \vdash \xi: \mathrm{QUAL} \rrbracket \delta=\mathcal{T} \llbracket \Delta \vdash \xi: \mathrm{QUAL} \rrbracket \delta$, which follows trivially,

- $W_{\Gamma} \in$ WorldDesc $_{k}$, which follows from Lemma 9 applied to $\left(k, q_{\Gamma}, W_{\Gamma}, \gamma\right) \in \mathcal{G} \llbracket \Delta \vdash \Gamma \rrbracket \delta$,

- $\mathcal{P}\left(k, \mathcal{T} \llbracket \Delta \vdash \xi: \mathrm{QUAL} \rrbracket \delta, W_{\Gamma}\right)$, which follows from Lemma 18 applied to $\Delta \vdash \Gamma \preceq \xi$ and $\left(k, q_{\Gamma}, W_{\Gamma}, \gamma\right) \in \mathcal{G} \llbracket \Delta \vdash \Gamma \rrbracket \delta$ and $\mathcal{T} \llbracket \Delta \vdash \xi: \mathrm{QUAL} \rrbracket \delta$. 
(APAIR)

Case $\frac{\Delta \vdash \xi: \text { QUAL } \quad \Delta \vdash \Gamma \preceq \xi \quad \Delta ; \Gamma \vdash e_{1}: \tau_{1} \quad \Delta ; \Gamma \vdash e_{2}: \tau_{2}}{\Delta ; \Gamma \vdash\left\langle\left\langle e_{1}, e_{2}\right\rangle\right\rangle:{ }^{\xi}\left(\tau_{1} \circledast \tau_{2}\right)}:$

We are required to show $\llbracket \Delta ; \Gamma \vdash\left\langle\left\langle e_{1}, e_{2}\right\rangle\right\rangle:{ }^{\xi}\left(\tau_{1} \circledast \tau_{2}\right) \rrbracket$.

Consider arbitrary $k, \delta, q_{\Gamma}, W_{\Gamma}$, and $\gamma$ such that

- $k \geq 0$,

- $\delta \in \mathcal{D} \llbracket \Delta \rrbracket$, and

- $\left(k, q_{\Gamma}, W_{\Gamma}, \gamma\right) \in \mathcal{G} \llbracket \Delta \vdash \Gamma \rrbracket \delta$.

Let $e_{s}=\gamma\left(\left\langle\left\langle e_{1}, e_{2}\right\rangle\right\rangle\right) \equiv\left\langle\left\langle\gamma\left(e_{1}\right), \gamma\left(e_{2}\right)\right\rangle\right\rangle$ and $W_{s}=W_{\Gamma}$.

We are required to show that $\operatorname{Comp}\left(k, W_{s}, e_{s}, \mathcal{T} \llbracket \Delta \vdash \xi_{\tau_{1}} \circledast \tau_{2}: \mathrm{TYPE} \rrbracket \delta\right) \quad \equiv$ $\operatorname{Comp}\left(k, W_{\Gamma},\left\langle\left\langle\gamma\left(e_{1}\right), \gamma\left(e_{2}\right)\right\rangle, \mathcal{T} \llbracket \Delta \vdash \xi_{\tau_{1}} \circledast \tau_{2}: \operatorname{TYPE} \rrbracket \delta\right)\right.$.

Consider arbitrary $j, W_{r}, w_{s}, w_{f}$, and $e_{f}$ such that

- $j<k$,

- $w_{s}:_{k}\left(W_{s} \odot_{k} W_{r}\right) \equiv w_{s}:_{k}\left(W_{\Gamma} \odot_{k} W_{r}\right)$,

- $\left(w_{s}, e_{s}\right) \equiv\left(w_{s},\left\langle\left\langle\gamma_{1}\left(e_{1}\right), \gamma_{2}\left(e_{2}\right)\right\rangle\right\rangle\right) \longmapsto^{j}\left(w_{f}, e_{f}\right)$, and

- $\operatorname{irred}\left(w_{f}, e_{f}\right)$.

Since $\left\langle\left\langle\gamma_{1}\left(e_{1}\right), \gamma_{2}\left(e_{2}\right)\right\rangle\right\rangle$ is a value, we have $\operatorname{irred}\left(w_{s},\left\langle\left\langle\gamma_{1}\left(e_{1}\right), \gamma_{2}\left(e_{2}\right)\right\rangle\right\rangle\right)$.

Hence, $j=0$ and $w_{f} \equiv w_{s}$ and $e_{f} \equiv\left\langle\left\langle\gamma_{1}\left(e_{1}\right), \gamma_{2}\left(e_{2}\right)\right\rangle\right.$.

Let $W_{f}=W_{\Gamma}$ and $q_{f}=\mathcal{T} \llbracket \Delta \vdash \xi: \mathrm{QUAL} \rrbracket \delta$.

We are required to show that

- $w_{f}:_{k-0}\left(W_{f} \odot_{k-0} W_{r}\right)$ $\equiv w_{s}:_{k}\left(W_{\Gamma} \odot_{k} W_{r}\right)$ which follows from above,

- $\left(k-0, q_{f}, W_{f}, e_{f}\right) \in \mathcal{T} \llbracket \Delta \vdash \xi_{\tau_{1}} \circledast \tau_{2}:$ TYPE $\rrbracket \delta$

$\equiv\left(k, \mathcal{T} \llbracket \Delta \vdash \xi:\right.$ QUAL $\left.\rrbracket \delta, W_{\Gamma},\left\langle\left\langle\gamma\left(e_{1}\right), \gamma\left(e_{2}\right)\right\rangle\right\rangle\right) \in \mathcal{T} \llbracket \Delta \vdash \xi_{\tau_{1}} \circledast \tau_{2}:$ TYPE $\rrbracket \delta$

$\equiv\left(k, \mathcal{T} \llbracket \Delta \vdash \xi: \mathrm{QUAL} \rrbracket \delta, W_{\Gamma},\left\langle\left\langle\gamma\left(e_{1}\right), \gamma\left(e_{2}\right)\right\rangle\right\rangle\right)$

$\in\{(k, q, W, v) \mid$

$q=\mathcal{T} \llbracket \Delta \vdash \xi: \mathrm{QUAL} \rrbracket \delta \wedge$

$(k, q, W, v) \in \mathcal{T} \llbracket \Delta \vdash \tau_{1} \circledast \tau_{2}:$ PRETYPE $\left.\rrbracket \delta\right\}$

$\equiv\left(k, \mathcal{T} \llbracket \Delta \vdash \xi: \mathrm{QUAL} \rrbracket \delta, W_{\Gamma},\left\langle\left\langle\gamma\left(e_{1}\right), \gamma\left(e_{2}\right)\right\rangle\right\rangle\right)$

$\in\left\{\left(k, q, W,\left\langle\left\langle e_{1}, e_{2}\right\rangle\right\rangle\right) \mid W \in\right.$ WorldDesc $_{k} \wedge \mathcal{P}(k, q, W) \wedge$

$q=\mathcal{T} \llbracket \Delta \vdash \xi: \mathrm{QUAL} \rrbracket \delta \wedge$

$\forall i<k$.

$\operatorname{Comp}(i, \mid W\rfloor_{i}, e_{1}, \mathcal{T} \llbracket \Delta \vdash \tau_{1}:$ TYPE $\left.\| \delta\right) \wedge$

$\operatorname{Comp}\left(i,\lfloor W\rfloor_{i}, e_{2}, \mathcal{T} \llbracket \Delta \vdash \tau_{2}:\right.$ TYPE $\left.\left.\rrbracket \delta\right)\right\}$,

which follows from

- $\mathcal{T} \llbracket \Delta \vdash \xi: \mathrm{QUAL} \rrbracket \delta=\mathcal{T} \llbracket \Delta \vdash \xi: \mathrm{QUAL} \rrbracket \delta$, which follows trivially,

- $W_{\Gamma} \in$ WorldDesc $_{k}$, which follows from Lemma 9 applied to $\left(k, q_{\Gamma}, W_{\Gamma}, \gamma\right) \in \mathcal{G} \llbracket \Delta \vdash \Gamma \rrbracket \delta$,

- $\mathcal{P}\left(k, \mathcal{T} \llbracket \Delta \vdash \xi:\right.$ QUAL $\left.\rrbracket \delta, W_{\Gamma}\right)$, which follows from Lemma 18 applied to $\Delta \vdash \Gamma \preceq \xi$ and $\left(k, q_{\Gamma}, W_{\Gamma}, \gamma\right) \in \mathcal{G} \llbracket \Delta \vdash \Gamma \rrbracket \delta$ and $\mathcal{T} \llbracket \Delta \vdash \xi: \mathrm{QUAL} \rrbracket \delta$,

- $\forall i<k$. . .

Consider arbitrary $i$ such that

- $i<k$. 
We are required to show that $\operatorname{Comp}\left(i,\left\lfloor W_{\Gamma}\right\rfloor_{i}, \gamma\left(e_{1}\right), \mathcal{T} \llbracket \Delta \vdash \tau_{1}:\right.$ TYPE $\left.\rrbracket \delta\right)$ and $\operatorname{Comp}\left(i,\left\lfloor W_{\Gamma}\right\rfloor_{i}, \gamma\left(e_{2}\right), \mathcal{T} \llbracket \Delta \vdash \tau_{2}: \operatorname{TYPE} \rrbracket \delta\right)$.

Applying the induction hypothesis to $\Delta ; \Gamma \vdash e_{1}: \tau_{1}$, we conclude that $\llbracket \Delta ; \Gamma \vdash e_{1}: \tau_{1} \rrbracket$. Instantiate this with $i, \delta, q_{\Gamma},\left\lfloor W_{\Gamma}\right\rfloor_{i}$, and $\gamma$. Note that

- $k \geq 0$,

- $\delta \in \mathcal{D} \llbracket \Delta \rrbracket$, and

- $\left(i, q_{\Gamma},\left\lfloor W_{\Gamma}\right\rfloor_{i}, \gamma\right) \in \mathcal{G} \llbracket \Delta \vdash \Gamma \rrbracket \delta$, which follows from Lemma 9 applied to $i<k$ and $\left(k, q_{\Gamma}, W_{\Gamma}, \gamma\right) \in \mathcal{G} \llbracket \Delta \vdash \Gamma \rrbracket \delta$.

Hence, $\operatorname{Comp}\left(i,\left\lfloor W_{\Gamma}\right\rfloor_{i}, \gamma\left(e_{1}\right), \mathcal{T} \llbracket \Delta \vdash \tau_{1}:\right.$ TYPE $\left.\rrbracket \delta\right)$.

Applying the induction hypothesis to $\Delta ; \Gamma \vdash e_{2}: \tau_{2}$, we conclude that $\llbracket \Delta ; \Gamma \vdash e_{2}: \tau_{2} \rrbracket$. Instantiate this with $i, \delta, q_{\Gamma},\left\lfloor W_{\Gamma}\right\rfloor_{i}$, and $\gamma$. Note that

- $k \geq 0$,

- $\delta \in \mathcal{D} \llbracket \Delta \rrbracket$, and

- $\left(i, q_{\Gamma},\left\lfloor W_{\Gamma}\right\rfloor_{i}, \gamma\right) \in \mathcal{G} \llbracket \Delta \vdash \Gamma \rrbracket \delta$, which follows from Lemma 9 applied to $i<k$ and $\left(k, q_{\Gamma}, W_{\Gamma}, \gamma\right) \in \mathcal{G} \llbracket \Delta \vdash \Gamma \rrbracket \delta$.

Hence, $\operatorname{Comp}\left(i,\left\lfloor W_{\Gamma}\right\rfloor_{i}, \gamma\left(e_{2}\right), \mathcal{T} \llbracket \Delta \vdash \tau_{2}:\right.$ TYPE $\left.\rrbracket \delta\right)$.

Hence, $\quad \operatorname{Comp}\left(i,\left\lfloor W_{\Gamma}\right\rfloor_{i}, \gamma\left(e_{1}\right), \mathcal{T} \llbracket \Delta \vdash \tau_{1}:\right.$ TYPE $\left.\rrbracket \delta\right) \quad$ and

$\operatorname{Comp}\left(i,\left\lfloor W_{\Gamma}\right\rfloor_{i}, \gamma\left(e_{2}\right), \mathcal{T} \llbracket \Delta \vdash \tau_{2}: \mathrm{TYPE} \rrbracket \delta\right)$. 
(FST)

Case $\frac{\Delta ; \Gamma \vdash e_{1}:{ }^{\xi}\left(\tau_{1} \circledast \tau_{2}\right)}{\Delta ; \Gamma \vdash \text { fst } e_{1}: \tau_{1}}:$

We are required to show $\llbracket \Delta ; \Gamma \vdash$ fst $e_{1}: \tau_{1} \rrbracket$.

Consider arbitrary $k, \delta, q_{\Gamma}, W_{\Gamma}$, and $\gamma$ such that

- $k \geq 0$,

- $\delta \in \mathcal{D} \llbracket \Delta \rrbracket$, and

- $\left(k, q_{\Gamma}, W_{\Gamma}, \gamma\right) \in \mathcal{G} \llbracket \Delta \vdash \Gamma \rrbracket \delta$.

Let $e_{s}=\gamma\left(\right.$ fst $\left.e_{1}\right) \equiv$ fst $\gamma\left(e_{1}\right)$ and $W_{s}=W_{\Gamma}$.

We are required to show that $\operatorname{Comp}\left(k, W_{s}, e_{s}, \mathcal{T} \llbracket \Delta \vdash \tau_{1}: \mathrm{TYPE} \rrbracket \delta\right) \quad \equiv$ $\operatorname{Comp}\left(k, W_{\Gamma}\right.$, fst $\gamma\left(e_{1}\right), \mathcal{T} \llbracket \Delta \vdash \tau_{1}:$ TYPE $\left.\rrbracket \delta\right)$.

Consider arbitrary $j, W_{r}, w_{s}, w_{f}$, and $e_{f}$ such that

- $j<k$,

- $w_{s}:_{k}\left(W_{s} \odot_{k} W_{r}\right) \equiv w_{s}:_{k}\left(W_{\Gamma} \odot_{k} W_{r}\right)$,

- $\left(w_{s}, e_{s}\right)=\left(w_{s}, \operatorname{drop} \gamma\left(e_{1}\right)\right) \longmapsto^{j}\left(w_{f}, e_{f}\right)$, and

- $\operatorname{irred}\left(w_{f}, e_{f}\right)$.

Hence, by inspection of the operational semantics, it follows that there exist $j_{1}, w_{f_{1}}$, and $e_{f_{1}}$ such that

- $\left(w_{s}, \gamma\left(e_{1}\right)\right) \longmapsto{ }^{j_{1}}\left(w_{f_{1}}, e_{f_{1}}\right)$,

- $\operatorname{irred}\left(w_{f_{1}}, e_{f_{1}}\right)$, and

- $j_{1} \leq j$.

Applying the induction hypothesis to $\Delta ; \Gamma \vdash e_{1}:{ }^{\tau_{1} \circledast \tau_{2}}$, we conclude that $\llbracket \Delta ; \Gamma \vdash e_{1}: \xi_{\tau_{1}} \circledast \tau_{2} \rrbracket$. Instantiate this with $k, \delta, q_{\Gamma}, W_{\Gamma}$, and $\gamma$. Note that

- $k \geq 0$,

- $\delta \in \mathcal{D} \llbracket \Delta \rrbracket$, and

- $\left(k, q_{\Gamma}, W_{\Gamma}, \gamma\right) \in \mathcal{G} \llbracket \Delta \vdash \Gamma \rrbracket \delta$.

Hence, $\operatorname{Comp}\left(k, W_{\Gamma}, \gamma\left(e_{1}\right), \mathcal{T} \llbracket \Delta \vdash \xi_{\tau_{1}} \circledast \tau_{2}:\right.$ TYPE $\left.\rrbracket \delta\right)$.

Instantiate this with $j_{1}, W_{r}, w_{s}, w_{f_{1}}$, and $e_{f_{1}}$. Note that

- $j_{1}<k$, which follows from $j_{1} \leq j$ and $j<k$,

- $w_{s}:_{k}\left(W_{\Gamma} \odot_{k} W_{r}\right)$, which follows from above,

- $\left(w_{s}, \gamma\left(e_{1}\right)\right) \longmapsto{ }^{j_{1}}\left(w_{f_{1}}, e_{f_{1}}\right)$,

- $\operatorname{irred}\left(w_{f_{1}}, e_{f_{1}}\right)$.

Hence, there exists $W_{f_{1}}$ and $q_{f_{1}}$ such that

- $w_{f_{1}}:_{k-j_{1}}\left(W_{f_{1}} \odot_{k-j_{1}} W_{r}\right)$, and

- $\left(k-j_{1}, q_{f_{1}}, W_{f_{1}}, e_{f_{1}}\right)$

$\in \mathcal{T} \llbracket \Delta \vdash \xi_{\tau_{1} \circledast \tau_{2}: \text { TYPE } \rrbracket \delta}$

$\equiv\left\{\left(k, q, W,\left\langle\left\langle e_{1}, e_{2}\right\rangle\right\rangle\right) \mid W \in\right.$ WorldDesc $_{k} \wedge \mathcal{P}(k, q, W) \wedge$

$q=\mathcal{T} \llbracket \Delta \vdash \xi: \mathrm{QUAL} \rrbracket \delta \wedge$

$\forall i<k$.

$\operatorname{Comp}\left(i,\lfloor W\rfloor_{i}, e_{1}, \mathcal{T} \llbracket \Delta \vdash \tau_{1}:\right.$ TYPE $\left.\rrbracket \delta\right) \wedge$

$\operatorname{Comp}\left(i,\lfloor W\rfloor_{i}, e_{2}, \mathcal{T} \llbracket \Delta \vdash \tau_{2}:\right.$ TYPE $\left.\left.\rrbracket \delta\right)\right\}$. 
Hence, $e_{f_{1}} \equiv\left\langle\left\langle e_{f_{11}}, e_{f_{12}}\right\rangle\right\rangle$ and $q_{f_{1}}=\mathcal{T} \llbracket \Delta \vdash \xi: \mathrm{QUAL} \rrbracket \delta$.

Note that

$$
\begin{aligned}
\left(w_{s}, e_{s}\right) & \equiv\left(w_{s}, \text { fst } \gamma\left(e_{1}\right)\right) \\
& \longmapsto w^{j}\left(w_{f_{1}}, \text { fst } e_{f_{1}}\right) \\
& \equiv\left(w_{f_{1}}, \text { fst }\left\langle\left\langle e_{f_{11}}, e_{f_{12}}\right\rangle\right)\right. \\
& \longmapsto{ }^{1}\left(w_{f_{1}}, e_{f_{11}}\right) \\
& \longmapsto^{j_{2}}\left(w_{f}, e_{f}\right)
\end{aligned}
$$

and $\operatorname{irred}\left(w_{f}, e_{f}\right)$, where $j=j_{1}+1+j_{2}$.

Note that $\left\lfloor\left(W_{f_{1}} \odot_{k-j_{1}} W_{r}\right)\right\rfloor_{k-j_{1}-1} \equiv\left(\left\lfloor W_{f_{1}}\right\rfloor_{k-j_{1}-1} \odot_{k-j_{1}-1} W_{r}\right)$, which follows from

$$
\begin{aligned}
& \left\lfloor\left(W_{f_{1}} \odot_{k-j_{1}} W_{r}\right)\right\rfloor_{k-j_{1}-1} \\
& \equiv\left(W_{f_{1}} \odot_{k-j_{1}-1} W_{r}\right) \\
& \quad \text { which follows from Req } 4 \text { (join-closed) } \\
& \equiv\left(\left\lfloor W_{f_{1}}\right\rfloor_{k-j_{1}-1} \odot_{k-j_{1}-1} W_{r}\right) \\
& \quad \text { which follows from Req } 5 \text { (join-aprx). }
\end{aligned}
$$

Instantiate $\left(k-j_{1}, q_{f_{1}}, W_{f_{1}},\left\langle\left\langle e_{f_{11}}, e_{f_{12}}\right\rangle\right) \in \mathcal{T} \llbracket \Delta \vdash^{\xi} \tau_{1} \circledast \tau_{2}:\right.$ TYPE $\rrbracket \delta$ with $k-j_{1}-1$. Note that

- $k-j_{1}-1<k-j_{1}$.

Hence, $\operatorname{Comp}\left(k-j_{1}-1,\left\lfloor W_{f_{1}}\right\rfloor_{k-j_{1}-1}, e_{f_{11}}, \mathcal{T} \llbracket \Delta \vdash \tau_{1}:\right.$ TYPE $\left.\rrbracket \delta\right)$.

Instantiate this with $j_{2}, W_{r}, w_{f_{1}}, w_{f}$, and $e_{f}$. Note that

- $k-j_{1}-1<j_{2}$, which follows from $j_{2}=j-j_{1}-1$ and $j<k$,

- $w_{f_{1}}:_{k-j_{1}-1}\left(\left\lfloor W_{f_{1}}\right\rfloor_{k-j_{1}-1} \odot_{k-j_{1}-1} W_{r}\right)$, which follows from

$$
\begin{aligned}
& w_{f_{1}}: k-j_{1}\left(W_{f_{1}} \odot_{k-j_{1}} W_{r}\right) \\
& \text { which follows from above } \\
& \Rightarrow w_{f_{1}}: k-j_{1}-1\left(W_{f_{1}} \odot_{k-j_{1}} W_{r}\right) \\
& \text { which follows from Req } 2 \text { (models-closed) } \\
& \Leftrightarrow w_{f_{1}}: k-j_{1}-1\left\lfloor\left(W_{f_{1}} \odot_{k-j_{1}} W_{r}\right)\right\rfloor_{k-j_{1}-1} \\
& \text { which follows from Req } 3 \text { (models-aprx) }
\end{aligned}
$$

$$
\left\lfloor\left(W_{f_{1}} \odot_{k-j_{1}} W_{r}\right)\right\rfloor_{k-j_{1}-1} \equiv\left(\left\lfloor W_{f_{1}}\right\rfloor_{k-j_{1}-1} \odot_{k-j_{1}-1} W_{r}\right)
$$

$$
\text { which follows from above, }
$$

- $\left(w_{f_{1}}, e_{f_{11}}\right) \longmapsto^{j_{2}}\left(w_{f}, e_{f}\right)$, and

- $\operatorname{irred}\left(w_{f}, e_{f}\right)$.

Hence, there exists $W_{f^{\prime}}$ and $q_{f^{\prime}}$ such that

- $w_{f}:_{k-j_{1}-1-j_{2}}\left(W_{f^{\prime}} \odot_{k-j_{1}-1-j_{2}} W_{r}\right)$, and

- $\left(k-j_{1}-1-j_{2}, q_{f^{\prime}}, W_{f^{\prime}}, e_{f}\right) \in \mathcal{T} \llbracket \Delta \vdash \tau_{1}:$ TYPE $\rrbracket \delta$.

Let $W_{f}=W_{f^{\prime}}$ and $q_{f}=q_{f^{\prime}}$.

We are required to show that

- $w_{f}:_{k-j}\left(W_{f} \odot_{k-j} W_{r}\right)$ $\equiv w_{f}:_{k-j_{1}-1-j_{2}}\left(W_{f^{\prime}} \odot_{k-j_{1}-1-j_{2}} W_{r}\right)$, which follows from above,

- $\left(k-j, q_{f}, W_{f}, e_{f}\right) \in \mathcal{T} \llbracket \Delta \vdash \tau_{1}:$ TYPE $\rrbracket \delta$ $\equiv\left(k-j_{1}-1-j_{2}, q_{f^{\prime}}, W_{f^{\prime}}, e_{f}\right) \in \mathcal{T} \llbracket \Delta \vdash \tau_{1}:$ TYPE $\rrbracket \delta$, which follows from above. 
$(\mathrm{SND})$

Case $\frac{\Delta ; \Gamma \vdash e_{1}:{ }^{\xi}\left(\tau_{1} \circledast \tau_{2}\right)}{\Delta ; \Gamma \vdash \text { snd } e_{1}: \tau_{2}}$ :

We are required to show $\llbracket \Delta ; \Gamma \vdash$ snd $e_{1}: \tau_{2} \rrbracket$.

Consider arbitrary $k, \delta, q_{\Gamma}, W_{\Gamma}$, and $\gamma$ such that

- $k \geq 0$,

- $\delta \in \mathcal{D} \llbracket \Delta \rrbracket$, and

- $\left(k, q_{\Gamma}, W_{\Gamma}, \gamma\right) \in \mathcal{G} \llbracket \Delta \vdash \Gamma \rrbracket \delta$.

Let $e_{s}=\gamma\left(\right.$ snd $\left.e_{1}\right) \equiv \operatorname{snd} \gamma\left(e_{1}\right)$ and $W_{s}=W_{\Gamma}$.

We are required to show that $\operatorname{Comp}\left(k, W_{s}, e_{s}, \mathcal{T} \llbracket \Delta \vdash \tau_{2}: \mathrm{TYPE} \rrbracket \delta\right) \quad \equiv$ $\operatorname{Comp}\left(k, W_{\Gamma}\right.$, snd $\gamma\left(e_{1}\right), \mathcal{T} \llbracket \Delta \vdash \tau_{2}:$ TYPE $\left.\rrbracket \delta\right)$.

Consider arbitrary $j, W_{r}, w_{s}, w_{f}$, and $e_{f}$ such that

- $j<k$,

- $w_{s}:_{k}\left(W_{s} \odot_{k} W_{r}\right) \equiv w_{s}:_{k}\left(W_{\Gamma} \odot_{k} W_{r}\right)$,

- $\left(w_{s}, e_{s}\right)=\left(w_{s}, \operatorname{drop} \gamma\left(e_{1}\right)\right) \longmapsto^{j}\left(w_{f}, e_{f}\right)$, and

- $\operatorname{irred}\left(w_{f}, e_{f}\right)$.

Hence, by inspection of the operational semantics, it follows that there exist $j_{1}, w_{f_{1}}$, and $e_{f_{1}}$ such that

- $\left(w_{s}, \gamma\left(e_{1}\right)\right) \longmapsto{ }^{j_{1}}\left(w_{f_{1}}, e_{f_{1}}\right)$,

- $\operatorname{irred}\left(w_{f_{1}}, e_{f_{1}}\right)$, and

- $j_{1} \leq j$.

Applying the induction hypothesis to $\Delta ; \Gamma \vdash e_{1}:{ }^{\tau_{1} \circledast \tau_{2}}$, we conclude that $\llbracket \Delta ; \Gamma \vdash e_{1}: \xi_{\tau_{1}} \circledast \tau_{2} \rrbracket$. Instantiate this with $k, \delta, q_{\Gamma}, W_{\Gamma}$, and $\gamma$. Note that

- $k \geq 0$,

- $\delta \in \mathcal{D} \llbracket \Delta \rrbracket$, and

- $\left(k, q_{\Gamma}, W_{\Gamma}, \gamma\right) \in \mathcal{G} \llbracket \Delta \vdash \Gamma \rrbracket \delta$.

Hence, $\operatorname{Comp}\left(k, W_{\Gamma}, \gamma\left(e_{1}\right), \mathcal{T} \llbracket \Delta \vdash \xi_{\tau_{1}} \circledast \tau_{2}:\right.$ TYPE $\left.\rrbracket \delta\right)$.

Instantiate this with $j_{1}, W_{r}, w_{s}, w_{f_{1}}$, and $e_{f_{1}}$. Note that

- $j_{1}<k$, which follows from $j_{1} \leq j$ and $j<k$,

- $w_{s}:_{k}\left(W_{\Gamma} \odot_{k} W_{r}\right)$, which follows from above,

- $\left(w_{s}, \gamma\left(e_{1}\right)\right) \longmapsto{ }^{j_{1}}\left(w_{f_{1}}, e_{f_{1}}\right)$,

- $\operatorname{irred}\left(w_{f_{1}}, e_{f_{1}}\right)$.

Hence, there exists $W_{f_{1}}$ and $q_{f_{1}}$ such that

- $w_{f_{1}}:_{k-j_{1}}\left(W_{f_{1}} \odot_{k-j_{1}} W_{r}\right)$, and

- $\left(k-j_{1}, q_{f_{1}}, W_{f_{1}}, e_{f_{1}}\right)$

$\in \mathcal{T} \llbracket \Delta \vdash \xi_{\tau_{1} \circledast \tau_{2}: \text { TYPE } \rrbracket \delta}$

$\equiv\left\{\left(k, q, W,\left\langle\left\langle e_{1}, e_{2}\right\rangle\right\rangle\right) \mid W \in\right.$ WorldDesc $_{k} \wedge \mathcal{P}(k, q, W) \wedge$

$q=\mathcal{T} \llbracket \Delta \vdash \xi: \mathrm{QUAL} \rrbracket \delta \wedge$

$\forall i<k$.

$\operatorname{Comp}\left(i,\lfloor W\rfloor_{i}, e_{1}, \mathcal{T} \llbracket \Delta \vdash \tau_{1}:\right.$ TYPE $\left.\rrbracket \delta\right) \wedge$

$\operatorname{Comp}\left(i,\lfloor W\rfloor_{i}, e_{2}, \mathcal{T} \llbracket \Delta \vdash \tau_{2}:\right.$ TYPE $\left.\left.\rrbracket \delta\right)\right\}$. 
Hence, $e_{f_{1}} \equiv\left\langle\left\langle e_{f_{11}}, e_{f_{12}}\right\rangle\right\rangle$ and $q_{f_{1}}=\mathcal{T} \llbracket \Delta \vdash \xi: \mathrm{QUAL} \rrbracket \delta$.

Note that

$$
\begin{aligned}
\left(w_{s}, e_{s}\right) & \equiv\left(w_{s}, \text { snd } \gamma\left(e_{1}\right)\right) \\
& \longmapsto w^{j}\left(w_{f_{1}}, \text { snd } e_{f_{1}}\right) \\
& \equiv\left(w_{f_{1}}, \text { snd }\left\langle\left\langle e_{f_{11}}, e_{f_{12}}\right\rangle\right)\right) \\
& \longmapsto{ }^{1}\left(w_{f_{1}}, e_{f_{12}}\right) \\
& \longmapsto^{j_{2}}\left(w_{f}, e_{f}\right)
\end{aligned}
$$

and $\operatorname{irred}\left(w_{f}, e_{f}\right)$, where $j=j_{1}+1+j_{2}$.

Note that $\left\lfloor\left(W_{f_{1}} \odot_{k-j_{1}} W_{r}\right)\right\rfloor_{k-j_{1}-1} \equiv\left(\left\lfloor W_{f_{1}}\right\rfloor_{k-j_{1}-1} \odot_{k-j_{1}-1} W_{r}\right)$, which follows from

$$
\begin{aligned}
& \left\lfloor\left(W_{f_{1}} \odot_{k-j_{1}} W_{r}\right)\right\rfloor_{k-j_{1}-1} \\
& \equiv\left(W_{f_{1}} \odot_{k-j_{1}-1} W_{r}\right) \\
& \quad \text { which follows from Req } 4 \text { (join-closed) } \\
& \equiv\left(\left\lfloor W_{f_{1}}\right\rfloor_{k-j_{1}-1} \odot_{k-j_{1}-1} W_{r}\right) \\
& \quad \text { which follows from Req } 5 \text { (join-aprx). }
\end{aligned}
$$

Instantiate $\left(k-j_{1}, q_{f_{1}}, W_{f_{1}},\left\langle\left\langle e_{f_{11}}, e_{f_{12}}\right\rangle\right) \in \mathcal{T} \llbracket \Delta \vdash^{\xi} \tau_{1} \circledast \tau_{2}:\right.$ TYPE $\rrbracket \delta$ with $k-j_{1}-1$. Note that

- $k-j_{1}-1<k-j_{1}$.

Hence, $\operatorname{Comp}\left(k-j_{1}-1,\left\lfloor W_{f_{1}}\right\rfloor_{k-j_{1}-1}, e_{f_{12}}, \mathcal{T} \llbracket \Delta \vdash \tau_{2}:\right.$ TYPE $\left.\rrbracket \delta\right)$.

Instantiate this with $j_{2}, W_{r}, w_{f_{1}}, w_{f}$, and $e_{f}$. Note that

- $k-j_{1}-1<j_{2}$, which follows from $j_{2}=j-j_{1}-1$ and $j<k$,

- $w_{f_{1}}:_{k-j_{1}-1}\left(\left\lfloor W_{f_{1}}\right\rfloor_{k-j_{1}-1} \odot_{k-j_{1}-1} W_{r}\right)$, which follows from

$$
\begin{aligned}
& w_{f_{1}}: k-j_{1}\left(W_{f_{1}} \odot_{k-j_{1}} W_{r}\right) \\
& \text { which follows from above } \\
& \Rightarrow w_{f_{1}}: k-j_{1}-1\left(W_{f_{1}} \odot_{k-j_{1}} W_{r}\right) \\
& \text { which follows from Req } 2 \text { (models-closed) } \\
& \Leftrightarrow w_{f_{1}}: k-j_{1}-1\left\lfloor\left(W_{f_{1}} \odot_{k-j_{1}} W_{r}\right)\right\rfloor_{k-j_{1}-1} \\
& \text { which follows from Req } 3 \text { (models-aprx) }
\end{aligned}
$$

$$
\left\lfloor\left(W_{f_{1}} \odot_{k-j_{1}} W_{r}\right)\right\rfloor_{k-j_{1}-1} \equiv\left(\left\lfloor W_{f_{1}}\right\rfloor_{k-j_{1}-1} \odot_{k-j_{1}-1} W_{r}\right)
$$

$$
\text { which follows from above, }
$$

- $\left(w_{f_{1}}, e_{f_{11}}\right) \longmapsto^{j_{2}}\left(w_{f}, e_{f}\right)$, and

- $\operatorname{irred}\left(w_{f}, e_{f}\right)$.

Hence, there exists $W_{f^{\prime}}$ and $q_{f^{\prime}}$ such that

- $w_{f}: k-j_{1}-1-j_{2}\left(W_{f^{\prime}} \odot_{k-j_{1}-1-j_{2}} W_{r}\right)$, and

- $\left(k-j_{1}-1-j_{2}, q_{f^{\prime}}, W_{f^{\prime}}, e_{f}\right) \in \mathcal{T} \llbracket \Delta \vdash \tau_{2}:$ TYPE $\rrbracket \delta$.

Let $W_{f}=W_{f^{\prime}}$ and $q_{f}=q_{f^{\prime}}$.

We are required to show that

- $w_{f}:_{k-j}\left(W_{f} \odot_{k-j} W_{r}\right)$ $\equiv w_{f}:_{k-j_{1}-1-j_{2}}\left(W_{f^{\prime}} \odot_{k-j_{1}-1-j_{2}} W_{r}\right)$, which follows from above,

- $\left(k-j, q_{f}, W_{f}, e_{f}\right) \in \mathcal{T} \llbracket \Delta \vdash \tau_{2}:$ TYPE $\rrbracket \delta$ $\equiv\left(k-j_{1}-1-j_{2}, q_{f^{\prime}}, W_{f^{\prime}}, e_{f}\right) \in \mathcal{T} \llbracket \Delta \vdash \tau_{2}:$ TYPE $\rrbracket \delta$, which follows from above. 
(ABORT)

Case $\frac{\Delta ; \Gamma \vdash e_{1}:{ }^{\xi} \mathbf{0} \quad \Delta \vdash \tau: \text { TYPE }}{\Delta ; \Gamma \vdash \text { abort } e_{1}: \tau}$ :

We are required to show $\llbracket \Delta ; \Gamma \vdash$ abort $e_{1}: \tau \rrbracket$.

Consider arbitrary $k, \delta, q_{\Gamma}, W_{\Gamma}$, and $\gamma$ such that

- $k \geq 0$,

- $\delta \in \mathcal{D} \llbracket \Delta \rrbracket$, and

- $\left(k, q_{\Gamma}, W_{\Gamma}, \gamma\right) \in \mathcal{G} \llbracket \Delta \vdash \Gamma \rrbracket \delta$.

Let $e_{s}=\gamma\left(\right.$ abort $\left.e_{1}\right) \equiv$ abort $\gamma\left(e_{1}\right)$ and $W_{s}=W_{\Gamma}$.

We are required to show that $\operatorname{Comp}\left(k, W_{s}, e_{s}, \mathcal{T} \llbracket \Delta \vdash \tau: \mathrm{TYPE} \rrbracket \delta\right) \quad \equiv$ $\operatorname{Comp}\left(k, W_{\Gamma}\right.$, abort $\gamma\left(e_{1}\right), \mathcal{T} \llbracket \Delta \vdash \tau:$ TYPE $\left.\rrbracket \delta\right)$.

Consider arbitrary $j, W_{r}, w_{s}, w_{f}$, and $e_{f}$ such that

- $j<k$,

- $w_{s}:_{k}\left(W_{s} \odot_{k} W_{r}\right) \equiv w_{s}:_{k}\left(W_{\Gamma} \odot_{k} W_{r}\right)$,

- $\left(w_{s}, e_{s}\right)=\left(w_{s}\right.$, abort $\left.\gamma\left(e_{1}\right)\right) \longmapsto j\left(w_{f}, e_{f}\right)$, and

- $\operatorname{irred}\left(w_{f}, e_{f}\right)$.

Hence, by inspection of the operational semantics, it follows that there exist $j_{1}, w_{f_{1}}$, and $e_{f_{1}}$ such that

- $\left(w_{s}, \gamma\left(e_{1}\right)\right) \longmapsto j^{j_{1}}\left(w_{f_{1}}, e_{f_{1}}\right)$,

- $\operatorname{irred}\left(w_{f_{1}}, e_{f_{1}}\right)$, and

- $j_{1} \leq j$.

Applying the induction hypothesis to $\Delta ; \Gamma \vdash e_{1}:{ }^{\xi} \mathbf{0}$, we conclude that $\llbracket \Delta ; \Gamma \vdash e_{1}:{ }^{\xi} \mathbf{0} \rrbracket$. Instantiate this with $k, \delta, q_{\Gamma}, W_{\Gamma}$, and $\gamma$. Note that

- $k \geq 0$,

- $\delta \in \mathcal{D} \llbracket \Delta \rrbracket$, and

- $\left(k, q_{\Gamma}, W_{\Gamma}, \gamma\right) \in \mathcal{G} \llbracket \Delta \vdash \Gamma \rrbracket \delta$.

Hence, $\operatorname{Comp}\left(k, W_{\Gamma}, \gamma\left(e_{1}\right), \mathcal{T} \llbracket \Delta \vdash \xi_{0}:\right.$ TYPE $\left.\rrbracket \delta\right)$.

Instantiate this with $j_{1}, W_{r}, w_{s}, w_{f_{1}}$, and $e_{f_{1}}$. Note that

- $j_{1}<k$, which follows from $j_{1} \leq j$ and $j<k$,

- $w_{s}:_{k}\left(W_{\Gamma} \odot_{k} W_{r}\right)$, which follows from above,

- $\left(w_{s}, \gamma\left(e_{1}\right)\right) \longmapsto{ }^{j_{1}}\left(w_{f_{1}}, e_{f_{1}}\right)$,

- $\operatorname{irred}\left(w_{f_{1}}, e_{f_{1}}\right)$.

Hence, there exists $W_{f_{1}}$ and $q_{f_{1}}$ such that

- $w_{f_{1}}:_{k-j_{1}}\left(W_{f_{1}} \odot_{k-j_{1}} W_{r}\right)$, and

- $\left(k-j_{1}, q_{f_{1}}, W_{f_{1}}, e_{f_{1}}\right)$

$\in \mathcal{T} \llbracket \Delta \vdash^{\xi} \mathbf{0}:$ TYPE $\rrbracket \delta$

$\equiv\{(k, q, W, v) \mid$

$q=\mathcal{T} \llbracket \Delta \vdash \xi: \mathrm{QUAL} \rrbracket \delta \wedge$

$(k, q, W, v) \in \mathcal{T} \llbracket \Delta \vdash \mathbf{0}:$ PRETYPE $\rrbracket \delta\}$

$\equiv\{(k, q, W, v) \mid$

$q=\mathcal{T} \llbracket \Delta \vdash \xi: \mathrm{QUAL} \rrbracket \delta \wedge$

$(k, q, W, v) \in\{\}\}$

$\equiv\{\}$. 
Note that $\left(k-j_{1}, q_{f_{1}}, W_{f_{1}}, e_{f_{1}}\right) \in\{\}$ implies False.

Hence, $\operatorname{Comp}\left(k, W_{\Gamma}\right.$, abort $\gamma\left(e_{1}\right), \mathcal{T} \llbracket \Delta \vdash \tau:$ TYPE $\left.\rrbracket \delta\right)$. 
(INL)

Case $\frac{\Delta \vdash \xi: \text { QUAL } \quad \Delta ; \Gamma \vdash v_{1}: \tau_{1} \quad \Delta \vdash \tau_{1} \preceq \xi \quad \Delta \vdash \tau_{2}: \text { TYPE }}{\Delta ; \Gamma \vdash \operatorname{inl} v_{1}:{ }^{\xi} \tau_{1} \oplus \tau_{2}}:$

We are required to show $\llbracket \Delta ; \Gamma \vdash \operatorname{inl} v_{1}:{ }^{\xi} \tau_{1} \oplus \tau_{2} \rrbracket$.

Consider arbitrary $k, \delta, q_{\Gamma}, W_{\Gamma}$, and $\gamma$ such that

- $k \geq 0$,

- $\delta \in \mathcal{D} \llbracket \Delta \rrbracket$, and

- $\left(k, q_{\Gamma}, W_{\Gamma}, \gamma\right) \in \mathcal{G} \llbracket \Delta \vdash \Gamma \rrbracket \delta$.

Let $e_{s}=\gamma\left(\operatorname{inl} v_{1}\right) \equiv \operatorname{inl} \gamma\left(v_{1}\right)$ and $W_{s}=W_{\Gamma}$.

We are required to show that $\operatorname{Comp}\left(k, W_{s}, e_{s}, \mathcal{T} \llbracket \Delta \vdash{ }^{\xi} \tau_{1} \oplus \tau_{2}:\right.$ TYPE $\left.\rrbracket \delta\right) \equiv$ $\operatorname{Comp}\left(k, W_{\Gamma},\langle\rangle, \mathcal{T} \llbracket \Delta \vdash{ }^{\tau_{1}} \oplus \tau_{2}: \operatorname{TYPE} \rrbracket \delta\right)$.

Consider arbitrary $j, W_{r}, w_{s}, w_{f}$, and $e_{f}$ such that

- $j<k$,

- $w_{s}:_{k}\left(W_{s} \odot_{k} W_{r}\right) \equiv w_{s}:_{k}\left(W_{\Gamma} \odot_{k} W_{r}\right)$,

- $\left(w_{s}, e_{s}\right) \equiv\left(w_{s}, \operatorname{inl} \gamma\left(v_{1}\right)\right) \longmapsto j\left(w_{f}, e_{f}\right)$, and

- $\operatorname{irred}\left(w_{f}, e_{f}\right)$.

Since $\operatorname{inl} \gamma\left(v_{1}\right)$ is a value, we have $\operatorname{irred}\left(w_{s}, \operatorname{inl} \gamma\left(v_{1}\right)\right)$.

Hence, $j=0$ and $w_{f} \equiv w_{s}$ and $e_{f} \equiv \operatorname{inl} \gamma\left(v_{1}\right)$.

Applying the induction hypothesis to $\Delta ; \Gamma \vdash v_{1}: \tau_{1}$, we conclude that $\llbracket \Delta ; \Gamma \vdash v_{1}: \tau_{1} \rrbracket$. Instantiate this with $k, \delta, q_{\Gamma}, W_{\Gamma}$, and $\gamma$. Note that

- $k \geq 0$,

- $\delta \in \mathcal{D} \llbracket \Delta \rrbracket$, and

- $\left(k, q_{\Gamma}, W_{\Gamma}, \gamma\right) \in \mathcal{G} \llbracket \Delta \vdash \Gamma \rrbracket \delta$.

Hence, $\operatorname{Comp}\left(k, W_{\Gamma}, \gamma\left(v_{1}\right), \mathcal{T} \llbracket \Delta \vdash \tau_{1}:\right.$ TYPE $\left.\rrbracket \delta\right)$.

Instantiate this with $0, W_{r}, w_{s}, w_{s}$, and $\gamma\left(v_{1}\right)$. Note that

- $0<k$, which follows from $j=0$ and $j<k$,

- $w_{s}:_{k}\left(W_{\Gamma} \odot_{k} W_{r}\right)$, which follows from above,

- $\left(w_{s}, \gamma\left(v_{1}\right)\right) \longmapsto^{0}\left(w_{s}, \gamma\left(v_{1}\right)\right)$, and

- $\operatorname{irred}\left(w_{s}, \gamma\left(v_{1}\right)\right)$, which follows from the fact that $\gamma\left(v_{1}\right)$ is a value.

Hence, there exists $W_{f_{1}}$ and $q_{f_{1}}$ such that

- $w_{s}:_{k-0}\left(W_{f_{1}} \odot_{k-0} W_{r}\right)$, and

- $\left(k-0, q_{f_{1}}, W_{f_{1}}, \gamma\left(v_{1}\right)\right) \in \mathcal{T} \llbracket \Delta \vdash \tau_{1}:$ TYPE $\rrbracket \delta$.

Let $W_{f}=W_{f_{1}}$ and $q_{f}=\mathcal{T} \llbracket \Delta \vdash \xi: \mathrm{QUAL} \rrbracket \delta$.

We are required to show that

- $w_{f}: k-0\left(W_{f} \odot_{k-0} W_{r}\right)$

$\equiv w_{s}: k\left(W_{f_{1}} \odot_{k} W_{r}\right)$,

which follows from above, and 
- $\left(k-0, q_{f}, W_{f}, e_{f}\right) \in \mathcal{T} \llbracket \Delta \vdash \xi_{\tau_{1}} \oplus \tau_{2}:$ TYPE $\rrbracket \delta$

$\equiv\left(k, \mathcal{T} \llbracket \Delta \vdash \xi: \mathrm{QUAL} \rrbracket \delta, W_{f_{1}}, \operatorname{inl} \gamma\left(v_{1}\right)\right)$

$\in\{(k, q, W, v) \mid$

$q=\mathcal{T} \llbracket \Delta \vdash \xi: \mathrm{QUAL} \rrbracket \delta \wedge$

$\left.(k, q, W, v) \in \mathcal{T} \llbracket \Delta \vdash \tau_{1} \oplus \tau_{2}: \mathrm{PRETYPE} \rrbracket \delta\right\}$

$\equiv\left(k, \mathcal{T} \llbracket \Delta \vdash \xi: \mathrm{QUAL} \rrbracket \delta, W_{f_{1}}, \operatorname{inl} \gamma\left(v_{1}\right)\right)$

$\in\left\{\left(k, q, W, \operatorname{inl} v_{1}\right) \mid\right.$

$q=\mathcal{T} \llbracket \Delta \vdash \xi: \mathrm{QUAL} \rrbracket \delta \wedge$

$\left(k, q_{1}, W, v_{1}\right) \in \mathcal{T} \llbracket \Delta \vdash \tau_{1}:$ TYPE $\rrbracket \delta \wedge$

$\left.q_{1} \preceq q\right\} \cup$

$\left\{\left(k, q, W, \operatorname{inr} v_{2}\right) \mid\right.$

$q=\mathcal{T} \llbracket \Delta \vdash \xi: \mathrm{QUAL} \rrbracket \delta \wedge$

$\left(k, q_{2}, W, v_{2}\right) \in \mathcal{T} \llbracket \Delta \vdash \tau_{2}:$ TYPE $\rrbracket \delta \wedge$

$\left.q_{2} \preceq q\right\}$

which follows from

- $\mathcal{T} \llbracket \Delta \vdash \xi: \mathrm{QUAL} \rrbracket \delta=\mathcal{T} \llbracket \Delta \vdash \xi: \mathrm{QUAL} \rrbracket \delta$, which follows trivially,

- $\left(k, q_{f_{1}}, W_{f_{1}}, \gamma\left(v_{1}\right)\right) \in \mathcal{T} \llbracket \Delta \vdash \tau_{1}:$ TYPE $\rrbracket \delta$, which follows from above, and

- $q_{f_{1}} \preceq \mathcal{T} \llbracket \Delta \vdash \xi: \mathrm{QUAL} \rrbracket \delta$, which follows from Lemma 15 applied to $\Delta \vdash \tau \preceq \xi$ and $\left(k, q_{f_{1}}, W_{f_{1}}, \gamma\left(v_{1}\right)\right) \in \mathcal{T} \llbracket \Delta \vdash \tau_{1}:$ TYPE $\rrbracket$ and $\mathcal{T} \llbracket \Delta \vdash \xi:$ QUAL $\rrbracket$. 
(INR)

Case $\frac{\Delta \vdash \xi: \text { QUAL } \quad \Delta \vdash \tau_{1}: \text { TYPE } \quad \Delta ; \Gamma \vdash v_{2}: \tau_{2} \quad \Delta \vdash \tau_{2} \preceq \xi}{\Delta ; \Gamma \vdash \text { inr } v_{2}:{ }^{\xi} \tau_{1} \oplus \tau_{2}}:$

We are required to show $\llbracket \Delta ; \Gamma \vdash \operatorname{inr} v_{2}:{ }^{\xi} \tau_{1} \oplus \tau_{2} \rrbracket$.

Consider arbitrary $k, \delta, q_{\Gamma}, W_{\Gamma}$, and $\gamma$ such that

- $k \geq 0$,

- $\delta \in \mathcal{D} \llbracket \Delta \rrbracket$, and

- $\left(k, q_{\Gamma}, W_{\Gamma}, \gamma\right) \in \mathcal{G} \llbracket \Delta \vdash \Gamma \rrbracket \delta$.

Let $e_{s}=\gamma\left(\operatorname{inr} v_{2}\right) \equiv \operatorname{inr} \gamma\left(v_{2}\right)$ and $W_{s}=W_{\Gamma}$.

We are required to show that $\operatorname{Comp}\left(k, W_{s}, e_{s}, \mathcal{T} \llbracket \Delta \vdash \xi \tau_{1} \oplus \tau_{2}: \mathrm{TYPE} \rrbracket \delta\right) \quad \equiv$ $\operatorname{Comp}\left(k, W_{\Gamma},\langle\rangle, \mathcal{T} \llbracket \Delta \vdash \xi_{\tau_{1}} \oplus \tau_{2}:\right.$ TYPE $\left.\rrbracket \delta\right)$.

Consider arbitrary $j, W_{r}, w_{s}, w_{f}$, and $e_{f}$ such that

- $j<k$,

- $w_{s}:_{k}\left(W_{s} \odot_{k} W_{r}\right) \equiv w_{s}:_{k}\left(W_{\Gamma} \odot_{k} W_{r}\right)$,

- $\left(w_{s}, e_{s}\right) \equiv\left(w_{s}, \operatorname{inr} \gamma\left(v_{2}\right)\right) \longmapsto j\left(w_{f}, e_{f}\right)$, and

- $\operatorname{irred}\left(w_{f}, e_{f}\right)$.

Since $\operatorname{inr} \gamma\left(v_{2}\right)$ is a value, we have $\operatorname{irred}\left(w_{s}, \operatorname{inr} \gamma\left(v_{2}\right)\right)$.

Hence, $j=0$ and $w_{f} \equiv w_{s}$ and $e_{f} \equiv \operatorname{inr} \gamma\left(v_{2}\right)$.

Applying the induction hypothesis to $\Delta ; \Gamma \vdash v_{2}: \tau_{2}$, we conclude that $\llbracket \Delta ; \Gamma \vdash v_{2}: \tau_{2} \rrbracket$. Instantiate this with $k, \delta, q_{\Gamma}, W_{\Gamma}$, and $\gamma$. Note that

- $k \geq 0$,

- $\delta \in \mathcal{D} \llbracket \Delta \rrbracket$, and

- $\left(k, q_{\Gamma}, W_{\Gamma}, \gamma\right) \in \mathcal{G} \llbracket \Delta \vdash \Gamma \rrbracket \delta$.

Hence, $\operatorname{Comp}\left(k, W_{\Gamma}, \gamma\left(v_{2}\right), \mathcal{T} \llbracket \Delta \vdash \tau_{2}:\right.$ TYPE $\left.\rrbracket \delta\right)$.

Instantiate this with $0, W_{r}, w_{s}, w_{s}$, and $\gamma\left(v_{2}\right)$. Note that

- $0<k$, which follows from $j=0$ and $j<k$,

- $w_{s}:_{k}\left(W_{\Gamma} \odot_{k} W_{r}\right)$, which follows from above,

- $\left(w_{s}, \gamma\left(v_{2}\right)\right) \longmapsto^{0}\left(w_{s}, \gamma\left(v_{2}\right)\right)$, and

- $\operatorname{irred}\left(w_{s}, \gamma\left(v_{2}\right)\right)$, which follows from the fact that $\gamma\left(v_{2}\right)$ is a value.

Hence, there exists $W_{f_{2}}$ and $q_{f_{2}}$ such that

- $w_{s}:_{k-0}\left(W_{f_{2}} \odot_{k-0} W_{r}\right)$, and

- $\left(k-0, q_{f_{2}}, W_{f_{2}}, \gamma\left(v_{2}\right)\right) \in \mathcal{T} \llbracket \Delta \vdash \tau_{2}:$ TYPE $\rrbracket \delta$.

Let $W_{f}=W_{f_{2}}$ and $q_{f}=\mathcal{T} \llbracket \Delta \vdash \xi:$ QUAL $\rrbracket \delta$.

We are required to show that

- $w_{f}:_{k-0}\left(W_{f} \odot_{k-0} W_{r}\right)$

$\equiv w_{s}: k\left(W_{f_{2}} \odot_{k} W_{r}\right)$

which follows from above, and 
- $\left(k-0, q_{f}, W_{f}, e_{f}\right) \in \mathcal{T} \llbracket \Delta \vdash \xi_{\tau_{1}} \oplus \tau_{2}:$ TYPE $\rrbracket \delta$

$\equiv\left(k, \mathcal{T} \llbracket \Delta \vdash \xi: \mathrm{QUAL} \rrbracket \delta, W_{f_{2}}, \operatorname{inr} \gamma\left(v_{2}\right)\right)$

$\in\{(k, q, W, v) \mid$

$q=\mathcal{T} \llbracket \Delta \vdash \xi: \mathrm{QUAL} \rrbracket \delta \wedge$

$\left.(k, q, W, v) \in \mathcal{T} \llbracket \Delta \vdash \tau_{1} \oplus \tau_{2}: \mathrm{PRETYPE} \rrbracket \delta\right\}$

$\equiv\left(k, \mathcal{T} \llbracket \Delta \vdash \xi: \mathrm{QUAL} \rrbracket \delta, W_{f_{2}}, \operatorname{inr} \gamma\left(v_{2}\right)\right)$

$\in\left\{\left(k, q, W, \operatorname{inl} v_{1}\right) \mid\right.$

$q=\mathcal{T} \llbracket \Delta \vdash \xi: \mathrm{QUAL} \rrbracket \delta \wedge$

$\left(k, q_{1}, W, v_{1}\right) \in \mathcal{T} \llbracket \Delta \vdash \tau_{1}:$ TYPE $\rrbracket \delta \wedge$

$\left.q_{1} \preceq q\right\} \cup$

$\left\{\left(k, q, W, \operatorname{inr} v_{2}\right) \mid\right.$

$q=\mathcal{T} \llbracket \Delta \vdash \xi: \mathrm{QUAL} \rrbracket \delta \wedge$

$\left(k, q_{2}, W, v_{2}\right) \in \mathcal{T} \llbracket \Delta \vdash \tau_{2}: \mathrm{TYPE} \rrbracket \delta \wedge$

$\left.q_{2} \preceq q\right\}$

which follows from

- $\mathcal{T} \llbracket \Delta \vdash \xi: \mathrm{QUAL} \rrbracket \delta=\mathcal{T} \llbracket \Delta \vdash \xi: \mathrm{QUAL} \rrbracket \delta$, which follows trivially,

- $\left(k, q_{f_{2}}, W_{f_{2}}, \gamma\left(v_{2}\right)\right) \in \mathcal{T} \llbracket \Delta \vdash \tau_{2}:$ TYPE $\rrbracket \delta$, which follows from above, and

- $q_{f_{2}} \preceq \mathcal{T} \llbracket \Delta \vdash \xi: \mathrm{QUAL} \rrbracket \delta$, which follows from Lemma 15 applied to $\Delta \vdash \tau \preceq \xi$ and $\left(k, q_{f_{2}}, W_{f_{2}}, \gamma\left(v_{2}\right)\right) \in \mathcal{T} \llbracket \Delta \vdash \tau_{2}:$ TYPE $\rrbracket \delta$ and $\mathcal{T} \llbracket \Delta \vdash \xi: \mathrm{QUAL} \rrbracket$. 
(CASE)

Case $\frac{\Delta \vdash \Gamma \sim \Gamma_{1} \boxplus \Gamma_{2} \quad \Delta ; \Gamma_{1} \vdash e_{1}:{ }^{\xi} \tau_{1} \oplus \tau_{2} \quad \Delta ; \Gamma_{2}, x_{1}: \tau_{1}: e_{21}: \tau \quad \Delta ; \Gamma_{2}, x_{2}: \tau_{2}: e_{22}: \tau}{\Delta ; \Gamma \vdash \operatorname{case} e_{1} \text { of } \operatorname{inl} x_{1} \Rightarrow e_{21} \| \operatorname{inr} x_{2} \Rightarrow e_{22}: \tau}$ :

We are required to show $\llbracket \Delta ; \Gamma \vdash$ case $e_{1}$ of inl $x_{1} \Rightarrow e_{21} \|$ inr $x_{2} \Rightarrow e_{22}: \tau \rrbracket$.

Consider arbitrary $k, \delta, q_{\Gamma}, W_{\Gamma}$, and $\gamma$ such that

- $k \geq 0$,

- $\delta \in \mathcal{D} \llbracket \Delta \rrbracket$, and

- $\left(k, q_{\Gamma}, W_{\Gamma}, \gamma\right) \in \mathcal{G} \llbracket \Delta \vdash \Gamma \rrbracket \delta$.

Applying Lemma 20 to $\left(k, q_{\Gamma}, W_{\Gamma}, \gamma\right) \in \mathcal{G} \llbracket \Delta \vdash \Gamma \rrbracket \delta$ and $\Delta \vdash \Gamma \leadsto \Gamma_{1} \boxplus \Gamma_{2}$, we conclude that there exist $q_{\Gamma_{1}}, W_{\Gamma_{1}}, \gamma_{1}, q_{\Gamma_{2}}, W_{\Gamma_{2}}$, and $\gamma_{2}$, such that

- $\left(k, q_{\Gamma_{1}}, W_{\Gamma_{1}}, \gamma_{1}\right) \in \mathcal{G} \llbracket \Delta \vdash \Gamma_{1} \rrbracket \delta$,

- $\left(k, q_{\Gamma_{2}}, W_{\Gamma_{2}}, \gamma_{2}\right) \in \mathcal{G} \llbracket \Delta \vdash \Gamma_{2} \rrbracket \delta$,

- $\gamma \in \gamma_{1} \boxplus \gamma_{2}$,

- $q_{\Gamma_{1}} \preceq q_{\Gamma}$,

- $q_{\Gamma_{2}} \preceq q_{\Gamma}$, and

- $\left(W_{\Gamma_{1}} \odot_{k} W_{\Gamma_{2}}=W_{\Gamma}\right)$.

Note that $\gamma\left(e_{1}\right) \equiv \gamma_{1}\left(e_{1}\right)$ and $\gamma\left(e_{21}\right) \equiv \gamma_{2}\left(e_{21}\right)$ and $\gamma\left(e_{22}\right) \equiv \gamma_{2}\left(e_{22}\right)$.

Let $e_{s}=\gamma\left(\operatorname{case} e_{1}\right.$ of inl $\left.x_{1} \Rightarrow e_{21} \| \operatorname{inr} x_{2} \Rightarrow e_{22}\right) \equiv \operatorname{case} \gamma\left(e_{1}\right)$ of inl $x_{1} \Rightarrow \gamma\left(e_{21}\right) \|$ inr $x_{2} \Rightarrow \gamma\left(e_{22}\right) \equiv$ case $\gamma_{1}\left(e_{1}\right)$ of inl $x_{1} \Rightarrow \gamma_{2}\left(e_{21}\right) \|$ inr $x_{2} \Rightarrow \gamma_{2}\left(e_{22}\right)$ and $W_{s}=W_{\Gamma}$.

We are required to show that $\operatorname{Comp}\left(k, W_{s}, e_{s}, \mathcal{T} \llbracket \Delta \vdash \tau: \mathrm{TYPE} \rrbracket \delta\right) \quad \equiv$ $\operatorname{Comp}\left(k, W_{\Gamma}\right.$, case $\gamma_{1}\left(e_{1}\right)$ of inl $x_{1} \Rightarrow \gamma_{2}\left(e_{21}\right) \|$ inr $x_{2} \Rightarrow \gamma_{2}\left(e_{22}\right), \mathcal{T} \llbracket \Delta \vdash \tau:$ TYPE $\left.\delta\right)$.

Consider arbitrary $j, W_{r}, w_{s}, w_{f}$, and $e_{f}$ such that

- $j<k$,

- $w_{s}:_{k}\left(W_{s} \odot_{k} W_{r}\right) \equiv w_{s}:_{k}\left(W_{\Gamma} \odot_{k} W_{r}\right)$, noting that

$$
\begin{gathered}
w_{s}:_{k}\left(W_{\Gamma} \odot_{k} W_{r}\right) \\
\equiv w_{s}: k\left(\left(W_{\Gamma_{1}} \odot_{k} W_{\Gamma_{2}}\right) \odot_{k} W_{r}\right) \\
\text { which follows from above, }
\end{gathered}
$$

- $\left(w_{s}, e_{s}\right) \equiv\left(w_{s}\right.$, case $\gamma_{1}\left(e_{1}\right)$ of inl $x_{1} \Rightarrow \gamma_{2}\left(e_{21}\right) \|$ inr $\left.x_{2} \Rightarrow \gamma_{2}\left(e_{22}\right)\right) \longmapsto^{j}\left(w_{f}, e_{f}\right)$, and

- $\operatorname{irred}\left(w_{f}, e_{f}\right)$.

Hence, by inspection of the operational semantics, it follows that there exist $j_{1}, w_{f_{1}}$, and $e_{f_{1}}$ such that

- $\left(w_{s}, \gamma_{1}\left(e_{1}\right)\right) \longmapsto^{j_{1}}\left(w_{f_{1}}, e_{f_{1}}\right)$,

- $\operatorname{irred}\left(w_{f_{1}}, e_{f_{1}}\right)$, and

- $j_{1} \leq j$.

Note that $\left(\left(W_{\Gamma_{1}} \odot_{k} W_{\Gamma_{2}}\right) \odot_{k} W_{r}\right) \equiv\left(W_{\Gamma_{1}} \odot_{k}\left(W_{\Gamma_{2}} \odot_{k} W_{r}\right)\right)$, which follows from

$$
\begin{aligned}
& \left(\left(W_{\Gamma_{1}} \odot_{k} W_{\Gamma_{2}}\right) \odot_{k} W_{r}\right) \\
& \equiv\left(W_{\Gamma_{1}} \odot_{k}\left(W_{\Gamma_{2}} \odot_{k} W_{r}\right)\right)
\end{aligned}
$$

which follows from Reqs 6,7 , and 8 (join-commut, join-assocl, and join-assocr).

Applying the induction hypothesis to $\Delta ; \Gamma_{1} \vdash \quad e_{1}: \xi_{\tau_{1}} \oplus \tau_{2}$, we conclude that $\llbracket \Delta ; \Gamma_{1} \vdash e_{1}:{ }^{\xi} \tau_{1} \oplus \tau_{2} \rrbracket$.

Instantiate this with $k, \delta, q_{\Gamma_{1}}, W_{\Gamma_{1}}$, and $\gamma_{1}$. Note that 
- $k \geq 0$,

- $\delta \in \mathcal{D} \llbracket \Delta \rrbracket$, and

- $\left(k, q_{\Gamma_{1}}, W_{\Gamma_{1}}, \gamma_{1}\right) \in \mathcal{G} \llbracket \Delta \vdash \Gamma_{1} \rrbracket \delta$.

Hence, $\operatorname{Comp}\left(k, W_{\Gamma_{1}}, \gamma_{1}\left(e_{1}\right), \mathcal{T} \llbracket \Delta \vdash \xi_{\tau_{1}} \oplus \tau_{2}: \operatorname{TYPE} \rrbracket \delta\right)$.

Instantiate this with $j_{1},\left(W_{\Gamma_{2}} \odot_{k} W_{r}\right), w_{s}, w_{f_{1}}$, and $e_{f_{1}}$. Note that

- $j_{1}<k$, which follows from $j_{1} \leq j$ and $j<k$,

- $w_{s}:_{k}\left(W_{\Gamma_{1}} \odot_{k}\left(W_{\Gamma_{2}} \odot_{k} W_{r}\right)\right)$, which follows from

$$
w_{s}:_{k}\left(\left(W_{\Gamma_{1}} \odot_{k} W_{\Gamma_{2}}\right) \odot_{k} W_{r}\right)
$$

which follows from above

$\left(\left(W_{\Gamma_{1}} \odot_{k} W_{\Gamma_{2}}\right) \odot_{k} W_{r}\right) \equiv\left(W_{\Gamma_{1}} \odot_{k}\left(W_{\Gamma_{2}} \odot_{k} W_{r}\right)\right)$

which follows from above,

- $\left(w_{s}, \gamma_{1}\left(e_{1}\right)\right) \longmapsto j^{j_{1}}\left(w_{f_{1}}, e_{f_{1}}\right)$, and

- $\operatorname{irred}\left(w_{f_{1}}, e_{f_{1}}\right)$.

Hence, there exists $W_{f_{1}}$ and $q_{f_{1}}$ such that

- $w_{f_{1}}:_{k-j_{1}}\left(W_{f_{1}} \odot_{k-j_{1}}\left(W_{\Gamma_{2}} \odot_{k} W_{r}\right)\right)$, and

- $\left(k-j_{1}, q_{f_{1}}, W_{f_{1}}, e_{f_{1}}\right)$

$\in \mathcal{T} \llbracket \Delta \vdash \xi_{\tau_{1} \oplus \tau_{2}: \text { TYPE } \rrbracket \delta}$

$\equiv\left\{\left(k, q, W, \operatorname{inl} v_{1}\right) \mid\right.$

$q=\mathcal{T} \llbracket \Delta \vdash \xi: \mathrm{QUAL} \rrbracket \delta \wedge$

$\left(k, q_{1}, W, v_{1}\right) \in \mathcal{T} \llbracket \Delta \vdash \tau_{1}: \mathrm{TYPE} \rrbracket \delta \wedge$

$\left.q_{1} \preceq q\right\} \cup$

$\left\{\left(k, q, W, \operatorname{inr} v_{2}\right) \mid\right.$

$q=\mathcal{T} \llbracket \Delta \vdash \xi: \mathrm{QUAL} \rrbracket \delta \wedge$

$\left(k, q_{2}, W, v_{2}\right) \in \mathcal{T} \llbracket \Delta \vdash \tau_{2}: \mathrm{TYPE} \rrbracket \delta \wedge$

$\left.q_{2} \preceq q\right\}$.

Hence, $e_{f_{1}} \equiv \operatorname{inl} v_{f_{11}}$ or $e_{f_{1}} \equiv \operatorname{inr} v_{f_{12}}$. and $q_{f_{1}}=\mathcal{T} \llbracket \Delta \vdash \xi: \mathrm{QUAL} \rrbracket \delta$.

Case $e_{f_{1}} \equiv \operatorname{inl} v_{f_{11}}$ :

Hence, $\left(k-j_{1}, q_{f_{11}}, W_{f_{1}}, v_{f_{11}}\right) \in \mathcal{T} \llbracket \Delta \vdash \tau_{1}:$ TYPE $\rrbracket \delta$ and $q_{f_{11}} \preceq q_{f_{1}}$.

Note that

$$
\begin{aligned}
\left(w_{s}, e_{s}\right) & \equiv\left(w_{s}, \text { case } \gamma_{1}\left(e_{1}\right) \text { of inl } x_{1} \Rightarrow \gamma_{2}\left(e_{21}\right) \| \operatorname{inr} x_{2} \Rightarrow \gamma_{2}\left(e_{22}\right)\right) \\
& \longmapsto^{j_{1}}\left(w_{f_{1}}, \text { case } e_{f_{1}} \text { of inl } x_{1} \Rightarrow \gamma_{2}\left(e_{21}\right) \| \operatorname{inr} x_{2} \Rightarrow \gamma_{2}\left(e_{22}\right)\right) \\
& \equiv\left(w_{f_{1}}, \text { case inl } v_{f_{11}} \text { of inl } x_{1} \Rightarrow \gamma_{2}\left(e_{21}\right) \| \operatorname{inr} x_{2} \Rightarrow \gamma_{2}\left(e_{22}\right)\right) \\
& \longmapsto^{1}\left(w_{f_{1}}, \gamma_{2}\left(e_{21}\right)\left[v_{f_{11}} / x_{1}\right]\right) \\
& \longmapsto^{j_{2}}\left(w_{f}, e_{f}\right)
\end{aligned}
$$

and $\operatorname{irred}\left(w_{f}, e_{f}\right)$, where $j=j_{1}+1+j_{2}$.

Note that $\left\lfloor\left(W_{f_{1}} \odot_{k-j_{1}}\left(W_{\Gamma_{2}} \odot_{k} W_{r}\right)\right)\right\rfloor_{k-j_{1}-1} \equiv\left(\left(W_{\Gamma_{2}} \odot_{k-j_{1}-1} W_{f_{1}}\right) \odot_{k-j_{1}-1} W_{r}\right)$, which follows from

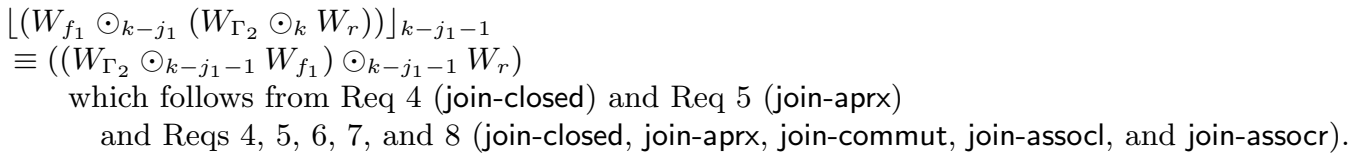

Applying the induction hypothesis to $\Delta ; \Gamma_{2}, x_{1}: \tau_{1} \vdash e_{21}: \tau$, we conclude that $\llbracket \Delta ; \Gamma_{2}, x_{1}: \tau_{1} \vdash e_{21}: \tau \rrbracket$.

Instantiate this with $k-j_{1}-1, \delta,\left(q_{\Gamma_{2}} \sqcap q_{f_{11}}\right),\left(W_{\Gamma_{2}} \odot_{k-j_{1}-1} W_{f_{1}}\right)$, and $\gamma_{2}\left[x_{1} \mapsto v_{f_{11}}\right]$.

Note that 
- $k-j_{1}-1 \geq 0$, which follows from $j_{1}+1+j_{2}=j$ and $j<k$,

- $\delta \in \mathcal{D} \llbracket \Delta \rrbracket$, which follows from above,

- $\left(k-j_{1}-1,\left(q_{\Gamma_{2}} \sqcap q_{f_{11}}\right),\left(W_{\Gamma_{2}} \odot_{k-j_{1}-1} W_{f_{1}}\right), \gamma_{2}\left[x_{1} \mapsto v_{f_{11}}\right]\right) \in \mathcal{G} \llbracket \Delta \vdash \Gamma_{2}, x_{1}: \tau_{1} \rrbracket \delta$, which follows from

- $\left(k-j_{1}-1, q_{\Gamma_{2}},\left\lfloor W_{\Gamma_{2}}\right\rfloor_{k-j_{1}-1}, \gamma_{2}\right) \in \mathcal{G} \llbracket \Delta \vdash \Gamma_{2} \rrbracket \delta$, which in turn follows from Lemma 9 applied to $k-j_{1}-1 \leq k$ and $\left(k, q_{\Gamma_{2}}, W_{\Gamma_{2}}, \gamma_{2}\right) \in \mathcal{G} \llbracket \Delta \vdash \Gamma_{2} \rrbracket \delta$,

- $\left(k-j_{1}-1, q_{f_{11}},\left\lfloor W_{f_{1}}\right\rfloor_{k-j_{1}-1}, v_{f_{11}}\right) \in \mathcal{T} \llbracket \Delta \vdash \tau_{1}:$ TYPE $\rrbracket \delta$, which follows from Fact 6 applied to $\left(k-j_{1}, q_{f_{11}}, W_{f_{1}}, v_{f_{11}}\right) \in \mathcal{T} \llbracket \Delta \vdash \tau_{1}$ : TYPE $\rrbracket \in$ Type (which follows from Lemma 8) instantiated with $k-j_{1}-1$, noting that $k-j_{1}-1 \leq k-j_{1}$,

- $q_{\Gamma_{2}} \preceq\left(q_{\Gamma_{2}} \sqcap q_{f_{11}}\right)$, which follows from the definition of $\sqcap$,

- $q_{f_{11}} \preceq\left(q_{\Gamma_{2}} \sqcap q_{f_{11}}\right)$, which follows from the definition of $\sqcap$, and

- $\left(W_{\Gamma_{2}} \odot_{k-j_{1}-1} W_{f_{1}}\right)=\left(\left\lfloor W_{\Gamma_{2}}\right\rfloor_{k-j_{1}-1} \odot_{k-j_{1}-1}\left\lfloor W_{f_{1}}\right\rfloor_{k-j_{1}-1}\right)$, which follows from $\left(W_{\Gamma_{2}} \odot_{k-j_{1}-1} W_{f_{1}}\right)$$$
\equiv\left(\left\lfloor W_{\Gamma_{2}}\right\rfloor_{k-j_{1}-1} \odot_{k-j_{1}-1}\left\lfloor W_{f_{1}}\right\rfloor_{k-j_{1}-1}\right)
$$

which follows from Req 5 (join-aprx).

Hence, $\operatorname{Comp}\left(k-j_{1}-1,\left(W_{\Gamma_{2}} \odot_{k-j_{1}-1} W_{f_{1}}\right), \gamma_{2}\left[x_{1} \mapsto v_{f_{11}}\right], \mathcal{T} \llbracket \Delta \vdash \tau:\right.$ TYPE $\left.\rrbracket\right)$.

Instantiate this with $j_{2}, W_{r}, w_{f_{1}}, w_{f}$, and $e_{f}$. Note that

- $j_{2}<k-j_{1}-1$, which follows from $j_{2}=j-j_{1}-1$ and $j<k$,

- $w_{f_{1}}:_{k-j_{1}-1}\left(\left(W_{\Gamma_{2}} \odot_{k-j_{1}-1} W_{f_{1}}\right) \odot_{k-j_{1}-1} W_{r}\right)$, which follows from

$w_{f_{1}}: k-j_{1}\left(W_{f_{1}} \odot_{k-j_{1}}\left(W_{\Gamma_{2}} \odot_{k} W_{r}\right)\right)$

which follows from above

$\Rightarrow w_{f_{1}}: k-j_{1}-1\left(W_{f_{1}} \odot_{k-j_{1}}\left(W_{\Gamma_{2}} \odot_{k} W_{r}\right)\right)$

which follows from Req 2 (models-closed)

$\Leftrightarrow w_{f_{1}}: k-j_{1}-1\left\lfloor\left(W_{f_{1}} \odot_{k-j_{1}}\left(W_{\Gamma_{2}} \odot_{k} W_{r}\right)\right)\right\rfloor_{k-j_{1}-1}$

which follows from Req 3 (models-aprx)

$\left\lfloor\left(W_{f_{1}} \odot_{k-j_{1}}\left(W_{\Gamma_{2}} \odot_{k} W_{r}\right)\right)\right\rfloor_{k-j_{1}-1} \equiv\left(\left(W_{\Gamma_{2}} \odot_{k-j_{1}-1} W_{f_{1}}\right) \odot_{k-j_{1}-1} W_{r}\right)$ which follows from above,

- $\left(w_{f_{1}}, \gamma_{2}\left[x_{1} \mapsto v_{f_{11}}\right]\left(e_{21}\right)\right) \equiv\left(w_{f_{1}}, \gamma_{2}\left(e_{21}\right)\left[v_{f_{11}} / x_{1}\right]\right) \longmapsto j^{j_{2}}\left(w_{f}, e_{f}\right)$,

- $\operatorname{irred}\left(w_{f}, e_{f}\right)$.

Hence, there exists $W_{f_{2}}$ and $q_{f_{2}}$ such that

- $w_{f}: k-j_{1}-1-j_{2}\left(W_{f_{2}} \odot_{k-j_{1}-1-j_{2}} W_{r}\right)$, and

- $\left(k-j_{1}-1-j_{2}, q_{f_{2}}, W_{f_{2}}, e_{f}\right) \in \mathcal{T} \llbracket \Delta, \vdash \tau:$ TYPE $\rrbracket \delta$.

Let $W_{f}=W_{f_{2}}$ and $q_{f}=q_{f_{2}}$.

We are required to show that

- $w_{f}: k-j\left(W_{f} \odot_{k-j} W_{r}\right)$ $\equiv w_{f}:_{k-j_{1}-1-j_{2}}\left(W_{f_{2}} \odot_{k-j_{1}-1-j_{2}} W_{r}\right)$, which follows from above,

- $\left(k-j, q_{f}, W_{f}, e_{f}\right) \in \mathcal{T} \llbracket \Delta \vdash \tau \rrbracket \delta$

$\equiv\left(k-j_{1}-1-j_{2}, q_{f_{2}}, W_{f_{2}}, e_{f}\right) \in \mathcal{T} \llbracket \Delta \vdash \tau \rrbracket \delta$, which follows from above.

Case $e_{f_{1}} \equiv \operatorname{inr} v_{f_{12}}$ :

Symmetric.

\section{End Case}


(ALL)

Case $\frac{\Delta \vdash \xi: \text { QUAL } \quad \Delta \vdash \Gamma \preceq \xi \quad \Delta, \alpha: \kappa ; \Gamma \vdash e: \tau}{\Delta ; \Gamma \vdash \Lambda . e:{ }^{\xi} \forall \alpha: \kappa . \tau}$ :

We are required to show $\llbracket \Delta ; \Gamma \vdash \Lambda . e:{ }^{\xi} \forall \alpha: \kappa . \tau \rrbracket$.

Consider arbitrary $k, \delta, q_{\Gamma}, W_{\Gamma}$, and $\gamma$ such that

- $k \geq 0$,

- $\delta \in \mathcal{D} \llbracket \Delta \rrbracket$, and

- $\left(k, q_{\Gamma}, W_{\Gamma}, \gamma\right) \in \mathcal{G} \llbracket \Delta \vdash \Gamma \rrbracket \delta$.

Let $e_{s}=\gamma(\Lambda . e) \equiv \Lambda . \gamma(e)$ and $W_{s}=W_{\Gamma}$.

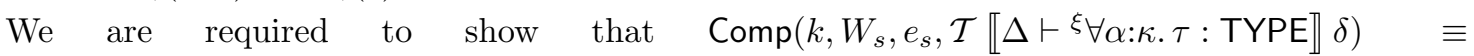
$\operatorname{Comp}\left(k, W_{\Gamma}, \Lambda . \gamma(e), \mathcal{T} \llbracket \Delta \vdash \xi \forall \alpha: \kappa . \tau:\right.$ TYPE $\left.\rrbracket \delta\right)$.

Consider arbitrary $j, W_{r}, w_{s}, w_{f}$, and $e_{f}$ such that

- $j<k$,

- $w_{s}:_{k}\left(W_{s} \odot_{k} W_{r}\right) \equiv w_{s}:_{k}\left(W_{\Gamma} \odot_{k} W_{r}\right)$,

- $\left(w_{s}, e_{s}\right) \equiv\left(w_{s}, \Lambda \cdot \gamma(e)\right) \longmapsto^{j}\left(w_{f}, e_{f}\right)$, and

- $\operatorname{irred}\left(w_{f}, e_{f}\right)$.

Since $\Lambda . \gamma(e)$ is a value, we have $\operatorname{irred}\left(w_{s}, \Lambda . \gamma(e)\right)$.

Hence, $j=0$ and $w_{f} \equiv w_{s}$ and $e_{f} \equiv \Lambda . \gamma(e)$.

Let $W_{f}=W_{\Gamma}$ and $q_{f}=\mathcal{T} \llbracket \Delta \vdash \xi: \mathrm{QUAL} \rrbracket \delta$.

We are required to show that

- $w_{f}: k-0\left(W_{f} \odot_{k-0} W_{r}\right)$

$\equiv w_{s}:_{k}\left(W_{\Gamma} \odot_{k} W_{r}\right)$,

which follows from above,

- $\left(k-0, q_{f}, W_{f}, e_{f}\right) \in \mathcal{T} \llbracket \Delta \vdash \xi \forall \alpha: \kappa . \tau: \mathrm{TYPE} \rrbracket \delta$

$\equiv\left(k, \mathcal{T} \llbracket \Delta \vdash \xi: \mathrm{QUAL} \rrbracket \delta, W_{\Gamma}, \Lambda . \gamma(e)\right) \in \mathcal{T} \llbracket \Delta \vdash \xi \forall \alpha: \kappa . \tau: \mathrm{TYPE} \rrbracket \delta$

$\equiv\left(k, \mathcal{T} \llbracket \Delta \vdash \xi: \mathrm{QUAL} \rrbracket \delta, W_{\Gamma}, \Lambda \cdot \gamma(e)\right)$

$\in\{(k, q, W, v) \mid$

$q=\mathcal{T} \llbracket \Delta \vdash \xi: \mathrm{QUAL} \rrbracket \delta \wedge$

$(k, q, W, v) \in \mathcal{T} \llbracket \Delta \vdash \forall \alpha: \kappa . \tau: \mathrm{PRETYPE} \rrbracket \delta\}$

$\equiv\left(k, \mathcal{T} \llbracket \Delta \vdash \xi: \mathrm{QUAL} \rrbracket \delta, W_{\Gamma}, \Lambda . \gamma(e)\right)$

$\in\left\{(k, q, W, \Lambda . e) \mid W \in\right.$ WorldDesc $_{k} \wedge \mathcal{P}(k, q, W) \wedge$

$q=\mathcal{T} \llbracket \Delta \vdash \xi: \mathrm{QUAL} \rrbracket \delta \wedge$

$\forall \mathcal{I}$.

$\mathcal{I} \in \mathcal{K} \llbracket \kappa \rrbracket \Rightarrow$

$\forall i<k$.

$\left.\operatorname{Comp}\left(i,\lfloor W\rfloor_{i}, e, \mathcal{T} \llbracket \Delta, \alpha: \kappa \vdash \tau: \operatorname{TYPE} \rrbracket \delta[\alpha \mapsto \mathcal{I}]\right)\right\}$,

which follows from

- $\mathcal{T} \llbracket \Delta \vdash \xi: \mathrm{QUAL} \rrbracket \delta=\mathcal{T} \llbracket \Delta \vdash \xi: \mathrm{QUAL} \rrbracket \delta$, which follows trivially,

- $W_{\Gamma} \in$ WorldDesc $_{k}$, which follows from Lemma 9 applied to $\left(k, q_{\Gamma}, W_{\Gamma}, \gamma\right) \in \mathcal{G} \llbracket \Delta \vdash \Gamma \rrbracket \delta$,

- $\mathcal{P}\left(k, \mathcal{T} \llbracket \Delta \vdash \xi: \mathrm{QUAL} \rrbracket \delta, W_{\Gamma}\right)$, which follows from Lemma 18 applied to $\Delta \vdash \Gamma \preceq \xi$ and $\left(k, q_{\Gamma}, W_{\Gamma}, \gamma\right) \in \mathcal{G} \llbracket \Delta \vdash \Gamma \rrbracket \delta$ and $\mathcal{T} \llbracket \Delta \vdash \xi: \mathrm{QUAL} \rrbracket \delta$,

- $\forall \mathcal{I}$. ...

Consider arbitrary $\mathcal{I}$ and $i$ such that

- $\mathcal{I} \in \mathcal{K} \llbracket \kappa \rrbracket$, and 
- $i<k$.

We are required to show that $\operatorname{Comp}\left(i,\left\lfloor W_{\Gamma}\right\rfloor_{i}, \gamma(e), \mathcal{T} \llbracket \Delta, \alpha: \kappa \vdash \tau: \operatorname{TYPE} \rrbracket \delta[\alpha \mapsto \mathcal{I}]\right)$.

Applying the induction hypothesis to $\Delta, \alpha: \kappa ; \Gamma \vdash e: \tau$, we conclude that $\llbracket \Delta, \alpha: \kappa ; \Gamma \vdash e: \tau \rrbracket$.

Instantiate this with $i, \delta[\alpha \mapsto \mathcal{I}], q_{\Gamma},\left\lfloor W_{\Gamma}\right\rfloor_{i}$, and $\gamma$. Note that

- $i \geq 0$,

- $\delta[\alpha \mapsto \mathcal{I}] \in \mathcal{D} \llbracket \Delta, \alpha: \kappa \rrbracket$, which follows from

- $\delta \in \mathcal{D} \llbracket \Delta \rrbracket$, and

- $\mathcal{I} \in \mathcal{K} \llbracket \kappa \rrbracket$, and

- $\left(i, q_{\Gamma},\left\lfloor W_{\Gamma}\right\rfloor_{i}, \gamma\right) \in \mathcal{G} \llbracket \Delta, \alpha: \kappa \vdash \Gamma \rrbracket \delta[\alpha \mapsto \mathcal{I}]$, which follows from $\left(i, q_{\Gamma},\left\lfloor W_{\Gamma}\right\rfloor_{i}, \gamma\right) \in$ $\mathcal{G} \llbracket \Delta \vdash \Gamma \rrbracket \delta$, which follows from Lemma 9 applied to $i<k$ and $\left(k, q_{\Gamma}, W_{\Gamma}, \gamma\right) \in$ $\mathcal{G} \llbracket \Delta \vdash \Gamma \rrbracket \delta$.

Hence, $\operatorname{Comp}\left(i,\left\lfloor W_{\Gamma}\right\rfloor_{i}, \gamma(e), \mathcal{T} \llbracket \Delta, \alpha: \kappa \vdash \tau: \operatorname{TYPE} \rrbracket \delta[\alpha \mapsto \mathcal{I}]\right)$. 
(INST)

Case $\frac{\Delta ; \Gamma \vdash e_{1}:{ }^{\xi} \forall \alpha: \kappa . \tau \quad \Delta \vdash \iota: \kappa}{\Delta ; \Gamma \vdash e_{1}[]: \tau[\iota / \alpha]}:$

We are required to show $\llbracket \Delta ; \Gamma \vdash e_{1}[]: \tau[\iota / \alpha] \rrbracket$.

Consider arbitrary $k, \delta, q_{\Gamma}, W_{\Gamma}$, and $\gamma$ such that

- $k \geq 0$,

- $\delta \in \mathcal{D} \llbracket \Delta \rrbracket$, and

- $\left(k, q_{\Gamma}, W_{\Gamma}, \gamma\right) \in \mathcal{G} \llbracket \Delta \vdash \Gamma \rrbracket \delta$.

Let $e_{s}=\gamma\left(e_{1}[]\right) \equiv \gamma\left(e_{1}\right)[]$ and $W_{s}=W_{\Gamma}$.

We are required to show that $\operatorname{Comp}\left(k, W_{s}, e_{s}, \mathcal{T} \llbracket \Delta \vdash \tau[\iota / \alpha]: \operatorname{TYPE} \rrbracket \delta\right) \quad \equiv$ $\operatorname{Comp}\left(k, W_{\Gamma}, \gamma\left(e_{1}\right)[], \mathcal{T} \llbracket \Delta \vdash \tau[\iota / \alpha]:\right.$ TYPE $\left.\rrbracket \delta\right)$

Consider arbitrary $j, W_{r}, w_{s}, w_{f}$, and $e_{f}$ such that

- $j<k$,

- $w_{s}:_{k}\left(W_{s} \odot_{k} W_{r}\right) \equiv w_{s}:_{k}\left(W_{\Gamma} \odot_{k} W_{r}\right)$,

- $\left(w_{s}, e_{s}\right) \equiv\left(w_{s}, \gamma\left(e_{1}\right)[]\right) \longmapsto^{j}\left(w_{f}, e_{f}\right)$, and

- $\operatorname{irred}\left(w_{f}, e_{f}\right)$.

Hence, by inspection of the operational semantics, it follows that there exist $j_{1}, w_{f_{1}}$, and $e_{f_{1}}$ such that

- $\left(w_{s}, \gamma\left(e_{1}\right)\right) \longmapsto{ }^{j_{1}}\left(w_{f_{1}}, e_{f_{1}}\right)$,

- $\operatorname{irred}\left(w_{f_{1}}, e_{f_{1}}\right)$, and

- $j_{1} \leq j$.

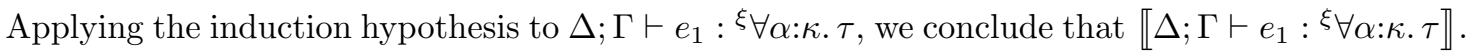
Instantiate this with $k, \delta, q_{\Gamma}, W_{\Gamma}$, and $\gamma$. Note that

- $k \geq 0$,

- $\delta \in \mathcal{D} \llbracket \Delta \rrbracket$, and

- $\left(k, q_{\Gamma}, W_{\Gamma}, \gamma\right) \in \mathcal{G} \llbracket \Delta \vdash \Gamma \rrbracket \delta$.

Hence, $\operatorname{Comp}\left(k, W_{\Gamma}, \gamma\left(e_{1}\right), \mathcal{T} \llbracket \Delta \vdash \xi_{\forall \alpha: \kappa . \tau}:\right.$ TYPE $\left.\rrbracket \delta\right)$.

Instantiate this with $j_{1}, W_{r}, w_{s}, w_{f_{1}}$, and $e_{f_{1}}$. Note that

- $j_{1}<k$, which follows from $j_{1} \leq j$ and $j<k$,

- $w_{s}:_{k}\left(W_{\Gamma} \odot_{k} W_{r}\right)$, which follows from above,

- $\left(w_{s}, \gamma\left(e_{1}\right)\right) \longmapsto{ }^{j_{1}}\left(w_{f_{1}}, e_{f_{1}}\right)$,

- $\operatorname{irred}\left(w_{f_{1}}, e_{f_{1}}\right)$.

Hence, there exists $W_{f_{1}}$ and $q_{f_{1}}$ such that

- $w_{f_{1}}:_{k-j_{1}}\left(W_{f_{1}} \odot_{k-j_{1}} W_{r}\right)$, and

- $\left(k-j_{1}, q_{f_{1}}, W_{f_{1}}, e_{f_{1}}\right)$

$\in \mathcal{T} \llbracket \Delta \vdash \xi_{\forall \alpha: \kappa . \tau}: \mathrm{TYPE} \rrbracket \delta$

$\equiv\left\{(k, q, W, \Lambda . e) \mid W \in\right.$ WorldDesc $_{k} \wedge \mathcal{P}(k, q, W) \wedge$

$q=\mathcal{T} \llbracket \Delta \vdash \xi: \mathrm{QUAL} \rrbracket \delta \wedge$

$\forall \mathcal{I}$.

$\mathcal{I} \in \mathcal{K} \llbracket \kappa \rrbracket \Rightarrow$

$\forall j<k$.

$\left.\operatorname{Comp}\left(i,\lfloor W\rfloor_{i}, e, \mathcal{T} \llbracket \Delta, \alpha: \kappa \vdash \tau: \mathrm{TYPE} \rrbracket \delta[\alpha \mapsto \mathcal{I}]\right)\right\}$. 
Hence, $e_{f_{1}} \equiv \Lambda . e_{f_{11}}$ and $q_{f_{1}}=\mathcal{T} \llbracket \Delta \vdash \xi: \mathrm{QUAL} \rrbracket \delta$.

Note that

$$
\begin{aligned}
\left(w_{s}, e_{s}\right) & \equiv\left(w_{s}, \gamma\left(e_{1}\right)[]\right) \\
& \longmapsto w^{j_{1}}\left(w_{f_{1}}, e_{f_{1}}[]\right) \\
& \equiv\left(w_{f_{1}},\left(\Lambda . e_{f_{11}}\right)[]\right) \\
& \longmapsto{ }^{1}\left(w_{f_{1}}, e_{f_{11}}\right) \\
& \longmapsto{ }^{j_{2}}\left(w_{f}, e_{f}\right)
\end{aligned}
$$

and $\operatorname{irred}\left(w_{f}, e_{f}\right)$, where $j=j_{1}+1+j_{2}$.

Note that $\left\lfloor\left(W_{f_{1}} \odot_{k-j_{1}} W_{r}\right)\right\rfloor_{k-j_{1}-1} \equiv\left(\left\lfloor W_{f_{1}}\right\rfloor_{k-j_{1}-1} \odot_{k-j_{1}-1} W_{r}\right)$, which follows from

$$
\begin{aligned}
& \left\lfloor\left(W_{f_{1}} \odot_{k-j_{1}} W_{r}\right)\right\rfloor_{k-j_{1}-1} \\
& \equiv\left(W_{f_{1}} \odot_{k-j_{1}-1} W_{r}\right) \\
& \quad \text { which follows from Req } 4 \text { (join-closed) } \\
& \equiv\left(\left\lfloor W_{f_{1}}\right\rfloor_{k-j_{1}-1} \odot_{k-j_{1}-1} W_{r}\right) \\
& \quad \text { which follows from Req } 5 \text { (join-aprx). }
\end{aligned}
$$

Instantiate $\left(k-j_{1}, q_{f_{1}}, W_{f_{1}}, \Lambda . e_{f_{11}}\right) \in \mathcal{T} \llbracket \Delta \vdash \xi_{\forall} \forall: \kappa . \tau:$ TYPE $\rrbracket \delta$ with $k-j_{1}-1$ and $\mathcal{T} \llbracket \Delta \vdash \iota: \kappa \rrbracket \delta$. Note that

- $\mathcal{T} \llbracket \Delta \vdash \iota: \kappa \rrbracket \delta \in \mathcal{K} \llbracket \kappa \rrbracket$, which follows from Lemma 8 applied to $\Delta \vdash \iota: \kappa$ and $\delta \in \mathcal{D} \llbracket \Delta \rrbracket$, and

- $k-j_{1}-1<k-j_{1}$.

Hence, $\operatorname{Comp}\left(k-j_{1}-1,\left\lfloor W_{f_{1}}\right\rfloor_{k-j_{1}-1}, e_{f_{11}}, \mathcal{T} \llbracket \Delta, \alpha: \kappa \vdash \tau:\right.$ TYPE $\left.\rrbracket \delta[\alpha \mapsto \mathcal{T} \llbracket \Delta \vdash \iota: \kappa \rrbracket \delta]\right)$.

Instantiate this with $j_{2}, W_{r}, w_{f_{1}}, w_{f}$, and $e_{f}$. Note that

- $k-j_{1}-1<j_{2}$, which follows from $j_{2}=j-j_{1}-1$ and $j<k$,

- $w_{f_{1}}: k-j_{1}-1\left(\left\lfloor W_{f_{1}}\right\rfloor_{k-j_{1}-1} \odot_{k-j_{1}-1} W_{r}\right)$, which follows from

$$
\begin{aligned}
& w_{f_{1}}: k-j_{1}\left(W_{f_{1}} \odot_{k-j_{1}} W_{r}\right) \\
& \text { which follows from above } \\
& \Rightarrow w_{f_{1}}: k-j_{1}-1\left(W_{f_{1}} \odot_{k-j_{1}} W_{r}\right) \\
& \quad \text { which follows from Req } 2 \text { (models-closed) } \\
& \Leftrightarrow w_{f_{1}}: k-j_{1}-1\left\lfloor\left(W_{f_{1}} \odot_{k-j_{1}} W_{r}\right)\right\rfloor_{k-j_{1}-1} \\
& \quad \text { which follows from Req } 3 \text { (models-aprx) }
\end{aligned}
$$

$$
\left\lfloor\left(W_{f_{1}} \odot_{k-j_{1}} W_{r}\right)\right\rfloor_{k-j_{1}-1} \equiv\left(\left\lfloor W_{f_{1}}\right\rfloor_{k-j_{1}-1} \odot_{k-j_{1}-1} W_{r}\right)
$$

$$
\text { which follows from above, }
$$

- $\left(w_{f_{1}}, e_{f_{11}}\right) \longmapsto^{j_{2}}\left(w_{f}, e_{f}\right)$, and

- $\operatorname{irred}\left(w_{f}, e_{f}\right)$.

Hence, there exists $W_{f^{\prime}}$ and $q_{f^{\prime}}$ such that

- $w_{f}:_{k-j_{1}-1-j_{2}}\left(W_{f^{\prime}} \odot_{k-j_{1}-1-j_{2}} W_{r}\right)$, and

- $\left(k-j_{1}-1-j_{2}, q_{f^{\prime}}, W_{f^{\prime}}, e_{f}\right) \in \mathcal{T} \llbracket \Delta, \alpha: \kappa \vdash \tau: \operatorname{TYPE} \rrbracket \delta[\alpha \mapsto \mathcal{T} \llbracket \Delta \vdash \iota: \kappa \rrbracket \delta]$.

Let $W_{f}=W_{f^{\prime}}$ and $q_{f}=q_{f^{\prime}}$.

We are required to show that

- $w_{f}:_{k-j}\left(W_{f} \odot_{k-j} W_{r}\right)$

$\equiv w_{f}: k-j_{1}-1-j_{2}\left(W_{f^{\prime}} \odot_{k-j_{1}-1-j_{2}} W_{r}\right)$,

which follows from above,

- $\left(k-j, q_{f}, W_{f}, e_{f}\right) \in \mathcal{T} \llbracket \Delta \vdash \tau[\iota / \alpha]:$ TYPE $\rrbracket \delta$

$\equiv\left(k-j_{1}-1-j_{2}, q_{f^{\prime}}, W_{f^{\prime}}, e_{f}\right) \in \mathcal{T} \llbracket \Delta \vdash \tau[\iota / \alpha]:$ TYPE $\delta$,

which follows from $\left(k-j_{1}-1-j_{2}, q_{f^{\prime}}, W_{f^{\prime}}, e_{f}\right) \in \mathcal{T} \llbracket \Delta, \alpha: \kappa \vdash \tau:$ TYPE $\delta[\alpha \mapsto \mathcal{T} \llbracket \Delta \vdash \iota: \kappa \rrbracket \delta]$ and $\mathcal{T} \llbracket \Delta, \alpha: \kappa \vdash \tau:$ TYPE $\rrbracket \delta[\alpha \mapsto \mathcal{T} \llbracket \Delta \vdash \iota: \kappa \rrbracket \delta]=\mathcal{T} \llbracket \Delta \vdash \tau[\iota / \alpha]:$ TYPE $\rrbracket \delta$, which in turn follows from Lemma 10 applied to $\Delta \vdash \iota: \kappa$ and $\delta \in \mathcal{D} \llbracket \Delta \rrbracket$. 
(PACK)

Case $\frac{\Delta \vdash \xi: \text { QUAL } \quad \Delta \vdash \iota: \kappa \quad \Delta ; \Gamma \vdash v_{1}: \tau[\iota / \alpha] \quad \Delta \vdash \tau[\iota / \alpha] \preceq \xi}{\Delta ; \Gamma \vdash\left\ulcorner v_{1}\right\urcorner:{ }^{\xi} \exists \alpha: \kappa . \tau}:$

We are required to show $\llbracket \Delta ; \Gamma \vdash\left\ulcorner v_{1}\right\urcorner: \xi_{\exists} \exists: \kappa . \tau \rrbracket$.

Consider arbitrary $k, \delta, q_{\Gamma}, W_{\Gamma}$, and $\gamma$ such that

- $k \geq 0$,

- $\delta \in \mathcal{D} \llbracket \Delta \rrbracket$, and

- $\left(k, q_{\Gamma}, W_{\Gamma}, \gamma\right) \in \mathcal{G} \llbracket \Delta \vdash \Gamma \rrbracket \delta$.

Let $e_{s}=\gamma\left(\left\ulcorner v_{1}\right\urcorner\right) \equiv\left\ulcorner\gamma\left(v_{1}\right)\right\urcorner$ and $W_{s}=W_{\Gamma}$.

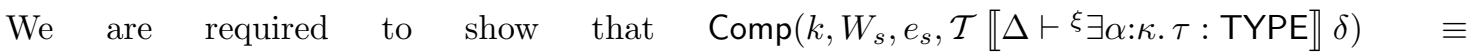
$\operatorname{Comp}\left(k, W_{\Gamma},\left\ulcorner\gamma\left(v_{1}\right)\right\urcorner, \mathcal{T} \llbracket \Delta \vdash \xi \exists \alpha: \kappa . \tau: \mathrm{TYPE} \rrbracket \delta\right)$.

Consider arbitrary $j, W_{r}, w_{s}, w_{f}$, and $e_{f}$ such that

- $j<k$,

- $w_{s}:_{k}\left(W_{s} \odot_{k} W_{r}\right) \equiv w_{s}:_{k}\left(W_{\Gamma} \odot_{k} W_{r}\right)$,

- $\left(w_{s}, e_{s}\right) \equiv\left(w_{s},\left\ulcorner\gamma\left(v_{1}\right)\right\urcorner\right) \longmapsto{ }^{j}\left(w_{f}, e_{f}\right)$, and

- $\operatorname{irred}\left(w_{f}, e_{f}\right)$.

Since $\left\ulcorner\gamma\left(v_{1}\right)\right\urcorner$ is a value, we have $\operatorname{irred}\left(w_{s},\left\ulcorner\gamma\left(v_{1}\right)\right\urcorner\right)$.

Hence, $j=0$ and $w_{f} \equiv w_{s}$ and $e_{f} \equiv\left\ulcorner\gamma\left(v_{1}\right)\right\urcorner$.

Applying the induction hypothesis to $\Delta ; \Gamma \vdash v_{1}: \tau[\iota / \alpha]$, we conclude that $\llbracket \Delta ; \Gamma \vdash v_{1}: \tau[\iota / \alpha] \rrbracket$. Instantiate this with $k, \delta, q_{\Gamma}, W_{\Gamma}$, and $\gamma$. Note that

- $k \geq 0$,

- $\delta \in \mathcal{D} \llbracket \Delta \rrbracket$, and

- $\left(k, q_{\Gamma}, W_{\Gamma}, \gamma\right) \in \mathcal{G} \llbracket \Delta \vdash \Gamma \rrbracket \delta$.

Hence, $\operatorname{Comp}\left(k, W_{\Gamma}, \gamma\left(v_{1}\right), \mathcal{T} \llbracket \Delta \vdash \tau[\iota / \alpha]:\right.$ TYPE $\left.\rrbracket \delta\right)$.

Instantiate this with $0, W_{r}, w_{s}, w_{s}$, and $\gamma\left(v_{1}\right)$. Note that

- $0<k$, which follows from $j=0$ and $j<k$,

- $w_{s}:_{k}\left(W_{\Gamma} \odot_{k} W_{r}\right)$, which follows from above,

- $\left(w_{s}, \gamma\left(v_{1}\right)\right) \longmapsto^{0}\left(w_{s}, \gamma\left(v_{1}\right)\right)$, and

- $\operatorname{irred}\left(w_{s}, \gamma\left(v_{1}\right)\right)$, which follows from the fact that $\gamma\left(v_{1}\right)$ is a value.

Hence, there exists $W_{f_{1}}$ and $q_{f_{1}}$ such that

- $w_{s}:_{k-0}\left(W_{f_{1}} \odot_{k-0} W_{r}\right)$, and

- $\left(k-0, q_{f_{1}}, W_{f_{1}}, \gamma\left(v_{1}\right)\right) \in \mathcal{T} \llbracket \Delta \vdash \tau[\iota / \alpha]:$ TYPE $\rrbracket$.

Let $W_{f}=W_{f_{1}}$ and $q_{f}=\mathcal{T} \llbracket \Delta \vdash \xi:$ QUAL $\rrbracket \delta$.

We are required to show that

- $w_{f}:_{k-0}\left(W_{f} \odot_{k-0} W_{r}\right)$

$\equiv w_{s}: k\left(W_{f_{1}} \odot_{k} W_{r}\right)$

which follows from above, and 
- $\left(k-0, q_{f}, W_{f}, e_{f}\right) \in \mathcal{T} \llbracket \Delta \vdash \xi \exists \alpha: \kappa . \tau:$ TYPE $\rrbracket \delta$

$\equiv\left(k, \mathcal{T} \llbracket \Delta \vdash \xi:\right.$ QUAL $\left.\rrbracket \delta, W_{f_{1}},\left\ulcorner\gamma\left(v_{1}\right)\right\urcorner\right)$

$\in\{(k, q, W, v) \mid$

$q=\mathcal{T} \llbracket \Delta \vdash \xi: \mathrm{QUAL} \rrbracket \delta \wedge$

$(k, q, W, v) \in \mathcal{T} \llbracket \Delta \vdash \exists \alpha: \kappa . \tau: \mathrm{PRETYPE} \rrbracket \delta\}$

$\equiv\left(k, \mathcal{T} \llbracket \Delta \vdash \xi: \mathrm{QUAL} \rrbracket \delta, W_{f_{1}},\left\ulcorner\gamma\left(v_{1}\right)\right\urcorner\right)$

$\in\left\{(k, q, W,\ulcorner v\urcorner) \mid W \in\right.$ WorldDesc $_{k} \wedge \mathcal{P}(k, q, W) \wedge$

$q=\mathcal{T} \llbracket \Delta \vdash \xi: \mathrm{QUAL} \rrbracket \delta \wedge$

$\exists \mathcal{I}, q^{\prime}$.

$\mathcal{I} \in \mathcal{K} \llbracket \kappa \rrbracket \wedge$

$q^{\prime} \preceq q \wedge$

$\forall i<k$.

which follows from

$\left.\left(i, q^{\prime},\lfloor W\rfloor_{i}, v\right) \in \mathcal{T} \llbracket \Delta, \alpha: \kappa \vdash \tau: \operatorname{TYPE} \rrbracket \delta[\alpha \mapsto \mathcal{I}]\right\}$,

- $\mathcal{T} \llbracket \Delta \vdash \xi: \mathrm{QUAL} \rrbracket \delta=\mathcal{T} \llbracket \Delta \vdash \xi: \mathrm{QUAL} \rrbracket \delta$, which follows trivially,

- $W_{f_{1}} \in$ WorldDesc $_{k}$, which follows from Fact 6 to $\left(k, q_{f_{1}}, W_{f_{1}}, \gamma\left(v_{1}\right)\right) \in$ $\mathcal{T} \llbracket \Delta \vdash \tau[\iota / \alpha]:$ TYPE $\rrbracket \delta \in$ Type,

- $\mathcal{P}\left(k, \mathcal{T} \llbracket \Delta \vdash \xi: \mathrm{QUAL} \rrbracket \delta, W_{f_{1}}\right)$, which follows from Corollary 16 applied to $\Delta \vdash \tau[\iota / \alpha] \preceq$ $\xi$ and $\left(k, q_{f_{1}}, W_{f_{1}}, \gamma\left(v_{1}\right)\right) \in \mathcal{T} \llbracket \Delta \vdash \tau[\iota / \alpha]:$ TYPE $\rrbracket \delta$ and $\mathcal{T} \llbracket \Delta \vdash \xi:$ QUAL $\rrbracket \delta$, and

- $\exists \mathcal{I}, q^{\prime}$. . .

Take $q^{\prime}=q_{f_{1}}$ and $\mathcal{I}=\mathcal{T} \llbracket \Delta \vdash \iota: \kappa \rrbracket \delta$. Note that

- $\mathcal{T} \llbracket \Delta \vdash \iota: \kappa \rrbracket \delta \in \mathcal{K} \llbracket \kappa \rrbracket$, which follows from Lemma 8 applied to $\delta \in \mathcal{D} \llbracket \Delta \rrbracket$ and $\Delta \vdash \iota: \kappa$,

- $q_{f_{1}} \preceq \mathcal{T} \llbracket \Delta \vdash \xi:$ QUAL $\rrbracket \delta$, which follows from Lemma 15 applied to $\Delta \vdash \tau[\iota / \alpha] \preceq \xi$ and $\left(k, q_{f_{1}}, W_{f_{1}}, \gamma\left(v_{1}\right)\right) \in \mathcal{T} \llbracket \Delta \vdash \tau[\iota / \alpha]:$ TYPE $\rrbracket \delta$ and $\mathcal{T} \llbracket \Delta \vdash \xi:$ QUAL $\rrbracket \delta$, and

- $\forall i<k$. ...

Consider arbitrary $i$ such that

- $i<k$.

We are required to show that $\left(i, q_{f_{1}},\left\lfloor W_{f_{1}}\right\rfloor_{i}, \gamma\left(v_{1}\right)\right) \in \mathcal{T} \llbracket \Delta, \alpha: \kappa \vdash \tau:$ TYPE $\rrbracket \delta[\alpha \mapsto$ $\mathcal{T} \llbracket \Delta \vdash \iota: \kappa \rrbracket \delta]$.

Applying Lemma 8 to $\Delta \vdash \tau[\iota / \alpha]$ : TYPE, we conclude that $\mathcal{T} \llbracket \Delta \vdash \tau[\iota / \alpha]:$ TYPE $\rrbracket \delta \in$ Type.

Applying Fact 6 to $\left(k, q_{f_{1}}, W_{f_{1}}, \gamma\left(v_{1}\right)\right) \in \mathcal{T} \llbracket \Delta \vdash \tau[\iota / \alpha]:$ TYPE $\rrbracket \delta \in$ Type instantiated with $i$, noting that

- $i \leq k$, which follows from $i<k$,

we conclude that $\left(i, q_{f_{1}},\left\lfloor W_{f_{1}}\right\rfloor_{i}, \gamma\left(v_{1}\right)\right) \in \mathcal{T} \llbracket \Delta \vdash \tau[\iota / \alpha]:$ TYPE $\rrbracket \delta$.

Applying Lemma 10 to $\Delta \vdash \iota: \kappa$ and $\delta \in \mathcal{D} \llbracket \Delta \rrbracket$, we conclude that $\mathcal{T} \llbracket \Delta, \alpha: \kappa \vdash \tau:$ TYPE $\rrbracket \delta[\alpha \mapsto \mathcal{T} \llbracket \Delta \vdash \iota: \kappa \rrbracket \delta]=\mathcal{T} \llbracket \Delta \vdash \tau[\iota / \alpha]:$ TYPE $\rrbracket \delta$.

Hence, we conclude that $\left(i, q_{f_{1}},\left\lfloor W_{f_{1}}\right\rfloor_{i}, \gamma\left(v_{1}\right)\right) \in \mathcal{T} \llbracket \Delta, \alpha: \kappa \vdash \tau:$ TYPE $\rrbracket \delta[\alpha \mapsto$ $\mathcal{T} \llbracket \Delta \vdash \iota: \kappa \rrbracket \delta]$.

(LET-PACK)

Case $\frac{\Delta \vdash \Gamma \sim \Gamma_{1} \boxplus \Gamma_{2} \quad \Delta ; \Gamma_{1} \vdash e_{1}:{ }^{\xi} \exists \alpha: \kappa \cdot \tau_{1} \quad \Delta \vdash \Gamma_{2} \quad \Delta \vdash \tau_{2}: \text { TYPE } \quad \Delta, \alpha: \kappa ; \Gamma_{2}, x: \tau_{1} \vdash e_{2}: \tau_{2}}{\Delta ; \Gamma \vdash \text { let }\ulcorner x\urcorner=e_{1} \text { in } e_{2}: \tau_{2}}$ :

We are required to show $\llbracket \Delta ; \Gamma \vdash$ let $\ulcorner x\urcorner=e_{1}$ in $e_{2}: \tau_{2} \rrbracket$.

Consider arbitrary $k, \delta, q_{\Gamma}, W_{\Gamma}$, and $\gamma$ such that 
- $k \geq 0$,

- $\delta \in \mathcal{D} \llbracket \Delta \rrbracket$, and

- $\left(k, q_{\Gamma}, W_{\Gamma}, \gamma\right) \in \mathcal{G} \llbracket \Delta \vdash \Gamma \rrbracket \delta$.

Applying Lemma 20 to $\left(k, q_{\Gamma}, W_{\Gamma}, \gamma\right) \in \mathcal{G} \llbracket \Delta \vdash \Gamma \rrbracket \delta$ and $\Delta \vdash \Gamma \leadsto \Gamma_{1} \boxplus \Gamma_{2}$, we conclude that there exist $q_{\Gamma_{1}}, W_{\Gamma_{1}}, \gamma_{1}, q_{\Gamma_{2}}, W_{\Gamma_{2}}$, and $\gamma_{2}$, such that

- $\left(k, q_{\Gamma_{1}}, W_{\Gamma_{1}}, \gamma_{1}\right) \in \mathcal{G} \llbracket \Delta \vdash \Gamma_{1} \rrbracket \delta$,

- $\left(k, q_{\Gamma_{2}}, W_{\Gamma_{2}}, \gamma_{2}\right) \in \mathcal{G} \llbracket \Delta \vdash \Gamma_{2} \rrbracket \delta$,

- $\gamma \in \gamma_{1} \boxplus \gamma_{2}$,

- $q_{\Gamma_{1}} \preceq q_{\Gamma}$,

- $q_{\Gamma_{2}} \preceq q_{\Gamma}$, and

- $\left(W_{\Gamma_{1}} \odot_{k} W_{\Gamma_{2}}=W_{\Gamma}\right)$.

Note that $\gamma\left(e_{1}\right) \equiv \gamma_{1}\left(e_{1}\right)$ and $\gamma\left(e_{2}\right) \equiv \gamma_{2}\left(e_{2}\right)$.

Let $e_{s}=\gamma\left(\right.$ let $\ulcorner x\urcorner=e_{1}$ in $\left.e_{2}\right) \equiv$ let $\ulcorner x\urcorner=\gamma\left(e_{1}\right)$ in $\gamma\left(e_{2}\right) \equiv$ let $\ulcorner x\urcorner=\gamma_{1}\left(e_{1}\right)$ in $\gamma_{2}\left(e_{2}\right)$ and $W_{s}=W_{\Gamma}$.

We are required to show that $\operatorname{Comp}\left(k, W_{s}, e_{s}, \mathcal{T} \llbracket \Delta \vdash \tau_{2}: \operatorname{TYPE} \rrbracket \delta\right) \equiv \operatorname{Comp}\left(k, W_{\Gamma}\right.$, let $\ulcorner x\urcorner=$ $\gamma_{1}\left(e_{1}\right)$ in $\gamma_{2}\left(e_{2}\right), \mathcal{T} \llbracket \Delta \vdash \tau_{2}:$ TYPE $\left.\rrbracket \delta\right)$.

Consider arbitrary $j, W_{r}, w_{s}, w_{f}$, and $e_{f}$ such that

- $j<k$,

- $w_{s}:_{k}\left(W_{s} \odot_{k} W_{r}\right) \equiv w_{s}:_{k}\left(W_{\Gamma} \odot_{k} W_{r}\right)$, noting that

$$
\begin{aligned}
& w_{s}:_{k}\left(W_{\Gamma} \odot_{k} W_{r}\right) \\
& \equiv w_{s}: k\left(\left(W_{\Gamma_{1}} \odot_{k} W_{\Gamma_{2}}\right) \odot_{k} W_{r}\right) \\
& \text { which follows from above, }
\end{aligned}
$$

- $\left(w_{s}, e_{s}\right) \equiv\left(w_{s}\right.$, let $\ulcorner x\urcorner=\gamma_{1}\left(e_{1}\right)$ in $\left.\gamma_{2}\left(e_{2}\right)\right) \longmapsto^{j}\left(w_{f}, e_{f}\right)$, and

- $\operatorname{irred}\left(w_{f}, e_{f}\right)$.

Hence, by inspection of the operational semantics, it follows that there exist $j_{1}, w_{f_{1}}$, and $e_{f_{1}}$ such that

- $\left(w_{s}, \gamma_{1}\left(e_{1}\right)\right) \longmapsto^{j_{1}}\left(w_{f_{1}}, e_{f_{1}}\right)$,

- $\operatorname{irred}\left(w_{f_{1}}, e_{f_{1}}\right)$, and

- $j_{1} \leq j$.

Note that $\left(\left(W_{\Gamma_{1}} \odot_{k} W_{\Gamma_{2}}\right) \odot_{k} W_{r}\right) \equiv\left(W_{\Gamma_{1}} \odot_{k}\left(W_{\Gamma_{2}} \odot_{k} W_{r}\right)\right)$, which follows from

$$
\begin{aligned}
& \left(\left(W_{\Gamma_{1}} \odot_{k} W_{\Gamma_{2}}\right) \odot_{k} W_{r}\right) \\
& \equiv\left(W_{\Gamma_{1}} \odot_{k}\left(W_{\Gamma_{2}} \odot_{k} W_{r}\right)\right)
\end{aligned}
$$

which follows from Reqs 6,7 , and 8 (join-commut, join-assocl, and join-assocr).

Applying the induction hypothesis to $\Delta ; \Gamma_{1} \vdash e_{1}: \xi_{\exists} \exists: \kappa . \tau_{1}$, we conclude that $\llbracket \Delta ; \Gamma_{1} \vdash e_{1}: \xi \exists \alpha: \kappa . \tau_{1} \rrbracket$.

Instantiate this with $k, \delta, q_{\Gamma_{1}}, W_{\Gamma_{1}}$, and $\gamma_{1}$. Note that

- $k \geq 0$,

- $\delta \in \mathcal{D} \llbracket \Delta \rrbracket$, and

- $\left(k, q_{\Gamma_{1}}, W_{\Gamma_{1}}, \gamma_{1}\right) \in \mathcal{G} \llbracket \Delta \vdash \Gamma_{1} \rrbracket \delta$.

Hence, $\operatorname{Comp}\left(k, W_{\Gamma_{1}}, \gamma_{1}\left(e_{1}\right), \mathcal{T} \llbracket \Delta \vdash^{\xi} \exists \alpha: \kappa . \tau_{1}:\right.$ TYPE $\left.\rrbracket \delta\right)$.

Instantiate this with $j_{1},\left(W_{\Gamma_{2}} \odot_{k} W_{r}\right), w_{s}, w_{f_{1}}$, and $e_{f_{1}}$. Note that 
- $j_{1}<k$, which follows from $j_{1} \leq j$ and $j<k$,

- $w_{s}:_{k}\left(W_{\Gamma_{1}} \odot_{k}\left(W_{\Gamma_{2}} \odot_{k} W_{r}\right)\right)$, which follows from $w_{s}:_{k}\left(\left(W_{\Gamma_{1}} \odot_{k} W_{\Gamma_{2}}\right) \odot_{k} W_{r}\right)$ which follows from above

$\left(\left(W_{\Gamma_{1}} \odot_{k} W_{\Gamma_{2}}\right) \odot_{k} W_{r}\right) \equiv\left(W_{\Gamma_{1}} \odot_{k}\left(W_{\Gamma_{2}} \odot_{k} W_{r}\right)\right)$ which follows from above,

- $\left(w_{s}, \gamma_{1}\left(e_{1}\right)\right) \longmapsto^{j_{1}}\left(w_{f_{1}}, e_{f_{1}}\right)$,

- $\operatorname{irred}\left(w_{f_{1}}, e_{f_{1}}\right)$.

Hence, there exists $W_{f_{1}}$ and $q_{f_{1}}$ such that

- $w_{f_{1}}: k-j_{1}\left(W_{f_{1}} \odot_{k-j_{1}}\left(W_{\Gamma_{2}} \odot_{k} W_{r}\right)\right)$, and

- $\left(k-j_{1}, q_{f_{1}}, W_{f_{1}}, e_{f_{1}}\right)$ $\in \mathcal{T} \llbracket \Delta \vdash \xi \exists \alpha: \kappa . \tau_{1}:$ TYPE $\rrbracket \delta$ $\equiv\left\{(k, q, W,\ulcorner v\urcorner) \mid W \in\right.$ WorldDesc $_{k} \wedge \mathcal{P}(k, q, W) \wedge$ $q=\mathcal{T} \llbracket \Delta \vdash \xi: \mathrm{QUAL} \rrbracket \delta \wedge$ $\exists \mathcal{I}, q^{\prime}$.

$\mathcal{I} \in \mathcal{K} \llbracket \kappa \rrbracket \wedge$

$q^{\prime} \preceq q \wedge$

$\forall i<k$.

$$
\left.\left(i, q^{\prime},\lfloor W\rfloor_{i}, v\right) \in \mathcal{T} \llbracket \Delta, \alpha: \kappa \vdash \tau: \operatorname{TYPE} \rrbracket \delta[\alpha \mapsto \mathcal{I}]\right\} .
$$

Hence, $e_{f_{1}} \equiv\left\ulcorner v_{f_{11}}\right\urcorner$ and $q_{f_{1}}=\mathcal{T} \llbracket \Delta \vdash \xi:$ QUAL $\rrbracket \delta$ and there exists $\mathcal{I}_{11}$ and $q_{11}^{\prime}$ such that

- $\mathcal{I}_{11} \in \mathcal{K} \llbracket \kappa \rrbracket$,

- $q_{11}^{\prime} \preceq q_{f_{1}}$, and

- $\forall i<k-j_{1} .\left(i, q_{11}^{\prime},\left\lfloor W_{f_{1}}\right\rfloor_{i}, v_{f_{11}}\right) \in \mathcal{T} \llbracket \Delta, \alpha: \kappa \vdash \tau_{1}: \operatorname{TYPE} \rrbracket \delta\left[\alpha \mapsto \mathcal{I}_{11}\right]$.

Note that

$$
\begin{aligned}
\left(w_{s}, e_{s}\right) & \equiv\left(w_{s}, \text { let }\ulcorner x\urcorner=\gamma_{1}\left(e_{1}\right) \text { in } \gamma_{2}\left(e_{2}\right)\right) \\
& \longmapsto w^{j_{1}}\left(w_{f_{1}}, \text { let }\ulcorner x\urcorner=e_{f_{1}} \text { in } \gamma_{2}\left(e_{2}\right)\right) \\
& \equiv\left(w_{f_{1}}, \text { let }\ulcorner x\urcorner=\left\ulcorner v_{f_{11}}\right\urcorner \text { in } \gamma_{2}\left(e_{2}\right)\right) \\
& \longmapsto{ }^{1}\left(w_{f_{1}}, \gamma_{2}\left(e_{2}\right)\left[v_{f_{11}} / x\right]\right) \\
& \longmapsto{ }^{j_{2}}\left(w_{f}, e_{f}\right)
\end{aligned}
$$

and $\operatorname{irred}\left(w_{f}, e_{f}\right)$, where $j=j_{1}+1+j_{2}$.

Note that $\left\lfloor\left(W_{f_{1}} \odot_{k-j_{1}}\left(W_{\Gamma_{2}} \odot_{k} W_{r}\right)\right)\right\rfloor_{k-j_{1}-1} \equiv\left(\left(W_{\Gamma_{2}} \odot_{k-j_{1}-1} W_{f_{1}}\right) \odot_{k-j_{1}-1} W_{r}\right)$, which follows from

$$
\begin{aligned}
& \left\lfloor\left(W_{f_{1}} \odot_{k-j_{1}}\left(W_{\Gamma_{2}} \odot_{k} W_{r}\right)\right)\right\rfloor_{k-j_{1}-1} \\
& \equiv\left(W_{f_{1}} \odot_{k-j_{1}-1}\left(W_{\Gamma_{2}} \odot_{k} W_{r}\right)\right) \\
& \quad \text { which follows from Req } 4 \text { (join-closed }) \\
& \equiv\left(\left(W_{\Gamma_{2}} \odot_{k-j_{1}-1} W_{f_{1}}\right) \odot_{k-j_{1}-1} W_{r}\right)
\end{aligned}
$$

which follows from Reqs 4, 5, 6, 7, and 8 (join-closed, join-aprx, join-commut, join-assocl, and join-assocr).

Applying the induction hypothesis to $\Delta, \alpha: \kappa ; \Gamma_{2}, x: \tau_{1} \vdash e_{2}: \tau_{2}$, we conclude that $\llbracket \Delta, \alpha: \kappa ; \Gamma_{2}, x: \tau_{1} \vdash e_{2}: \tau_{2} \rrbracket$.

Instantiate this with $k-j_{1}-1, \delta\left[\alpha \mapsto \mathcal{I}_{11}\right],\left(q_{\Gamma_{2}} \sqcap q_{11}^{\prime}\right),\left(W_{\Gamma_{2}} \odot_{k-j_{1}-1} W_{f_{1}}\right)$, and $\gamma_{2}\left[x \mapsto v_{f_{11}}\right]$. Note that

- $k-j_{1}-1 \geq 0$, which follows from $j_{1}+1+j_{2}=j$ and $j<k$,

- $\delta\left[\alpha \mapsto \mathcal{I}_{11}\right] \in \mathcal{D} \llbracket \Delta, \alpha: \kappa \rrbracket$, which follows from

- $\delta \in \mathcal{D} \llbracket \Delta \rrbracket$, and 
- $\mathcal{I}_{11} \in \mathcal{K} \llbracket \kappa \rrbracket$, and

- $\left(k-j_{1}-1,\left(q_{\Gamma_{2}} \sqcap q_{11}^{\prime}\right),\left(W_{\Gamma_{2}} \odot_{k-j_{1}-1} W_{f_{1}}\right), \gamma_{2}\left[x \mapsto v_{f_{11}}\right]\right) \in \mathcal{G} \llbracket \Delta, \alpha: \kappa \vdash \Gamma_{2} \rrbracket \delta\left[\alpha \mapsto \mathcal{I}^{\prime}\right]$, which follows from

- $\left(k-j_{1}-1, q_{\Gamma_{2}},\left\lfloor W_{\Gamma_{2}}\right\rfloor_{k-j_{1}-1}, \gamma_{2}\right) \in \mathcal{G} \llbracket \Delta, \alpha: \kappa \vdash \Gamma_{2}, x: \tau_{1} \rrbracket \delta\left[\alpha \mapsto \mathcal{I}_{11}\right]$, which follows from $\left(k-j_{1}-1, q_{\Gamma_{2}},\left\lfloor W_{\Gamma_{2}}\right\rfloor_{k-j_{1}-1}, \gamma_{2}\right) \in \mathcal{G} \llbracket \Delta \vdash \Gamma_{2} \rrbracket \delta$, which in turn follows from Lemma 9 applied to $k-j_{1}-1 \leq k$ and $\left(k, q_{\Gamma_{2}}, W_{\Gamma_{2}}, \gamma_{2}\right) \in \mathcal{G} \llbracket \Delta \vdash \Gamma_{2} \rrbracket \delta$,

- $\left(k-j_{1}-1, q_{11}^{\prime},\left\lfloor W_{f_{1}}\right\rfloor_{k-j_{1}-1}, v_{f_{11}}\right) \in \mathcal{T} \llbracket \Delta, \alpha: \kappa \vdash \tau_{1}: \operatorname{TYPE} \rrbracket \delta\left[\alpha \mapsto \mathcal{I}_{11}\right]$, which follows from $k-j_{1}-1<k-j_{1}$ and $\forall i<k-j_{1} .\left(i, q_{11}^{\prime},\left\lfloor W_{f_{1}}\right\rfloor_{i}, v_{f_{11}}\right) \in$ $\mathcal{T} \llbracket \Delta, \alpha: \kappa \vdash \tau_{1}:$ TYPE $\rrbracket \delta\left[\alpha \mapsto \mathcal{I}_{11}\right]$,

- $q_{\Gamma_{2}} \preceq\left(q_{\Gamma_{2}} \sqcap q_{11}^{\prime}\right)$, which follows from the definition of $\sqcap$,

- $q_{11}^{\prime} \preceq\left(q_{\Gamma_{2}} \sqcap q_{11}^{\prime}\right)$, which follows from the definition of $\sqcap$, and

- $\left(W_{\Gamma_{2}} \odot_{k-j_{1}-1} W_{f_{1}}\right)=\left(\left\lfloor W_{\Gamma_{2}}\right\rfloor_{k-j_{1}-1} \odot_{k-j_{1}-1}\left\lfloor W_{f_{1}}\right\rfloor_{k-j_{1}-1}\right)$, which follows from

$$
\begin{aligned}
& \left(W_{\Gamma_{2}} \odot_{k-j_{1}-1} W_{f_{1}}\right) \\
& \equiv\left(\left\lfloor W_{\Gamma_{2}}\right\rfloor_{k-j_{1}-1} \odot_{k-j_{1}-1}\left\lfloor W_{f_{1}}\right\rfloor_{k-j_{1}-1}\right) \\
& \quad \text { which follows from Req } 5 \text { (join-aprx). }
\end{aligned}
$$

Hence, $\operatorname{Comp}\left(k-j_{1}-1,\left(W_{\Gamma_{2}} \odot_{k-j_{1}-1} W_{f_{1}}\right), \gamma_{2}\left[x \mapsto v_{f_{11}}\right], \mathcal{T} \llbracket \Delta, \alpha: \kappa \vdash \tau_{2}: \operatorname{TYPE} \rrbracket \delta\left[\alpha \mapsto \mathcal{I}_{11}\right]\right)$.

Instantiate this with $j_{2}, w_{f_{1}}, W_{r}, w_{f}$, and $e_{f}$. Note that

- $j_{2}<k-j_{1}-1$, which follows from $j_{2}=j-j_{1}-1$ and $j<k$,

- $w_{f_{1}}: k-j_{1}-1\left(\left(W_{\Gamma_{2}} \odot_{k-j_{1}-1} W_{f_{1}}\right) \odot_{k-j_{1}-1} W_{r}\right)$, which follows from

$w_{f_{1}}: k-j_{1}\left(W_{f_{1}} \odot_{k-j_{1}}\left(W_{\Gamma_{2}} \odot_{k} W_{r}\right)\right)$

which follows from above

$\Rightarrow w_{f_{1}}: k-j_{1}-1\left(W_{f_{1}} \odot_{k-j_{1}}\left(W_{\Gamma_{2}} \odot_{k} W_{r}\right)\right)$

which follows from Req 2 (models-closed)

$\Leftrightarrow w_{f_{1}: k-j_{1}-1}\left\lfloor\left(W_{f_{1}} \odot_{k-j_{1}}\left(W_{\Gamma_{2}} \odot_{k} W_{r}\right)\right)\right\rfloor_{k-j_{1}-1}$

which follows from Req 3 (models-aprx)

$\left\lfloor\left(W_{f_{1}} \odot_{k-j_{1}}\left(W_{\Gamma_{2}} \odot_{k} W_{r}\right)\right)\right\rfloor_{k-j_{1}-1} \equiv\left(\left(W_{\Gamma_{2}} \odot_{k-j_{1}-1} W_{f_{1}}\right) \odot_{k-j_{1}-1} W_{r}\right)$

which follows from above,

- $\left(w_{f_{1}}, \gamma_{2}\left[x \mapsto v_{f_{11}}\right]\left(e_{2}\right)\right) \equiv\left(w_{f_{1}}, \gamma_{2}\left(e_{2}\right)\left[v_{f_{11}} / x\right]\right) \longmapsto{ }^{j_{2}}\left(w_{f}, e_{f}\right)$,

- $\operatorname{irred}\left(w_{f}, e_{f}\right)$.

Hence, there exists $W_{f_{2}}$ and $q_{f_{2}}$ such that

- $w_{f}: k-j_{1}-1-j_{2}\left(W_{f_{2}} \odot_{k-j_{1}-1-j_{2}} W_{r}\right)$, and

- $\left(k-j_{1}-1-j_{2}, q_{f_{2}}, W_{f_{2}}, e_{f}\right) \in \mathcal{T} \llbracket \Delta, \alpha: \kappa \vdash \tau_{2}:$ TYPE $\rrbracket \delta\left[\alpha \mapsto \mathcal{I}_{11}\right]$.

Let $W_{f}=W_{f_{2}}$ and $q_{f}=q_{f_{2}}$.

We are required to show that

- $w_{f}: k-j\left(W_{f} \odot_{k-j} W_{r}\right)$

$\equiv w_{f}:_{k-j_{1}-1-j_{2}}\left(W_{f_{2}} \odot_{k-j_{1}-1-j_{2}} W_{r}\right)$,

which follows from above,

- $\left(k-j, q_{f}, W_{f}, e_{f}\right) \in \mathcal{T} \llbracket \Delta \vdash \tau_{2} \rrbracket \delta$

$\equiv\left(k-j_{1}-1-j_{2}, q_{f_{2}}, W_{f_{2}}, e_{f}\right) \in \mathcal{T} \llbracket \Delta \vdash \tau_{2} \rrbracket \delta$,

which follows from $\left(k-j_{1}-1-j_{2}, q_{f_{2}}, W_{f_{2}}, e_{f}\right) \in \mathcal{T} \llbracket \Delta, \alpha: \kappa \vdash \tau_{2}:$ TYPE $\rrbracket \delta\left[\alpha \mapsto \mathcal{I}_{11}\right]$ and $\Delta \vdash \tau_{2}:$ TYPE. 
(COPY)

Case $\frac{\Delta ; \Gamma \vdash e_{1}: \tau \quad \Delta \vdash \tau \preceq \mathrm{R}}{\Delta ; \Gamma \vdash \operatorname{copy} e_{1}:{ }^{\mathrm{L}} \tau \otimes \tau}:$

We are required to show $\llbracket \Delta ; \Gamma \vdash \operatorname{copy} e_{1}:{ }^{\mathrm{L}} \tau \otimes \tau \rrbracket$.

Consider arbitrary $k, \delta, q_{\Gamma}, W_{\Gamma}$, and $\gamma$ such that

- $k \geq 0$,

- $\delta \in \mathcal{D} \llbracket \Delta \rrbracket$, and

- $\left(k, q_{\Gamma}, W_{\Gamma}, \gamma\right) \in \mathcal{G} \llbracket \Delta \vdash \Gamma \rrbracket \delta$.

Let $e_{s}=\gamma\left(\operatorname{copy} e_{1}\right) \equiv \operatorname{copy} \gamma\left(e_{1}\right)$ and $W_{s}=W_{\Gamma}$.

We are required to show that $\operatorname{Comp}\left(k, W_{s}, e_{s}, \mathcal{T} \llbracket \Delta \vdash{ }^{\mathrm{L}} \tau \otimes \tau: \mathrm{TYPE} \rrbracket \delta\right) \quad \equiv$ $\operatorname{Comp}\left(k, W_{\Gamma}, \operatorname{copy} \gamma\left(e_{1}\right), \mathcal{T} \llbracket \Delta \vdash{ }^{\mathrm{L}} \tau \otimes \tau:\right.$ TYPE $\left.\rrbracket \delta\right)$.

Consider arbitrary $j, W_{r}, w_{s}, w_{f}$, and $e_{f}$ such that

- $j<k$,

- $w_{s}:_{k}\left(W_{s} \odot_{k} W_{r}\right) \equiv w_{s}:_{k}\left(W_{\Gamma} \odot_{k} W_{r}\right)$,

- $\left(w_{s}, e_{s}\right)=\left(w_{s}, \operatorname{copy} \gamma\left(e_{1}\right)\right) \longmapsto^{j}\left(w_{f}, e_{f}\right)$, and

- $\operatorname{irred}\left(w_{f}, e_{f}\right)$.

Hence, by inspection of the operational semantics, it follows that there exist $j_{1}, w_{f_{1}}$, and $e_{f_{1}}$ such that

- $\left(w_{s}, \gamma\left(e_{1}\right)\right) \longmapsto{ }^{j_{1}}\left(w_{f_{1}}, e_{f_{1}}\right)$,

- $\operatorname{irred}\left(w_{f_{1}}, e_{f_{1}}\right)$, and

- $j_{1} \leq j$.

Applying the induction hypothesis to $\Delta ; \Gamma \vdash e_{1}: \tau$, we conclude that $\llbracket \Delta ; \Gamma \vdash e_{1}: \tau \rrbracket$. Instantiate this with $k, \delta, q_{\Gamma}, W_{\Gamma}$, and $\gamma$. Note that

- $k \geq 0$,

- $\delta \in \mathcal{D} \llbracket \Delta \rrbracket$, and

- $\left(k, q_{\Gamma}, W_{\Gamma}, \gamma\right) \in \mathcal{G} \llbracket \Delta \vdash \Gamma \rrbracket \delta$.

Hence, $\operatorname{Comp}\left(k, W_{\Gamma}, \gamma\left(e_{1}\right), \mathcal{T} \llbracket \Delta \vdash \tau:\right.$ TYPE $\left.\rrbracket \delta\right)$.

Instantiate this with $j_{1}, W_{r}, w_{s}, w_{f_{1}}$, and $e_{f_{1}}$. Note that

- $j_{1}<k$, which follows from $j_{1} \leq j$ and $j<k$,

- $w_{s}:_{k}\left(W_{\Gamma} \odot_{k} W_{r}\right)$, which follows from above,

- $\left(w_{s}, \gamma\left(e_{1}\right)\right) \longmapsto^{j_{1}}\left(w_{f_{1}}, e_{f_{1}}\right)$,

- $\operatorname{irred}\left(w_{f_{1}}, e_{f_{1}}\right)$.

Hence, there exists $W_{f_{1}}$ and $q_{f_{1}}$ such that

- $w_{f_{1}}:_{k-j_{1}}\left(W_{f_{1}} \odot_{k-j_{1}} W_{r}\right)$, and

- $\left(k-j_{1}, q_{f_{1}}, W_{f_{1}}, e_{f_{1}}\right) \in \mathcal{T} \llbracket \Delta \vdash \tau:$ TYPE $\rrbracket \delta$.

Hence, $e_{f_{1}} \equiv v_{f_{1}}$.

Note that $\mathcal{P}\left(k-j_{1}, \mathrm{R}, W_{f_{1}}\right)$, which follows from Corollary 16 applied to $\Delta \vdash \tau \preceq \mathrm{R}$ and $(k-$ $\left.j_{1}, q_{f_{1}}, W_{f_{1}}, v_{f_{1}}\right) \in \mathcal{T} \llbracket \Delta \vdash \tau: \mathrm{TYPE} \rrbracket \delta$ and $\mathrm{R}=\mathcal{T} \llbracket \Delta \vdash \mathrm{R}: \mathrm{QUAL} \rrbracket \delta$.

Note that $\mathcal{P}\left(k-j_{1}-1, \mathrm{R}, W_{f_{1}}\right)$, which follows from Req 10 (qualpred-closed) and $\mathcal{P}\left(k-j_{1}, \mathrm{R}, W_{f_{1}}\right)$. Note that 


$$
\begin{aligned}
\left(w_{s}, e_{s}\right) & \equiv\left(w_{s}, \operatorname{copy} \gamma\left(e_{1}\right)\right) \\
& \longmapsto^{j_{1}}\left(w_{f_{1}}, \operatorname{copy} e_{f_{1}}\right) \\
& \equiv\left(w_{f_{1}}, \operatorname{copy} v_{f_{1}}\right) \\
& \longmapsto^{1}\left(w_{f_{1}},\left\langle v_{f_{1}}, v_{f_{1}}\right\rangle\right) \\
& \longmapsto^{j-j_{1}-1}\left(w_{f}, e_{f}\right) .
\end{aligned}
$$

Since $\left\langle v_{f_{1}}, v_{f_{1}}\right\rangle$ is value, we have $\operatorname{irred}\left(w_{f_{1}},\left\langle v_{f_{1}}, v_{f_{1}}\right\rangle\right)$.

Hence, $j-j_{1}-1=0$ (and $j=j_{1}+1$ ) and $w_{f} \equiv w_{f_{1}}$ and $e_{f} \equiv\left\langle v_{f_{1}}, v_{f_{1}}\right\rangle$.

Note that $\left\lfloor\left(W_{f_{1}} \odot_{k-j_{1}} W_{r}\right)\right\rfloor_{k-j_{1}-1} \equiv\left(\left\lfloor W_{f_{1}}\right\rfloor_{k-j_{1}-1} \odot_{k-j_{1}-1} W_{r}\right)$, which follows from

$$
\begin{aligned}
& \left\lfloor\left(W_{f_{1}} \odot_{k-j_{1}} W_{r}\right)\right\rfloor_{k-j_{1}-1} \\
& \equiv\left(W_{f_{1}} \odot_{k-j_{1}-1} W_{r}\right) \\
& \quad \text { which follows from Req } 4 \text { (join-closed) } \\
& \equiv\left(\left\lfloor W_{f_{1}}\right\rfloor_{k-j_{1}-1} \odot_{k-j_{1}-1} W_{r}\right) \\
& \quad \text { which follows from Req } 5 \text { (join-aprx). }
\end{aligned}
$$

Let $W_{f}=\left\lfloor W_{f_{1}}\right\rfloor_{k-j_{1}-1}$ and $q_{f}=\mathrm{L}$.

We are required to show that

- $w_{f}:_{k-j}\left(W_{f} \odot_{k-j} W_{r}\right)$

$$
\equiv w_{f_{1}}:_{k-j_{1}-1}\left(\left\lfloor W_{f_{1}}\right\rfloor_{k-j_{1}-1} \odot_{k-j_{1}-1} W_{r}\right) \text {, }
$$

which follows from

$$
\begin{gathered}
w_{f_{1}}: k-j_{1}\left(W_{f_{1}} \odot_{k-j_{1}} W_{r}\right) \\
\text { which follows from above } \\
\Rightarrow w_{f_{1}}: k-j_{1}-1\left(W_{f_{1}} \odot_{k-j_{1}} W_{r}\right) \\
\text { which follows from Req } 2 \text { (models-closed) } \\
\Leftrightarrow w_{f_{1}}: k-j_{1}-1\left\lfloor\left(W_{f_{1}} \odot_{k-j_{1}} W_{r}\right)\right\rfloor_{k-j_{1}-1} \\
\text { which follows from Req } 3 \text { (models-aprx) }
\end{gathered}
$$

$\left\lfloor\left(W_{f_{1}} \odot_{k-j_{1}} W_{r}\right)\right\rfloor_{k-j_{1}-1} \equiv\left(\left\lfloor W_{f_{1}}\right\rfloor_{k-j_{1}-1} \odot_{k-j_{1}-1} W_{r}\right)$ which follows from above,

- $\left(k-j, q_{f}, W_{f}, e_{f}\right) \in \mathcal{T} \llbracket \Delta \vdash{ }^{\mathrm{L}} \tau \otimes \tau: \mathrm{TYPE} \rrbracket \delta$

$$
\begin{aligned}
& \equiv\left(k-j_{1}-1, \mathrm{~L},\left\lfloor W_{f_{1}}\right\rfloor_{k-j_{1}-1},\left\langle v_{f_{1}}, v_{f_{1}}\right\rangle\right) \in \mathcal{T} \llbracket \Delta \vdash{ }^{\mathrm{L}} \tau \otimes \tau: \mathrm{TYPE} \rrbracket \delta \\
& \equiv\left(k-j_{1}-1, \mathrm{~L},\left\lfloor W_{f_{1}}\right\rfloor_{k-j_{1}-1},\left\langle v_{f_{1}}, v_{f_{1}}\right\rangle\right) \\
& \in\left\{\left(k, q, W,\left\langle v_{1}, v_{2}\right\rangle\right) \mid\right. \\
& q=\mathcal{T} \llbracket \Delta \vdash \mathrm{L}: \mathrm{QUAL} \rrbracket \delta \wedge \\
& \left(k, q_{1}, W_{1}, v_{1}\right) \in \mathcal{T} \llbracket \Delta \vdash \tau: \mathrm{TYPE} \rrbracket \delta \wedge \\
& \left(k, q_{2}, W_{2}, v_{2}\right) \in \mathcal{T} \llbracket \Delta \vdash \tau: \mathrm{TYPE} \rrbracket \delta \wedge \\
& \\
& q_{1} \preceq q \wedge q_{2} \preceq q \wedge \\
& \left.\left(W_{1} \odot_{k} W_{2}=W\right)\right\},
\end{aligned}
$$

which follows from

- $\mathrm{L}=\mathcal{T} \llbracket \Delta \vdash \mathrm{L}: \mathrm{QUAL} \rrbracket \delta$, which follows trivially,

- $\left(k-j_{1}-1, q_{f_{1}},\left\lfloor W_{f_{1}}\right\rfloor_{k-j_{1}-1}, v_{f_{1}}\right) \in \mathcal{T} \llbracket \Delta \vdash \tau:$ TYPE $\rrbracket$, which follows from Lemma 8 and Fact 6 applied to $k-j_{1}-1 \leq k-j_{1}$ and $\left(k-j_{1}, q_{f_{1}}, W_{f_{1}}, v_{f_{1}}\right) \in \mathcal{T} \llbracket \Delta \vdash \tau:$ TYPE $\rrbracket$, which in turn follows from above,

- $\left(k-j_{1}-1, q_{f_{1}},\left\lfloor W_{f_{1}}\right\rfloor_{k-j_{1}-1}, v_{f_{1}}\right) \in \mathcal{T} \llbracket \Delta \vdash \tau:$ TYPE $\rrbracket$, which follows from Lemma 8 and Fact 6 applied to $k-j_{1}-1 \leq k-j_{1}$ and $\left(k-j_{1}, q_{f_{1}}, W_{f_{1}}, v_{f_{1}}\right) \in \mathcal{T} \llbracket \Delta \vdash \tau:$ TYPE $\rrbracket$, which in turn follows from above,

- $q_{f_{1}} \preceq \mathrm{L}$, which follows trivially,

- $q_{f_{1}} \preceq \mathrm{L}$, which follows trivially, and, 
- $\left\lfloor W_{f_{1}}\right\rfloor_{k-j_{1}-1}=\left(\left\lfloor W_{f_{1}}\right\rfloor_{k-j_{1}-1} \odot_{k-j_{1}-1}\left\lfloor W_{f_{1}}\right\rfloor_{k-j_{1}-1}\right)$, which follows from $\left\lfloor W_{f_{1}}\right\rfloor_{k-j_{1}-1}$ $\equiv\left(W_{f_{1}} \odot_{k-j_{1}-1} W_{f_{1}}\right)$ which follows from Req 15 (qualpred-rel-join) $\equiv\left(\left\lfloor W_{f_{1}}\right\rfloor_{k-j_{1}-1} \odot_{k-j_{1}-1}\left\lfloor W_{f_{1}}\right\rfloor_{k-j_{1}-1}\right)$ which follows from Req 5 (join-aprx). 
(DROP)

Case $\frac{\Delta ; \Gamma \vdash e_{1}: \tau \quad \Delta \vdash \tau \preceq \mathrm{A}}{\Delta ; \Gamma \vdash \operatorname{drop} e_{1}:{ }^{\mathrm{L}} \mathbf{1}_{\otimes}}:$

We are required to show $\llbracket \Delta ; \Gamma \vdash \operatorname{drop} e_{1}:{ }^{\mathrm{L}} \mathbf{1}_{\otimes} \rrbracket$.

Consider arbitrary $k, \delta, q_{\Gamma}, W_{\Gamma}$, and $\gamma$ such that

- $k \geq 0$,

- $\delta \in \mathcal{D} \llbracket \Delta \rrbracket$, and

- $\left(k, q_{\Gamma}, W_{\Gamma}, \gamma\right) \in \mathcal{G} \llbracket \Delta \vdash \Gamma \rrbracket \delta$.

Let $e_{s}=\gamma\left(\operatorname{drop} e_{1}\right) \equiv \operatorname{drop} \gamma\left(e_{1}\right)$ and $W_{s}=W_{\Gamma}$.

We are required to show that $\operatorname{Comp}\left(k, W_{s}, e_{s}, \mathcal{T} \llbracket \Delta \vdash^{\mathrm{L}} \mathbf{1}_{\otimes}: \mathrm{TYPE} \rrbracket \delta\right) \quad \equiv$ $\operatorname{Comp}\left(k, W_{\Gamma}, \operatorname{drop} \gamma\left(e_{1}\right), \mathcal{T} \llbracket \Delta \vdash{ }^{\mathrm{L}} \mathbf{1}_{\otimes}:\right.$ TYPE $\left.\rrbracket \delta\right)$.

Consider arbitrary $j, W_{r}, w_{s}, w_{f}$, and $e_{f}$ such that

- $j<k$,

- $w_{s}:_{k}\left(W_{s} \odot_{k} W_{r}\right) \equiv w_{s}:_{k}\left(W_{\Gamma} \odot_{k} W_{r}\right)$,

- $\left(w_{s}, e_{s}\right)=\left(w_{s}, \operatorname{drop} \gamma\left(e_{1}\right)\right) \longmapsto^{j}\left(w_{f}, e_{f}\right)$, and

- $\operatorname{irred}\left(w_{f}, e_{f}\right)$.

Hence, by inspection of the operational semantics, it follows that there exist $j_{1}, w_{f_{1}}$, and $e_{f_{1}}$ such that

- $\left(w_{s}, \gamma\left(e_{1}\right)\right) \longmapsto{ }^{j_{1}}\left(w_{f_{1}}, e_{f_{1}}\right)$,

- $\operatorname{irred}\left(w_{f_{1}}, e_{f_{1}}\right)$, and

- $j_{1} \leq j$.

Applying the induction hypothesis to $\Delta ; \Gamma \vdash e_{1}: \tau$, we conclude that $\llbracket \Delta ; \Gamma \vdash e_{1}: \tau \rrbracket$. Instantiate this with $k, \delta, q_{\Gamma}, W_{\Gamma}$, and $\gamma$. Note that

- $k \geq 0$,

- $\delta \in \mathcal{D} \llbracket \Delta \rrbracket$, and

- $\left(k, q_{\Gamma}, W_{\Gamma}, \gamma\right) \in \mathcal{G} \llbracket \Delta \vdash \Gamma \rrbracket \delta$.

Hence, $\operatorname{Comp}\left(k, W_{\Gamma}, \gamma\left(e_{1}\right), \mathcal{T} \llbracket \Delta \vdash \tau:\right.$ TYPE $\left.\rrbracket \delta\right)$.

Instantiate this with $j_{1}, W_{r}, w_{s}, w_{f_{1}}$, and $e_{f_{1}}$. Note that

- $j_{1}<k$, which follows from $j_{1} \leq j$ and $j<k$,

- $w_{s}:_{k}\left(W_{\Gamma} \odot_{k} W_{r}\right)$, which follows from above,

- $\left(w_{s}, \gamma\left(e_{1}\right)\right) \longmapsto^{j_{1}}\left(w_{f_{1}}, e_{f_{1}}\right)$,

- $\operatorname{irred}\left(w_{f_{1}}, e_{f_{1}}\right)$.

Hence, there exists $W_{f_{1}}$ and $q_{f_{1}}$ such that

- $w_{f_{1}}:_{k-j_{1}}\left(W_{f_{1}} \odot_{k-j_{1}} W_{r}\right)$, and

- $\left(k-j_{1}, q_{f_{1}}, W_{f_{1}}, e_{f_{1}}\right) \in \mathcal{T} \llbracket \Delta \vdash \tau:$ TYPE $\rrbracket \delta$.

Hence, $e_{f_{1}} \equiv v_{f_{1}}$.

Note that $\mathcal{P}\left(k-j_{1}, \mathrm{~A}, W_{f_{1}}\right)$, which follows from Corollary 16 applied to $\Delta \vdash \tau \preceq \mathrm{A}$ and $(k-$ $\left.j_{1}, q_{f_{1}}, W_{f_{1}}, v_{f_{1}}\right) \in \mathcal{T} \llbracket \Delta \vdash \tau: \mathrm{TYPE} \rrbracket \delta$ and $\mathrm{A}=\mathcal{T} \llbracket \Delta \vdash \mathrm{A}: \mathrm{QUAL} \rrbracket \delta$.

Note that $\mathcal{P}\left(k-j_{1}-1, \mathrm{~A}, W_{f_{1}}\right)$, which follows from Req 10 (qualpred-closed) and $\mathcal{P}\left(k-j_{1}, \mathrm{~A}, W_{f_{1}}\right)$. Note that 


$$
\begin{aligned}
\left(w_{s}, e_{s}\right) & \equiv\left(w_{s}, \operatorname{drop} \gamma\left(e_{1}\right)\right) \\
& \longmapsto^{j_{1}}\left(w_{f_{1}}, \operatorname{drop} e_{f_{1}}\right) \\
& \equiv\left(w_{f_{1}}, \operatorname{drop} v_{f_{1}}\right) \\
& \longmapsto^{1}\left(w_{f_{1}},\langle\rangle\right) \\
& \longmapsto^{j-j_{1}-1}\left(w_{f}, e_{f}\right) .
\end{aligned}
$$

Since \langle\rangle is value, we have $\operatorname{irred}\left(w_{f_{1}},\langle\rangle\right)$.

Hence, $j-j_{1}-1=0\left(\right.$ and $\left.j=j_{1}+1\right)$ and $w_{f} \equiv w_{f_{1}}$ and $e_{f} \equiv\langle\rangle$.

Note that $\left\lfloor\left(W_{f_{1}} \odot_{k-j_{1}} W_{r}\right)\right\rfloor_{k-j_{1}-1} \equiv\left(W_{f_{1}} \odot_{k-j_{1}-1}\left(\left\lfloor\mathcal{U}_{\odot}\right\rfloor_{k-j_{1}-1} \odot_{k-j_{1}-1} W_{r}\right)\right)$, which follows from

$$
\begin{aligned}
& \left\lfloor\left(W_{f_{1}} \odot_{k-j_{1}} W_{r}\right)\right\rfloor_{k-j_{1}-1} \\
& \equiv\left(\mathcal{U}_{\odot} \odot_{k-j_{1}-1}\left(W_{f_{1}} \odot_{k-j_{1}} W_{r}\right)\right) \\
& \quad \text { which follows from Req } 9(\text { join-unit-left }) \\
& \equiv\left(\left\lfloor\mathcal{U}_{\odot}\right\rfloor_{k-j_{1}-1} \odot_{k-j_{1}-1}\left(W_{f_{1}} \odot_{k-j_{1}} W_{r}\right)\right) \\
& \quad \text { which follows from Req } 5(\text { join-aprx }) \\
& \equiv\left(W_{f_{1}} \odot_{k-j_{1}-1}\left(\left\lfloor\mathcal{U}_{\odot}\right\rfloor_{k-j_{1}-1} \odot_{k-j_{1}-1} W_{r}\right)\right)
\end{aligned}
$$

which follows from Reqs $4,5,6,7$, and 8 (join-closed, join-aprx, join-commut, join-assocl, and join-assocr).

Let $W_{f}=\left\lfloor\mathcal{U}_{\odot}\right\rfloor_{k-j_{1}-1}$ and $q_{f}=\mathrm{L}$.

We are required to show that

- $w_{f}: k-j\left(W_{f} \odot_{k-j} W_{r}\right)$

$$
\equiv w_{f_{1}}:_{k-j_{1}-1}\left(\left\lfloor\mathcal{U}_{\odot}\right\rfloor_{k-j_{1}-1} \odot_{k-j_{1}-1} W_{r}\right)
$$

which follows from

$$
\begin{gathered}
w_{f_{1}}: k_{k-j_{1}}\left(W_{f_{1}} \odot_{k-j_{1}} W_{r}\right) \\
\text { which follows from above } \\
\Rightarrow w_{f_{1}}: k-j_{1}-1\left(W_{f_{1}} \odot_{k-j_{1}} W_{r}\right) \\
\text { which follows from Req } 2 \text { (models-closed) } \\
\Leftrightarrow w_{f_{1}}: k_{-j}-1\left\lfloor\left(W_{f_{1}} \odot_{k-j_{1}} W_{r}\right)\right\rfloor_{k-j_{1}-1} \\
\quad \text { which follows from Req } 3 \text { (models-aprx) }
\end{gathered}
$$$$
\left\lfloor\left(W_{f_{1}} \odot_{k-j_{1}} W_{r}\right)\right\rfloor_{k-j_{1}-1} \equiv\left(W_{f_{1}} \odot_{k-j_{1}-1}\left(\left\lfloor\mathcal{U}_{\odot}\right\rfloor_{k-j_{1}-1} \odot_{k-j_{1}-1} W_{r}\right)\right)
$$
which follows from above

$$
\begin{gathered}
w_{f_{1}}: k-j_{1}-1 \\
\Rightarrow w_{f_{1}}: k-j_{1}-1 \\
\text { which follows from Req } 16 \text { (qualpred-aff-models), }
\end{gathered}
$$

- $\left(k-j, q_{f}, W_{f}, e_{f}\right) \in \mathcal{T} \llbracket \Delta \vdash{ }^{\mathrm{L}} \mathbf{1}_{\otimes}:$ TYPE $\rrbracket \delta$

$$
\begin{gathered}
\equiv\left(k-j_{1}-1, \mathrm{~L},\left\lfloor\mathcal{U}_{\odot}\right\rfloor_{k-j_{1}-1},\langle\rangle\right) \in \mathcal{T} \llbracket \Delta \vdash{ }^{\mathrm{L}} \mathbf{1}_{\otimes}: \text { TYPE } \rrbracket \delta \\
\equiv\left(k-j_{1}-1, \mathrm{~L},\left\lfloor\mathcal{U}_{\odot}\right\rfloor_{k-j_{1}-1},\langle\rangle\right) \\
\in\{(k, q, W,\langle\rangle) \mid \\
q=\mathcal{T} \llbracket \Delta \vdash \mathrm{L}: \mathrm{QUAL} \rrbracket \delta \wedge \\
\left.\quad W=\left\lfloor\mathcal{U}_{\odot}\right\rfloor_{k}\right\},
\end{gathered}
$$

which follows from

- $\mathrm{L}=\mathcal{T} \llbracket \Delta \vdash \mathrm{L}: \mathrm{QUAL} \rrbracket \delta$, which follows trivially, and

- $\left\lfloor\mathcal{U}_{\odot}\right\rfloor_{k-j_{1}-1}=\left\lfloor\mathcal{U}_{\odot}\right\rfloor_{k-j_{1}-1}$ which follows trivially. 
(WEAK)

Case $\frac{\Delta \vdash \Gamma \sim \Gamma_{1} \boxplus \Gamma_{2} \quad \Delta ; \Gamma_{1} \vdash e_{1}: \tau \quad \Delta \vdash \Gamma_{2} \preceq \mathrm{A}}{\Delta ; \Gamma \vdash e_{1}: \tau}$ :

We are required to show $\llbracket \Delta ; \Gamma \vdash e: \tau \rrbracket$.

Consider arbitrary $k, \delta, q_{\Gamma}, W_{\Gamma}$, and $\gamma$ such that

- $k \geq 0$,

- $\delta \in \mathcal{D} \llbracket \Delta \rrbracket$, and

- $\left(k, q_{\Gamma}, W_{\Gamma}, \gamma\right) \in \mathcal{G} \llbracket \Delta \vdash \Gamma \rrbracket \delta$.

Applying Lemma 20 to $\left(k, q_{\Gamma}, W_{\Gamma}, \gamma\right) \in \mathcal{G} \llbracket \Delta \vdash \Gamma \rrbracket \delta$ and $\Delta \vdash \Gamma \leadsto \Gamma_{1} \boxplus \Gamma_{2}$, we conclude that there exist $q_{\Gamma_{1}}, W_{\Gamma_{1}}, \gamma_{1}, q_{\Gamma_{2}}, W_{\Gamma_{2}}$ and $\gamma_{2}$, such that

- $\left(k, q_{\Gamma_{1}}, W_{\Gamma_{1}}, \gamma_{1}\right) \in \mathcal{G} \llbracket \Delta \vdash \Gamma_{1} \rrbracket \delta$,

- $\left(k, q_{\Gamma_{2}}, W_{\Gamma_{2}}, \gamma_{2}\right) \in \mathcal{G} \llbracket \Delta \vdash \Gamma_{2} \rrbracket \delta$,

- $\gamma \in \gamma_{1} \boxplus \gamma_{2}$,

- $q_{\Gamma_{1}} \preceq q_{\Gamma}$,

- $q_{\Gamma_{2}} \preceq q_{\Gamma}$, and

- $\left(W_{\Gamma_{1}} \odot_{k} W_{\Gamma_{2}}=W_{\Gamma}\right)$.

Note that $\gamma\left(e_{1}\right) \equiv \gamma_{1}\left(e_{1}\right)$.

We are required to show that $\operatorname{Comp}\left(k, W_{s}, e_{s}, \mathcal{T} \llbracket \Delta \vdash \tau: \mathrm{TYPE} \rrbracket \delta\right) \quad \equiv$ $\operatorname{Comp}\left(k, W_{\Gamma}, \gamma_{1}\left(e_{1}\right), \mathcal{T} \llbracket \Delta \vdash \tau:\right.$ TYPE $\left.\rrbracket \delta\right)$.

Consider arbitrary $j, W_{r}, w_{s}, w_{f}$, and $e_{f}$ such that

- $j<k$,

- $w_{s}:_{k}\left(W_{s} \odot_{k} W_{r}\right) \equiv w_{s}:_{k}\left(W_{\Gamma} \odot_{k} W_{r}\right)$, noting that

$$
\begin{gathered}
w_{s}:_{k}\left(W_{\Gamma} \odot_{k} W_{r}\right) \\
\equiv w_{s}:_{k}\left(\left(W_{\Gamma_{1}} \odot_{k} W_{\Gamma_{2}}\right) \odot_{k} W_{r}\right) \\
\text { which follows from above, }
\end{gathered}
$$

- $\left(w_{s}, e_{s}\right) \equiv\left(w_{s}, \gamma_{1}\left(e_{1}\right)\right) \longmapsto^{j}\left(w_{f}, e_{f}\right)$, and

- $\operatorname{irred}\left(w_{f}, e_{f}\right)$.

Note that $\left(\left(W_{\Gamma_{1}} \odot_{k} W_{\Gamma_{2}}\right) \odot_{k} W_{r}\right) \equiv\left(W_{\Gamma_{2}} \odot_{k}\left(W_{\Gamma_{1}} \odot_{k} W_{r}\right)\right)$, which follows from

$$
\begin{aligned}
& \left(\left(W_{\Gamma_{1}} \odot_{k} W_{\Gamma_{2}}\right) \odot_{k} W_{r}\right) \\
& \equiv\left(W_{\Gamma_{2}} \odot_{k}\left(W_{\Gamma_{1}} \odot_{k} W_{r}\right)\right)
\end{aligned}
$$

which follows from Reqs 6,7 , and 8 (join-commut, join-assocl, and join-assocr).

Note that $\mathcal{P}\left(k, \mathrm{~A}, W_{\Gamma_{2}}\right)$, which follows from Corollary 18 applied to $\Delta \vdash \Gamma_{2} \preceq \mathrm{A}$ and $\mathrm{A}=$ $\mathcal{T} \llbracket \Delta \vdash \mathrm{A}: \mathrm{QUAL} \rrbracket \delta$.

Note that $w_{s}:_{k}\left(W_{\Gamma_{1}} \odot_{k} W_{r}\right)$, which follows from

$$
\begin{aligned}
& w_{s}: k\left(\left(W_{\Gamma_{1}} \odot_{k} W_{\Gamma_{2}}\right) \odot_{k} W_{r}\right) \quad \text { which follows from above } \\
& \left(\left(W_{\Gamma_{1}} \odot_{k} W_{\Gamma_{2}}\right) \odot_{k} W_{r}\right) \equiv\left(W_{\Gamma_{2}} \odot_{k}\left(W_{\Gamma_{1}} \odot_{k} W_{r}\right)\right) \\
& \quad \text { which follows from above } \\
& w_{s}:_{k}\left(W_{\Gamma_{2}} \odot_{k}\left(W_{\Gamma_{1}} \odot_{k} W_{r}\right)\right) \\
& \Rightarrow w_{s}: k\left(W_{\Gamma_{1}} \odot_{k} W_{r}\right) \\
& \quad \text { which follows from Req } 16 \text { (qualpred-aff-models). }
\end{aligned}
$$

Applying the induction hypothesis to $\Delta ; \Gamma_{1} \vdash e_{1}: \tau$, we conclude that $\llbracket \Delta ; \Gamma_{1} \vdash e_{1}: \tau \rrbracket$. Instantiate this with $k, \delta, q_{\Gamma_{1}}, W_{\Gamma_{1}}$, and $\gamma_{1}$. Note that 
- $k \geq 0$,

- $\delta \in \mathcal{D} \llbracket \Delta \rrbracket$, and

- $\left(k, q_{\Gamma_{1}}, W_{\Gamma_{1}}, \gamma_{1}\right) \in \mathcal{G} \llbracket \Delta \vdash \Gamma_{1} \rrbracket \delta$.

Hence, $\operatorname{Comp}\left(k, W_{\Gamma_{1}}, \gamma_{1}\left(e_{1}\right), \mathcal{T} \llbracket \Delta \vdash \tau: \operatorname{TYPE} \rrbracket \delta\right)$.

Instantiate this with $j_{1}, W_{r}, w_{s}, w_{f}$, and $e_{f}$. Note that

- $j<k$,

- $w_{s}: k\left(W_{\Gamma_{1}} \odot_{k} W_{r}\right)$, which follows from above,

- $\left(w_{s}, \gamma_{1}\left(e_{1}\right)\right) \longmapsto j\left(w_{f}, e_{f}\right)$,

- $\operatorname{irred}\left(w_{f}, e_{f}\right)$.

Hence, there exists $W_{f}$ and $q_{f}$ such that

- $w_{f}:_{k-j}\left(W_{f} \odot_{k-j} W_{r}\right)$, and

- $\left(k-j, q_{f}, W_{f}, e_{f}\right) \in \mathcal{T} \llbracket \Delta \vdash \tau: \mathrm{TYPE} \rrbracket \delta$. 
Case (UserPVal) ...: $\ldots$

Case (UserExp) ...: ..

End Case 


\section{A.7.6 Type Safety}

\section{Theorem 22 (Core Language Type Safety)}

If $\bullet \bullet \vdash e: \tau$ and $w: \mathcal{U}_{\odot}$ and $(w, e) \longmapsto *\left(w^{\prime}, e^{\prime}\right)$,

then either $e^{\prime} \equiv v^{\prime}$ or $\exists w^{\prime \prime}, e^{\prime \prime} .\left(w^{\prime}, e^{\prime}\right) \longmapsto\left(w^{\prime \prime}, e^{\prime \prime}\right)$.

\section{Proof}

Let $\bullet ; \bullet \bullet: \tau$ and $w: \mathcal{U}_{\odot}$ and $(w, e) \longmapsto^{*}\left(w^{\prime}, e^{\prime}\right)$.

Either $\operatorname{irred}\left(w^{\prime}, e^{\prime}\right)$ or $\neg$ irred $\left(w^{\prime}, e^{\prime}\right)$.

Suppose $\neg$ irred $\left(w^{\prime}, e^{\prime}\right)$.

Then $\exists w^{\prime \prime}, e^{\prime \prime} .\left(w^{\prime}, e^{\prime}\right) \longmapsto\left(w^{\prime \prime}, e^{\prime \prime}\right)$.

Suppose $\operatorname{irred}\left(w^{\prime}, e^{\prime}\right)$.

Note that there exists $i$ such that $(w, e) \longmapsto^{i}\left(w^{\prime}, e^{\prime}\right)$, which follows from $(w, e) \longmapsto^{*}\left(w^{\prime}, e^{\prime}\right)$.

Applying Theorem 21 to $\bullet ; \bullet \vdash e: \tau$, we conclude that $\llbracket \bullet ; \bullet \vdash e: \tau \rrbracket$.

This is equivalent to

$$
\begin{aligned}
& \forall k \geq 0 . \forall \delta, q_{\Gamma}, W_{\Gamma}, \gamma . \\
& \quad \delta \in \mathcal{D} \llbracket \bullet \rrbracket \wedge \\
& \quad\left(k, q_{\Gamma}, W_{\Gamma}, \gamma\right) \in \mathcal{G} \llbracket \bullet \vdash \bullet \rrbracket \delta \Rightarrow \\
& \quad \operatorname{Comp}\left(k, W_{\Gamma}, \gamma(e), \mathcal{T} \llbracket \bullet \vdash \tau: \text { TYPE } \rrbracket \delta\right)
\end{aligned}
$$

Instantiate this with $i+1, \emptyset, \mathbf{U},\left\lfloor\mathcal{U}_{\odot}\right\rfloor_{i+1}$, and $\emptyset$. Note that

- $i+1 \geq 0$

- $\emptyset \in \mathcal{D} \llbracket \bullet \rrbracket$, and

- $\left(i+1, \mathrm{U},\left\lfloor\mathcal{U}_{\odot}\right\rfloor_{i+1}, \emptyset\right) \in \mathcal{G} \llbracket \bullet \vdash \bullet \rrbracket \emptyset$.

Hence, we conclude that $\operatorname{Comp}\left(i+1,\left\lfloor\mathcal{U}_{\odot}\right\rfloor_{i+1}, e, \mathcal{T} \llbracket \bullet \vdash \tau:\right.$ TYPE $\left.\emptyset\right)$.

This is equivalent to

$$
\begin{aligned}
& \forall j<i+1, W_{r}, w_{s}, w_{f}, e_{f} . \\
& \left(\left\lfloor\mathcal{U}_{\odot}\right\rfloor_{i+1} \odot_{i+1} W_{r}\right) \text { defined } \wedge \\
& w_{s}:_{i+1}\left(\left\lfloor\mathcal{U}_{\odot}\right\rfloor_{i+1} \odot_{i+1} W_{r}\right) \wedge \\
& \left(w_{s}, e\right) \longmapsto^{j}\left(w_{f}, e_{f}\right) \wedge \\
& i r r e d\left(w_{f}, e_{f}\right) \Rightarrow \\
& \exists W_{f}, q_{f} \cdot \\
& \quad\left(W_{f} \odot_{i+1-j} W_{r}\right) \text { defined } \wedge \\
& \quad w_{f}:_{i+1-j}\left(W_{f} \odot_{i+1-j} W_{r}\right) \wedge \\
& \quad\left(i+1-j, q_{f}, W_{f}, e_{f}\right) \in \mathcal{T} \llbracket \bullet \vdash \tau: \text { TYPE } \emptyset
\end{aligned}
$$

Instantiate this with $i, \mathcal{U}_{\odot}, w, w^{\prime}$, and $e^{\prime}$. Note that

- $i<i+1$,

- $\left(\left[\mathcal{U}_{\odot}\right\rfloor_{i+1} \odot_{i+1} \mathcal{U}_{\odot}\right)$ defined, which follows from

$$
\begin{aligned}
& \left\lfloor\mathcal{U}_{\odot}\right\rfloor_{i+1} \\
& \equiv\left(\mathcal{U}_{\odot} \odot_{i+1} \mathcal{U}_{\odot}\right) \\
& \quad \text { which follows from Req } 9 \text { (join-unit-left) } \\
& \equiv\left(\left\lfloor\mathcal{U}_{\odot}\right\rfloor_{i+1} \odot_{i+1} \mathcal{U}_{\odot}\right) \\
& \quad \text { which follows from Req } 5 \text { (join-aprx), }
\end{aligned}
$$


- $w:_{i+1}\left(\left\lfloor\mathcal{U}_{\odot}\right\rfloor_{i+1} \odot_{i+1} \mathcal{U}_{\odot}\right)$, which follows from

$$
\begin{aligned}
w: & \mathcal{U}_{\odot} \\
& \text { which follows from above } \\
\Rightarrow & w:_{i+1} \mathcal{U}_{\odot} \\
& \text { which follows from } i+1 \geq 0 \\
\Leftrightarrow & w:_{i+1}\left\lfloor\mathcal{U}_{\odot}\right\rfloor_{i+1} \\
& \text { which follows from Req } 3 \text { (models-aprx) } \\
\equiv & w:_{i+1}\left(\mathcal{U}_{\odot} \odot_{i+1} \mathcal{U}_{\odot}\right) \\
& \text { which follows from Req } 9 \text { (join-unit-left) } \\
\equiv & w:_{i+1}\left(\left[\mathcal{U}_{\odot}\right\rfloor_{i+1} \odot_{i+1} \mathcal{U}_{\odot}\right) \\
& \text { which follows from Req } 5 \text { (join-aprx), }
\end{aligned}
$$

- $(w, e) \longmapsto^{i}\left(w^{\prime}, e^{\prime}\right)$, which follows from above, and

- $\operatorname{irred}\left(w^{\prime}, e^{\prime}\right)$, which follows from above.

Hence, we conclude that there exists $W^{\prime}$ and $q^{\prime}$ such that

- $\left(W^{\prime} \odot_{i+1-i} \mathcal{U}_{\odot}\right)$ defined,

- $w^{\prime}:_{i+1-i}\left(W^{\prime} \odot_{i+1-i} \mathcal{U}_{\odot}\right)$, and

- $\left(i+1-i, q^{\prime}, W^{\prime}, e^{\prime}\right) \in \mathcal{T} \llbracket \bullet \vdash \tau:$ TYPE $\rrbracket$.

Applying Lemma 8 to $\emptyset \in \mathcal{D} \llbracket \bullet \rrbracket$ and $\bullet \vdash \tau:$ TYPE, we conclude that $\mathcal{T} \llbracket \bullet \vdash \tau:$ TYPE $\emptyset \in$ Type.

Hence, $\mathcal{T} \llbracket \bullet \vdash \tau:$ TYPE $\rrbracket \in$ Type $\subseteq$ CandUberType ${ }_{\omega}=2^{\text {CandAtom }_{\omega}}$.

Hence, $\left(i+1-i, q^{\prime}, W^{\prime}, e^{\prime}\right) \in$ CandAtom $_{\omega}=\bigcup_{k \geq 0}$ CandAtom $_{k}$.

Hence, $e^{\prime} \in$ CValues.

Hence, $e^{\prime} \equiv v^{\prime}$. 


\title{
B Recursive Types and Functions Extension
}

\section{B.1 Syntax}

\author{
Type Level: \\ PreTypes $\quad \bar{\tau}::=\ldots \mid \mu \alpha$ :PRETYPE. $\tau$ \\ Expression Level: \\ Values $\quad v \quad:=\ldots \mid$ fold $v$ \\ Expressions $e \quad:=\ldots \mid$ unfold $e$
}

Figure 16: Rec Extension - Syntax 


\section{B.2 Operational Semantics}

$$
\begin{gathered}
\text { Evaluation Contexts } E \quad::=\ldots \mid \text { unfold } E \\
\text { (unfold) }(w, \text { unfold }(\text { fold } v)) \quad \longmapsto \quad(w, v)
\end{gathered}
$$

Figure 17: Rec Extension - Operational Semantics 


\section{B.3 Static Semantics}

$\Delta \vdash \iota: \kappa$

$$
\begin{aligned}
& \text { (RECPTY) } \\
& \frac{\Delta, \alpha: \text { PRETYPE } \vdash \tau: \text { TYPE }}{\Delta \vdash \mu \alpha: \text { PRETYPE. } \tau: \text { PRETYPE }}
\end{aligned}
$$

Figure 18: Rec Extension - Static Semantics (I)

$$
\Delta ; \Gamma \vdash e: \tau
$$

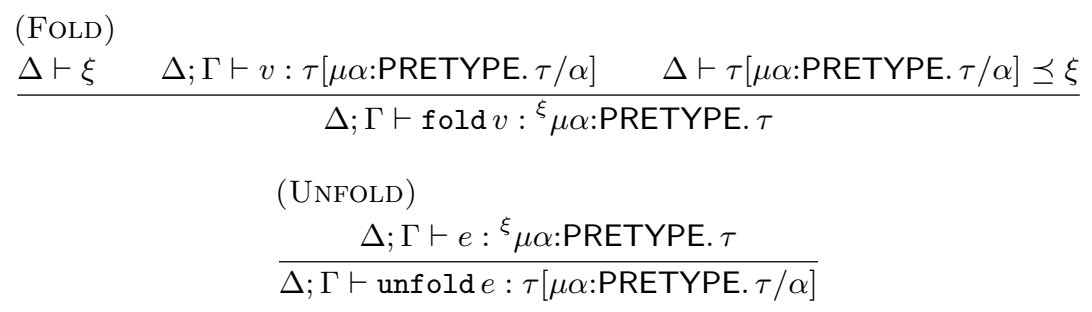

Figure 19: Rec Extension - Static Semantics (V) 


\section{B.4 Desugar}

\section{B.4.1 Y}

Syntax

$\mathrm{Y} \equiv \Lambda . \lambda f .(\lambda x . f((\operatorname{unfold} x) x))(\operatorname{fold}(\lambda x . f((\operatorname{unfold} x) x)))$

\section{Static Semantics}

$\frac{(\mathrm{Y})}{\Delta ; \bullet \vdash \mathrm{Y}:{ }^{\mathrm{L}} \forall \alpha: \text { TYPE. }^{\mathrm{L}}\left({ }^{\mathrm{U}}(\alpha \multimap \alpha) \multimap \alpha\right)} \equiv \frac{\vdots}{\Delta ; \bullet \vdash \ldots:{ }^{\mathrm{L}} \forall \alpha: \text { TYPE. }{ }^{\mathrm{L}}\left({ }^{\mathrm{U}}(\alpha \multimap \alpha) \multimap \alpha\right)}=\mathfrak{D}_{0}$

\begin{tabular}{|c|c|c|c|}
\hline \multicolumn{4}{|l|}{$(\mathrm{ALL})$} \\
\hline & $(\mathrm{FN})$ & & \\
\hline & $\Delta \vdash \mathrm{L}: \mathrm{QUAL}$ & $\Delta \vdash \bullet \preceq \mathrm{L}$ & $\overline{\Delta, \alpha: \text { TYPE } ; \bullet, f:{ }^{\cup}(\alpha \multimap \alpha) \vdash \ldots: \alpha}=\mathfrak{D}_{1}$ \\
\hline$\Delta \vdash \bullet \preceq \mathrm{L}$ & \multicolumn{3}{|c|}{$\Delta, \alpha:$ TYPE $; \bullet \vdash \lambda f \ldots:^{\mathrm{L}}\left({ }^{\mathrm{U}}(\alpha \multimap \alpha) \multimap \alpha\right)$} \\
\hline \multicolumn{4}{|c|}{$\overline{\Delta \Delta ; \bullet \vdash \Lambda . \lambda f \ldots . . .{ }^{\mathrm{L}} \forall \alpha: \text { TYPE. }{ }^{\mathrm{L}}\left({ }^{\mathrm{U}}(\alpha \multimap \alpha) \multimap \alpha\right)}$} \\
\hline
\end{tabular}

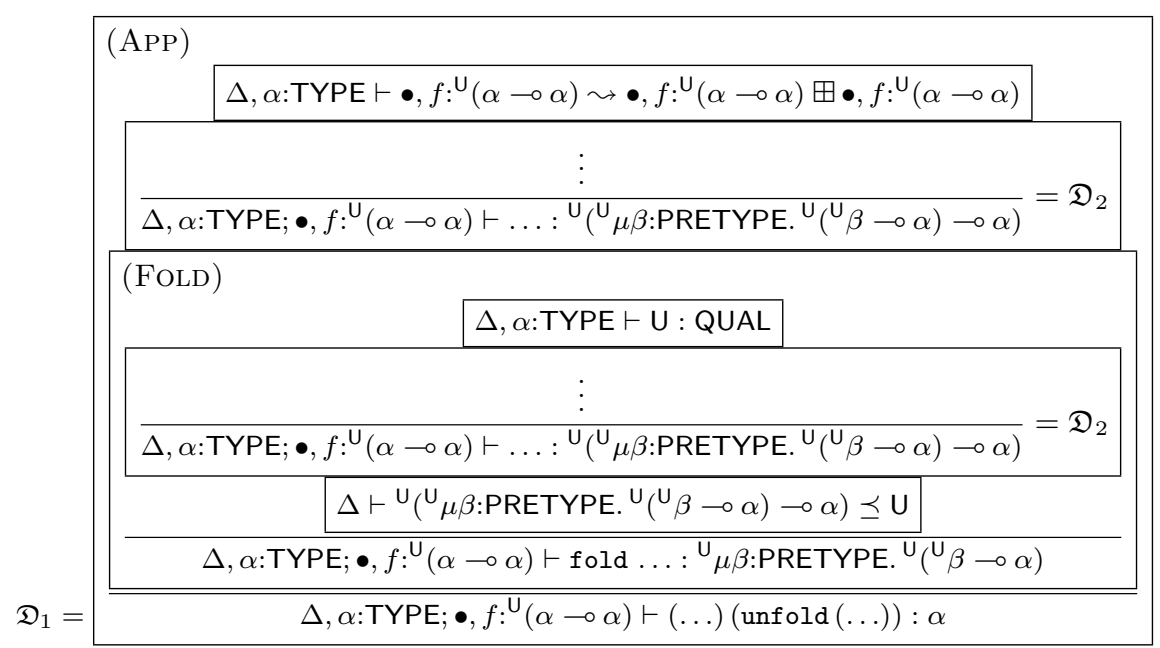

\begin{tabular}{|c|c|}
\hline \multicolumn{2}{|c|}{$(\mathrm{FN})$} \\
\hline$\Delta, \alpha:$ TYPE $\vdash \mathrm{U}:$ QUAL & $\Delta, \alpha:$ TYPE $\vdash \bullet, f: \cup^{\cup}(\alpha \multimap \alpha) \preceq \mathrm{U}$ \\
\hline \multicolumn{2}{|c|}{ 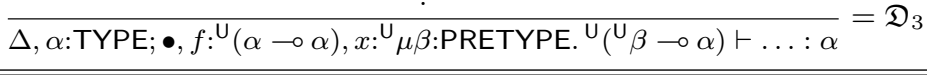 } \\
\hline \multicolumn{2}{|c|}{$\Delta, \alpha:$ TYPE $; \bullet, f:{ }^{\mathrm{U}}(\alpha \multimap \alpha) \vdash \lambda x \ldots .:{ }^{\mathrm{U}}\left({ }^{\mathrm{U}} \mu \beta:\right.$ PRETYPE. $\left.{ }^{\mathrm{U}}\left({ }^{\mathrm{U}} \beta \multimap \alpha\right) \multimap \alpha\right)$} \\
\hline
\end{tabular}




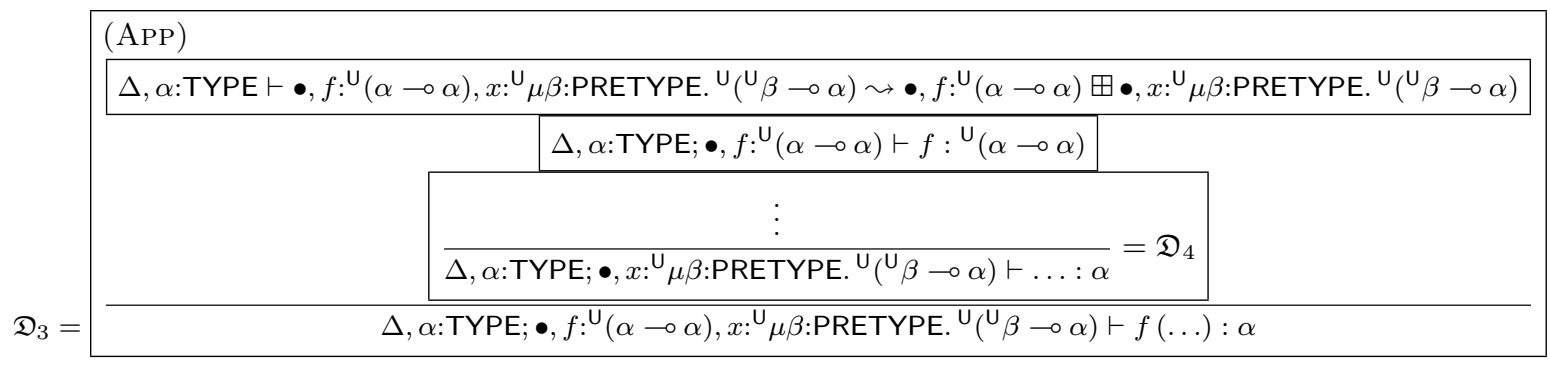

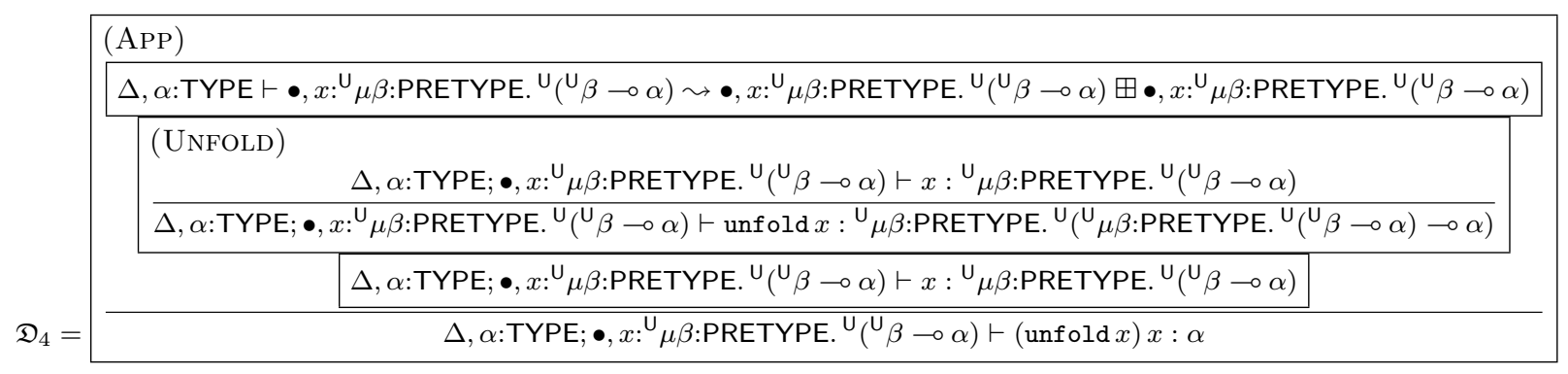

B.4.2 fix

Syntax

$$
\text { fix } f(x) . e \equiv(\mathrm{Y}[])(\lambda f \cdot \lambda x \cdot e)
$$

\section{Static Semantics}

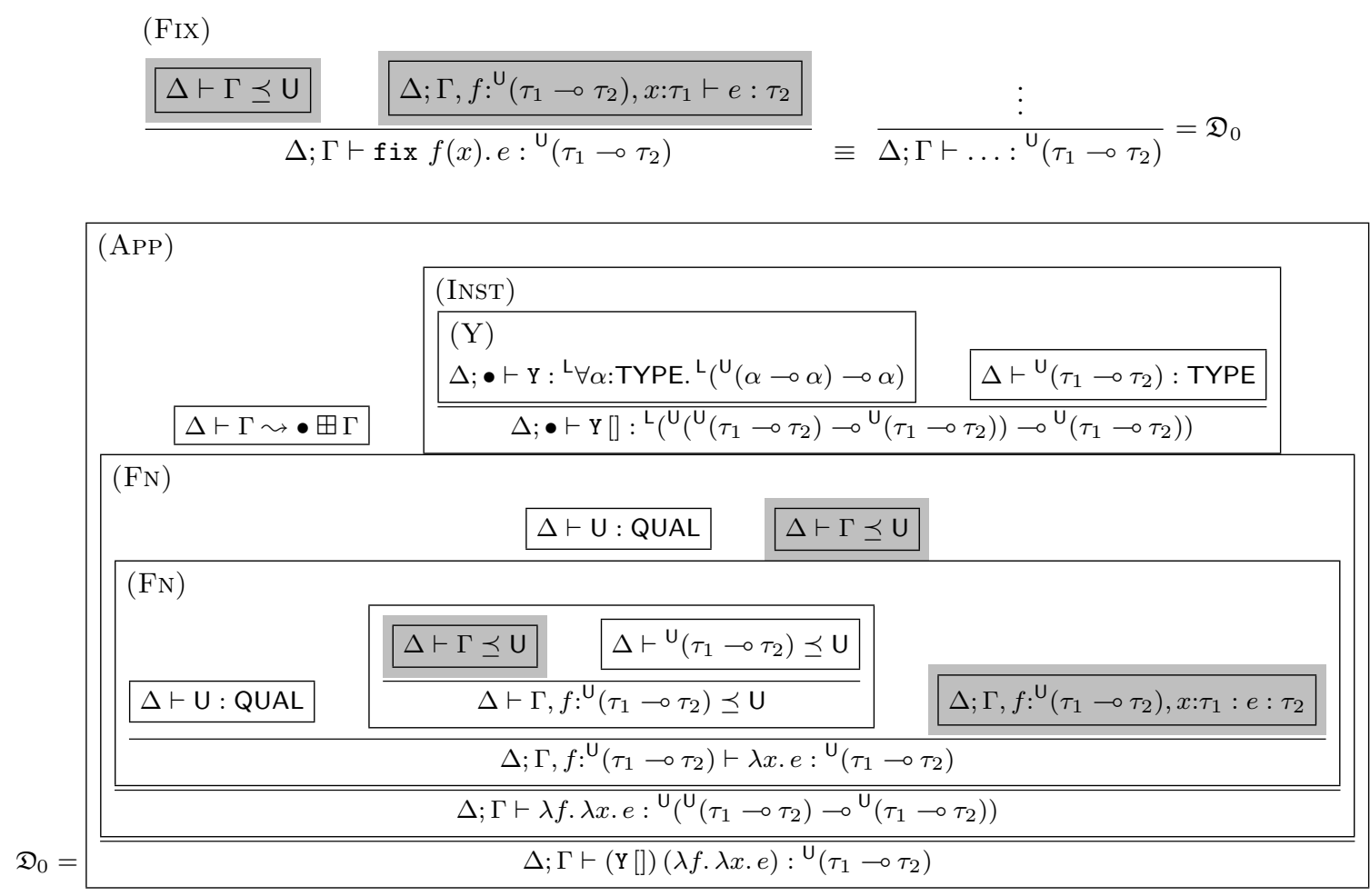




\section{B.5 Model}

$$
\begin{aligned}
& \mathcal{T}_{\mu} \llbracket k^{\prime}, \frac{\Delta, \alpha: \text { PRETYPE } \vdash \tau: \text { TYPE }}{\Delta \vdash \mu \alpha: \text { PRETYPE. } \tau: \operatorname{PRETYPE}} \rrbracket \delta= \\
& \left\{(k, q, W, \text { fold } v) \mid k \leq k^{\prime} \wedge W \in W_{\text {orldDesc }} \wedge \mathcal{P}(k, q, W) \wedge\right. \\
& \exists q^{\prime} \text {. } \\
& q^{\prime} \preceq q \wedge \\
& \forall i<k \text {. } \\
& \text { let } \mathcal{I}=\mathcal{T}_{\mu} \llbracket i, \Delta \vdash \mu \alpha: \text { PRETYPE. } \tau \text { : PRETYPE } \rrbracket \delta \text { in } \\
& \left.\left(i, q^{\prime},\lfloor W\rfloor_{i}, v\right) \in \mathcal{T} \llbracket \Delta, \alpha: \operatorname{PRETYPE} \vdash \tau: \text { TYPE } \rrbracket \delta[\alpha \mapsto \mathcal{I}]\right\} \\
& \mathcal{T} \llbracket \Delta \vdash \mu \alpha: \text { PRETYPE. } \tau: \text { PRETYPE } \rrbracket \delta=\bigcup_{k^{\prime}>0} \mathcal{T}_{\mu} \llbracket k^{\prime}, \Delta \vdash \mu \alpha: \text { PRETYPE. } \tau: \text { PRETYPE } \rrbracket \delta
\end{aligned}
$$

Figure 20: Rec Extension - Semantic Interpretations (III) 


\section{B.6 Proofs}

\section{B.6.1 Validity of Kinding Rules}

Lemma 23 (Rec Extension: $\mathcal{T}_{\mu} \llbracket k^{\prime}, \Delta \vdash \mu \alpha$ :PRETYPE. $\tau$ :PRETYPE $\rrbracket \in \mathcal{K} \llbracket$ PRETYPE $\rrbracket$ )

Let $\delta \in \mathcal{D} \llbracket \Delta \rrbracket$ and $\Delta \vdash \mu \alpha:$ PRETYPE. $\tau$ : PRETYPE.

Then forall $k^{\prime}, \mathcal{T}_{\mu} \llbracket k^{\prime}, \Delta \vdash \mu \alpha$ :PRETYPE. $\tau$ : PRETYPE $\rrbracket \in \mathcal{K} \llbracket$ PRETYPE $\rrbracket$.

Proof (Ref Extension Language: $\mathcal{T}_{\mu} \llbracket k^{\prime}, \Delta \vdash \mu \alpha: \operatorname{PRETYPE} . \tau: \operatorname{PRETYPE} \rrbracket \delta \in \mathcal{K} \llbracket \mathrm{PRETYPE} \rrbracket$ )

Recall from Lemma 8, it suffices to prove the following:

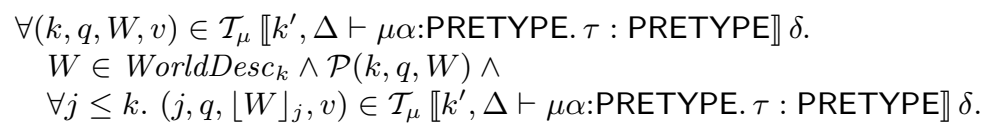

Proceed by induction on the derivation $\Delta \vdash \mu \alpha$ :PRETYPE. $\tau$ : PRETYPE.

(RECPTy)

Case $\frac{\Delta, \alpha: \text { PRETYPE } \vdash \tau: \text { TYPE }}{\Delta \vdash \mu \alpha: \text { PRETYPE. } \tau: \text { PRETYPE }}:$

Consider arbitrary $k^{\prime}$.

Recall that

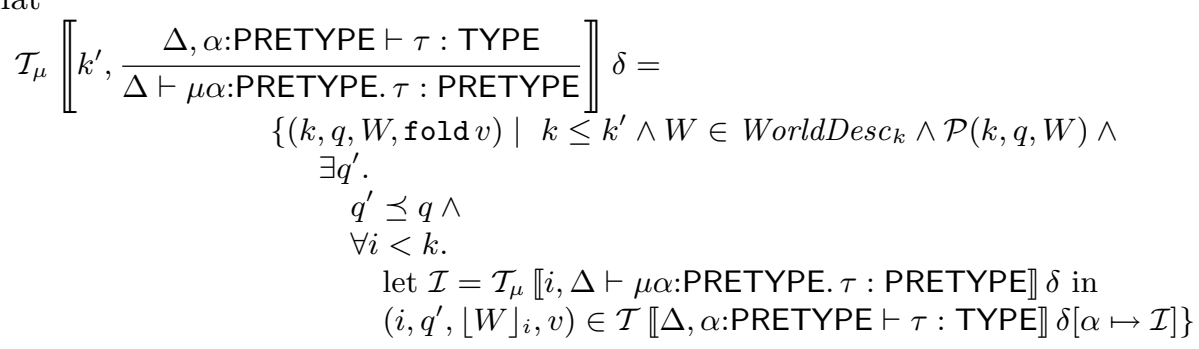

Consider arbitrary $(k, q, W, v) \in \mathcal{T}_{\mu} \llbracket k^{\prime}, \Delta \vdash \mu \alpha$ :PRETYPE. $\tau$ : PRETYPE $\rrbracket \delta$.

Hence, $k \leq k^{\prime}$ and $v \equiv$ fold $v_{\mu}$ and $W \in$ WorldDesc $_{k}$ and $\mathcal{P}(k, q, W)$ and there exists $q_{\mu}^{\prime}$ such that

- $q_{\mu}^{\prime} \preceq q$,

- $\forall i<k$.

let $\mathcal{I}=\mathcal{T}_{\mu} \llbracket i, \Delta \vdash \mu \alpha:$ PRETYPE. $\tau$ :PRETYPE $\rrbracket \delta$ in

$\left(i, q_{\mu}^{\prime},\lfloor W\rfloor_{i}, v_{\mu}\right) \in \mathcal{T} \llbracket \Delta, \alpha: \mathrm{PRETYPE} \vdash \tau: \mathrm{TYPE} \rrbracket \delta[\alpha \mapsto \mathcal{I}]$.

We are required to show that

- $W \in$ WorldDesc $_{k}$, which follows from above, and

- $\mathcal{P}(k, q, W)$, which follows from above.

Consider $j \leq k$.

We are required to show that $\left(j, q,\lfloor W\rfloor_{j}\right.$, fold $\left.v_{\mu}\right) \in \mathcal{T}_{\mu} \llbracket k^{\prime}, \Delta \vdash \mu \alpha$ :PRETYPE. $\tau$ : PRETYPE $\rrbracket \delta$.

Note that $j \leq k^{\prime}$, which follows from $j \leq k$ and $k \leq k^{\prime}$.

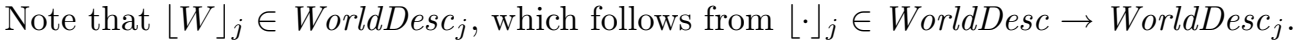

Note that $\mathcal{P}\left(j, q,\lfloor W\rfloor_{j}\right)$, which follows from Req 10 (qualpred-closed) and Req 11 (qualpred-aprx).

Take $q^{\prime}=q_{\mu}^{\prime}$. Note that

- $q^{\prime} \preceq q \equiv q_{\mu}^{\prime} \preceq q$, which follows from above, 
- $\forall i<j$.

let $\mathcal{I}=\mathcal{T}_{\mu} \llbracket i, \Delta \vdash \mu \alpha:$ PRETYPE. $\tau:$ PRETYPE $\rrbracket \delta$ in

$\left(i, q^{\prime},\lfloor W\rfloor_{i}, v_{\mu}\right) \in \mathcal{T} \llbracket \Delta, \alpha: \operatorname{PRETYPE} \vdash \tau: \operatorname{TYPE} \rrbracket \delta[\alpha \mapsto \mathcal{I}]:$

Consider arbitrary $i<j$.

Let $\mathcal{I}=\mathcal{T}_{\mu} \llbracket i, \Delta \vdash \mu \alpha$ :PRETYPE. $\tau$ : PRETYPE $\rrbracket \delta$.

We are required to show that $\left(i, q^{\prime},\lfloor W\rfloor_{i}, v_{\mu}\right) \equiv\left(i, q_{\mu}^{\prime},\lfloor W\rfloor_{i}, v_{\mu}\right) \in$ $\mathcal{T} \llbracket \Delta, \alpha: \kappa \vdash \tau:$ TYPE $\rrbracket \delta[\alpha \mapsto \mathcal{I}]$.

Instantiate $\forall i<k$. let $\mathcal{I}=\mathcal{T}_{\mu} \llbracket i, \Delta \vdash \mu \alpha$ :PRETYPE. $\tau$ : PRETYPE $\rrbracket \delta$ in $\left(i, q_{\mu}^{\prime},\lfloor W\rfloor_{i}, v_{\mu}\right) \in$ $\mathcal{T} \llbracket \Delta, \alpha$ :PRETYPE $\vdash \tau:$ TYPE $\rrbracket \delta[\alpha \mapsto \mathcal{I}]$ with $i$, noting that $i<k$, which follows from $i<j$ and $j \leq k$.

Hence, $\left(i, q_{\mu}^{\prime},\lfloor W\rfloor_{i}, v_{\mu}\right) \in \mathcal{T} \llbracket \Delta, \alpha: \operatorname{PRETYPE} \vdash \tau: \operatorname{TYPE} \rrbracket \delta[\alpha \mapsto \mathcal{I}]$.

Note that $\left\lfloor\lfloor W\rfloor_{j}\right\rfloor_{i} \equiv\lfloor W\rfloor_{i}$, which follows from Req 1 (aprx-idem).

Hence, $\left(i,\left\lfloor\lfloor W\rfloor_{j}\right\rfloor_{i}, v\right) \in \mathcal{T} \llbracket \Delta, \alpha$ :PRETYPE $\vdash \tau: \operatorname{TYPE} \rrbracket \delta[\alpha \mapsto \mathcal{I}]$.

\section{End Case}

Lemma 24 (Rec Extension: $\mathcal{T} \llbracket \Delta \vdash \iota: \kappa \rrbracket \delta \in \mathcal{K} \llbracket \kappa \rrbracket)$

Let $\delta \in \mathcal{D} \llbracket \Delta \rrbracket$ and $\Delta \vdash \iota: \kappa$.

Then $\mathcal{T} \llbracket \Delta \vdash \iota: \kappa \rrbracket \delta \in \mathcal{K} \llbracket \kappa \rrbracket$.

Proof (Ref Extension: $\mathcal{T} \llbracket \Delta \vdash \iota: \kappa \rrbracket \delta \in \mathcal{K} \llbracket \kappa \rrbracket$ )

Recall from Lemma 8, for $\kappa \equiv$ QUAL, it suffices to prove the following:

$$
\mathcal{T} \llbracket \Delta \vdash \xi: \mathrm{QUAL} \rrbracket \delta \in \text { Quals. }
$$

Recall from Lemma 8, for $\kappa \equiv$ PRETYPE, it suffices to prove the following:

$$
\begin{aligned}
\forall(k, q, W, v) \in \mathcal{T} \llbracket \Delta \vdash \bar{\tau}: \mathrm{PRETYPE} \rrbracket & \delta . \quad W \in \text { WorldDesc }_{k} \wedge \mathcal{P}(k, q, W) \wedge \\
& \forall j \leq k .\left(j, q,\lfloor W\rfloor_{j}, v\right) \in \mathcal{T} \llbracket \Delta \vdash \bar{\tau}: \mathrm{PRETYPE} \rrbracket \delta .
\end{aligned}
$$

Recall from Lemma 8, for $\kappa \equiv$ TYPE, it suffices to prove the following:

$$
\begin{aligned}
\exists q^{\prime} \in \text { Quals. } \forall(k, q, W, v) \in \mathcal{T} \llbracket \Delta \vdash \tau: \text { TYPE } \rrbracket & \delta . \\
& \forall \in \text { WorldDesc }_{k} \wedge \mathcal{P}(k, q, W) \wedge \\
& \forall=q^{\prime} .\left(j, q,\lfloor W\rfloor_{j}, v\right) \in \mathcal{T} \llbracket \Delta \vdash \bar{\tau}: \mathrm{PRETYPE} \rrbracket \delta \wedge \\
&
\end{aligned}
$$

Proceed by induction on the derivation $\Delta \vdash \iota: \kappa$.

(RECPTY)

Case $\frac{\Delta, \alpha: \text { PRETYPE } \vdash \tau: \text { TYPE }}{\Delta \vdash \mu \alpha: \text { PRETYPE. } \tau: \text { PRETYPE }}:$

Recall that

$$
\mathcal{T} \llbracket \Delta \vdash \mu \alpha \text { :PRETYPE. } \tau \text { : PRETYPE } \rrbracket \delta=\bigcup_{k^{\prime} \geq 0} \mathcal{T}_{\mu} \llbracket k^{\prime}, \Delta \vdash \mu \alpha: \text { PRETYPE. } \tau: \text { PRETYPE } \rrbracket \delta
$$

Consider arbitrary $(k, q, W, v) \in \mathcal{T} \llbracket \Delta \vdash \mu \alpha$ :PRETYPE. $\tau$ : PRETYPE $\rrbracket \delta$.

Hence, there exists $k^{\prime} \geq 0$ such that

- $(k, q, W, v) \in \mathcal{T}_{\mu} \llbracket k^{\prime}, \Delta \vdash \mu \alpha:$ PRETYPE. $\tau$ : PRETYPE $\rrbracket \delta$.

Applying Lemma 23 to $\Delta \vdash \mu \alpha$ :PRETYPE. $\tau$ : PRETYPE, we conclude that $\mathcal{T}_{\mu} \llbracket k^{\prime}, \Delta \vdash \mu \alpha:$ PRETYPE. $\tau$ : PRETYPE $\rrbracket \delta \in$ PreType.

Applying Fact 5 to $(k, q, W, v) \in \mathcal{T}_{\mu} \llbracket k^{\prime}, \Delta \vdash \mu \alpha:$ PRETYPE. $\tau:$ PRETYPE $\rrbracket \delta \in$ PreType, we conclude that $W \in$ WorldDesc $_{k}$ and $\mathcal{P}(k, q, W)$ and $\forall j \leq k .\left(j, q,\lfloor W\rfloor_{j}, v\right) \in$ $\mathcal{T}_{\mu} \llbracket k^{\prime}, \Delta \vdash \mu \alpha$ :PRETYPE. $\tau:$ PRETYPE $\rrbracket \delta$.

We are required to show that 
- $W \in W_{\text {orldDesc }}$, which follows from above,

- $\mathcal{P}(k, q, W)$, which follows from above,

Consider arbitrary $j \leq k$.

We are required to show that $\left(j, q,\lfloor W\rfloor_{j}, v\right) \in \mathcal{T} \llbracket \Delta \vdash \mu \alpha$ :PRETYPE. $\tau$ : PRETYPE $\rrbracket$.

Note that $\left(j, q,\lfloor W\rfloor_{j}, v\right) \in \mathcal{T}_{\mu} \llbracket k^{\prime}, \Delta \vdash \mu \alpha$ :PRETYPE. $\tau$ : PRETYPE $\rrbracket \delta$, which follows from above. Hence, $\left(j, q,\lfloor W\rfloor_{j}, v\right) \in \mathcal{T} \llbracket \Delta \vdash \mu \alpha$ :PRETYPE. $\tau$ : PRETYPE $\rrbracket$.

\section{End Case}


Lemma 25 (Rec Extension: $\mathcal{T} \llbracket \Delta \vdash \iota: \kappa \rrbracket \delta$ (type-level substitution))

Let $\Delta, \Delta^{\prime} \vdash \mu \alpha$ :PRETYPE. $\tau:$ PRETYPE and $\delta \in \mathcal{D} \llbracket \Delta, \Delta^{\prime} \rrbracket$.

Let $\mathcal{I}=\mathcal{T}_{\mu} \llbracket k^{\prime}, \Delta, \Delta^{\prime} \vdash \mu \alpha:$ PRETYPE. $\tau:$ PRETYPE $\rrbracket \delta$.

Then $\left\lfloor\mathcal{T} \llbracket \Delta, \alpha: \text { PRETYPE, } \Delta^{\prime} \vdash \tau: \text { TYPE } \rrbracket \delta[\alpha \mapsto \mathcal{I}]\right\rfloor_{k^{\prime}+1}=\left\lfloor\mathcal{T} \llbracket \Delta, \Delta^{\prime} \vdash \tau[\mu \alpha: \text { PRETYPE. } \tau / \alpha]: \text { TYPE } \rrbracket \delta\right\rfloor_{k^{\prime}+1}$

Proof (Rec Extension: $\mathcal{T} \llbracket \Delta \vdash \iota: \kappa \rrbracket \delta$ (type-level substitution))

Let $\Delta, \Delta^{\prime} \vdash \mu \alpha$ :PRETYPE. $\tau$ : PRETYPE and $\delta \in \mathcal{D} \llbracket \Delta, \Delta^{\prime} \rrbracket$.

Let $\mathcal{I}=\mathcal{T}_{\mu} \llbracket k^{\prime}, \Delta, \Delta^{\prime} \vdash \mu \alpha:$ PRETYPE. $\tau:$ PRETYPE $\rrbracket \delta$.

Proceed by induction on the derivation $\Delta, \alpha$ :PRETYPE, $\Delta^{\prime} \vdash \tau$ : TYPE.

Case (UserPTy)...:

Case (UserTerm)...:

End Case 


\section{B.6.2 Validity of Typing Rules}

\section{Theorem 26 (Rec Extension Soundness)}

$$
\text { If } \Delta ; \Gamma \vdash e: \tau \text {, then } \llbracket \Delta ; \Gamma \vdash e: \tau \rrbracket \text {. }
$$

\section{Proof}

By induction on the derivation $\Delta ; \Gamma \vdash e: \tau$.

(FOLD)

Case $\frac{\Delta \vdash \xi \quad \Delta ; \Gamma \vdash v_{1}: \tau[\mu \alpha: \text { PRETYPE. } \tau / \alpha] \quad \Delta \vdash \tau[\mu \alpha: \text { PRETYPE. } \tau / \alpha] \preceq \xi}{\Delta ; \Gamma \vdash \text { fold } v_{1}:{ }^{\xi} \mu \alpha: \text { PRETYPE. } \tau}:$

We are required to show $\llbracket \Delta ; \Gamma \vdash$ fold $v_{1}:{ }^{\xi} \mu \alpha:$ PRETYPE. $\tau \rrbracket$.

Consider arbitrary $k, \delta, q_{\Gamma}, W_{\Gamma}$, and $\gamma$ such that

- $k \geq 0$,

- $\delta \in \mathcal{D} \llbracket \Delta \rrbracket$, and

- $\left(k, q_{\Gamma}, W_{\Gamma}, \gamma\right) \in \mathcal{G} \llbracket \Delta \vdash \Gamma \rrbracket \delta$.

Let $e_{s}=\gamma\left(\right.$ fold $\left.v_{1}\right) \equiv \operatorname{fold} \gamma\left(v_{1}\right)$ and $W_{s}=W_{\Gamma}$.

We are required to show that $\operatorname{Comp}\left(k, W_{s}, e_{s}, \mathcal{T} \llbracket \Delta \vdash^{\xi} \mu \alpha: \operatorname{PRETYPE} . \tau: \operatorname{TYPE} \rrbracket \delta\right) \equiv$ $\operatorname{Comp}\left(k, W_{\Gamma}\right.$, fold $\gamma\left(v_{1}\right), \mathcal{T} \llbracket \Delta \vdash{ }^{\xi} \mu \alpha:$ PRETYPE. $\tau$ : TYPE $\left.\rrbracket \delta\right)$.

Consider arbitrary $j, W_{r}, w_{s}, w_{f}$, and $e_{f}$ such that

- $j<k$,

- $w_{s}:_{k}\left(W_{s} \odot_{k} W_{r}\right) \equiv w_{s}:_{k} W_{\Gamma} \odot_{k} W_{r}$

- $\left(w_{s}, e_{s}\right) \equiv\left(w_{s}\right.$, fold $\left.\gamma\left(v_{1}\right)\right) \longmapsto{ }^{j}\left(w_{f}, e_{f}\right)$, and

- $\operatorname{irred}\left(w_{f}, e_{f}\right)$.

Since fold $\gamma\left(v_{1}\right)$ is a value, we have $\operatorname{irred}\left(w_{s}\right.$, fold $\left.\gamma\left(v_{1}\right)\right)$.

Hence, $j=0$ and $w_{f} \equiv w_{s}$ and $e_{f} \equiv \operatorname{fold} \gamma\left(v_{1}\right)$.

Applying the induction hypothesis to $\Delta ; \Gamma \vdash v_{1}: \tau[\mu \alpha$ :PRETYPE. $\tau / \alpha]$, we conclude that $\llbracket \Delta ; \Gamma \vdash v_{1}: \tau[\mu \alpha:$ PRETYPE. $\tau / \alpha] \rrbracket$.

Instantiate this with $k, \delta, q_{\Gamma}, W_{\Gamma}$, and $\gamma$. Note that

- $k \geq 0$,

- $\delta \in \mathcal{D} \llbracket \Delta \rrbracket$, and

- $\left(k, q_{\Gamma}, W_{\Gamma}, \gamma\right) \in \mathcal{G} \llbracket \Delta \vdash \Gamma \rrbracket \delta$.

Hence, $\operatorname{Comp}\left(k, W_{\Gamma}, \gamma\left(v_{1}\right), \mathcal{T} \llbracket \Delta \vdash \tau[\mu \alpha: \operatorname{PRETYPE} . \tau / \alpha]:\right.$ TYPE $\left.\rrbracket \delta\right)$.

Instantiate this with $0, W_{r}, w_{s}, w_{s}$, and $\gamma\left(v_{1}\right)$. Note that

- $0<k$, which follows from $j=0$ and $j<k$,

- $w_{s}:_{k}\left(W_{\Gamma} \odot_{k} W_{r}\right)$, which follows from above,

- $\left(w_{s}, \gamma\left(v_{1}\right)\right) \longmapsto{ }^{0}\left(w_{s}, \gamma\left(v_{1}\right)\right)$, and

- $\operatorname{irred}\left(w_{s}, \gamma\left(v_{1}\right)\right)$, which follows from the fact that $\gamma\left(v_{1}\right)$ is a value.

Hence, there exists $W_{f_{1}}$ and $q_{f_{1}}$ such that

- $w_{s}:_{k-0}\left(W_{f_{1}} \odot_{k-0} W_{r}\right)$, and

- $\left(k-0, q_{f_{1}}, W_{f_{1}}, \gamma\left(v_{1}\right)\right) \in \mathcal{T} \llbracket \Delta \vdash \tau[\mu \alpha:$ PRETYPE. $\tau / \alpha]:$ TYPE $\rrbracket$. 
Let $W_{f}=W_{f_{1}}$ and $q_{f}=\mathcal{T} \llbracket \Delta \vdash \xi: \mathrm{QUAL} \rrbracket \delta$. We are required to show that

- $w_{f}: k-0\left(W_{f} \odot_{k-0} W_{r}\right)$

$\equiv w_{s}:_{k}\left(W_{f_{1}} \odot_{k} W_{r}\right)$,

which follows from above, and

- $\left(k-0, q_{f}, W_{f}, e_{f}\right) \in \mathcal{T} \llbracket \Delta \vdash{ }^{\xi} \mu \alpha:$ PRETYPE. $\tau:$ TYPE $\rrbracket \delta$

$\equiv\left(k, \mathcal{T} \llbracket \Delta \vdash \xi: \mathrm{QUAL} \rrbracket \delta, W_{f_{1}}\right.$, fold $\left.\gamma\left(v_{1}\right)\right)$

$\in\{(k, q, W, v) \mid$

$q=\mathcal{T} \llbracket \Delta \vdash \xi: \mathrm{QUAL} \rrbracket \delta \wedge$

$(k, q, W, v) \in \mathcal{T} \llbracket \Delta \vdash \mu \alpha: \mathrm{PRETYPE} . \tau: \mathrm{PRETYPE} \rrbracket \delta\}$

$\equiv\left(k, \mathcal{T} \llbracket \Delta \vdash \xi: \mathrm{QUAL} \rrbracket \delta, W_{f_{1}}\right.$, fold $\left.\gamma\left(v_{1}\right)\right)$

$\in\{(k, q, W, v) \mid$

$q=\mathcal{T} \llbracket \Delta \vdash \xi: \mathrm{QUAL} \rrbracket \delta \wedge$

$(k, q, W, v) \in \bigcup_{k^{\prime}>0} \mathcal{T}_{\mu} \llbracket k^{\prime}, \Delta \vdash \mu \alpha:$ PRETYPE. $\tau:$ PRETYPE $\left.\rrbracket \delta\right\}$

$\equiv\left(k, \mathcal{T} \llbracket \Delta \vdash \xi: \mathrm{QUAL} \rrbracket \delta, W_{f_{1}}\right.$, fold $\left.\gamma\left(v_{1}\right)\right)$

$\in\left\{(k, q, W\right.$, fold $v) \mid \exists k^{\prime} . k \leq k^{\prime} \wedge W \in$ WorldDesc $_{k} \wedge \mathcal{P}(k, q, W) \wedge$

$q=\mathcal{T} \llbracket \Delta \vdash \xi: \mathrm{QUAL} \rrbracket \delta \wedge$

$\exists q^{\prime}$.

$q^{\prime} \preceq q \wedge$

$\forall i<k$.

let $\mathcal{I}=\mathcal{T}_{\mu} \llbracket i, \Delta \vdash \mu \alpha:$ PRETYPE. $\tau:$ PRETYPE $\rrbracket \delta$ in

which follows from

$\left(i, q^{\prime},\lfloor W\rfloor_{i}, v\right) \in \mathcal{T} \llbracket \Delta, \alpha:$ PRETYPE $\vdash \tau:$ TYPE $\left.\rrbracket \delta[\alpha \mapsto \mathcal{I}]\right\}$

- $\exists k^{\prime} . k \leq k$

Take $k^{\prime}=k$. Note that $k \leq k$ follows trivially.

- $\mathcal{T} \llbracket \Delta \vdash \xi: \mathrm{QUAL} \rrbracket \delta=\mathcal{T} \llbracket \Delta \vdash \xi: \mathrm{QUAL} \rrbracket \delta$, which follows trivially,

- $W_{f_{1}} \in$ WorldDesc $_{k}$, which follows from Fact 6 to $\left(k, q_{f_{1}}, W_{f_{1}}, \gamma\left(v_{1}\right)\right) \in$ $\mathcal{T} \llbracket \Delta \vdash \tau[\mu \alpha:$ PRETYPE. $\tau / \alpha]:$ TYPE $\delta$,

- $\mathcal{P}\left(k, \mathcal{T} \llbracket \Delta \vdash \xi: \mathrm{QUAL} \rrbracket \delta, W_{f_{1}}\right)$, which follows from Corollary 16 applied to $\Delta \quad \vdash \quad \tau[\mu \alpha$ :PRETYPE. $\tau / \alpha] \preceq \xi$ and $\left(k, q_{f_{1}}, W_{f_{1}}, \gamma\left(v_{1}\right)\right) \quad \in$ $\mathcal{T} \llbracket \Delta \vdash \tau[\mu \alpha$ :PRETYPE. $\tau / \alpha]:$ TYPE $\rrbracket \delta$ and $\mathcal{T} \llbracket \Delta \vdash \xi:$ QUAL $\rrbracket \delta$, and

- $\exists q^{\prime} . \ldots$

Take $q^{\prime}=q_{f_{1}}$. Note that

- $q_{f_{1}} \preceq \mathcal{T} \llbracket \Delta \vdash \xi: \mathrm{QUAL} \rrbracket \delta$, which follows from Lemma 15 applied to $\Delta \vdash \tau[\mu \alpha:$ PRETYPE. $\tau / \alpha] \preceq \xi$ and $\left(k, q_{f_{1}}, W_{f_{1}}, \gamma\left(v_{1}\right)\right) \quad \in$ $\mathcal{T} \llbracket \Delta \vdash \tau[\mu \alpha:$ PRETYPE. $\tau / \alpha]:$ TYPE $\rrbracket \delta$ and $\mathcal{T} \llbracket \Delta \vdash \xi:$ QUAL $\rrbracket \delta$,

- $\forall i<k . \ldots$

Consider arbitrary $i$ such that

- $i<k$.

Let $\mathcal{I}=\mathcal{T}_{\mu} \llbracket i, \Delta \vdash \mu \alpha:$ PRETYPE. $\tau:$ TYPE $\rrbracket \delta$.

We are required to show that $\left(i, q_{f_{1}},\left\lfloor W_{f_{1}}\right\rfloor_{i}, v_{1}\right) \quad \in$ $\mathcal{T} \llbracket \Delta, \alpha: \operatorname{PRETYPE} \vdash \tau:$ TYPE $\rrbracket \delta[\alpha \mapsto \mathcal{I}]$.

Note that $\mathcal{T}_{\mu} \llbracket i, \Delta \vdash \mu \alpha:$ PRETYPE. $\tau$ : PRETYPE $\rrbracket \delta \in \mathcal{K} \llbracket$ PRETYPE $\rrbracket$, which follows from Lemma 23 applied to $\delta \in \mathcal{D} \llbracket \Delta \rrbracket$ and $\Delta \vdash \mu \alpha$ :PRETYPE. $\tau$ : PRETYPE.

Applying Lemma 8 to $\Delta \vdash \tau[\mu \alpha$ :PRETYPE. $\tau / \alpha]$ : TYPE, we conclude that $\mathcal{T} \llbracket \Delta \vdash \tau[\mu \alpha:$ PRETYPE. $\tau / \alpha]:$ TYPE $\rrbracket \delta \in$ Type.

Applying Fact 6 to $\left(k, q_{f_{1}}, W_{f_{1}}, \gamma\left(v_{1}\right)\right) \in \mathcal{T} \llbracket \Delta \vdash \tau[\mu \alpha:$ PRETYPE. $\tau / \alpha]:$ TYPE $\rrbracket \delta \in$ Type instantiated with $i$, noting that 
- $i \leq k$, which follows from $i<k$,

we conclude that $\left(i, q_{f_{1}},\left\lfloor W_{f_{1}}\right\rfloor_{i}, \gamma\left(v_{1}\right)\right) \in \mathcal{T} \llbracket \Delta \vdash \tau[\mu \alpha$ :PRETYPE. $\tau / \alpha]:$ TYPE $\rrbracket \delta$. Hence, $\quad\left(i, q_{f_{1}},\left\lfloor W_{f_{1}}\right\rfloor_{i}, \gamma\left(v_{1}\right)\right) \in\lfloor\mathcal{T} \llbracket \Delta \vdash \tau[\mu \alpha \text { :PRETYPE. } \tau / \alpha]: \operatorname{TYPE} \rrbracket \delta\rfloor_{i+1}$, which follows from the definition $\lfloor\cdot\rfloor_{k}$.

Applying Lemma 25 to $\Delta \vdash \mu \alpha$ :PRETYPE. $\tau$ : PRETYPE and $\delta \in$ $\mathcal{D} \llbracket \Delta \rrbracket$, we conclude that $\lfloor\mathcal{T} \llbracket \Delta, \alpha: \operatorname{PRETYPE} \vdash \tau: \operatorname{TYPE} \rrbracket \delta[\alpha \mapsto \mathcal{I}]\rfloor_{i+1}=$ $\lfloor\mathcal{T} \llbracket \Delta \vdash \tau[\mu \alpha: \text { PRETYPE. } \tau / \alpha]: \text { TYPE } \rrbracket \delta\rfloor_{i+1}$.

Hence, we conclude that $\left(i, q_{f_{1}},\left\lfloor W_{f_{1}}\right\rfloor_{j}, \gamma\left(v_{1}\right)\right) \quad \in$ $\lfloor\mathcal{T} \llbracket \Delta, \alpha: \text { PRETYPE } \vdash \tau: \text { TYPE } \rrbracket \delta[\alpha \mapsto \mathcal{I}]\rfloor_{i+1}$.

Hence, we conclude that $\left(i, q_{f_{1}},\left\lfloor W_{f_{1}}\right\rfloor_{i}, \gamma\left(v_{1}\right)\right) \quad \in$ $\mathcal{T} \llbracket \Delta, \alpha:$ PRETYPE $\vdash \tau:$ TYPE $\rrbracket \delta[\alpha \mapsto \mathcal{I}]$, which follows from the definition of $\lfloor\cdot\rfloor_{k}$. 
(UNFOLD)

Case $\frac{\Delta ; \Gamma \vdash e_{1}:{ }^{\xi} \mu \alpha: \text { PRETYPE. } \tau}{\Delta ; \Gamma \vdash \text { unfold } e_{1}: \tau[\mu \alpha: \text { PRETYPE. } \tau / \alpha]}:$

We are required to show $\llbracket \Delta ; \Gamma \vdash$ unfold $e_{1}: \tau[\mu \alpha$ :PRETYPE. $\tau / \alpha] \rrbracket$.

Consider arbitrary $k, \delta, q_{\Gamma}, W_{\Gamma}$, and $\gamma$ such that

- $k \geq 0$,

- $\delta \in \mathcal{D} \llbracket \Delta \rrbracket$, and

- $\left(k, q_{\Gamma}, W_{\Gamma}, \gamma\right) \in \mathcal{G} \llbracket \Delta \vdash \Gamma \rrbracket \delta$.

Let $e_{s}=\gamma\left(\right.$ unfold $\left.e_{1}\right) \equiv \operatorname{unfold} \gamma\left(e_{1}\right)$ and $W_{s}=W_{\Gamma}$.

We are required to show that $\operatorname{Comp}\left(k, W_{s}, e_{s}, \mathcal{T} \llbracket \Delta \vdash \tau[\mu \alpha: \operatorname{PRETYPE} . \tau / \alpha]:\right.$ TYPE $\left.\rrbracket\right) \equiv$ $\operatorname{Comp}\left(k, W_{\Gamma}\right.$, unfold $\gamma\left(e_{1}\right), \mathcal{T} \llbracket \Delta \vdash \tau[\mu \alpha$ :PRETYPE. $\tau / \alpha]:$ TYPE $\left.\rrbracket \delta\right)$.

Consider arbitrary $j, W_{r}, w_{s}, w_{f}$, and $e_{f}$ such that

- $j<k$,

- $w_{s}:_{k}\left(W_{s} \odot_{k} W_{r}\right) \equiv w_{s}:_{k}\left(W_{\Gamma} \odot_{k} W_{r}\right)$,

- $\left(w_{s}, e_{s}\right)=\left(w_{s}\right.$, unfold $\left.\gamma\left(e_{1}\right)\right) \longmapsto^{j}\left(w_{f}, e_{f}\right)$, and

- $\operatorname{irred}\left(w_{f}, e_{f}\right)$.

Hence, by inspection of the operational semantics, it follows that there exist $j_{1}, w_{f_{1}}$, and $e_{f_{1}}$ such that

- $\left(w_{s}, \gamma\left(e_{1}\right)\right) \longmapsto{ }^{j_{1}}\left(w_{f_{1}}, e_{f_{1}}\right)$,

- $\operatorname{irred}\left(w_{f_{1}}, e_{f_{1}}\right)$, and

- $j_{1} \leq j$.

Applying the induction hypothesis to $\Delta ; \Gamma \vdash e_{1}:{ }^{\xi} \mu \alpha$ :PRETYPE. $\tau$, we conclude that $\llbracket \Delta ; \Gamma \vdash e_{1}:{ }_{\mu \alpha}$ PRETYPE. $\tau \rrbracket$.

Instantiate this with $k, \delta, q_{\Gamma}, W_{\Gamma}$, and $\gamma$. Note that

- $k \geq 0$,

- $\delta \in \mathcal{D} \llbracket \Delta \rrbracket$, and

- $\left(k, q_{\Gamma}, W_{\Gamma},, \gamma\right) \in \mathcal{G} \llbracket \Delta \vdash \Gamma \rrbracket \delta$.

Hence, $\operatorname{Comp}\left(k, W_{\Gamma}, \gamma\left(e_{1}\right), \mathcal{T} \llbracket \Delta \vdash{ }^{\xi} \mu \alpha:\right.$ PRETYPE. $\tau:$ TYPE $\left.\rrbracket \delta\right)$.

Instantiate this with $j_{1}, W_{r}, w_{s}, w_{f_{1}}$, and $e_{f_{1}}$. Note that

- $j_{1}<k$, which follows from $j_{1} \leq j$ and $j<k$,

- $w_{s}:_{k}\left(W_{\Gamma} \odot_{k} W_{r}\right)$, which follows from above,

- $\left(w_{s}, \gamma\left(e_{1}\right)\right) \longmapsto{ }^{j_{1}}\left(w_{f_{1}}, e_{f_{1}}\right)$,

- $\operatorname{irred}\left(w_{f_{1}}, e_{f_{1}}\right)$.

Hence, there exists $W_{f_{1}}$ and $q_{f_{1}}$ such that

- $w_{f_{1}}:_{k-j_{1}}\left(W_{f_{1}} \odot_{k-j_{1}} W_{r}\right)$, and

- $\left(k-j_{1}, q_{f_{1}}, W_{f_{1}}, e_{f_{1}}\right)$

$\in \mathcal{T} \llbracket \Delta \vdash^{\xi} \mu \alpha:$ PRETYPE. $\tau:$ TYPE $\| \delta$

$\equiv\left\{(k, q, W\right.$, fold $v) \mid \exists k^{\prime} . k \leq k^{\prime} \wedge W \in$ WorldDesc $_{k} \wedge \mathcal{P}(k, q, W) \wedge$ $q=\mathcal{T} \llbracket \Delta \vdash \xi: \mathrm{QUAL} \rrbracket \delta \wedge$

$\exists q^{\prime}$.

$q^{\prime} \preceq q \wedge$

$\forall i<k$.

let $\mathcal{I}=\mathcal{T}_{\mu} \llbracket i, \Delta \vdash \mu \alpha$ :PRETYPE. $\tau$ : PRETYPE $\rrbracket \delta$ in

$\left.\left(i, q^{\prime},\lfloor W\rfloor_{i}, v\right) \in \mathcal{T} \llbracket \Delta, \alpha: \mathrm{PRETYPE} \vdash \tau: \mathrm{TYPE} \rrbracket \delta[\alpha \mapsto \mathcal{I}]\right\}$ 
Hence, $e_{f_{1}} \equiv$ fold $v_{f_{11}}$ and $q_{f_{1}}=\mathcal{T} \llbracket \Delta \vdash \xi:$ QUAL $\rrbracket \delta$ and there exists $q^{\prime}$ such that $q^{\prime} \preceq$ $q_{f_{1}}$ and $\forall i<k-j_{1}$. let $\mathcal{I}=\mathcal{T}_{\mu} \llbracket i, \Delta \vdash \mu \alpha$ :PRETYPE. $\tau$ :PRETYPE $\rrbracket \delta$ in $\left(i, q^{\prime},\lfloor W\rfloor_{i}, v\right) \in$ $\mathcal{T} \llbracket \Delta, \alpha: \operatorname{PRETYPE} \vdash \tau:$ TYPE $\rrbracket \delta[\alpha \mapsto \mathcal{I}]$.

Note that

$$
\begin{aligned}
\left(w_{s}, e_{s}\right) & \equiv\left(w_{s}, \text { unfold } \gamma\left(e_{1}\right)\right) \\
& \longmapsto\left(w_{f_{1}}, \text { unfold } e_{f_{1}}\right) \\
& \equiv\left(w_{f_{1}}, \text { unfold }\left(\text { fold } v_{f_{11}}\right)\right) \\
& \longmapsto\left(w_{f_{1}}, v_{f_{11}}\right) \\
& \longmapsto{ }^{j-j_{1}-1}\left(w_{f}, e_{f}\right)
\end{aligned}
$$

Since $\left(w_{f_{1}}, v_{f_{11}}\right)$ is a value, we have $\operatorname{irred}\left(w_{f_{1}}, v_{f_{11}}\right)$.

Hence, $j-j_{1}-1=0$ (and $j=j_{1}+1$ ) and $w_{f} \equiv w_{f_{1}}$ and $e_{f} \equiv v_{f_{11}}$.

Note that $\left\lfloor\left(W_{f_{1}} \odot_{k-j_{1}} W_{r}\right)\right\rfloor_{k-j_{1}-1} \equiv\left(\left\lfloor W_{f_{1}}\right\rfloor_{k-j_{1}-1} \odot_{k-j_{1}-1} W_{r}\right)$, which follows from

$$
\begin{aligned}
& \left\lfloor\left(W_{f_{1}} \odot_{k-j_{1}} W_{r}\right)\right\rfloor_{k-j_{1}-1} \\
& \equiv\left(W_{f_{1}} \odot_{k-j_{1}-1} W_{r}\right) \\
& \quad \text { which follows from Req } 4 \text { (join-closed) } \\
& \equiv\left(\left\lfloor W_{f_{1}}\right\rfloor_{k-j_{1}-1} \odot_{k-j_{1}-1} W_{r}\right) \\
& \quad \text { which follows from Req } 5 \text { (join-aprx). }
\end{aligned}
$$

Let $W_{f}=\left\lfloor W_{f_{1}}\right\rfloor_{k-j_{1}-1}$ and $q_{f}=q^{\prime}$.

We are required to show that

- $w_{f}:_{k-j}\left(W_{f} \odot_{k-j} W_{r}\right)$

$$
\equiv w_{f_{1}}: k-j_{1}-1\left(\left\lfloor W_{f_{1}}\right\rfloor_{k-j_{1}-1} \odot_{k-j_{1}-1} W_{r}\right) \text {, }
$$

which follows from

$$
\begin{aligned}
& w_{f_{1}}: k-j_{1}\left(W_{f_{1}} \odot_{k-j_{1}} W_{r}\right) \\
& \quad \text { which follows from above } \\
& \Rightarrow w_{f_{1}}: k-j_{1}-1\left(W_{f_{1}} \odot_{k-j_{1}} W_{r}\right) \\
& \quad \text { which follows from Req } 2 \text { (models-closed) } \\
& \Leftrightarrow w_{f_{1}}: k-j_{1}-1\left\lfloor\left(W_{f_{1}} \odot_{k-j_{1}} W_{r}\right)\right\rfloor_{k-j_{1}-1} \\
& \quad \text { which follows from Req } 3 \text { (models-aprx) } \\
& \\
& \left.\qquad\left(W_{f_{1}} \odot_{k-j_{1}} W_{r}\right)\right\rfloor_{k-j_{1}-1} \equiv\left(\left\lfloor W_{f_{1}}\right\rfloor_{k-j_{1}-1} \odot_{k-j_{1}-1} W_{r}\right) \\
& \quad \text { which follows from above, }
\end{aligned}
$$

- $\left(k-j, q_{f}, W_{f}, e_{f}\right) \in \mathcal{T} \llbracket \Delta \vdash \tau[\mu \alpha:$ PRETYPE. $\tau / \alpha]:$ TYPE $\rrbracket \delta$

$$
\equiv\left(k-j_{1}-1, q^{\prime},\left\lfloor W_{f_{1}}\right\rfloor_{k-j_{1}-1}, v_{f_{11}}\right) \in \mathcal{T} \llbracket \Delta \vdash \tau[\mu \alpha: \text { PRETYPE. } \tau / \alpha]: \text { TYPE } \rrbracket \delta
$$

Instantiate $\forall i<k-j_{1}$. let $\mathcal{I}=\mathcal{T}_{\mu} \llbracket i, \Delta \vdash \mu \alpha$ :PRETYPE. $\tau$ : PRETYPE $\rrbracket \delta$ in $\left(i, q^{\prime},\lfloor W\rfloor_{i}, v\right) \in$ $\mathcal{T} \llbracket \Delta, \alpha: \operatorname{PRETYPE} \vdash \tau: \mathrm{TYPE} \rrbracket \delta[\alpha \mapsto \mathcal{I}]$ with $k-j_{1}-1$. Note that

- $k-j_{1}-1<k-j_{1}$.

Let $\mathcal{I}=\mathcal{T}_{\mu} \llbracket k-j_{1}-1, \Delta \vdash \mu \alpha:$ PRETYPE. $\tau: \mathrm{PRETYPE} \rrbracket \delta$.

Hence, we conclude that $\left(k-j_{1}-1, q^{\prime},\left\lfloor W_{f_{1}}\right\rfloor_{k-j_{1}-1}, v_{f_{11}}\right) \in \mathcal{T} \llbracket \Delta, \alpha: \kappa \vdash \tau: \operatorname{TYPE} \rrbracket \delta[\alpha \mapsto \mathcal{I}]$. Hence, $\left(k-j_{1}-1, q^{\prime},\left\lfloor W_{f_{1}}\right\rfloor_{k-j_{1}-1}, v_{f_{11}}\right) \in\lfloor\mathcal{T} \llbracket \Delta, \alpha: \kappa \vdash \tau: \text { TYPE } \rrbracket[\alpha \mapsto \mathcal{I}]\rfloor_{k-j_{1}}$, which follows from the definition $\lfloor\cdot\rfloor_{k}$.

Applying Lemma 25 to $\Delta \vdash \mu \alpha$ :PRETYPE. $\tau$ : PRETYPE and $\delta \in$ $\mathcal{D} \llbracket \Delta \rrbracket$, we conclude that $\lfloor\mathcal{T} \llbracket \Delta, \alpha: \text { PRETYPE } \vdash \tau: \text { TYPE } \rrbracket \delta[\alpha \quad \mapsto \quad \mathcal{I}]\rfloor_{k-j_{1}}=$ $\lfloor\mathcal{T} \llbracket \Delta \vdash \tau[\mu \alpha: \text { PRETYPE. } \tau / \alpha]: \text { TYPE } \rrbracket \delta\rfloor_{k-j_{1}}$.

Hence, we conclude that $\left(k-j_{1}-1, q^{\prime},\left\lfloor W_{f_{1}}\right\rfloor_{k-j_{1}-1}, v_{f_{11}}\right) \quad \in$ $\lfloor\mathcal{T} \llbracket \Delta \vdash \tau[\mu \alpha: \text { PRETYPE. } \tau / \alpha]: \text { TYPE } \rrbracket \delta\rfloor_{k-j_{1}}$.

Hence, we conclude that $\left(k-j_{1}-1, q^{\prime},\left\lfloor W_{f_{1}}\right\rfloor_{k-j_{1}-1}, v_{f_{11}}\right) \quad \in$ $\mathcal{T} \llbracket \Delta \vdash \tau[\mu \alpha:$ PRETYPE. $\tau / \alpha]:$ TYPE $\rrbracket \delta$, which follows from the definition of $\lfloor\cdot\rfloor_{k}$.

\section{End Case}




\section{References}

\section{C.1 Syntax}

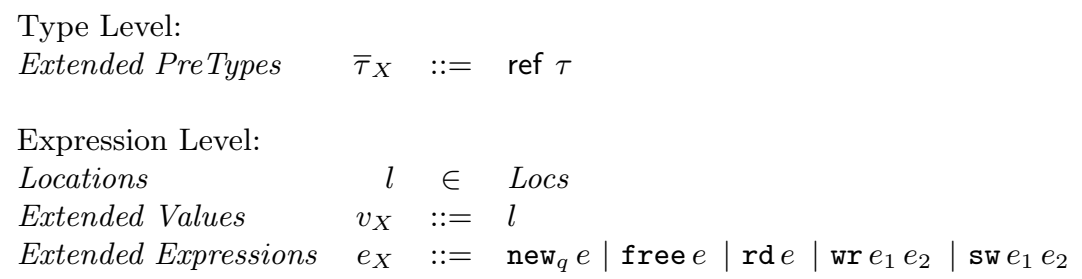

Figure 21: Ref Extension - Syntax 


\section{C.2 Operational Semantics}

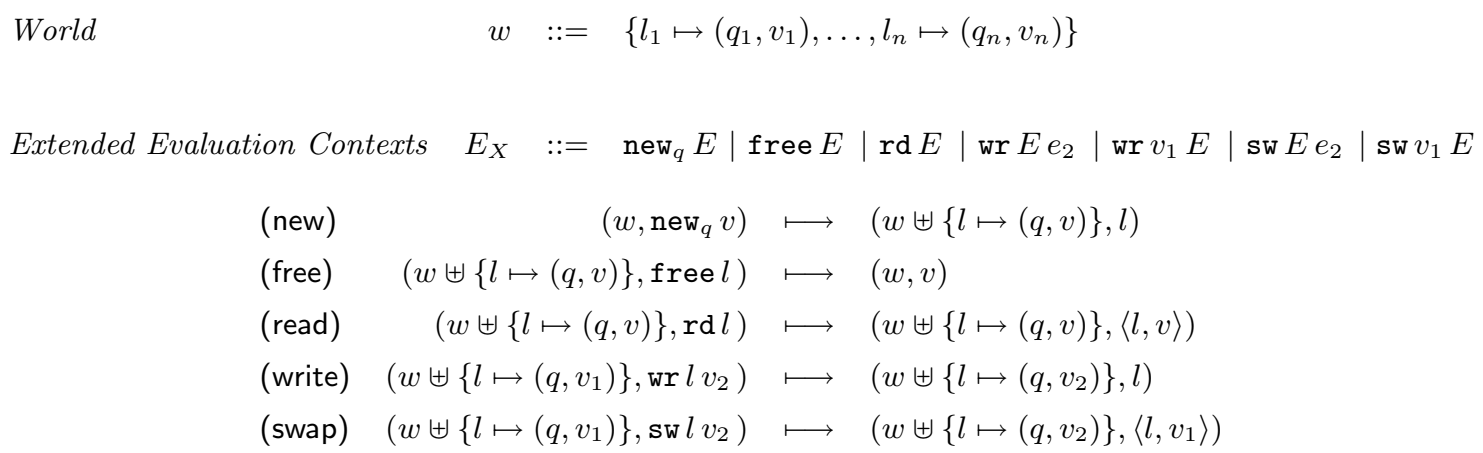

Figure 22: Ref Extension - Operational Semantics 


\section{C.3 Static Semantics}

$\Delta \vdash \iota: \kappa$

$$
\begin{aligned}
& (\text { REFPTY }) \\
& \frac{\Delta \vdash \tau: \text { TYPE }}{\Delta \vdash \operatorname{ref} \tau: \text { PRETYPE }}
\end{aligned}
$$

Figure 23: Ref Extension - Static Semantics (I)

$$
\begin{aligned}
& \Delta ; \Gamma \vdash e: \tau \\
& (\operatorname{New}(\mathrm{U}, \mathrm{A})) \\
& (\operatorname{NEW}(\mathrm{R}, \mathrm{L})) \\
& \text { (FREE) } \\
& \frac{q \preceq \mathrm{A} \quad \Delta ; \Gamma \vdash e: \tau \quad \Delta \vdash \tau \preceq \mathrm{A}}{\Delta ; \Gamma \vdash \text { new }_{q} e:{ }^{q} \operatorname{ref} \tau} \\
& \frac{\mathrm{R} \preceq q \quad \Delta ; \Gamma \vdash e: \tau}{\Delta ; \Gamma \vdash \text { new }_{q} e:{ }^{q} \operatorname{ref} \tau} \\
& \frac{\Delta ; \Gamma \vdash e:{ }^{\xi} \text { ref } \tau \quad \Delta \vdash \mathrm{A} \preceq \xi}{\Delta ; \Gamma \vdash \text { free } e: \tau} \\
& \text { (READ) } \\
& \frac{\Delta ; \Gamma \vdash e:{ }^{\xi} \operatorname{ref} \tau \quad \Delta \vdash \tau \preceq \mathrm{R}}{\Delta ; \Gamma \vdash \operatorname{rd} e:{ }^{\mathrm{L}}\left({ }^{\xi} \text { ref } \tau \otimes \tau\right)} \\
& \frac{\Delta \vdash \Gamma \sim \Gamma_{1} \boxplus \Gamma_{2} \quad \Delta ; \Gamma_{1} \vdash e_{1}:{ }^{\xi} \text { ref } \tau_{1} \quad \Delta \vdash \tau_{1} \preceq \mathrm{A} \quad \Delta \vdash \mathrm{A} \preceq \xi \quad \Delta ; \Gamma_{2} \vdash e_{2}: \tau_{2} \quad \Delta \vdash \tau_{2} \preceq \xi}{\Delta ; \Gamma \vdash \text { wr } e_{1} e_{2}:{ }^{\xi} \text { ref } \tau_{2}} \\
& \text { (WRITE }(\text { WEAK })) \\
& \frac{\Delta \vdash \Gamma \leadsto \Gamma_{1} \boxplus \Gamma_{2} \quad \Delta ; \Gamma_{1} \vdash e_{1}:{ }^{\xi} \operatorname{ref} \tau \quad \Delta \vdash \tau \preceq \mathrm{A} \quad \Delta ; \Gamma_{2} \vdash e_{2}: \tau}{\Delta ; \Gamma \vdash \text { wr } e_{1} e_{2}:{ }^{\xi} \operatorname{ref} \tau} \\
& (\operatorname{SwAP}(\operatorname{Strong})) \\
& \frac{\Delta \vdash \Gamma \leadsto \Gamma_{1} \boxplus \Gamma_{2} \quad \Delta ; \Gamma_{1} \vdash e_{1}:{ }^{\xi} \text { ref } \tau_{1} \quad \Delta \vdash \mathrm{A} \preceq \xi \quad \Delta ; \Gamma_{2} \vdash e_{2}: \tau_{2} \quad \Delta \vdash \tau_{2} \preceq \xi}{\Delta ; \Gamma \vdash \operatorname{sw} e_{1} e_{2}:{ }^{\mathrm{L}}\left({ }^{\xi} \text { ref } \tau_{2} \otimes \tau_{1}\right)} \\
& (\operatorname{SWAP}(\mathrm{WEAK})) \\
& \frac{\Delta \vdash \Gamma \leadsto \Gamma_{1} \boxplus \Gamma_{2} \quad \Delta ; \Gamma_{1} \vdash e_{1}:{ }^{\xi} \text { ref } \tau \quad \Delta ; \Gamma_{2} \vdash e_{2}: \tau}{\Delta ; \Gamma \vdash \operatorname{sw} e_{1} e_{2}:{ }^{\mathrm{L}}\left({ }^{\xi} \text { ref } \tau \otimes \tau\right)}
\end{aligned}
$$

Figure 24: Ref Extension - Static Semantics (V) 


\section{C.4 Desugar}

C.4.1 wr

Syntax

wr $e_{1} e_{2} \equiv \operatorname{let}\langle r, x\rangle=\operatorname{sw} e_{1} e_{2}$ in $r$

\section{Operational Semantics}

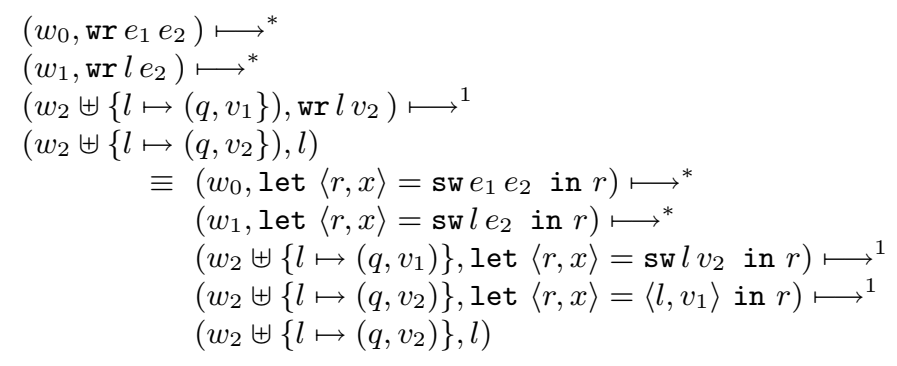

\section{Static Semantics}

(Write(Strong))

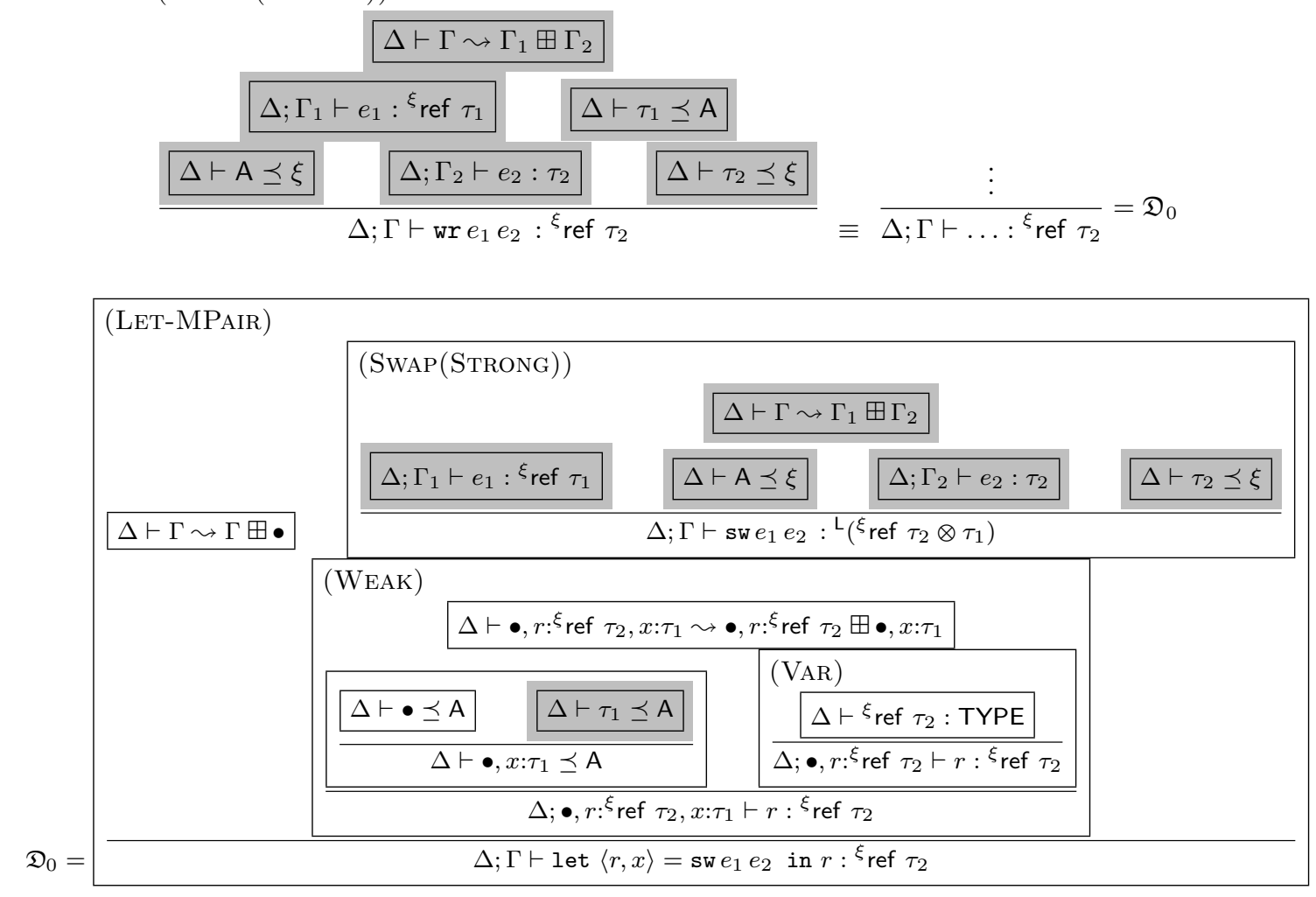




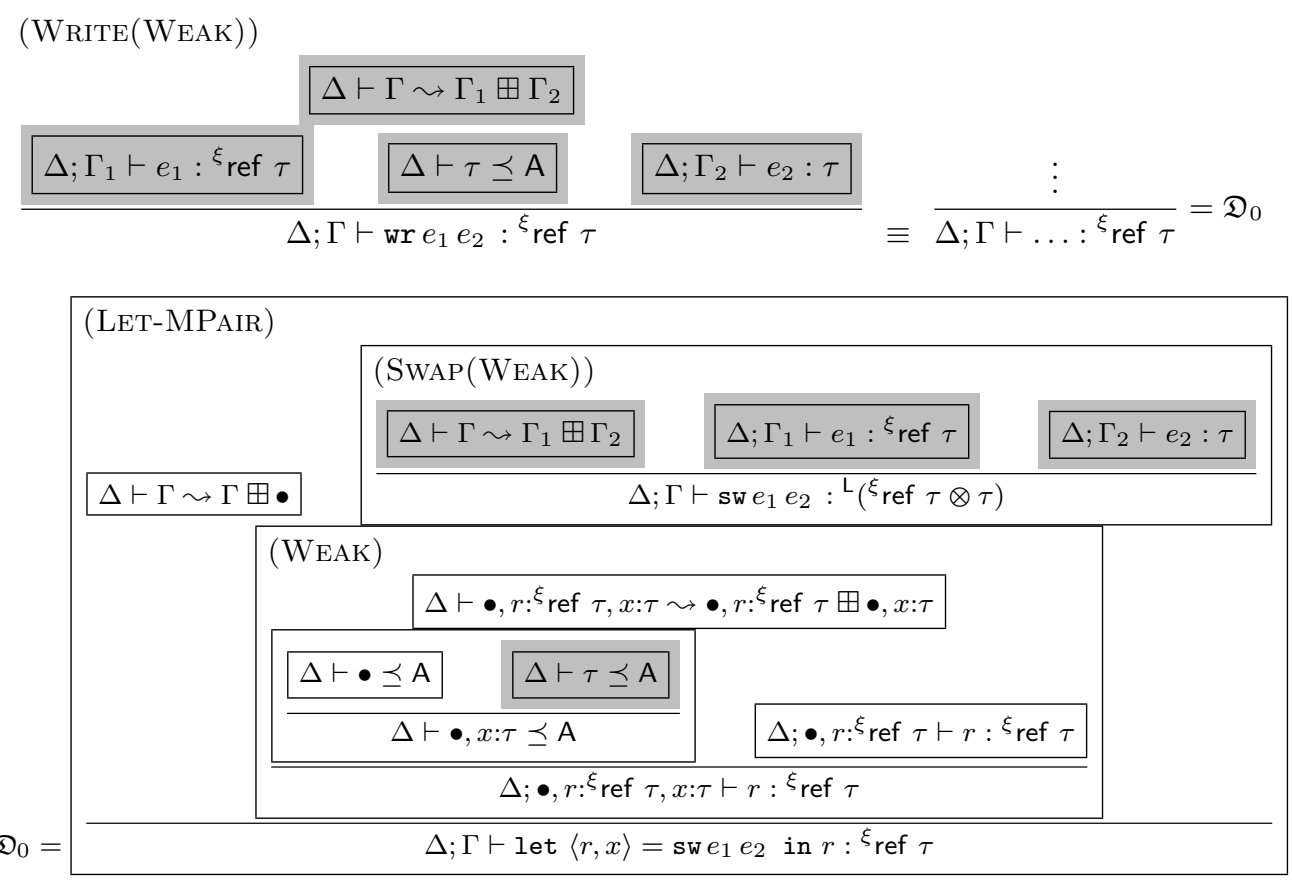




\title{
C.5 Model
}

\author{
World Description (Notation) $\quad W \quad::=\quad\{(l \mapsto(q, \chi), \ldots\}$ \\ $W \in$ CandWorldDesc $_{k}=$ Locs $\rightarrow$ Quals $\times$ CandUberType ${ }_{k}$ \\ $W \in$ CandWorldDesc $_{\omega}=$ Locs $\rightarrow$ Quals $\times$ CandUberType ${ }_{\omega}$ \\ $\bigcup_{k \geq 0}$ CandWorldDesc $_{k} \subseteq$ CandWorldDesc $\omega$ \\ $\lfloor W\rfloor_{k} \quad \stackrel{\text { def }}{=} \quad\left\{l \mapsto\left(q,\lfloor\chi\rfloor_{k}\right) \mid l \in \operatorname{dom}(W) \wedge W(l)=(q, \chi)\right\}$ \\ $\lfloor W\rfloor_{k} \in$ CandWorldDesc $_{\omega} \rightarrow$ CandWorldDesc $_{k}$ \\ $\mathcal{P}(k, q, W) \stackrel{\text { def }}{=} \forall l \in \operatorname{dom}(W) . W^{\text {qual }}(l) \preceq q$ \\ $\mathcal{P}(k, q, W) \in \mathbb{N} \times$ Quals $\times$ CandWorldDesc $\omega \rightarrow \mathbb{P}$ \\ $\mathcal{R}(W) \stackrel{\text { def }}{=} \forall l \in \operatorname{dom}(W) .\left(W^{\text {qual }}(l) \preceq \mathrm{A} \Rightarrow \forall\left({ }_{-}, q^{\prime},,_{-},-\right) \in W^{\text {type }}(l) . q^{\prime} \preceq \mathrm{A}\right)$ \\ $\mathcal{R}(W) \in$ CandWorldDesc $_{\omega} \rightarrow \mathbb{P}$
}

Figure 25: Ref Extension - Semantic Interpretations (Ia) 
$W \in$ WorldDesc $_{k}=\left\{W \in\right.$ Locs $\rightarrow$ Quals $\times$ Type $\left._{k} \mid \mathcal{R}(W)\right\} \subseteq$ CandWorldDesc $_{k}$

$$
\lfloor W\rfloor_{k} \quad \in \quad \text { WorldDesc } \rightarrow \text { WorldDesc }_{k}
$$

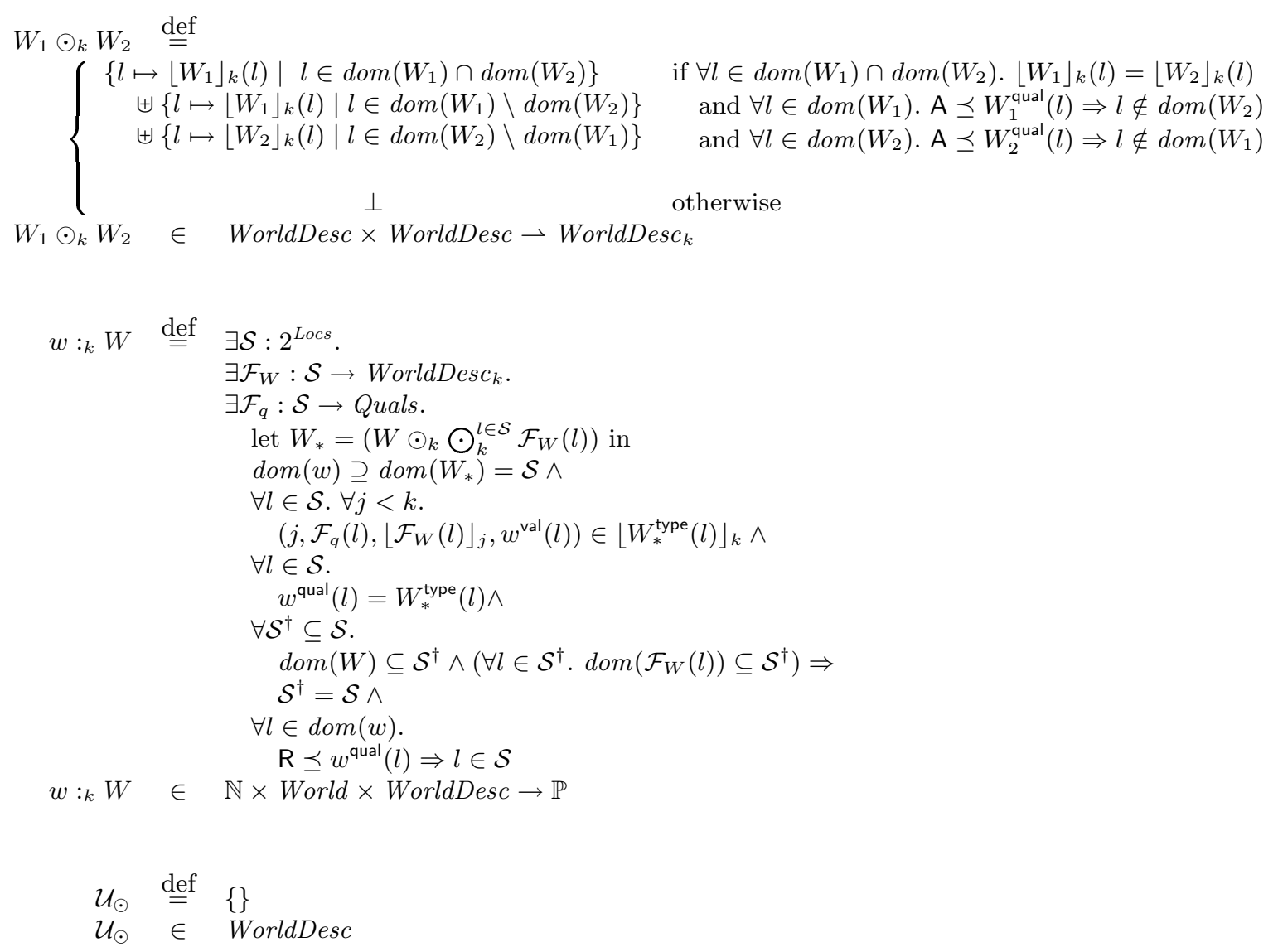

Figure 26: Ref Extension - Semantic Interpretations (Ib) 


$$
\mathcal{T} \llbracket \frac{\Delta \vdash \tau: \text { TYPE }}{\Delta \vdash \operatorname{ref} \tau: \text { PRETYPE }} \rrbracket \delta=\quad \begin{aligned}
\{( & , q,\{l \mapsto(q, \chi)\}, l) \mid \\
& \chi=\lfloor\mathcal{T} \llbracket \Delta \vdash \tau: \text { TYPE } \rrbracket \delta\rfloor_{k} \wedge \\
& \left.\left(q \preceq \mathrm{A} \Rightarrow \forall\left({ }_{-}, q^{\prime},{ }_{-},-\right) \in \chi . q^{\prime} \preceq \mathrm{A}\right)\right\}
\end{aligned}
$$

Figure 27: Ref Extension - Semantic Interpretations (III) 


\section{C.6 Requirements}

- Req 1 (aprx-idem)

$\left\lfloor\lfloor W\rfloor_{k_{2}}\right\rfloor_{k_{1}}=\lfloor W\rfloor_{\min \left(k_{1}, k_{2}\right)}$.

Proof

Immediate from the definition of $\lfloor W\rfloor_{k}$.

- Req 2 (models-closed)

if $j \leq k$ and $w:{ }_{k} W$, then $w::_{j} W$.

Proof

Immediate from the definition of $w:_{k} W$ and Req 5 (join-aprx) and Fact 6.

(Note that Req 2 (models-closed) does not require Req 3 (models-aprx).)

- Req 3 (models-aprx)

$w:_{k} W$ iff $w:_{k}\lfloor W\rfloor_{k}$.

Proof

Note that $\operatorname{dom}(W)=\operatorname{dom}\left(\lfloor W\rfloor_{k}\right)$.

Immediate from the definition of $w: k W$ and Req 5 (join-aprx).

(Note that Req 5 (join-aprx) does not require Req 3 (models-aprx).)

- Req 4 (join-closed)

if $j \leq k$ and $\left(W_{1} \odot_{k} W_{2}=W_{3}\right)$, then $\left(W_{1} \odot_{j} W_{2}=\left\lfloor W_{3}\right\rfloor_{j}\right)$.

Proof

Immediate from the definition of $W_{1} \odot_{k} W_{2}$.

- Req 5 (join-aprx)

$\left(W_{1} \odot_{k} W_{2}=W_{3}\right)$ iff $\left(\left\lfloor W_{1}\right\rfloor_{k} \odot_{k} W_{2}=W_{3}\right)$ iff $\left(W_{1} \odot_{k}\left\lfloor W_{2}\right\rfloor_{k}=W_{3}\right)$ iff $\left(\left\lfloor W_{1}\right\rfloor_{k} \odot_{k}\left\lfloor W_{2}\right\rfloor_{k}=W_{3}\right)$.

Proof

Immediate from the definitions of $W_{1} \odot_{k} W_{2}$ and $\lfloor W\rfloor_{k}$.

- Req 6 (join-commut)

if $\left(W_{1} \odot_{k} W_{2}=W_{3}\right)$, then $\left(W_{2} \odot_{k} W_{1}=W_{3}\right)$.

Proof

Immediate from the definition of $W_{1} \odot_{k} W_{2}$.

- Req 7 (join-assocl)

if $\left(W_{2} \odot_{k} W_{3}=W_{23}\right)$ and $\left(W_{1} \odot_{k} W_{23}=W_{123}\right)$,

then there exists $W_{12}$ such that $\left(W_{1} \odot_{k} W_{2}=W_{12}\right)$ and $\left(W_{12} \odot_{k} W_{3}=W_{123}\right)$.

Proof

Immediate from the definition of $W_{1} \odot_{k} W_{2}$.

- Req 8 (join-assocr)

if $\left(W_{1} \odot_{k} W_{1}=W_{12}\right)$ and $\left(W_{12} \odot_{k} W_{3}=W_{123}\right)$,

then there exists $W_{23}$ such that $\left(W_{2} \odot_{k} W_{3}=W_{23}\right)$ and $\left(W_{1} \odot_{k} W_{23}=W_{123}\right)$.

Proof 
Immediate from the definition of $W_{1} \odot_{k} W_{2}$.

- Req 9 (join-unit-left)

$\left(\mathcal{U}_{\odot} \odot_{k} W=\lfloor W\rfloor_{k}\right)$.

Proof

Immediate from the definitions of $W_{1} \odot_{k} W_{2}$ and $\mathcal{U}_{\odot}$.

- Req 10 (qualpred-closed)

if $j \leq k$ and $\mathcal{P}(k, q, W)$, then $\mathcal{P}(j, q, W)$.

Proof

Immediate from the definition of $\mathcal{P}(k, q, W)$.

- Req 11 (qualpred-aprx)

$\mathcal{P}(k, q, W)$ iff $\mathcal{P}\left(k, q,\lfloor W\rfloor_{k}\right)$.

Proof

Immediate from the definitions of $\mathcal{P}(k, q, W)$ and $\lfloor W\rfloor_{k}$.

- Req 12 (qualpred-join)

if $\mathcal{P}\left(k, q, W_{1}\right)$ and $\mathcal{P}\left(k, q, W_{2}\right)$ and $\left(W_{1} \odot_{k} W_{2}=W_{3}\right)$, then $\mathcal{P}\left(k, q, W_{3}\right)$.

Proof

Immediate from the definitions of $\mathcal{P}(k, q, W)$ and $W_{1} \odot_{k} W_{2}$.

- Req 13 (qualpred-qualsub)

if $\mathcal{P}(k, q, W)$ and $q \preceq q^{\prime}$, then $\mathcal{P}\left(k, q^{\prime}, W\right)$.

Proof

Immediate from the definition of $\mathcal{P}(k, q, W)$.

- Req 14 (qualpred-unr-unit)

$\mathcal{P}\left(k, \mathrm{U}, \mathcal{U}_{\odot}\right)$.

Proof

$$
\mathcal{P}\left(k, \mathrm{U}, \mathcal{U}_{\odot}\right)=\forall l \in \operatorname{dom}(\{\}) .\{\}^{\text {qual }}(l) \preceq \mathrm{U} \equiv \text { True. }
$$

- Req 15 (qualpred-rel-join)

if $\mathcal{P}(k, \mathrm{R}, W)$, then $\left.\left(W \odot_{k} W\right)=\lfloor W\rfloor_{k}\right)$.

Proof

Immediate from $\mathcal{P}(k, \mathrm{R}, W)=\forall l \in \operatorname{dom}(W) . W^{\text {qual }}(l) \preceq \mathrm{R}$ and the definition of $W_{1} \odot_{k} W_{2}$.

- Req 16 (qualpred-aff-models)

if $\mathcal{P}\left(k, \mathrm{~A}, W_{1}\right)$ and $\left(W_{1} \odot_{k} W_{2}=W_{3}\right)$ and $w:_{k} W_{3}$, then $w:_{k} W_{2}$.

Proof

Note that

$$
\begin{aligned}
w:{ }_{k} W_{3} \equiv & \exists \mathcal{S}_{3}: 2^{\text {Locs }} . \\
& \exists \mathcal{F}_{3 W}: \mathcal{S}_{3} \rightarrow \text { WorldDesc } \\
& \exists \mathcal{F}_{3 q}: \mathcal{S}_{3} \rightarrow \text { Quals. }
\end{aligned}
$$


We are required to show that

$$
\begin{aligned}
w:{ }_{k} W_{2} \equiv & \exists \mathcal{S}_{2}: 2^{\text {Locs }} . \\
& \exists \mathcal{F}_{2 W}: \mathcal{S}_{2} \rightarrow \text { WorldDesc } k \\
& \exists \mathcal{F}_{2 q}: \mathcal{S}_{2} \rightarrow \text { Quals. }
\end{aligned}
$$

Take

$$
\begin{aligned}
\mathcal{S}_{2}=\min \subseteq\left\{\mathcal{S} \in 2^{\text {Locs }} \mid \operatorname{dom}\left(W_{2}\right) \subseteq \mathcal{S} \wedge\left(\forall l \in \mathcal{S} . \operatorname{dom}\left(\mathcal{F}_{3 W}(l)\right) \subseteq \mathcal{S}\right)\right\} \\
\mathcal{F}_{2 W}(l)=\left\{\mathcal{F}_{3 W}(l) \text { if } l \in \mathcal{S}_{2}\right. \\
\mathcal{F}_{2 q}(l)=\left\{\mathcal{F}_{3 q}(l) \text { if } l \in \mathcal{S}_{2}\right.
\end{aligned}
$$

- Req 17 (qualpred-lin)

$\mathcal{P}(k, \mathrm{~L}, W)$.

Proof

$$
\mathcal{P}(k, \mathrm{~L}, W)=\forall l \in \operatorname{dom}(W) . W^{\text {qual }}(l) \preceq \mathrm{L} \equiv \text { True. }
$$




\section{C.7 Proofs}

\section{C.7.1 Validity of Kinding Rules}

Lemma 27 (Ref Extension: $\mathcal{T} \llbracket \Delta \vdash \iota: \kappa \rrbracket \delta \in \mathcal{K} \llbracket \kappa \rrbracket)$

Let $\delta \in \mathcal{D} \llbracket \Delta \rrbracket$ and $\Delta \vdash \iota: \kappa$.

Then $\mathcal{T} \llbracket \Delta \vdash \iota: \kappa \rrbracket \delta \in \mathcal{K} \llbracket \kappa \rrbracket$.

Proof (Ref Extension: $\mathcal{T} \llbracket \Delta \vdash \iota: \kappa \rrbracket \delta \in \mathcal{K} \llbracket \kappa \rrbracket)$

Recall from Lemma 8 , for $\kappa \equiv$ QUAL, it suffices to prove the following:

$$
\mathcal{T} \llbracket \Delta \vdash \xi: \mathrm{QUAL} \rrbracket \delta \in \text { Quals. }
$$

Recall from Lemma 8, for $\kappa \equiv$ PRETYPE, it suffices to prove the following:

$$
\begin{aligned}
\forall(k, q, W, v) \in \mathcal{T} \llbracket \Delta \vdash \bar{\tau}: \mathrm{PRETYPE} \rrbracket \delta . & W \in \text { WorldDesc }_{k} \wedge \mathcal{P}(k, q, W) \wedge \\
& \forall j \leq k .\left(j, q,\lfloor W\rfloor_{j}, v\right) \in \mathcal{T} \llbracket \Delta \vdash \bar{\tau}: \mathrm{PRETYPE} \rrbracket \delta .
\end{aligned}
$$

Recall from Lemma 8 , for $\kappa \equiv$ TYPE, it suffices to prove the following:

$$
\begin{aligned}
\exists q^{\prime} \in \text { Quals. } \forall(k, q, W, v) \in \mathcal{T} \llbracket \Delta \vdash \tau: \text { TYPE } \rrbracket & \delta . \\
& W \in \text { WorldDesc }_{k} \wedge \mathcal{P}(k, q, W) \wedge \\
& \forall j .\left(j, q,\lfloor W\rfloor_{j}, v\right) \in \mathcal{T} \llbracket \Delta \vdash \bar{\tau}: \mathrm{PRETYPE} \rrbracket \delta \wedge \\
& q=q^{\prime} .
\end{aligned}
$$

Proceed by induction on the derivation $\Delta \vdash \iota: \kappa$.

(REFPTy)

Case $\frac{\Delta \vdash \tau: \text { TYPE }}{\Delta \vdash \operatorname{ref} \tau: \text { PRETYPE }}$ :

Recall that

$$
\mathcal{T} \llbracket \frac{\Delta \vdash \tau: \text { TYPE }}{\Delta \vdash \operatorname{ref} \tau: \text { PRETYPE }} \rrbracket \delta=\left\{\begin{array}{c}
(k, q,\{l \mapsto(q, \chi)\}, l) \mid \\
\chi=\lfloor\mathcal{T} \llbracket \Delta \vdash \tau: \text { TYPE } \rrbracket \delta\rfloor_{k} \wedge \\
\left.\left(q \preceq \mathrm{A} \Rightarrow \forall\left({ }_{-}, q^{\prime},{ }_{-},-\right) \in \chi \cdot q^{\prime} \preceq \mathrm{A}\right)\right\}
\end{array}\right.
$$

Consider arbitrary $(k, q, W, v) \in \mathcal{T} \llbracket \Delta \vdash$ ref $\tau:$ PRETYPE $\rrbracket \delta$.

Hence, $v \equiv l$ and $W \equiv\{l \mapsto(q, \chi)\}$ and $\chi=\lfloor\mathcal{T} \llbracket \Delta \vdash \tau: \mathrm{TYPE} \rrbracket \delta\rfloor_{k}$ and $\left(q \preceq \mathrm{A} \Rightarrow \forall\left({ }_{-}, q^{\prime},{ }_{-},{ }_{-}\right) \in\right.$ $\left.\chi \cdot q^{\prime} \preceq \mathrm{A}\right)$.

Applying the induction hypothesis to $\Delta \vdash \tau:$ TYPE, we conclude that $\mathcal{T} \llbracket \Delta \vdash \tau:$ TYPE $\llbracket \delta \in$ Type. Note that $\mathcal{T} \llbracket \Delta \vdash \tau:$ TYPE $\rrbracket \delta \in$ CandUberType $\omega_{\omega}$ and $\forall k \geq 0 .\lfloor\mathcal{T} \llbracket \Delta \vdash \tau: \text { TYPE } \rrbracket \delta\rfloor_{k} \in$ Type $_{k}$, which follows from the definition of Type.

We are required to show that

- $\{l \mapsto(q, \chi)\} \in$ WorldDesc $_{k}$, which follows from

$\{l \mapsto(q, \chi)\} \in$ Locs $\rightarrow$ Quals $\times$ Type $_{k}$ which follows from $\chi \equiv\lfloor\mathcal{T} \llbracket \Delta \vdash \tau: \text { TYPE } \rrbracket \delta\rfloor_{k} \in$ Type $_{k}$

$\left(q \preceq \mathrm{A} \Rightarrow \forall\left({ }_{-}, q^{\prime},_{-},-\right) \in \chi \cdot q^{\prime} \preceq \mathrm{A}\right)$ which follows from above

$\equiv\left(\{l \mapsto(q, \chi)\}^{\text {qual }}(l) \preceq \mathrm{A} \Rightarrow \forall\left({ }_{-}, q^{\prime},{ }_{-},-\right) \in\{l \mapsto(q, \chi)\}^{\text {type }}(l) . q^{\prime} \preceq \mathrm{A}\right)$ which follows from the fact that $\{l \mapsto(q, \chi)\}^{\text {qual }}(l) \equiv q$ and $\{l \mapsto(q, \chi)\}^{\text {type }}(l) \equiv \chi$

$\equiv \forall l^{\prime} \in \operatorname{dom}(\{l \mapsto(q, \chi)\}) .\left(\{l \mapsto(q, \chi)\}^{\text {qual }}\left(l^{\prime}\right) \preceq \mathrm{A} \Rightarrow \forall\left({ }_{-}, q^{\prime},,_{-},-\right) \in\{l \mapsto(q, \chi)\}^{\text {type }}\left(l^{\prime}\right) . q^{\prime} \preceq \mathrm{A}\right)$ which follows from the fact that $\operatorname{dom}(\{l \mapsto(q, \chi)\}) \equiv\{l\}$

$\equiv \mathcal{R}(\{l \mapsto(q, \chi)\})$

which follows from the definition of $\mathcal{R}(\cdot)$

$\{l \mapsto(q, \chi)\} \in\left\{W \in\right.$ Locs $\rightarrow$ Quals $\times$ Type $\left._{k} \mid \mathcal{R}(W)\right\}$

which follows from above

$\equiv\{l \mapsto(q, \chi)\} \in$ WorldDesc $_{k}$

which follows from the definition of WorldDesc $k$. 
- $\mathcal{P}(k, q,\{l \mapsto(q, \chi)\})$, which follows from

$$
\begin{aligned}
& \equiv q \preceq q \\
& \quad \text { which follows from reflexivity of } \preceq \\
& \equiv\left({ }^{\text {qual }}\{l \mapsto(q, \chi)\}\right)(l) \preceq q \\
& \left.\quad \text { which follows from the fact that }{ }^{\text {qual }}\{l \mapsto(q, \chi)\}\right)(l) \equiv q \\
& \left.\equiv \forall l^{\prime} \in \operatorname{dom}(\{l \mapsto(q, \chi)\}) \text {. ( }{ }^{\text {qual }}\{l \mapsto(q, \chi)\}\right)\left(l^{\prime}\right) \preceq q \\
& \quad \text { which follows from the fact that } \operatorname{dom}(\{l \mapsto(q, \chi)\}) \equiv\{l\} \\
& \equiv \mathcal{P}(k, q,\{l \mapsto(q, \chi)\}) \\
& \quad \text { which follows from the definition of } \mathcal{P}(\cdot, \cdot, \cdot) .
\end{aligned}
$$

Consider arbitrary $j \leq k$.

We are required to show that $\left(j, q,\lfloor\{l \mapsto(q, \chi)\}\rfloor_{j}, l\right) \in \mathcal{T} \llbracket \Delta \vdash \operatorname{ref} \tau: \operatorname{PRETYPE} \rrbracket \delta$.

Note that $\lfloor\{l \mapsto(q, \chi)\}\rfloor_{j} \equiv\left\{l \mapsto\left(q,\lfloor\chi\rfloor_{j}\right)\right\}$, which follows from the definition of $\lfloor\cdot\rfloor_{k}$.

Hence, we are required to show that $\left(j, q,\left\{l \mapsto\left(q,\lfloor\chi\rfloor_{j}\right)\right\}, l\right) \in \mathcal{T} \llbracket \Delta \vdash \operatorname{ref} \tau: \operatorname{PRETYPE} \rrbracket \delta$. Note that

- $\lfloor\chi\rfloor_{j}=\lfloor\mathcal{T} \llbracket \Delta \vdash \tau: \operatorname{TYPE} \rrbracket \delta\rfloor_{j}$, which follows from

$$
\begin{aligned}
& \chi=\lfloor\mathcal{T} \llbracket \Delta \vdash \tau: \text { TYPE } \rrbracket \delta\rfloor_{k} \\
& \text { which follows from above } \\
& \Rightarrow\left.\lfloor\rfloor_{j}=\lfloor\mathcal{T} \llbracket \Delta \vdash \tau: \text { TYPE } \llbracket \delta\rfloor_{k}\right\rfloor_{j} \\
& \equiv\lfloor\chi\rfloor_{j}=\lfloor\mathcal{T} \llbracket \Delta \vdash \tau: \text { TYPE } \rrbracket \delta\rfloor_{j} \\
& \text { which follows from Fact } 2 .
\end{aligned}
$$

- $\left(q \preceq \mathrm{A} \Rightarrow \forall\left({ }_{-}, q^{\prime},,_{-},{ }_{-}\right) \in\lfloor\chi\rfloor_{j} . q^{\prime} \preceq \mathrm{A}\right)$, which follows from Fact 1 and $\left(q \preceq \mathrm{A} \Rightarrow \forall\left({ }_{-}, q^{\prime},,_{-},{ }_{-}\right) \in\right.$ $\left.\chi \cdot q^{\prime} \preceq \mathrm{A}\right)$, which in turn follows from above.

\section{End Case}




\section{C.7.2 Validity of Typing Rules}

\section{Theorem 28 (Ref Extension Soundness)}

$$
\text { If } \Delta ; \Gamma \vdash e: \tau \text {, then } \llbracket \Delta ; \Gamma \vdash e: \tau \rrbracket \text {. }
$$

\section{Proof}

By induction on the derivation $\Delta ; \Gamma \vdash e: \tau$.

$(\operatorname{New}(\mathrm{U}, \mathrm{A}))$

Case $\frac{q \preceq \mathrm{A} \quad \Delta ; \Gamma \vdash e: \tau \quad \Delta \vdash \tau \preceq \mathrm{A}}{\Delta ; \Gamma \vdash \operatorname{new}_{q} e:{ }^{q} \operatorname{ref} \tau}$ :

We are required to show $\llbracket \Delta ; \Gamma \vdash$ new $_{q} e_{1}:{ }^{\xi}$ ref $\tau \rrbracket$.

Consider arbitrary $k, \delta, q_{\Gamma}, W_{\Gamma}$, and $\gamma$ such that

- $k \geq 0$,

- $\delta \in \mathcal{D} \llbracket \Delta \rrbracket$, and

- $\left(k, q_{\Gamma}, W_{\Gamma}, \gamma\right) \in \mathcal{G} \llbracket \Delta \vdash \Gamma \rrbracket \delta$.

Let $e_{s}=\gamma\left(\right.$ new $\left._{q} e_{1}\right) \equiv$ new $_{q} \gamma\left(e_{1}\right)$ and $W_{s}=W_{\Gamma}$.

We are required to show that $\operatorname{Comp}\left(k, W_{s}, e_{s}, \mathcal{T} \llbracket \Delta \vdash{ }^{\left.U_{\text {ref }} \tau: \operatorname{TYPE} \rrbracket \delta\right) \equiv}\right.$ $\operatorname{Comp}\left(k, W_{\Gamma}, \operatorname{new}_{q} \gamma\left(e_{1}\right), \mathcal{T} \llbracket \Delta \vdash{ }^{\mathrm{ref}} \tau: \operatorname{TYPE} \rrbracket \delta\right)$.

Consider arbitrary $j, W_{r}, w_{s}, w_{f}$, and $e_{f}$ such that

- $j<k$,

- $w_{s}:_{k}\left(W_{s} \odot_{k} W_{r}\right) \equiv w_{s}:_{k}\left(W_{\Gamma} \odot_{k} W_{r}\right)$,

- $\left(w_{s}, e_{s}\right)=\left(w_{s}\right.$, new $\left._{q} \gamma\left(e_{1}\right)\right) \longmapsto j\left(w_{f}, e_{f}\right)$, and

- $\operatorname{irred}\left(w_{f}, e_{f}\right)$.

Hence, by inspection of the operational semantics, it follows that there exist $j_{1}, w_{f_{1}}$, and $e_{f_{1}}$ such that

- $\left(w_{s}, \gamma\left(e_{1}\right)\right) \longmapsto{ }^{j_{1}}\left(w_{f_{1}}, e_{f_{1}}\right)$,

- $\operatorname{irred}\left(w_{f_{1}}, e_{f_{1}}\right)$, and

- $j_{1} \leq j$.

Applying the induction hypothesis to $\Delta ; \Gamma \vdash e_{1}: \tau$, we conclude that $\llbracket \Delta ; \Gamma \vdash e_{1}: \tau \rrbracket$. Instantiate this with $k, \delta, q_{\Gamma}, W_{\Gamma}$, and $\gamma$. Note that

- $k \geq 0$,

- $\delta \in \mathcal{D} \llbracket \Delta \rrbracket$, and

- $\left(k, q_{\Gamma}, W_{\Gamma}, \gamma\right) \in \mathcal{G} \llbracket \Delta \vdash \Gamma \rrbracket \delta$.

Hence, $\operatorname{Comp}\left(k, W_{\Gamma}, \gamma\left(e_{1}\right), \mathcal{T} \llbracket \Delta \vdash \tau:\right.$ TYPE $\left.\rrbracket \delta\right)$.

Instantiate this with $j_{1}, W_{r}, w_{s}, w_{f_{1}}$, and $e_{f_{1}}$. Note that

- $j_{1}<k$, which follows from $j_{1} \leq j$ and $j<k$,

- $w_{s}:_{k}\left(W_{\Gamma} \odot_{k} W_{r}\right)$, which follows from above,

- $\left(w_{s}, \gamma\left(e_{1}\right)\right) \longmapsto{ }^{j_{1}}\left(w_{f_{1}}, e_{f_{1}}\right)$,

- $\operatorname{irred}\left(w_{f_{1}}, e_{f_{1}}\right)$.

Hence, there exists $W_{f_{1}}$ and $q_{f_{1}}$ such that 
- $w_{f_{1}}:_{k-j_{1}}\left(W_{f_{1}} \odot_{k-j_{1}} W_{r}\right)$, and

- $\left(k-j_{1}, q_{f_{1}}, W_{f_{1}}, e_{f_{1}}\right) \in \mathcal{T} \llbracket \Delta \vdash \tau:$ TYPE $\rrbracket \delta$.

Hence, $e_{f_{1}} \equiv v_{f_{1}}$.

Note that

$$
\begin{aligned}
\left(w_{s}, e_{s}\right) & \equiv\left(w_{s}, \operatorname{new}_{q} \gamma\left(e_{1}\right)\right) \\
& \longmapsto^{j_{1}}\left(w_{f_{1}}, \text { new }_{q} e_{f_{1}}\right) \\
& \equiv\left(w_{f_{1}}, \text { new }_{q} v_{f_{1}}\right) \\
& \longmapsto^{1}\left(w_{f_{1}} \uplus\left\{l_{f} \mapsto\left(q, v_{f_{1}}\right)\right\}, l_{f}\right) \quad l_{f} \notin \operatorname{dom}\left(w_{f_{1}}\right) \\
& \longmapsto^{j-j_{1}-1}\left(w_{f}, e_{f}\right) .
\end{aligned}
$$

Since $l_{f}$ is value, we have $\operatorname{irred}\left(w_{f_{1}} \uplus\left\{l_{f} \mapsto\left(q, v_{f_{1}}\right)\right\}, l_{f}\right)$.

Hence, $j-j_{1}-1=0$ (and $j=j_{1}+1$ ) and $w_{f} \equiv w_{f_{1}} \uplus\left\{l_{f} \mapsto v_{f_{1}}\right\}$ and $e_{f} \equiv l_{f}$.

Note that

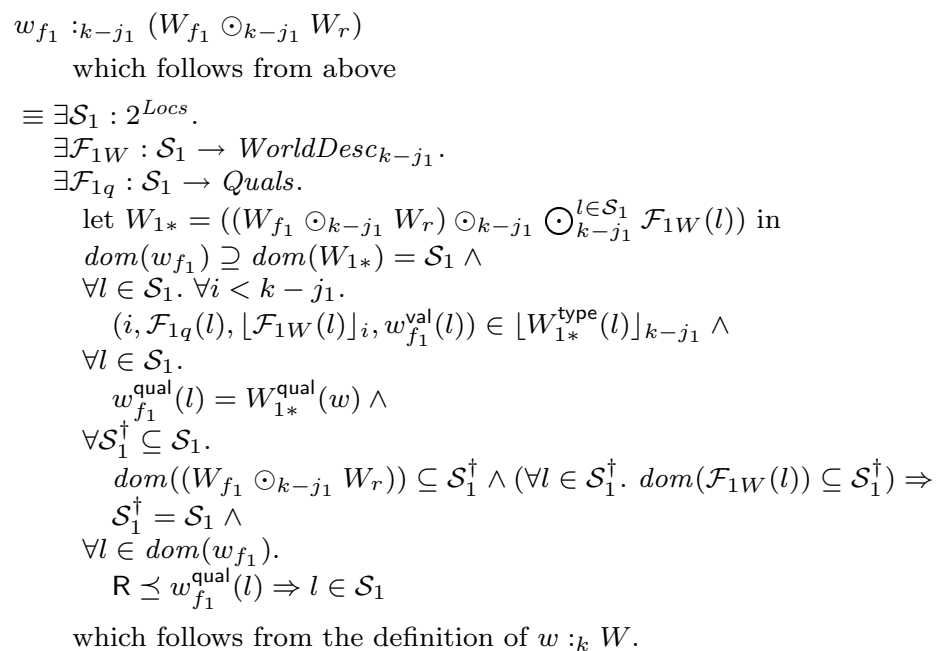

Note that

$$
\begin{aligned}
& \operatorname{dom}\left(w_{f_{1}}\right) \supseteq \operatorname{dom}\left(W_{1 *}\right)=\mathcal{S}_{1} \\
& \quad \text { which follows from above }\left(w_{f_{1}}:_{k-j_{1}}\left(W_{f_{1}} \odot_{k-j_{1}} W_{r}\right)\right) \\
& \equiv \operatorname{dom}\left(w_{f_{1}}\right) \supseteq \operatorname{dom}\left(W_{1 *}\right)=\operatorname{dom}\left(W_{f_{1}}\right) \cup \operatorname{dom}\left(W_{r}\right) \cup \bigcup^{l \in \mathcal{S}_{1}} \operatorname{dom}\left(\mathcal{F}_{1 W}(l)\right)=\mathcal{S}_{1} \\
& \quad \text { which follows from above }\left(W_{1 *}=\ldots\right) \text { and } \operatorname{dom}\left(W_{1} \odot_{k} W_{2}\right)=\operatorname{dom}\left(W_{1}\right) \cup \operatorname{dom}\left(W_{2}\right) .
\end{aligned}
$$

Furthermore, note that $l_{f} \notin \operatorname{dom}\left(W_{1 *}\right)$ and $l_{f} \notin d o m\left(W_{r}\right)$ and $l_{f} \notin \mathcal{S}_{1}$, which follows from $l_{f} \notin \operatorname{dom}\left(w_{f_{1}}\right)$.

Note that either $q=\mathrm{U}$ or $q=\mathrm{A}$, which follows from $q \preceq \mathrm{A}$.

Case $q=\mathrm{U}$ :

Let $\chi_{f}=\lfloor\mathcal{T} \llbracket \Delta \vdash \tau: \mathrm{TYPE} \rrbracket \delta\rfloor_{k-j}$.

Let $W_{f}=\left\{l_{f} \mapsto\left(\mathrm{U}, \chi_{f}\right)\right\}$.

Let $q_{f}=\mathrm{U}$.

Note that $\left(\left\{l_{f} \mapsto\left(\mathrm{U}, \chi_{f}\right)\right\} \odot_{k-j} W_{r}\right)$ defined, which follows from $l_{f} \notin \operatorname{dom}\left(W_{r}\right)$.

We are required to show that

- $w_{f}:_{k-j}\left(W_{f} \odot_{k-j} W_{r}\right)$

$$
\equiv w_{f_{1}} \uplus\left\{l_{f} \mapsto v_{f_{1}}\right\}:_{k-j}\left(\left\{l_{f} \mapsto\left(\mathrm{U}, \chi_{f}\right)\right\} \odot_{k-j} W_{r}\right),
$$


which is equivalent to

$$
\begin{aligned}
& w_{f_{1}} \uplus\left\{l_{f} \mapsto\left(\mathrm{U}, v_{f_{1}}\right)\right\}:_{k-j}\left(\left\{l_{f} \mapsto\left(\mathrm{U}, \chi_{f}\right)\right\} \odot_{k-j} W_{r}\right) \\
& \equiv \exists \mathcal{S}: 2^{\text {Locs }} \text {. } \\
& \exists \mathcal{F}_{W}: \mathcal{S} \rightarrow \text { WorldDesc }_{k-j} \text {. } \\
& \exists \mathcal{F}_{q}: \mathcal{S} \rightarrow \text { Quals. } \\
& \text { let } W_{*}=\left(\left(\left\{l_{f} \mapsto\left(\mathbf{U}, \chi_{f}\right)\right\} \odot_{k-j} W_{r}\right) \odot_{k-j} \bigodot_{k-j}^{l \in \mathcal{S}} \mathcal{F}_{W}(l)\right) \text { in } \\
& \operatorname{dom}\left(\left(w_{f_{1}} \uplus\left\{l_{f} \mapsto\left(\mathrm{U}, v_{f_{1}}\right)\right\}\right)\right) \supseteq \operatorname{dom}\left(W_{*}\right)=\mathcal{S} \wedge \\
& \forall l \in \mathcal{S} . \forall i<k-j \text {. } \\
& \left(i, \mathcal{F}_{q}(l),\left\lfloor\mathcal{F}_{W}(l)\right\rfloor_{i},\left(w_{f_{1}} \uplus\left\{l_{f} \mapsto\left(\mathrm{U}, v_{f_{1}}\right)\right\}\right)^{\mathrm{val}}(l)\right) \in\left\lfloor W_{*}^{\text {type }}(l)\right\rfloor_{k-j} \wedge \\
& \forall l \in \mathcal{S} \text {. } \\
& \left(w_{f_{1}} \uplus\left\{l_{f} \mapsto\left(\mathrm{U}, v_{f_{1}}\right)\right)^{\text {qual }}(l)=W_{*}^{\text {qual }}(l) \wedge\right. \\
& \forall \mathcal{S}^{\dagger} \subseteq \mathcal{S} \\
& \operatorname{dom}\left(\left(\left\{l_{f} \mapsto\left(\mathrm{U}, \chi_{f}\right)\right\} \odot_{k-j} W_{r}\right)\right) \subseteq \mathcal{S}^{\dagger} \wedge\left(\forall l \in \mathcal{S}^{\dagger} . \operatorname{dom}\left(\mathcal{F}_{W}(l)\right) \subseteq \mathcal{S}^{\dagger}\right) \Rightarrow \\
& \mathcal{S}^{\dagger}=\mathcal{S} \wedge \\
& \forall l \in \operatorname{dom}\left(\left(w_{f_{1}} \uplus\left\{l_{f} \mapsto\left(\mathrm{U}, v_{f_{1}}\right)\right\}\right)\right) . \\
& \mathrm{R} \preceq\left(w_{f_{1}} \uplus\left\{l_{f} \mapsto\left(\mathrm{U}, v_{f_{1}}\right)\right\}\right)^{\text {qual }}(l) \Rightarrow l \in \mathcal{S} \\
& \text { which follows from the definition of } w:_{k} W \text {. }
\end{aligned}
$$

Take

$$
\mathcal{S}=\left\{l_{f}\right\} \uplus \mathcal{S}_{1}
$$

It remains to show that

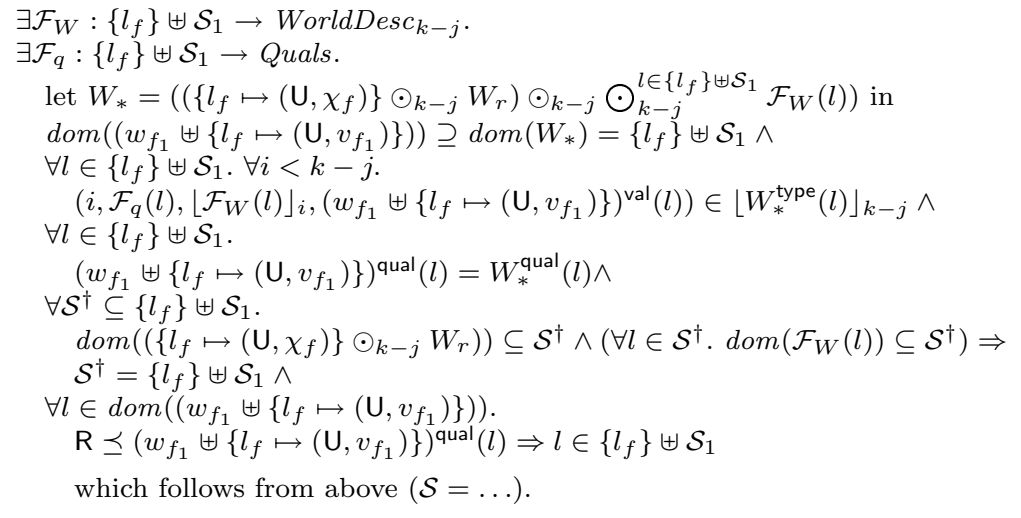

Take

and

$$
\mathcal{F}_{W}(l)= \begin{cases}\left\lfloor W_{f_{1}}\right\rfloor_{k-j} & \text { if } l \in\left\{l_{f}\right\} \\ \left\lfloor\mathcal{F}_{1 W}(l)\right\rfloor_{k-j} & \text { if } l \in \mathcal{S}_{1}\end{cases}
$$

Note that

$$
\mathcal{F}_{q}(l)= \begin{cases}q_{f_{1}} & \text { if } l \in\left\{l_{f}\right\} \\ \mathcal{F}_{1 q}(l) & \text { if } l \in \mathcal{S}_{1}\end{cases}
$$

$W_{f_{1}} \in$ WorldDesc $_{k-j_{1}}$

which follows from Fact 6 applied to $\left(k-j_{1}, q_{f_{1}}, W_{f_{1}}, v_{f_{1}}\right) \in \mathcal{T} \llbracket \Delta \vdash \tau:$ TYPE $\delta \in$ Type,

which in turn follows from Lemma 8 applied to $\mathcal{T} \llbracket \Delta \vdash \tau:$ TYPE $\rrbracket \delta$

$\Rightarrow\left\lfloor W_{f_{1}}\right\rfloor_{k-j} \in$ WorldDesc $_{k-j}$

which follows from $\lfloor\cdot\rfloor_{k-j} \in$ WorldDesc $\rightarrow$ WorldDesc $_{k-j}$

$\equiv \mathcal{F}_{W}\left(l_{f}\right) \in$ WorldDesc $_{k-j}$

which follows from above $\left(\mathcal{F}_{W}(l)=\ldots\right)$.

Note that

$\forall l \in \mathcal{S}_{1} . \mathcal{F}_{1 W}(l) \in$ WorldDesc $_{k-j_{1}}$

which follows from above $\left(w_{f_{1}}: k-j_{1}\left(W_{f_{1}} \odot_{k-j_{1}} W_{r}\right)\right) \Rightarrow \forall l \in \mathcal{S}_{1} .\left\lfloor\mathcal{F}_{1 W}(l)\right\rfloor_{k-j} \in$ WorldDesc $_{k-j}$ which follows from $\lfloor\cdot\rfloor_{k} \in$ WorldDesc $\rightarrow$ WorldDesc $_{k}$

$\equiv \forall l \in \mathcal{S}_{1} . \mathcal{F}_{W}(l) \in$ WorldDesc $_{k-j}$

which follows from above $\left(\mathcal{F}_{W}(l)=\ldots\right)$.

Hence, $\mathcal{F}_{W}:\left\{l_{f}\right\} \uplus \mathcal{S}_{1} \rightarrow$ WorldDesc $_{k-j}$. 
Trivially, $\mathcal{F}_{q}:\left\{l_{f}\right\} \uplus \mathcal{S}_{1} \rightarrow$ Quals.

It remains to show that

$$
\begin{aligned}
& \text { let } W_{*}=\left(\left(\left\{l_{f} \mapsto\left(\mathrm{U}, \chi_{f}\right)\right\} \odot_{k-j} W_{r}\right) \odot_{k-j} \bigodot_{k-j}^{l \in\left\{l_{f}\right\} \uplus \mathcal{S}_{1}} \mathcal{F}_{W}(l)\right) \text { in } \\
& \operatorname{dom}\left(\left(w_{f_{1}} \uplus\left\{l_{f} \mapsto\left(\mathrm{U}, v_{f_{1}}\right)\right\}\right)\right) \supseteq \operatorname{dom}\left(W_{*}\right)=\left\{l_{f}\right\} \uplus \mathcal{S}_{1} \wedge \\
& \forall l \in\left\{l_{f}\right\} \uplus \mathcal{S}_{1} . \forall i<k-j . \\
& \quad\left(i, \mathcal{F}_{q}(l),\left\lfloor\mathcal{F}_{W}(l)\right\rfloor_{i},\left(w_{f_{1}} \uplus\left\{l_{f} \mapsto\left(\mathrm{U}, v_{f_{1}}\right)\right\}\right)^{\text {val }}(l)\right) \in\left\lfloor W_{*}^{\text {type }}(l)\right\rfloor_{k-j} \wedge \\
& \forall l \in\left\{l_{f}\right\} \uplus \mathcal{S}_{1} . \\
& \quad\left(w_{f_{1}} \uplus\left\{l_{f} \mapsto\left(\mathrm{U}, v_{f_{1}}\right)\right\}\right)^{\text {qual }}(l)=W_{*}^{\text {qual }}(l) \wedge \\
& \forall \mathcal{S}^{\dagger} \subseteq\left\{l_{f}\right\} \uplus \mathcal{S}_{1} . \\
& \quad \operatorname{dom}\left(\left(\left\{l_{f} \mapsto\left(\mathrm{U}, \chi_{f}\right)\right\} \odot_{k-j} W_{r}\right)\right) \subseteq \mathcal{S}^{\dagger} \wedge\left(\forall l \in \mathcal{S}^{\dagger} . \operatorname{dom}\left(\mathcal{F}_{W}(l)\right) \subseteq \mathcal{S}^{\dagger}\right) \Rightarrow \mathcal{S}^{\dagger}=\left\{l_{f}\right\} \uplus \mathcal{S}_{1} \wedge \\
& \forall l \in \operatorname{dom}\left(\left(w_{f_{1}} \uplus\left\{l_{f} \mapsto\left(\mathrm{U}, v_{f_{1}}\right)\right\}\right)\right) . \\
& \quad \mathrm{R} \preceq\left(w_{f_{1}} \uplus\left\{l_{f} \mapsto\left(\mathrm{U}, v_{f_{1}}\right)\right\}\right)^{\text {qual }}(l) \Rightarrow l \in\left\{l_{f}\right\} \uplus \mathcal{S}_{1} \\
& \quad \text { which follows from above. }
\end{aligned}
$$

Note that $\left(\left\{l_{f} \mapsto\left(\mathrm{U}, \chi_{f}\right)\right\} \odot_{k-j} W_{1 *}\right)$ defined, which follows from $l_{f} \notin \operatorname{dom}\left(W_{1 *}\right)$.

Furthermore, $\operatorname{dom}\left(\left(\left\{l_{f} \mapsto\left(\mathrm{U}, \chi_{f}\right)\right\} \odot_{k-j} W_{1 *}\right)\right)=\left\{l_{f}\right\} \uplus \operatorname{dom}\left(W_{1 *}\right)$.

Note that

$$
\begin{aligned}
& \left(\left\{l_{f} \mapsto\left(\mathrm{U}, \chi_{f}\right)\right\} \odot_{k-j} W_{1 *}\right) \\
& \equiv\left(\left\{l_{f} \mapsto\left(\mathrm{U}, \chi_{f}\right)\right\} \odot_{k-j}\left(\left(W_{f_{1}} \odot_{k-j_{1}} W_{r}\right) \odot_{k-j_{1}} \bigodot_{k-j_{1}}^{l \in \mathcal{S}_{1}} \mathcal{F}_{1 W}(l)\right)\right) \\
& \quad \text { which follows from above }\left(W_{1 *}=\ldots\right) \\
& \equiv\left(\left\{l_{f} \mapsto\left(\mathrm{U}, \chi_{f}\right)\right\} \odot_{k-j}\left\lfloor\left(\left(W_{f_{1}} \odot_{k-j_{1}} W_{r}\right) \odot_{k-j_{1}} \bigodot_{k-j_{1}}^{l \in \mathcal{S}_{1}} \mathcal{F}_{1 W}(l)\right)\right\rfloor_{k-j}\right) \\
& \quad \text { which follows from Req } 5(\text { join-aprx }) \\
& \equiv\left(\left\{l_{f} \mapsto\left(\mathrm{U}, \chi_{f}\right)\right\} \bigodot_{k-j}\left(\left(\left\lfloor W_{f_{1}}\right\rfloor_{k-j} \bigodot_{k-j} W_{r}\right) \odot_{k-j} \bigodot_{k-j}^{l \in \mathcal{S}_{1}}\left\lfloor\mathcal{F}_{1 W}(l)\right\rfloor k-j\right)\right) \\
& \quad \text { which follows from Req } 4(\text { join-closed }) \text { and Req } 5(\text { join-aprx }) \\
& \equiv\left(\left\{l_{f} \mapsto\left(\mathrm{U}, \chi_{f}\right)\right\} \odot_{k-j}\left(\left(\mathcal{F}_{W}\left(l_{f}\right) \odot_{k-j} W_{r}\right) \odot_{k-j} \bigodot_{k-j}^{l \in \mathcal{S}_{1}} \mathcal{F}_{W}(l)\right)\right) \\
& \quad \text { which follows from above }\left(\mathcal{F}_{W}(l)=\ldots\right) \\
& \equiv\left(\left(\left\{l_{f} \mapsto\left(\mathrm{U}, \chi_{f}\right)\right\} \odot_{k-j} W_{r}\right) \odot_{k-j}\left(\mathcal{F}_{W}\left(l_{f}\right) \odot_{k-j} \bigodot_{k-j}^{l \in \mathcal{S}_{1}} \mathcal{F}_{W}(l)\right)\right) \\
& \quad \text { which follows from Reqs } 6,7, \text { and } 8(\text { join-commut, join-assocl, and join-assocr })
\end{aligned}
$$$$
\equiv\left(\left(\left\{l_{f} \mapsto\left(\mathrm{U}, \chi_{f}\right)\right\} \odot_{k-j} W_{r}\right) \odot_{k-j} \bigodot_{k-j}^{l \in l_{f} \uplus \mathcal{S}_{1}} \mathcal{F}_{W}(l)\right)
$$$$
\text { which follows from simplifications of } \bigodot_{k-j}^{l \in\left\{l_{f}\right\} \uplus \mathcal{S}_{1}} \mathcal{F}_{W}(l) \text {. }
$$

Hence, $W_{*}=\left(\left(\left\{l_{f} \mapsto\left(\mathrm{U}, \chi_{f}\right)\right\} \odot_{k-j} W_{r}\right) \odot_{k-j} \bigodot_{k-j}^{l \in l_{f} \uplus \mathcal{S}_{1}} \mathcal{F}_{W}(l)\right)$ is defined.

Furthermore, $W_{*}=\left(\left\{l_{f} \mapsto\left(\mathrm{U}, \chi_{f}\right)\right\} \odot_{k-j} W_{1 *}\right)$ and $\operatorname{dom}\left(W_{*}\right)=\operatorname{dom}\left(\left(\left\{l_{f} \mapsto\right.\right.\right.$ $\left.\left.\left.\left(\mathrm{U}, \chi_{f}\right)\right\} \odot_{k-j} W_{1 *}\right)\right)=\left\{l_{f}\right\} \uplus \operatorname{dom}\left(W_{1 *}\right)$.

Note that

$$
\begin{aligned}
& \operatorname{dom}\left(w_{f_{1}}\right) \supseteq \operatorname{dom}\left(W_{1 *}\right)=\mathcal{S}_{1} \\
& \quad \text { which follows from above }\left(w_{f_{1}}: k-j\right. \\
& \left.\left.\equiv \operatorname{dom}\left(w_{f_{1}}\right) \uplus\left\{l_{f}\right\} \supseteq\left\{l_{f}\right\} \uplus \operatorname{dom}\left(W_{f_{1}}\right)=\left\{l_{f-j}\right\} \uplus W_{r}\right)\right) \\
& \quad \text { which follows from } l_{f} \notin \operatorname{dom}\left(w_{f_{1}}\right) \text { and } l_{f} \notin \operatorname{dom}\left(W_{1 *}\right) \text { and } l_{f} \notin \mathcal{S}_{1} \\
& \equiv \operatorname{dom}\left(w_{f_{1}}\right) \uplus\left\{l_{f}\right\} \supseteq \operatorname{dom}\left(W_{*}\right)=\left\{l_{f}\right\} \uplus \mathcal{S}_{1} \\
& \quad \text { which follows from above }\left(\operatorname{dom}\left(W_{*}\right)=\ldots\right) \\
& \equiv \operatorname{dom}\left(\left(w_{f_{1}} \uplus\left\{l_{f} \mapsto\left(\mathrm{U}, v_{f_{1}}\right)\right\}\right)\right) \supseteq \operatorname{dom}\left(W_{*}\right)=\left\{l_{f}\right\} \uplus \mathcal{S}_{1} \\
& \quad \text { which follows from simplifications of } \operatorname{dom}\left(\left(w_{f_{1}} \uplus\left\{l_{f} \mapsto\left(\mathrm{U}, v_{f_{1}}\right)\right\}\right)\right) .
\end{aligned}
$$

It remains to show that

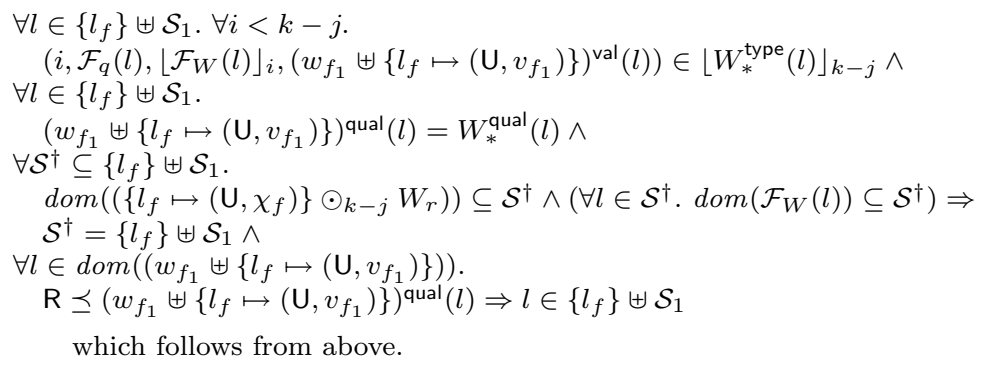

We are required to show that 
- $\forall l \in\left\{l_{f}\right\} \uplus \mathcal{S}_{1} . \forall i<k-j$.

$$
\left(i, \mathcal{F}_{q}(l),\left\lfloor\mathcal{F}_{W}(l)\right\rfloor_{i},\left(w_{f_{1}} \uplus\left\{l_{f} \mapsto\left(\mathrm{U}, v_{f_{1}}\right)\right\}\right)^{\mathrm{val}}(l)\right) \in\left\lfloor W_{*}^{\text {type }}(l)\right\rfloor_{k-j}
$$

Note that

$$
\begin{aligned}
& \forall l \in\left\{l_{f}\right\} \uplus \mathcal{S}_{1} . \forall i<k-j . \\
& \left(i, \mathcal{F}_{q}(l),\left\lfloor\mathcal{F}_{W}(l)\right\rfloor_{i},\left(w_{f_{1}} \uplus\left\{l_{f} \mapsto\left(\mathrm{U}, v_{f_{1}}\right)\right\}\right)^{\mathrm{val}}(l)\right) \in\left\lfloor W_{*}^{\text {type }}(l)\right\rfloor_{k-j} \\
& \equiv \forall l \in\left\{l_{f}\right\} . \forall i<k-j . \\
& \left(i, \mathcal{F}_{q}(l),\left\lfloor\mathcal{F}_{W}(l)\right\rfloor_{i},\left(w_{f_{1}} \uplus\left\{l_{f} \mapsto\left(\mathrm{U}, v_{f_{1}}\right)\right\}\right)^{\mathrm{val}}(l)\right) \in\left\lfloor W_{*}^{\text {type }}(l)\right\rfloor_{k-j} \wedge \\
& \forall l \in \mathcal{S}_{1} . \forall i<k-j \text {. } \\
& \left(i, \mathcal{F}_{q}(l),\left\lfloor\mathcal{F}_{W}(l)\right\rfloor_{i},\left(w_{f_{1}} \uplus\left\{l_{f} \mapsto\left(\mathrm{U}, v_{f_{1}}\right)\right\}\right)^{\mathrm{val}}(l)\right) \in\left\lfloor W_{*}^{\text {type }}(l)\right\rfloor_{k-j} \\
& \text { which follows from simplifications of } \forall l \in\left\{l_{f}\right\} \uplus \mathcal{S}_{1} \text {. ... . . . } \\
& \equiv \forall i<k-j \text {. } \\
& \left(i, \mathcal{F}_{q}\left(l_{f}\right),\left\lfloor\mathcal{F}_{W}\left(l_{f}\right)\right\rfloor_{i},\left(w_{f_{1}} \uplus\left\{l_{f} \mapsto\left(\mathrm{U}, v_{f_{1}}\right)\right\}\right)^{\mathrm{val}}\left(l_{f}\right)\right) \in\left\lfloor W_{*}^{\mathrm{type}}\left(l_{f}\right)\right\rfloor_{k-j} \wedge \\
& \forall l \in \mathcal{S}_{1} . \forall i<k-j \text {. } \\
& \left(i, \mathcal{F}_{q}(l),\left\lfloor\mathcal{F}_{W}(l)\right\rfloor_{i},\left(w_{f_{1}} \uplus\left\{l_{f} \mapsto\left(\mathrm{U}, v_{f_{1}}\right)\right\}\right)^{\mathrm{val}}(l)\right) \in\left\lfloor W_{*}^{\mathrm{type}}(l)\right\rfloor_{k-j} \\
& \text { which follows from simplifications of } \forall l \in\left\{l_{f}\right\} . \ldots l \ldots \\
& \equiv \forall i<k-j \text {. } \\
& \left(i, q_{f_{1}},\left\lfloor\left\lfloor W_{f_{1}}\right\rfloor_{k-j}\right\rfloor_{i},\left(w_{f_{1}} \uplus\left\{l_{f} \mapsto\left(\mathrm{U}, v_{f_{1}}\right)\right\}\right)^{\mathrm{val}}\left(l_{f}\right)\right) \in\left\lfloor W_{*}^{\text {type }}\left(l_{f}\right)\right\rfloor_{k-j} \wedge \\
& \forall l \in \mathcal{S}_{1} . \forall i<k-j \text {. } \\
& \left(i, \mathcal{F}_{1 q}(l),\left\lfloor\left\lfloor\mathcal{F}_{1 W}(l)\right\rfloor_{k-j}\right\rfloor_{i},\left(w_{f_{1}} \uplus\left\{l_{f} \mapsto\left(\mathrm{U}, v_{f_{1}}\right)\right\}\right)^{\mathrm{val}}(l)\right) \in\left\lfloor W_{*}^{\text {type }}(l)\right\rfloor_{k-j} \\
& \text { which follows from above }\left(\mathcal{F}_{W}(l)=\ldots \text { and } \mathcal{F}_{q}(l)=\ldots\right) \\
& \equiv \forall i<k-j \text {. } \\
& \left(i, q_{f_{1}},\left\lfloor W_{f_{1}}\right\rfloor_{i},\left(w_{f_{1}} \uplus\left\{l_{f} \mapsto\left(\mathrm{U}, v_{f_{1}}\right)\right\}\right)^{\mathrm{val}}\left(l_{f}\right)\right) \in\left\lfloor W_{*}^{\mathrm{type}}\left(l_{f}\right)\right\rfloor_{k-j} \wedge \\
& \forall l \in \mathcal{S}_{1} . \forall i<k-j . \\
& \left(i, \mathcal{F}_{1 q}(l),\left\lfloor\mathcal{F}_{1 W}(l)\right\rfloor_{i},\left(w_{f_{1}} \uplus\left\{l_{f} \mapsto\left(\mathrm{U}, v_{f_{1}}\right)\right\}\right)^{\mathrm{val}}(l)\right) \in\left\lfloor W_{*}^{\text {type }}(l)\right\rfloor_{k-j} \\
& \text { which follows from Req } 1 \text { (aprx-idem) } \\
& \equiv \forall i<k-j \text {. } \\
& \left(i, q_{f_{1}},\left\lfloor W_{f_{1}}\right\rfloor_{i}, v_{f_{1}}\right) \in\left\lfloor W_{*}^{\text {type }}\left(l_{f}\right)\right\rfloor_{k-j} \wedge \\
& \forall l \in \mathcal{S}_{1} . \forall i<k-j \text {. } \\
& \left(i, \mathcal{F}_{1 q}(l),\left\lfloor\mathcal{F}_{1 W}(l)\right\rfloor_{i},\left(w_{f_{1}} \uplus\left\{l_{f} \mapsto\left(\mathrm{U}, v_{f_{1}}\right)\right\}\right)^{\mathrm{val}}(l)\right) \in\left\lfloor W_{*}^{\text {type }}(l)\right\rfloor_{k-j} \\
& \text { which follows from simplifications of }\left(w_{f_{1}} \uplus\left\{l_{f} \mapsto\left(\mathrm{U}, v_{f_{1}}\right)\right\}\right)^{\mathrm{val}}\left(l_{f}\right) \equiv v_{f_{1}} \\
& \equiv \forall i<k-j \text {. } \\
& \left(i, q_{f_{1}},\left\lfloor W_{f_{1}}\right\rfloor_{i}, v_{f_{1}}\right) \in\left\lfloor W_{*}^{\text {type }}\left(l_{f}\right)\right\rfloor_{k-j} \wedge \\
& \forall l \in \mathcal{S}_{1} . \forall i<k-j \text {. } \\
& \left(i, \mathcal{F}_{1 q}(l),\left\lfloor\mathcal{F}_{1 W}(l)\right\rfloor_{i}, w_{f_{1}}^{\text {val }}(l)\right) \in\left\lfloor W_{*}^{\text {type }}(l)\right\rfloor_{k-j} \\
& \forall l \in \mathcal{S}_{1} \ldots\left(w_{f_{1}} \uplus\left\{l_{f} \mapsto\left(\mathrm{U}, v_{f_{1}}\right)\right\}\right)^{\mathrm{val}}(l) \ldots \equiv \forall l \in \mathcal{S}_{1} \ldots w_{f_{1}}^{\mathrm{val}}(l) \ldots
\end{aligned}
$$

We are required to show that 
- $\forall i<k-j$.

$\left(i, q_{f_{1}},\left\lfloor W_{f_{1}}\right\rfloor_{i}, v_{f_{1}}\right) \in\left\lfloor\left\lfloor\chi_{f}\right\rfloor_{k-j}\right\rfloor_{k-j}$

which follows from

$$
\begin{aligned}
& \left(k-j_{1}, q_{f_{1}}, W_{f_{1}}, v_{f_{1}}\right) \in \mathcal{T} \llbracket \Delta \vdash \tau: \text { TYPE } \rrbracket \delta \\
& \text { which follows from above } \\
& \Rightarrow \forall i<k-j_{1} .\left(i, q_{f_{1}},\left\lfloor W_{f_{1}}\right\rfloor_{i}, v_{f_{1}}\right) \in \mathcal{T} \llbracket \Delta \vdash \tau: \text { TYPE } \rrbracket \\
& \quad \text { which follows from Lemma } 8 \text { and Fact } 6 \\
& \Rightarrow \forall i<k-j .\left(i, q_{f_{1}},\left\lfloor W_{f_{1}}\right\rfloor_{i}, v_{f_{1}}\right) \in \mathcal{T} \llbracket \Delta \vdash \tau: \text { TYPE } \rrbracket \\
& \quad \text { which follows } k-j<k-j_{1} \\
& \Rightarrow \forall i<k-j .\left(i, q_{f_{1}},\left\lfloor W_{f_{1}}\right\rfloor_{i}, v_{f_{1}}\right) \in\lfloor\mathcal{T} \llbracket \Delta \vdash \tau: \text { TYPE } \rrbracket\rfloor_{k-j} \\
& \quad \text { which follows from } j<k \wedge(j, q, W, v) \in \chi \Rightarrow(j, q, W, v) \in\lfloor\chi\rfloor_{k} \\
& \Rightarrow \forall i<k-j .\left(i, q_{f_{1}},\left\lfloor W_{f_{1}}\right\rfloor_{i}, v_{f_{1}}\right) \in \chi \\
& \quad \text { which follows from above }\left(\chi_{f}=\ldots\right) \\
& \Rightarrow \forall i<k-j .\left(i, q_{f_{1}},\left\lfloor W_{f_{1}}\right\rfloor_{i}, v_{f_{1}}\right) \in\left\lfloor\chi_{f}\right\rfloor_{k-j} \\
& \quad \text { which follows from } j<k \wedge(j, q, W, v) \in \chi \Rightarrow(j, q, W, v) \in\lfloor\chi\rfloor_{k} \\
& \Rightarrow \forall i<k-j .\left(i, q_{f_{1}},\left\lfloor W_{f_{1}}\right\rfloor_{i}, v_{f_{1}}\right) \in\left\lfloor\left\lfloor\chi_{f}\right\rfloor_{k-j}\right\rfloor_{k-j} \\
& \quad \text { which follows from } j<k \wedge(j, q, W, v) \in \chi \Rightarrow(j, q, W, v) \in\lfloor\chi\rfloor_{k} .
\end{aligned}
$$

- $\forall l \in \mathcal{S}_{1} . \forall i<k-j$.

$$
\left(i, \mathcal{F}_{1 q}(l),\left\lfloor\mathcal{F}_{1 W}(l)\right\rfloor_{i}, w_{f_{1}}^{\text {val }}(l)\right) \in\left\lfloor\left\lfloor W_{1 *}\right\rfloor_{k-j}^{\text {type }}(l)\right\rfloor_{k-j}
$$

which follows from

$$
\begin{aligned}
\forall l \in \mathcal{S}_{1} . \forall i<k-j_{1} .\left(i, \mathcal{F}_{1 q}(l),\left\lfloor\mathcal{F}_{1 W}(l)\right\rfloor_{i}, w_{f_{1}}^{\text {val }}(l)\right) \in\left\lfloor W_{1 *}^{\text {type }}(l)\right\rfloor_{k-j_{1}} \\
\quad \text { which follows from above }\left(w_{f_{1}}: k-j_{1}\left(W_{f_{1}} \odot{ }_{k-j_{1}} W_{r}\right)\right) \\
\Rightarrow \forall l \in \mathcal{S}_{1} . \forall i<k-j .\left(i, \mathcal{F}_{1 q}(l),\left\lfloor\mathcal{F}_{1 W}(l)\right\rfloor_{i}, w_{f_{1}}^{\text {val }}(l)\right) \in\left\lfloor W_{1 *}^{\text {type }}(l)\right\rfloor_{k-j_{1}} \\
\quad \text { which follows from } k-j<k-j_{1} \\
\Rightarrow \forall l \in \mathcal{S}_{1} . \forall i<k-j .\left(i, \mathcal{F}_{1 q}(l),\left\lfloor\mathcal{F}_{1 W}(l)\right\rfloor_{i}, w_{f_{1}}(l)\right) \in\left\lfloor\left\lfloor W_{1 *}^{\text {type }}(l)\right\rfloor_{k-j_{1}}\right\rfloor_{k-j} \\
\quad \text { which follows from } j<k \wedge(j, q, W, v) \in \chi \Rightarrow(j, q, W, v) \in\lfloor\chi\rfloor_{k} \\
\equiv \forall l \in \mathcal{S}_{1} . \forall i<k-j .\left(i, \mathcal{F}_{1 q}(l),\left\lfloor\mathcal{F}_{1 W}(l)\right\rfloor_{i}, w_{f_{1}}(l)\right) \in\left\lfloor W_{1 *}^{\text {type }}(l)\right\rfloor_{k-j} \\
\quad \text { which follows from Req } 1(\text { aprx-idem) } \\
\equiv \forall l \in \mathcal{S}_{1} . \forall i<k-j .\left(i, \mathcal{F}_{1 q}(l),\left\lfloor\mathcal{F}_{1 W}(l)\right\rfloor_{i}, w_{f_{1}}(l)\right) \in\left\lfloor W_{1 *}\right\rfloor_{k-j}^{\text {type }}(l) \\
\quad \text { which follows from the definition of }\lfloor W\rfloor_{k} \\
\Rightarrow \forall l \in \mathcal{S}_{1} . \forall i<k-j .\left(i, \mathcal{F}_{1 q}(l),\left\lfloor\mathcal{F}_{1 W}(l)\right\rfloor_{i}, w_{f_{1}}(l)\right) \in\left\lfloor\left\lfloor W_{1 *}\right\rfloor_{k-j}^{\text {type }}(l)\right\rfloor_{k-j} \\
\quad \text { which follows from } j<k \wedge(j, q, W, v) \in \chi \Rightarrow(j, q, W, v) \in\lfloor\chi\rfloor_{k} .
\end{aligned}
$$

- $\forall l \in\left\{l_{f}\right\} \uplus \mathcal{S}_{1}$.

$$
\left(w_{f_{1}} \uplus\left\{l_{f} \mapsto\left(\mathrm{U}, v_{f_{1}}\right)\right\}\right)^{\text {qual }}(l)=W_{*}^{\text {qual }}(l)
$$

Note that

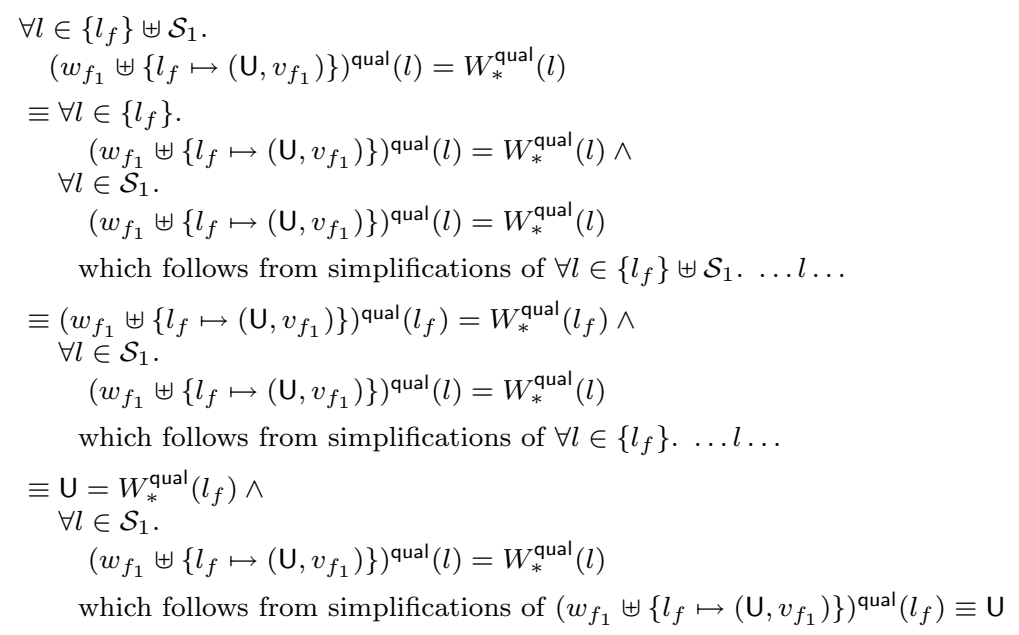




$$
\begin{aligned}
& \begin{aligned}
& \mathrm{U}=W_{*}^{\text {qual }} \\
& \forall l \in \mathcal{S}_{1}
\end{aligned} \\
& w_{f_{1}}^{\text {qual }}(l)=W_{*}^{\text {qual }}(l) \\
& \text { which follows from simplifications of } \\
& \forall l \in \mathcal{S}_{1} \ldots\left(w_{f_{1}} \uplus\left\{l_{f} \mapsto\left(\mathrm{U}, v_{f_{1}}\right)\right\}\right)^{\text {qual }}(l) \ldots \equiv \forall l \in \mathcal{S}_{1} \ldots w_{f_{1}}^{\text {qual }}(l) \ldots \\
& \equiv \mathrm{U}=\left(\left\{l_{f} \mapsto\left(\mathrm{U}, \chi_{f}\right)\right\} \odot_{k-j} W_{1 *}\right)^{\text {qual }}\left(l_{f}\right) \wedge \\
& \forall l \in \mathcal{S}_{1} \text {. } \\
& w_{f_{1}}^{\text {qual }}(l)=\left(\left\{l_{f} \mapsto\left(\mathrm{U}, \chi_{f}\right)\right\} \odot_{k-j} W_{1 *}\right)^{\text {qual }}(l) \\
& \text { which follows from above }\left(W_{*}=\ldots\right) \\
& \equiv \mathrm{U}=\mathrm{U} \wedge \\
& w_{f_{1}}^{\text {qual }}(l)=\left(\left\{l_{f} \mapsto\left(\mathrm{U}, \chi_{f}\right)\right\} \odot_{k-j} W_{1 *}\right)^{\text {qual }}(l) \\
& \text { which follows from simplifications of }\left(\left\{l_{f} \mapsto\left(\mathrm{U}, \chi_{f}\right)\right\} \odot_{k-j} W_{1 *}\right)^{\text {qual }}\left(l_{f}\right) \equiv \mathrm{U} \\
& \equiv \mathrm{U}=\mathrm{U} \wedge \\
& \forall l \in \mathcal{S}_{1} . \\
& w_{f_{1}}^{\text {qual }}(l)=W_{1 *}^{\text {qual }}(l) \\
& \forall l \in \mathcal{S}_{1} \ldots\left(\left\{l_{f} \mapsto\left(\mathrm{U}, \chi_{f}\right)\right\} \odot_{k-j} W_{1 *}\right)^{\text {qual }}(l) \ldots \equiv \forall l \in \mathcal{S}_{1} \ldots W_{1 *}^{\text {qual }}(l) \ldots
\end{aligned}
$$

We are required to show that

- $\mathrm{U}=\mathrm{U}$

which follows trivially,

- $\forall l \in \mathcal{S}_{1}$.

$$
w_{f_{1}}^{\text {qual }}(l)=W_{1 *}^{\text {qual }}(l)
$$

which follows from

$$
\begin{aligned}
\forall l \in & \mathcal{S}_{1} \cdot w_{f_{1}}^{\text {qual }}(l)=W_{1 *}^{\text {qual }}(l) \\
& \text { which follows from above }\left(w_{f_{1}}:_{k-j_{1}}\left(W_{f_{1}} \odot_{k-j_{1}} W_{r}\right)\right) .
\end{aligned}
$$

- $\forall \mathcal{S}^{\dagger} \subseteq\left\{l_{f}\right\} \uplus \mathcal{S}_{1}$.

$$
\begin{aligned}
& \operatorname{dom}\left(\left(\left\{l_{f} \mapsto\left(\mathrm{U}, \chi_{f}\right)\right\} \odot_{k-j} W_{r}\right)\right) \subseteq \mathcal{S}^{\dagger} \wedge \\
& \left(\forall l \in \mathcal{S}^{\dagger} . \operatorname{dom}\left(\mathcal{F}_{W}(l)\right) \subseteq \mathcal{S}^{\dagger}\right) \Rightarrow \\
& \mathcal{S}^{\dagger}=\left\{l_{f}\right\} \uplus \mathcal{S}_{1}
\end{aligned}
$$

Consider arbitrary $\mathcal{S}^{\dagger}$ such that

- $\mathcal{S}^{\dagger} \subseteq\left\{l_{f}\right\} \uplus \mathcal{S}_{1}$,

- $\operatorname{dom}\left(\left(\left\{l_{f} \mapsto\left(\mathrm{U}, \chi_{f}\right)\right\} \odot_{k-j} W_{r}\right)\right) \subseteq \mathcal{S}^{\dagger}$, and

- $\forall l \in \mathcal{S}^{\dagger} . \operatorname{dom}\left(\mathcal{F}_{W}(l)\right) \subseteq \mathcal{S}^{\dagger}$.

Note that $\left\{l_{f}\right\} \uplus \operatorname{dom}\left(W_{r}\right) \subseteq \mathcal{S}^{\dagger}$, which follows from $\operatorname{dom}\left(\left(\left\{l_{f} \mapsto\left(\mathrm{U}, \chi_{f}\right)\right\} \odot_{k-j} W_{r}\right)\right) \subseteq$ $\mathcal{S}^{\dagger}$ and $l_{f} \notin \operatorname{dom}\left(W_{r}\right)$.

Note that $l_{f} \in \mathcal{S}^{\dagger}$, which follows from $\left\{l_{f}\right\} \uplus W_{r} \subseteq \mathcal{S}^{\dagger}$.

Let $\mathcal{S}_{1}^{\dagger}=\mathcal{S}^{\dagger} \backslash\left\{l_{f}\right\}$.

Note that $\mathcal{S}^{\dagger}=\left\{l_{f}\right\} \uplus \mathcal{S}_{1}^{\dagger}$.

Note that

- $\mathcal{S}_{1}^{\dagger} \subseteq \mathcal{S}_{1}$, which follows from $\mathcal{S}^{\dagger} \subseteq\left\{l_{f}\right\} \uplus \mathcal{S}_{1}$,

- $\operatorname{dom}\left(W_{r}\right) \subseteq \mathcal{S}_{1}^{\dagger}$, which follows from $\left\{l_{f}\right\} \uplus \operatorname{dom}\left(W_{r}\right) \subseteq \mathcal{S}^{\dagger}$,

- $\operatorname{dom}\left(\mathcal{F}_{W}\left(l_{f}\right)\right) \subseteq\left\{l_{f}\right\} \uplus \mathcal{S}_{1}^{\dagger}$, which follows from $\forall l \in \mathcal{S}^{\dagger}$. $\operatorname{dom}\left(\mathcal{F}_{W}(l)\right) \subseteq \mathcal{S}^{\dagger}$, and, furthermore, $\operatorname{dom}\left(\left\lfloor W_{f_{1}}\right\rfloor_{k-j}\right) \subseteq\left\{l_{f}\right\} \uplus \mathcal{S}_{1}^{\dagger}$, which follows from the definition of $\mathcal{F}_{W}$, and, furthermore, $\operatorname{dom}\left(W_{f_{1}}\right) \subseteq\left\{l_{f}\right\} \uplus \mathcal{S}_{1}^{\dagger}$,

- $\forall l \in \mathcal{S}_{1}^{\dagger} . \operatorname{dom}\left(\mathcal{F}_{W}(l)\right) \subseteq\left\{l_{f}\right\} \uplus \mathcal{S}_{1}^{\dagger}$, which follows from $\forall l \in \mathcal{S}^{\dagger} . \operatorname{dom}\left(\mathcal{F}_{W}(l)\right) \subseteq \mathcal{S}^{\dagger}$, and, furthermore, $\forall l \in \mathcal{S}_{1}^{\dagger}$. $\operatorname{dom}\left(\left\lfloor\mathcal{F}_{1 W}(l)\right\rfloor_{k-j}\right) \subseteq\left\{l_{f}\right\} \uplus \mathcal{S}_{1}^{\dagger}$, which follows from the definition of $\mathcal{F}_{W}$. 
Recall that $\operatorname{dom}\left(W_{f_{1}}\right) \subseteq \mathcal{S}_{1}$ and $\forall l \in \mathcal{S}_{1}$. $\operatorname{dom}\left(\mathcal{F}_{1 W}(l)\right) \subseteq \mathcal{S}_{1}$, which follows from $w_{f_{1}}: k-j_{1}\left(W_{f_{1}} \odot_{k-j_{1}} W_{r}\right)$.

Hence, $\operatorname{dom}\left(W_{f_{1}}\right) \subseteq \mathcal{S}_{1}^{\dagger}$, which follows from $\operatorname{dom}\left(W_{f_{1}}\right) \subseteq\left\{l_{f}\right\} \uplus \mathcal{S}_{1}^{\dagger}$ and $\operatorname{dom}\left(W_{f_{1}}\right) \subseteq$ $\mathcal{S}_{1}$ and $l_{f} \notin \mathcal{S}_{1}$.

Hence, $\forall l \in \mathcal{S}_{1}^{\dagger} . \quad \operatorname{dom}\left(\mathcal{F}_{1 W}(l)\right) \subseteq \mathcal{S}_{1}^{\dagger}$, which follows from $\forall l \quad \in$ $\mathcal{S}_{1}^{\dagger} \cdot \operatorname{dom}\left(\left\lfloor\mathcal{F}_{1 W}(l)\right\rfloor_{k-j}\right) \subseteq\left\{l_{f}\right\} \uplus \mathcal{S}_{1}^{\dagger}$ and $\forall l \in \mathcal{S}_{1} \cdot \operatorname{dom}\left(\mathcal{F}_{1 W}(l)\right) \subseteq \mathcal{S}_{1}\left(\right.$ and $\left.\mathcal{S}_{1}^{\dagger} \subseteq \mathcal{S}_{1}\right)$ and $l_{f} \notin \mathcal{S}_{1}$.

Instantiate $\left(\forall \mathcal{S}_{1}^{\dagger} \subseteq \mathcal{S}_{1} \ldots\right)$ of $w_{f_{1}}:_{k-j_{1}}\left(W_{f_{1}} \odot_{k-j_{1}} W_{r}\right)$ with $\mathcal{S}_{1}^{\dagger}$. Note that

- $\mathcal{S}_{1}^{\dagger} \subseteq \mathcal{S}_{1}$, which follows from above,

- $\operatorname{dom}\left(\left(W_{f_{1}} \odot_{k-j_{1}} \operatorname{dom}\left(W_{r}\right)\right)\right) \subseteq \mathcal{S}_{1}^{\dagger}$, which follows from $\operatorname{dom}\left(W_{f_{1}}\right) \subseteq \mathcal{S}_{1}^{\dagger}$, which follows from above, and $\operatorname{dom}\left(W_{r}\right) \subseteq \mathcal{S}_{1}^{\dagger}$, which follows from above,

- $\forall l \in \mathcal{S}_{1}^{\dagger} \cdot \operatorname{dom}\left(\mathcal{F}_{1 W}(l)\right) \subseteq \mathcal{S}_{1}^{\dagger}$, which follows from above.

Hence, we conclude that $\mathcal{S}_{1}^{\dagger}=\mathcal{S}_{1}$.

Hence, $\mathcal{S}^{\dagger}=\left\{l_{f}\right\} \uplus \mathcal{S}_{1}^{\dagger}=\left\{l_{f}\right\} \uplus \mathcal{S}_{1}$.

- $\forall l \in \operatorname{dom}\left(\left(w_{f_{1}} \uplus\left\{l_{f} \mapsto\left(\mathrm{U}, v_{f_{1}}\right)\right\}\right)\right)$.

$\mathrm{R} \preceq\left(w_{f_{1}} \uplus\left\{l_{f} \mapsto\left(\mathrm{U}, v_{f_{1}}\right)\right\}\right)^{)^{\text {qual }}}(l) \Rightarrow l \in\left\{l_{f}\right\} \uplus \mathcal{S}_{1}$

Note that

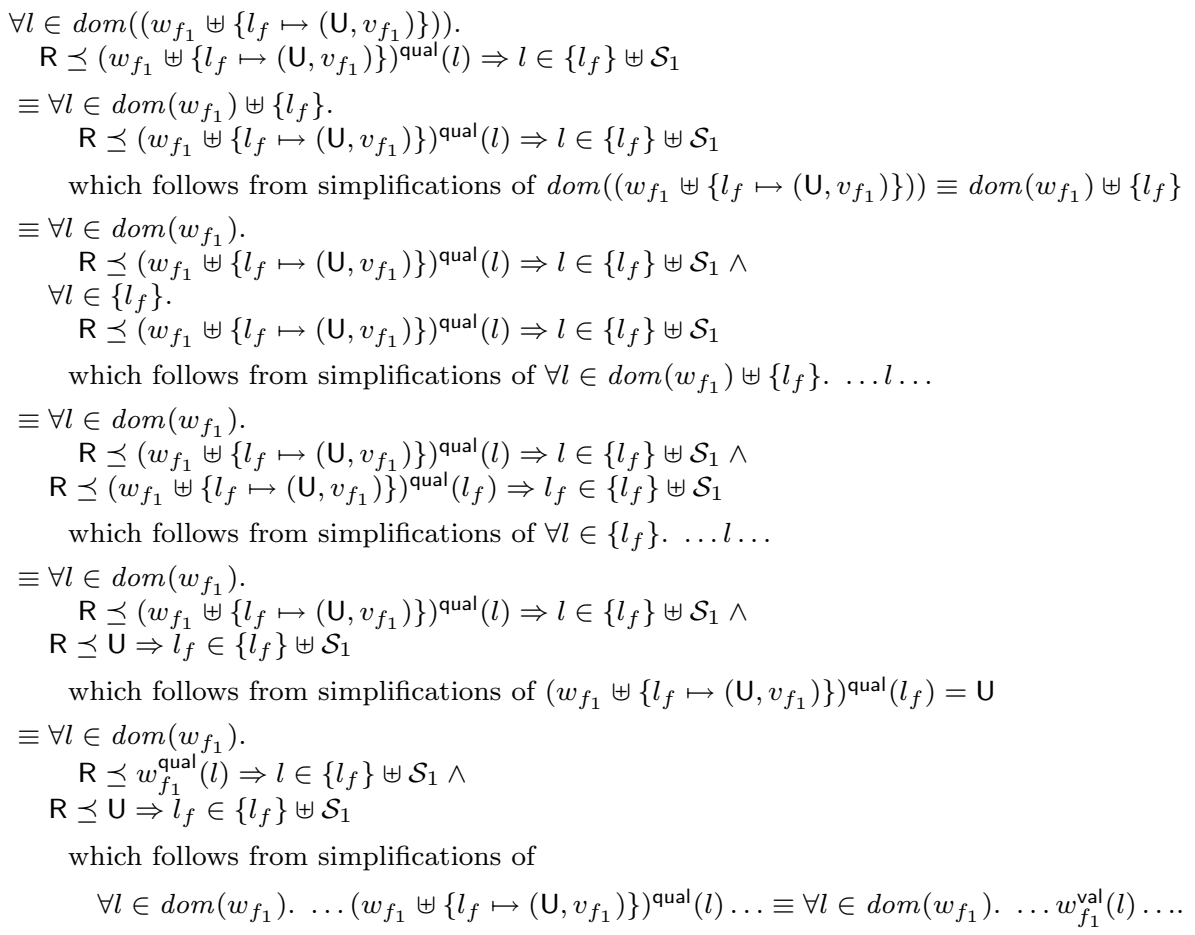

We are required to show that

- $\forall l \in \operatorname{dom}\left(w_{f_{1}}\right) . \mathrm{R} \preceq w_{f_{1}}^{\text {qual }}(l) \Rightarrow l \in\left\{l_{f}\right\} \uplus \mathcal{S}_{1}$

which follows from

$$
\begin{aligned}
& \forall l \in \operatorname{dom}\left(w_{f_{1}}\right) . \mathrm{R} \preceq w_{f_{1}}^{\text {qual }}(l) \Rightarrow l \in \mathcal{S}_{1} \\
& \quad \text { which follows from } w_{f_{1}}: k-j_{1} \\
& \Rightarrow \quad\left(W_{f_{1}} \odot_{k-j_{1}} W_{r}\right) \\
& \forall l \in \operatorname{dom}\left(w_{f_{1}}\right) . \mathrm{R} \preceq w_{f_{1}}^{\text {qual }}(l) \Rightarrow l \in\left\{l_{f}\right\} \uplus \mathcal{S}_{1} \\
& \text { which follows from } l_{f} \notin \mathcal{S}_{1} .
\end{aligned}
$$

- $\mathrm{R} \preceq \mathrm{U} \Rightarrow l_{f} \in\left\{l_{f}\right\} \uplus \mathcal{S}_{1}$

which follows trivially. 
- $\left(k-j, q_{f}, W_{f}, e_{f}\right) \in \mathcal{T} \llbracket \Delta \vdash \mathrm{U}_{\text {ref }} \tau: \mathrm{TYPE} \rrbracket \delta$

$$
\begin{aligned}
& \equiv\left(k-j, \mathrm{U},\left\{l_{f} \mapsto\left(\mathrm{U}, \chi_{f}\right)\right\}, l_{f}\right) \in \mathcal{T} \llbracket \Delta \vdash \mathrm{U}_{\text {ref }} \tau: \mathrm{TYPE} \rrbracket \delta \\
& \equiv\left(k-j, \mathrm{U},\left\{l_{f} \mapsto\left(\mathrm{U}, \chi_{f}\right)\right\}, l_{f}\right)
\end{aligned}
$$$$
\in\{(k, q, W, v) \mid
$$$$
q=\mathcal{T} \llbracket \Delta \vdash \mathrm{U}: \mathrm{QUAL} \rrbracket \delta \wedge
$$$$
(k, q, W, v) \in \mathcal{T} \llbracket \Delta \vdash \operatorname{ref} \tau: \mathrm{PRETYPE} \rrbracket \delta\}
$$

$\equiv\left(k-j, \mathrm{U},\left\{l_{f} \mapsto\left(\mathrm{U}, \chi_{f}\right)\right\}, l_{f}\right)$

$\in\{(k, q,\{l \mapsto(q, \chi)\}, l) \mid$

$$
\begin{aligned}
& q=\mathcal{T} \llbracket \Delta \vdash \mathrm{U}: \mathrm{QUAL} \rrbracket \delta \wedge \\
& \chi=\lfloor\mathcal{T} \llbracket \Delta \vdash \tau: \mathrm{TYPE} \rrbracket \delta\rfloor_{k} \wedge \\
& \left.\left(q \preceq \mathrm{A} \Rightarrow \forall\left({ }_{-}, q^{\prime},{ }_{-},{ }_{-}\right) \in \chi \cdot q^{\prime} \preceq \mathrm{A}\right)\right\},
\end{aligned}
$$

which follows from

- $\mathrm{U}=\mathcal{T} \llbracket \Delta \vdash \mathrm{U}: \mathrm{QUAL} \rrbracket \delta$, which follows trivially,

- $\chi_{f}=\lfloor\mathcal{T} \llbracket \Delta \vdash \tau \text { : TYPE } \rrbracket \delta\rfloor_{k-j}$, which follows trivially,

- $\left(\mathrm{U} \preceq \mathrm{A} \Rightarrow \forall\left({ }_{-}, q^{\prime},{ }_{-},{ }_{-}\right) \in \chi_{f} . q^{\prime} \preceq \mathrm{A}\right)$ $\equiv\left(\mathrm{U} \preceq \mathrm{A} \Rightarrow \forall\left({ }_{-}, q^{\prime},{ }_{-},-\right) \in\lfloor\mathcal{T} \llbracket \Delta \vdash \tau: \mathrm{TYPE} \rrbracket \delta\rfloor_{k-j} . q^{\prime} \preceq \mathrm{A}\right)$

Consider arbitrary $\left({ }_{-}, q^{\prime},,_{-},-\right) \in\lfloor\mathcal{T} \llbracket \Delta \vdash \tau: \text { TYPE } \rrbracket \delta\rfloor_{k-j}$.

Note that $\left({ }_{-}, q^{\prime},,_{-},\right) \in \mathcal{T} \llbracket \Delta \vdash \tau:$ TYPE $\rrbracket \delta$, which follows from the definition of $\lfloor\cdot\rfloor_{k}$. Note that $q^{\prime} \preceq \mathrm{A}$, which follows from Lemma 15 applied to $\Delta \vdash \tau \preceq \mathrm{A}$ and $\left(., q^{\prime},{ }_{-},-\right) \in$ $\mathcal{T} \llbracket \Delta \vdash \tau: \mathrm{TYPE} \rrbracket \delta$ and $\mathrm{A}=\mathcal{T} \llbracket \Delta \vdash \mathrm{A}: \mathrm{QUAL} \rrbracket \delta$.

Case $q=\mathrm{A}$ : Symmetric.

\section{End Case}


$(\operatorname{NEW}(\mathrm{R}, \mathrm{L}))$

\section{Case $\frac{\mathrm{R} \preceq q \quad \Delta ; \Gamma \vdash e: \tau}{\Delta ; \Gamma \vdash \text { new }_{q} e:{ }^{q} \operatorname{ref} \tau}$ :}

We are required to show $\llbracket \Delta ; \Gamma \vdash$ new $_{q} e_{1}: \xi_{\text {ref }} \tau \rrbracket$.

Consider arbitrary $k, \delta, q_{\Gamma}, W_{\Gamma}$, and $\gamma$ such that

- $k \geq 0$,

- $\delta \in \mathcal{D} \llbracket \Delta \rrbracket$, and

- $\left(k, q_{\Gamma}, W_{\Gamma}, \gamma\right) \in \mathcal{G} \llbracket \Delta \vdash \Gamma \rrbracket \delta$.

Let $e_{s}=\gamma\left(\right.$ new $\left._{q} e_{1}\right) \equiv \operatorname{new}_{q} \gamma\left(e_{1}\right)$ and $W_{s}=W_{\Gamma}$.

We are required to show that $\operatorname{Comp}\left(k, W_{s}, e_{s}, \mathcal{T} \llbracket \Delta \vdash{ }^{\text {ref }} \tau:\right.$ TYPE $\left.\rrbracket \delta\right) \quad \equiv$ $\operatorname{Comp}\left(k, W_{\Gamma}, \operatorname{new}_{q} \gamma\left(e_{1}\right), \mathcal{T} \llbracket \Delta \vdash \mathrm{U}_{\text {ref }} \tau:\right.$ TYPE $\left.\rrbracket \delta\right)$.

Consider arbitrary $j, W_{r}, w_{s}, w_{f}$, and $e_{f}$ such that

- $j<k$,

- $w_{s}:_{k}\left(W_{s} \odot_{k} W_{r}\right) \equiv w_{s}:_{k}\left(W_{\Gamma} \odot_{k} W_{r}\right)$,

- $\left(w_{s}, e_{s}\right)=\left(w_{s}\right.$, new $\left._{q} \gamma\left(e_{1}\right)\right) \longmapsto^{j}\left(w_{f}, e_{f}\right)$, and

- $\operatorname{irred}\left(w_{f}, e_{f}\right)$.

Hence, by inspection of the operational semantics, it follows that there exist $j_{1}, w_{f_{1}}$, and $e_{f_{1}}$ such that

- $\left(w_{s}, \gamma\left(e_{1}\right)\right) \longmapsto{ }^{j_{1}}\left(w_{f_{1}}, e_{f_{1}}\right)$,

- $\operatorname{irred}\left(w_{f_{1}}, e_{f_{1}}\right)$, and

- $j_{1} \leq j$.

Applying the induction hypothesis to $\Delta ; \Gamma \vdash e_{1}: \tau$, we conclude that $\llbracket \Delta ; \Gamma \vdash e_{1}: \tau \rrbracket$. Instantiate this with $k, \delta, q_{\Gamma}, W_{\Gamma}$, and $\gamma$. Note that

- $k \geq 0$,

- $\delta \in \mathcal{D} \llbracket \Delta \rrbracket$, and

- $\left(k, q_{\Gamma}, W_{\Gamma}, \gamma\right) \in \mathcal{G} \llbracket \Delta \vdash \Gamma \rrbracket \delta$.

Hence, $\operatorname{Comp}\left(k, W_{\Gamma}, \gamma\left(e_{1}\right), \mathcal{T} \llbracket \Delta \vdash \tau:\right.$ TYPE $\left.\rrbracket \delta\right)$.

Instantiate this with $j_{1}, W_{r}, w_{s}, w_{f_{1}}$, and $e_{f_{1}}$. Note that

- $j_{1}<k$, which follows from $j_{1} \leq j$ and $j<k$,

- $w_{s}:_{k}\left(W_{\Gamma} \odot_{k} W_{r}\right)$, which follows from above,

- $\left(w_{s}, \gamma\left(e_{1}\right)\right) \longmapsto{ }^{j_{1}}\left(w_{f_{1}}, e_{f_{1}}\right)$,

- $\operatorname{irred}\left(w_{f_{1}}, e_{f_{1}}\right)$.

Hence, there exists $W_{f_{1}}$ and $q_{f_{1}}$ such that

- $w_{f_{1}}:_{k-j_{1}}\left(W_{f_{1}} \odot_{k-j_{1}} W_{r}\right)$, and

- $\left(k-j_{1}, q_{f_{1}}, W_{f_{1}}, e_{f_{1}}\right) \in \mathcal{T} \llbracket \Delta \vdash \tau:$ TYPE $\rrbracket \delta$.

Hence, $e_{f_{1}} \equiv v_{f_{1}}$.

Note that

$$
\begin{aligned}
\left(w_{s}, e_{s}\right) & \equiv\left(w_{s}, \operatorname{new}_{q} \gamma\left(e_{1}\right)\right) \\
& \longmapsto^{j_{1}}\left(w_{f_{1}}, \text { new }_{q} e_{f_{1}}\right) \\
& \equiv\left(w_{f_{1}}, \text { new }_{q} v_{f_{1}}\right) \\
& \longmapsto^{1}\left(w_{f_{1}} \uplus\left\{l_{f} \mapsto\left(q, v_{f_{1}}\right)\right\}, l_{f}\right) \quad l_{f} \notin \operatorname{dom}\left(w_{f_{1}}\right) \\
& \longmapsto^{j-j_{1}-1}\left(w_{f}, e_{f}\right) .
\end{aligned}
$$


Since $l_{f}$ is value, we have $\operatorname{irred}\left(w_{f_{1}} \uplus\left\{l_{f} \mapsto\left(q, v_{f_{1}}\right)\right\}, l_{f}\right)$.

Hence, $j-j_{1}-1=0$ (and $j=j_{1}+1$ ) and $w_{f} \equiv w_{f_{1}} \uplus\left\{l_{f} \mapsto v_{f_{1}}\right\}$ and $e_{f} \equiv l_{f}$.

Note that

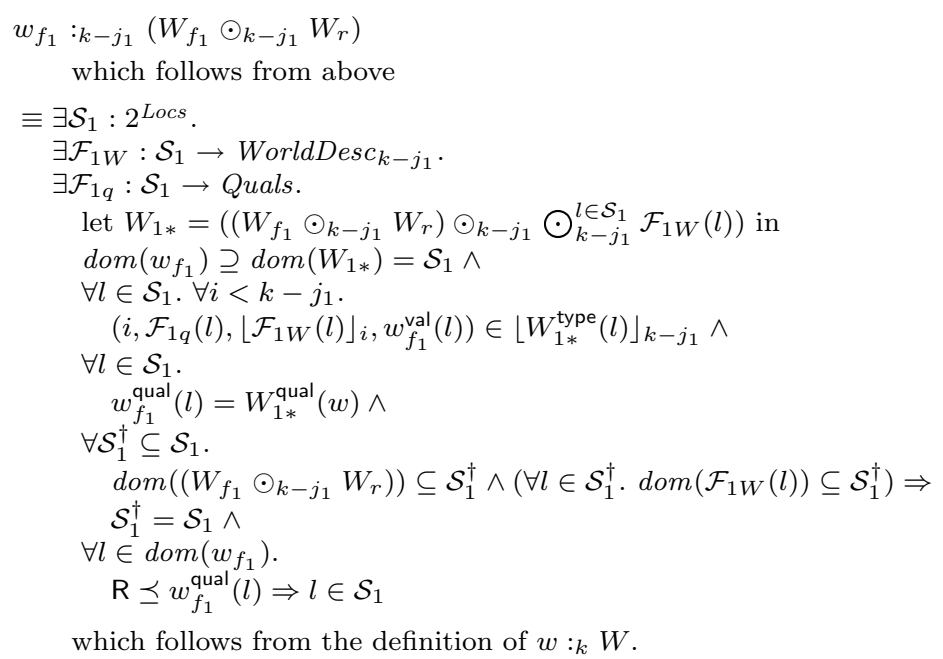

Note that

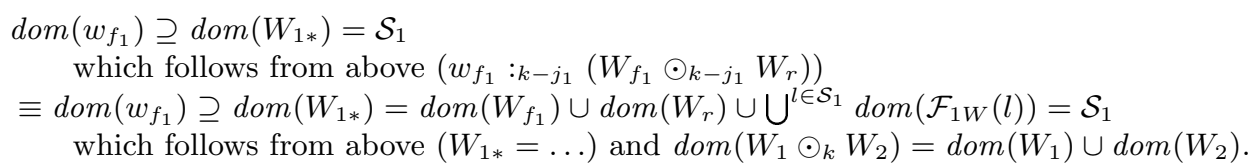

Furthermore, note that $l_{f} \notin \operatorname{dom}\left(W_{1 *}\right)$ and $l_{f} \notin \operatorname{dom}\left(W_{r}\right)$ and $l_{f} \notin \mathcal{S}_{1}$, which follows from $l_{f} \notin \operatorname{dom}\left(w_{f_{1}}\right)$.

Note that either $q=\mathrm{L}$ or $q=\mathrm{R}$, which follows from $\mathrm{R} \preceq q$.

Case $q=\mathrm{L}$ :

Let $\chi_{f}=\lfloor\mathcal{T} \llbracket \Delta \vdash \tau: \text { TYPE } \rrbracket \delta\rfloor_{k-j}$.

Let $W_{f}=\left\{l_{f} \mapsto\left(\mathrm{L}, \chi_{f}\right)\right\}$.

Let $q_{f}=\mathrm{L}$.

Note that $\left(\left\{l_{f} \mapsto\left(\mathrm{L}, \chi_{f}\right)\right\} \odot_{k-j} W_{r}\right)$ defined, which follows from $l_{f} \notin \operatorname{dom}\left(W_{r}\right)$.

We are required to show that

- $w_{f}:_{k-j}\left(W_{f} \odot_{k-j} W_{r}\right)$ $\equiv w_{f_{1}} \uplus\left\{l_{f} \mapsto v_{f_{1}}\right\}:_{k-j}\left(\left\{l_{f} \mapsto\left(\mathrm{L}, \chi_{f}\right)\right\} \odot_{k-j} W_{r}\right)$, which is equivalent to

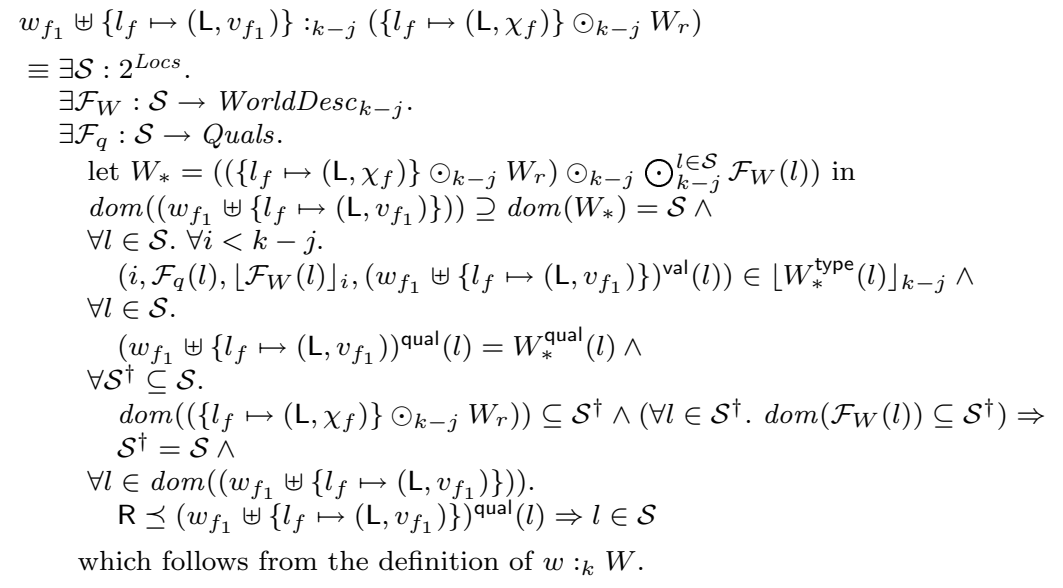


Take

$$
\mathcal{S}=\left\{l_{f}\right\} \uplus \mathcal{S}_{1} .
$$

It remains to show that

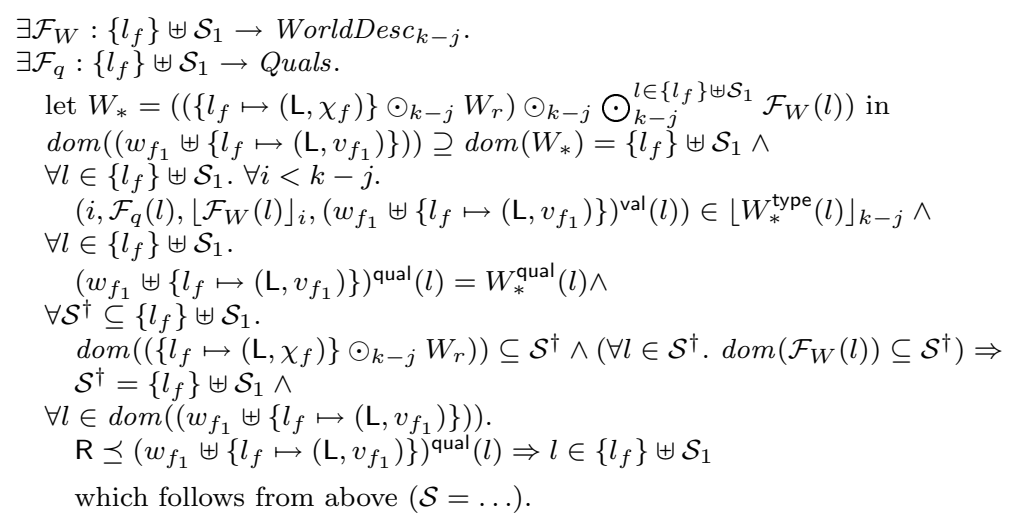

Take

and

$$
\mathcal{F}_{W}(l)= \begin{cases}\left\lfloor W_{f_{1}}\right\rfloor_{k-j} & \text { if } l \in\left\{l_{f}\right\} \\ \left\lfloor\mathcal{F}_{1 W}(l)\right\rfloor_{k-j} & \text { if } l \in \mathcal{S}_{1}\end{cases}
$$

Note that

$W_{f_{1}} \in$ WorldDesc $_{k-j_{1}}$

which follows from Fact 6 applied to $\left(k-j_{1}, q_{f_{1}}, W_{f_{1}}, v_{f_{1}}\right) \in \mathcal{T} \llbracket \Delta \vdash \tau:$ TYPE $\rrbracket \in$ Type, which in turn follows from Lemma 8 applied to $\mathcal{T} \llbracket \Delta \vdash \tau:$ TYPE $\rrbracket \delta$

$\Rightarrow\left\lfloor W_{f_{1}}\right\rfloor_{k-j} \in$ WorldDesc $_{k-j}$

which follows from $\lfloor\cdot\rfloor_{k-j} \in$ WorldDesc $\rightarrow$ WorldDesc $_{k-j}$

$\equiv \mathcal{F}_{W}\left(l_{f}\right) \in$ WorldDesc $_{k-j}$

which follows from above $\left(\mathcal{F}_{W}(l)=\ldots\right)$.

Note that

$\forall l \in \mathcal{S}_{1} . \mathcal{F}_{1 W}(l) \in$ WorldDesc $_{k-j_{1}}$

which follows from above $\left(w_{f_{1}}: k-j_{1}\left(W_{f_{1}} \odot_{k-j_{1}} W_{r}\right)\right) \Rightarrow \forall l \in \mathcal{S}_{1} .\left\lfloor\mathcal{F}_{1 W}(l)\right\rfloor_{k-j} \in$ WorldDesc $_{k-j}$ which follows from $\lfloor\cdot\rfloor_{k} \in$ WorldDesc $\rightarrow$ WorldDesc $_{k}$

$\equiv \forall l \in \mathcal{S}_{1} . \mathcal{F}_{W}(l) \in$ WorldDesc $_{k-j}$

which follows from above $\left(\mathcal{F}_{W}(l)=\ldots\right)$.

Hence, $\mathcal{F}_{W}:\left\{l_{f}\right\} \uplus \mathcal{S}_{1} \rightarrow$ WorldDesc $_{k-j}$.

Trivially, $\mathcal{F}_{q}:\left\{l_{f}\right\} \uplus \mathcal{S}_{1} \rightarrow$ Quals.

It remains to show that

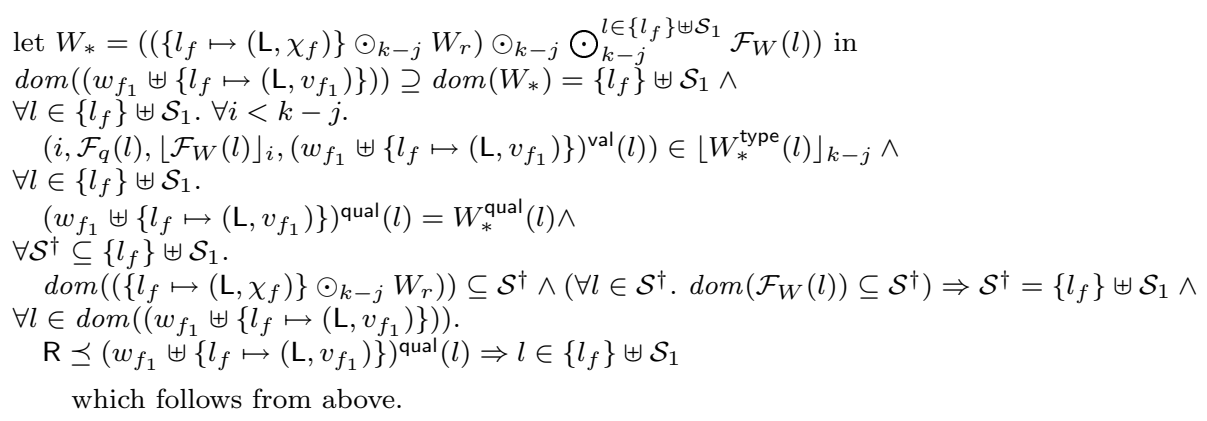

Note that $\left(\left\{l_{f} \mapsto\left(\mathrm{L}, \chi_{f}\right)\right\} \odot_{k-j} W_{1 *}\right)$ defined, which follows from $l_{f} \notin \operatorname{dom}\left(W_{1 *}\right)$.

Furthermore, $\operatorname{dom}\left(\left(\left\{l_{f} \mapsto\left(\mathrm{L}, \chi_{f}\right)\right\} \odot_{k-j} W_{1 *}\right)\right)=\left\{l_{f}\right\} \uplus \operatorname{dom}\left(W_{1 *}\right)$.

Note that 


$$
\begin{aligned}
& \left(\left\{l_{f} \mapsto\left(\mathrm{L}, \chi_{f}\right)\right\} \odot_{k-j} W_{1 *}\right) \\
& \equiv\left(\left\{l_{f} \mapsto\left(\mathrm{L}, \chi_{f}\right)\right\} \odot_{k-j}\left(\left(W_{f_{1}} \odot_{k-j_{1}} W_{r}\right) \odot_{k-j_{1}} \bigodot_{k-j_{1}}^{l \in \mathcal{S}_{1}} \mathcal{F}_{1 W}(l)\right)\right) \\
& \quad \text { which follows from above }\left(W_{1 *}=\ldots\right) \\
& \equiv\left(\left\{l_{f} \mapsto\left(\mathrm{L}, \chi_{f}\right)\right\} \odot_{k-j}\left\lfloor\left(\left(W_{f_{1}} \odot_{k-j_{1}} W_{r}\right) \odot_{k-j_{1}} \bigodot_{k-j_{1}}^{l \in \mathcal{S}_{1}} \mathcal{F}_{1 W}(l)\right)\right\rfloor_{k-j}\right) \\
& \quad \text { which follows from Req } 5(\text { join-aprx }) \\
& \equiv\left(\left\{l_{f} \mapsto\left(\mathrm{L}, \chi_{f}\right)\right\} \odot_{k-j}\left(\left(\left\lfloor W_{f_{1}}\right\rfloor_{k-j} \odot_{k-j} W_{r}\right) \odot_{k-j} \bigodot_{k-j}^{l \in \mathcal{S}_{1}}\left\lfloor\mathcal{F}_{1 W}(l)\right\rfloor_{k-j}\right)\right) \\
& \quad \text { which follows from Req } 4(\text { join-closed }) \text { and Req } 5(\text { join-aprx }) \\
& \equiv\left(\left\{l_{f} \mapsto\left(\mathrm{L}, \chi_{f}\right)\right\} \odot_{k-j}\left(\left(\mathcal{F}_{W}\left(l_{f}\right) \odot_{k-j} W_{r}\right) \odot_{k-j} \bigodot_{k-j}^{l \in \mathcal{S}_{1}} \mathcal{F}_{W}(l)\right)\right) \\
& \quad \text { which follows from above }\left(\mathcal{F}_{W}(l)=\ldots\right) \\
& \equiv\left(\left(\left\{l_{f} \mapsto\left(\mathrm{L}, \chi_{f}\right)\right\} \odot_{k-j} W_{r}\right) \odot_{k-j}\left(\mathcal{F}_{W}\left(l_{f}\right) \odot_{k-j} \bigodot_{k-j}^{l \in \mathcal{S}_{1}} \mathcal{F}_{W}(l)\right)\right) \\
& \quad \text { which follows from Reqs } 6,7, \text { and } 8(\text { join-commut, join-assocl, and join-assocr }) \\
& \equiv\left(\left(\left\{l_{f} \mapsto\left(\mathrm{L}, \chi_{f}\right)\right\} \odot_{k-j} W_{r}\right) \odot_{k-j} \bigodot_{k-j}^{l \in l_{f} \uplus \mathcal{S}_{1}} \mathcal{F}_{W}(l)\right) \\
& \quad \text { which follows from simplifications of } \bigodot_{k-j}^{l \in\left\{l_{f}\right\} \uplus \mathcal{S}_{1}} \mathcal{F}_{W}(l) .
\end{aligned}
$$

Hence, $W_{*}=\left(\left(\left\{l_{f} \mapsto\left(\mathrm{L}, \chi_{f}\right)\right\} \odot_{k-j} W_{r}\right) \odot_{k-j} \bigodot_{k-j}^{l \in l_{f} \uplus \mathcal{S}_{1}} \mathcal{F}_{W}(l)\right)$ is defined.

Furthermore, $W_{*}=\left(\left\{l_{f} \mapsto\left(\mathrm{L}, \chi_{f}\right)\right\} \odot_{k-j} W_{1 *}\right)$ and $\operatorname{dom}\left(W_{*}\right)=\operatorname{dom}\left(\left(\left\{l_{f} \mapsto\right.\right.\right.$ $\left.\left.\left.\left(\mathrm{L}, \chi_{f}\right)\right\} \odot_{k-j} W_{1 *}\right)\right)=\left\{l_{f}\right\} \uplus \operatorname{dom}\left(W_{1 *}\right)$.

Note that

$$
\begin{aligned}
& \operatorname{dom}\left(w_{f_{1}}\right) \supseteq \operatorname{dom}\left(W_{1 *}\right)=\mathcal{S}_{1} \\
& \quad \text { which follows from above }\left(w_{f_{1}}: k_{k-j}\left(W_{f_{1}} \odot_{k-j} W_{r}\right)\right) \\
& \equiv \operatorname{dom}\left(w_{f_{1}}\right) \uplus\left\{l_{f}\right\} \supseteq\left\{l_{f}\right\} \uplus \operatorname{dom}\left(W_{1 *}\right)=\left\{l_{f}\right\} \uplus \mathcal{S}_{1} \\
& \quad \text { which follows from } l_{f} \notin \operatorname{dom}\left(w_{f_{1}}\right) \text { and } l_{f} \notin \operatorname{dom}\left(W_{1 *}\right) \text { and } l_{f} \notin \mathcal{S}_{1} \\
& \equiv \operatorname{dom}\left(w_{f_{1}}\right) \uplus\left\{l_{f}\right\} \supseteq \operatorname{dom}\left(W_{*}\right)=\left\{l_{f}\right\} \uplus \mathcal{S}_{1} \\
& \quad \text { which follows from above }\left(\operatorname{dom}\left(W_{*}\right)=\ldots\right) \\
& \equiv \operatorname{dom}\left(\left(w_{f_{1}} \uplus\left\{l_{f} \mapsto\left(\mathrm{L}, v_{f_{1}}\right)\right\}\right)\right) \supseteq \operatorname{dom}\left(W_{*}\right)=\left\{l_{f}\right\} \uplus \mathcal{S}_{1} \\
& \quad \text { which follows from simplifications of } \operatorname{dom}\left(\left(w_{f_{1}} \uplus\left\{l_{f} \mapsto\left(\mathrm{L}, v_{f_{1}}\right)\right\}\right)\right) .
\end{aligned}
$$

It remains to show that

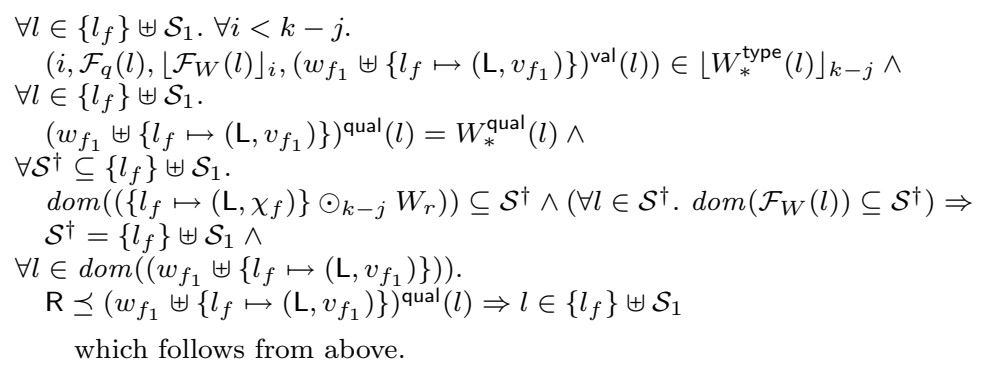

We are required to show that

- $\forall l \in\left\{l_{f}\right\} \uplus \mathcal{S}_{1} . \forall i<k-j$.

$$
\left(i, \mathcal{F}_{q}(l),\left\lfloor\mathcal{F}_{W}(l)\right\rfloor_{i},\left(w_{f_{1}} \uplus\left\{l_{f} \mapsto\left(\mathrm{L}, v_{f_{1}}\right)\right\}\right)^{\text {val }}(l)\right) \in\left\lfloor W_{*}^{\text {type }}(l)\right\rfloor_{k-j}
$$

Note that

$$
\begin{aligned}
& \forall l \in\left\{l_{f}\right\} \uplus \mathcal{S}_{1} . \forall i<k-j . \\
& \left(i, \mathcal{F}_{q}(l),\left\lfloor\mathcal{F}_{W}(l)\right\rfloor_{i},\left(w_{f_{1}} \uplus\left\{l_{f} \mapsto\left(\mathrm{L}, v_{f_{1}}\right)\right\}\right)^{\mathrm{val}}(l)\right) \in\left\lfloor W_{*}^{\text {type }}(l)\right\rfloor_{k-j} \\
& \equiv \forall l \in\left\{l_{f}\right\} . \forall i<k-j \text {. } \\
& \left(i, \mathcal{F}_{q}(l),\left\lfloor\mathcal{F}_{W}(l)\right\rfloor_{i},\left(w_{f_{1}} \uplus\left\{l_{f} \mapsto\left(\mathrm{L}, v_{f_{1}}\right)\right\}\right)^{\mathrm{val}}(l)\right) \in\left\lfloor W_{*}^{\text {type }}(l)\right\rfloor_{k-j} \wedge \\
& \forall l \in \mathcal{S}_{1} . \forall i<k-j . \\
& \left(i, \mathcal{F}_{q}(l),\left\lfloor\mathcal{F}_{W}(l)\right\rfloor_{i},\left(w_{f_{1}} \uplus\left\{l_{f} \mapsto\left(\mathrm{L}, v_{f_{1}}\right)\right\}\right)^{\mathrm{val}}(l)\right) \in\left\lfloor W_{*}^{\text {type }}(l)\right\rfloor_{k-j} \\
& \text { which follows from simplifications of } \forall l \in\left\{l_{f}\right\} \uplus \mathcal{S}_{1} \ldots l \ldots \\
& \equiv \forall i<k-j \text {. } \\
& \left(i, \mathcal{F}_{q}\left(l_{f}\right),\left\lfloor\mathcal{F}_{W}\left(l_{f}\right)\right\rfloor_{i},\left(w_{f_{1}} \uplus\left\{l_{f} \mapsto\left(\mathrm{L}, v_{f_{1}}\right)\right\}\right)^{\text {val }}\left(l_{f}\right)\right) \in\left\lfloor W_{*}^{\text {type }}\left(l_{f}\right)\right\rfloor_{k-j} \wedge \\
& \forall l \in \mathcal{S}_{1} . \forall i<k-j . \\
& \left(i, \mathcal{F}_{q}(l),\left\lfloor\mathcal{F}_{W}(l)\right\rfloor_{i},\left(w_{f_{1}} \uplus\left\{l_{f} \mapsto\left(\mathrm{L}, v_{f_{1}}\right)\right\}\right)^{\mathrm{val}}(l)\right) \in\left\lfloor W_{*}^{\text {type }}(l)\right\rfloor_{k-j} \\
& \text { which follows from simplifications of } \forall l \in\left\{l_{f}\right\} . . . l \ldots
\end{aligned}
$$




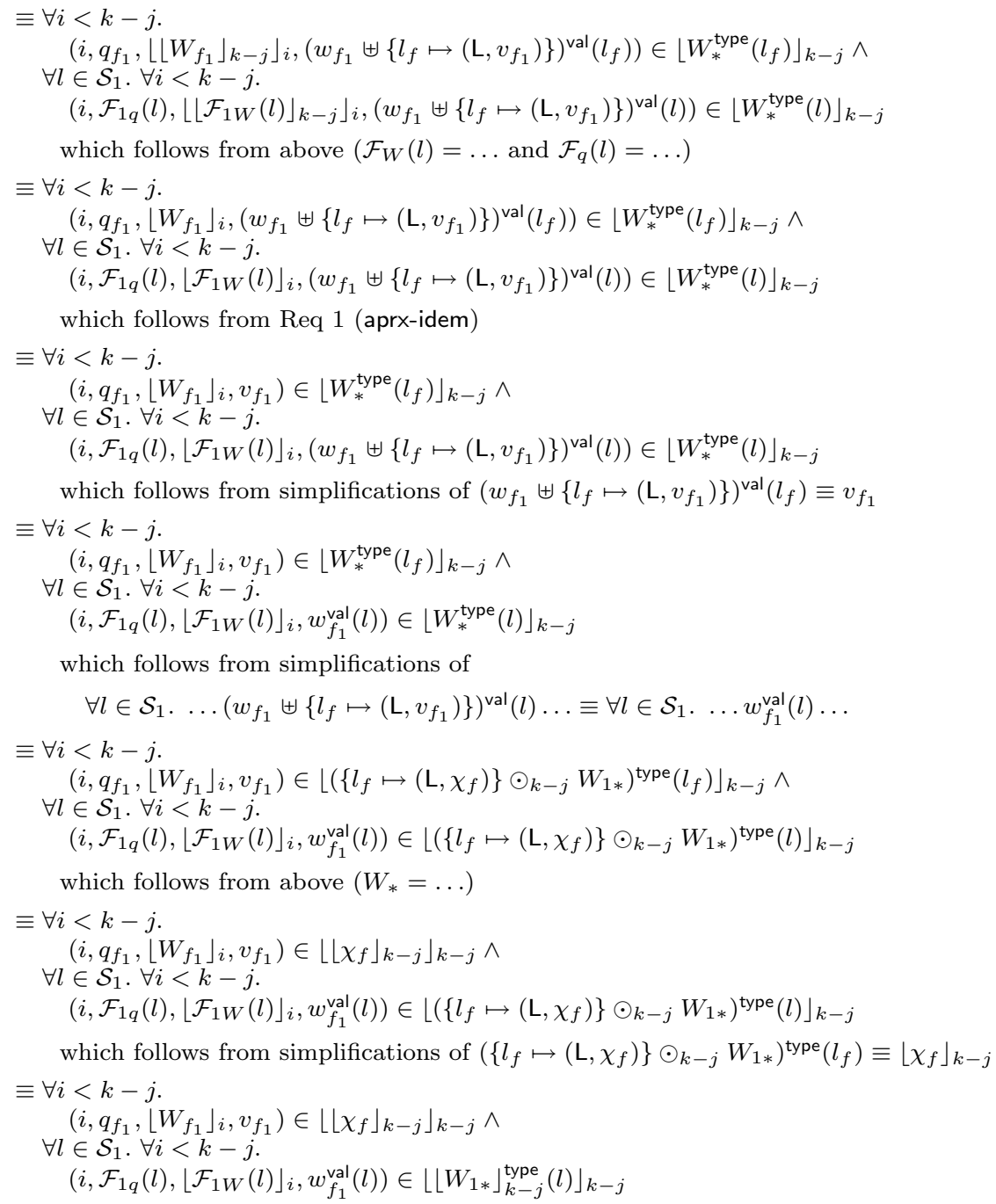

which follows from simplifications of

$\forall l \in \mathcal{S}_{1} \ldots\left(\left\{l_{f} \mapsto\left(\mathrm{L}, \chi_{f}\right)\right\} \odot_{k-j} W_{1 *}\right)^{\mathrm{type}}(l) \ldots \equiv \forall l \in \mathcal{S}_{1} \ldots\left\lfloor W_{1 *}\right\rfloor_{k-j}^{\mathrm{type}}(l) \ldots$.

We are required to show that

- $\forall i<k-j$.

$\left(i, q_{f_{1}},\left\lfloor W_{f_{1}}\right\rfloor_{i}, v_{f_{1}}\right) \in\left\lfloor\left\lfloor\chi_{f}\right\rfloor_{k-j}\right\rfloor_{k-j}$

which follows from 


$$
\begin{aligned}
& \left(k-j_{1}, q_{f_{1}}, W_{f_{1}}, v_{f_{1}}\right) \in \mathcal{T} \llbracket \Delta \vdash \tau: \text { TYPE } \rrbracket \delta \\
& \text { which follows from above } \\
& \Rightarrow \forall i<k-j_{1} \cdot\left(i, q_{f_{1}},\left\lfloor W_{f_{1}}\right\rfloor_{i}, v_{f_{1}}\right) \in \mathcal{T} \llbracket \Delta \vdash \tau: \text { TYPE } \rrbracket \delta \\
& \quad \text { which follows from Lemma } 8 \text { and Fact } 6 \\
& \Rightarrow \forall i<k-j .\left(i, q_{f_{1}},\left\lfloor W_{f_{1}}\right\rfloor_{i}, v_{f_{1}}\right) \in \mathcal{T} \llbracket \Delta \vdash \tau: \text { TYPE } \rrbracket \delta \\
& \quad \text { which follows } k-j<k-j_{1} \\
& \Rightarrow \forall i<k-j .\left(i, q_{f_{1}},\left\lfloor W_{f_{1}}\right\rfloor_{i}, v_{f_{1}}\right) \in\lfloor\mathcal{T} \llbracket \Delta \vdash \tau: \text { TYPE } \rrbracket \delta\rfloor_{k-j} \\
& \quad \text { which follows from } j<k \wedge(j, q, W, v) \in \chi \Rightarrow(j, q, W, v) \in\lfloor\chi\rfloor_{k} \\
& \Rightarrow \forall i<k-j .\left(i, q_{f_{1}},\left\lfloor W_{f_{1}}\right\rfloor_{i}, v_{f_{1}}\right) \in \chi_{f} \\
& \quad \text { which follows from above }\left(\chi_{f}=\ldots\right) \\
& \Rightarrow \forall i<k-j \cdot\left(i, q_{f_{1}},\left\lfloor W_{f_{1}}\right\rfloor_{i}, v_{f_{1}}\right) \in\left\lfloor\chi_{f}\right\rfloor_{k-j} \\
& \quad \text { which follows from } j<k \wedge(j, q, W, v) \in \chi \Rightarrow(j, q, W, v) \in\lfloor\chi\rfloor_{k} \\
& \Rightarrow \forall i<k-j .\left(i, q_{f_{1}},\left\lfloor W_{f_{1}}\right\rfloor_{i}, v_{f_{1}}\right) \in\left\lfloor\left\lfloor\chi_{f}\right\rfloor_{k-j}\right\rfloor_{k-j} \\
& \quad \text { which follows from } j<k \wedge(j, q, W, v) \in \chi \Rightarrow(j, q, W, v) \in\lfloor\chi\rfloor_{k} .
\end{aligned}
$$

- $\forall l \in \mathcal{S}_{1} . \forall i<k-j$.

$$
\left(i, \mathcal{F}_{1 q}(l),\left\lfloor\mathcal{F}_{1 W}(l)\right\rfloor_{i}, w_{f_{1}}^{\text {val }}(l)\right) \in\left\lfloor\left\lfloor W_{1 *}\right\rfloor_{k-j}^{\text {type }}(l)\right\rfloor_{k-j}
$$

which follows from

$$
\begin{aligned}
\forall l \in \mathcal{S}_{1} . \forall i<k-j_{1} .\left(i, \mathcal{F}_{1 q}(l),\left\lfloor\mathcal{F}_{1 W}(l)\right\rfloor_{i}, w_{f_{1}}^{\text {val }}(l)\right) \in\left\lfloor W_{1 *}^{\text {type }}(l)\right\rfloor_{k-j_{1}} \\
\text { which follows from above }\left(w_{f_{1}}:_{k-j_{1}}\left(W_{f_{1}} \odot_{k-j_{1}} W_{r}\right)\right) \\
\Rightarrow \forall l \in \mathcal{S}_{1} . \forall i<k-j .\left(i, \mathcal{F}_{1 q}(l),\left\lfloor\mathcal{F}_{1 W}(l)\right\rfloor_{i}, w_{f_{1}}^{\text {val }}(l)\right) \in\left\lfloor W_{1 *}^{\text {type }}(l)\right\rfloor_{k-j_{1}} \\
\quad \text { which follows from } k-j<k-j_{1} \\
\Rightarrow \forall l \in \mathcal{S}_{1} . \forall i<k-j .\left(i, \mathcal{F}_{1 q}(l),\left\lfloor\mathcal{F}_{1 W}(l)\right\rfloor_{i}, w_{f_{1}}(l)\right) \in\left\lfloor\left\lfloor W_{1 *}^{\text {type }}(l)\right\rfloor_{k-j_{1}}\right\rfloor_{k-j} \\
\quad \text { which follows from } j<k \wedge(j, q, W, v) \in \chi \Rightarrow(j, q, W, v) \in\lfloor\chi\rfloor_{k} \\
\equiv \forall l \in \mathcal{S}_{1} . \forall i<k-j .\left(i, \mathcal{F}_{1 q}(l),\left\lfloor\mathcal{F}_{1 W}(l)\right\rfloor_{i}, w_{f_{1}}(l)\right) \in\left\lfloor W_{1 *}^{\text {type }}(l)\right\rfloor_{k-j} \\
\quad \text { which follows from Req } 1(\text { aprx-idem }) \\
\equiv \forall l \in \mathcal{S}_{1} . \forall i<k-j .\left(i, \mathcal{F}_{1 q}(l),\left\lfloor\mathcal{F}_{1 W}(l)\right\rfloor_{i}, w_{f_{1}}(l)\right) \in\left\lfloor W_{1 *}\right\rfloor_{k-j}^{\text {type }}(l) \\
\quad \text { which follows from the definition of }\lfloor W\rfloor_{k} \\
\Rightarrow \forall l \in \mathcal{S}_{1} . \forall i<k-j .\left(i, \mathcal{F}_{1 q}(l),\left\lfloor\mathcal{F}_{1 W}(l)\right\rfloor_{i}, w_{f_{1}}(l)\right) \in\left\lfloor\left\lfloor W_{1 *}\right\rfloor_{k-j}^{\text {type }}(l)\right\rfloor_{k-j} \\
\quad \text { which follows from } j<k \wedge(j, q, W, v) \in \chi \Rightarrow(j, q, W, v) \in\lfloor\chi\rfloor_{k} .
\end{aligned}
$$

- $\forall l \in\left\{l_{f}\right\} \uplus \mathcal{S}_{1}$.

$$
\left(w_{f_{1}} \uplus\left\{l_{f} \mapsto\left(\mathrm{L}, v_{f_{1}}\right)\right\}\right)^{\text {qual }}(l)=W_{*}^{\text {qual }}(l)
$$

Note that

$$
\begin{aligned}
\forall l \in\{ & \left\{l_{f}\right\} \uplus \mathcal{S}_{1} . \\
& \left(w_{f_{1}} \uplus\left\{l_{f} \mapsto\left(\mathrm{L}, v_{f_{1}}\right)\right\}\right)^{\text {qual }}(l)=W_{*}^{\text {qual }}(l) \\
\equiv & \forall l \in\left\{l_{f}\right\} . \\
& \left(w_{f_{1}} \uplus\left\{l_{f} \mapsto\left(\mathrm{L}, v_{f_{1}}\right)\right\}\right)^{\text {qual }}(l)=W_{*}^{\text {qual }}(l) \wedge \\
\forall l \in \mathcal{S}_{1} . & \\
& \left(w_{f_{1}} \uplus\left\{l_{f} \mapsto\left(\mathrm{L}, v_{f_{1}}\right)\right\}\right)^{\text {qual }}(l)=W_{*}^{\text {qual }}(l) \\
& \text { which follows from simplifications of } \forall l \in\left\{l_{f}\right\} \uplus \mathcal{S}_{1} \ldots \ldots l \ldots \\
\equiv & \left(w_{f_{1}} \uplus\left\{l_{f} \mapsto\left(\mathrm{L}, v_{f_{1}}\right)\right\}\right)^{\text {qual }}\left(l_{f}\right)=W_{*}^{\text {qual }}\left(l_{f}\right) \wedge \\
\forall l \in \mathcal{S}_{1} . & \left(w_{f_{1}} \uplus\left\{l_{f} \mapsto\left(\mathrm{L}, v_{f_{1}}\right)\right\}\right)^{\text {qual }}(l)=W_{*}^{\text {qual }}(l) \\
& \text { which follows from simplifications of } \forall l \in\left\{l_{f}\right\} \ldots l \ldots \\
\equiv & \mathrm{L}=W_{*}^{\text {qual }}\left(l_{f}\right) \wedge \\
\forall l \in \mathcal{S}_{1} . & \left(w_{f_{1}} \uplus\left\{l_{f} \mapsto\left(\mathrm{L}, v_{f_{1}}\right)\right\}\right)^{\text {qual }}(l)=W_{*}^{\text {qual }}(l) \\
& \text { which follows from simplifications of }\left(w_{f_{1}} \uplus\left\{l_{f} \mapsto\left(\mathrm{L}, v_{f_{1}}\right)\right\}\right)^{\text {qual }}\left(l_{f}\right) \equiv \mathrm{L} \\
\equiv & \mathrm{L}=W_{*}^{\text {qual }}\left(l_{f}\right) \wedge \\
\forall l \in \mathcal{S}_{1} . & w_{f_{1}}^{\text {qual }}(l)=W_{*}^{\text {qual }}(l) \\
& \text { which follows from simplifications of } \\
& \forall l \in \mathcal{S}_{1} \ldots\left(w_{f_{1}} \uplus\left\{l_{f} \mapsto\left(\mathrm{L}, v_{f_{1}}\right)\right\}\right)^{\text {qual }}(l) \ldots \equiv \forall l \in \mathcal{S}_{1} \ldots w_{f_{1}}^{\text {qual }}(l) \ldots
\end{aligned}
$$




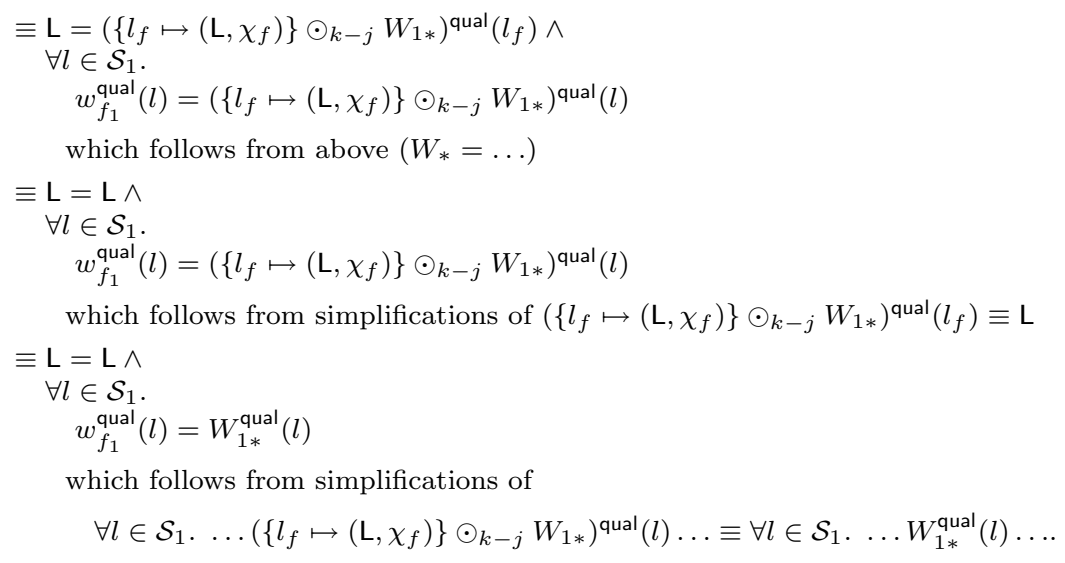

We are required to show that

- $\mathrm{L}=\mathrm{L}$

which follows trivially,

- $\forall l \in \mathcal{S}_{1}$.

$$
w_{f_{1}}^{\text {qual }}(l)=W_{1 *}^{\text {qual }}(l)
$$

which follows from

$$
\begin{aligned}
\forall l \in \mathcal{S}_{1} \cdot w_{f_{1}}^{\text {qual }}(l)=W_{1 *}^{\text {qual }}(l) \\
\quad \text { which follows from above }\left(w_{f_{1}: k-j_{1}}\left(W_{f_{1}} \odot_{k-j_{1}} W_{r}\right)\right) .
\end{aligned}
$$

- $\forall \mathcal{S}^{\dagger} \subseteq\left\{l_{f}\right\} \uplus \mathcal{S}_{1}$.

$\operatorname{dom}\left(\left(\left\{l_{f} \mapsto\left(\mathrm{L}, \chi_{f}\right)\right\} \odot_{k-j} W_{r}\right)\right) \subseteq \mathcal{S}^{\dagger} \wedge$

$\left(\forall l \in \mathcal{S}^{\dagger} . \operatorname{dom}\left(\mathcal{F}_{W}(l)\right) \subseteq \mathcal{S}^{\dagger}\right) \Rightarrow$

$\mathcal{S}^{\dagger}=\left\{l_{f}\right\} \uplus \mathcal{S}_{1}$

Consider arbitrary $\mathcal{S}^{\dagger}$ such that

- $\mathcal{S}^{\dagger} \subseteq\left\{l_{f}\right\} \uplus \mathcal{S}_{1}$,

- $\operatorname{dom}\left(\left(\left\{l_{f} \mapsto\left(\mathrm{L}, \chi_{f}\right)\right\} \odot_{k-j} W_{r}\right)\right) \subseteq \mathcal{S}^{\dagger}$, and

- $\forall l \in \mathcal{S}^{\dagger} . \operatorname{dom}\left(\mathcal{F}_{W}(l)\right) \subseteq \mathcal{S}^{\dagger}$.

Note that $\left\{l_{f}\right\} \uplus \operatorname{dom}\left(W_{r}\right) \subseteq \mathcal{S}^{\dagger}$, which follows from $\operatorname{dom}\left(\left(\left\{l_{f} \mapsto\left(\mathrm{L}, \chi_{f}\right)\right\} \odot_{k-j} W_{r}\right)\right) \subseteq$ $\mathcal{S}^{\dagger}$ and $l_{f} \notin \operatorname{dom}\left(W_{r}\right)$.

Note that $l_{f} \in \mathcal{S}^{\dagger}$, which follows from $\left\{l_{f}\right\} \uplus W_{r} \subseteq \mathcal{S}^{\dagger}$.

Let $\mathcal{S}_{1}^{\dagger}=\mathcal{S}^{\dagger} \backslash\left\{l_{f}\right\}$.

Note that $\mathcal{S}^{\dagger}=\left\{l_{f}\right\} \uplus \mathcal{S}_{1}^{\dagger}$.

Note that

- $\mathcal{S}_{1}^{\dagger} \subseteq \mathcal{S}_{1}$, which follows from $\mathcal{S}^{\dagger} \subseteq\left\{l_{f}\right\} \uplus \mathcal{S}_{1}$,

- $\operatorname{dom}\left(W_{r}\right) \subseteq \mathcal{S}_{1}^{\dagger}$, which follows from $\left\{l_{f}\right\} \uplus \operatorname{dom}\left(W_{r}\right) \subseteq \mathcal{S}^{\dagger}$,

- $\operatorname{dom}\left(\mathcal{F}_{W}\left(l_{f}\right)\right) \subseteq\left\{l_{f}\right\} \uplus \mathcal{S}_{1}^{\dagger}$, which follows from $\forall l \in \mathcal{S}^{\dagger}$. $\operatorname{dom}\left(\mathcal{F}_{W}(l)\right) \subseteq \mathcal{S}^{\dagger}$, and, furthermore, $\operatorname{dom}\left(\left\lfloor W_{f_{1}}\right\rfloor_{k-j}\right) \subseteq\left\{l_{f}\right\} \uplus \mathcal{S}_{1}^{\dagger}$, which follows from the definition of $\mathcal{F}_{W}$, and, furthermore, $\operatorname{dom}\left(W_{f_{1}}\right) \subseteq\left\{l_{f}\right\} \uplus \mathcal{S}_{1}^{\dagger}$,

- $\forall l \in \mathcal{S}_{1}^{\dagger} . \operatorname{dom}\left(\mathcal{F}_{W}(l)\right) \subseteq\left\{l_{f}\right\} \uplus \mathcal{S}_{1}^{\dagger}$, which follows from $\forall l \in \mathcal{S}^{\dagger} . \operatorname{dom}\left(\mathcal{F}_{W}(l)\right) \subseteq \mathcal{S}^{\dagger}$, and, furthermore, $\forall l \in \mathcal{S}_{1}^{\dagger}$. $\operatorname{dom}\left(\left\lfloor\mathcal{F}_{1 W}(l)\right\rfloor_{k-j}\right) \subseteq\left\{l_{f}\right\} \uplus \mathcal{S}_{1}^{\dagger}$, which follows from the definition of $\mathcal{F}_{W}$.

Recall that $\operatorname{dom}\left(W_{f_{1}}\right) \subseteq \mathcal{S}_{1}$ and $\forall l \in \mathcal{S}_{1} \cdot \operatorname{dom}\left(\mathcal{F}_{1 W}(l)\right) \subseteq \mathcal{S}_{1}$, which follows from $w_{f_{1}}: k-j_{1}\left(W_{f_{1}} \odot_{k-j_{1}} W_{r}\right)$.

Hence, $\operatorname{dom}\left(W_{f_{1}}\right) \subseteq \mathcal{S}_{1}^{\dagger}$, which follows from $\operatorname{dom}\left(W_{f_{1}}\right) \subseteq\left\{l_{f}\right\} \uplus \mathcal{S}_{1}^{\dagger}$ and $\operatorname{dom}\left(W_{f_{1}}\right) \subseteq$ $\mathcal{S}_{1}$ and $l_{f} \notin \mathcal{S}_{1}$. 
Hence, $\quad \forall l \in \mathcal{S}_{1}^{\dagger} . \quad \operatorname{dom}\left(\mathcal{F}_{1 W}(l)\right) \subseteq \mathcal{S}_{1}^{\dagger}$, which follows from $\forall l \quad \in$ $\mathcal{S}_{1}^{\dagger} \cdot \operatorname{dom}\left(\left\lfloor\mathcal{F}_{1 W}(l)\right\rfloor_{k-j}\right) \subseteq\left\{l_{f}\right\} \uplus \mathcal{S}_{1}^{\dagger}$ and $\forall l \in \mathcal{S}_{1} \cdot \operatorname{dom}\left(\mathcal{F}_{1 W}(l)\right) \subseteq \mathcal{S}_{1}\left(\right.$ and $\left.\mathcal{S}_{1}^{\dagger} \subseteq \mathcal{S}_{1}\right)$ and $l_{f} \notin \mathcal{S}_{1}$.

Instantiate $\left(\forall \mathcal{S}_{1}^{\dagger} \subseteq \mathcal{S}_{1} \ldots\right)$ of $w_{f_{1}}: k-j_{1}\left(W_{f_{1}} \odot_{k-j_{1}} W_{r}\right)$ with $\mathcal{S}_{1}^{\dagger}$. Note that

- $\mathcal{S}_{1}^{\dagger} \subseteq \mathcal{S}_{1}$, which follows from above,

- $\operatorname{dom}\left(\left(W_{f_{1}} \odot_{k-j_{1}} \operatorname{dom}\left(W_{r}\right)\right)\right) \subseteq \mathcal{S}_{1}^{\dagger}$, which follows from $\operatorname{dom}\left(W_{f_{1}}\right) \subseteq \mathcal{S}_{1}^{\dagger}$, which follows from above, and $\operatorname{dom}\left(W_{r}\right) \subseteq \mathcal{S}_{1}^{\dagger}$, which follows from above,

- $\forall l \in \mathcal{S}_{1}^{\dagger} . \operatorname{dom}\left(\mathcal{F}_{1 W}(l)\right) \subseteq \mathcal{S}_{1}^{\dagger}$, which follows from above.

Hence, we conclude that $\mathcal{S}_{1}^{\dagger}=\mathcal{S}_{1}$.

Hence, $\mathcal{S}^{\dagger}=\left\{l_{f}\right\} \uplus \mathcal{S}_{1}^{\dagger}=\left\{l_{f}\right\} \uplus \mathcal{S}_{1}$.

- $\forall l \in \operatorname{dom}\left(\left(w_{f_{1}} \uplus\left\{l_{f} \mapsto\left(\mathrm{L}, v_{f_{1}}\right)\right\}\right)\right)$.

$\mathrm{R} \preceq\left(w_{f_{1}} \uplus\left\{l_{f} \mapsto\left(\mathrm{L}, v_{f_{1}}\right)\right\}\right)^{\text {qual }}(l) \Rightarrow l \in\left\{l_{f}\right\} \uplus \mathcal{S}_{1}$

Note that

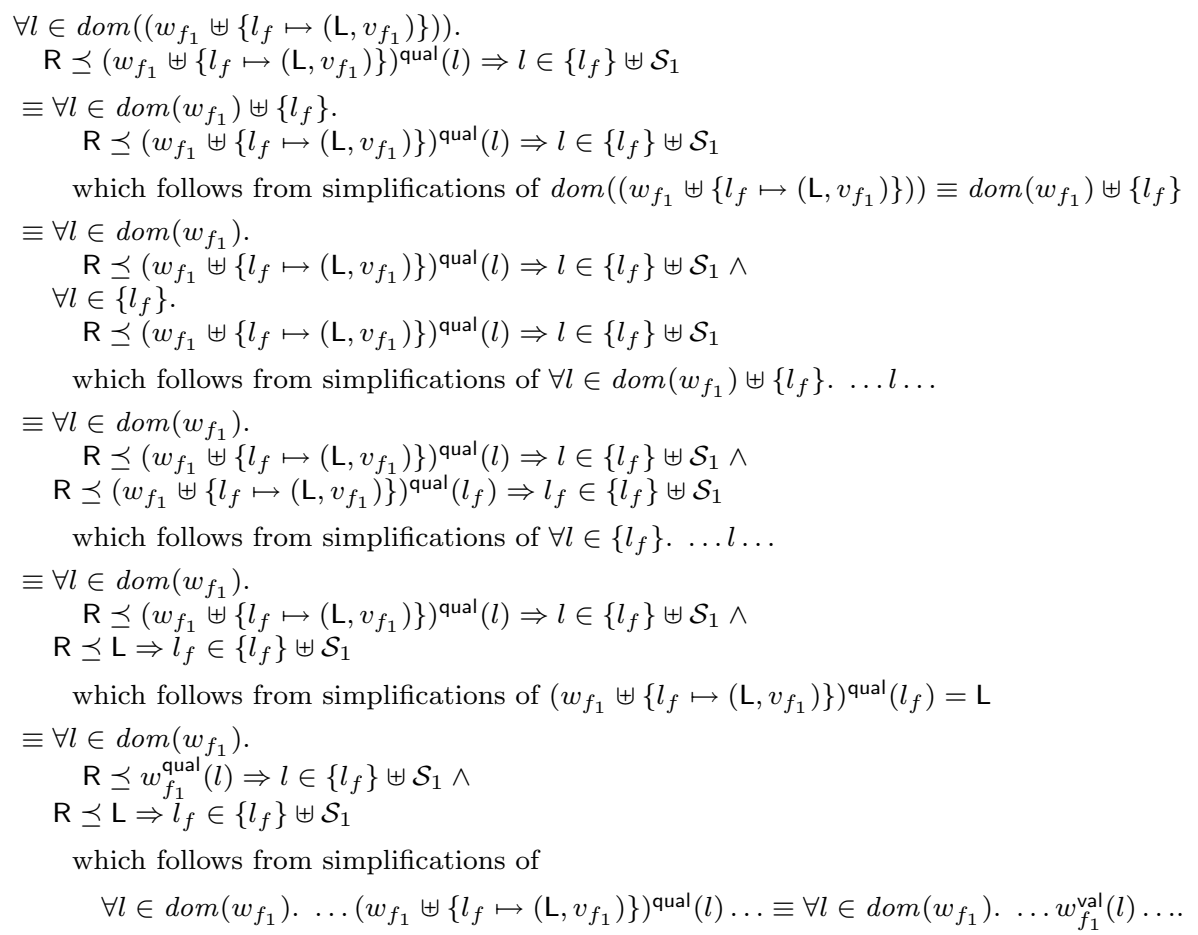

We are required to show that

- $\forall l \in \operatorname{dom}\left(w_{f_{1}}\right) . \mathrm{R} \preceq w_{f_{1}}^{\text {qual }}(l) \Rightarrow l \in\left\{l_{f}\right\} \uplus \mathcal{S}_{1}$

which follows from

$$
\begin{aligned}
& \forall l \in \operatorname{dom}\left(w_{f_{1}}\right) . \mathrm{R} \preceq w_{f_{1}}^{\text {qual }}(l) \Rightarrow l \in \mathcal{S}_{1} \\
& \text { which follows from } w_{f_{1}}: k-j_{1} \\
& \left.\Rightarrow W_{f_{1}} \odot_{k-j_{1}} W_{r}\right) \\
& \forall l \in \operatorname{dom}\left(w_{f_{1}}\right) . \mathrm{R} \preceq w_{f_{1}}^{\text {qual }}(l) \Rightarrow l \in\left\{l_{f}\right\} \uplus \mathcal{S}_{1} \\
& \text { which follows from } l_{f} \notin \mathcal{S}_{1} .
\end{aligned}
$$

- $\mathrm{R} \preceq \mathrm{L} \Rightarrow l_{f} \in\left\{l_{f}\right\} \uplus \mathcal{S}_{1}$ which follows trivially. 
- $\left(k-j, q_{f}, W_{f}, e_{f}\right) \in \mathcal{T} \llbracket \Delta \vdash{ }^{\mathrm{L}} \operatorname{ref} \tau: \mathrm{TYPE} \rrbracket \delta$

$\equiv\left(k-j, \mathrm{~L},\left\{l_{f} \mapsto\left(\mathrm{L}, \chi_{f}\right)\right\}, l_{f}\right) \in \mathcal{T} \llbracket \Delta \vdash \mathrm{L}_{\text {ref }} \tau: \mathrm{TYPE} \rrbracket \delta$

$\equiv\left(k-j, \mathrm{~L},\left\{l_{f} \mapsto\left(\mathrm{L}, \chi_{f}\right)\right\}, l_{f}\right)$

$\in\{(k, q, W, v) \mid$

$q=\mathcal{T} \llbracket \Delta \vdash \mathrm{L}: \mathrm{QUAL} \rrbracket \delta \wedge$

$(k, q, W, v) \in \mathcal{T} \llbracket \Delta \vdash \operatorname{ref} \tau: \mathrm{PRETYPE} \rrbracket \delta\}$

$\equiv\left(k-j, \mathrm{~L},\left\{l_{f} \mapsto\left(\mathrm{L}, \chi_{f}\right)\right\}, l_{f}\right)$

$\in\{(k, q,\{l \mapsto(q, \chi)\}, l) \mid$

$q=\mathcal{T} \llbracket \Delta \vdash \mathrm{L}: \mathrm{QUAL} \rrbracket \delta \wedge$

$\chi=\lfloor\mathcal{T} \llbracket \Delta \vdash \tau: \text { TYPE } \rrbracket \delta\rfloor_{k} \wedge$

$\left.\left(q \preceq \mathrm{A} \Rightarrow \forall\left({ }_{-}, q^{\prime},{ }_{-},{ }_{-}\right) \in \chi . q^{\prime} \preceq \mathrm{A}\right)\right\}$,

which follows from

- $\mathrm{L}=\mathcal{T} \llbracket \Delta \vdash \mathrm{L}: \mathrm{QUAL} \rrbracket \delta$, which follows trivially,

- $\chi_{f}=\lfloor\mathcal{T} \llbracket \Delta \vdash \tau \text { : TYPE } \rrbracket \delta\rfloor_{k-j}$, which follows trivially,

- $(\mathrm{L} \preceq \mathrm{A} \Rightarrow \ldots)$, which follows trivially.

Case $q=\mathrm{R}$ : Symmetric.

\section{End Case}


(FREE)

Case $\frac{\Delta ; \Gamma \vdash e_{1}: \xi_{\text {ref }} \tau \quad \Delta \vdash \mathrm{A} \preceq \xi}{\Delta ; \Gamma \vdash \text { free } e_{1}: \tau}$ :

We are required to show $\llbracket \Delta ; \Gamma \vdash$ free $e_{1}: \tau \rrbracket$.

Consider arbitrary $k, \delta, q_{\Gamma}, W_{\Gamma}$, and $\gamma$ such that

- $k \geq 0$,

- $\delta \in \mathcal{D} \llbracket \Delta \rrbracket$, and

- $\left(k, q_{\Gamma}, W_{\Gamma}, \gamma\right) \in \mathcal{G} \llbracket \Delta \vdash \Gamma \rrbracket \delta$.

Let $e_{s}=\gamma\left(\right.$ free $\left.e_{1}\right) \equiv$ free $\gamma\left(e_{1}\right)$ and $W_{s}=W_{\Gamma}$.

We are required to show that $\operatorname{Comp}\left(k, W_{s}, e_{s}, \mathcal{T} \llbracket \Delta \vdash \tau: \mathrm{TYPE} \rrbracket \delta\right) \quad \equiv$ $\operatorname{Comp}\left(k, W_{\Gamma}\right.$, free $\gamma\left(e_{1}\right), \mathcal{T} \llbracket \Delta \vdash \tau:$ TYPE $\left.\rrbracket\right)$.

Consider arbitrary $j, W_{r}, w_{s}, w_{f}$, and $e_{f}$ such that

- $j<k$,

- $w_{s}:_{k}\left(W_{s} \odot_{k} W_{r}\right) \equiv w_{s}:_{k}\left(W_{\Gamma} \odot_{k} W_{r}\right)$,

- $\left(w_{s}, e_{s}\right)=\left(w_{s}\right.$, free $\left.\gamma\left(e_{1}\right)\right) \longmapsto j\left(w_{f}, e_{f}\right)$, and

- $\operatorname{irred}\left(w_{f}, e_{f}\right)$.

Hence, by inspection of the operational semantics, it follows that there exist $j_{1}, w_{f_{1}}$, and $e_{f_{1}}$ such that

- $\left(w_{s}, \gamma\left(e_{1}\right)\right) \longmapsto{ }^{j_{1}}\left(w_{f_{1}}, e_{f_{1}}\right)$,

- $\operatorname{irred}\left(w_{f_{1}}, e_{f_{1}}\right)$, and

- $j_{1} \leq j$.

Applying the induction hypothesis to $\Delta ; \Gamma \vdash e_{1}:{ }^{\xi}$ ref $\tau$, we conclude that $\llbracket \Delta ; \Gamma \vdash e_{1}:{ }^{\xi}$ ref $\tau \rrbracket$. Instantiate this with $k, \delta, q_{\Gamma}, W_{\Gamma}$, and $\gamma$. Note that

- $k \geq 0$,

- $\delta \in \mathcal{D} \llbracket \Delta \rrbracket$, and

- $\left(k, q_{\Gamma}, W_{\Gamma}, \gamma\right) \in \mathcal{G} \llbracket \Delta \vdash \Gamma \rrbracket \delta$.

Hence, $\operatorname{Comp}\left(k, W_{\Gamma}, \gamma\left(e_{1}\right), \mathcal{T} \llbracket \Delta \vdash \xi_{\text {ref }} \tau:\right.$ TYPE $\left.\rrbracket \delta\right)$.

Instantiate this with $j_{1}, W_{r}, w_{s}, w_{f_{1}}$, and $e_{f_{1}}$. Note that

- $j_{1}<k$, which follows from $j_{1} \leq j$ and $j<k$,

- $w_{s}:_{k}\left(W_{\Gamma} \odot_{k} W_{r}\right)$, which follows from above,

- $\left(w_{s}, \gamma\left(e_{1}\right)\right) \longmapsto{ }^{j_{1}}\left(w_{f_{1}}, e_{f_{1}}\right)$,

- $\operatorname{irred}\left(w_{f_{1}}, e_{f_{1}}\right)$.

Hence, there exists $W_{f_{1}}$ and $q_{f_{1}}$ such that

- $w_{f_{1}}:_{k-j_{1}}\left(W_{f_{1}} \odot_{k-j_{1}} W_{r}\right)$, and

- $\left(k-j_{1}, q_{f_{1}}, W_{f_{1}}, e_{f_{1}}\right)$

$\in \mathcal{T} \llbracket \Delta \vdash \xi_{\text {ref }} \tau:$ TYPE $\| \delta$

$\equiv\{(\bar{k}, q,\{l \mapsto(q, \chi)\}, l) \mid$

$q=\mathcal{T} \llbracket \Delta \vdash \xi: \mathrm{QUAL} \rrbracket \delta \wedge$

$\chi=\lfloor\mathcal{T} \llbracket \Delta \vdash \tau: \text { TYPE } \llbracket \delta\rfloor_{k} \wedge$

$\left.\left(q \preceq \mathrm{A} \Rightarrow \forall\left({ }_{-}, q^{\prime},{ }_{-},,_{-}\right) \in \chi \cdot q^{\prime} \preceq \mathrm{A}\right)\right\}$, 
Hence, $e_{f_{1}} \equiv l_{f_{1}}$ and $q_{f_{1}}=\mathcal{T} \llbracket \Delta \vdash \xi: \mathrm{QUAL} \rrbracket \delta$ and $W_{f_{1}} \equiv\left\{l_{f_{1}} \mapsto\left(q_{f_{1}}, \chi_{f_{1}}\right)\right\}$ and $\chi_{f_{1}}=$ $\lfloor\mathcal{T} \llbracket \Delta \vdash \tau: \mathrm{TYPE} \rrbracket \delta\rfloor_{k-j_{1}}$ and $\left(q_{f_{1}} \preceq \mathrm{A} \Rightarrow \forall\left({ }_{-}, q^{\prime},{ }_{-},{ }_{-}\right) \in \chi_{f_{1}} . q^{\prime} \preceq \mathrm{A}\right)$.

Note that $\mathrm{A} \preceq q_{f_{1}}$, which follows from Lemma 12 applied to $\Delta \vdash \mathrm{A} \preceq \xi$ and $\mathrm{A}=$ $\mathcal{T} \llbracket \Delta \vdash \mathrm{A}: \mathrm{QUAL} \rrbracket \delta$ and $q_{f_{1}}=\mathcal{T} \llbracket \Delta \vdash \xi: \mathrm{QUAL} \rrbracket \delta$.

Note that $l_{f_{1}} \notin \operatorname{dom}\left(W_{r}\right)$, which follows from $\mathrm{A} \preceq q_{f_{1}}=W_{f_{1}}^{\text {qual }}\left(l_{f_{1}}\right)$ and $\left(W_{f_{1}} \odot_{k-j_{1}} W_{r}\right)$ defined.

Note that

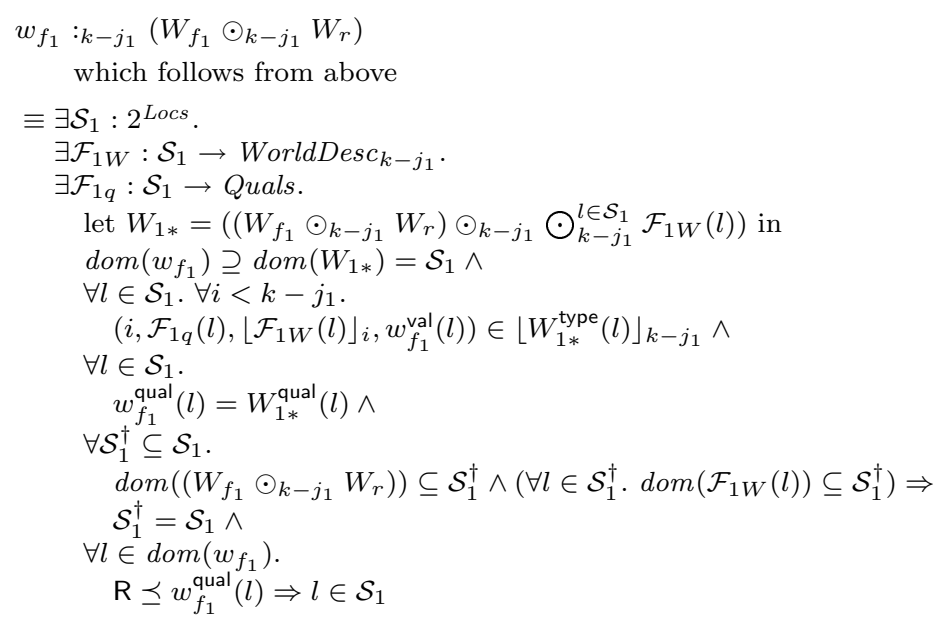

Note that

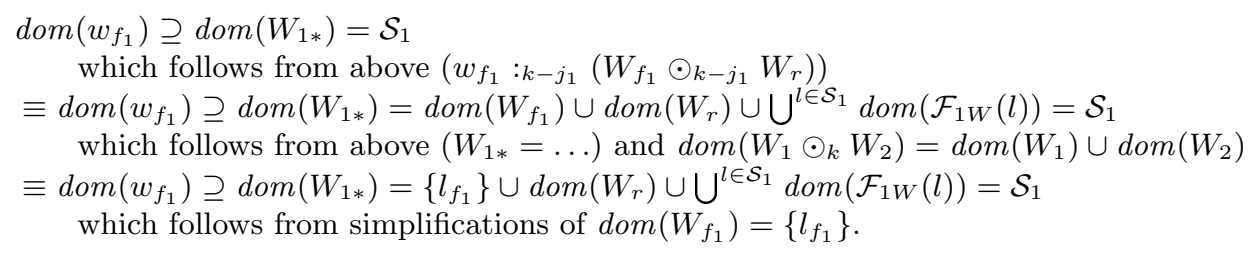

Hence, $l_{f_{1}} \in \operatorname{dom}\left(w_{f_{1}}\right)$ and $l_{f_{1}} \in \mathcal{S}_{1}$.

Note that

$$
\begin{aligned}
& \forall l \in \mathcal{S}_{1} . w_{f_{1}}^{\text {qual }}(l)=W_{1 *}^{\text {qual }}(l) \\
& \quad \text { which follows from above }\left(w_{f_{1}}:_{k-j_{1}}\left(W_{f_{1}} \odot_{k-j_{1}} W_{r}\right)\right) \\
& \Rightarrow w_{f_{1}}^{\text {qual }}\left(l_{f_{1}}\right)=W_{1 *}^{\text {qual }}\left(l_{f_{1}}\right) \\
& \quad \text { which follows from } l_{f_{1}} \in \mathcal{S}_{1} \\
& \equiv w_{f_{1}}^{\text {qual }}\left(l_{f_{1}}\right)=\left(\left(W_{f_{1}} \odot_{k-j_{1}} W_{r}\right) \odot_{k-j_{1}} \bigodot_{k-j_{1}}^{l \in \mathcal{S}_{1}} \mathcal{F}_{1 W}(l)\right)^{\text {qual }}\left(l_{f_{1}}\right) \\
& \quad \text { which follows from above }\left(W_{1 *}=\ldots\right) \\
& \equiv w_{f_{1}}^{\text {qual }}\left(l_{f_{1}}\right)=\left(\left(\left\{l_{f_{1}} \mapsto\left(q_{f_{1}}, \chi_{f_{1}}\right)\right\} \odot_{k-j_{1}} W_{r}\right) \odot_{k-j_{1}} \bigodot_{k-j_{1}}^{l \in \mathcal{S}_{1}} \mathcal{F}_{1 W}(l)\right)^{\text {qual }}\left(l_{f_{1}}\right) \\
& \quad \text { which follows from above }\left(W_{f_{1}}=\ldots\right) \\
& \equiv w_{f_{1}}^{\text {qual }}\left(l_{f_{1}}\right)=q_{f_{1}} \\
& \quad \text { which follows from the definition of }\left(W_{1} \odot_{k} W_{2}\right) .
\end{aligned}
$$

Note that $w_{f_{1}} \equiv w_{f_{11}} \uplus\left\{l_{f_{1}} \mapsto\left(q_{f_{1}}, v_{f_{11}}\right)\right\}$, which follows from $l_{f_{1}} \in \operatorname{dom}\left(w_{f_{1}}\right)$ and $w_{f_{1}}^{\text {qual }}\left(l_{f_{1}}\right)=q_{f_{1}}$. Note that

$$
\begin{aligned}
\left(w_{s}, e_{s}\right) & \equiv\left(w_{s}, \text { free } \gamma\left(e_{1}\right)\right) \\
& \longmapsto{ }^{j}\left(w_{f_{1}}, \text { free } e_{f_{1}}\right) \\
& \equiv\left(w_{f_{1}}, \text { free } l_{f_{1}}\right) \\
& \equiv\left(w_{f_{11}} \uplus\left\{l_{f_{1}} \mapsto\left(q_{f_{1}}, v_{f_{11}}\right)\right\}, \text { free } l_{f_{1}}\right) \\
& \longmapsto \stackrel{1}{\equiv}\left(w_{f_{11}}, v_{f_{11}}\right)
\end{aligned}
$$

Since $v_{f_{11}}$ is a value, we have $\operatorname{irred}\left(w_{f_{11}}, v_{f_{11}}\right)$. 
Hence, $j-j_{1}-1=0\left(\right.$ and $\left.j=j_{1}+1\right)$, and $w_{f} \equiv w_{f_{11}}$ and $e_{f} \equiv v_{f_{11}}$.

Note that

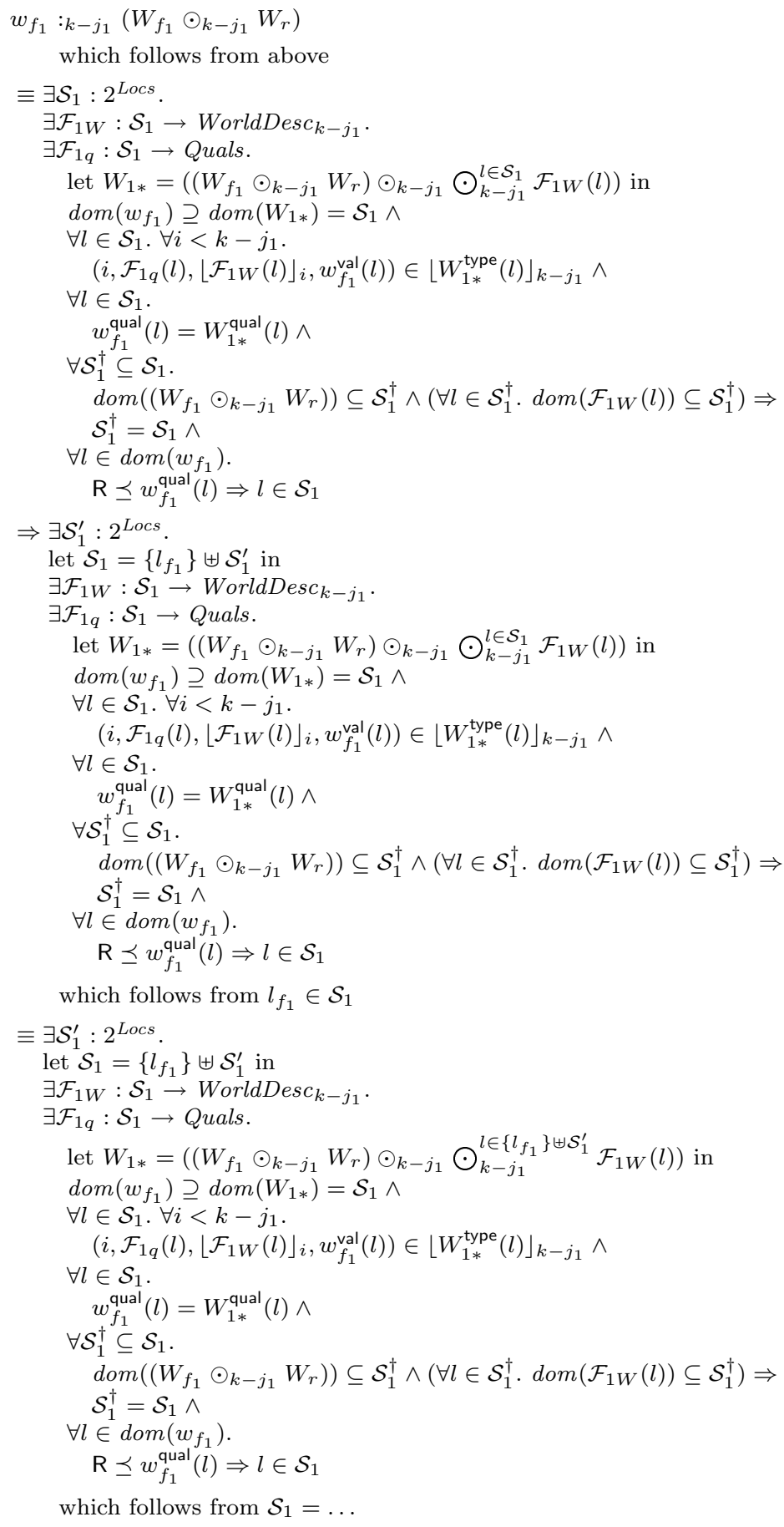




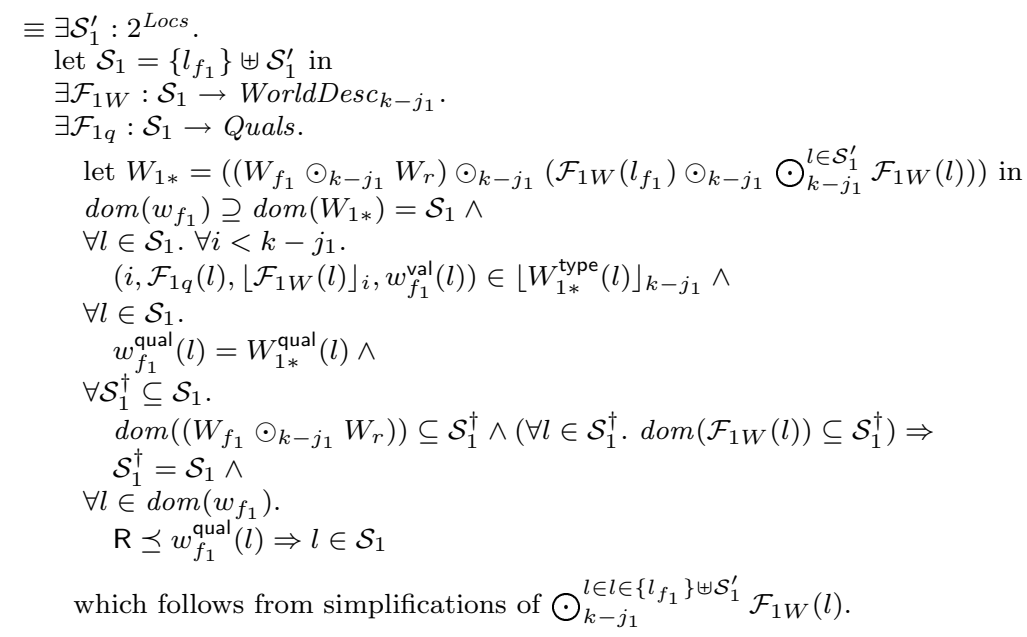

Let $W_{f}=\left\lfloor\mathcal{F}_{1 W}\left(l_{f_{1}}\right)\right\rfloor_{k-j}$.

Let $q_{f}=\mathcal{F}_{1 q}\left(l_{f_{1}}\right)$.

Note that $\left(\left\lfloor\mathcal{F}_{1 W}\left(l_{f_{1}}\right)\right\rfloor_{k-j} \odot_{k-j} W_{r}\right)$ is defined, which follows from Reqs $4,5,6,7$, and 8 (joinclosed, join-aprx, join-commut, join-assocl, and join-assocr $)$ and $\left(\mathcal{F}_{1 W}\left(l_{f_{1}}\right) \odot_{k-j_{1}} W_{r}\right)$ defined, which in turn follows from $W_{1 *}$ defined.

We are required to show that

- $w_{f}:_{k-j}\left(W_{f} \odot_{k-j} W_{r}\right)$

$\equiv w_{f_{11}}: k-j\left(\left\lfloor\mathcal{F}_{1 W}\left(l_{f_{1}}\right)\right\rfloor_{k-j} \odot_{k-j} W_{r}\right)$

which is equivalent to

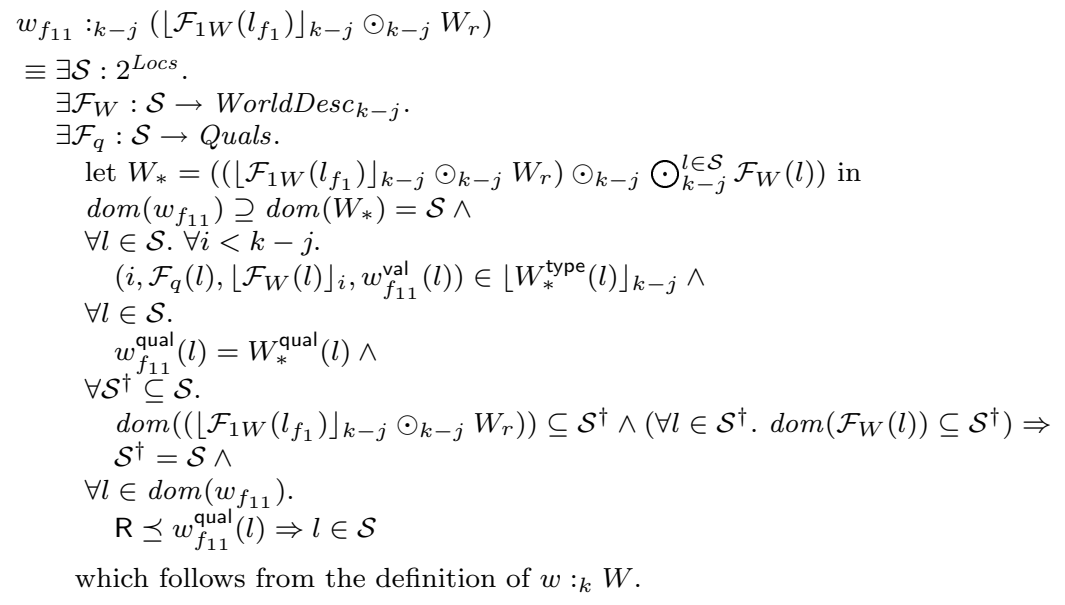

Take

$$
\mathcal{S}=\mathcal{S}_{1}^{\prime}
$$


It remains to show that

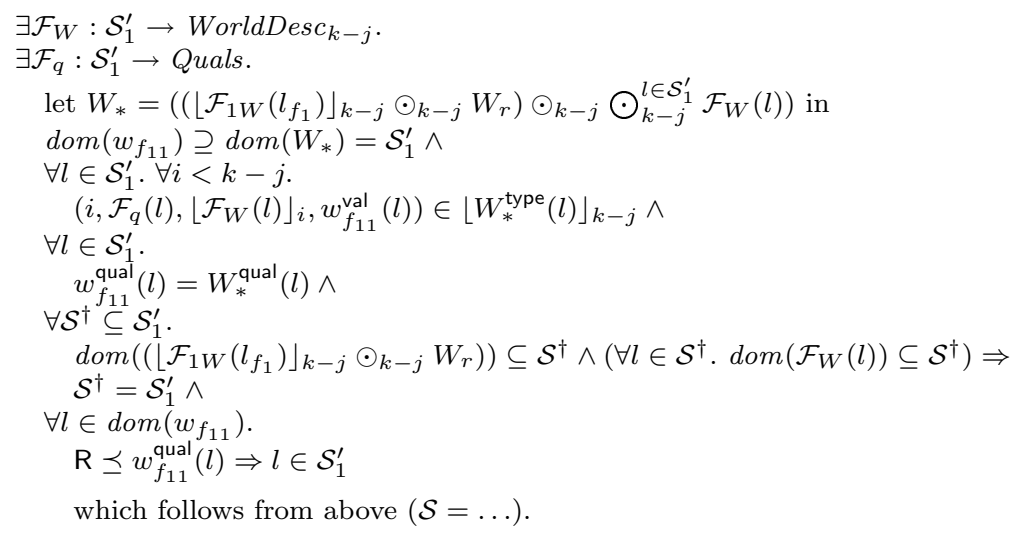

Take

$$
\mathcal{F}_{W}(l)=\left\{\left\lfloor\mathcal{F}_{1 W}(l)\right\rfloor_{k-j} \quad \text { if } l \in \mathcal{S}_{1}^{\prime}\right.
$$

and

$$
\mathcal{F}_{q}(l)=\left\{\mathcal{F}_{1 q}(l) \quad \text { if } l \in \mathcal{S}_{1}^{\prime}\right.
$$

Note that

$$
\begin{aligned}
\forall l \in \mathcal{S}_{1} . \mathcal{F}_{1 W}(l) \in \text { WorldDesc }_{k-j_{1}} \\
\text { which follows from above }\left(w_{f_{1}}: k-j_{1}\right. \\
\Rightarrow \forall l \in \mathcal{S}_{1}^{\prime} . \mathcal{F}_{1 W}(l) \in \text { WorldDesc }_{k-j_{1}} \\
\quad \text { which follows from } \mathcal{S}_{1}^{\prime} \subseteq \mathcal{S}_{1} \\
\Rightarrow \forall l \in \mathcal{S}_{1}^{\prime} .\left\lfloor\mathcal{F}_{1 W}(l)\right\rfloor_{k-j} \in \text { WorldDesc }_{k-j} \\
\quad \text { which follows from }\lfloor\cdot\rfloor_{k} \in \text { WorldDesc } \rightarrow \text { WorldDesc }_{k} \\
\equiv \forall l \in \mathcal{S}_{1}^{\prime} . \mathcal{F}_{W}(l) \in \text { WorldDesc }_{k-j} \\
\quad \text { which follows from above }\left(\mathcal{F}_{W}(l)=\ldots\right) .
\end{aligned}
$$

Hence, $\mathcal{F}_{W}: \mathcal{S}_{1}^{\prime} \rightarrow$ WorldDesc $_{k-j}$.

Trivially, $\mathcal{F}_{q}: \mathcal{S}_{1}^{\prime} \rightarrow$ Quals.

It remains to show that

$$
\begin{aligned}
& \text { let } W_{*}=\left(\left(\left\lfloor\mathcal{F}_{1 W}\left(l_{f_{1}}\right)\right\rfloor_{k-j} \odot_{k-j} W_{r}\right) \odot_{k-j} \bigodot_{k-j}^{l \in \mathcal{S}_{1}^{\prime}}\left\lfloor\mathcal{F}_{1 W}(l)\right\rfloor_{k-j}\right) \text { in } \\
& \operatorname{dom}\left(w_{f_{11}}\right) \supseteq \operatorname{dom}\left(W_{*}\right)=\mathcal{S}_{1}^{\prime} \wedge \\
& \forall l \in \mathcal{S}_{1}^{\prime} . \forall i<k-j . \\
& \quad\left(i, \mathcal{F}_{1 q}(l),\left\lfloor\left\lfloor\mathcal{F}_{1 W}(l)\right\rfloor_{k-j}\right\rfloor_{i}, w_{f_{11}}^{\text {val }}(l)\right) \in\left\lfloor W_{*}^{\text {type }}(l)\right\rfloor_{k-j} \wedge \\
& \forall l \in \mathcal{S}_{1}^{\prime} . \\
& \quad w_{f_{11}^{\text {qual }}}(l)=W_{*}^{\text {qual }}(l) \wedge \\
& \forall \mathcal{S}^{\dagger} \subseteq \mathcal{S}_{1}^{\prime} \\
& \quad \operatorname{dom}\left(\left(\left\lfloor\mathcal{F}_{1 W}\left(l_{f_{1}}\right)\right\rfloor_{k-j} \odot_{k-j} W_{r}\right)\right) \subseteq \mathcal{S}^{\dagger} \wedge\left(\forall l \in \mathcal{S}^{\dagger} . \operatorname{dom}\left(\left\lfloor\mathcal{F}_{1 W}(l)\right\rfloor_{k-j}\right) \subseteq \mathcal{S}^{\dagger}\right) \Rightarrow \\
& \quad \mathcal{S}^{\dagger}=\mathcal{S}_{1}^{\prime} \wedge \\
& \forall l \in \operatorname{dom}\left(w_{f_{11}}\right) . \\
& \quad \mathrm{R} \preceq w_{f_{11}}^{\text {qual }}(l) \Rightarrow l \in \mathcal{S}_{1}^{\prime} \\
& \quad \text { which follows from above }\left(\mathcal{F}_{W}(l)=\ldots \text { and } \mathcal{F}_{q}(l)=\ldots\right) .
\end{aligned}
$$

Note that

$$
\begin{aligned}
& \left\lfloor W_{1 *}\right\rfloor_{k-j} \\
& \equiv\left\lfloor\left(\left(W_{f_{1}} \odot_{k-j_{1}} W_{r}\right) \odot_{k-j_{1}}\left(\mathcal{F}_{1 W}\left(l_{f_{1}}\right) \odot_{k-j_{1}} \bigodot_{k-j_{1}}^{l \in \mathcal{S}_{1}^{\prime}} \mathcal{F}_{1 W}(l)\right)\right)\right\rfloor_{k-j} \\
& \quad \text { which follows from above }\left(W_{1 *}=\ldots\right) \\
& \equiv\left(\left(W_{f_{1}} \odot_{k-j} W_{r}\right) \odot_{k-j}\left(\left\lfloor\mathcal{F}_{1 W}\left(l_{f_{1}}\right)\right\rfloor_{k-j} \odot_{k-j} \bigodot_{k-j}^{l \in \mathcal{S}_{1}^{\prime}}\left\lfloor\mathcal{F}_{1 W}(l)\right\rfloor_{k-j}\right)\right) \\
& \quad \text { which follows from Req } 4(\text { join-closed }) \text { and Req } 5(\text { join-aprx }) \\
& \equiv\left(W_{f_{1}} \odot_{k-j}\left(\left(\left\lfloor\mathcal{F}_{1 W}\left(l_{f_{1}}\right)\right\rfloor_{k-j} \odot_{k-j} W_{r}\right) \odot_{k-j} \bigodot_{k-j}^{l \in \mathcal{S}_{1}^{\prime}}\left\lfloor\mathcal{F}_{1 W}(l)\right\rfloor_{k-j}\right)\right)
\end{aligned}
$$

which follows from Reqs 6,7 , and 8 (join-commut, join-assocl, and join-assocr). 
Hence, $W_{*}=\left(\left(\left\lfloor\mathcal{F}_{1 W}\left(l_{f_{1}}\right)\right\rfloor_{k-j} \odot_{k-j} W_{r}\right) \odot_{k-j} \bigodot_{k-j}^{l \in \mathcal{S}_{1}^{\prime}}\left\lfloor\mathcal{F}_{1 W}(l)\right\rfloor_{k-j}\right)$ is defined.

Furthermore, $\left(W_{f_{1}} \odot_{k-j} W_{*}\right)=\left\lfloor W_{1 *}\right\rfloor_{k-j}$.

Note that $W_{*}=\left\lfloor W_{1 *}\right\rfloor_{k-j} \backslash\left\{l_{f_{1}}\right\}$, which follows from $W_{f_{1}}=\left\{l_{f_{1}} \mapsto\left(q_{f_{1}}, \chi_{f_{1}}\right)\right\}$ and $\mathrm{A} \preceq q_{f_{1}}$ and the definition of $\left(\cdot \odot_{k} \cdot\right)$.

Furthermore, $\operatorname{dom}\left(W_{*}\right)=\operatorname{dom}\left(W_{1 *}\right) \backslash\left\{l_{f_{1}}\right\}$, which follows from

$$
\begin{aligned}
& \operatorname{dom}\left(\left(W_{f_{1}} \odot_{k-j} W_{*}\right)\right)=\operatorname{dom}\left(\left\lfloor W_{1 *}\right\rfloor_{k-j}\right) \\
& \quad \text { which follows from }\left(W_{f_{1}} \odot_{k-j} W_{*}\right)=\left\lfloor W_{1 *}\right\rfloor_{k-j} \\
& \equiv \operatorname{dom}\left(\left(\left\{l_{f_{1}} \mapsto\left(q_{f_{1}}, \chi_{f_{1}}\right)\right\} \odot_{k-j} W_{*}\right)\right)=\operatorname{dom}\left(\left\lfloor W_{1 *}\right\rfloor_{k-j}\right) \\
& \quad \text { which follows from } W_{f_{1}}=\left\{l_{f_{1}} \mapsto\left(q_{f_{1}}, \chi_{f_{1}}\right)\right\} \\
& \equiv\left\{l_{f_{1}}\right\} \uplus \operatorname{dom}\left(W_{*}\right)=\operatorname{dom}\left(\left\lfloor W_{1 *}\right\rfloor_{k-j}\right) \\
& \quad \text { which follows from } \mathrm{A} \preceq q_{f_{1}} \text { and } \\
& \quad\left(\left\{l_{f_{1}} \mapsto\left(q_{f_{1}}, \chi_{f_{1}}\right)\right\} \bigodot_{k-j} W_{*}\right) \text { defined } \\
& \equiv \operatorname{dom}\left(W_{*}\right)=\operatorname{dom}\left(\left\lfloor W_{1 *}\right\rfloor k-j\right) \backslash\left\{l_{f_{1}}\right\} \\
& \quad \text { which follows from simplifications of } A \uplus B=C \Rightarrow B=C \backslash A \\
& \equiv \operatorname{dom}\left(W_{*}\right)=\operatorname{dom}\left(W_{1 *}\right) \backslash\left\{l_{f_{1}}\right\} .
\end{aligned}
$$

Note that

$$
\begin{aligned}
& \operatorname{dom}\left(w_{f_{1}}\right) \supseteq \operatorname{dom}\left(W_{1 *}\right)=\mathcal{S}_{1} \\
& \quad \text { which follows from above }\left(w_{f_{1}}: k-j\right. \\
& \left.\left.\equiv \operatorname{dom}\left(w_{f_{11}} \uplus\left\{l_{f_{1}} \mapsto\left(q_{f_{1}}, v_{f_{11}}\right)\right\}\right) \supseteq \operatorname{dom}\left(W_{1 *}\right)=\mathcal{S}_{r}\right)\right) \\
& \quad \text { which follows from above }\left(w_{f_{1}} \equiv w_{f_{11}} \uplus\left\{l_{f_{1}} \mapsto\left(q_{f_{1}}, v_{f_{11}}\right)\right\}\right) \\
& \equiv \operatorname{dom}\left(w_{f_{11}}\right) \uplus\left\{l_{f_{1}}\right\} \supseteq \operatorname{dom}\left(W_{1 *}\right)=\mathcal{S}_{1} \\
& \quad \text { which follows from simplifications of } \operatorname{dom}\left(w_{f_{11}} \uplus\left\{l_{f_{1}} \mapsto\left(q_{f_{1}}, v_{f_{11}}\right)\right\}\right) \\
& \equiv \operatorname{dom}\left(w_{f_{11}}\right) \uplus\left\{l_{f_{1}}\right\} \supseteq \operatorname{dom}\left(W_{1 *}\right)=\left\{l_{f_{1}}\right\} \uplus \mathcal{S}_{1}^{\prime} \\
& \quad \text { which follows from above }\left(\mathcal{S}_{1}=\ldots\right) \\
& \equiv \operatorname{dom}\left(w_{f_{11}}\right) \supseteq \operatorname{dom}\left(W_{1 *}\right) \backslash\left\{l_{f_{1}}\right\}=\mathcal{S}_{1}^{\prime} \\
& \quad \text { which follows from simplifications of } A \uplus B \supseteq C=B \uplus D \Rightarrow A \supseteq C \backslash B=D \\
& \equiv \operatorname{dom}\left(w_{f_{11}}\right) \supseteq \operatorname{dom}\left(W_{*}\right)=\mathcal{S}_{1}^{\prime} \\
& \quad \text { which follows from above }\left(\operatorname{dom}\left(W_{*}\right)=\ldots\right) .
\end{aligned}
$$

It remains to show that

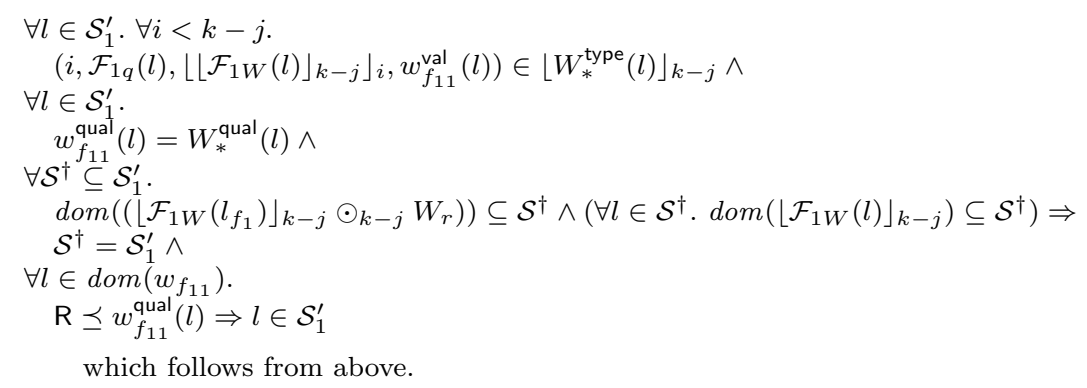

We are required to show that

- $\forall l \in \mathcal{S}_{1}^{\prime} . \forall i<k-j$.

$$
\left(i, \mathcal{F}_{1 q}(l),\left\lfloor\left\lfloor\mathcal{F}_{1 W}(l)\right\rfloor_{k-j}\right\rfloor_{i}, w_{f_{11}}^{\text {val }}(l)\right) \in\left\lfloor W_{*}^{\text {type }}(l)\right\rfloor_{k-j}
$$

Note that

$$
\begin{aligned}
\forall l \in & \mathcal{S}_{1}^{\prime} . \forall i<k-j . \\
& \left(i, \mathcal{F}_{1 q}(l),\left\lfloor\left\lfloor\mathcal{F}_{1 W}(l)\right\rfloor_{k-j}\right\rfloor_{i}, w_{f_{11}}(l)\right) \in\left\lfloor W_{*}^{\mathrm{type}}(l)\right\rfloor_{k-j} \\
\equiv & \forall l \in \mathcal{S}_{1}^{\prime} . \forall i<k-j . \\
& \left(i, \mathcal{F}_{1 q}(l),\left\lfloor\mathcal{F}_{1 W}(l)\right\rfloor_{i}, w_{f_{11}}(l)\right) \in\left\lfloor W_{*}^{\mathrm{type}}(l)\right\rfloor_{k-j} \\
& \text { which follows from Req } 1 \text { (aprx-idem) } \\
\equiv & \forall l \in \mathcal{S}_{1}^{\prime} . \forall i<k-j . \\
& \left(i, \mathcal{F}_{1 q}(l),\left\lfloor\mathcal{F}_{1 W}(l)\right\rfloor_{i}, w_{f_{11}}(l)\right) \in\left\lfloor\left(\left\lfloor W_{1 *}\right\rfloor_{k-j} \backslash\left\{l_{f_{1}}\right\}\right)^{\mathrm{type}}(l)\right\rfloor_{k-j} \\
& \text { which follows from above }\left(W_{*}=\ldots\right)
\end{aligned}
$$




$$
\begin{aligned}
\equiv & \forall l \in \mathcal{S}_{1}^{\prime} . \forall i<k-j . \\
& \quad\left(i, \mathcal{F}_{1 q}(l),\left\lfloor\mathcal{F}_{1 W}(l)\right\rfloor_{i}, w_{f_{11}}(l)\right) \in\left\lfloor\left\lfloor W_{1 *}\right\rfloor_{k-j}^{\text {type }}(l)\right\rfloor_{k-j} \\
& \text { which follows from simplifications of } l_{f_{1}} \notin \mathcal{S}_{1}^{\prime} \\
\equiv & \forall l \in \mathcal{S}_{1}^{\prime} . \forall i<k-j . \\
& \left(i, \mathcal{F}_{1 q}(l),\left\lfloor\mathcal{F}_{1 W}(l)\right\rfloor_{i}, w_{f_{11}}(l)\right) \in\left\lfloor W_{1 *}^{\mathrm{type}}(l)\right\rfloor_{k-j} \\
& \text { which follows from the definition of }\lfloor W\rfloor_{k} \text { and Fact } 2 .
\end{aligned}
$$

We are required to show that

- $\forall l \in \mathcal{S}_{1}^{\prime} . \forall i<k-j$.

$\left(i, \mathcal{F}_{1 q}(l),\left\lfloor\mathcal{F}_{1 W}(l)\right\rfloor_{i}, w_{f_{11}}^{\text {val }}(l)\right) \in\left\lfloor W_{1 *}^{\text {type }}(l)\right\rfloor_{k-j}$

which follows from

$\forall l \in \mathcal{S}_{1} . \forall i<k-j_{1} .\left(i, \mathcal{F}_{1 q}(l),\left\lfloor\mathcal{F}_{1 W}(l)\right\rfloor_{i}, w_{f_{1}}^{\text {val }}(l)\right) \in\left\lfloor W_{1 *}^{\text {type }}(l)\right\rfloor_{k-j_{1}}$ which follows from above $\left(w_{f_{1}}:_{k-j_{1}}\left(W_{f_{1}} \odot_{k-j_{1}} W_{r}\right)\right)$

$\Rightarrow \forall l \in \mathcal{S}_{1}^{\prime} . \forall i<k-j_{1} .\left(i, \mathcal{F}_{1 q}(l),\left\lfloor\mathcal{F}_{1 W}(l)\right\rfloor_{i}, w_{f_{1}}^{\text {val }}(l)\right) \in\left\lfloor W_{1 *}^{\text {type }}(l)\right\rfloor_{k-j_{1}}$ which follows from $\mathcal{S}_{1}^{\prime} \subseteq \mathcal{S}_{1}$

$\Rightarrow \forall l \in \mathcal{S}_{1}^{\prime} . \forall i<k-j .\left(i, \overline{\mathcal{F}}_{1 q}(l),\left\lfloor\mathcal{F}_{1 W}(l)\right\rfloor_{i}, w_{f_{1}}^{\text {val }}(l)\right) \in\left\lfloor W_{1 *}^{\text {type }}(l)\right\rfloor_{k-j_{1}}$ which follows from $k-j<k-j_{1}$

$\equiv \forall l \in \mathcal{S}_{1}^{\prime} . \forall i<k-j .\left(i, \mathcal{F}_{1 q}(l),\left\lfloor\mathcal{F}_{1 W}(l)\right\rfloor_{i},\left(w_{f_{11}} \uplus\left\{l_{f_{1}} \mapsto\left(q_{f_{1}}, v_{f_{11}}\right)\right\}\right)^{\mathrm{val}}(l)\right) \in\left\lfloor W_{1 *}^{\mathrm{type}}(l)\right\rfloor_{k-j_{1}}$ which follows from above $\left(w_{f_{1}}=w_{f_{11}} \uplus\left\{l_{f_{1}} \mapsto\left(q_{f_{1}}, v_{f_{11}}\right)\right\}\right)$

$\equiv \forall l \in \mathcal{S}_{1}^{\prime} . \forall i<k-j .\left(i, \mathcal{F}_{1 q}(l),\left\lfloor\mathcal{F}_{1 W}(l)\right\rfloor_{i}, w_{f_{11}}^{\text {val }}(l)\right) \in\left\lfloor W_{1 *}^{\text {type }}(l)\right\rfloor_{k-j_{1}}$ which follows from simplifications of

$\forall l \in \mathcal{S}_{1}^{\prime} . \ldots\left(w_{f_{11}} \uplus\left\{l_{f_{1}} \mapsto\left(q_{f_{1}}, v_{f_{11}}\right)\right\}\right)^{\mathrm{val}}(l) \ldots \equiv \forall l \in \mathcal{S}_{1} \ldots w_{f_{11}}^{\mathrm{val}}(l) \ldots$

$\Rightarrow \forall l \in \mathcal{S}_{1}^{\prime} . \forall i<k-j .\left(i, \mathcal{F}_{1 q}(l),\left\lfloor\mathcal{F}_{1 W}(l)\right\rfloor_{i}, w_{f_{11}}^{\text {val }}(l)\right) \in\left\lfloor\left\lfloor W_{1 *}^{\text {type }}(l)\right\rfloor_{k-j_{1}}\right\rfloor_{k-j}$ which follows from $j<k \wedge(j, q, W, v) \in \chi \Rightarrow(j, q, W, v) \in\lfloor\chi\rfloor_{k}$

$\equiv \forall l \in \mathcal{S}_{1}^{\prime} . \forall i<k-j .\left(i, \mathcal{F}_{1 q}(l),\left\lfloor\mathcal{F}_{1 W}(l)\right\rfloor_{i}, w_{f_{11}}^{\text {val }}(l)\right) \in\left\lfloor W_{1 *}^{\text {type }}(l)\right\rfloor_{k-j}$ which follows from Fact 2.

- $\forall l \in \mathcal{S}_{1}^{\prime}$.

$$
w_{f_{11}}^{\text {qual }}(l)=W_{*}^{\text {qual }}(l)
$$

Note that

$$
\begin{aligned}
& \forall l \in \mathcal{S}_{1}^{\prime} . \\
& w_{f_{11}}^{\text {qual }}(l)=W_{*}^{\text {qual }}(l) \\
& \equiv \forall l \in \mathcal{S}_{1}^{\prime} . \\
& \quad w_{f_{11}}^{\text {qual }}(l)=\left(\left\lfloor W_{1 *}\right\rfloor_{k-j} \backslash\left\{l_{f_{1}}\right\}\right)^{\text {qual }}(l) \\
& \quad \text { which follows from above }\left(W_{*}=\ldots\right) \\
& \equiv \forall l \in \mathcal{S}_{1}^{\prime} . \\
& \quad w_{f_{11}}^{\text {qual }}(l)=\left\lfloor W_{1 *}\right\rfloor_{k-j}^{\text {qual }}(l) \\
& \quad \text { which follows from simplifications of } l_{f_{1}} \notin \mathcal{S}_{1}^{\prime} \\
& \equiv \forall \in \mathcal{S}_{1}^{\prime} . \\
& \quad w_{f_{11}}^{\text {qual }}(l)=W_{1 *}^{\text {qual }}(l) \\
& \quad \text { which follows from the definition of }\lfloor W\rfloor_{k} .
\end{aligned}
$$

We are required to show that

- $\forall l \in \mathcal{S}_{1}^{\prime}$.

$$
w_{f_{11}}^{\text {qual }}(l)=W_{1 *}^{\text {qual }}(l)
$$

which follows from 


$$
\begin{aligned}
& \forall l \in \mathcal{S}_{1} . w_{f_{1}}^{\text {qual }}(l)=W_{1 *}^{\text {qual }}(l) \\
& \quad \text { which follows from above }\left(w_{f_{1}: k-j}\left(W_{f_{1}} \odot_{k-j} W_{r}\right)\right) \\
& \Rightarrow \forall l \in \mathcal{S}_{1}^{\prime} \cdot w_{f_{1}}^{\text {qual }}(l)=W_{1 * 1}^{\text {qual }}(l) \\
& \quad \text { hhich follows from } \mathcal{S}_{1}^{\prime} \subseteq \mathcal{S}_{1} \\
& \equiv \forall l \in \mathcal{S}_{1}^{\prime} .\left(w_{f_{11}} \uplus\left\{l_{f_{1}} \mapsto\left(q_{f_{1}}, v_{f_{11}}\right)\right\}\right)^{\text {qual }}(l)=W_{1 *}^{\text {qual }}(l) \\
& \quad \text { which follows from above }\left(w_{f_{1}}=w_{f_{11}} \uplus\left\{l_{f_{1}} \mapsto\left(q_{f_{1}}, v_{f_{11}}\right)\right\}\right) \\
& \equiv \forall l \in \mathcal{S}_{1}^{\prime} . w_{f_{11}}^{\text {qual }}(l)=W_{1 *}^{\text {qual }}(l) \\
& \quad \text { which follows from simplifications of } \\
& \quad \forall l \in \mathcal{S}_{1}^{\prime} \ldots\left(w_{f_{11}} \uplus\left\{l_{f_{1}} \mapsto\left(q_{f_{1}}, v_{f_{11}}\right)\right\}\right)^{\text {qual }}(l) \ldots \equiv \forall l \in \mathcal{S}_{1} \ldots w_{f_{11}}^{\text {qual }}(l) \ldots
\end{aligned}
$$

- $\forall \mathcal{S}^{\dagger} \subseteq \mathcal{S}_{1}^{\prime}$.

$\operatorname{dom}\left(\left(\left\lfloor\mathcal{F}_{1 W}\left(l_{f_{1}}\right)\right\rfloor_{k-j} \odot_{k-j} W_{r}\right)\right) \subseteq \mathcal{S}^{\dagger} \wedge\left(\forall l \in \mathcal{S}^{\dagger} . \operatorname{dom}\left(\left\lfloor\mathcal{F}_{1 W}(l)\right\rfloor_{k-j}\right) \subseteq \mathcal{S}^{\dagger}\right) \Rightarrow$ $\mathcal{S}^{\dagger}=\mathcal{S}_{1}^{\prime}$

Consider arbitrary $\mathcal{S}^{\dagger}$ such that

- $\mathcal{S}^{\dagger} \subseteq \mathcal{S}_{1}^{\prime}$,

- $\operatorname{dom}\left(\left(\left\lfloor\mathcal{F}_{1 W}\left(l_{f_{1}}\right)\right\rfloor_{k-j} \odot_{k-j} W_{r}\right)\right) \subseteq \mathcal{S}^{\dagger}$,

- $\forall l \in \mathcal{S}^{\dagger} . \operatorname{dom}\left(\left\lfloor\mathcal{F}_{1 W}(l)\right\rfloor_{k-j}\right) \subseteq \mathcal{S}^{\dagger}$.

Note that $l_{f_{1}} \notin \mathcal{S}^{\dagger}$, which follows from $\mathcal{S}^{\dagger} \subseteq \mathcal{S}_{1}^{\prime}$ and $l_{f_{1}} \notin \mathcal{S}_{1}^{\prime}$.

Let $\mathcal{S}_{1}^{\dagger}=\left\{l_{f_{1}}\right\} \uplus \mathcal{S}^{\dagger}$.

Note that

- $\mathcal{S}_{1}^{\dagger} \subseteq \mathcal{S}_{1}$, which follows from $\mathcal{S}^{\dagger} \subseteq \mathcal{S}_{1}^{\prime}$ and $\mathcal{S}_{1}=\left\{l_{f_{1}}\right\} \uplus \mathcal{S}_{1}^{\prime}$,

- $\left\{l_{f_{1}}\right\} \uplus \operatorname{dom}\left(W_{r}\right) \subseteq \mathcal{S}_{1}^{\dagger}$, which follows from $\operatorname{dom}\left(\left(\left\lfloor\mathcal{F}_{1 W}\left(l_{f_{1}}\right)\right\rfloor_{k-j} \odot_{k-j} W_{r}\right)\right) \subseteq \mathcal{S}^{\dagger}$ and $l_{f_{1}} \notin \operatorname{dom}\left(W_{r}\right)$,

- $\forall l \in \mathcal{S}_{1}^{\dagger} \cdot \operatorname{dom}\left(\mathcal{F}_{1 W}(l)\right) \subseteq \mathcal{S}_{1}^{\dagger}$, which follows from

- $\forall l \in\left\{l_{f_{1}}\right\} . \operatorname{dom}\left(\mathcal{F}_{1 W}(l)\right) \subseteq \mathcal{S}_{1}^{\dagger}$, which follows from $\operatorname{dom}\left(\left(\left\lfloor\mathcal{F}_{1 W}\left(l_{f_{1}}\right)\right\rfloor_{k-j} \odot_{k-j}\right.\right.$ $\left.\left.W_{r}\right)\right) \subseteq \mathcal{S}^{\dagger}$

- $\forall l \in \mathcal{S}^{\dagger} . \operatorname{dom}\left(\mathcal{F}_{1 W}(l)\right) \subseteq \mathcal{S}_{1}^{\dagger}$, which follows from $\forall l \quad \in$ $\mathcal{S}^{\dagger} . \operatorname{dom}\left(\left\lfloor\mathcal{F}_{1 W}(l)\right\rfloor_{k-j}\right) \subseteq \mathcal{S}^{\dagger}$.

Instantiate $\left(\forall \mathcal{S}_{1}^{\dagger} \subseteq \mathcal{S}_{1} \ldots\right)$ of $w_{f_{1}}: k-j_{1}\left(W_{f_{1}} \odot_{k-j_{1}} W_{r}\right)$ with $\mathcal{S}_{1}^{\dagger}$. Note that

- $\mathcal{S}_{1}^{\dagger} \subseteq \mathcal{S}_{1}$, which follows from above,

- $\operatorname{dom}\left(\left(\left\{l_{f_{1}} \mapsto\left(q_{f_{1}}, \chi_{f_{1}}\right)\right\} \odot_{k-j} \operatorname{dom}\left(W_{r}\right)\right)\right) \subseteq \mathcal{S}_{1}^{\dagger}$, which follows from $\left\{l_{f_{1}}\right\} \uplus$ $\operatorname{dom}\left(W_{r}\right) \subseteq \mathcal{S}_{1}^{\dagger}$, which follows from above,

- $\forall l \in \mathcal{S}_{1}^{\dagger} \cdot \operatorname{dom}\left(\mathcal{F}_{1 W}(l)\right) \subseteq \mathcal{S}_{1}^{\dagger}$, which follows from above.

Hence, we conclude that $\mathcal{S}_{1}^{\dagger}=\mathcal{S}_{1}$.

Hence, $\left\{l_{f_{1}}\right\} \uplus \mathcal{S}^{\dagger}=\left\{l_{f_{1}}\right\} \uplus \mathcal{S}_{1}^{\prime}$, which follows from $\mathcal{S}_{1}^{\dagger}=\left\{l_{f_{1}}\right\} \uplus \mathcal{S}^{\dagger}$ and $\mathcal{S}_{1}=\left\{l_{f_{1}}\right\} \uplus \mathcal{S}_{1}^{\prime}$. Hence, $\mathcal{S}^{\dagger}=\mathcal{S}_{1}^{\prime}$.

- $\forall l \in \operatorname{dom}\left(w_{f_{11}}\right)$.

$\mathrm{R} \preceq w_{f_{11}}^{\text {qual }}(l) \Rightarrow l \in \mathcal{S}_{1}^{\prime}$

which follows from 
$\forall l \in \operatorname{dom}\left(w_{f_{1}}\right) . \mathrm{R} \preceq w_{f_{1}}^{\text {qual }}(l) \Rightarrow l \in \mathcal{S}_{1}$

which follows from above $\left(w_{f_{1}}: k-j_{1}\left(W_{f_{1}} \odot_{k-j_{1}} W_{r}\right)\right)$

$\equiv \forall l \in \operatorname{dom}\left(w_{f_{1}}\right) . \mathrm{R} \preceq w_{f_{1}}^{\text {qual }}(l) \Rightarrow l \in\left\{l_{f_{1}}\right\} \uplus \mathcal{S}_{1}^{\prime}$

which follows from above $\left(\mathcal{S}_{1}=\left\{l_{f_{1}}\right\} \uplus \mathcal{S}_{1}^{\prime}\right)$

$\equiv \forall l \in \operatorname{dom}\left(\left(w_{f_{11}} \uplus\left\{l_{f_{1}} \mapsto\left(q_{f_{1}}, v_{f_{11}}\right)\right\}\right)\right) . \mathrm{R} \preceq\left(w_{f_{11}} \uplus\left\{l_{f_{1}} \mapsto\left(q_{f_{1}}, v_{f_{11}}\right)\right\}\right)^{\text {qual }}(l) \Rightarrow l \in\left\{l_{f_{1}}\right\} \uplus \mathcal{S}_{1}^{\prime}$

which follows from above $\left(w_{f_{1}}=w_{f_{11}} \uplus\left\{l_{f_{1}} \mapsto\left(q_{f_{1}}, v_{f_{11}}\right)\right\}\right)$

$\equiv \forall l \in \operatorname{dom}\left(w_{f_{11}}\right) \uplus\left\{l_{f_{1}}\right\} . \mathrm{R} \preceq\left(w_{f_{11}} \uplus\left\{l_{f_{1}} \mapsto\left(q_{f_{1}}, v_{f_{11}}\right)\right\}\right)^{\text {qual }}(l) \Rightarrow l \in\left\{l_{f_{1}}\right\} \uplus \mathcal{S}_{1}^{\prime}$

which follows from simplifications of $\operatorname{dom}\left(w_{f_{11}} \uplus\left\{l_{f_{1}} \mapsto\left(q_{f_{1}}, v_{f_{11}}\right)\right\}\right)$

$\Rightarrow \forall l \in \operatorname{dom}\left(w_{f_{11}}\right) . \mathrm{R} \preceq\left(w_{f_{11}} \uplus\left\{l_{f_{1}} \mapsto\left(q_{f_{1}}, v_{f_{11}}\right)\right\}\right)^{\text {qual }}(l) \Rightarrow l \in \mathcal{S}_{1}^{\prime}$

which follows from $\operatorname{dom}\left(w_{f_{11}}\right) \subseteq \operatorname{dom}\left(w_{f_{11}}\right) \uplus\left\{l_{f_{1}}\right\}$

$\equiv \forall l \in \operatorname{dom}\left(w_{f_{11}}\right) . \mathrm{R} \preceq w_{f_{11}}^{\text {qual }}(l) \Rightarrow l \in \mathcal{S}_{1}^{\prime}$

which follows from simplifications of

$\forall l \in \operatorname{dom}\left(w_{f_{11}}\right) \ldots\left(w_{f_{11}} \uplus\left\{l_{f_{1}} \mapsto\left(q_{f_{1}}, v_{f_{11}}\right)\right\}\right)^{\text {qual }}(l) \ldots \equiv \forall l \in \operatorname{dom}\left(w_{f_{11}}\right) \ldots w_{f_{11}}^{\text {qual }}(l) \ldots$

- $\left(k-j, q_{f}, W_{f}, e_{f}\right) \in \mathcal{T} \llbracket \Delta \vdash \tau:$ TYPE $\rrbracket \delta$

$\equiv\left(k-j, \mathcal{F}_{1 q}\left(l_{f_{1}}\right),\left\lfloor\mathcal{F}_{1 W}\left(l_{f_{1}}\right)\right\rfloor_{k-j}, v_{f_{11}}\right) \in \mathcal{T} \llbracket \Delta \vdash \tau:$ TYPE $\rrbracket \delta$

which follows from

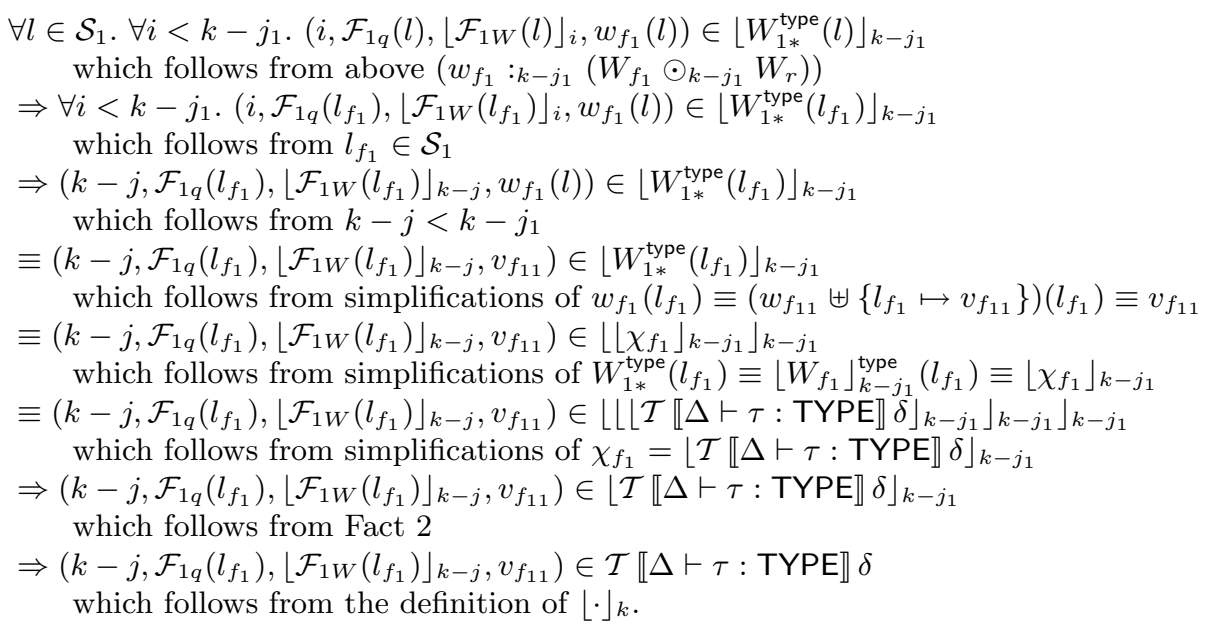


(READ)

Case $\frac{\Delta ; \Gamma \vdash e:{ }^{\xi} \text { ref } \tau \quad \Delta \vdash \tau \preceq \mathrm{R}}{\Delta ; \Gamma \vdash \operatorname{rd} e:{ }^{\mathrm{L}}\left({ }^{\xi} \text { ref } \tau \otimes \tau\right)}:$

We are required to show $\llbracket \Delta ; \Gamma \vdash \operatorname{rd} e_{1}:{ }^{\mathrm{L}}\left({ }^{\xi}\right.$ ref $\left.\tau \otimes \tau\right) \rrbracket$.

Consider arbitrary $k, \delta, q_{\Gamma}, W_{\Gamma}$, and $\gamma$ such that

- $k \geq 0$,

- $\delta \in \mathcal{D} \llbracket \Delta \rrbracket$, and

- $\left(k, q_{\Gamma}, W_{\Gamma}, \gamma\right) \in \mathcal{G} \llbracket \Delta \vdash \Gamma \rrbracket \delta$.

Let $e_{s}=\gamma\left(r \mathrm{~d} e_{1}\right) \equiv \operatorname{rd} \gamma\left(e_{1}\right)$ and $W_{s}=W_{\Gamma}$.

We are required to show that $\operatorname{Comp}\left(k, W_{s}, e_{s}, \mathcal{T} \llbracket \Delta \vdash^{\mathrm{L}}\left(\xi_{\text {ref }} \tau \otimes \tau\right): \mathrm{TYPE} \rrbracket \delta\right) \quad \equiv$ $\operatorname{Comp}\left(k, W_{\Gamma}, \operatorname{rd} \gamma\left(e_{1}\right), \mathcal{T} \llbracket \Delta \vdash^{\mathrm{L}}\left(\xi_{\text {ref }} \tau \otimes \tau\right): \mathrm{TYPE} \rrbracket \delta\right)$.

Consider arbitrary $j, W_{r}, w_{s}, w_{f}$, and $e_{f}$ such that

- $j<k$,

- $w_{s}:_{k}\left(W_{s} \odot_{k} W_{r}\right) \equiv w_{s}:_{k}\left(W_{\Gamma} \odot_{k} W_{r}\right)$,

- $\left(w_{s}, e_{s}\right)=\left(w_{s}, \mathrm{rd} \gamma\left(e_{1}\right)\right) \longmapsto^{j}\left(w_{f}, e_{f}\right)$, and

- $\operatorname{irred}\left(w_{f}, e_{f}\right)$.

Hence, by inspection of the operational semantics, it follows that there exist $j_{1}, w_{f_{1}}$, and $e_{f_{1}}$ such that

- $\left(w_{s}, \gamma\left(e_{1}\right)\right) \longmapsto{ }^{j_{1}}\left(w_{f_{1}}, e_{f_{1}}\right)$,

- $\operatorname{irred}\left(w_{f_{1}}, e_{f_{1}}\right)$, and

- $j_{1} \leq j$.

Applying the induction hypothesis to $\Delta ; \Gamma \vdash e_{1}:{ }^{\xi}$ ref $\tau$, we conclude that $\llbracket \Delta ; \Gamma \vdash e_{1}:{ }^{\xi}$ ref $\tau \rrbracket$. Instantiate this with $k, \delta, q_{\Gamma}, W_{\Gamma}$, and $\gamma$. Note that

- $k \geq 0$,

- $\delta \in \mathcal{D} \llbracket \Delta \rrbracket$, and

- $\left(k, q_{\Gamma}, W_{\Gamma}, \gamma\right) \in \mathcal{G} \llbracket \Delta \vdash \Gamma \rrbracket \delta$.

Hence, $\operatorname{Comp}\left(k, W_{\Gamma}, \gamma\left(e_{1}\right), \mathcal{T} \llbracket \Delta \vdash \xi_{\text {ref }} \tau:\right.$ TYPE $\left.\rrbracket \delta\right)$.

Instantiate this with $j_{1}, W_{r}, w_{s}, w_{f_{1}}$, and $e_{f_{1}}$. Note that

- $j_{1}<k$, which follows from $j_{1} \leq j$ and $j<k$,

- $w_{s}:_{k}\left(W_{\Gamma} \odot_{k} W_{r}\right)$, which follows from above,

- $\left(w_{s}, \gamma\left(e_{1}\right)\right) \longmapsto{ }^{j_{1}}\left(w_{f_{1}}, e_{f_{1}}\right)$,

- $\operatorname{irred}\left(w_{f_{1}}, e_{f_{1}}\right)$.

Hence, there exists $W_{f_{1}}$ and $q_{f_{1}}$ such that

- $w_{f_{1}}:_{k-j_{1}}\left(W_{f_{1}} \odot_{k-j_{1}} W_{r}\right)$, and

- $\left(k-j_{1}, q_{f_{1}}, W_{f_{1}}, e_{f_{1}}\right)$

$\in \mathcal{T} \llbracket \Delta \vdash \xi_{\text {ref }} \tau:$ TYPE $\| \delta$

$\equiv\{(k, q,\{l \mapsto(q, \chi)\}, l) \mid$

$q=\mathcal{T} \llbracket \Delta \vdash \xi: \mathrm{QUAL} \rrbracket \delta \wedge$

$\chi=\lfloor\mathcal{T} \llbracket \Delta \vdash \tau: \text { TYPE } \llbracket \delta\rfloor_{k} \wedge$

$\left.\left(q \preceq \mathrm{A} \Rightarrow \forall\left({ }_{-}, q^{\prime},,_{-},{ }_{-}\right) \in \chi \cdot q^{\prime} \preceq \mathrm{A}\right)\right\}$, 
Hence, $e_{f_{1}} \equiv l_{f_{1}}$ and $q_{f_{1}}=\mathcal{T} \llbracket \Delta \vdash \xi:$ QUAL $\rrbracket \delta$ and $W_{f_{1}} \equiv\left\{l_{f_{1}} \mapsto\left(q_{f_{1}}, \chi_{f_{1}}\right)\right\}$ and $\chi_{f_{1}}=$ $\lfloor\mathcal{T} \llbracket \Delta \vdash \tau: \mathrm{TYPE} \rrbracket \delta\rfloor_{k-j_{1}}$ and $\left(q_{f_{1}} \preceq \mathrm{A} \Rightarrow \forall\left({ }_{-}, q^{\prime},{ }_{-},{ }_{-}\right) \in \chi_{f_{1}} \cdot q^{\prime} \preceq \mathrm{A}\right)$.

Note that

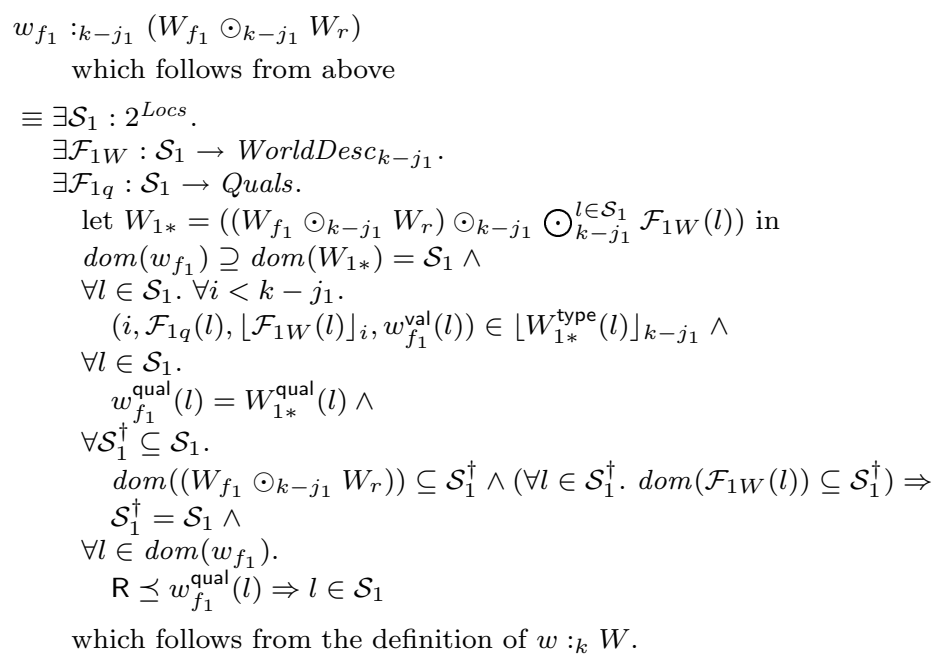

Note that

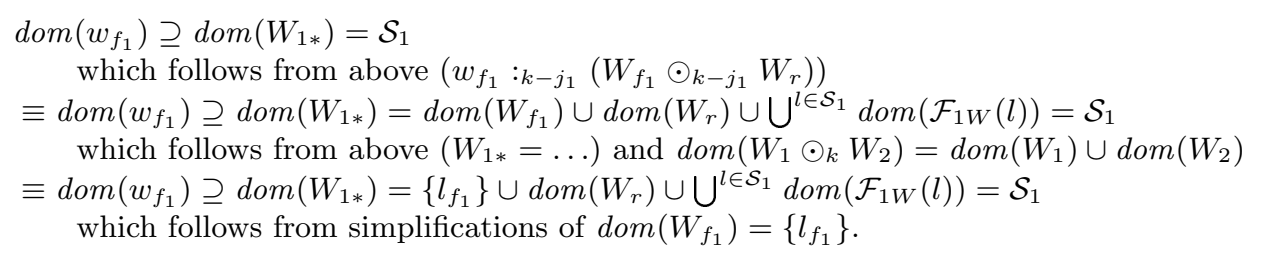

Hence, $l_{f_{1}} \in \operatorname{dom}\left(w_{f_{1}}\right)$ and $l_{f_{1}} \in \mathcal{S}_{1}$.

Note that

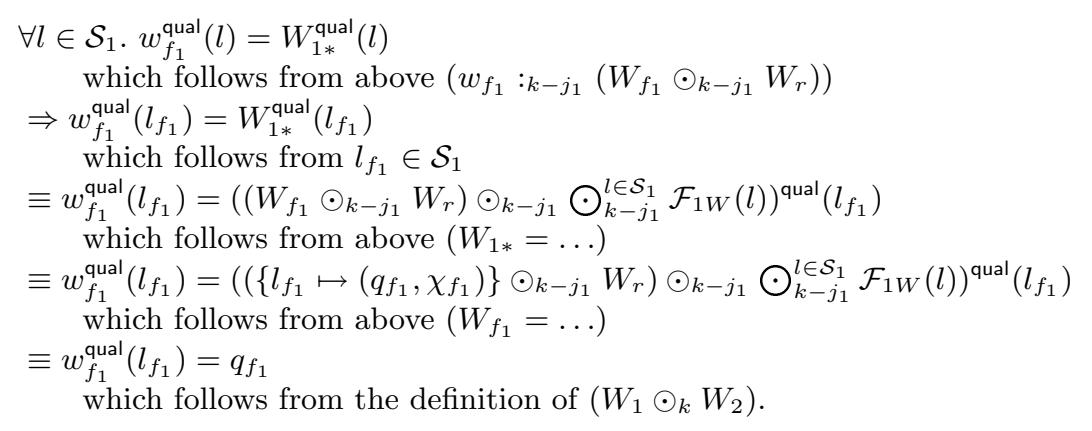

Note that $w_{f_{1}} \equiv w_{f_{11}} \uplus\left\{l_{f_{1}} \mapsto\left(q_{f_{1}}, v_{f_{11}}\right)\right\}$, which follows from $l_{f_{1}} \in \operatorname{dom}\left(w_{f_{1}}\right)$ and $w_{f_{1}}^{\text {qual }}\left(l_{f_{1}}\right)=q_{f_{1}}$. Note that

$$
\begin{aligned}
\left(w_{s}, e_{s}\right) & \equiv\left(w_{s}, \operatorname{rd} \gamma\left(e_{1}\right)\right) \\
& \longmapsto{ }^{j_{1}}\left(w_{f_{1}}, \operatorname{rd} e_{f_{1}}\right) \\
& \equiv\left(w_{f_{1}}, \operatorname{rd} l_{f_{1}}\right) \\
& \equiv\left(w_{f_{11}} \uplus\left\{l_{f_{1}} \mapsto\left(q_{f_{1}}, v_{f_{11}}\right)\right\}, \operatorname{rd} l_{f_{1}}\right) \\
& \longmapsto{ }^{1} \equiv\left(w_{f_{11}} \uplus\left\{l_{f_{1}} \mapsto\left(q_{f_{1}}, v_{f_{11}}\right)\right\},\left\langle l_{f_{1}}, v_{f_{11}}\right\rangle\right) \\
& \longmapsto{ }^{j-j_{1}-1}\left(w_{f}, e_{f}\right) .
\end{aligned}
$$

Since $\left\langle l_{f_{1}}, v_{f_{11}}\right\rangle$ is a value, we have $\operatorname{irred}\left(w_{f_{11}} \uplus\left\{l_{f_{1}} \mapsto\left(q_{f_{1}}, v_{f_{11}}\right)\right\},\left\langle l_{f_{1}}, v_{f_{11}}\right\rangle\right)$.

Hence, $j-j_{1}-1=0$ (and $j=j_{1}+1$ ), and $w_{f} \equiv w_{f_{11}} \uplus\left\{l_{f_{1}} \mapsto\left(q_{f_{1}}, v_{f_{11}}\right)\right\} \equiv w_{f_{1}}$ and $e_{f} \equiv v_{f_{11}}$. 
Note that

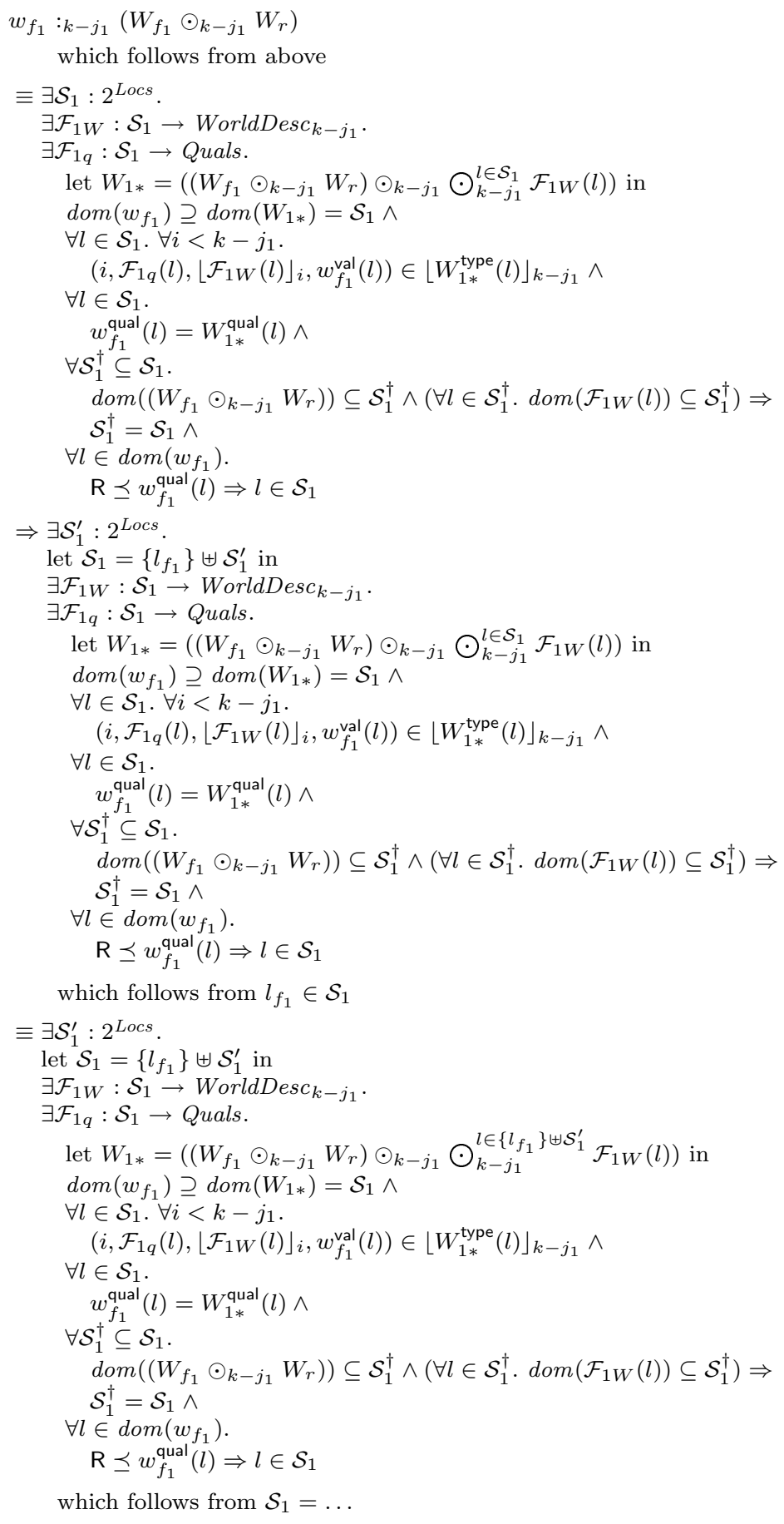




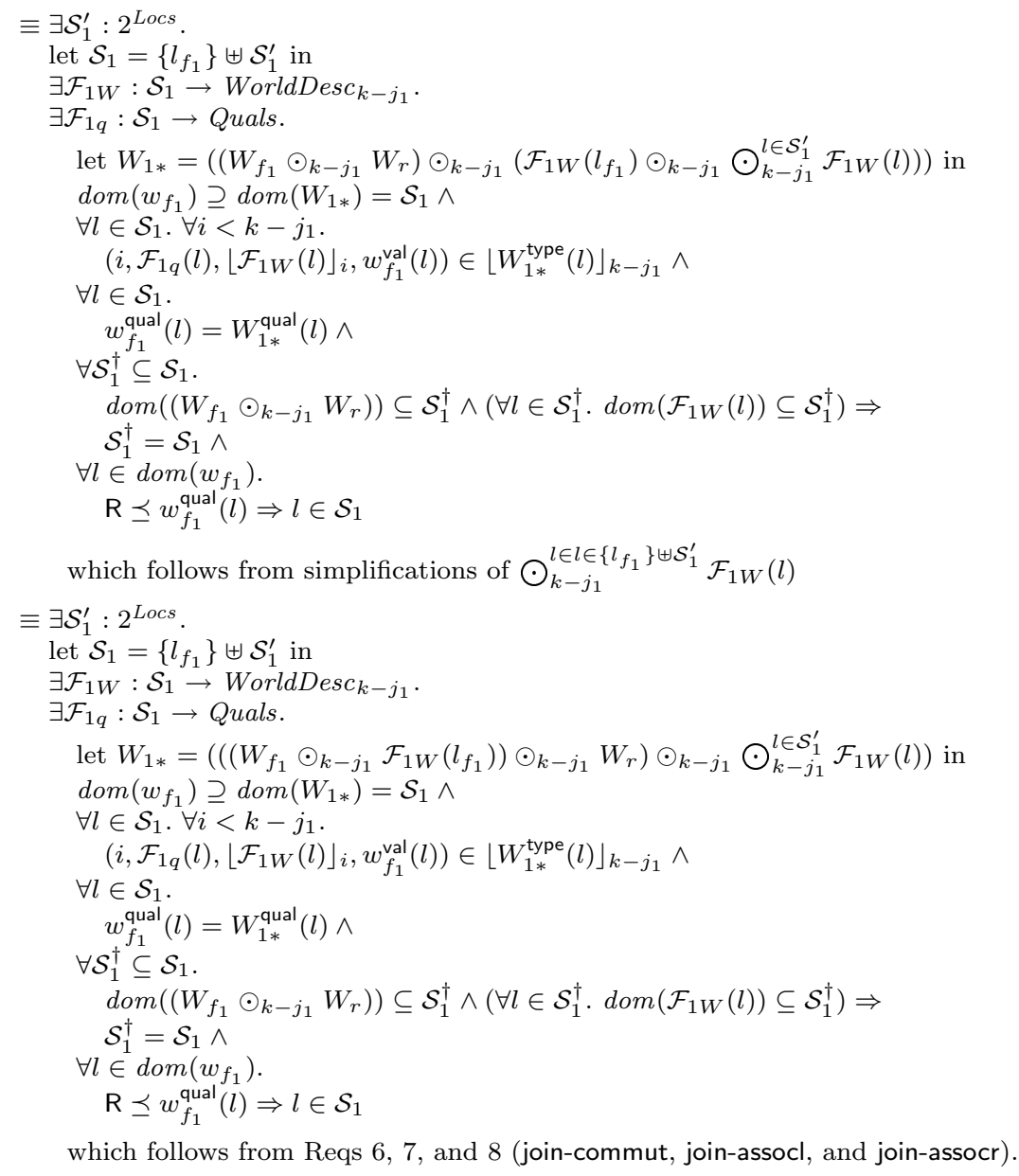

Note that $\left(W_{f_{1}} \odot_{k-j_{1}-1} \mathcal{F}_{1 W}\left(l_{f_{1}}\right)\right)$ is defined, which follows from Req 4 (join-closed) and $\left(W_{f_{1}} \odot_{k-j_{1}}\right.$ $\left.\mathcal{F}_{1}\left(l_{f_{1 W}}\right)\right)$ defined, which in turn follows from $W_{1 *}$ defined.

Note that 


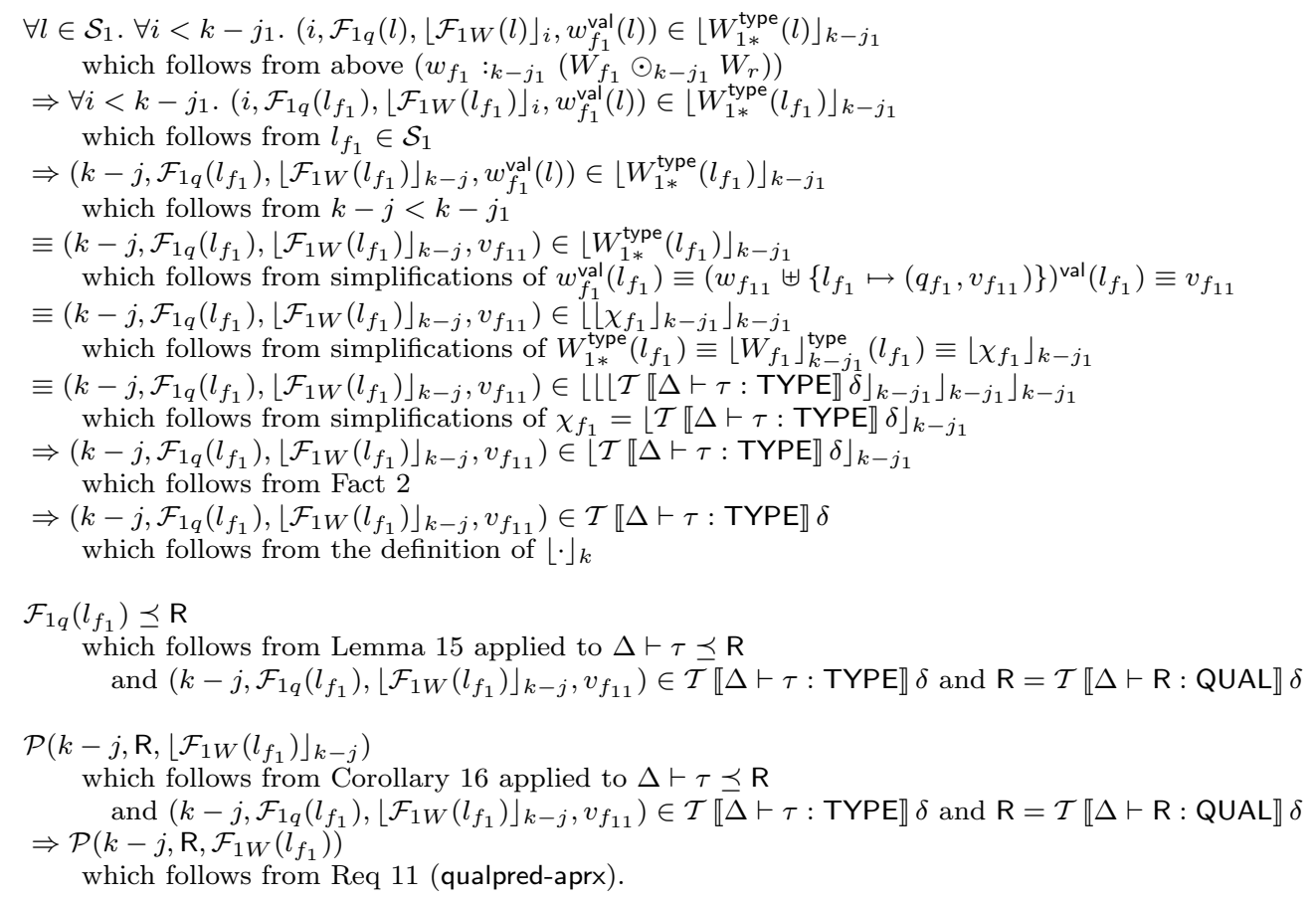

Let $W_{f}=\left(W_{f_{1}} \odot_{k-j} \mathcal{F}_{1 W}\left(l_{f_{1}}\right)\right)$.

Let $q_{f}=\mathrm{L}$.

Note that $\left(\left(W_{f_{1}} \odot_{k-j} \mathcal{F}_{1 W}\left(l_{f_{1}}\right)\right) \odot_{k-j} W_{r}\right)$ is defined, which follows from Reqs $4,5,6,7$, and 8 (joinclosed, join-aprx, join-commut, join-assocl, and join-assocr $)$ and $\left(\left(W_{f_{1}} \odot_{k-j_{1}} \mathcal{F}_{1 W}\left(l_{f_{1}}\right)\right) \odot_{k-j_{1}} W_{r}\right)$ defined, which in turn follows from $W_{1 *}$ defined.

We are required to show that

- $w_{f}: k-j\left(W_{f} \odot_{k-j} W_{r}\right)$

$\equiv w_{f_{1}}: k-j\left(\left(W_{f_{1}} \odot_{k-j} \mathcal{F}_{1 W}\left(l_{f_{1}}\right)\right) \odot_{k-j} W_{r}\right)$,

which is equivalent to

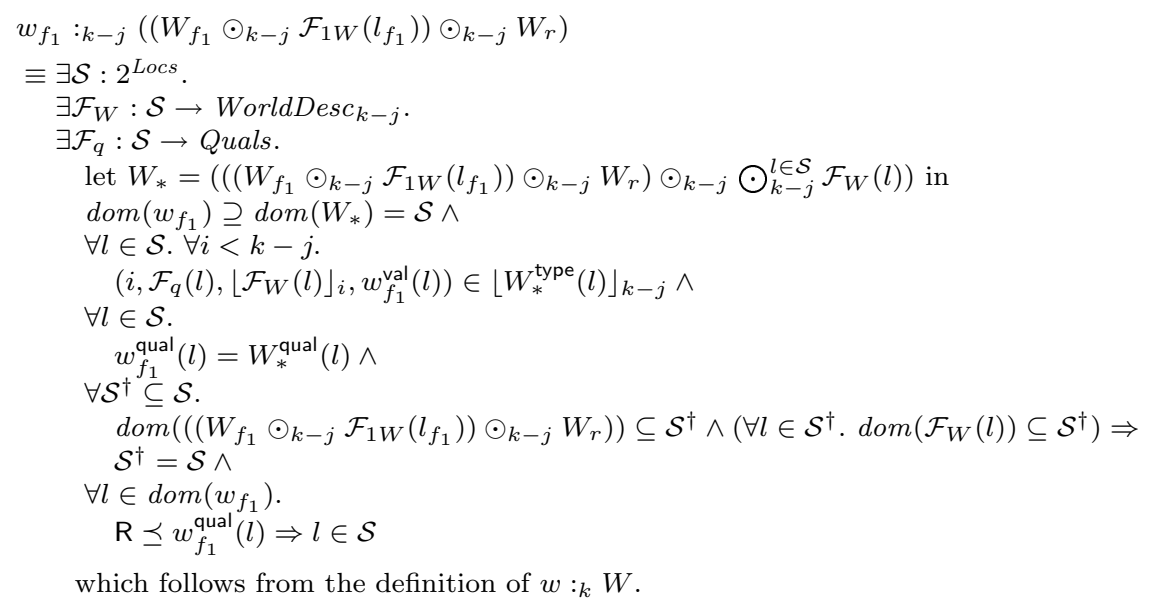

Take

$$
\mathcal{S}=\mathcal{S}_{1}=\left\{l_{f_{1}}\right\} \uplus \mathcal{S}_{1}^{\prime} .
$$


It remains to show that

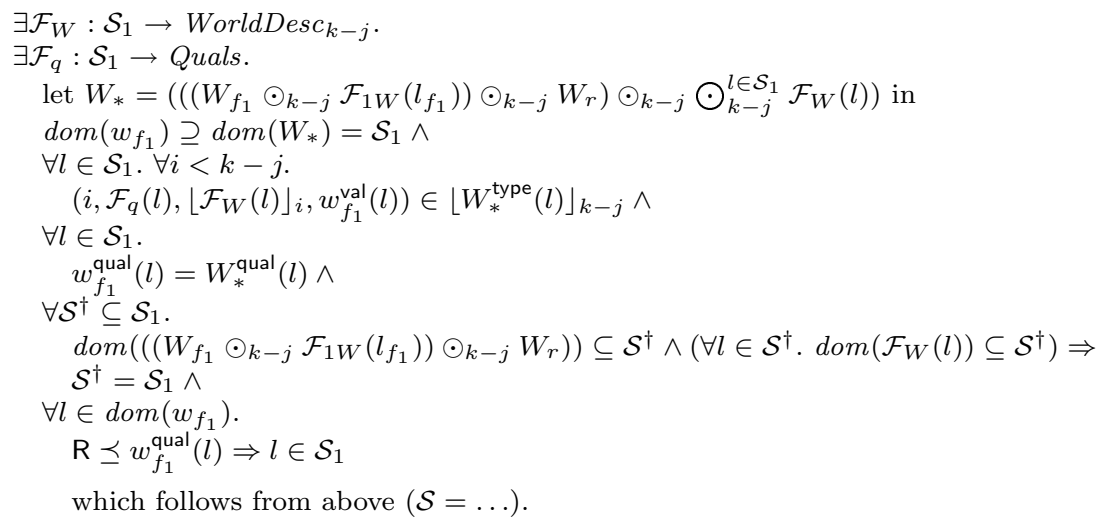

Take

$$
\mathcal{F}_{W}(l)=\left\{\left\lfloor\mathcal{F}_{1 W}(l)\right\rfloor_{k-j} \quad \text { if } l \in \mathcal{S}_{1}\right.
$$

and

$$
\mathcal{F}_{q}(l)=\left\{\mathcal{F}_{1 q}(l) \quad \text { if } l \in \mathcal{S}_{1}\right.
$$

Note that

$$
\begin{aligned}
\forall l \in \mathcal{S}_{1} . \mathcal{F}_{1 W}(l) \in \text { WorldDesc }_{k-j_{1}} \\
\text { which follows from above }\left(w_{f_{1}}:_{k-j_{1}}\left(W_{f_{1}} \odot_{k-j_{1}} W_{r}\right)\right) \\
\Rightarrow \forall l \in \mathcal{S}_{1} .\left\lfloor\mathcal{F}_{1 W}(l)\right\rfloor_{k-j} \in \text { WorldDesc }_{k-j} \\
\quad \text { which follows from }\lfloor\cdot\rfloor_{k} \in \text { WorldDesc } \rightarrow \text { WorldDesc }_{k} \\
\equiv \forall l \in \mathcal{S}_{1} . \mathcal{F}_{W}(l) \in \text { WorldDesc }_{k-j} \\
\quad \text { which follows from above }\left(\mathcal{F}_{W}(l)=\ldots\right) .
\end{aligned}
$$

Hence, $\mathcal{F}_{W}: \mathcal{S}_{1} \rightarrow$ WorldDesc $_{k-j}$.

Trivially, $\mathcal{F}_{q}: \mathcal{S}_{1} \rightarrow$ Quals .

It remains to show that

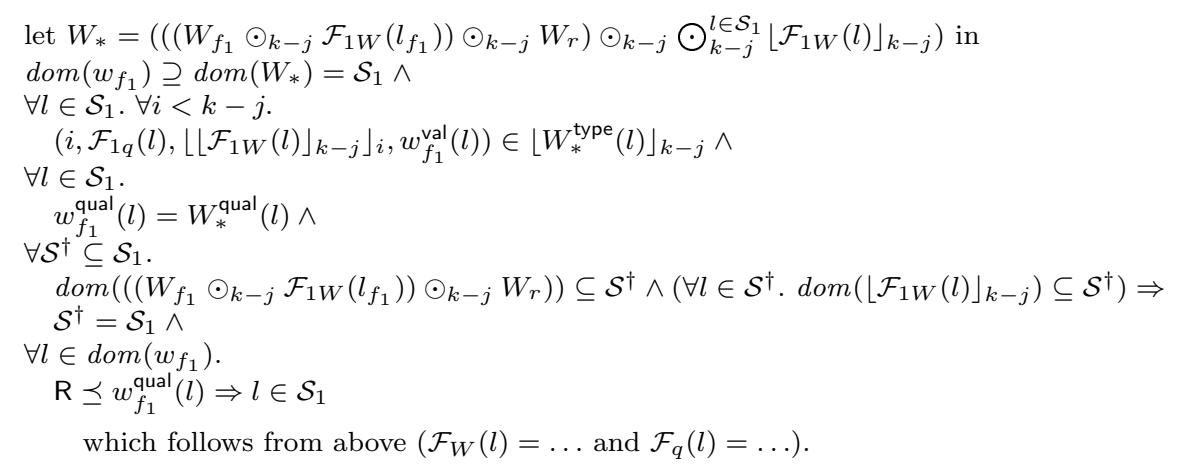

Note that 


$$
\begin{aligned}
& \left\lfloor W_{1 *}\right\rfloor_{k-j} \\
& \equiv\left\lfloor\left(\left(\left(W_{f_{1}} \odot_{k-j_{1}} \mathcal{F}_{1 W}\left(l_{f_{1}}\right)\right) \odot_{k-j_{1}} W_{r}\right) \odot_{k-j_{1}} \bigodot_{k-j_{1}}^{l \in \mathcal{S}_{1}^{\prime}} \mathcal{F}_{1 W}(l)\right)\right\rfloor_{k-j} \\
& \text { which follows from above }\left(W_{1 *}=\ldots\right) \\
& \equiv\left(\left(\left(W_{f_{1}} \odot_{k-j}\left\lfloor\mathcal{F}_{1 W}\left(l_{f_{1}}\right)\right\rfloor_{k-j}\right) \odot_{k-j} W_{r}\right) \odot_{k-j} \bigodot_{k-j}^{l \in \mathcal{S}_{1}^{\prime}} \mathcal{F}_{1 W}(l)\right) \\
& \text { which follows from Req } 4 \text { (join-closed) and Req } 5 \text { (join-aprx) } \\
& \equiv\left(\left(\left(W_{f_{1}} \odot_{k-j}\left(\mathcal{F}_{1 W}\left(l_{f_{1}}\right) \odot_{k-j} \mathcal{F}_{1 W}\left(l_{f_{1}}\right)\right)\right) \odot_{k-j} W_{r}\right) \odot_{k-j} \bigodot_{k-j}^{l \in \mathcal{S}_{1}^{\prime}} \mathcal{F}_{1 W}(l)\right) \\
& \text { which follows from } \mathcal{P}\left(k-j, \mathrm{R}, \mathcal{F}_{1 W}\left(l_{f_{1}}\right)\right) \text { and Req } 15 \text { (qualpred-rel-join) } \\
& \equiv\left(\left(\left(W_{f_{1}} \odot_{k-j} \mathcal{F}_{1 W}\left(l_{f_{1}}\right)\right) \odot_{k-j} W_{r}\right) \odot_{k-j}\left(\mathcal{F}_{1 W}\left(l_{f_{1}}\right) \odot_{k-j} \bigodot_{k-j}^{l \in \mathcal{S}_{1}^{\prime}} \mathcal{F}_{1 W}(l)\right)\right) \\
& \text { which follows from Reqs } 6,7 \text {, and } 8 \text { (join-commut, join-assocl, and join-assocr) } \\
& \equiv\left(\left(\left(W_{f_{1}} \odot_{k-j} \mathcal{F}_{1 W}\left(l_{f_{1}}\right)\right) \odot_{k-j} W_{r}\right) \odot_{k-j} \bigodot_{k-j}^{l \in\left\{l_{f_{1}}\right\} \uplus \mathcal{S}_{1}^{\prime}} \mathcal{F}_{1 W}(l)\right) \\
& \text { which follows from simplifications of } \bigodot_{k-j}^{l \in\left\{l_{f}\right\} \uplus \mathcal{S}_{1}^{\prime}} \mathcal{F}_{W}(l) \\
& \equiv\left(\left(\left(W_{f_{1}} \odot_{k-j} \mathcal{F}_{1 W}\left(l_{f_{1}}\right)\right) \odot_{k-j} W_{r}\right) \odot_{k-j} \bigodot_{k-j}^{l \in \mathcal{S}_{1}} \mathcal{F}_{1 W}(l)\right) \\
& \text { which follows from } \mathcal{S}_{1}=\ldots \\
& \equiv\left(\left(\left(W_{f_{1}} \odot_{k-j} \mathcal{F}_{1 W}\left(l_{f_{1}}\right)\right) \odot_{k-j} W_{r}\right) \odot_{k-j} \bigodot_{k-j}^{l \in \mathcal{S}_{1}}\left\lfloor\mathcal{F}_{1 W}(l)\right\rfloor_{k-j}\right) \\
& \text { which follows from Req } 4 \text { (join-closed) and Req } 5 \text { (join-aprx). }
\end{aligned}
$$

Hence, $W_{*}=\left(\left(\left(W_{f_{1}} \odot_{k-j} \mathcal{F}_{1 W}\left(l_{f_{1}}\right)\right) \odot_{k-j} W_{r}\right) \odot_{k-j} \bigodot_{k-j}^{l \in \mathcal{S}_{1}}\left\lfloor\mathcal{F}_{1 W}(l)\right\rfloor_{k-j}\right)$ is defined. Furthermore, $W_{*}=\left\lfloor W_{1 *}\right\rfloor_{k-j}$ and $\operatorname{dom}\left(W_{*}\right)=\operatorname{dom}\left(\left\lfloor W_{1 *}\right\rfloor_{k-j}\right)=\operatorname{dom}\left(W_{1 *}\right)$.

Note that

$$
\begin{aligned}
& \operatorname{dom}\left(w_{f_{1}}\right) \supseteq \operatorname{dom}\left(W_{1 *}\right)=\mathcal{S}_{1} \\
& \quad \text { which follows from above }\left(w_{f_{1}}:_{k-j_{1}}\left(W_{f_{1}} \odot_{k-j_{1}} W_{r}\right)\right) \\
& \equiv \operatorname{dom}\left(w_{f_{1}}\right) \supseteq \operatorname{dom}\left(W_{*}\right)=\mathcal{S}_{1} \\
& \quad \text { which follows from above }\left(\operatorname{dom}\left(W_{*}\right)=\ldots\right) .
\end{aligned}
$$

It remains to show that

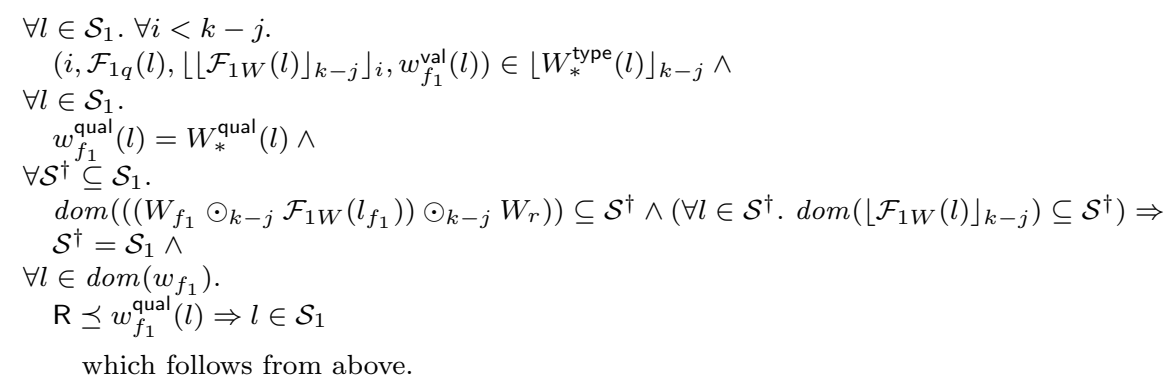

We are required to show that

- $\forall l \in \mathcal{S}_{1} . \forall i<k-j$.

$$
\left(i, \mathcal{F}_{1 q}(l),\left\lfloor\left\lfloor\mathcal{F}_{1 W}(l)\right\rfloor_{k-j}\right\rfloor_{i}, w_{f_{1}}^{\mathrm{val}}(l)\right) \in\left\lfloor W_{*}^{\text {type }}(l)\right\rfloor_{k-j}
$$

Note that

$$
\begin{aligned}
& \forall l \in \mathcal{S}_{1} . \forall i<k-j . \\
& \left(i, \mathcal{F}_{1 q}(l),\left\lfloor\left\lfloor\mathcal{F}_{1 W}(l)\right\rfloor_{k-j}\right\rfloor_{i}, w_{f_{1}}^{\mathrm{val}}(l)\right) \in\left\lfloor W_{*}^{\text {type }}(l)\right\rfloor_{k-j} \\
& \equiv \forall l \in \mathcal{S}_{1} . \forall i<k-j . \\
& \left(i, \mathcal{F}_{1 q}(l),\left\lfloor\mathcal{F}_{1 W}(l)\right\rfloor_{i}, w_{f_{1}}^{\text {val }}(l)\right) \in\left\lfloor W_{*}^{\text {type }}(l)\right\rfloor_{k-j} \\
& \text { which follows from Req } 1 \text { (aprx-idem) } \\
& \equiv \forall l \in \mathcal{S}_{1} . \forall i<k-j . \\
& \left(i, \mathcal{F}_{1 q}(l),\left\lfloor\mathcal{F}_{1 W}(l)\right\rfloor_{i}, w_{f_{1}}(l)\right) \in\left\lfloor\left\lfloor W_{1 *}\right\rfloor_{k-j}^{\text {type }}(l)\right\rfloor_{k-j} \\
& \text { which follows from above }\left(W_{*}=\ldots\right) \\
& \equiv \forall l \in \mathcal{S}_{1} . \forall i<k-j . \quad\left(i, \mathcal{F}_{1 q}(l),\left\lfloor\mathcal{F}_{1 W}(l)\right\rfloor_{i}, w_{f_{1}}(l)\right) \in\left\lfloor W_{1 *}^{\text {type }}(l)\right\rfloor_{k-j} \\
& \text { which follows from the definition of }\lfloor W\rfloor_{k} \text { and Fact } 2 \text {. }
\end{aligned}
$$

We are required to show that 
- $\forall l \in \mathcal{S}_{1} . \forall i<k-j . \quad\left(i, \mathcal{F}_{1 q}(l),\left\lfloor\mathcal{F}_{1 W}(l)\right\rfloor_{i}, w_{f_{1}}(l)\right) \in\left\lfloor W_{1 *}^{\text {type }}(l)\right\rfloor_{k-j}$ which follows from

$$
\begin{aligned}
\forall l \in \mathcal{S}_{1} . \forall i<k-j_{1} .\left(i, \mathcal{F}_{1 q}(l),\left\lfloor\mathcal{F}_{1 W}(l)\right\rfloor_{i}, w_{f_{1}}(l)\right) \in\left\lfloor W_{1 *}^{\text {type }}(l)\right\rfloor_{k-j_{1}} \\
\text { which follows from above }\left(w_{f_{1}}: k-j_{1}\left(W_{f_{1}} \odot_{k-j_{1}} W_{r}\right)\right) \\
\Rightarrow \forall l \in \mathcal{S}_{1} . \forall i<k-j .\left(i, \mathcal{F}_{1 q}(l),\left\lfloor\mathcal{F}_{1 W}(l)\right\rfloor_{i}, w_{f_{1}}(l)\right) \in\left\lfloor W_{1 *}^{\text {type }}(l)\right\rfloor_{k-j_{1}} \\
\quad \text { which follows from } k-j<k-j_{1} \\
\Rightarrow \forall l \in \mathcal{S}_{1} . \forall i<k-j .\left(i, \mathcal{F}_{1 q}(l),\left\lfloor\mathcal{F}_{1 W}(l)\right\rfloor_{i}, w_{f_{1}}(l)\right) \in\left\lfloor\left\lfloor W_{1 *}^{\text {type }}(l)\right\rfloor_{k-j_{1}}\right\rfloor_{k-j} \\
\quad \text { which follows from } j<k \wedge(j, q, W, v) \in \chi \Rightarrow(j, q, W, v) \in\lfloor\chi\rfloor_{k} \\
\equiv \forall l \in \mathcal{S}_{1} . \forall i<k-j .\left(i, \mathcal{F}_{1 q}(l),\left\lfloor\mathcal{F}_{1 W}(l)\right\rfloor_{i}, w_{f_{1}}(l)\right) \in\left\lfloor W_{1 *}^{\text {type }}(l)\right\rfloor_{k-j} \\
\quad \text { which follows from Fact } 2 .
\end{aligned}
$$

- $\forall l \in \mathcal{S}_{1}$.

$$
w_{f_{1}}^{\text {qual }}(l)=W_{*}^{\text {qual }}(l)
$$

Note that

$$
\begin{aligned}
& \forall l \in \mathcal{S}_{1} . \\
& w_{f_{1}}^{\text {qual }}(l)=W_{*}^{\text {qual }}(l) \\
& \equiv \forall l \in \mathcal{S}_{1} . \\
& \quad w_{f_{1}}^{\text {qual }}(l)=\left\lfloor W_{1 *}\right\rfloor_{k-j}^{\text {qual }}(l) \\
& \quad \text { which follows from above }\left(W_{*}=\ldots\right) \\
& \equiv l \in \mathcal{S}_{1} . \\
& \quad w_{f_{1}}^{\text {qual }}(l)=W_{1 *}^{\text {qual }}(l) \\
& \quad \text { which follows from the definition of }\lfloor W\rfloor_{k} .
\end{aligned}
$$

We are required to show that

- $\forall l \in \mathcal{S}_{1}$.

$$
w_{f_{1}}^{\text {qual }}(l)=W_{1 *}^{\text {qual }}(l)
$$

which follows from

$$
\begin{aligned}
\forall l \in \mathcal{S}_{1} \cdot w_{f_{1}}^{\text {qual }}(l)=W_{1 *}^{\text {qual }}(l) \\
\quad \text { which follows from above }\left(w_{f_{1}}: k-j\right. \\
\left.\quad\left(W_{f_{1}} \odot_{k-j} W_{r}\right)\right) .
\end{aligned}
$$

- $\forall \mathcal{S}^{\dagger} \subseteq \mathcal{S}_{1}$

$$
\begin{aligned}
& \operatorname{dom}\left(\left(\left(W_{f_{1}} \odot_{k-j} \mathcal{F}_{1 W}\left(l_{f_{1}}\right)\right) \odot_{k-j} W_{r}\right)\right) \subseteq \mathcal{S}^{\dagger} \wedge\left(\forall l \in \mathcal{S}^{\dagger} . \operatorname{dom}\left(\left\lfloor\mathcal{F}_{1 W}(l)\right\rfloor_{k-j}\right) \subseteq \mathcal{S}^{\dagger}\right) \Rightarrow \\
& \mathcal{S}^{\dagger}=\mathcal{S}_{1}
\end{aligned}
$$

Consider arbitrary $\mathcal{S}^{\dagger}$ such that

- $\mathcal{S}^{\dagger} \subseteq \mathcal{S}_{1}$,

- $\operatorname{dom}\left(\left(\left(W_{f_{1}} \odot_{k-j} \mathcal{F}_{1 W}\left(l_{f_{1}}\right)\right) \odot_{k-j} W_{r}\right)\right) \subseteq \mathcal{S}^{\dagger}$,

- $\forall l \in \mathcal{S}^{\dagger} . \operatorname{dom}\left(\left\lfloor\mathcal{F}_{1 W}(l)\right\rfloor_{k-j}\right) \subseteq \mathcal{S}^{\dagger}$.

Instantiate $\left(\forall \mathcal{S}_{1}^{\dagger} \subseteq \mathcal{S}_{1} \ldots\right)$ of $w_{f_{1}}: k-j_{1}\left(W_{f_{1}} \odot_{k-j_{1}} W_{r}\right)$ with $\mathcal{S}^{\dagger}$. Note that

- $\mathcal{S}^{\dagger} \subseteq \mathcal{S}_{1}$, which follows from above,

- $\operatorname{dom}\left(\left(W_{f_{1}} \odot_{k-j_{1}} W_{r}\right)\right) \subseteq \mathcal{S}^{\dagger}$, which follows from $\operatorname{dom}\left(\left(\left(W_{f_{1}} \odot_{k-j} \mathcal{F}_{1 W}\left(l_{f_{1}}\right)\right) \odot_{k-j} W_{r}\right)\right) \subseteq \mathcal{S}^{\dagger}$

which follows from above

$\equiv \operatorname{dom}\left(\left(\left(W_{f_{1}} \odot_{k-j} W_{r}\right) \odot_{k-j} \mathcal{F}_{1 W}\left(l_{f_{1}}\right)\right)\right) \subseteq \mathcal{S}^{\dagger}$

which follows from Reqs 6, 7, and 8 (join-commut, join-assocl, and join-assocr)

$\equiv \operatorname{dom}\left(\left(W_{f_{1}} \odot_{k-j} W_{r}\right)\right) \cup \operatorname{dom}\left(\mathcal{F}_{1 W}\left(l_{f_{1}}\right)\right) \subseteq \mathcal{S}^{\dagger}$

which follows from $\operatorname{dom}\left(W_{1} \odot_{k} W_{2}\right)=\operatorname{dom}\left(W_{1}\right) \cup \operatorname{dom}\left(W_{2}\right)$

$\Rightarrow \operatorname{dom}\left(\left(W_{f_{1}} \odot_{k-j} W_{r}\right)\right) \subseteq \mathcal{S}^{\dagger}$

$\equiv \operatorname{dom}\left(\left(W_{f_{1}} \odot_{k-j_{1}} W_{r}\right)\right) \subseteq \mathcal{S}^{\dagger}$

which follows from $\operatorname{dom}\left(\left(W_{f_{1}} \odot_{k-j} W_{r}\right)\right)=\operatorname{dom}\left(\left(W_{f_{1}} \odot_{k-j_{1}} W_{r}\right)\right)$. 
- $\forall l \in \mathcal{S}^{\dagger} . \operatorname{dom}\left(\mathcal{F}_{1 W}(l)\right) \subseteq \mathcal{S}^{\dagger}$ which follows from $\forall l \in \mathcal{S}^{\dagger} . \operatorname{dom}\left(\left\lfloor\mathcal{F}_{1 W}(l)\right\rfloor_{k-j}\right) \subseteq \mathcal{S}^{\dagger}$ Hence, we conclude that $\mathcal{S}^{\dagger}=\mathcal{S}_{1}$.

- $\forall l \in \operatorname{dom}\left(w_{f_{1}}\right)$.

$$
\mathrm{R} \preceq w_{f_{1}}^{\text {qual }}(l) \Rightarrow l \in \mathcal{S}_{1}
$$

which follows from

$$
\forall l \in \operatorname{dom}\left(w_{f_{1}}\right) . \mathrm{R} \preceq w_{f_{1}}^{\text {qual }}(l) \Rightarrow l \in \mathcal{S}_{1}
$$

which follows from above $\left(w_{f_{1}}:_{k-j}\left(W_{f_{1}} \odot_{k-j} W_{r}\right)\right)$.

- $\left(k-j, q_{f}, W_{f}, e_{f}\right) \in \mathcal{T} \llbracket \Delta \vdash^{\mathrm{L}}\left({ }^{\xi}\right.$ ref $\left.\tau \otimes \tau\right):$ TYPE $\rrbracket \delta$

$\equiv\left(k-j, \mathrm{~L},\left(W_{f_{1}} \odot_{k-j} \mathcal{F}_{1 W}\left(l_{f_{1}}\right)\right),\left\langle l_{f_{1}}, v_{f_{11}}\right\rangle\right) \in \mathcal{T} \llbracket \Delta \vdash^{\mathrm{L}}\left(\xi_{\text {ref }} \tau \otimes \tau\right):$ TYPE $\rrbracket \delta$

$\equiv\left(k-j, \mathrm{~L},\left(W_{f_{1}} \odot_{k-j} \mathcal{F}_{1 W}\left(l_{f_{1}}\right)\right),\left\langle l_{f_{1}}, v_{f_{11}}\right\rangle\right)$ $\in\left\{\left(k, q, W,\left\langle v_{1}, v_{2}\right\rangle\right) \mid\right.$

$q=\mathcal{T} \llbracket \Delta \vdash \mathrm{L}: \mathrm{QUAL} \rrbracket \delta \wedge$

$\left(k, q_{1}, W_{1}, v_{1}\right) \in \mathcal{T} \llbracket \Delta \vdash \xi_{\text {ref }} \tau:$ TYPE $\rrbracket \delta \wedge$

$\left(k, q_{2}, W_{2}, v_{2}\right) \in \mathcal{T} \llbracket \Delta \vdash \tau: \mathrm{TYPE} \rrbracket \delta \wedge$

$q_{1} \preceq q \wedge q_{1} \preceq q \wedge$

$\left.\left(W_{1} \odot_{k} W_{2}=W\right)\right\}$,

which follows from

- $\mathrm{L}=\mathcal{T} \llbracket \Delta \vdash \mathrm{L}: \mathrm{QUAL} \rrbracket \delta$, which follows trivially,

- $\left(k-j, q_{f_{1}},\left\lfloor W_{f_{1}}\right\rfloor_{k-j}, l_{f_{1}}\right) \in \mathcal{T} \llbracket \Delta \vdash \xi_{\text {ref }} \tau:$ TYPE $\rrbracket \delta$, which follows from Lemma 8 and Fact 6 applied to $k-j \leq k-j_{1}$ and $\left(k-j_{1}, q_{f_{1}}, W_{f_{1}}, l_{f_{1}}\right) \in \mathcal{T} \llbracket \Delta \vdash \xi_{\text {ref }} \tau:$ TYPE $\rrbracket \delta$,

- $\left(k-j, \mathcal{F}_{1 q}\left(l_{f_{1}}\right),\left\lfloor\mathcal{F}_{1 W}\left(l_{f_{1}}\right)\right\rfloor_{k-j}, v_{f_{11}}\right) \in \mathcal{T} \llbracket \Delta \vdash \tau:$ TYPE $\rrbracket$, which follows from above,

- $q_{f_{1}} \preceq \mathrm{L}$, which follows trivially,

- $\mathcal{F}_{1 q}\left(l_{f_{1}}\right) \preceq \mathrm{L}$, which follows trivially,

- $\left(W_{f_{1}} \odot_{k-j} \mathcal{F}_{1 W}\left(l_{f_{1}}\right)\right)=\left(\left\lfloor W_{f_{1}}\right\rfloor_{k-j} \odot_{k-j}\left\lfloor\mathcal{F}_{1 W}\left(l_{f_{1}}\right)\right\rfloor_{k-j}\right)$, which follows from Req 5 (join-aprx). 
(SWAP (STRONG))

Case $\frac{\Delta \vdash \Gamma \sim \Gamma_{1} \boxplus \Gamma_{2} \quad \Delta ; \Gamma_{1} \vdash e_{1}:{ }^{\xi} \text { ref } \tau_{1} \quad \Delta \vdash \mathrm{A} \preceq \xi \quad \Delta ; \Gamma_{2} \vdash e_{2}: \tau_{2} \quad \Delta \vdash \tau_{2} \preceq \xi}{\Delta ; \Gamma \vdash \text { sw } e_{1} e_{2}:{ }^{\mathrm{L}}\left({ }^{\xi} \text { ref } \tau_{2} \otimes \tau_{1}\right)}:$

We are required to show $\llbracket \Delta ; \Gamma \vdash \mathrm{sw} e_{1} e_{2}:{ }^{\mathrm{L}}\left(\xi_{\text {ref }} \tau_{2} \otimes \tau_{1}\right) \rrbracket$.

Consider arbitrary $k, \delta, q_{\Gamma}, W_{\Gamma}$, and $\gamma$ such that

- $k \geq 0$,

- $\delta \in \mathcal{D} \llbracket \Delta \rrbracket$, and

- $\left(k, q_{\Gamma}, W_{\Gamma}, \gamma\right) \in \mathcal{G} \llbracket \Delta \vdash \Gamma \rrbracket \delta$.

Applying Lemma 20 to $\left(k, q_{\Gamma}, W_{\Gamma}, \gamma\right) \in \mathcal{G} \llbracket \Delta \vdash \Gamma \rrbracket \delta$ and $\Delta \vdash \Gamma \leadsto \Gamma_{1} \boxplus \Gamma_{2}$, we conclude that there exist $q_{\Gamma_{1}}, W_{\Gamma_{1}}, \gamma_{1}, q_{\Gamma_{2}}, W_{\Gamma_{2}}$ and $\gamma_{2}$, such that

- $\left(k, q_{\Gamma_{1}}, W_{\Gamma_{1}}, \gamma_{1}\right) \in \mathcal{G} \llbracket \Delta \vdash \Gamma_{1} \rrbracket \delta$,

- $\left(k, q_{\Gamma_{2}}, W_{\Gamma_{2}}, \gamma_{2}\right) \in \mathcal{G} \llbracket \Delta \vdash \Gamma_{2} \rrbracket \delta$,

- $\gamma \in \gamma_{1} \boxplus \gamma_{2}$,

- $q_{\Gamma_{1}} \preceq q_{\Gamma}$,

- $q_{\Gamma_{2}} \preceq q_{\Gamma}$, and

- $\left(W_{\Gamma_{1}} \odot_{k} W_{\Gamma_{2}}=W_{\Gamma}\right)$.

Note that $\gamma\left(e_{1}\right) \equiv \gamma_{1}\left(e_{1}\right)$ and $\gamma\left(e_{2}\right) \equiv \gamma_{2}\left(e_{2}\right)$.

Let $e_{s}=\gamma\left(\right.$ sw $\left.e_{1} e_{2}\right) \equiv \operatorname{sw} \gamma\left(e_{1}\right) \gamma\left(e_{2}\right) \equiv \operatorname{sw} \gamma_{1}\left(e_{1}\right) \gamma_{2}\left(e_{2}\right)$ and $W_{s}=W_{\Gamma}$.

We are required to show that $\operatorname{Comp}\left(k, W_{s}, e_{s}, \mathcal{T} \llbracket \Delta ; \Gamma \vdash \operatorname{sw} e_{1} e_{2}:{ }^{\mathrm{L}}\left({ }^{\xi} \operatorname{ref} \tau_{2} \otimes \tau_{1}\right) \rrbracket \delta\right) \equiv$ $\operatorname{Comp}\left(k, W_{\Gamma}\right.$, sw $\gamma_{1}\left(e_{1}\right) \gamma_{2}\left(e_{2}\right), \mathcal{T} \llbracket \Delta ; \Gamma \vdash$ sw $\left.e_{1} e_{2}:{ }^{\mathrm{L}}\left(\xi_{\text {ref }} \tau_{2} \otimes \tau_{1}\right) \rrbracket \delta\right)$.

Consider arbitrary $j, W_{r}, w_{s}, w_{f}$, and $e_{f}$ such that

- $j<k$,

- $w_{s}:_{k}\left(W_{s} \odot_{k} W_{r}\right) \equiv w_{s}:_{k}\left(W_{\Gamma} \odot_{k} W_{r}\right)$, noting that

$$
\begin{gathered}
w_{s}:_{k}\left(W_{\Gamma} \odot_{k} W_{r}\right) \\
\equiv w_{s}:_{k}\left(\left(W_{\Gamma_{1}} \odot_{k} W_{\Gamma_{2}}\right) \bigodot_{k} W_{r}\right) \\
\text { which follows from above, }
\end{gathered}
$$

- $\left(w_{s}, e_{s}\right) \equiv\left(w_{s}\right.$, sw $\left.\gamma_{1}\left(e_{1}\right) \gamma_{2}\left(e_{2}\right)\right) \longmapsto j\left(w_{f}, e_{f}\right)$, and

- $\operatorname{irred}\left(w_{f}, e_{f}\right)$.

Hence, by inspection of the operational semantics, it follows that there exist $j_{1}, w_{f_{1}}$, and $e_{f_{1}}$ such that

- $\left(w_{s}, \gamma_{1}\left(e_{1}\right)\right) \longmapsto^{j_{1}}\left(w_{f_{1}}, e_{f_{1}}\right)$,

- $\operatorname{irred}\left(w_{f_{1}}, e_{f_{1}}\right)$, and

- $j_{1} \leq j$.

Note that $\left(\left(W_{\Gamma_{1}} \odot_{k} W_{\Gamma_{2}}\right) \odot_{k} W_{r}\right) \equiv\left(W_{\Gamma_{1}} \odot_{k}\left(W_{\Gamma_{2}} \odot_{k} W_{r}\right)\right)$, which follows from

$$
\begin{aligned}
& \left(\left(W_{\Gamma_{1}} \odot_{k} W_{\Gamma_{2}}\right) \odot_{k} W_{r}\right) \\
& \equiv\left(W_{\Gamma_{1}} \odot_{k}\left(W_{\Gamma_{2}} \odot_{k} W_{r}\right)\right)
\end{aligned}
$$

which follows from Reqs 6,7 , and 8 (join-commut, join-assocl, and join-assocr).

Applying the induction hypothesis to $\Delta ; \Gamma_{1} \vdash e_{1}:{ }^{\xi}$ ref $\tau_{1}$, we conclude that $\llbracket \Delta ; \Gamma_{1} \vdash e_{1}:{ }^{\xi}$ ref $\tau_{1} \rrbracket$. Instantiate this with $k, \delta, q_{\Gamma_{1}}, W_{\Gamma_{1}}$, and $\gamma_{1}$. Note that

- $k \geq 0$,

- $\delta \in \mathcal{D} \llbracket \Delta \rrbracket$, and 
- $\left(k, q_{\Gamma_{1}}, W_{\Gamma_{1}}, \gamma_{1}\right) \in \mathcal{G} \llbracket \Delta \vdash \Gamma_{1} \rrbracket \delta$.

Hence, $\operatorname{Comp}\left(k, W_{\Gamma_{1}}, \gamma_{1}\left(e_{1}\right), \mathcal{T} \llbracket \Delta \vdash \xi_{\text {ref }} \tau_{1}:\right.$ TYPE $\left.\rrbracket \delta\right)$.

Instantiate this with $j_{1},\left(W_{\Gamma_{2}} \odot_{k} W_{r}\right), w_{s}, w_{f_{1}}$, and $e_{f_{1}}$. Note that

- $j_{1}<k$, which follows from $j_{1} \leq j$ and $j<k$,

- $w_{s}:_{k}\left(W_{\Gamma_{1}} \odot_{k}\left(W_{\Gamma_{2}} \odot_{k} W_{r}\right)\right)$, which follows from

$w_{s}:_{k}\left(\left(W_{\Gamma_{1}} \odot_{k} W_{\Gamma_{2}}\right) \odot_{k} W_{r}\right)$

which follows from above

$\left(\left(W_{\Gamma_{1}} \odot_{k} W_{\Gamma_{2}}\right) \odot_{k} W_{r}\right) \equiv\left(W_{\Gamma_{1}} \odot_{k}\left(W_{\Gamma_{2}} \odot_{k} W_{r}\right)\right)$

which follows from above,

- $\left(w_{s}, \gamma_{1}\left(e_{1}\right)\right) \longmapsto^{j_{1}}\left(w_{f_{1}}, e_{f_{1}}\right)$,

- $\operatorname{irred}\left(w_{f_{1}}, e_{f_{1}}\right)$.

Hence, there exists $W_{f_{1}}$ and $q_{f_{1}}$ such that

- $w_{f_{1}}:_{k-j_{1}}\left(W_{f_{1}} \odot_{k-j_{1}}\left(W_{\Gamma_{2}} \odot_{k} W_{r}\right)\right)$, and

- $\left(k-j_{1}, q_{f_{1}}, W_{f_{1}}, e_{f_{1}}\right)$

$\in \mathcal{T} \llbracket \Delta \vdash \xi_{\text {ref }} \tau_{1}:$ TYPE $\rrbracket \delta$

$\equiv\{(k, q,\{l \mapsto(q, \chi)\}, l) \mid$

$q=\mathcal{T} \llbracket \Delta \vdash \xi: \mathrm{QUAL} \rrbracket \delta \wedge$

$\chi=\left\lfloor\mathcal{T} \llbracket \Delta \vdash \tau_{1}: \text { TYPE } \rrbracket \delta\right\rfloor_{k} \wedge$

$\left.\left(q \preceq \mathrm{A} \Rightarrow \forall\left({ }_{-}, q^{\prime},,_{-}\right) \in \chi \cdot q^{\prime} \preceq \mathrm{A}\right)\right\}$.

Hence, $e_{f_{1}} \equiv l_{f_{1}}$ and $q_{f_{1}}=\mathcal{T} \llbracket \Delta \vdash \xi:$ QUAL $\rrbracket \delta$ and $W_{f_{1}} \equiv\left\{l_{f_{1}} \mapsto\left(q_{f_{1}}, \chi_{f_{1}}\right)\right\}$ and $\chi_{f_{1}}=$ $\left\lfloor\mathcal{T} \llbracket \Delta \vdash \tau_{1}: \mathrm{TYPE} \rrbracket \delta\right\rfloor_{k-j_{1}}$ and $\left(q_{f_{1}} \preceq \mathrm{A} \Rightarrow \forall\left({ }_{-}, q^{\prime},{ }_{-},{ }_{-}\right) \in \chi_{f_{1}} \cdot q^{\prime} \preceq \mathrm{A}\right)$.

Note that $\mathrm{A} \preceq q_{f_{1}}$, which follows from Lemma 12 applied to $\Delta \vdash \mathrm{A} \preceq \xi$ and $\mathrm{A}=$ $\mathcal{T} \llbracket \Delta \vdash \mathrm{A}: \mathrm{QUAL} \rrbracket \delta$ and $q_{f_{1}}=\mathcal{T} \llbracket \Delta \vdash \xi: \mathrm{QUAL} \rrbracket \delta$.

Note that

$$
\begin{aligned}
\left(w_{s}, e_{s}\right) & \equiv\left(w_{s}, \text { sw } \gamma_{1}\left(e_{1}\right) \gamma_{2}\left(e_{2}\right)\right) \\
& \longmapsto j^{j_{1}}\left(w_{f_{1}}, \text { sw } e_{f_{1}} \gamma_{2}\left(e_{2}\right)\right) \\
& \equiv\left(w_{f_{1}}, \text { sw } l_{f_{1}} \gamma_{2}\left(e_{2}\right)\right) \\
& \longmapsto{ }^{j-j_{1}}\left(w_{f}, e_{f}\right)
\end{aligned}
$$

and $\operatorname{irred}\left(w_{f}, e_{f}\right)$.

Hence, by inspection of the operational semantics, it follows that there exist $j_{2}, w_{f_{2}}$, and $e_{f_{2}}$ such that

- $\left(w_{f_{1}}, \gamma_{2}\left(e_{2}\right)\right) \longmapsto{ }^{j_{2}}\left(w_{f_{2}}, e_{f_{2}}\right)$,

- $\operatorname{irred}\left(w_{f_{2}}, e_{f_{2}}\right)$, and

- $j_{2} \leq j-j_{1}$.

Note that $\left(W_{f_{1}} \odot_{k-j_{1}}\left(W_{\Gamma_{2}} \odot_{k} W_{r}\right)\right) \equiv\left(\left\lfloor W_{\Gamma_{2}}\right\rfloor_{k-j_{1}} \odot_{k-j_{1}}\left(W_{f_{1}} \odot_{k-j_{1}} W_{r}\right)\right)$, which follows from $\left(W_{f_{1}} \odot_{k-j_{1}}\left(W_{\Gamma_{2}} \odot_{k} W_{r}\right)\right)$

$\equiv\left(\left\lfloor W_{\Gamma_{2}}\right\rfloor_{k-j_{1}} \odot_{k-j_{1}}\left(W_{f_{1}} \odot_{k-j_{1}} W_{r}\right)\right)$

which follows from Reqs 4, 5, 6, 7, and 8 (join-closed, join-aprx, join-commut, join-assocl, and join-assocr).

Applying the induction hypothesis to $\Delta ; \Gamma_{2} \vdash e_{2}: \tau_{2}$, we conclude that $\llbracket \Delta ; \Gamma_{2} \vdash e_{2}: \tau_{2} \rrbracket$. Instantiate this with $k-j_{1}, \delta, q_{\Gamma_{2}},\left\lfloor W_{\Gamma_{2}}\right\rfloor_{k-j_{1}}$, and $\gamma_{2}$. Note that

- $k-j_{1} \geq 0$, which follows from $j_{1}<k$,

- $\delta \in \mathcal{D} \llbracket \Delta \rrbracket$, and 
- $\left(k-j_{1}, q_{\Gamma_{2}},\left\lfloor W_{\Gamma_{2}}\right\rfloor_{k-j_{1}}, \gamma_{2}\right) \in \mathcal{G} \llbracket \Delta \vdash \Gamma_{2} \rrbracket \delta$, which follows from Lemma 9 applied to $k-j_{1} \leq k$ and $\left(k, q_{\Gamma_{2}}, W_{\Gamma_{2}}, \gamma_{2}\right) \in \mathcal{G} \llbracket \Delta \vdash \Gamma_{2} \rrbracket \delta$.

Hence, $\operatorname{Comp}\left(k-j_{1}, \gamma_{2}\left(e_{2}\right),\left\lfloor W_{\Gamma_{2}}\right\rfloor_{k-j_{1}}, \mathcal{T} \llbracket \Delta \vdash \tau_{2}:\right.$ TYPE $\left.\rrbracket \delta\right)$.

Instantiate this with $j_{2},\left(W_{f_{1}} \odot_{k-j_{1}} W_{r}\right), w_{f_{1}}, w_{f_{2}}$, and $e_{f_{2}}$. Note that

- $j_{2}<k-j_{1}$, which follows from $j_{2} \leq j-j_{1}$ and $j<k$,

- $w_{f_{1}}: k-j_{1}\left(\left\lfloor W_{\Gamma_{2}}\right\rfloor_{k-j_{1}} \odot_{k-j_{1}}\left(W_{f_{1}} \odot_{k-j_{1}} W_{r}\right)\right)$, which follows from

$w_{f_{1}}: k-j_{1}\left(W_{f_{1}} \odot_{k-j_{1}}\left(W_{\Gamma_{2}} \odot_{k} W_{r}\right)\right)$

which follows from above

$\left(W_{f_{1}} \odot_{k-j_{1}}\left(W_{\Gamma_{2}} \odot_{k} W_{r}\right)\right) \equiv\left(\left\lfloor W_{\Gamma_{2}}\right\rfloor_{k-j_{1}} \odot_{k-j_{1}}\left(W_{f_{1}} \odot_{k-j_{1}} W_{r}\right)\right)$

which follows from above,

- $\left(w_{f_{1}}, \gamma_{2}\left(e_{2}\right)\right) \longmapsto{ }^{j_{2}}\left(w_{f_{2}}, e_{f_{2}}\right)$, and

- $\operatorname{irred}\left(w_{f_{2}}, e_{f_{2}}\right)$.

Hence, there exists $W_{f_{2}}$ and $q_{f_{2}}$ such that

- $w_{f_{2}}:_{k-j_{1}-j_{2}}\left(W_{f_{2}} \odot_{k-j_{1}-j_{2}}\left(W_{f_{1}} \odot_{k-j_{1}} W_{r}\right)\right)$, and

- $\left(k-j_{1}-j_{2}, q_{f_{2}}, W_{f_{2}}, e_{f_{2}}\right) \in \mathcal{T} \llbracket \Delta \vdash \tau_{2}:$ TYPE $\rrbracket \delta$.

Hence, $e_{f_{2}} \equiv v_{f_{2}}$.

Note that $l_{f_{1}} \notin \operatorname{dom}\left(W_{f_{2}} \odot_{k-j_{1}-j_{2}} W_{r}\right)$, which follows from $\mathrm{A} \preceq q_{f_{1}}=W_{f_{1}}^{\text {qual }}\left(l_{f_{1}}\right)$ and $\left(W_{f_{2}} \odot_{k-j_{1}-j_{2}}\left(W_{f_{1}} \odot_{k-j_{1}} W_{r}\right)\right) \equiv\left(W_{f_{1}} \odot_{k-j_{1}-j_{2}}\left(W_{f_{2}} \odot_{k-j_{1}-j_{2}} W_{r}\right)\right)$ defined.

Hence, $l_{f_{1}} \notin \operatorname{dom}\left(W_{f_{2}}\right) \cup \operatorname{dom}\left(W_{r}\right)$, which follows from $\operatorname{dom}\left(W_{1} \odot_{k} W_{2}\right)=\operatorname{dom}\left(W_{1}\right) \cup \operatorname{dom}\left(W_{2}\right)$.

Note that

$$
\begin{aligned}
& w_{f_{2}}: k-j_{1}-j_{2}\left(W_{f_{2}} \odot_{k-j_{1}-j_{2}}\left(W_{f_{1}} \odot_{k-j_{1}} W_{r}\right)\right) \\
& \text { which follows from above } \\
& \equiv \exists \mathcal{S}_{2}: 2^{\text {Locs }} \text {. } \\
& \exists \mathcal{F}_{2 W}: \mathcal{S}_{2} \rightarrow \text { WorldDesc }_{k-j_{1}-j_{2}} \text {. } \\
& \exists \mathcal{F}_{2 q}: \mathcal{S}_{2} \rightarrow \text { Quals. } \\
& \text { let } W_{2 *}=\left(\left(W_{f_{2}} \odot_{k-j_{1}-j_{2}}\left(W_{f_{1}} \odot_{k-j_{1}} W_{r}\right)\right) \odot_{k-j_{1}-j_{2}} \odot_{k-j_{1}-j_{2}}^{l \in \mathcal{F}_{2}} \mathcal{F}_{2 W}(l)\right) \text { in } \\
& \operatorname{dom}\left(w_{f_{2}}\right) \supseteq \operatorname{dom}\left(W_{2 *}\right)=\mathcal{S}_{2} \wedge \\
& \forall l \in \mathcal{S}_{2} . \forall i<k-j_{1}-j_{2} \text {. } \\
& \left(i, \mathcal{F}_{2 q}(l),\left\lfloor\mathcal{F}_{2 W}(l)\right\rfloor_{i}, w_{f_{2}}^{\text {val }}(l)\right) \in\left\lfloor W_{2 *}^{\text {type }}(l)\right\rfloor_{k-j_{1}-j_{2}} \wedge \\
& \forall l \in \mathcal{S}_{2} \text {. } \\
& w_{f_{2}}^{\text {qual }}(l)=W_{2 *}^{\text {qual }}(l) \wedge \\
& \forall \mathcal{S}_{2}^{\dagger} \subseteq \mathcal{S}_{2} . \\
& \operatorname{dom}\left(\left(W_{f_{2}} \odot_{k-j_{1}-j_{2}}\left(W_{f_{1}} \odot_{k-j_{1}} W_{r}\right)\right)\right) \subseteq \mathcal{S}_{2}^{\dagger} \wedge\left(\forall l \in \mathcal{S}_{2}^{\dagger} \cdot \operatorname{dom}\left(\mathcal{F}_{2 W}(l)\right) \subseteq \mathcal{S}_{2}^{\dagger}\right) \Rightarrow \\
& \mathcal{S}_{2}^{\dagger}=\mathcal{S}_{2} \wedge \\
& \forall l \in \operatorname{dom}\left(w_{f_{2}}\right) \text {. } \\
& \mathrm{R} \preceq w_{f_{2}}^{\text {qual }}(l) \Rightarrow l \in \mathcal{S}_{2} \\
& \text { which follows from the definition of } w:_{k} W \text {. }
\end{aligned}
$$

Note that

$$
\begin{aligned}
& \operatorname{dom}\left(w_{f_{2}}\right) \supseteq \operatorname{dom}\left(W_{2 *}\right)=\mathcal{S}_{2} \\
& \quad \text { which follows from above }\left(w_{f_{2}}: k-j_{1}-j_{2}\left(W_{f_{2}} \odot_{k-j_{1}-j_{2}}\left(W_{f_{1}} \odot_{k-j_{1}} W_{r}\right)\right)\right) \\
& \equiv \operatorname{dom}\left(w_{f_{2}}\right) \supseteq \operatorname{dom}\left(W_{2 *}\right)=\operatorname{dom}\left(W_{f_{2}}\right) \cup \operatorname{dom}\left(W_{f_{1}}\right) \cup \operatorname{dom}\left(W_{r}\right) \cup \bigcup^{l \in \mathcal{S}_{2}} \operatorname{dom}\left(\mathcal{F}_{2 W}(l)\right)=\mathcal{S}_{2} \\
& \quad \text { which follows from above }\left(W_{2 *}=\ldots\right) \text { and } \operatorname{dom}\left(W_{1} \odot_{k} W_{2}\right)=\operatorname{dom}\left(W_{1}\right) \cup \operatorname{dom}\left(W_{2}\right) \\
& \equiv \operatorname{dom}\left(w_{f_{2}}\right) \supseteq \operatorname{dom}\left(W_{2 *}\right)=\operatorname{dom}\left(W_{f_{2}}\right) \cup\left\{l_{f_{1}}\right\} \cup \operatorname{dom}\left(W_{r}\right) \cup \bigcup^{l \in \mathcal{S}_{2}} \operatorname{dom}\left(\mathcal{F}_{2 W}(l)\right)=\mathcal{S}_{2} \\
& \quad \text { which follows from simplifications of } \operatorname{dom}\left(W_{f_{1}}\right)=\left\{l_{f_{1}}\right\} .
\end{aligned}
$$

Hence, $l_{f_{1}} \in \operatorname{dom}\left(w_{f_{2}}\right)$ and $l_{f_{1}} \in \mathcal{S}_{2}$.

Note that 


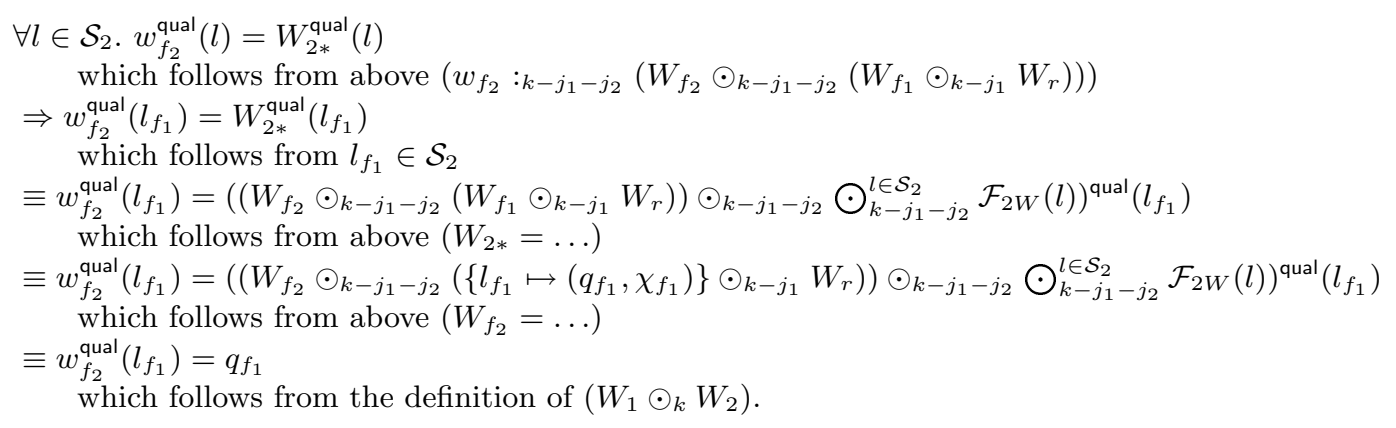

Note that $w_{f_{2}} \equiv w_{f_{21}} \uplus\left\{l_{f_{1}} \mapsto\left(q_{f_{1}}, v_{f_{11}}\right)\right\}$, which follows from $l_{f_{1}} \in \operatorname{dom}\left(w_{f_{2}}\right)$ and $w_{f_{2}}^{\text {qual }}\left(l_{f_{1}}\right)=q_{f_{1}}$. Note that

$$
\begin{aligned}
\left(w_{s}, e_{s}\right) & \equiv\left(w_{s}, \operatorname{sw} \gamma_{1}\left(e_{1}\right) \gamma_{2}\left(e_{2}\right)\right) \\
& \longmapsto{ }^{j_{1}}\left(w_{f_{1}}, \text { sw } e_{f_{1}} \gamma_{2}\left(e_{2}\right)\right) \\
& \equiv\left(w_{f_{1}}, \text { sw } l_{f_{1}} \gamma_{2}\left(e_{2}\right)\right) \\
& \longmapsto{ }^{j_{2}}\left(w_{f_{2}}, \text { sw } l_{f_{1}} e_{f_{2}}\right) \\
& \equiv\left(w_{f_{2}}, \operatorname{sw} l_{f_{1}} v_{f_{2}}\right) \\
& \equiv\left(w_{f_{21}} \uplus\left\{l_{f_{1}} \mapsto\left(q_{f_{1}}, v_{f_{11}}\right)\right\}, \text { sw } l_{f_{1}} v_{f_{2}}\right) \\
& \longmapsto{ }^{1}\left(w_{f_{21}} \uplus\left\{l_{f_{1}} \mapsto\left(q_{f_{1}}, v_{f_{2}}\right)\right\},\left\langle l_{f_{1}}, v_{f_{11}}\right\rangle\right) \\
& \longmapsto{ }^{j-j_{1}-j_{2}-1}\left(w_{f}, e_{f}\right) .
\end{aligned}
$$

Since $\left\langle l_{f_{1}}, v_{f_{11}}\right\rangle$ is value, we have $\operatorname{irred}\left(w_{f_{21}} \uplus\left\{l_{f_{1}} \mapsto\left(q_{f_{1}}, v_{f_{2}}\right)\right\},\left\langle l_{f_{1}}, v_{f_{11}}\right\rangle\right)$.

Hence, $j-j_{1}-j_{2}-1=0$ (and $\left.j=j_{1}+j_{2}+1\right)$ and $w_{f} \equiv w_{f_{21}} \uplus\left\{l_{f_{1}} \mapsto\left(q_{f_{1}}, v_{f_{2}}\right)\right\}$ and $e_{f} \equiv\left\langle l_{f_{1}}, v_{f_{11}}\right\rangle$.

Note that

$$
\begin{aligned}
& \forall l \in \mathcal{S}_{2} . \forall i<k-j_{1}-j_{2} .\left(i, \mathcal{F}_{2 q}(l),\left\lfloor\mathcal{F}_{2 W}(l)\right\rfloor_{i}, w_{f_{2}}^{\text {val }}(l)\right) \in\left\lfloor W_{2 *}^{\text {type }}(l)\right\rfloor_{k-j_{1}-j_{2}} \\
& \text { which follows from above }\left(w_{f_{2}}: k-j_{1}-j_{2}\left(W_{f_{2}} \odot_{k-j_{1}-j_{2}}\left(W_{f_{1}} \odot_{k-j_{1}} W_{r}\right)\right)\right) \\
& \Rightarrow \forall i<k-j_{1}-j_{2} .\left(i, \mathcal{F}_{2 q}\left(l_{f_{1}}\right),\left\lfloor\mathcal{F}_{2 W}\left(l_{f_{1}}\right)\right\rfloor_{i}, w_{f_{2}}^{\mathrm{val}}\left(l_{f_{1}}\right)\right) \in\left\lfloor W_{2 *}^{\mathrm{type}}\left(l_{f_{1}}\right)\right\rfloor_{k-j_{1}-j_{2}} \\
& \text { which follows from } l_{f_{1}} \in \mathcal{S}_{2} \\
& \equiv \forall i<k-j_{1}-j_{2} .\left(i, \mathcal{F}_{2 q}\left(l_{f_{1}}\right),\left\lfloor\mathcal{F}_{2 W}\left(l_{f_{1}}\right)\right\rfloor_{i},\left(w_{f_{21}} \uplus\left\{l_{f_{1}} \mapsto\left(q_{f_{1}}, v_{f_{11}}\right)\right\}\right)^{\mathrm{val}}\left(l_{f_{1}}\right)\right) \in\left\lfloor W_{2 *}^{\mathrm{type}}\left(l_{f_{1}}\right)\right\rfloor_{k-j_{1}-j_{2}} \\
& \text { which follows from }\left(w_{f_{2}}=\ldots\right) \\
& \equiv \forall i<k-j_{1}-j_{2} .\left(i, \mathcal{F}_{2 q}\left(l_{f_{1}}\right),\left\lfloor\mathcal{F}_{2 W}\left(l_{f_{1}}\right)\right\rfloor_{i}, v_{f_{11}}\right) \in\left\lfloor W_{2 *}^{\text {type }}\left(l_{f_{1}}\right)\right\rfloor_{k-j_{1}-j_{2}} \\
& \text { which follows from }\left(w_{f_{21}} \uplus\left\{l_{f_{1}} \mapsto\left(q_{f_{1}}, v_{f_{11}}\right)\right\}\right)^{\mathrm{val}}\left(l_{f_{1}}\right)=v_{f_{11}} \\
& \Rightarrow\left(k-j, \mathcal{F}_{2 q}\left(l_{f_{1}}\right),\left\lfloor\mathcal{F}_{2 W}\left(l_{f_{1}}\right)\right\rfloor_{k-j}, v_{f_{11}}\right) \in\left\lfloor W_{2 *}^{\text {type }}\left(l_{f_{1}}\right)\right\rfloor_{k-j_{1}-j_{2}} \\
& \text { which follows from } k-j<k-j_{1}-j_{2} \\
& \equiv\left(k-j, \mathcal{F}_{2 q}\left(l_{f_{1}}\right),\left\lfloor\mathcal{F}_{2 W}\left(l_{f_{1}}\right)\right\rfloor_{k-j}, v_{f_{11}}\right) \in\left\lfloor\left\lfloor\chi_{f_{1}}\right\rfloor_{k-j_{1}-j_{2}}\right\rfloor_{k-j_{1}-j_{2}} \\
& \text { which follows from simplifications of } W_{2 *}^{\text {type }}\left(l_{f_{1}}\right) \equiv\left\lfloor W_{f_{1}}\right\rfloor_{k-j_{1}-j_{2}}^{\text {type }}\left(l_{f_{1}}\right) \equiv\left\lfloor\chi_{f_{1}}\right\rfloor_{k-j_{1}-j_{2}} \\
& \equiv\left(k-j, \mathcal{F}_{2 q}\left(l_{f_{1}}\right),\left\lfloor\mathcal{F}_{2 W}\left(l_{f_{1}}\right)\right\rfloor_{k-j}, v_{f_{11}}\right) \in\left\lfloor\left\lfloor\left\lfloor\mathcal{T} \llbracket \Delta \vdash \tau_{1}: \text { TYPE } \rrbracket \delta\right\rfloor_{k-j_{1}}\right\rfloor_{k-j_{1}-j_{2}}\right\rfloor_{k-j_{1}-j_{2}} \\
& \text { which follows from simplifications of } \chi_{f_{1}}=\left\lfloor\mathcal{T} \llbracket \Delta \vdash \tau_{1}: \text { TYPE } \rrbracket \delta\right\rfloor_{k-j_{1}} \\
& \Rightarrow\left(k-j, \mathcal{F}_{2 q}\left(l_{f_{1}}\right),\left\lfloor\mathcal{F}_{2 W}\left(l_{f_{1}}\right)\right\rfloor_{k-j}, v_{f_{11}}\right) \in\left\lfloor\mathcal{T} \llbracket \Delta \vdash \tau_{1}: \text { TYPE } \rrbracket\right\rfloor_{k-j_{1}-j_{2}} \\
& \text { which follows from Fact } 2 \\
& \Rightarrow\left(k-j, \mathcal{F}_{2 q}\left(l_{f_{1}}\right),\left\lfloor\mathcal{F}_{2 W}\left(l_{f_{1}}\right)\right\rfloor_{k-j}, v_{f_{11}}\right) \in \mathcal{T} \llbracket \Delta \vdash \tau_{1}: \text { TYPE } \rrbracket \delta \\
& \text { which follows from the definition of }\lfloor\cdot\rfloor_{k} \text {. }
\end{aligned}
$$

Note that

$$
\begin{aligned}
& w_{f_{2}}:_{k-j_{1}-j_{2}}\left(W_{f_{2}} \odot_{k-j_{1}-j_{2}}\left(W_{f_{1}} \odot_{k-j_{1}} W_{r}\right)\right) \\
& \text { which follows from above }
\end{aligned}
$$




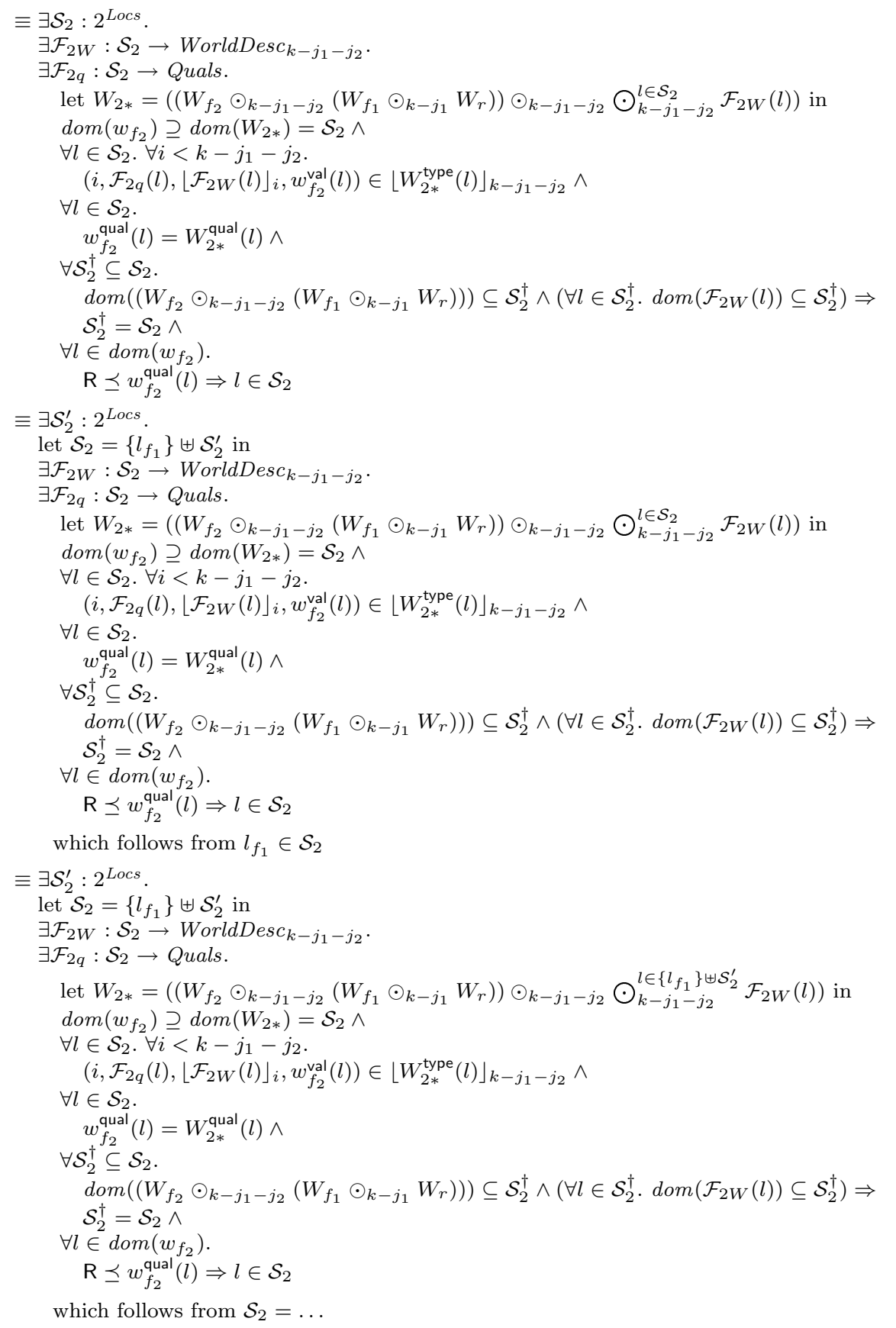




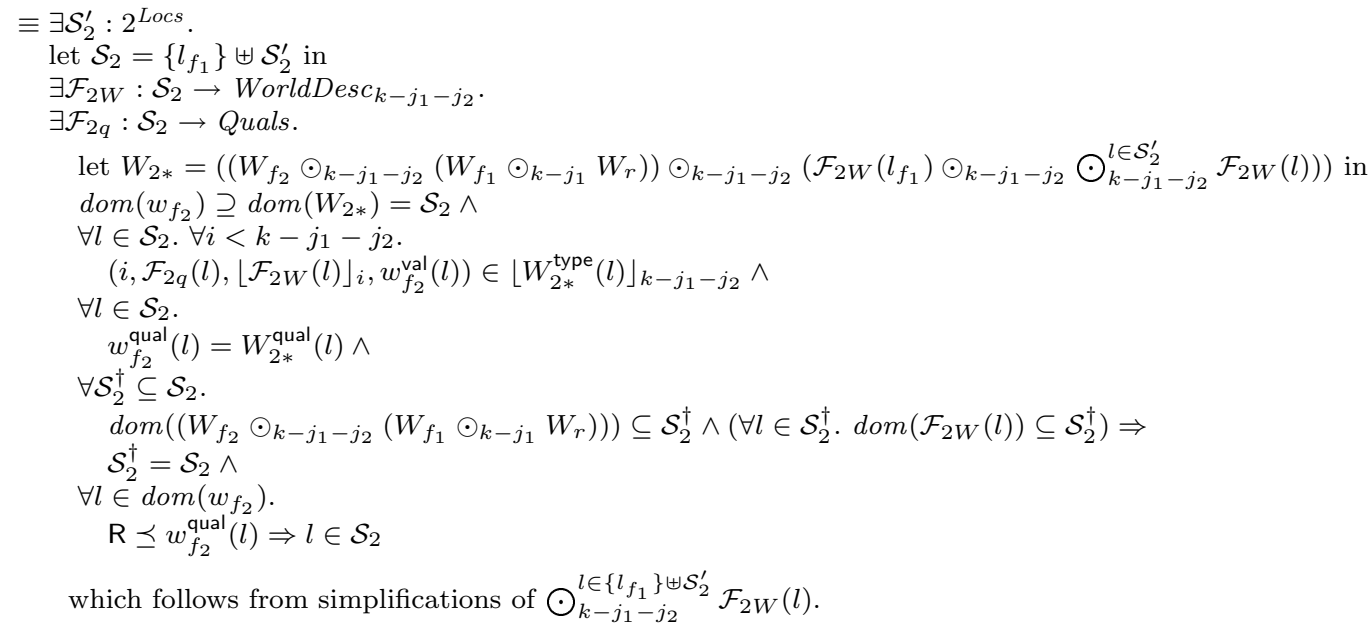

Let $\chi_{f_{1}}^{\prime}=\left\lfloor\mathcal{T} \llbracket \Delta \vdash \tau_{2}: \text { TYPE } \rrbracket \delta\right\rfloor_{k-j}$.

Let $W_{f_{1}}^{\prime}=\left\{l_{f_{1}} \mapsto\left(q_{f_{1}}, \chi_{f_{1}}^{\prime}\right)\right\}$.

Note that $\left(W_{f_{1}}^{\prime} \odot_{k-j} \mathcal{F}_{2 W}\left(l_{f_{1}}\right)\right)$ is defined, which follows from $\mathrm{A} \preceq q_{f_{1}}$ and $\left(W_{f_{1}} \odot_{k-j} \mathcal{F}_{2 W}\left(l_{f_{1}}\right)\right)$ defined, which in turn follows from Reqs $4,5,6,7$, and 8 (join-closed, join-aprx, join-commut, join-assocl, and join-assocr)and $W_{2 *}$ defined.

Let $W_{f}=\left(W_{f_{1}}^{\prime} \odot_{k-j} \mathcal{F}_{2 W}\left(l_{f_{1}}\right)\right)$.

Let $q_{f}=\mathrm{L}$.

Note that $\left(\left(W_{f_{1}}^{\prime} \odot_{k-j} \mathcal{F}_{2 W}\left(l_{f_{1}}\right)\right) \odot_{k-j} W_{r}\right)$ is defined, which follows from $\mathrm{A} \preceq q_{f_{1}}$ and $\left(\left(W_{f_{1}} \odot_{k-j}\right.\right.$ $\left.\mathcal{F}_{2 W}\left(l_{f_{1}}\right)\right) \odot_{k-j} W_{r}$ ) defined, which in turn follows from Reqs $4,5,6,7$, and 8 (join-closed, join-aprx, join-commut, join-assocl, and join-assocr)and $W_{2 *}$ defined.

Note that $l_{f_{1}} \notin \operatorname{dom}\left(\mathcal{F}_{2 W}\left(l_{f_{1}}\right) \odot_{k-j} W_{r}\right)$, which follows from $\mathrm{A} \preceq q_{f_{1}}=W_{f_{1}}^{\text {qqual }}\left(l_{f_{1}}\right)$ and $\left(\left(W_{f_{1}}^{\prime} \odot_{k-j}\right.\right.$ $\left.\left.\mathcal{F}_{2 W}\left(l_{f_{1}}\right)\right) \odot_{k-j} W_{r}\right) \equiv\left(W_{f_{1}}^{\prime} \odot_{k-j}\left(\mathcal{F}_{2 W}\left(l_{f_{1}}\right) \odot_{k-j} W_{r}\right)\right)$ defined.

Hence, $l_{f_{1}} \notin \operatorname{dom}\left(\mathcal{F}_{2 W}\left(l_{f_{1}}\right)\right) \cup \operatorname{dom}\left(W_{r}\right)$, which follows from $\operatorname{dom}\left(W_{1} \odot_{k} W_{2}\right)=\operatorname{dom}\left(W_{1}\right) \cup$ $\operatorname{dom}\left(W_{2}\right)$.

We are required to show that

- $w_{f}: k-j\left(W_{f} \odot_{k-j} W_{r}\right)$

$\equiv w_{f_{21}} \uplus\left\{l_{f_{1}} \mapsto\left(q_{f_{1}}, v_{f_{2}}\right)\right\}: k-j\left(\left(W_{f_{1}}^{\prime} \odot_{k-j} \mathcal{F}_{2 W}\left(l_{f_{1}}\right)\right) \odot_{k-j} W_{r}\right)$,

which is equivalent to

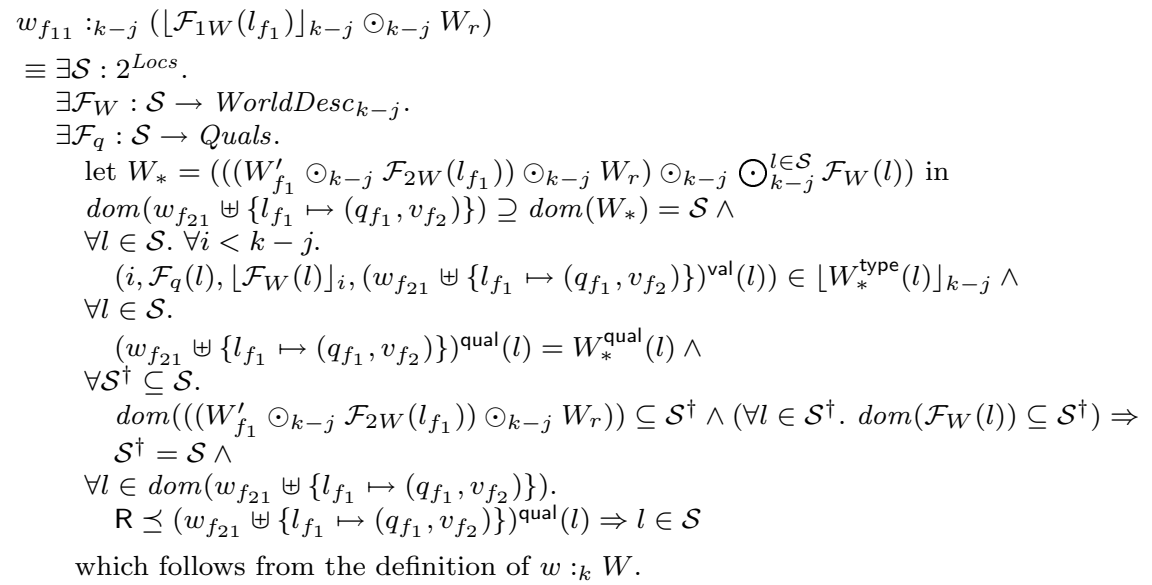

Take 
It remains to show that

$$
\mathcal{S}=\mathcal{S}_{2}=\left\{l_{f_{1}}\right\} \uplus \mathcal{S}_{2}^{\prime} .
$$

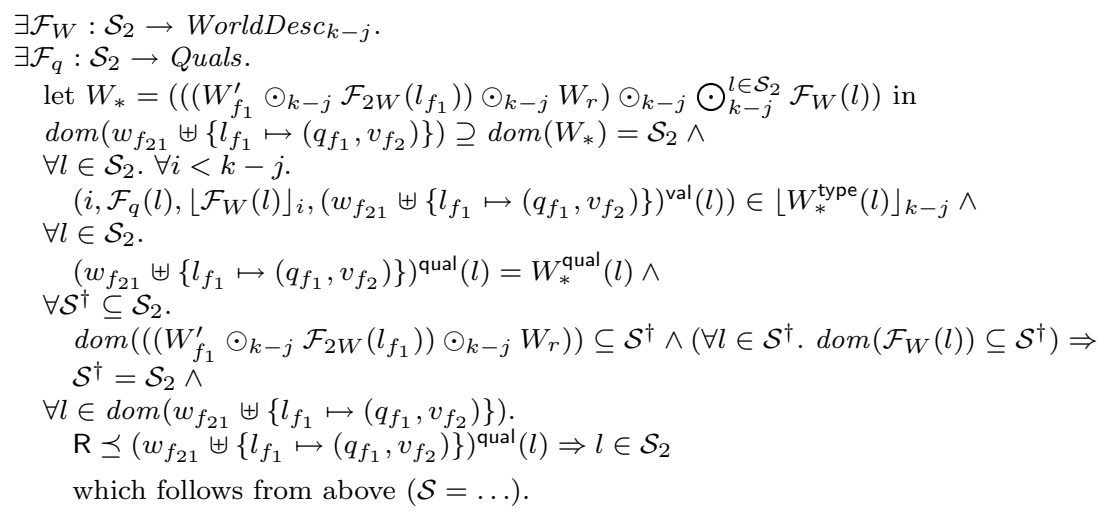

Take

and

$$
\mathcal{F}_{W}(l)= \begin{cases}\left\lfloor W_{f_{2}}\right\rfloor_{k-j} & \text { if } l \in\left\{l_{f_{1}}\right\} \\ \left\lfloor\mathcal{F}_{2 W}(l)\right\rfloor_{k-j} & \text { if } l \in \mathcal{S}_{2}^{\prime}\end{cases}
$$

Note that

$$
\mathcal{F}_{q}(l)= \begin{cases}q_{f_{2}} & \text { if } l \in\left\{l_{f_{1}}\right\} \\ \mathcal{F}_{2 q}(l) & \text { if } l \in \mathcal{S}_{2}^{\prime}\end{cases}
$$

$W_{f_{2}} \in$ WorldDesc $_{k-j_{1}-j_{2}}$

which follows from Fact 6 applied to $\left(k-j_{1}-j_{2}, q_{f_{2}}, W_{f_{2}}, v_{f_{2}}\right) \in \mathcal{T} \llbracket \Delta \vdash \tau_{2}:$ TYPE $\rrbracket \in \in$ Type, which in turn follows from Lemma 8 applied to $\mathcal{T} \llbracket \Delta \vdash \tau_{2}:$ TYPE $\rrbracket \delta$

$\Rightarrow\left\lfloor W_{f_{2}}\right\rfloor_{k-j} \in$ WorldDesc $_{k-j}$

which follows from $\lfloor\cdot\rfloor_{k} \in$ WorldDesc $\rightarrow$ WorldDesc $_{k}$

$\equiv \mathcal{F}_{W}\left(l_{f_{1}}\right) \in$ WorldDesc $_{k-j}$

which follows from above $\left(\mathcal{F}_{W}(l)=\ldots\right)$.

Note that

$$
\begin{aligned}
\forall l \in \mathcal{S}_{2} . \mathcal{F}_{2 W}(l) \in \text { WorldDesc }_{k-j_{1}-j_{2}} \\
\quad \text { which follows from above }\left(w_{f_{2}}: k-j_{1}-j_{2}\left(W_{f_{2}} \odot_{k-j_{1}-j_{2}}\left(W_{f_{1}} \odot_{k-j_{1}} W_{r}\right)\right)\right) \\
\Rightarrow \forall l \in \mathcal{S}_{2}^{\prime} . \mathcal{F}_{2 W}(l) \in \text { WorldDesc }_{k-j_{1}-j_{2}} \\
\quad \text { which follows from } \mathcal{S}_{2} \subseteq \mathcal{S}_{2} \\
\Rightarrow \forall l \in \mathcal{S}_{2}^{\prime} .\left\lfloor\mathcal{F}_{2 W}(l)\right\rfloor_{k-j} \in \text { WorldDesc }_{k-j} \\
\quad \text { which follows from }\lfloor\cdot\rfloor_{k} \in \text { WorldDesc } \rightarrow \text { WorldDesc }_{k} \\
\Rightarrow \forall l \in \mathcal{S}_{2}^{\prime} . \mathcal{F}_{W}(l) \in \text { WorldDesc }_{k-j} \\
\quad \text { which follows from above }\left(\mathcal{F}_{W}(l)=\ldots\right) .
\end{aligned}
$$

Hence, $\mathcal{F}_{W}: \mathcal{S}_{2} \rightarrow$ WorldDesc $c_{k-j}$.

Trivially, $\mathcal{F}_{q}: \mathcal{S}_{2} \rightarrow$ Quals.

It remains to show that

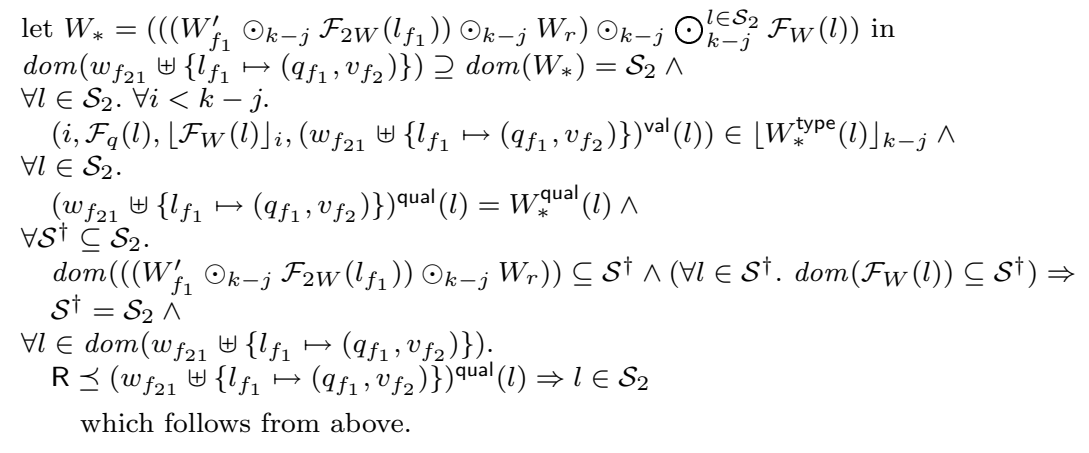


Note that

$$
\begin{aligned}
& \left\lfloor W_{2 *}\right\rfloor_{k-j} \\
& \equiv \quad\left\lfloor\left(\left(W_{f_{2}} \odot_{k-j_{1}-j_{2}}\left(W_{f_{1}} \odot_{k-j_{1}} W_{r}\right)\right) \odot_{k-j_{1}-j_{2}}\left(\mathcal{F}_{2 W}\left(l_{f_{1}}\right) \odot_{k-j_{1}-j_{2}} \bigodot_{k-j_{1}-j_{2}}^{l \in \mathcal{F}_{2}^{\prime}} \mathcal{F}_{2 W}(l)\right)\right)\right\rfloor_{k-j} \\
& \quad \text { which follows from above }\left(W_{2 *}=\ldots\right) \\
& \equiv\left(\left(\left\lfloor W_{f_{2}}\right\rfloor_{k-j} \odot_{k-j}\left(W_{f_{1}} \odot_{k-j} W_{r}\right)\right) \odot_{k-j}\left(\mathcal{F}_{2 W}\left(l_{f_{1}}\right) \odot_{k-j} \bigodot_{k-j}^{l \in \mathcal{S}_{2}^{\prime}}\left\lfloor\mathcal{F}_{2 W}(l)\right\rfloor_{k-j}\right)\right) \\
& \quad \text { which follows from Req } 4 \text { (join-closed) and Req } 5(\text { join-aprx) } \\
& \equiv\left(\left(\left(W_{f_{1}} \odot_{k-j} \mathcal{F}_{2 W}\left(l_{f_{1}}\right)\right) \odot_{k-j} W_{r}\right) \odot_{k-j}\left(\left\lfloor W_{f_{2}}\right\rfloor_{k-j} \odot_{k-j} \bigodot_{k-j}^{l \in \mathcal{S}_{2}^{\prime}}\left\lfloor\mathcal{F}_{2 W}(l)\right\rfloor_{k-j}\right)\right) \\
& \quad \text { which follows from Reqs } 6,7, \text { and } 8(\text { join-commut, join-assocl, and join-assocr }) \\
& \equiv\left(\left(\left(W_{f_{1}} \odot_{k-j} \mathcal{F}_{2 W}\left(l_{f_{1}}\right)\right) \odot_{k-j} W_{r}\right) \odot_{k-j}\left(\mathcal{F}_{W}\left(l_{f_{1}}\right) \odot_{k-j} \bigodot_{k-j}^{l \in \mathcal{S}_{2}^{\prime}} \mathcal{F}_{W}(l)\right)\right) \\
& \quad \text { which follows from above }\left(\mathcal{F}_{W}(l)=\ldots\right) \\
& \equiv\left(\left(\left(W_{f_{1}} \odot_{k-j} \mathcal{F}_{2 W}\left(l_{f_{1}}\right)\right) \odot_{k-j} W_{r}\right) \odot_{k-j} \bigodot_{k-j}^{l \in\left\{l_{f_{1}}\right\} \uplus \mathcal{S}_{2}^{\prime}} \mathcal{F}_{W}(l)\right) \\
& \quad \text { which follows from simplifications of } \bigodot_{k-j}^{l \in\left\{l_{f_{1}}\right\} \uplus \mathcal{S}_{2}^{\prime}} \mathcal{F}_{W}(l) \\
& \equiv\left(\left(\left(W_{f_{1}} \odot_{k-j} \mathcal{F}_{2 W}\left(l_{f_{1}}\right)\right) \odot_{k-j} W_{r}\right) \odot_{k-j} \bigodot_{k-j}^{l \in \mathcal{S}_{2}} \mathcal{F}_{W}(l)\right) \\
& \quad \text { which follows from above }\left(\mathcal{S}_{2}=\ldots\right) .
\end{aligned}
$$

Hence, $W_{*}=\left(\left(\left(W_{f_{1}}^{\prime} \odot_{k-j} \mathcal{F}_{2 W}\left(l_{f_{1}}\right)\right) \odot_{k-j} W_{r}\right) \odot_{k-j} \bigodot_{k-j}^{l \in \mathcal{S}_{2}} \mathcal{F}_{W}(l)\right)$ is defined, which follows from $W_{f_{1}}=\left\{l_{f_{1}} \mapsto\left(q_{f_{1}}, \chi_{f_{1}}\right)\right\}$ and $W_{f_{1}}^{\prime}=\left\{l_{f_{1}} \mapsto\left(q_{f_{1}}, \chi_{f_{1}}^{\prime}\right)\right\}$ and and $\mathrm{A} \preceq q_{f_{1}}$ and the definition of $\left(\cdot \odot_{k} \cdot\right)$ and $\left\lfloor W_{2 *}\right\rfloor_{k-j}=\ldots$.

Note that $W_{*}=\left(W_{f_{1}}^{\prime} \odot_{k-j}\left(\left\lfloor W_{2 *}\right\rfloor_{k-j} \backslash\left\{l_{f_{1}}\right\}\right)\right)$, which follows from $W_{f_{1}}=\left\{l_{f_{1}} \mapsto\left(q_{f_{1}}, \chi_{f_{1}}\right)\right\}$ and $W_{f_{1}}^{\prime}=\left\{l_{f_{1}} \mapsto\left(q_{f_{1}}, \chi_{f_{1}}^{\prime}\right)\right\}$ and and $\mathrm{A} \preceq q_{f_{1}}$ and the definition of $\left(\cdot \odot_{k} \cdot\right)$ and $\left\lfloor W_{2 *}\right\rfloor_{k-j}=$ -...

Furthermore, $\operatorname{dom}\left(W_{*}\right)=\operatorname{dom}\left(W_{2 *}\right)$.

Note that

$$
\begin{aligned}
& \operatorname{dom}\left(w_{f_{2}}\right) \supseteq \operatorname{dom}\left(W_{2 *}\right)=\mathcal{S}_{2} \\
& \quad \text { which follows from above }\left(w_{f_{2}}: k-j_{1}-j_{2}\left(W_{f_{2}} \odot_{k-j_{1}-j_{2}}\left(W_{f_{1}} \odot_{k-j_{1}} W_{r}\right)\right)\right) \\
& \equiv \operatorname{dom}\left(w_{f_{21}} \uplus\left\{l_{f_{1}} \mapsto\left(q_{f_{1}}, v_{f_{11}}\right)\right\}\right) \supseteq \operatorname{dom}\left(W_{2 *}\right)=\mathcal{S}_{2} \\
& \quad \text { which follows from above }\left(w_{f_{2}}=\ldots\right) \\
& \equiv \operatorname{dom}\left(w_{f_{21}} \uplus\left\{l_{f_{1}} \mapsto\left(q_{f_{1}}, v_{f_{2}}\right)\right\}\right) \supseteq \operatorname{dom}\left(W_{2 *}\right)=\mathcal{S}_{2} \\
& \quad \text { which follows from } \operatorname{dom}\left(w_{f_{21}} \uplus\left\{l_{f_{1}} \mapsto\left(q_{f_{1}}, v_{f_{11}}\right)\right\}\right)=\operatorname{dom}\left(w_{f_{21}} \uplus\left\{l_{f_{1}} \mapsto\left(q_{f_{1}}, v_{f_{2}}\right)\right\}\right) \\
& \equiv \operatorname{dom}\left(w_{f_{21}} \uplus\left\{l_{f_{1}} \mapsto\left(q_{f_{1}}, v_{f_{2}}\right)\right\}\right) \supseteq \operatorname{dom}\left(W_{*}\right)=\mathcal{S}_{2} \\
& \quad \text { which follows from above }\left(\operatorname{dom}\left(W_{*}\right)=\ldots\right) .
\end{aligned}
$$

It remains to show that

$$
\begin{aligned}
& \forall l \in \mathcal{S}_{2} . \forall i<k-j . \\
& \quad\left(i, \mathcal{F}_{q}(l),\left\lfloor\mathcal{F}_{W}(l)\right\rfloor_{i},\left(w_{f_{21}} \uplus\left\{l_{f_{1}} \mapsto\left(q_{f_{1}}, v_{f_{2}}\right)\right\}\right)^{\text {val }}(l)\right) \in\left\lfloor W_{*}^{\text {type }}(l)\right\rfloor k-j \wedge \\
& \forall l \in \mathcal{S}_{2} . \\
& \quad\left(w_{f_{21}} \uplus\left\{l_{f_{1}} \mapsto\left(q_{f_{1}}, v_{f_{2}}\right)\right\}\right)^{\text {qual }}(l)=W_{*}^{\text {qual }}(l) \wedge \\
& \forall \mathcal{S}^{\dagger} \subseteq \mathcal{S}_{2} . \\
& \quad d o m\left(\left(\left(W_{f_{1}}^{\prime} \odot_{k-j} \mathcal{F}_{2 W}\left(l_{f_{1}}\right)\right) \odot_{k-j} W_{r}\right)\right) \subseteq \mathcal{S}^{\dagger} \wedge\left(\forall l \in \mathcal{S}^{\dagger} . \operatorname{dom}\left(\mathcal{F}_{W}(l)\right) \subseteq \mathcal{S}^{\dagger}\right) \Rightarrow \\
& \quad \mathcal{S}^{\dagger}=\mathcal{S}_{2} \wedge \\
& \forall l \in \operatorname{dom}\left(w_{f_{21}} \uplus\left\{l_{f_{1}} \mapsto\left(q_{f_{1}}, v_{f_{2}}\right)\right\}\right) . \\
& \quad \mathrm{R} \preceq\left(w_{f_{21}} \uplus\left\{l_{f_{1}} \mapsto\left(q_{f_{1}}, v_{f_{2}}\right)\right\}\right)^{\text {qual }}(l) \Rightarrow l \in \mathcal{S}_{2} \\
& \quad \text { which follows from above. }
\end{aligned}
$$

We are required to show that

- $\forall l \in \mathcal{S}_{2} . \forall i<k-j$.

$$
\left(i, \mathcal{F}_{q}(l),\left\lfloor\mathcal{F}_{W}(l)\right\rfloor_{i},\left(w_{f_{21}} \uplus\left\{l_{f_{1}} \mapsto\left(q_{f_{1}}, v_{f_{2}}\right)\right\}\right)^{\mathrm{val}}(l)\right) \in\left\lfloor W_{*}^{\text {type }}(l)\right\rfloor_{k-j}
$$

Note that

$$
\begin{aligned}
& \forall l \in \mathcal{S}_{2} . \forall i<k-j . \\
& \quad\left(i, \mathcal{F}_{q}(l),\left\lfloor\mathcal{F}_{W}(l)\right\rfloor_{i},\left(w_{f_{21}} \uplus\left\{l_{f_{1}} \mapsto\left(q_{f_{1}}, v_{f_{2}}\right)\right\}\right)^{\mathrm{val}}(l)\right) \in\left\lfloor W_{*}^{\text {type }}(l)\right\rfloor_{k-j} \\
& \equiv \forall l \in\left\{l_{f_{1}}\right\} \uplus \mathcal{S}_{2}^{\prime} . \forall i<k-j . \\
& \quad\left(i, \mathcal{F}_{q}(l),\left\lfloor\mathcal{F}_{W}(l)\right\rfloor_{i},\left(w_{f_{21}} \uplus\left\{l_{f_{1}} \mapsto\left(q_{f_{1}}, v_{f_{2}}\right)\right\}\right)^{\mathrm{val}}(l)\right) \in\left\lfloor W_{*}^{\text {type }}(l)\right\rfloor_{k-j} \\
& \quad \text { which follows from above }\left(\mathcal{S}_{1}=\ldots\right)
\end{aligned}
$$




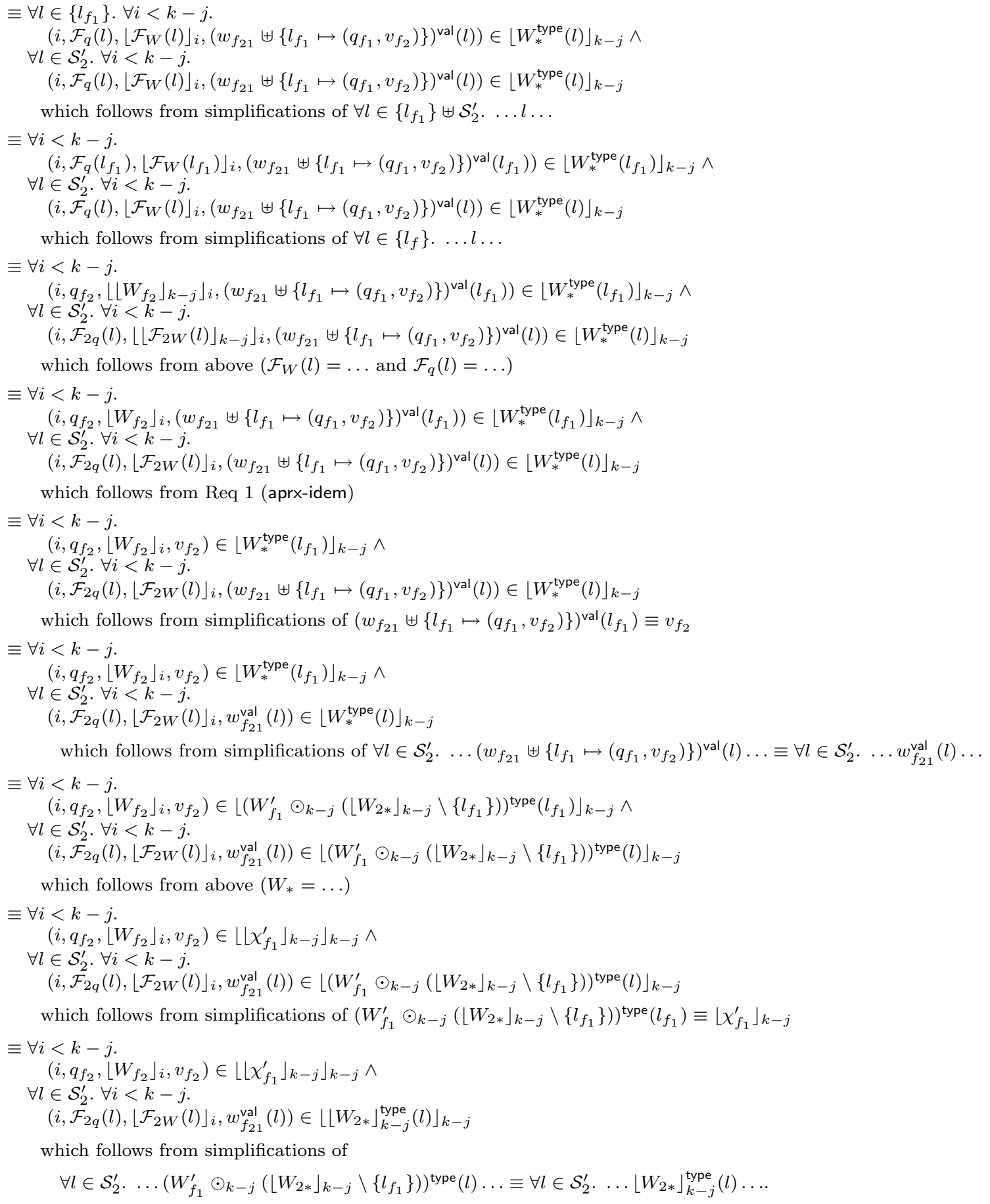

We are required to show that

- $\forall i<k-j$.

$\left(i, q_{f_{2}},\left\lfloor W_{f_{2}}\right\rfloor_{i}, v_{f_{2}}\right) \in\left\lfloor\left\lfloor\chi_{f_{1}}^{\prime}\right\rfloor_{k-j}\right\rfloor_{k-j}$ which follows from 


$$
\begin{aligned}
& \left(k-j_{1}-j_{2}, q_{f_{2}}, W_{f_{2}}, v_{f_{2}}\right) \in \mathcal{T} \llbracket \Delta \vdash \tau_{2}: \text { TYPE } \rrbracket \delta \\
& \text { which follows from above } \\
& \Rightarrow \forall i<k-j_{1}-j_{2} .\left(i, q_{f_{2}},\left\lfloor W_{f_{2}}\right\rfloor_{i}, v_{f_{2}}\right) \in \mathcal{T} \llbracket \Delta \vdash \tau_{2}: \text { TYPE } \rrbracket \delta \\
& \quad \text { which follows from Lemma } 8 \text { and Fact } 6 \\
& \Rightarrow \quad \forall i<k-j \cdot\left(i, q_{f_{2}},\left\lfloor W_{f_{2}}\right\rfloor_{i}, v_{f_{2}}\right) \in \mathcal{T} \llbracket \Delta \vdash \tau_{2}: \text { TYPE } \rrbracket \delta \\
& \quad \text { which follows } k-j<k-j_{1}-j_{2} \\
& \Rightarrow \quad \forall i<k-j \cdot\left(i, q_{f_{2}},\left\lfloor W_{f_{2}}\right\rfloor_{i}, v_{f_{2}}\right) \in\left\lfloor\mathcal{T} \llbracket \Delta \vdash \tau_{2}: \text { TYPE } \rrbracket \delta\right\rfloor_{k-j} \\
& \quad \text { which follows from } j<k \wedge(j, q, W, v) \in \chi \Rightarrow(j, q, W, v) \in\lfloor\chi\rfloor_{k} \\
& \Rightarrow \quad \forall i<k-j \cdot\left(i, q_{f_{2}},\left\lfloor W_{f_{2}}\right\rfloor_{i}, v_{f_{2}}\right) \in \chi_{f_{1}}^{\prime} \\
& \quad \text { which follows from above }\left(\chi_{f_{1}}^{\prime}=\ldots\right) \\
& \Rightarrow \quad \forall i<k-j \cdot\left(i, q_{f_{2}},\left\lfloor W_{f_{2}}\right\rfloor_{i}, v_{f_{2}}\right) \in\left\lfloor\chi_{f_{1}}^{\prime}\right\rfloor_{k-j} \\
& \quad \text { which follows from } j<k \wedge(j, q, W, v) \in \chi \Rightarrow(j, q, W, v) \in\lfloor\chi\rfloor_{k} \\
& \Rightarrow \quad \forall i<k-j \cdot\left(i, q_{f_{2}},\left\lfloor W_{f_{2}}\right\rfloor_{i}, v_{f_{2}}\right) \in\left\lfloor\left\lfloor\chi_{f_{1}}^{\prime}\right\rfloor_{k-j}\right\rfloor_{k-j} \\
& \quad \text { which follows from } j<k \wedge(j, q, W, v) \in \chi \Rightarrow(j, q, W, v) \in\lfloor\chi\rfloor_{k} .
\end{aligned}
$$

- $\forall l \in \mathcal{S}_{2}^{\prime} . \forall i<k-j$.

$\left(i, \mathcal{F}_{2 q}(l),\left\lfloor\mathcal{F}_{2 W}(l)\right\rfloor_{i}, w_{f_{21}}^{\text {val }}(l)\right) \in\left\lfloor\left\lfloor W_{2 *}\right\rfloor_{k-j}^{\text {type }}(l)\right\rfloor_{k-j}$

which follows from

$\forall l \in \mathcal{S}_{2} . \forall i<k-j_{1}-j_{2} .\left(i, \mathcal{F}_{2 q}(l),\left\lfloor\mathcal{F}_{2 W}(l)\right\rfloor_{i}, w_{f_{2}}^{\mathrm{val}}(l)\right) \in\left\lfloor W_{2 *}^{\text {type }}(l)\right\rfloor_{k-j_{1}-j_{2}}$

which follows from above $\left(w_{f_{2}}:_{k-j_{1}-j_{2}}\left(W_{f_{2}} \odot_{k-j_{1}-j_{2}}\left(W_{f_{1}} \odot_{k-j_{1}} W_{r}\right)\right)\right)$

$\Rightarrow \forall l \in \mathcal{S}_{2}^{\prime} . \forall i<k-j_{1}-j_{2} .\left(i, \mathcal{F}_{2 q}(l),\left\lfloor\mathcal{F}_{2 W}(l)\right\rfloor_{i}, w_{f_{2}}^{\mathrm{val}}(l)\right) \in\left\lfloor W_{2 *}^{\mathrm{type}}(l)\right\rfloor_{k-j_{1}-j_{2}}$

which follows from $\mathcal{S}_{2}^{\prime} \subseteq \mathcal{S}_{2}$

$\Rightarrow \forall l \in \mathcal{S}_{2}^{\prime} . \forall i<k-j .\left(i, \mathcal{F}_{2 q}(l),\left\lfloor\mathcal{F}_{2 W}(l)\right\rfloor_{i}, w_{f_{2}}^{\text {val }}(l)\right) \in\left\lfloor W_{2 *}^{\text {type }}(l)\right\rfloor_{k-j_{1}-j_{2}}$ which follows from $k-j<k-j_{1}-j_{2}$

$\equiv \forall l \in \mathcal{S}_{2}^{\prime} . \forall i<k-j .\left(i, \mathcal{F}_{2 q}(l),\left\lfloor\mathcal{F}_{2 W}(l)\right\rfloor_{i},\left(w_{f_{21}} \uplus\left\{l_{f_{1}} \mapsto\left(q_{f_{1}}, v_{f_{11}}\right)\right\}\right)^{\mathrm{val}}(l)\right) \in\left\lfloor W_{2 *}^{\text {type }}(l)\right\rfloor_{k-j_{1}-j_{2}}$ which follows from above $\left(w_{f_{2}}=\ldots\right)$

$\equiv \forall l \in \mathcal{S}_{2}^{\prime} . \forall i<k-j .\left(i, \mathcal{F}_{2 q}(l),\left\lfloor\mathcal{F}_{2 W}(l)\right\rfloor_{i}, w_{f_{21}}^{\text {val }}(l)\right) \in\left\lfloor W_{2 *}^{\text {type }}(l)\right\rfloor_{k-j_{1}-j_{2}}$ which follows from simplifications of

$\forall l \in \mathcal{S}_{2}^{\prime} . \ldots\left(w_{f_{21}} \uplus\left\{l_{f_{1}} \mapsto\left(q_{f_{1}}, v_{f_{11}}\right)\right\}\right)^{\mathrm{val}}(l) \ldots \equiv \forall l \in \mathcal{S}_{2}^{\prime} \ldots w_{f_{21}}^{\mathrm{val}}(l) \ldots$

$\Rightarrow \forall l \in \mathcal{S}_{2}^{\prime} . \forall i<k-j .\left(i, \mathcal{F}_{2 q}(l),\left\lfloor\mathcal{F}_{2 W}(l)\right\rfloor_{i}, w_{f_{21}}^{\mathrm{val}}(l)\right) \in\left\lfloor\left\lfloor W_{2 *}^{\text {type }}(l)\right\rfloor_{k-j_{1}-j_{2}}\right\rfloor_{k-j}$ which follows from $j<k \wedge(j, q, W, v) \in \chi \Rightarrow(j, q, W, v) \in\lfloor\chi\rfloor_{k}$

$\equiv \forall l \in \mathcal{S}_{2}^{\prime} . \forall i<k-j .\left(i, \mathcal{F}_{2 q}(l),\left\lfloor\mathcal{F}_{2 W}(l)\right\rfloor_{i}, w_{f_{21}}^{\text {val }}(l)\right) \in\left\lfloor W_{2 *}^{\text {type }}(l)\right\rfloor_{k-j}$ which follows from Fact 2 .

- $\forall l \in \mathcal{S}_{2}$.

$\left(w_{f_{21}} \uplus\left\{l_{f_{1}} \mapsto\left(q_{f_{1}}, v_{f_{2}}\right)\right\}\right)^{\text {qual }}(l)=W_{*}^{\text {qual }}(l)$

which follows from

$\forall l \in \mathcal{S}_{2} .\left(w_{f_{21}} \uplus\left\{l_{f_{1}} \mapsto\left(q_{f_{1}}, v_{f_{11}}\right)\right\}\right)^{\text {qual }}(l)=W_{2 *}^{\text {qual }}(l)$

which follows from above $\left(w_{f_{2}}: k-j_{1}-j_{2}\left(W_{f_{2}} \odot_{k-j_{1}-j_{2}}\left(W_{f_{1}} \odot_{k-j_{1}} W_{r}\right)\right)\right)$

$\equiv \forall l \in \mathcal{S}_{2} .\left(w_{f_{21}} \uplus\left\{l_{f_{1}} \mapsto\left(q_{f_{1}}, v_{f_{2}}\right)\right\}\right)^{\text {qual }}(l)=W_{2 *}^{\text {qual }}(l)$

which follows from $\forall l \in \mathcal{S}_{2} .\left(w_{f_{21}} \uplus\left\{l_{f_{1}} \mapsto\left(q_{f_{1}}, v_{f_{11}}\right)\right\}\right)^{\text {qual }}(l)=\left(w_{f_{21}} \uplus\left\{l_{f_{1}} \mapsto\left(q_{f_{1}}, v_{f_{2}}\right)\right\}\right)^{\text {qual }}(l)$

$\equiv \forall l \in \mathcal{S}_{2} .\left(w_{f_{21}} \uplus\left\{l_{f_{1}} \mapsto\left(q_{f_{1}}, v_{f_{2}}\right)\right\}\right)^{\text {qual }}(l)=W_{*}^{\text {qual }}(l)$

which follows from $\forall l \in \mathcal{S}_{2} . W_{2 *}^{\text {qual }}(l)=\left(W_{f_{1}}^{\prime} \odot_{k-j}\left(\left\lfloor W_{2 *}\right\rfloor_{k-j} \backslash\left\{l_{f_{1}}\right\}\right)\right)^{\text {qual }}(l)=W_{*}^{\text {qual }}(l)$.

- $\forall \mathcal{S}^{\dagger} \subseteq \mathcal{S}_{2}$.

$\operatorname{dom}\left(\left(\left(W_{f_{1}}^{\prime} \odot_{k-j} \mathcal{F}_{2 W}\left(l_{f_{1}}\right)\right) \odot_{k-j} W_{r}\right)\right) \subseteq \mathcal{S}^{\dagger} \wedge\left(\forall l \in \mathcal{S}^{\dagger} . \operatorname{dom}\left(\mathcal{F}_{W}(l)\right) \subseteq \mathcal{S}^{\dagger}\right) \Rightarrow$ $\mathcal{S}^{\dagger}=\mathcal{S}_{2}$

Consider arbitrary $\mathcal{S}^{\dagger}$ such that

- $\mathcal{S}^{\dagger} \subseteq \mathcal{S}_{2}$,

- $\operatorname{dom}\left(\left(\left(W_{f_{1}}^{\prime} \odot_{k-j} \mathcal{F}_{2 W}\left(l_{f_{1}}\right)\right) \odot_{k-j} W_{r}\right)\right) \subseteq \mathcal{S}^{\dagger}$, and

- $\forall l \in \mathcal{S}^{\dagger} . \operatorname{dom}\left(\mathcal{F}_{W}(l)\right) \subseteq \mathcal{S}^{\dagger}$.

Note that $\left\{l_{f_{1}}\right\} \cup \operatorname{dom}\left(\mathcal{F}_{2 W}\left(l_{f_{1}}\right)\right) \cup \operatorname{dom}\left(W_{r}\right) \subseteq \mathcal{S}^{\dagger}$, which follows from $\operatorname{dom}\left(\left(\left(W_{f_{1}}^{\prime} \odot_{k-j}\right.\right.\right.$ $\left.\left.\left.\mathcal{F}_{2 W}\left(l_{f_{1}}\right)\right) \odot_{k-j} W_{r}\right)\right) \subseteq \mathcal{S}^{\dagger}$ and $\operatorname{dom}\left(W_{f_{1}}^{\prime}\right)=\left\{l_{f_{1}}\right\}$, 
Note that $l_{f_{1}} \in \mathcal{S}^{\dagger}$, which follows from $\left\{l_{f_{1}}\right\} \cup \operatorname{dom}\left(\mathcal{F}_{2 W}\left(l_{f_{1}}\right)\right) \cup \operatorname{dom}\left(W_{r}\right) \subseteq \mathcal{S}^{\dagger}$.

Note that $\operatorname{dom}\left(W_{f_{2}}\right) \in \mathcal{S}^{\dagger}$, which follows from

$$
\begin{aligned}
& \forall l \in \mathcal{S}^{\dagger} . \operatorname{dom}\left(\mathcal{F}_{W}(l)\right) \subseteq \mathcal{S}^{\dagger} \\
& \quad \text { which follows from above } \\
& \Rightarrow \operatorname{dom}\left(\mathcal{F}_{W}\left(l_{f_{1}}\right)\right) \subseteq \mathcal{S}^{\dagger} \\
& \quad \text { which follows from } l_{f_{1}} \in \mathcal{S}^{\dagger} \\
& \equiv \operatorname{dom}\left(\left\lfloor W_{f_{2}}\right\rfloor_{k-j}\right) \subseteq \mathcal{S}^{\dagger} \\
& \quad \text { which follows from above }\left(\mathcal{F}_{W}(l)=\ldots\right) \\
& \equiv \operatorname{dom}\left(W_{f_{2}}\right) \subseteq \mathcal{S}^{\dagger} \\
& \quad \text { which follows from } \operatorname{dom}\left(\left\lfloor W_{f_{2}}\right\rfloor_{k-j}\right)=\operatorname{dom}\left(W_{f_{2}}\right) .
\end{aligned}
$$

Let $\mathcal{S}^{\ddagger}=\mathcal{S}^{\dagger} \backslash\left\{l_{f_{1}}\right\}$.

Note that $\mathcal{S}^{\dagger}=\left\{l_{f_{1}}\right\} \uplus \mathcal{S}_{2}^{\ddagger}$.

Note that $\forall l \in \mathcal{S}_{2}^{\dagger} \cdot \operatorname{dom}\left(\mathcal{F}_{2 W}(l)\right) \subseteq \mathcal{S}_{2}^{\dagger}$, which follows from

$$
\begin{aligned}
& \operatorname{dom}\left(\mathcal{F}_{2 W}\left(l_{f_{1}}\right)\right) \subseteq \mathcal{S}^{\dagger} \wedge \forall l \in \mathcal{S}^{\dagger} . \operatorname{dom}\left(\mathcal{F}_{W}(l)\right) \subseteq \mathcal{S}^{\dagger} \\
& \quad \text { which follows from above } \\
& \Rightarrow \operatorname{dom}\left(\mathcal{F}_{2 W}\left(l_{f_{1}}\right) \subseteq \subseteq \mathcal{S}^{\dagger} \wedge \forall l \in \mathcal{S}^{\ddagger} . \operatorname{dom}\left(\mathcal{F}_{W}(l)\right) \subseteq \mathcal{S}^{\dagger}\right. \\
& \quad \text { which follows from } \mathcal{S}^{\ddagger} \subseteq \mathcal{S} \\
& \equiv \operatorname{dom}\left(\mathcal{F}_{2 W}\left(l_{f_{1}}\right) \subseteq \subseteq \mathcal{S}^{\dagger} \wedge \forall l \in \mathcal{S}^{\ddagger} . \operatorname{dom}\left(\left\lfloor\mathcal{F}_{2 W}(l)\right\rfloor_{k-j}\right) \subseteq \mathcal{S}^{\dagger}\right. \\
& \quad \text { which follows from above }\left(\mathcal{F}_{W}(l)=\ldots\right) \\
& \equiv \operatorname{dom}\left(\mathcal{F}_{2 W}\left(l_{f_{1}}\right)\right) \subseteq \mathcal{S}^{\dagger} \wedge \forall l \in \mathcal{S}^{\ddagger} \text { dom }\left(\mathcal{F}_{2 W}(l)\right) \subseteq \mathcal{S}^{\dagger} \\
& \quad \text { which follows from } \operatorname{dom}\left(\left\lfloor\mathcal{F}_{2 W}(l)\right\rfloor k-j\right)=\operatorname{dom}\left(\mathcal{F}_{2 W}(l)\right) \\
& \equiv \forall l \in\left\{l_{f_{1}}\right\} \uplus \mathcal{S}^{\ddagger} \text {.dom }\left(\mathcal{F}_{2 W}(l)\right) \subseteq \mathcal{S}^{\dagger} \\
& \quad \text { which follows from simplifications of } \forall l \in\left\{l_{f_{1}}\right\} \uplus \mathcal{S}^{\ddagger} \ldots l \ldots \\
& \equiv \forall l \in \mathcal{S}^{\dagger} . \operatorname{dom}\left(\mathcal{F}_{2 W}(l)\right) \subseteq \mathcal{S}^{\dagger} \quad \text { which follows from above }\left(\mathcal{S}^{\dagger}=\ldots\right) .
\end{aligned}
$$

Instantiate $\left(\forall \mathcal{S}_{2}^{\dagger} \subseteq \mathcal{S}_{2} . \ldots\right)$ of $w_{f_{2}}:_{k-j_{1}-j_{2}}\left(W_{f_{2}} \odot_{k-j_{1}-j_{2}}\left(W_{f_{1}} \odot_{k-j_{1}} W_{r}\right)\right)$ with $\mathcal{S}^{\dagger}$ Note that

- $\mathcal{S}^{\dagger} \subseteq \mathcal{S}_{2}$, which follows from above,

- $\operatorname{dom}\left(\left(W_{f_{2}} \odot_{k-j_{1}-j_{2}}\left(W_{f_{1}} \odot_{k-j_{1}} W_{r}\right)\right)\right) \subseteq \mathcal{S}_{2}^{\dagger}$, which follows from $\operatorname{dom}\left(W_{f_{2}}\right) \subseteq$ $\mathcal{S}_{2}^{\dagger}$, which follows from above, and $\left\{l_{f_{1}}\right\} \subseteq \mathcal{S}_{2}^{\dagger}$, which follows from above and $\operatorname{dom}\left(W_{f_{1}}\right)=\left\{l_{f_{1}}\right\}$, and $\operatorname{dom}\left(W_{r}\right) \subseteq \mathcal{S}_{2}^{\dagger}$, which follows from above, and

- $\forall l \in \mathcal{S}_{2}^{\dagger} \cdot \operatorname{dom}\left(\mathcal{F}_{2 W}(l)\right) \subseteq \mathcal{S}_{2}^{\dagger}$, which follows from above.

Hence, we conclude that $\mathcal{S}^{\dagger}=\mathcal{S}_{2}$.

- $\forall l \in \operatorname{dom}\left(w_{f_{21}} \uplus\left\{l_{f_{1}} \mapsto\left(q_{f_{1}}, v_{f_{2}}\right)\right\}\right)$.

$\mathrm{R} \preceq\left(w_{f_{21}} \uplus\left\{l_{f_{1}} \mapsto\left(q_{f_{1}}, v_{f_{2}}\right)\right\}\right)^{\text {qual }}(l) \Rightarrow l \in \mathcal{S}_{2}$

which follows from

$\forall l \in \operatorname{dom}\left(w_{f_{21}} \uplus\left\{l_{f_{1}} \mapsto\left(q_{f_{1}}, v_{f_{11}}\right)\right\}\right) . \mathrm{R} \preceq\left(w_{f_{21}} \uplus\left\{l_{f_{1}} \mapsto\left(q_{f_{1}}, v_{f_{11}}\right)\right\}\right)^{\text {qual }}(l) \Rightarrow l \in \mathcal{S}_{2}$

which follows from above $\left(w_{f_{2}}: k-j_{1}-j_{2}\left(W_{f_{2}} \odot_{k-j_{1}-j_{2}}\left(W_{f_{1}} \odot_{k-j_{1}} W_{r}\right)\right)\right)$

$\equiv \forall l \in \operatorname{dom}\left(w_{f_{21}} \uplus\left\{l_{f_{1}} \mapsto\left(q_{f_{1}}, v_{f_{2}}\right)\right\}\right) . \mathrm{R} \preceq\left(w_{f_{21}} \uplus\left\{l_{f_{1}} \mapsto\left(q_{f_{1}}, v_{f_{11}}\right)\right\}\right)^{\text {qual }}(l) \Rightarrow l \in \mathcal{S}_{2}$

which follows from $\operatorname{dom}\left(w_{f_{21}} \uplus\left\{l_{f_{1}} \mapsto\left(q_{f_{1}}, v_{f_{11}}\right)\right\}\right)=\operatorname{dom}\left(w_{f_{21}}\right) \uplus\left\{l_{f_{1}}\right\}$

$\equiv \forall l \in \operatorname{dom}\left(w_{f_{21}}\right) \uplus\left\{l_{f_{1}}\right\} . \mathbf{R} \preceq\left(w_{f_{21}} \uplus\left\{l_{f_{1}} \mapsto\left(q_{f_{1}}, v_{f_{2}}\right)\right\}\right)^{\text {qual }}(l) \Rightarrow l \in \mathcal{S}_{2}$

which follows from

$\forall l \in \operatorname{dom}\left(w_{f_{21}}\right) \uplus\left\{l_{f_{1}}\right\} .\left(w_{f_{21}} \uplus\left\{l_{f_{1}} \mapsto\left(q_{f_{1}}, v_{f_{11}}\right)\right\}\right)^{\text {qual }}(l)=\left(w_{f_{21}} \uplus\left\{l_{f_{1}} \mapsto\left(q_{f_{1}}, v_{f_{2}}\right)\right\}\right)^{\text {qual }}(l)$

$\equiv \forall l \in \operatorname{dom}\left(w_{f_{21}} \uplus\left\{l_{f_{1}} \mapsto\left(q_{f_{1}}, v_{f_{2}}\right)\right\}\right) . \mathrm{R} \preceq\left(w_{f_{21}} \uplus\left\{l_{f_{1}} \mapsto\left(q_{f_{1}}, v_{f_{2}}\right)\right\}\right)^{\text {qual }}(l) \Rightarrow l \in \mathcal{S}_{2}$

which follows from $\operatorname{dom}\left(w_{f_{21}}\right) \uplus\left\{l_{f_{1}}\right\}=\operatorname{dom}\left(w_{f_{21}} \uplus\left\{l_{f_{1}} \mapsto\left(q_{f_{1}}, v_{f_{2}}\right)\right\}\right)$. 
- $\left(k-j, q_{f}, W_{f}, e_{f}\right) \in \mathcal{T} \llbracket \Delta \vdash^{\mathrm{L}}\left(\xi_{\text {ref }} \tau_{2} \otimes \tau_{1}\right):$ TYPE $\rrbracket \delta$

$\equiv\left(k-j, \mathrm{~L},\left(W_{f_{1}}^{\prime} \odot_{k-j} \mathcal{F}_{2 W}\left(l_{f_{1}}\right)\right),\left\langle l_{f_{1}}, v_{f_{11}}\right\rangle\right) \in \mathcal{T} \llbracket \Delta \vdash^{\mathrm{L}}\left(\xi_{\text {ref }} \tau_{2} \otimes \tau_{1}\right):$ TYPE $\rrbracket \delta$

$\equiv\left(k-j, \mathrm{~L},\left(W_{f_{1}}^{\prime} \odot_{k-j} \mathcal{F}_{2 W}\left(l_{f_{1}}\right)\right),\left\langle l_{f_{1}}, v_{f_{11}}\right\rangle\right)$

$\in\left\{\left(k, q, W,\left\langle v_{1}, v_{2}\right\rangle\right) \mid\right.$

$q=\mathcal{T} \llbracket \Delta \vdash \mathrm{L}: \mathrm{QUAL} \rrbracket \delta \wedge$

$\left(k, q_{1}, W_{1}, v_{1}\right) \in \mathcal{T} \llbracket \Delta \vdash \xi_{\text {ref }} \tau_{2}:$ TYPE $\rrbracket \delta \wedge$

$\left(k, q_{2}, W_{2}, v_{2}\right) \in \mathcal{T} \llbracket \Delta \vdash \tau_{1}: \mathrm{TYPE} \rrbracket \delta \wedge$

$q_{1} \preceq q \wedge q_{1} \preceq q \wedge$

$\left.\left(W_{1} \odot_{k} W_{2}=W\right)\right\}$,

which follows from

- $\mathrm{L}=\mathcal{T} \llbracket \Delta \vdash \mathrm{L}: \mathrm{QUAL} \rrbracket \delta$, which follows trivially,

- $\left(k-j, q_{f_{1}}, W_{f_{1}}^{\prime}, l_{f_{1}}\right) \in \mathcal{T} \llbracket \Delta \vdash \xi_{\text {ref }} \tau_{2}:$ TYPE $\rrbracket \delta$

$\equiv\left(k-j, q_{f_{1}},\left\{l_{f_{1}} \mapsto\left(q_{f_{1}}, \chi_{f_{1}}^{\prime}\right)\right\}, l_{f_{1}}\right)$

$\in\{(k, q,\{l \mapsto(q, \chi)\}, l) \mid$

$q=\mathcal{T} \llbracket \Delta \vdash \xi: \mathrm{QUAL} \rrbracket \delta \wedge$

$\chi=\left\lfloor\mathcal{T} \llbracket \Delta \vdash \tau_{2}: \text { TYPE } \rrbracket \delta\right\rfloor_{k} \wedge$

$\left.\left(q \preceq \mathrm{A} \Rightarrow \forall\left({ }_{-}, q^{\prime},{ }_{-},-\right) \in \chi \cdot q^{\prime} \preceq \mathrm{A}\right)\right\}$,

which follows from

- $q_{f_{1}}=\mathcal{T} \llbracket \Delta \vdash \xi: \mathrm{QUAL} \rrbracket \delta$, which follows from above,

- $\chi_{f_{1}}^{\prime}=\left\lfloor\mathcal{T} \llbracket \Delta \vdash \tau_{2}: \mathrm{TYPE} \rrbracket \delta\right\rfloor_{k-j}$, which follows from above,

- $\left(q_{f_{1}} \preceq \mathrm{A} \Rightarrow \forall\left({ }_{-}, q^{\prime},_{-,},\right) \in \chi_{f_{1}}^{\prime} \cdot q^{\prime} \preceq \mathrm{A}\right)$

$\equiv\left(q_{f_{1}} \preceq \mathrm{A} \Rightarrow \forall\left({ }_{-}, q^{\prime},{ }_{-},{ }_{-}\right) \in\left\lfloor\mathcal{T} \llbracket \Delta \vdash \tau_{2}: \mathrm{TYPE} \rrbracket \delta\right\rfloor_{k-j} . q^{\prime} \preceq \mathrm{A}\right)$

Suppose $q_{f_{1}} \preceq \mathrm{A}$.

Consider arbitrary $\left(,, q^{\prime},{ }_{-},{ }_{-}\right) \in\left\lfloor\mathcal{T} \llbracket \Delta \vdash \tau_{2}: \text { TYPE } \rrbracket \delta\right\rfloor_{k-j}$.

Note that $\left({ }_{-}, q^{\prime},{ }_{-},{ }_{-}\right) \in \mathcal{T} \llbracket \Delta \vdash \tau_{2}:$ TYPE $\rrbracket \delta$, which follows from the definition of $\lfloor\cdot\rfloor_{k}$.

Note that $q^{\prime} \preceq q_{f_{1}}$, which follows from Lemma 15 applied to $\Delta \vdash \tau_{2} \preceq \xi$ and $\left({ }_{-}, q^{\prime},{ }_{-},{ }_{-}\right) \in \mathcal{T} \llbracket \Delta \vdash \tau_{2}:$ TYPE $\rrbracket \delta$ and $q_{f_{1}}=\mathcal{T} \llbracket \Delta \vdash \xi: \mathrm{QUAL} \rrbracket \delta$.

Note that $q^{\prime} \preceq \mathrm{A}$, which follows from $q^{\prime} \preceq q_{f_{1}}$ and $q_{f_{1}} \preceq \mathrm{A}$.

- $\left(k-j, \mathcal{F}_{2 q}\left(l_{f_{1}}\right),\left\lfloor\mathcal{F}_{2 W}\left(l_{f_{1}}\right)\right\rfloor_{k-j}, v_{f_{11}}\right) \in \mathcal{T} \llbracket \Delta \vdash \tau_{1}:$ TYPE $\rrbracket \delta$, which follows from above,

- $q_{f_{1}} \preceq \mathrm{L}$, which follows trivially,

- $\mathcal{F}_{2 q}\left(l_{f_{1}}\right) \preceq \mathrm{L}$, which follows trivially,

- $\left(W_{f_{1}}^{\prime} \odot_{k-j} \mathcal{F}_{2 W}\left(l_{f_{1}}\right)\right)=\left(W_{f_{1}}^{\prime} \odot_{k-j}\left\lfloor\mathcal{F}_{2 W}\left(l_{f_{1}}\right)\right\rfloor_{k-j}\right)$, which follows from Req 5 (join-aprx). 
$(\operatorname{SWAP}(\mathrm{WEAK}))$

Case $\frac{\Delta \vdash \Gamma \sim \Gamma_{1} \boxplus \Gamma_{2} \quad \Delta ; \Gamma_{1} \vdash e_{1}:{ }^{\xi} \text { ref } \tau \quad \Delta ; \Gamma_{2} \vdash e_{2}: \tau}{\Delta ; \Gamma \vdash \operatorname{sw} e_{1} e_{2}:{ }^{\mathrm{L}}\left({ }^{\xi} \text { ref } \tau \otimes \tau\right)}$ :

We are required to show $\llbracket \Delta ; \Gamma \vdash \mathrm{sw} e_{1} e_{2}:{ }^{\mathrm{L}}\left(\xi_{\text {ref }} \tau \otimes \tau\right) \rrbracket$.

Consider arbitrary $k, \delta, q_{\Gamma}, W_{\Gamma}$, and $\gamma$ such that

- $k \geq 0$,

- $\delta \in \mathcal{D} \llbracket \Delta \rrbracket$, and

- $\left(k, q_{\Gamma}, W_{\Gamma}, \gamma\right) \in \mathcal{G} \llbracket \Delta \vdash \Gamma \rrbracket \delta$.

Applying Lemma 20 to $\left(k, q_{\Gamma}, W_{\Gamma}, \gamma\right) \in \mathcal{G} \llbracket \Delta \vdash \Gamma \rrbracket \delta$ and $\Delta \vdash \Gamma \leadsto \Gamma_{1} \boxplus \Gamma_{2}$, we conclude that there exist $q_{\Gamma_{1}}, W_{\Gamma_{1}}, \gamma_{1}, q_{\Gamma_{2}}, W_{\Gamma_{2}}$ and $\gamma_{2}$, such that

- $\left(k, q_{\Gamma_{1}}, W_{\Gamma_{1}}, \gamma_{1}\right) \in \mathcal{G} \llbracket \Delta \vdash \Gamma_{1} \rrbracket \delta$,

- $\left(k, q_{\Gamma_{2}}, W_{\Gamma_{2}}, \gamma_{2}\right) \in \mathcal{G} \llbracket \Delta \vdash \Gamma_{2} \rrbracket \delta$,

- $\gamma \in \gamma_{1} \boxplus \gamma_{2}$,

- $q_{\Gamma_{1}} \preceq q_{\Gamma}$,

- $q_{\Gamma_{2}} \preceq q_{\Gamma}$, and

- $\left(W_{\Gamma_{1}} \odot_{k} W_{\Gamma_{2}}=W_{\Gamma}\right)$.

Note that $\gamma\left(e_{1}\right) \equiv \gamma_{1}\left(e_{1}\right)$ and $\gamma\left(e_{2}\right) \equiv \gamma_{2}\left(e_{2}\right)$.

Let $e_{s}=\gamma\left(\mathrm{sw} e_{1} e_{2}\right) \equiv \operatorname{sw} \gamma\left(e_{1}\right) \gamma\left(e_{2}\right) \equiv \operatorname{sw} \gamma_{1}\left(e_{1}\right) \gamma_{2}\left(e_{2}\right)$ and $W_{s}=W_{\Gamma}$.

We are required to show that $\operatorname{Comp}\left(k, W_{s}, e_{s}, \mathcal{T} \llbracket \Delta ; \Gamma \vdash \operatorname{sw} e_{1} e_{2}:{ }^{\mathrm{L}}\left({ }^{\xi}\right.\right.$ ref $\left.\left.\tau \otimes \tau\right) \rrbracket \delta\right) \equiv$ $\operatorname{Comp}\left(k, W_{\Gamma}, \mathrm{sw} \gamma_{1}\left(e_{1}\right) \gamma_{2}\left(e_{2}\right), \mathcal{T} \llbracket \Delta ; \Gamma \vdash \mathrm{sw} e_{1} e_{2}:{ }^{\mathrm{L}}\left(\xi_{\text {ref }} \tau \otimes \tau\right) \rrbracket \delta\right)$.

Consider arbitrary $j, W_{r}, w_{s}, w_{f}$, and $e_{f}$ such that

- $j<k$,

- $w_{s}:_{k}\left(W_{s} \odot_{k} W_{r}\right) \equiv w_{s}:_{k}\left(W_{\Gamma} \odot_{k} W_{r}\right)$, noting that

$$
\begin{gathered}
w_{s}:_{k}\left(W_{\Gamma} \odot_{k} W_{r}\right) \\
\equiv w_{s}:_{k}\left(\left(W_{\Gamma_{1}} \odot_{k} W_{\Gamma_{2}}\right) \bigodot_{k} W_{r}\right) \\
\text { which follows from above, }
\end{gathered}
$$

- $\left(w_{s}, e_{s}\right) \equiv\left(w_{s}\right.$, sw $\left.\gamma_{1}\left(e_{1}\right) \gamma_{2}\left(e_{2}\right)\right) \longmapsto j\left(w_{f}, e_{f}\right)$, and

- $\operatorname{irred}\left(w_{f}, e_{f}\right)$.

Hence, by inspection of the operational semantics, it follows that there exist $j_{1}, w_{f_{1}}$, and $e_{f_{1}}$ such that

- $\left(w_{s}, \gamma_{1}\left(e_{1}\right)\right) \longmapsto^{j_{1}}\left(w_{f_{1}}, e_{f_{1}}\right)$,

- $\operatorname{irred}\left(w_{f_{1}}, e_{f_{1}}\right)$, and

- $j_{1} \leq j$.

Note that $\left(\left(W_{\Gamma_{1}} \odot_{k} W_{\Gamma_{2}}\right) \odot_{k} W_{r}\right) \equiv\left(W_{\Gamma_{1}} \odot_{k}\left(W_{\Gamma_{2}} \odot_{k} W_{r}\right)\right)$, which follows from

$$
\begin{aligned}
& \left(\left(W_{\Gamma_{1}} \odot_{k} W_{\Gamma_{2}}\right) \odot_{k} W_{r}\right) \\
& \equiv\left(W_{\Gamma_{1}} \odot_{k}\left(W_{\Gamma_{2}} \odot_{k} W_{r}\right)\right)
\end{aligned}
$$

which follows from Reqs 6,7 , and 8 (join-commut, join-assocl, and join-assocr).

Applying the induction hypothesis to $\Delta ; \Gamma_{1} \vdash e_{1}:{ }^{\xi}$ ref $\tau$, we conclude that $\llbracket \Delta ; \Gamma_{1} \vdash e_{1}:{ }^{\xi}$ ref $\tau \rrbracket$. Instantiate this with $k, \delta, q_{\Gamma_{1}}, W_{\Gamma_{1}}$, and $\gamma_{1}$. Note that

- $k \geq 0$,

- $\delta \in \mathcal{D} \llbracket \Delta \rrbracket$, and 
- $\left(k, q_{\Gamma_{1}}, W_{\Gamma_{1}}, \gamma_{1}\right) \in \mathcal{G} \llbracket \Delta \vdash \Gamma_{1} \rrbracket \delta$.

Hence, $\operatorname{Comp}\left(k, W_{\Gamma_{1}}, \gamma_{1}\left(e_{1}\right), \mathcal{T} \llbracket \Delta \vdash \xi_{\text {ref }} \tau:\right.$ TYPE $\left.\rrbracket \delta\right)$.

Instantiate this with $j_{1},\left(W_{\Gamma_{2}} \odot_{k} W_{r}\right), w_{s}, w_{f_{1}}$, and $e_{f_{1}}$. Note that

- $j_{1}<k$, which follows from $j_{1} \leq j$ and $j<k$,

- $w_{s}:_{k}\left(W_{\Gamma_{1}} \odot_{k}\left(W_{\Gamma_{2}} \odot_{k} W_{r}\right)\right)$, which follows from

$w_{s}: k\left(\left(W_{\Gamma_{1}} \odot_{k} W_{\Gamma_{2}}\right) \odot_{k} W_{r}\right)$

which follows from above

$\left(\left(W_{\Gamma_{1}} \odot_{k} W_{\Gamma_{2}}\right) \odot_{k} W_{r}\right) \equiv\left(W_{\Gamma_{1}} \odot_{k}\left(W_{\Gamma_{2}} \odot_{k} W_{r}\right)\right)$

which follows from above,

- $\left(w_{s}, \gamma_{1}\left(e_{1}\right)\right) \longmapsto^{j_{1}}\left(w_{f_{1}}, e_{f_{1}}\right)$,

- $\operatorname{irred}\left(w_{f_{1}}, e_{f_{1}}\right)$.

Hence, there exists $W_{f_{1}}$ and $q_{f_{1}}$ such that

- $w_{f_{1}}: k_{k-j_{1}}\left(W_{f_{1}} \odot_{k-j_{1}}\left(W_{\Gamma_{2}} \odot_{k} W_{r}\right)\right)$, and

- $\left(k-j_{1}, q_{f_{1}}, W_{f_{1}}, e_{f_{1}}\right)$

$\in \mathcal{T} \llbracket \Delta \vdash \xi_{\text {ref }} \tau:$ TYPE $\| \delta$

$\equiv\{(k, q,\{l \mapsto(q, \chi)\}, l) \mid$

$q=\mathcal{T} \llbracket \Delta \vdash \xi: \mathrm{QUAL} \rrbracket \delta \wedge$

$\chi=\lfloor\mathcal{T} \llbracket \Delta \vdash \tau: \text { TYPE } \rrbracket \delta\rfloor_{k} \wedge$

$\left.\left(q \preceq \mathrm{A} \Rightarrow \forall\left({ }_{-}, q^{\prime},{ }_{-},-\right) \in \chi \cdot q^{\prime} \preceq \mathrm{A}\right)\right\}$.

Hence, $e_{f_{1}} \equiv l_{f_{1}}$ and $q_{f_{1}}=\mathcal{T} \llbracket \Delta \vdash \xi: \mathrm{QUAL} \rrbracket \delta$ and $W_{f_{1}} \equiv\left\{l_{f_{1}} \mapsto\left(q_{f_{1}}, \chi_{f_{1}}\right)\right\}$ and $\chi_{f_{1}}=$ $\lfloor\mathcal{T} \llbracket \Delta \vdash \tau: \mathrm{TYPE} \rrbracket \delta\rfloor_{k-j_{1}}$ and $\left(q_{f_{1}} \preceq \mathrm{A} \Rightarrow \forall\left({ }_{-}, q^{\prime},{ }_{-},{ }_{-}\right) \in \chi_{f_{1}} \cdot q^{\prime} \preceq \mathrm{A}\right)$.

Note that

$$
\begin{aligned}
\left(w_{s}, e_{s}\right) & \equiv\left(w_{s}, \operatorname{sw} \gamma_{1}\left(e_{1}\right) \gamma_{2}\left(e_{2}\right)\right) \\
& \longmapsto^{j_{1}}\left(w_{f_{1}}, \operatorname{sw} e_{f_{1}} \gamma_{2}\left(e_{2}\right)\right) \\
& \equiv\left(w_{f_{1}}, \mathbf{s w} l_{f_{1}} \gamma_{2}\left(e_{2}\right)\right) \\
& \longmapsto^{j-j_{1}}\left(w_{f}, e_{f}\right)
\end{aligned}
$$

and $\operatorname{irred}\left(w_{f}, e_{f}\right)$.

Hence, by inspection of the operational semantics, it follows that there exist $j_{2}, w_{f_{2}}$, and $e_{f_{2}}$ such that

- $\left(w_{f_{1}}, \gamma_{2}\left(e_{2}\right)\right) \longmapsto j^{j}\left(w_{f_{2}}, e_{f_{2}}\right)$,

- $\operatorname{irred}\left(w_{f_{2}}, e_{f_{2}}\right)$, and

- $j_{2} \leq j-j_{1}$.

Note that $\left(W_{f_{1}} \odot_{k-j_{1}}\left(W_{\Gamma_{2}} \odot_{k} W_{r}\right)\right) \equiv\left(\left\lfloor W_{\Gamma_{2}}\right\rfloor_{k-j_{1}} \odot_{k-j_{1}}\left(W_{f_{1}} \odot_{k-j_{1}} W_{r}\right)\right)$, which follows from $\left(W_{f_{1}} \odot_{k-j_{1}}\left(W_{\Gamma_{2}} \odot_{k} W_{r}\right)\right)$ $\equiv\left(\left\lfloor W_{\Gamma_{2}}\right\rfloor_{k-j_{1}} \odot_{k-j_{1}}\left(W_{f_{1}} \odot_{k-j_{1}} W_{r}\right)\right)$

which follows from Reqs 4, 5, 6, 7, and 8 (join-closed, join-aprx, join-commut, join-assocl, and join-assocr).

Applying the induction hypothesis to $\Delta ; \Gamma_{2} \vdash e_{2}: \tau$, we conclude that $\llbracket \Delta ; \Gamma_{2} \vdash e_{2}: \tau \rrbracket$.

Instantiate this with $k-j_{1}, \delta, q_{\Gamma_{2}},\left\lfloor W_{\Gamma_{2}}\right\rfloor_{k-j_{1}}$, and $\gamma_{2}$. Note that

- $k-j_{1} \geq 0$, which follows from $j_{1}<k$,

- $\delta \in \mathcal{D} \llbracket \Delta \rrbracket$, and

- $\left(k-j_{1}, q_{\Gamma_{2}},\left\lfloor W_{\Gamma_{2}}\right\rfloor_{k-j_{1}}, \gamma_{2}\right) \in \mathcal{G} \llbracket \Delta \vdash \Gamma_{2} \rrbracket \delta$, which follows from Lemma 9 applied to $k-j_{1} \leq k$ and $\left(k, q_{\Gamma_{2}}, W_{\Gamma_{2}}, \gamma_{2}\right) \in \mathcal{G} \llbracket \Delta \vdash \Gamma_{2} \rrbracket \delta$. 
Hence, $\operatorname{Comp}\left(k-j_{1}, \gamma_{2}\left(e_{2}\right),\left\lfloor W_{\Gamma_{2}}\right\rfloor_{k-j_{1}}, \mathcal{T} \llbracket \Delta \vdash \tau: \mathrm{TYPE} \rrbracket \delta\right)$.

Instantiate this with $j_{2},\left(W_{f_{1}} \odot_{k-j_{1}} W_{r}\right), w_{f_{1}}, w_{f_{2}}$, and $e_{f_{2}}$. Note that

- $j_{2}<k-j_{1}$, which follows from $j_{2} \leq j-j_{1}$ and $j<k$,

- $w_{f_{1}}: k-j_{1}\left(\left\lfloor W_{\Gamma_{2}}\right\rfloor_{k-j_{1}} \odot_{k-j_{1}}\left(W_{f_{1}} \odot_{k-j_{1}} W_{r}\right)\right)$, which follows from

$w_{f_{1}}: k-j_{1}\left(W_{f_{1}} \odot_{k-j_{1}}\left(W_{\Gamma_{2}} \odot_{k} W_{r}\right)\right)$

which follows from above

$\left(W_{f_{1}} \odot_{k-j_{1}}\left(W_{\Gamma_{2}} \odot_{k} W_{r}\right)\right) \equiv\left(\left\lfloor W_{\Gamma_{2}}\right\rfloor_{k-j_{1}} \odot_{k-j_{1}}\left(W_{f_{1}} \odot_{k-j_{1}} W_{r}\right)\right)$

which follows from above,

- $\left(w_{f_{1}}, \gamma_{2}\left(e_{2}\right)\right) \longmapsto^{j_{2}}\left(w_{f_{2}}, e_{f_{2}}\right)$, and

- $\operatorname{irred}\left(w_{f_{2}}, e_{f_{2}}\right)$.

Hence, there exists $W_{f_{2}}$ and $q_{f_{2}}$ such that

- $w_{f_{2}}: k-j_{1}-j_{2}\left(W_{f_{2}} \odot_{k-j_{1}-j_{2}}\left(W_{f_{1}} \odot_{k-j_{1}} W_{r}\right)\right)$, and

- $\left(k-j_{1}-j_{2}, q_{f_{2}}, W_{f_{2}}, e_{f_{2}}\right) \in \mathcal{T} \llbracket \Delta \vdash \tau:$ TYPE $\rrbracket \delta$.

Hence, $e_{f_{2}} \equiv v_{f_{2}}$.

Note that $l_{f_{1}} \notin \operatorname{dom}\left(W_{f_{2}} \odot_{k-j_{1}-j_{2}} W_{r}\right)$, which follows from $\mathrm{A} \preceq q_{f_{1}}=W_{f_{1}}^{\text {qual }}\left(l_{f_{1}}\right)$ and $\left(W_{f_{2}} \odot_{k-j_{1}-j_{2}}\left(W_{f_{1}} \odot_{k-j_{1}} W_{r}\right)\right) \equiv\left(W_{f_{1}} \odot_{k-j_{1}-j_{2}}\left(W_{f_{2}} \odot_{k-j_{1}-j_{2}} W_{r}\right)\right)$ defined.

Hence, $l_{f_{1}} \notin \operatorname{dom}\left(W_{f_{2}}\right) \cup \operatorname{dom}\left(W_{r}\right)$, which follows from $\operatorname{dom}\left(W_{1} \odot_{k} W_{2}\right)=\operatorname{dom}\left(W_{1}\right) \cup \operatorname{dom}\left(W_{2}\right)$. Note that

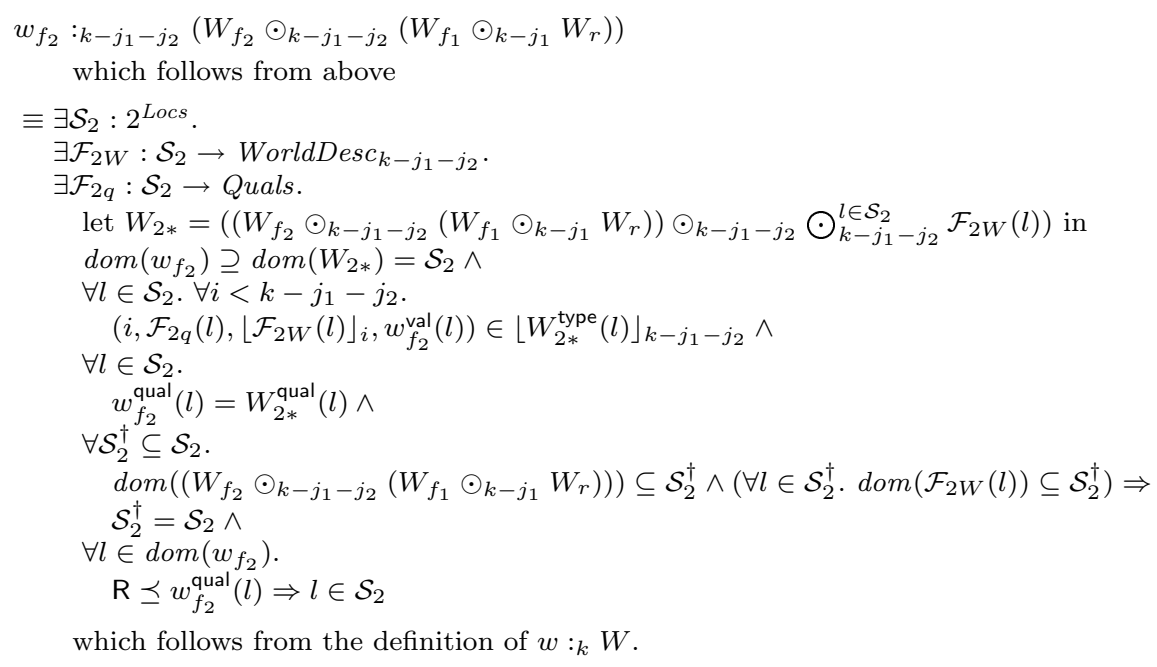

Note that

$$
\begin{aligned}
& \operatorname{dom}\left(w_{f_{2}}\right) \supseteq \operatorname{dom}\left(W_{2 *}\right)=\mathcal{S}_{2} \\
& \quad \text { which follows from above }\left(w_{f_{2}}: k-j_{1}-j_{2}\left(W_{f_{2}} \odot_{k-j_{1}-j_{2}}\left(W_{f_{1}} \odot_{k-j_{1}} W_{r}\right)\right)\right) \\
& \equiv \operatorname{dom}\left(w_{f_{2}}\right) \supseteq \operatorname{dom}\left(W_{2 *}\right)=\operatorname{dom}\left(W_{f_{2}}\right) \cup \operatorname{dom}\left(W_{f_{1}}\right) \cup \operatorname{dom}\left(W_{r}\right) \cup \bigcup^{l \in \mathcal{S}_{2}} \operatorname{dom}\left(\mathcal{F}_{2 W}(l)\right)=\mathcal{S}_{2} \\
& \quad \text { which follows from above }\left(W_{2 *}=\ldots\right) \text { and } \operatorname{dom}\left(W_{1} \odot_{k} W_{2}\right)=\operatorname{dom}\left(W_{1}\right) \cup \operatorname{dom}\left(W_{2}\right) \\
& \equiv \operatorname{dom}\left(w_{f_{2}}\right) \supseteq \operatorname{dom}\left(W_{2 *}\right)=\operatorname{dom}\left(W_{f_{2}}\right) \cup\left\{l_{f_{1}}\right\} \cup \operatorname{dom}\left(W_{r}\right) \cup \bigcup^{l \in \mathcal{S}_{2}} \operatorname{dom}\left(\mathcal{F}_{2 W}(l)\right)=\mathcal{S}_{2} \\
& \quad \text { which follows from simplifications of } \operatorname{dom}\left(W_{f_{1}}\right)=\left\{l_{f_{1}}\right\} .
\end{aligned}
$$

Hence, $l_{f_{1}} \in \operatorname{dom}\left(w_{f_{2}}\right)$ and $l_{f_{1}} \in \mathcal{S}_{2}$.

Note that 


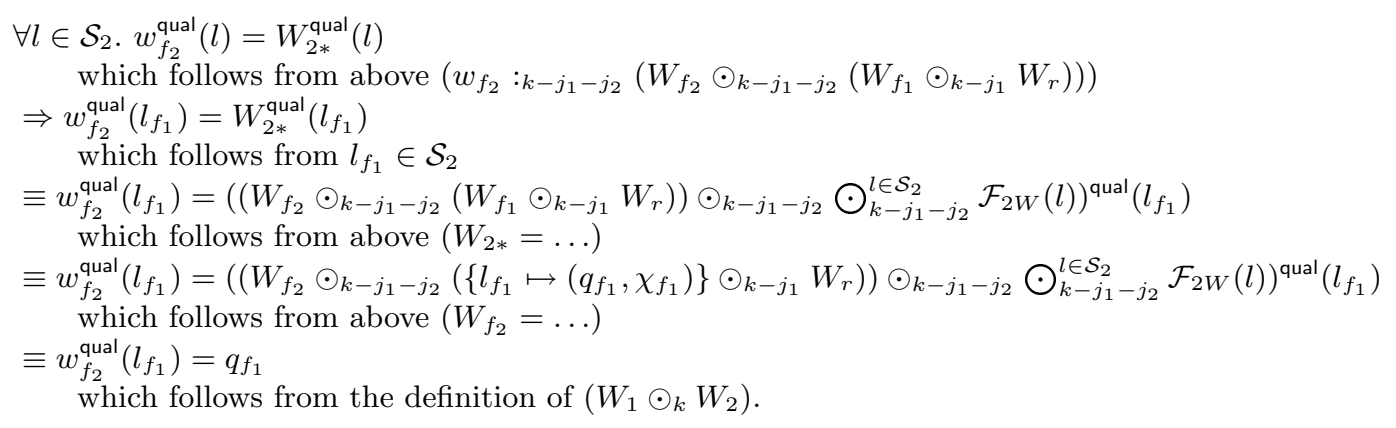

Note that $w_{f_{2}} \equiv w_{f_{21}} \uplus\left\{l_{f_{1}} \mapsto\left(q_{f_{1}}, v_{f_{11}}\right)\right\}$, which follows from $l_{f_{1}} \in \operatorname{dom}\left(w_{f_{2}}\right)$ and $w_{f_{2}}^{\text {qual }}\left(l_{f_{1}}\right)=q_{f_{1}}$. Note that

$$
\begin{aligned}
\left(w_{s}, e_{s}\right) & \equiv\left(w_{s}, \operatorname{sw} \gamma_{1}\left(e_{1}\right) \gamma_{2}\left(e_{2}\right)\right) \\
& \longmapsto{ }^{j_{1}}\left(w_{f_{1}}, \text { sw } e_{f_{1}} \gamma_{2}\left(e_{2}\right)\right) \\
& \equiv\left(w_{f_{1}}, \text { sw } l_{f_{1}} \gamma_{2}\left(e_{2}\right)\right) \\
& \longmapsto{ }^{j_{2}}\left(w_{f_{2}}, \text { sw } l_{f_{1}} e_{f_{2}}\right) \\
& \equiv\left(w_{f_{2}}, \operatorname{sw} l_{f_{1}} v_{f_{2}}\right) \\
& \equiv\left(w_{f_{21}} \uplus\left\{l_{f_{1}} \mapsto\left(q_{f_{1}}, v_{f_{11}}\right)\right\}, \text { sw } l_{f_{1}} v_{f_{2}}\right) \\
& \longmapsto{ }^{1}\left(w_{f_{21}} \uplus\left\{l_{f_{1}} \mapsto\left(q_{f_{1}}, v_{f_{2}}\right)\right\},\left\langle l_{f_{1}}, v_{f_{11}}\right\rangle\right) \\
& \longmapsto{ }^{j-j_{1}-j_{2}-1}\left(w_{f}, e_{f}\right) .
\end{aligned}
$$

Since $\left\langle l_{f_{1}}, v_{f_{11}}\right\rangle$ is value, we have $\operatorname{irred}\left(w_{f_{21}} \uplus\left\{l_{f_{1}} \mapsto\left(q_{f_{1}}, v_{f_{2}}\right)\right\},\left\langle l_{f_{1}}, v_{f_{11}}\right\rangle\right)$.

Hence, $j-j_{1}-j_{2}-1=0$ (and $\left.j=j_{1}+j_{2}+1\right)$ and $w_{f} \equiv w_{f_{21}} \uplus\left\{l_{f_{1}} \mapsto\left(q_{f_{1}}, v_{f_{2}}\right)\right\}$ and $e_{f} \equiv\left\langle l_{f_{1}}, v_{f_{11}}\right\rangle$.

Note that

$$
\begin{aligned}
& \forall l \in \mathcal{S}_{2} . \forall i<k-j_{1}-j_{2} .\left(i, \mathcal{F}_{2 q}(l),\left\lfloor\mathcal{F}_{2 W}(l)\right\rfloor_{i}, w_{f_{2}}^{\text {val }}(l)\right) \in\left\lfloor W_{2 *}^{\text {type }}(l)\right\rfloor_{k-j_{1}-j_{2}} \\
& \text { which follows from above }\left(w_{f_{2}}: k-j_{1}-j_{2}\left(W_{f_{2}} \odot_{k-j_{1}-j_{2}}\left(W_{f_{1}} \odot_{k-j_{1}} W_{r}\right)\right)\right) \\
& \Rightarrow \forall i<k-j_{1}-j_{2} .\left(i, \mathcal{F}_{2 q}\left(l_{f_{1}}\right),\left\lfloor\mathcal{F}_{2 W}\left(l_{f_{1}}\right)\right\rfloor_{i}, w_{f_{2}}^{\mathrm{val}}\left(l_{f_{1}}\right)\right) \in\left\lfloor W_{2 *}^{\mathrm{type}}\left(l_{f_{1}}\right)\right\rfloor_{k-j_{1}-j_{2}} \\
& \text { which follows from } l_{f_{1}} \in \mathcal{S}_{2} \\
& \equiv \forall i<k-j_{1}-j_{2} .\left(i, \mathcal{F}_{2 q}\left(l_{f_{1}}\right),\left\lfloor\mathcal{F}_{2 W}\left(l_{f_{1}}\right)\right\rfloor_{i},\left(w_{f_{21}} \uplus\left\{l_{f_{1}} \mapsto\left(q_{f_{1}}, v_{f_{11}}\right)\right\}\right)^{\mathrm{val}}\left(l_{f_{1}}\right)\right) \in\left\lfloor W_{2 *}^{\mathrm{type}}\left(l_{f_{1}}\right)\right\rfloor_{k-j_{1}-j_{2}} \\
& \text { which follows from above }\left(w_{f_{2}}=\ldots\right) \\
& \equiv \forall i<k-j_{1}-j_{2} \cdot\left(i, \mathcal{F}_{2 q}\left(l_{f_{1}}\right),\left\lfloor\mathcal{F}_{2 W}\left(l_{f_{1}}\right)\right\rfloor_{i}, v_{f_{11}}\right) \in\left\lfloor W_{2 *}^{\text {type }}\left(l_{f_{1}}\right)\right\rfloor_{k-j_{1}-j_{2}} \\
& \text { which follows from }\left(w_{f_{21}} \uplus\left\{l_{f_{1}} \mapsto\left(q_{f_{1}}, v_{f_{11}}\right)\right\}\right)^{\mathrm{val}}\left(l_{f_{1}}\right)=v_{f_{11}} \\
& \Rightarrow\left(k-j, \mathcal{F}_{2 q}\left(l_{f_{1}}\right),\left\lfloor\mathcal{F}_{2 W}\left(l_{f_{1}}\right)\right\rfloor_{k-j}, v_{f_{11}}\right) \in\left\lfloor W_{2 *}^{\text {type }}\left(l_{f_{1}}\right)\right\rfloor_{k-j_{1}-j_{2}} \\
& \text { which follows from } k-j<k-j_{1}-j_{2} \\
& \equiv\left(k-j, \mathcal{F}_{2 q}\left(l_{f_{1}}\right),\left\lfloor\mathcal{F}_{2 W}\left(l_{f_{1}}\right)\right\rfloor_{k-j}, v_{f_{11}}\right) \in\left\lfloor\left\lfloor\chi_{f_{1}}\right\rfloor_{k-j_{1}-j_{2}}\right\rfloor_{k-j_{1}-j_{2}} \\
& \text { which follows from simplifications of } W_{2 *}^{\text {type }}\left(l_{f_{1}}\right) \equiv\left\lfloor W_{f_{1}}\right\rfloor_{k-j_{1}-j_{2}}^{\text {type }}\left(l_{f_{1}}\right) \equiv\left\lfloor\chi_{f_{1}}\right\rfloor_{k-j_{1}-j_{2}} \\
& \equiv\left(k-j, \mathcal{F}_{2 q}\left(l_{f_{1}}\right),\left\lfloor\mathcal{F}_{2 W}\left(l_{f_{1}}\right)\right\rfloor_{k-j}, v_{f_{11}}\right) \in\left\lfloor\left\lfloor\left\lfloor\mathcal{T} \llbracket \Delta \vdash \tau_{1}: \text { TYPE } \rrbracket \delta\right\rfloor_{k-j_{1}}\right\rfloor_{k-j_{1}-j_{2}}\right\rfloor_{k-j_{1}-j_{2}} \\
& \text { which follows from simplifications of } \chi_{f_{1}}=\lfloor\mathcal{T} \llbracket \Delta \vdash \tau: \text { TYPE } \rrbracket \delta\rfloor_{k-j_{1}} \\
& \Rightarrow\left(k-j, \mathcal{F}_{2 q}\left(l_{f_{1}}\right),\left\lfloor\mathcal{F}_{2 W}\left(l_{f_{1}}\right)\right\rfloor_{k-j}, v_{f_{11}}\right) \in\lfloor\mathcal{T} \llbracket \Delta \vdash \tau: \text { TYPE } \rrbracket \delta\rfloor_{k-j_{1}-j_{2}} \\
& \text { which follows from Fact } 2 \\
& \Rightarrow\left(k-j, \mathcal{F}_{2 q}\left(l_{f_{1}}\right),\left\lfloor\mathcal{F}_{2 W}\left(l_{f_{1}}\right)\right\rfloor_{k-j}, v_{f_{11}}\right) \in \mathcal{T} \llbracket \Delta \vdash \tau: \text { TYPE } \rrbracket \delta \\
& \text { which follows from the definition of }\lfloor\cdot\rfloor_{k} \text {. }
\end{aligned}
$$

Note that

$$
\begin{aligned}
& w_{f_{2}}:_{k-j_{1}-j_{2}}\left(W_{f_{2}} \odot_{k-j_{1}-j_{2}}\left(W_{f_{1}} \odot_{k-j_{1}} W_{r}\right)\right) \\
& \text { which follows from above }
\end{aligned}
$$




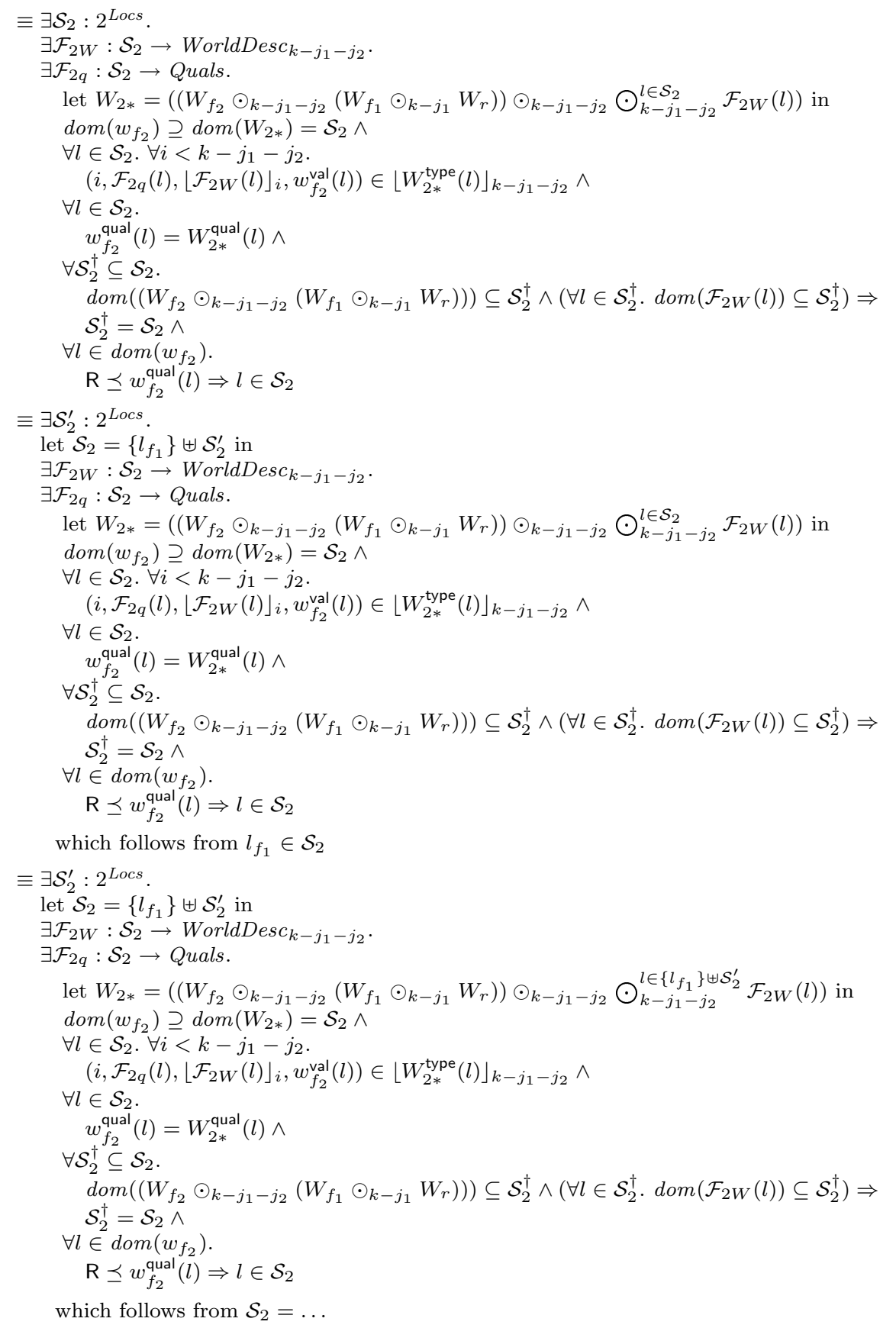




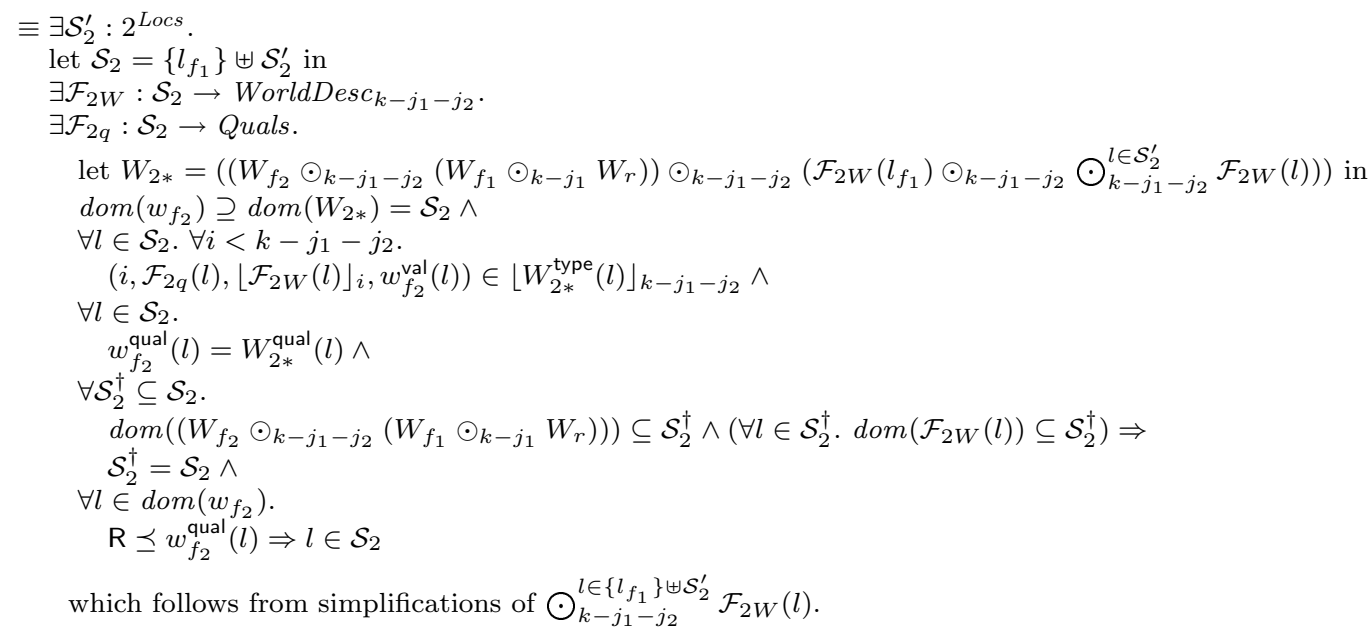

Note that $\left(W_{f_{1}} \odot_{k-j} \mathcal{F}_{2 W}\left(l_{f_{1}}\right)\right)$ is defined, which follows from Reqs $4,5,6,7$, and 8 (join-closed, join-aprx, join-commut, join-assocl, and join-assocr)and $W_{2 *}$ defined.

Let $W_{f}=\left(W_{f_{1}} \odot_{k-j} \mathcal{F}_{2 W}\left(l_{f_{1}}\right)\right)$.

Let $q_{f}=\mathrm{L}$.

Note that $\left(\left(W_{f_{1}} \odot_{k-j} \mathcal{F}_{2 W}\left(l_{f_{1}}\right)\right) \odot_{k-j} W_{r}\right)$ is defined, which follows from Reqs 4, 5, 6, 7, and 8 (join-closed, join-aprx, join-commut, join-assocl, and join-assocr)and $W_{2 *}$ defined.

We are required to show that

- $w_{f}:_{k-j}\left(W_{f} \odot_{k-j} W_{r}\right)$ $\equiv w_{f_{21}} \uplus\left\{l_{f_{1}} \mapsto\left(q_{f_{1}}, v_{f_{2}}\right)\right\}: k-j\left(\left(W_{f_{1}} \odot_{k-j} \mathcal{F}_{2 W}\left(l_{f_{1}}\right)\right) \odot_{k-j} W_{r}\right)$, which is equivalent to

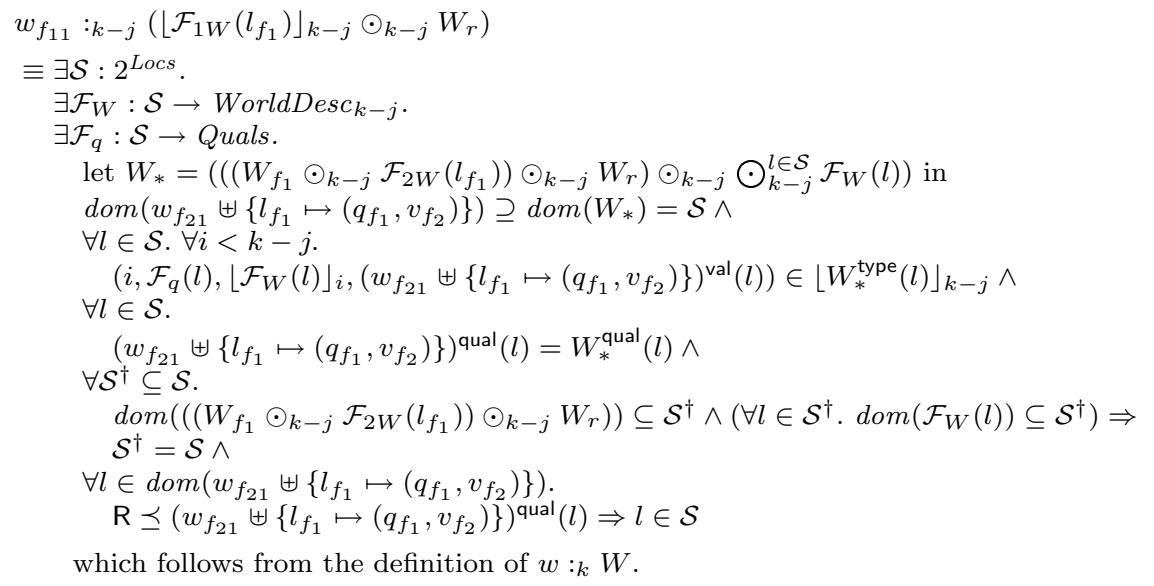

Take

$$
\mathcal{S}=\mathcal{S}_{2}=\left\{l_{f_{1}}\right\} \uplus \mathcal{S}_{2}^{\prime} .
$$


It remains to show that

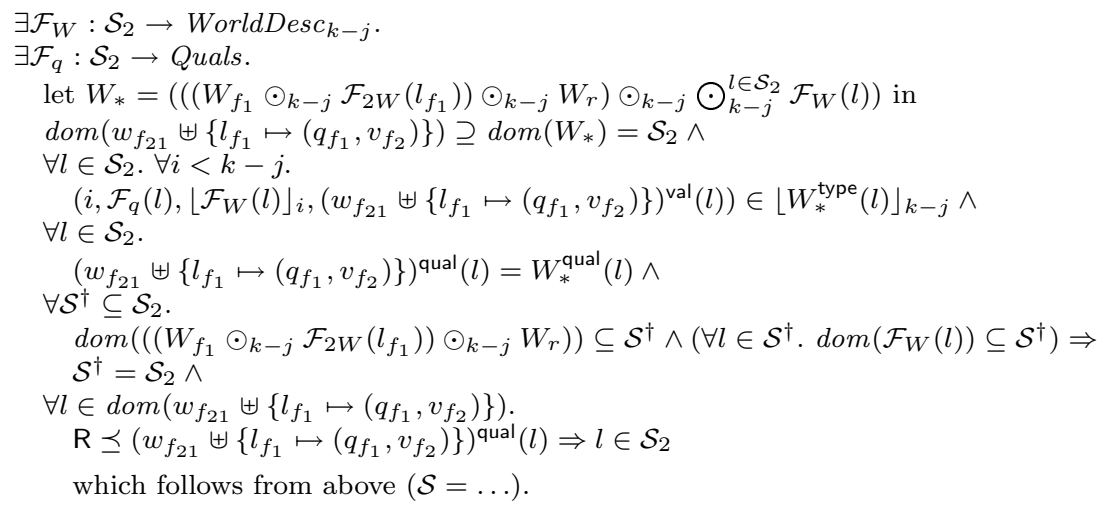

Take

$$
\mathcal{F}_{W}(l)= \begin{cases}\left\lfloor W_{f_{2}}\right\rfloor_{k-j} & \text { if } l \in\left\{l_{f_{1}}\right. \\ \left\lfloor\mathcal{F}_{2 W}(l)\right\rfloor_{k-j} & \text { if } l \in \mathcal{S}_{2}^{\prime}\end{cases}
$$

and

$$
\mathcal{F}_{q}(l)= \begin{cases}q_{f_{2}} & \text { if } l \in\left\{l_{f_{1}}\right\} \\ \mathcal{F}_{2 q}(l) & \text { if } l \in \mathcal{S}_{2}^{\prime}\end{cases}
$$

Note that

$W_{f_{2}} \in$ WorldDesc $_{k-j_{1}-j_{2}}$

which follows from Fact 6 applied to $\left(k-j_{1}-j_{2}, q_{f_{2}}, W_{f_{2}}, v_{f_{2}}\right) \in \mathcal{T} \llbracket \Delta \vdash \tau_{2}:$ TYPE $\rrbracket \delta \in$ Type,

which in turn follows from Lemma 8 applied to $\mathcal{T} \llbracket \Delta \vdash \tau_{2}:$ TYPE $\rrbracket$

$\Rightarrow\left\lfloor W_{f_{2}}\right\rfloor_{k-j} \in$ WorldDesc $_{k-j}$

which follows from $\lfloor\cdot\rfloor_{k} \in$ WorldDesc $\rightarrow$ WorldDesc $_{k}$

$\equiv \mathcal{F}_{W}\left(l_{f_{1}}\right) \in$ WorldDesc $_{k-j}$

which follows from above $\left(\mathcal{F}_{W}(l)=\ldots\right)$.

Note that

$$
\begin{aligned}
\forall l \in \mathcal{S}_{2} . \mathcal{F}_{2 W}(l) \in \text { WorldDesc }_{k-j_{1}-j_{2}} \\
\quad \text { which follows from above }\left(w_{f_{2}}: k-j_{1}-j_{2}\left(W_{f_{2}} \odot_{k-j_{1}-j_{2}}\left(W_{f_{1}} \odot_{k-j_{1}} W_{r}\right)\right)\right) \\
\forall l \in \mathcal{S}_{2}^{\prime} . \mathcal{F}_{2 W}(l) \in \text { WorldDesc }_{k-j_{1}-j_{2}} \\
\quad \text { which follows from } \mathcal{S}_{2} \subseteq \mathcal{S}_{2} \\
\Rightarrow \forall l \in \mathcal{S}_{2}^{\prime} .\left\lfloor\mathcal{F}_{2 W}(l)\right\rfloor_{k-j} \in \text { WorldDesc }_{k-j} \\
\quad \text { which follows from }\lfloor\cdot\rfloor_{k} \in \text { WorldDesc } \rightarrow \text { WorldDesc }_{k} \\
\Rightarrow \forall l \in \mathcal{S}_{2}^{\prime} . \mathcal{F}_{W}(l) \in \text { WorldDesc }_{k-j} \\
\quad \text { which follows from above }\left(\mathcal{F}_{W}(l)=\ldots\right) .
\end{aligned}
$$

Hence, $\mathcal{F}_{W}: \mathcal{S}_{2} \rightarrow$ WorldDesc $_{k-j}$.

Trivially, $\mathcal{F}_{q}: \mathcal{S}_{2} \rightarrow$ Quals.

It remains to show that

$$
\begin{aligned}
& \text { let } W_{*}=\left(\left(\left(W_{f_{1}} \odot_{k-j} \mathcal{F}_{2 W}\left(l_{f_{1}}\right)\right) \odot_{k-j} W_{r}\right) \odot_{k-j} \bigodot_{k-j}^{l \in \mathcal{S}_{2}} \mathcal{F}_{W}(l)\right) \text { in } \\
& \operatorname{dom}\left(w_{f_{21}} \uplus\left\{l_{f_{1}} \mapsto\left(q_{f_{1}}, v_{f_{2}}\right)\right\}\right) \supseteq \operatorname{dom}\left(W_{*}\right)=\mathcal{S}_{2} \wedge \\
& \forall l \in \mathcal{S}_{2} . \forall i<k-j . \\
& \quad\left(i, \mathcal{F}_{q}(l),\left\lfloor\mathcal{F}_{W}(l)\right\rfloor_{i},\left(w_{f_{21}} \uplus\left\{l_{f_{1}} \mapsto\left(q_{f_{1}}, v_{f_{2}}\right)\right\}\right)^{\text {val }}(l)\right) \in\left\lfloor W_{*}^{\text {type }}(l)\right\rfloor_{k-j} \wedge \\
& \forall l \in \mathcal{S}_{2} . \\
& \quad\left(w_{f_{21}} \uplus\left\{l_{f_{1}} \mapsto\left(q_{f_{1}}, v_{f_{2}}\right)\right\}\right)^{\text {qual }}(l)=W_{*}^{\text {qual }}(l) \wedge \\
& \forall \mathcal{S}^{\dagger} \subseteq \mathcal{S}_{2} . \\
& \left.\quad \operatorname{dom}\left(\left(W_{f_{1}} \odot_{k-j} \mathcal{F}_{2 W}\left(l_{f_{1}}\right)\right) \odot_{k-j} W_{r}\right)\right) \subseteq \mathcal{S}^{\dagger} \wedge\left(\forall l \in \mathcal{S}^{\dagger} . \operatorname{dom}\left(\mathcal{F}_{W}(l)\right) \subseteq \mathcal{S}^{\dagger}\right) \Rightarrow \\
& \quad \mathcal{S}^{\dagger}=\mathcal{S}_{2} \wedge \\
& \forall l \in \operatorname{dom}\left(w_{f_{21}} \uplus\left\{l_{f_{1}} \mapsto\left(q_{f_{1}}, v_{f_{2}}\right)\right\}\right) . \\
& \quad \mathrm{R} \preceq\left(w_{f_{21}} \uplus\left\{l_{f_{1}} \mapsto\left(q_{f_{1}}, v_{f_{2}}\right)\right\}\right)^{\text {qual }}(l) \Rightarrow l \in \mathcal{S}_{2} \\
& \quad \text { which follows from above. }
\end{aligned}
$$


Note that

$$
\begin{aligned}
& \left\lfloor W_{2 *}\right\rfloor_{k-j} \\
& \equiv\left\lfloor\left(\left(W_{f_{2}} \odot_{k-j_{1}-j_{2}}\left(W_{f_{1}} \odot_{k-j_{1}} W_{r}\right)\right) \odot_{k-j_{1}-j_{2}}\left(\mathcal{F}_{2 W}\left(l_{f_{1}}\right) \odot_{k-j_{1}-j_{2}} \bigodot_{k-j_{1}-j_{2}}^{l \in \mathcal{S}_{2}^{\prime}} \mathcal{F}_{2 W}(l)\right)\right)\right\rfloor_{k-j} \\
& \text { which follows from above }\left(W_{2 *}=\ldots\right) \\
& \equiv\left(\left(\left\lfloor W_{f_{2}}\right\rfloor_{k-j} \odot_{k-j}\left(W_{f_{1}} \odot_{k-j} W_{r}\right)\right) \odot_{k-j}\left(\mathcal{F}_{2 W}\left(l_{f_{1}}\right) \odot_{k-j} \bigodot_{k-j}^{l \in \mathcal{S}_{2}^{\prime}}\left\lfloor\mathcal{F}_{2 W}(l)\right\rfloor_{k-j}\right)\right) \\
& \text { which follows from Req } 4 \text { (join-closed) and Req } 5 \text { (join-aprx) } \\
& \equiv\left(\left(\left(W_{f_{1}} \odot_{k-j} \mathcal{F}_{2 W}\left(l_{f_{1}}\right)\right) \odot_{k-j} W_{r}\right) \odot_{k-j}\left(\left\lfloor W_{f_{2}}\right\rfloor_{k-j} \odot_{k-j} \bigodot_{k-j}^{l \in \mathcal{S}_{2}^{\prime}}\left\lfloor\mathcal{F}_{2 W}(l)\right\rfloor_{k-j}\right)\right) \\
& \text { which follows from Reqs } 6,7 \text {, and } 8 \text { (join-commut, join-assocl, and join-assocr) } \\
& \equiv\left(\left(\left(W_{f_{1}} \odot_{k-j} \mathcal{F}_{2 W}\left(l_{f_{1}}\right)\right) \odot_{k-j} W_{r}\right) \odot_{k-j}\left(\mathcal{F}_{W}\left(l_{f_{1}}\right) \odot_{k-j} \bigodot_{k-j}^{l \in \mathcal{S}_{2}^{\prime}} \mathcal{F}_{W}(l)\right)\right) \\
& \text { which follows from above }\left(\mathcal{F}_{W}(l)=\ldots\right) \\
& \equiv\left(\left(\left(W_{f_{1}} \odot_{k-j} \mathcal{F}_{2 W}\left(l_{f_{1}}\right)\right) \odot_{k-j} W_{r}\right) \odot_{k-j} \bigodot_{k-j}^{l \in\left\{l_{f_{1}}\right\} \uplus \mathcal{S}_{2}^{\prime}} \mathcal{F}_{W}(l)\right) \\
& \text { which follows from simplifications of } \bigodot_{k-j}^{l \in\left\{l_{f_{1}}\right\} \uplus \mathcal{S}_{2}^{\prime}} \mathcal{F}_{W}(l) \\
& \equiv\left(\left(\left(W_{f_{1}} \odot_{k-j} \mathcal{F}_{2 W}\left(l_{f_{1}}\right)\right) \odot_{k-j} W_{r}\right) \odot_{k-j} \bigodot_{k-j}^{l \in \mathcal{S}_{2}} \mathcal{F}_{W}(l)\right) \\
& \text { which follows from above }\left(\mathcal{S}_{2}=\ldots\right) \text {. }
\end{aligned}
$$

Hence, $W_{*}=\left(\left(\left(W_{f_{1}} \odot_{k-j} \mathcal{F}_{2 W}\left(l_{f_{1}}\right)\right) \odot_{k-j} W_{r}\right) \odot_{k-j} \bigodot_{k-j}^{l \in \mathcal{S}_{2}} \mathcal{F}_{W}(l)\right)$ is defined.

Furthermore, $W_{*}=\left\lfloor W_{2 *}\right\rfloor_{k-j}$ and $\operatorname{dom}\left(W_{*}\right)=\operatorname{dom}\left(W_{2 *}\right)$.

Note that

$$
\begin{aligned}
& \operatorname{dom}\left(w_{f_{2}}\right) \supseteq \operatorname{dom}\left(W_{2 *}\right)=\mathcal{S}_{2} \\
& \quad \text { which follows from above }\left(w_{f_{2}}: k-j_{1}-j_{2}\left(W_{f_{2}} \odot_{k-j_{1}-j_{2}}\left(W_{f_{1}} \odot_{k-j_{1}} W_{r}\right)\right)\right) \\
& \equiv \operatorname{dom}\left(w_{f_{21}} \uplus\left\{l_{f_{1}} \mapsto\left(q_{f_{1}}, v_{f_{11}}\right)\right\}\right) \supseteq \operatorname{dom}\left(W_{2 *}\right)=\mathcal{S}_{2} \\
& \quad \text { which follows from above }\left(w_{f_{2}}=\ldots\right) \\
& \equiv \operatorname{dom}\left(w_{f_{21}} \uplus\left\{l_{f_{1}} \mapsto\left(q_{f_{1}}, v_{f_{2}}\right)\right\}\right) \supseteq \operatorname{dom}\left(W_{2 *}\right)=\mathcal{S}_{2} \\
& \quad \text { which follows from } \operatorname{dom}\left(w_{f_{21}} \uplus\left\{l_{f_{1}} \mapsto\left(q_{f_{1}}, v_{f_{11}}\right)\right\}\right)=\operatorname{dom}\left(w_{f_{21}} \uplus\left\{l_{f_{1}} \mapsto\left(q_{f_{1}}, v_{f_{2}}\right)\right\}\right) \\
& \equiv \operatorname{dom}\left(w_{f_{21}} \uplus\left\{l_{f_{1}} \mapsto\left(q_{f_{1}}, v_{f_{2}}\right)\right\}\right) \supseteq \operatorname{dom}\left(W_{*}\right)=\mathcal{S}_{2} \\
& \quad \text { which follows from above }\left(\operatorname{dom}\left(W_{*}\right)=\ldots\right) .
\end{aligned}
$$

It remains to show that

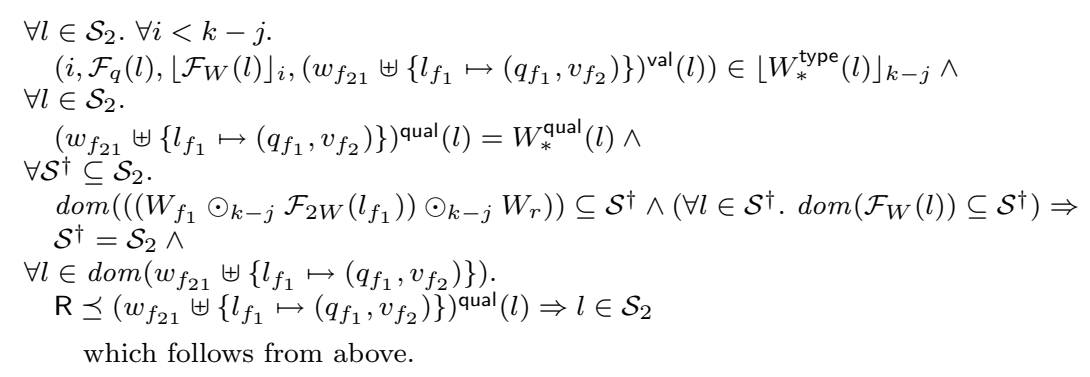

We are required to show that

- $\forall l \in \mathcal{S}_{2} . \forall i<k-j$.

$$
\left(i, \mathcal{F}_{q}(l),\left\lfloor\mathcal{F}_{W}(l)\right\rfloor_{i},\left(w_{f_{21}} \uplus\left\{l_{f_{1}} \mapsto\left(q_{f_{1}}, v_{f_{2}}\right)\right\}\right)^{\text {val }}(l)\right) \in\left\lfloor W_{*}^{\text {type }}(l)\right\rfloor_{k-j}
$$

Note that

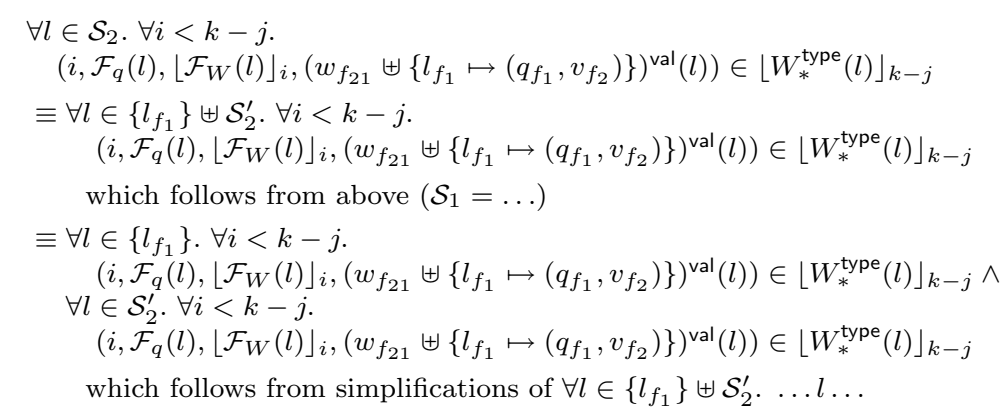




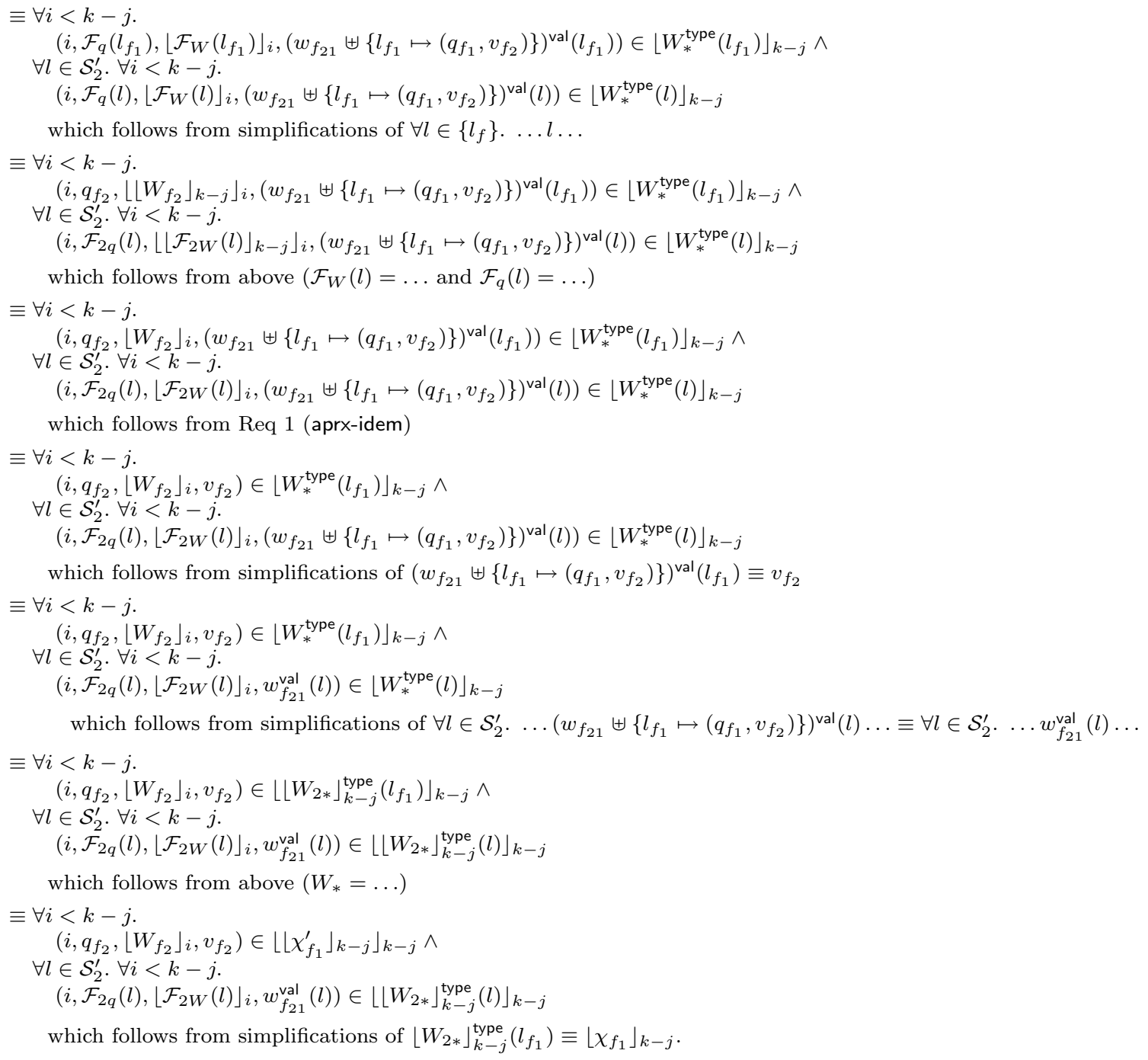

We are required to show that

- $\forall i<k-j$.

$\left(i, q_{f_{2}},\left\lfloor W_{f_{2}}\right\rfloor_{i}, v_{f_{2}}\right) \in\left\lfloor\left\lfloor\chi_{f_{1}}\right\rfloor_{k-j}\right\rfloor_{k-j}$ which follows from

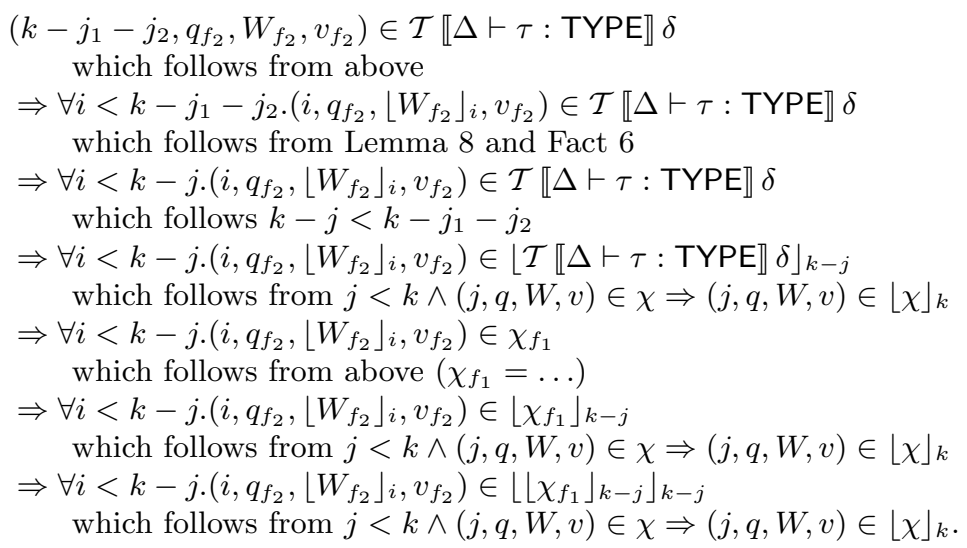


- $\forall l \in \mathcal{S}_{2}^{\prime} . \forall i<k-j$.

$\left(i, \mathcal{F}_{2 q}(l),\left\lfloor\mathcal{F}_{2 W}(l)\right\rfloor_{i}, w_{f_{21}}^{\text {val }}(l)\right) \in\left\lfloor\left\lfloor W_{2 *}\right\rfloor_{k-j}^{\text {type }}(l)\right\rfloor_{k-j}$

which follows from

$\forall l \in \mathcal{S}_{2} . \forall i<k-j_{1}-j_{2} .\left(i, \mathcal{F}_{2 q}(l),\left\lfloor\mathcal{F}_{2 W}(l)\right\rfloor_{i}, w_{f_{2}}^{\mathrm{val}}(l)\right) \in\left\lfloor W_{2 *}^{\mathrm{type}}(l)\right\rfloor_{k-j_{1}-j_{2}}$ which follows from above $\left(w_{f_{2}}: k-j_{1}-j_{2}\left(W_{f_{2}} \odot_{k-j_{1}-j_{2}}\left(W_{f_{1}} \odot_{k-j_{1}} W_{r}\right)\right)\right)$

$\Rightarrow \forall l \in \mathcal{S}_{2}^{\prime} . \forall i<k-j_{1}-j_{2} .\left(i, \mathcal{F}_{2 q}(l),\left\lfloor\mathcal{F}_{2 W}(l)\right\rfloor_{i}, w_{f_{2}}^{\text {val }}(l)\right) \in\left\lfloor W_{2 *}^{\text {type }}(l)\right\rfloor_{k-j_{1}-j_{2}}$ which follows from $\mathcal{S}_{2}^{\prime} \subseteq \mathcal{S}_{2}$

$\Rightarrow \forall l \in \mathcal{S}_{2}^{\prime} . \forall i<k-j .\left(i, \mathcal{F}_{2 q}(l),\left\lfloor\mathcal{F}_{2 W}(l)\right\rfloor_{i}, w_{f_{2}}^{\mathrm{val}}(l)\right) \in\left\lfloor W_{2 *}^{\mathrm{type}}(l)\right\rfloor_{k-j_{1}-j_{2}}$ which follows from $k-j<k-j_{1}-j_{2}$

$\equiv \forall l \in \mathcal{S}_{2}^{\prime} . \forall i<k-j .\left(i, \mathcal{F}_{2 q}(l),\left\lfloor\mathcal{F}_{2 W}(l)\right\rfloor_{i},\left(w_{f_{21}} \uplus\left\{l_{f_{1}} \mapsto\left(q_{f_{1}}, v_{f_{11}}\right)\right\}\right)^{\mathrm{val}}(l)\right) \in\left\lfloor W_{2 *}^{\text {type }}(l)\right\rfloor_{k-j_{1}-j_{2}}$ which follows from above $\left(w_{f_{2}}=\ldots\right)$

$\equiv \forall l \in \mathcal{S}_{2}^{\prime} . \forall i<k-j .\left(i, \mathcal{F}_{2 q}(l),\left\lfloor\mathcal{F}_{2 W}(l)\right\rfloor_{i}, w_{f_{21}}^{\mathrm{val}}(l)\right) \in\left\lfloor W_{2 *}^{\mathrm{type}}(l)\right\rfloor_{k-j_{1}-j_{2}}$ which follows from simplifications of

$\forall l \in \mathcal{S}_{2}^{\prime} \ldots\left(w_{f_{21}} \uplus\left\{l_{f_{1}} \mapsto\left(q_{f_{1}}, v_{f_{11}}\right)\right\}\right)^{\mathrm{val}}(l) \ldots \equiv \forall l \in \mathcal{S}_{2}^{\prime} \ldots w_{f_{21}}^{\mathrm{val}}(l) \ldots$

$\Rightarrow \forall l \in \mathcal{S}_{2}^{\prime} . \forall i<k-j .\left(i, \mathcal{F}_{2 q}(l),\left\lfloor\mathcal{F}_{2 W}(l)\right\rfloor_{i}, w_{f_{21}}^{\text {val }}(l)\right) \in\left\lfloor\left\lfloor W_{2 *}^{\text {type }}(l)\right\rfloor_{k-j_{1}-j_{2}}\right\rfloor_{k-j}$ which follows from $j<k \wedge(j, q, W, v) \in \chi \Rightarrow(j, q, W, v) \in\lfloor\chi\rfloor_{k}$

$\equiv \forall l \in \mathcal{S}_{2}^{\prime} . \forall i<k-j .\left(i, \mathcal{F}_{2 q}(l),\left\lfloor\mathcal{F}_{2 W}(l)\right\rfloor_{i}, w_{f_{21}}^{\text {val }}(l)\right) \in\left\lfloor W_{2 *}^{\text {type }}(l)\right\rfloor_{k-j}$ which follows from Fact 2.

- $\forall l \in \mathcal{S}_{2}$.

$\left(w_{f_{21}} \uplus\left\{l_{f_{1}} \mapsto\left(q_{f_{1}}, v_{f_{2}}\right)\right\}\right)^{\text {qual }}(l)=W_{*}^{\text {qual }}(l)$

which follows from

$\forall l \in \mathcal{S}_{2} .\left(w_{f_{21}} \uplus\left\{l_{f_{1}} \mapsto\left(q_{f_{1}}, v_{f_{11}}\right)\right\}\right)^{\text {qual }}(l)=W_{2 *}^{\text {qual }}(l)$

which follows from above $\left(w_{f_{2}}: k-j_{1}-j_{2}\left(W_{f_{2}} \odot_{k-j_{1}-j_{2}}\left(W_{f_{1}} \odot_{k-j_{1}} W_{r}\right)\right)\right)$

$\equiv \forall l \in \mathcal{S}_{2} .\left(w_{f_{21}} \uplus\left\{l_{f_{1}} \mapsto\left(q_{f_{1}}, v_{f_{2}}\right)\right\}\right)^{\text {qual }}(l)=W_{2 *}^{\text {qual }}(l)$

which follows from $\forall l \in \mathcal{S}_{2} .\left(w_{f_{21}} \uplus\left\{l_{f_{1}} \mapsto\left(q_{f_{1}}, v_{f_{11}}\right)\right\}\right)^{\text {qual }}(l)=\left(w_{f_{21}} \uplus\left\{l_{f_{1}} \mapsto\left(q_{f_{1}}, v_{f_{2}}\right)\right\}\right)^{\text {qual }}(l)$

$\equiv \forall l \in \mathcal{S}_{2} .\left(w_{f_{21}} \uplus\left\{l_{f_{1}} \mapsto\left(q_{f_{1}}, v_{f_{2}}\right)\right\}\right)^{\text {qual }}(l)=W_{*}^{\text {qual }}(l)$

which follows from $\forall l \in \mathcal{S}_{2} . W_{2 *}^{\text {qual }}(l)=\left\lfloor W_{2 *}\right\rfloor_{k-j}^{\text {qual }}(l)=W_{*}^{\text {qual }}(l)$.

- $\forall \mathcal{S}^{\dagger} \subseteq \mathcal{S}_{2}$.

$\operatorname{dom}\left(\left(\left(W_{f_{1}} \odot_{k-j} \mathcal{F}_{2 W}\left(l_{f_{1}}\right)\right) \odot_{k-j} W_{r}\right)\right) \subseteq \mathcal{S}^{\dagger} \wedge\left(\forall l \in \mathcal{S}^{\dagger} . \operatorname{dom}\left(\mathcal{F}_{W}(l)\right) \subseteq \mathcal{S}^{\dagger}\right) \Rightarrow$

$\mathcal{S}^{\dagger}=\mathcal{S}_{2}$

Consider arbitrary $\mathcal{S}^{\dagger}$ such that

- $\mathcal{S}^{\dagger} \subseteq \mathcal{S}_{2}$,

- $\operatorname{dom}\left(\left(\left(W_{f_{1}} \odot_{k-j} \mathcal{F}_{2 W}\left(l_{f_{1}}\right)\right) \odot_{k-j} W_{r}\right)\right) \subseteq \mathcal{S}^{\dagger}$, and

- $\forall l \in \mathcal{S}^{\dagger} . \operatorname{dom}\left(\mathcal{F}_{W}(l)\right) \subseteq \mathcal{S}^{\dagger}$.

Note that $\left\{l_{f_{1}}\right\} \cup \operatorname{dom}\left(\mathcal{F}_{2 W}\left(l_{f_{1}}\right)\right) \cup \operatorname{dom}\left(W_{r}\right) \subseteq \mathcal{S}^{\dagger}$, which follows from $\operatorname{dom}\left(\left(\left(W_{f_{1}} \odot_{k-j}\right.\right.\right.$ $\left.\left.\left.\mathcal{F}_{2 W}\left(l_{f_{1}}\right)\right) \odot_{k-j} W_{r}\right)\right) \subseteq \mathcal{S}^{\dagger}$ and $\operatorname{dom}\left(W_{f_{1}}\right)=\left\{l_{f_{1}}\right\}$,

Note that $l_{f_{1}} \in \mathcal{S}^{\dagger}$, which follows from $\left\{l_{f_{1}}\right\} \cup \operatorname{dom}\left(\mathcal{F}_{2 W}\left(l_{f_{1}}\right)\right) \cup \operatorname{dom}\left(W_{r}\right) \subseteq \mathcal{S}^{\dagger}$.

Note that $\operatorname{dom}\left(W_{f_{2}}\right) \in \mathcal{S}^{\dagger}$, which follows from

$$
\begin{aligned}
& \forall l \in \mathcal{S}^{\dagger} . \operatorname{dom}\left(\mathcal{F}_{W}(l)\right) \subseteq \mathcal{S}^{\dagger} \\
& \quad \text { which follows from above } \\
& \Rightarrow \operatorname{dom}\left(\mathcal{F}_{W}\left(l_{f_{1}}\right) \subseteq \subseteq \mathcal{S}^{\dagger}\right. \\
& \quad \text { which follows from } l_{f_{1}} \in \mathcal{S}^{\dagger} \\
& \equiv \operatorname{dom}\left(\left\lfloor W_{f_{2}}\right\rfloor_{k-j}\right) \subseteq \mathcal{S}^{\dagger} \\
& \quad \text { which follows from above }\left(\mathcal{F}_{W}(l)=\ldots\right) \\
& \equiv \operatorname{dom}\left(W_{f_{2}}\right) \subseteq \mathcal{S}^{\dagger} \\
& \quad \text { which follows from } \operatorname{dom}\left(\left\lfloor W_{f_{2}}\right\rfloor_{k-j}\right)=\operatorname{dom}\left(W_{f_{2}}\right) .
\end{aligned}
$$

Let $\mathcal{S}^{\ddagger}=\mathcal{S}^{\dagger} \backslash\left\{l_{f_{1}}\right\}$.

Note that $\mathcal{S}^{\dagger}=\left\{l_{f_{1}}\right\} \uplus \mathcal{S}_{2}^{\ddagger}$. 
Note that $\forall l \in \mathcal{S}_{2}^{\dagger}$. $\operatorname{dom}\left(\mathcal{F}_{2 W}(l)\right) \subseteq \mathcal{S}_{2}^{\dagger}$, which follows from

$$
\begin{aligned}
& \operatorname{dom}\left(\mathcal{F}_{2 W}\left(l_{f_{1}}\right)\right) \subseteq \mathcal{S}^{\dagger} \wedge \forall l \in \mathcal{S}^{\dagger} . \operatorname{dom}\left(\mathcal{F}_{W}(l)\right) \subseteq \mathcal{S}^{\dagger} \\
& \quad \text { which follows from above } \\
& \Rightarrow \operatorname{dom}\left(\mathcal{F}_{2 W}\left(l_{f_{1}}\right)\right) \subseteq \mathcal{S}^{\dagger} \wedge \forall l \in \mathcal{S}^{\ddagger} . \operatorname{dom}\left(\mathcal{F}_{W}(l)\right) \subseteq \mathcal{S}^{\dagger} \\
& \quad \text { which follows from } \mathcal{S}^{\ddagger} \subseteq \mathcal{S} \\
& \equiv \operatorname{dom}\left(\mathcal{F}_{2 W}\left(l_{f_{1}}\right)\right) \subseteq \mathcal{S}^{\dagger} \wedge \forall l \in \mathcal{S}^{\ddagger} . \operatorname{dom}\left(\left\lfloor\mathcal{F}_{2 W}(l)\right\rfloor_{k-j}\right) \subseteq \mathcal{S}^{\dagger} \\
& \quad \text { which follows from above }\left(\mathcal{F}_{W}(l)=\ldots\right) \\
& \equiv \operatorname{dom}\left(\mathcal{F}_{2 W}\left(l_{f_{1}}\right)\right) \subseteq \mathcal{S}^{\dagger} \wedge \forall l \in \mathcal{S}^{\ddagger} . \operatorname{dom}\left(\mathcal{F}_{2 W}(l)\right) \subseteq \mathcal{S}^{\dagger} \\
& \quad \text { which follows from } \operatorname{dom}\left(\left\lfloor\mathcal{F}_{2 W}(l)\right\rfloor k-j\right)=\operatorname{dom}\left(\mathcal{F}_{2 W}(l)\right) \\
& \equiv \forall l \in\left\{l_{f_{1}}\right\} \uplus \mathcal{S}^{\ddagger} \text {.dom }\left(\mathcal{F}_{2 W}(l)\right) \subseteq \mathcal{S}^{\dagger} \\
& \quad \text { which follows from simplifications of } \forall l \in\left\{l_{f_{1}}\right\} \uplus \mathcal{S}^{\ddagger} \ldots l \ldots \\
& \equiv \forall l \in \mathcal{S}^{\dagger} . \operatorname{dom}\left(\mathcal{F}_{2 W}(l)\right) \subseteq \mathcal{S}^{\dagger} \quad \text { which follows from above }\left(\mathcal{S}^{\dagger}=\ldots\right) .
\end{aligned}
$$

Instantiate $\left(\forall \mathcal{S}_{2}^{\dagger} \subseteq \mathcal{S}_{2} . \ldots\right)$ of $w_{f_{2}}: k-j_{1}-j_{2}\left(W_{f_{2}} \odot_{k-j_{1}-j_{2}}\left(W_{f_{1}} \odot_{k-j_{1}} W_{r}\right)\right)$ with $\mathcal{S}^{\dagger}$ Note that

- $\mathcal{S}^{\dagger} \subseteq \mathcal{S}_{2}$, which follows from above,

- $\operatorname{dom}\left(\left(W_{f_{2}} \odot_{k-j_{1}-j_{2}}\left(W_{f_{1}} \odot_{k-j_{1}} W_{r}\right)\right)\right) \subseteq \mathcal{S}_{2}^{\dagger}$, which follows from $\operatorname{dom}\left(W_{f_{2}}\right) \subseteq \mathcal{S}_{2}^{\dagger}$, which follows from above, and $\operatorname{dom}\left(W_{f_{1}}\right) \subseteq \mathcal{S}_{2}^{\dagger}$, which follows from above, and $\operatorname{dom}\left(W_{r}\right) \subseteq \mathcal{S}_{2}^{\dagger}$, which follows from above, and

- $\forall l \in \mathcal{S}_{2}^{\dagger} . \operatorname{dom}\left(\mathcal{F}_{2 W}(l)\right) \subseteq \mathcal{S}_{2}^{\dagger}$, which follows from above.

Hence, we conclude that $\mathcal{S}^{\dagger}=\mathcal{S}_{2}$.

- $\forall l \in \operatorname{dom}\left(w_{f_{21}} \uplus\left\{l_{f_{1}} \mapsto\left(q_{f_{1}}, v_{f_{2}}\right)\right\}\right)$.

$$
\mathrm{R} \preceq\left(w_{f_{21}} \uplus\left\{l_{f_{1}} \mapsto\left(q_{f_{1}}, v_{f_{2}}\right)\right\}\right)^{\text {qual }}(l) \Rightarrow l \in \mathcal{S}_{2}
$$

which follows from

$\forall l \in \operatorname{dom}\left(w_{f_{21}} \uplus\left\{l_{f_{1}} \mapsto\left(q_{f_{1}}, v_{f_{11}}\right)\right\}\right) . \mathrm{R} \preceq\left(w_{f_{21}} \uplus\left\{l_{f_{1}} \mapsto\left(q_{f_{1}}, v_{f_{11}}\right)\right\}\right)^{\text {qual }}(l) \Rightarrow l \in \mathcal{S}_{2}$

which follows from above $\left(w_{f_{2}}: k-j_{1}-j_{2}\left(W_{f_{2}} \odot_{k-j_{1}-j_{2}}\left(W_{f_{1}} \odot_{k-j_{1}} W_{r}\right)\right)\right)$

$\equiv \forall l \in \operatorname{dom}\left(w_{f_{21}} \uplus\left\{l_{f_{1}} \mapsto\left(q_{f_{1}}, v_{f_{2}}\right)\right\}\right) . \mathrm{R} \preceq\left(w_{f_{21}} \uplus\left\{l_{f_{1}} \mapsto\left(q_{f_{1}}, v_{f_{11}}\right)\right\}\right)^{\text {qual }}(l) \Rightarrow l \in \mathcal{S}_{2}$ which follows from $\operatorname{dom}\left(w_{f_{21}} \uplus\left\{l_{f_{1}} \mapsto\left(q_{f_{1}}, v_{f_{11}}\right)\right\}\right)=\operatorname{dom}\left(w_{f_{21}}\right) \uplus\left\{l_{f_{1}}\right\}$

$\equiv \forall l \in \operatorname{dom}\left(w_{f_{21}}\right) \uplus\left\{l_{f_{1}}\right\} . \mathrm{R} \preceq\left(w_{f_{21}} \uplus\left\{l_{f_{1}} \mapsto\left(q_{f_{1}}, v_{f_{2}}\right)\right\}\right)^{\text {qual }}(l) \Rightarrow l \in \mathcal{S}_{2}$

which follows from

$\forall l \in \operatorname{dom}\left(w_{f_{21}}\right) \uplus\left\{l_{f_{1}}\right\} .\left(w_{f_{21}} \uplus\left\{l_{f_{1}} \mapsto\left(q_{f_{1}}, v_{f_{11}}\right)\right\}\right)^{\text {qual }}(l)=\left(w_{f_{21}} \uplus\left\{l_{f_{1}} \mapsto\left(q_{f_{1}}, v_{f_{2}}\right)\right\}\right)^{\text {qual }}(l)$

$\equiv \forall l \in \operatorname{dom}\left(w_{f_{21}} \uplus\left\{l_{f_{1}} \mapsto\left(q_{f_{1}}, v_{f_{2}}\right)\right\}\right) . \mathrm{R} \preceq\left(w_{f_{21}} \uplus\left\{l_{f_{1}} \mapsto\left(q_{f_{1}}, v_{f_{2}}\right)\right\}\right)^{\text {qual }}(l) \Rightarrow l \in \mathcal{S}_{2}$ which follows from $\operatorname{dom}\left(w_{f_{21}}\right) \uplus\left\{l_{f_{1}}\right\}=\operatorname{dom}\left(w_{f_{21}} \uplus\left\{l_{f_{1}} \mapsto\left(q_{f_{1}}, v_{f_{2}}\right)\right\}\right)$.

- $\left(k-j, q_{f}, W_{f}, e_{f}\right) \in \mathcal{T} \llbracket \Delta \vdash^{\mathrm{L}}\left(\xi_{\text {ref }} \tau \otimes \tau\right):$ TYPE $\rrbracket \delta$

$\equiv\left(k-j, \mathrm{~L},\left(W_{f_{1}}^{\prime} \odot_{k-j} \mathcal{F}_{2 W}\left(l_{f_{1}}\right)\right),\left\langle l_{f_{1}}, v_{f_{11}}\right\rangle\right) \in \mathcal{T} \llbracket \Delta \vdash^{\mathrm{L}}\left(\xi_{\text {ref }} \tau \otimes \tau\right):$ TYPE $\rrbracket \delta$

$\equiv\left(k-j, \mathrm{~L},\left(W_{f_{1}}^{\prime} \odot_{k-j} \mathcal{F}_{2 W}\left(l_{f_{1}}\right)\right),\left\langle l_{f_{1}}, v_{f_{11}}\right\rangle\right)$

$\in\left\{\left(k, q, W,\left\langle v_{1}, v_{2}\right\rangle\right) \mid\right.$

$q=\mathcal{T} \llbracket \Delta \vdash \mathrm{L}: \mathrm{QUAL} \rrbracket \delta \wedge$

$\left(k, q_{1}, W_{1}, v_{1}\right) \in \mathcal{T} \llbracket \Delta \vdash \xi_{\text {ref }} \tau:$ TYPE $\rrbracket \delta \wedge$

$\left(k, q_{2}, W_{2}, v_{2}\right) \in \mathcal{T} \llbracket \Delta \vdash \tau: \mathrm{TYPE} \rrbracket \delta \wedge$

$q_{1} \preceq q \wedge q_{1} \preceq q \wedge$

$\left.\left(W_{1} \odot_{k} W_{2}=W\right)\right\}$,

which follows from

- $\mathrm{L}=\mathcal{T} \llbracket \Delta \vdash \mathrm{L}: \mathrm{QUAL} \rrbracket \delta$, which follows trivially,

- $\left(k-j, q_{f_{1}},\left\lfloor W_{f_{1}}\right\rfloor_{k-j}, l_{f_{1}}\right) \in \mathcal{T} \llbracket \Delta \vdash \xi_{\text {ref }} \tau:$ TYPE $\rrbracket \delta$, which follows from Lemma 8 and Fact 6 applied to $k-j \leq k-j_{1}$ and $\left(k-j_{1}, q_{f_{1}}, W_{f_{1}}, l_{f_{1}}\right) \in \mathcal{T} \llbracket \Delta \vdash \xi_{\text {ref }} \tau:$ TYPE $\rrbracket \delta$,

- $\left(k-j, \mathcal{F}_{2 q}\left(l_{f_{1}}\right),\left\lfloor\mathcal{F}_{2 W}\left(l_{f_{1}}\right)\right\rfloor_{k-j}, v_{f_{11}}\right) \in \mathcal{T} \llbracket \Delta \vdash \tau:$ TYPE $\rrbracket \delta$, which follows from above,

- $q_{f_{1}} \preceq \mathrm{L}$, which follows trivially,

- $\mathcal{F}_{2 q}\left(l_{f_{1}}\right) \preceq \mathrm{L}$, which follows trivially, 
- $\left(W_{f_{1}} \odot_{k-j} \mathcal{F}_{2 W}\left(l_{f_{1}}\right)\right)=\left(W_{f_{1}} \odot_{k-j}\left\lfloor\mathcal{F}_{2 W}\left(l_{f_{1}}\right)\right\rfloor_{k-j}\right)$, which follows from Req 5 (join-aprx).

End Case 


\section{C.7.3 Type Safety}

\section{Theorem 29 (Ref Extension Type Safety)}

If $\bullet ; \bullet e: \tau$ and $(\{\}, e) \longmapsto *\left(w^{\prime}, e^{\prime}\right)$,

then either $e^{\prime} \equiv v^{\prime}$ or $\exists w^{\prime \prime}, e^{\prime \prime} .\left(w^{\prime}, e^{\prime}\right) \longmapsto\left(w^{\prime \prime}, e^{\prime \prime}\right)$.

Proof

Let $\bullet ; \bullet e: \tau$ and $(\{\}, e) \longmapsto^{*}\left(w^{\prime}, e^{\prime}\right)$.

Note that $\forall k \geq 0 .\{\}: k\{\}$.

Applying Theorem 22 to $\bullet \bullet \vdash e: \tau$ and \{\}$:\{\}$ and $(\{\}, e) \longmapsto^{*}\left(w^{\prime}, e^{\prime}\right)$, we conclude that either $e^{\prime} \equiv v^{\prime}$ or $\exists w^{\prime \prime}, e^{\prime \prime} .\left(w^{\prime}, e^{\prime}\right) \longmapsto\left(w^{\prime \prime}, e^{\prime \prime}\right)$.

\section{Theorem 30 (Ref Extension Collection)}

If $\bullet \bullet \vdash e:{ }^{\mathrm{L}} \mathbf{1}_{\otimes}$ and $(\{\}, e) \longmapsto *\left(w_{f}, e_{f}\right)$ and $\operatorname{irred}\left(w_{f}, e_{f}\right)$,

then $\forall l \in \operatorname{dom}\left(w_{f}\right)$. $w_{f}^{\text {qual }}(l) \preceq \mathrm{A}$.

\section{Proof}

Let $\bullet ; \bullet e:{ }^{\mathrm{L}} \mathbf{1}_{\otimes}$ and $(\{\}, e) \longmapsto{ }^{*}\left(w_{f}, e_{f}\right)$ and $\operatorname{irred}\left(w_{f}, e_{f}\right)$.

Note that there exists $i$ such that $(\{\}, e) \longmapsto^{i}\left(w_{f}, e_{f}\right)$, which follows from $(\{\}, e) \longmapsto^{*}\left(w_{f}, e_{f}\right)$.

Applying Theorem 28 to $\bullet ; \bullet \cdot e:{ }^{\mathrm{L}} \mathbf{1}_{\otimes}$, we conclude that $\llbracket \bullet ; \bullet \vdash e:{ }^{\mathrm{L}} \mathbf{1}_{\otimes} \rrbracket$.

This is equivalent to

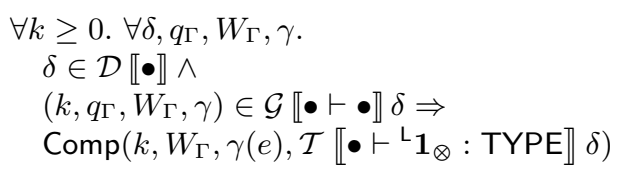

Instantiate this with $i+1, \emptyset, \mathbf{U},\{\}$, and $\emptyset$. Note that

- $i+1 \geq 0$

- $\emptyset \in \mathcal{D} \llbracket \bullet \rrbracket$, and

- $(i+1, \mathrm{U},\{\}, \emptyset) \in \mathcal{G} \llbracket \bullet \vdash \bullet \rrbracket$.

Hence, we conclude that $\operatorname{Comp}\left(i+1,\{\}, e, \mathcal{T} \llbracket \bullet \vdash^{\mathrm{L}} \mathbf{1}_{\otimes}: \operatorname{TYPE} \rrbracket \emptyset\right)$.

This is equivalent to

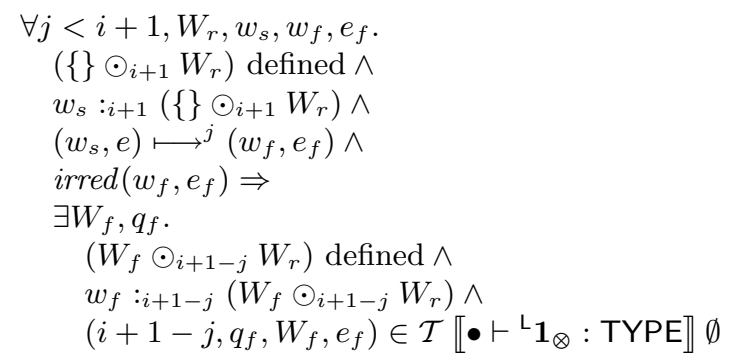

Instantiate this with $i,\{\},\{\}, w_{f}$, and $e_{f}$. Note that

- $i<i+1$,

- $\left(\{\} \odot_{i+1}\{\}\right) \equiv\{\}$ defined, 
- \{\}$:_{i+1}\left(\{\} \odot_{i+1}\{\}\right) \equiv\{\}:_{i+1}\{\}$,

- $(\{\}, e) \longmapsto^{i}\left(w_{f}, e_{f}\right)$, which follows from above, and

- $\operatorname{irred}\left(w_{f}, e_{f}\right)$, which follows from above.

Hence, we conclude that there exists $W_{f}$ and $q_{f}$ such that

- $\left(W_{f} \odot_{i+1-i}\{\}\right)$ defined,

- $w_{f}:_{i+1-i}\left(W_{f} \odot_{i+1-i}\{\}\right)$, and

- $\left(i+1-i, q_{f}, W_{f}, e_{f}\right)$

$\in \mathcal{T} \llbracket \bullet \vdash{ }^{\mathrm{L}} \mathbf{1}_{\otimes}:$ TYPE $\emptyset$

$\equiv\{(k, q, W,\langle\rangle) \mid$

$q=\mathcal{T} \llbracket \Delta \vdash \mathrm{L}: \mathrm{QUAL} \rrbracket \delta \wedge$

$W=\{\}\}$.

Hence, $e_{f} \equiv\langle\rangle$ and $q_{f}=\mathrm{L}$ and $W_{f}=\{\}$.

Note that

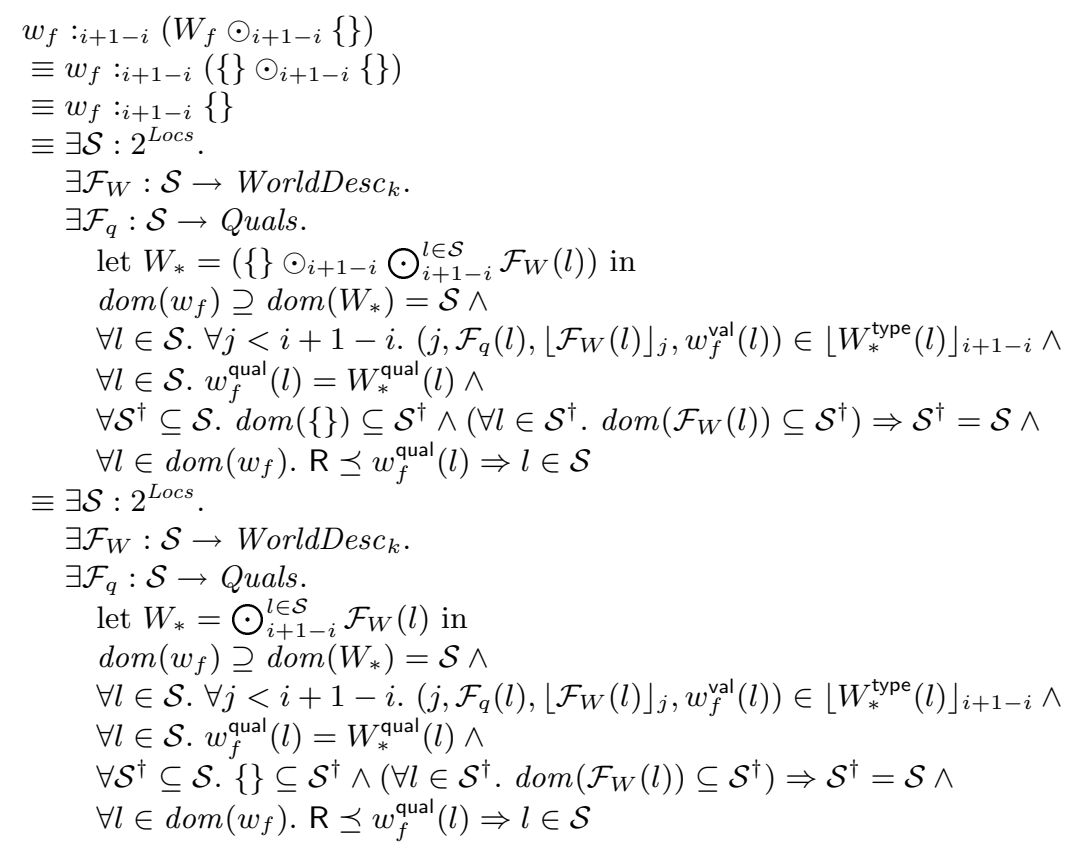

Hence, there exists $\mathcal{S}, \mathcal{F}_{W}$, and $\mathcal{F}_{q}$ such that

- $W_{*}=\bigodot_{i+1-i}^{l \in \mathcal{S}} \mathcal{F}_{W}(l)$ is defined,

- $\operatorname{dom}\left(w_{f}\right) \supseteq \operatorname{dom}\left(W_{*}\right)=\mathcal{S}$,

- $\forall l \in \mathcal{S} . \forall j<i+1-i .\left(j, \mathcal{F}_{q}(l),\left\lfloor\mathcal{F}_{W}(l)\right\rfloor_{j}, w_{f}^{\text {val }}(l)\right) \in\left\lfloor W_{*}^{\text {type }}(l)\right\rfloor_{i+1-i}$,

- $\forall l \in \mathcal{S} . w_{f}^{\text {qual }}(l)=W_{*}^{\text {qual }}(l)$,

- $\forall \mathcal{S}^{\dagger} \subseteq \mathcal{S} .\{\} \subseteq \mathcal{S}^{\dagger} \wedge\left(\forall l \in \mathcal{S}^{\dagger} . \operatorname{dom}\left(\mathcal{F}_{W}(l)\right) \subseteq \mathcal{S}^{\dagger}\right) \Rightarrow \mathcal{S}^{\dagger}=\mathcal{S}$, and

- $\forall l \in \operatorname{dom}\left(w_{f}\right) . \mathrm{R} \preceq w_{f}^{\text {qual }}(l) \Rightarrow l \in \mathcal{S}$. 
Instantiate $\left(\forall \mathcal{S}^{\dagger} \subseteq \mathcal{S}\right.$. ...) with \{\} . Note that

- \{\}$\subseteq \mathcal{S}$, which follows trivially,

- \{\}$\subseteq\{\}$, which follows trivially,

- $\forall l \in\{\} . \operatorname{dom}\left(\mathcal{F}_{1 W}(l)\right) \subseteq\{\}$, which follows trivially.

Hence, we conclude that \{\}$=\mathcal{S}$.

Hence, $\forall l \in \operatorname{dom}\left(w_{f}\right) . \mathrm{R} \preceq w_{f}^{\text {qual }}(l) \Rightarrow l \in\{\}$.

Consider arbitrary $l \in \operatorname{dom}\left(w_{f}\right)$.

We are required to show that $w_{f}^{\text {qual }}(l) \preceq \mathrm{A}$.

Suppose, by way of contradiction, that $\neg\left(w_{f}^{\text {qual }}(l) \preceq \mathrm{A}\right)$.

Hence, $\mathrm{R} \preceq w_{f}^{\text {qual }}(l)$.

Instantiate $\left(\forall l \in \operatorname{dom}\left(w_{f}\right) . \mathrm{R} \preceq w_{f}^{\text {qual }}(l) \Rightarrow l \in\{\}\right)$ with $l$, noting that $\mathrm{R} \preceq w_{f}^{\text {qual }}(l)$.

Hence, we conclude that $l \in\{\} . \Rightarrow \Leftarrow$.

Hence, $w_{f}^{\text {qual }}(l) \preceq \mathrm{A}$. 